\title{
Aeolian iron and its contribution to phytoplankton production in McMurdo Sound, southwest Ross Sea, Antarctica
}

\section{Victoria Holly Liberty Winton}

\author{
A thesis \\ submitted to Victoria University of Wellington \\ in partial fulfilment of the requirements for the degree of \\ Master of Science in Geology
}

Victoria University of Wellington

2012 



\begin{abstract}
Each summer the waters in McMurdo Sound (Lat. $77.5^{\circ} \mathrm{S}$; Long. $165^{\circ} \mathrm{E}$ ), south-western (SW) Ross Sea encounter vast phytoplankton blooms. This phenomenon is stimulated by the addition of bio-available iron $(\mathrm{Fe})$ to an environment where phytoplankton growth is otherwise Fe-limited. One possible source of such Fe is aeolian sand and dust (ASD) which accumulates on sea ice and is released into the ocean during the summer melt season.
\end{abstract}

The amount of bio-available Fe (i.e. the amount of Fe immedately accessible to phytoplankton) potentially supplied to the ocean by ASD depends on a number of factors including; the ASD flux into the ocean, its particle size distribution and Fe content. However, none of these parameters are well constrained in the SW Ross Sea region and, as a result, the significance of this Fe source in the biogeochemical cycle of phytoplankton growth remains to be quantified.

This study focuses on an area $\left(7400 \mathrm{~km}^{2}\right)$ of Southern McMurdo Sound, one of the few areas where direct sampling of ASD that has accumulated on sea ice is possible. To evaluate the flux and solubility of Fe contained in ASD into McMurdo Sound, the mass accumulation rate and particle size of 70 surface snow samples and 3 shallow $(3 \mathrm{~m})$ firn cores from the nearby McMurdo Ice Shelf covering the period 2000 - 2008 have been analysed. Selected samples were also measured for total and soluble $\mathrm{Fe}, \mathrm{Sr}$ and $\mathrm{Nd}$ isotopic ratios and mineralogy as a guide to Fe-fertilisation potential and provenance, respectively.

Mass and particle size data show an exponential decrease in mass accumulation rate (from $26.00 \mathrm{~g} \mathrm{~m}^{-2} \mathrm{yr}^{-1}$ to $0.70 \mathrm{~g} \mathrm{~m}^{-2} \mathrm{yr}^{-1}$ ) and a decrease in modal particle size (from 130 to $69 \mu \mathrm{m}$ ) over a distance of $120 \mathrm{~km}$ from Southern McMurdo Sound northwards to Granite Harbour. Both these trends are consistent with ASD being dispersed northwards across the sea ice by southerly storms from an area of the McMurdo Ice Shelf, where submarine freezing and surface ablation have resulted in a surface covered with debris from the sea floor, known as the 'dirty ice' or 'debris bands' (Lat. $77.929^{\circ} \mathrm{S}$; Long. $165.505^{\circ} \mathrm{E}$ ) in Southern McMurdo Sound. This assertion is further supported by the $\mathrm{Sr}$ and $\mathrm{Nd}$ isotopic signature of ASD 
matching local source rocks and the presence of vesicular glass of Southern McMurdo Sound in all samples which also points to the debris bands as the origin of ASD in McMurdo Sound.

Bio-available $\mathrm{Fe}$ is extremely difficult to quantify hence Fe solubility was used as an approximation in this thesis. Analysis of both total (i.e. particulate and soluble) and the percentage of soluble $\mathrm{Fe}$ in the $0.4-10 \mu \mathrm{m}$ dust size fraction (i.e. the fraction most likely to become bio-available) by solution ICP-MS shows a narrow range of values; $3.84 \pm 1.99 \mathrm{wt} \%$ and $9.42 \pm 0.70 \%$ respectively. Combining these values with mass accumulation rate estimates for the particles $0.4-10 \mu \mathrm{m}$ in size, gives an annual soluble Fe flux for the region $500 \mathrm{~km}^{2}$ north of the debris bands in McMurdo Sound of $0.55 \mathrm{mg} \mathrm{m}^{-2} \mathrm{yr}^{-1}\left(9.89 \mu \mathrm{mol} \mathrm{m}{ }^{-2}\right.$ $\mathrm{yr}^{-1}$ ), with spatial variability largely determined by differences in mass accumulation rate.

These fluxes are at least an order of magnitude greater than predicted in global dust deposition models for the Southern Ocean and measured in snow samples from East Antarctica. Furthermore, these values exceed the Fe threshold, estimated as $0.2 \mathrm{nM}$ (Boyd and Abraham, 2001), required for phytoplankton growth following the simple dust-biota model of Boyd et al. (2010) and assuming the release of captured ASD in snow is instantaneous. Whilst not constrained in the present study, ASD sourced from the debris bands may be sufficiently widely dispersed, particularly during storm years, to contribute to Fe-fertilisation up to $1200 \mathrm{~km}$ from Southern McMurdo Sound.

Short, $\sim 10$ year long, firn core records of mass accumulation and methylsuphonate concentration, a proxy for phytoplankton productivity, shows a close correspondence between the two during particularly stormy years. Whilst not demonstrating a cause-andeffect relationship, this observation suggests coastal ice cores may contain an important record of the interplay between climate, dust supply, Fe-fertilisation of near shore waters and phytoplankton productivity on decadal and longer timescales. 


\section{Contents page}

Abstract I

Table of contents

List of Figures $\quad$ VI

List of Tables $\quad$ X

List of abbreviations $\quad$ XI

$\begin{array}{lll}\text { Acknowledgements } & \text { XIII }\end{array}$

Chapter 1: Introduction $\quad 1$

1.1. Aeolian sediment and its biogeochemical importance in the SW Ross Sea 1

1.2. Research aims and objectives 3

1.3. Layout of this thesis 5

1.4. Analyses conducted in this thesis $\quad 6$

Chapter 2: The sedimentology of aeolian sand and dust in McMurdo Sound $\quad 7$

2.1. Sedimentology background

2.1.1. Introduction 7

2.1.2. Regional setting 9

2.1.3. Transport of sediment by wind 24

2.1.4. The deposition of aeolian sand and dust in Antarctica and the Southern
Ocean

2.1.5. The origin of aeolian dust in Antarctica 33

2.2. Spatial distribution of aeolian sand and dust accumulation in McMurdo Sound

2.2.1. Methodology 37

2.2.2. Results - spatial trends in aeolian sand and dust accumulation rate and texture 48

2.3. Temporal distribution of aeolian sand and dust in Windless Bight from firn cores

2.3.1. Methodology 61

2.3.2. Results - temporal trends in aeolian sand and dust accumulation rate and texture 66

2.4. Discussion - sedimentology and provenance of aeolian sand and dust

2.4.1. Annual aeolian sand and dust accumulation rate patterns in McMurdo Sound 77

2.4.2. Evidence for a single dominant source of aeolian sand and dust 77

2.4.3. Processes of aeolian sand and dust transport and particle size trends 84

2.4.4. Contribution of aeolian sand and dust to seafloor accumulation rate 89

2.4.5. Aeolian sand and dust transport during 2004 storm event 91 
3.1. Iron in the Ross Sea, Southern Ocean

3.1.1. Introduction $\quad 97$

3.1.2. Sources of iron in the Southern Ocean 100

3.1.3. Atmospheric iron and phytoplankton productivity 106

3.2. Development of a method for leaching iron from aeolian dust contained in snow and ice samples

3.2.1. Introduction 118

3.2.2. Methods, materials and dust leaching tests 119

$\begin{array}{ll}\text { 3.2.3. Results - Arizona test dust leaching tests } & 127\end{array}$

3.2.4. Discussion 134

3.2.5. Method arising from Arizona test dust leaching tests 137

3.2.6. Conclusions 139

3.3. Methodology - particulate and soluble iron methodology for Antarctic aeolian sand and dust

3.3.1. Samples 140

3.3.2. Soluble iron 141

3.3.3. Particulate iron 143

3.4. Results - particulate and soluble aeolian iron in McMurdo Sound

3.4.1. Particulate iron concentration in lithogenic particles 148

3.4.2. Soluble iron 149

3.4.3. The percentage of soluble iron 151

3.5. Discussion - particulate and soluble iron

3.5.1. Particulate iron concentration in lithogenic particles 155

3.5.2. Soluble iron 156

3.5.3. Percentage of soluble iron 158

$\begin{array}{ll}\text { 3.6. Conclusions } & 167\end{array}$

Chapter 4: Synthesis: The role of local dust-derived iron on setting the primary production of McMurdo Sound $\quad 168$

4.1. Introduction 168

4.2. Background 168

4.3. The role of aeolian sand and dust as a source of iron to the SW Ross Sea 175

4.4. Is there enough aeolian iron in McMurdo Sound to stimulate primary productivity? 184

4.5. Connections between aeolian sand and dust, chlorophyll- $a$ and methylsuphonate $\quad 190$

$\begin{array}{ll}\text { 4.6. Conclusions } & 200\end{array}$ 
5.1. Conclusions 201

5.2. Suggestions for future work 205

$\begin{array}{lr}\text { References } & 206\end{array}$

Appendices

A1: Surface snow aeolian sand and dust mass data 229

A2: Coulter-Counter Multi-sizer 3 methodology 233

A3: Particle size data for surface snow and firn core samples 235

A4: Downcore profiles in dust mass and stable isotopes at Windless Bight 252

A5: Published Fe fluxes in Antarctic snow, sea ice and dust 263

A6: Published fluxes for other Fe sources to the Southern Ocean 265

A7: Iron geochemistry data of McMurdo Sound dust 266

A8: Time series of dust accumulation, methylsuphonate and chlorophyll- $a$ data for SW

Ross Sea 2000-2008 


\section{List of Figures}

\section{Chapter 2}

Fig. 2.1: Map of Antarctica and McMurdo Sound with snow accumulation rates and dominant wind direction.

Fig. 2.2: Geological map of Southern McMurdo Sound.

Fig. 2.3: Aeolian sediment on sea ice in western and Southern McMurdo Sound during the summer.

Fig. 2.4: Wind roses illustrating the dominant southerly wind direction for the strongest storms in McMurdo Sound.

Fig. 2.5: Mean annual temperature and inter-annual variability from Scott Base AWS.

Fig. 2.6: Schematic of the timing of annual sea ice breakout, temperature and aeolian sand and dust accumulation on the surface snow of the sea ice in McMurdo Sound.

Fig. 2.7: Sea ice break up sequence in McMurdo Sound 2009-2010.

Fig. 2.8: Sea ice extent in McMurdo Sound during sampling in November 2009.

Fig. 2.9: Modes of transport of quartz spheres at different wind shear velocities. Source: Toscar and Pye (1987).

Fig. 2.10: Modes of particle transport by wind. Source: Pye (1989).

Fig. 2.11: Global dust deposition estimated from reanalysis models. Source: Mahowald (2005).

Fig. 2.12: Dust mass accumulation rates at various sites in Antarctica.

Fig. 2.13: Aeolian sand and dust trapped in surface snow Southern McMurdo Sound sea ice, November 2009.

Fig. 2.14: $\mathrm{Sr}$ and $\mathrm{Nd}$ isotopic composition of worldwide aerosols, loess and sand deposits. Source: Grousset and Biscaye (2005).

Fig. 2.15: Isotopic signature of non-glaciated areas in McMurdo Sound. Source: Delmonte (2003).

Fig. 2.16: LIMA satellite image of McMurdo Sound showing the location of samples in the present study.

Fig. 2.17: Snow sampling procedure for bag samples, Southern McMurdo Sound, November 2009.

Fig. 2.18: Millipore filtration apparatus.

Fig. 2.19: Siphoning dusty samples in the clean room, National Ice Core Facility, GNS Science.

Fig. 2.20: Reproducibility tests of glass bead particle size.

Fig. 2.21: WB1_25 example of the reproducibility from diluting concentrated samples for particle size analysis.

Fig. 2.22: Location of samples for sand and dust provenance analysis using $\mathrm{Sr}$ and $\mathrm{Nd}$ isotopic ratios.

Fig. 2.23: Aeolian sand and dust accumulation rates and dispersion model for McMurdo Sound.

Fig. 2.24: SEM images of calcite particles from MSI6 found in surface snow at Cape Evans. 
Fig. 2.25: EDS counts of elements on dust from Cape Evans.

Fig. 2.26: Same as Fig. 2.23 but for the fraction of dust $<10 \mu \mathrm{m}$.

Fig. 2.27: Ternary plots of dust from surface snow samples showing the proportion of sand, silt and clay.

Fig. 2.28: Percentage frequency curves of particle size distribution in surface snow on McMurdo Sound sea ice in 2009.

Fig. 2.29: North-south transect $X Y$ of particle size trends in the percentage of sand, the sand mode and the percentage of dust $<10 \mu \mathrm{m}$.

Fig. 2.30: Granite Harbour west-east transect $Y^{\prime}$ ' of particle size trends in the percentage of sand, the primary mode and the percentage of dust $<10 \mu \mathrm{m}$.

Fig. 2.31: Southern McMurdo Sound transects ZZ' and WW' of particle size trends in the percentage of sand, the primary mode and the percentage of dust $<10 \mu \mathrm{m}$.

Fig. 2.32: Isotopic composition of samples in the present study compared with PSA samples from the literature and the location of PSA samples in the Victoria Land coastline with proposed dust transport path in McMurdo Sound.

Fig. 2.33: Ice core sub-sampling. Source: Schuck (2009).

Fig. 2.34: Relationship between $\delta^{18} \mathrm{O}$ and $\delta \mathrm{D}$ for WB1 comparing results from Laser Absorption Spectroscopy verse Mass Spectrometry.

Fig. 2.35: Age model of Windless Bight firn cores showing downcore profiles of $\delta^{18} \mathrm{O}$ and dust concentration.

Fig. 2.36: Downcore sediment record in Windless Bights firn cores.

Fig. 2.37: Extending the dust record of McMurdo Sound: comparison of WB1 with WB06/07.

Fig. 2.38: Particle size distribution in firn cores along transect downwind from Black Island, McMurdo Ice Shelf.

Fig. 2.39: Ternary plots of dust of firn cores showing the proportion of sand, silt and clay.

Fig. 2.40: Size distribution of Antarctic background dust verse McMurdo Sound sand and dust.

Fig. 2.41: Sand and dust mineral composition at Granite Harbour.

Fig. 2.42: Mixing line between two end member sand and dust sources in McMurdo Sound.

Fig. 2.43: Schematic of aeolian sand and dust dispersal in McMurdo Sound.

Fig. 2.44: Sediment distribution and contribution to the ocean each summer.

Fig. 2.45: Back and forward air mass trajectories for the 24 hour period 15 May 1800 UTC and 0000 UTC 16 May imitated at $500 \mathrm{~m}$ above sea level, McMurdo Sound.

Fig. 2.46: Surface winds for the May 2004 and chlorophyll- $a$ concentration in the SW Ross Sea. 


\section{Chapter 3}

Fig. 3.1: Schematic illustrating Fe supply, cycling and the 'biological carbon pump' in Antarctic waters.

Fig. 3.2: Comparison of marine and terrestrial climate records over the last 1.1 Ma. Source: Martinez-Garcia et al. (2009).

Fig. 3.3: The percentage of soluble $\mathrm{Fe}$ in aerosols as a function of mineral aerosol atmospheric mass loading. Source: Baker et al. (2006a).

Fig. 3.4: The variation in surface area to volume ratio of a simple spherical particle with atmospheric concentration. Source: Baker et al. (2006a).

Fig. 3.5: Processing and fate of aeolian $\mathrm{Fe}$ in McMurdo Sound.

Fig. 3.6: Simple model of steady state aerosol Fe fractional solubility under oceanic control of Fe dissolution. Source: Baker and Croot (2010).

Fig. 3.7: Particle size distribution for ultra-fine and medium particle-sized Arizona test dust.

Fig. 3.8: Flow-through reactor apparatus for aerosol Fe solubility measurements.

Fig. 3.9: Repeat measurement of Fe concentration 10 days after initial measurements.

Fig. 3.10: Petrographic analysis of particle size of Arizona test dust on $10 \mu \mathrm{m}$ and $0.4 \mu \mathrm{m}$ filter membranes.

Fig. 3.11: The percentage of soluble $\mathrm{Fe}$ of $10 \mathrm{mg}$ aliquots of ultra-fine particle-sized Arizona test dust leached each in $180 \mathrm{ml}$ of MQ water at various time intervals.

Fig. 3.12: Arizona test dust Fe solubility verse dust recovery and days between leaching and ICP-MS measurement.

Fig. 3.13: Dust load verse Arizona test dust the percentage of soluble Fe leaching for 20 minutes in $180 \mathrm{ml}$ of MQ water.

Fig. 3.14: The percentage of soluble $\mathrm{Fe}$ of Arizona test dust determined for repeat experiments of successive aliquots of MQ water.

Fig. 3.15: Location of samples taken for Fe solubility and total Fe analyses.

Fig. 3.16: Particulate Fe analysis of fine particle-sized Arizona test dust filters and JB2 standard compared to the known Fe concentration for that sample.

Fig. 3.17: Transect XY of total digested Fe of McMurdo Sound samples.

Fig. 3.18: Fe concentration leached from McMurdo Sound dust samples along transect XY.

Fig. 3.19: Percent of instantaneous soluble Fe (particles $<0.4 \mu \mathrm{m}$ ) as a function of percent of dissolved Fe solubility for samples from McMurdo Sound, and a 1:1 line.

Fig. 3.20: Cumulative percent Fe solubility for successive leaches.

Fig. 3.21: The percentage of cumulative Fe solubility for Arizona test dust leaching at various time intervals.

Fig. 3.22: Cumulative percentage of Fe solubility in McMurdo Sound dust samples.

Fig. 3.23: Percentage of soluble Fe in snow melt (leaching the bulk sample).

Fig. 3.24: Sand and dust load versus soluble Fe concentration leached from McMurdo Sound dust samples.

Fig. 3.25: Physical and chemical properties of McMurdo Sound sand and dust from the debris band source to $300 \mathrm{~km}$ into SW Ross Sea.

Fig. 3.26: Particulate $\mathrm{Ca}$ concentrations in McMurdo Sound dust samples. 


\section{Chapter 4}

Fig. 4.1: Macronutrients in the surface waters of the global ocean.

Fig. 4.2: Seasonal evolution of phytoplankton productivity bloom in the SW Ross Sea November 2008 - March 2009.

Fig. 4.3: Satellite image showing katabatic winds from major outlet glaciers across the Ross Ice Shelf.

Fig. 4.4: Location of phytoplankton blooms and sea-ice in the SW Ross Sea.

Fig. 4.5: Area defined as the "broader McMurdo Sound region."

Fig. 4.6: Aeolian Fe flux in McMurdo Sound.

Fig. 4.7: Aeolian Fe dispersal $500 \mathrm{~km}$ from McMurdo Sound into the Ross Sea.

Fig. 4.8: Present day atmospheric Fe fluxes in Antarctica compared to local Fe derived from aeolian ASD in McMurdo Sound for the SW Ross Sea.

Fig. 4.9: The biological response of aeolian Fe to McMurdo Sound for background years during a severe storm event in 2004.

Fig. 4.10: SW Ross Sea polynya and ice cover during December 2004 and phytoplankton bloom on the surface of the ocean.

Fig. 4.11: Back trajectory analysis and cluster means for Gawn Ice Piedmont Glacier. Source: Markle (2011).

Fig. 4.12: Relationship between sea ice location and primary productivity in the SW Ross Sea.

Fig. 4.13: Chlorophyll- $a$, dust flux and MS concentration seasonal records for the SW Ross Sea. 


\section{List of Tables}

\section{Chapter 2}

Table 2.1: Aeolian sand and dust accumulation rates at various sites in Antarctica.

Table 2.2: $\mathrm{Sr}$ and $\mathrm{Nd}$ isotope geochemistry of McMurdo Sound sand and dust.

Table 2.3: Threshold velocities of McMurdo Sound sand and dust.

\section{Chapter 3}

Table 3.1: Annual Fe sources for the Southern Ocean south of $60^{\circ} \mathrm{S}$.

Table 3.2: Summary of leaching schemes employed in published research accounts.

Table 3.3: Triplicate analysis of major ion analysis of ultra-fine and medium Arizona test dust.

Table 3.4: Values of controlled parameters for Arizona test dust leaching experiments.

Table 3.5: Reproducibility of Arizona test dust blank leaching experiments.

Table 3.6: Inductively coupled plasma mass spectrometry instrumental error: accuracy and precision of iron standards.

Table 3.7: McMurdo Sound sand dust samples, collected using ultra clean methodology, used in dust leaching analyses.

Table 3.8: Savilex beaker acid washing procedure for total Fe digestion.

Table 3.9: Precision of the acid digestion method for measuring total $\mathrm{Fe}$ in Arizona test dust.

Table 3.10: Element concentrations determined by repeated measurement of JB2 rock standard with precision and accuracy of measurements.

Table 3.11: Detection limit of blank filter papers.

\section{Chapter 4}

Table 4.1: Present day atmospheric Fe fluxes in Antarctica.

Table 4.2: Fe budget for sea ice in the Ross Sea. Modified from: Lannuzel et al. (2007).

Table 4.3: Fe budget for Antarctic waters including a local dust source. Modified from: Measures and Vink (2001).

Table 4.4: Parameters used in the Boyd et al. (2010) dust-biota model. 


\section{List of abbreviations}

$\delta^{18} \mathrm{O}$

$\delta \mathrm{D}$

ALM

AMP

AR

ASD

ATD

AWS

DVDP

EAIS

EPICA

CHUR

$d$ excess

$\mathrm{dFe}$

EDX

EDS

$\mathrm{GH}$

GNS

HNLC

GIS

LIG

LIMA

LGR

LGM

LLS

LDPE

MAF

MIS

MDV

MLM

MS3

MQ oxygen isotope ratio

deuterium isotope ratio

Aqueous Liquid Module

Antarctic mescoscale prediction systems

analytical reagent

Aeolian sand and dust

Arizona test dust

Automatic Weather Station

Dry Valley Drilling Project

East Antarctic Ice Shelf

European Project for Ice Coring in Antarctica

chondritic uniform reservoir

deuterium excess

dissolved Fe

energy dispersive $\mathrm{x}$-ray spectrometry

energy dispersive spectrum

Granite Harbour

Geological and Nuclear Sciences

high nutrient low chlorophyll

Geographic Information Systems

Laboratory for Isotope Geology

Landsat Image Mosaic of Antarctica

Los Gatos Research

Last Glacial Maximum

laser light scattering

Low density polyethylene

Ministry of Agriculture and Forestry

marine isotope stage

McMurdo Dry Valleys

Micro Liquid Module

Multi-Sizer 3

Milli-Q 


\begin{tabular}{|c|c|}
\hline MVG & McMurdo Volcanic Group \\
\hline NGRIP & North Greenland Ice Core Drilling Project \\
\hline NIST & National Institute of Standards and Technology \\
\hline NBS & National Bureau of Standards \\
\hline $\mathrm{nM}$ & nanomolar \\
\hline PSA & potential source area \\
\hline $\mathrm{ppb}$ & parts per billion \\
\hline ppm & parts per million \\
\hline ppt & parts per trillion \\
\hline $\mathrm{R}^{2}$ & coefficient of determination \\
\hline SB & sub boiled \\
\hline $\mathrm{Sd}$ & standard deviation \\
\hline $\mathrm{SE}$ & standard error \\
\hline SeaWiFS & Sea-viewing Wide Field-of-view Sensor \\
\hline SEM & scanning electron microscope \\
\hline SMS & Southern McMurdo Sound \\
\hline SO & Southern Ocean \\
\hline SS & SeaStar \\
\hline SSIZ & seasonal sea ice zone \\
\hline SW & South-western \\
\hline TAM & Transantarctic Mountains \\
\hline TIMS & thermal ionisation mass spectrometer \\
\hline VLG & Victoria Lower Glacier \\
\hline VSMOW & Vienna Mean Standard Ocean Water \\
\hline $\mathrm{Wt}$ & weight \\
\hline$V^{*}{ }_{t}$ & threshold velocity for particle diameter \\
\hline $\mathrm{v}_{\mathrm{t}}$ & fluid threshold velocity \\
\hline WAIS & West Antarctic Ice Shelf \\
\hline WB & Windless Bight \\
\hline WRF & Weather and Research Forecasting \\
\hline VUW & Victoria University of Wellington \\
\hline XRD & $\mathrm{x}$-ray difractometer \\
\hline XRF & $\mathrm{x}$-ray fluorescence \\
\hline
\end{tabular}




\section{Acknowledgments}

Firstly and most importantly I'd like to thank my two supervisors Dr Nancy Bertler and Dr Gavin Dunbar for your support, time, encouragement, enthusiasm and open door policy for this project. Thank you Nancy for giving me the opportunity to carry out this project and for your passion and praise throughout the past two and a half years. Thank you Gavin for your interpretation with data, valuable suggestions regarding further work and your help improving my writing and editing skills. I would also like to thank Dr Marc-Alban Millet for your continued support with developing laboratory and analytical procedures not previously carried out at Victoria University of Wellington, for your willingness to help with data processing and interpretation, even on weekends, and for encouraging me to work efficiently.

Thank you to Dr Barbara Delmonte for inviting me to, and hosting me at the University of Milano-Bicocca, Italy to develop a modified method for Coulter-Counter MS3 analysis of coastal Antarctic dust, and for your continued support regarding dust provenance and particle size. Thank you to Dr Ana Aguilar-Islas for the use of your through flow reactor apparatus for dust leaching experiments, your advice on soluble iron method development and for collecting an additional 10 snow samples from Granite Harbour, McMurdo Sound. I would also like to thank Dr Cliff Atkins for supplying me with an additional 7 snow samples from the area between Marble Point and Granite Harbour, and for sedimentological discussions regarding aeolian dust in McMurdo Sound.

Thank you to Antarctica New Zealand for fieldwork and logistical support and to Dr Tim Haskell, Dr Pat Langhorn and the K131 team for hosting me in your event at Camp Haskell on the sea ice in November 2009.

Thank you to Dr Dan Zwartz for helping create a simple model for dust dispersion in McMurdo Sound; to Andy Phillips and Cedric Douence for liquid isotope analyser and stable isotope mass spectrometer training at GNS Science; to Dr Margaret Harper for helping me identify diatom species; to Dr Rob McKay for supplying me with a matlab script to construct wind roses; to Rebecca Pyne for laboratory assistance during her summer research scholarship; and to Professor Joel Baker and Dr Monica Handler for assisting me with ICPMS analysis. 
Thank you to Dr Kate Sinclair for your continued support and friendship in the Ice Core Lab and to my fellow students Brad Markle, Dr Rachel Rhodes, Lana Cohen and the rest of the Antarctic Research Centre team.

A final thank you to New Zealand Post and Antarctica New Zealand for making this project possible and to the following organisations for scholarships: Victoria University of Wellington (JL and Kathleen Stewart Postgraduate Research Experience Travel Award; Kathleen Stewart Scholarship; Antarctic Research Endowed Development Fund) and Golden Key (Asia-Pacific Travel Award).

This project was funded by Victoria University of Wellington, GNS Science, the Ministry for Science and Innovation (contracts ANZICE, VUW: VICX0704, and GCT, GNS Science: CO5X0902). Isotopic analyses for provenance characterisation were carried out at the Swedish Museum of Natural History and were supported by SYNTHESYS funding (project SE-TAF-212) made available by the European Community - Research Infrastructure Action under the FP6 "Structuring the European Research Area" Programme. 


\section{Chapter 1}

\section{Introduction}

\section{Note on terminology}

Aeolian or windblown dust is the $<63 \mu \mathrm{m}$ fraction of sediment transported by wind, and sand is the $63-2000 \mu \mathrm{m}$ fraction (Bagnold, 1941). Sediment analysed in this thesis includes both these particle size fractions and hence the term aeolian sand and dust (ASD) is used. Aerosols include both fine solid particles (lithogenic or non-lithogenic) and liquid droplets suspended in the atmosphere.

\subsection{Aeolian sediment and its biogeochemical importance in the SW Ross Sea}

Atmospheric deposition of mineral dust is a major source of micro-nutrient iron (Fe) to open ocean surface waters and is thought to play an important role in regulating marine phytoplankton and thus atmospheric $\mathrm{CO}_{2}$ concentration (Martin, 1990; Donghay et al., 1991; Duce and Tindale, 1991; Broecker and Henderson, 1998; Lefèvre and Watson, 1999; Archer and Johnson, 2000; Fung et al., 2000; Watson et al., 2000; Arrigo et al., 2002; 2008a). Dust may influence phytoplankton production on a variety of spatial and temporal scales, ranging from short-lived seasonal ice-edge blooms (Sedwick and DiTullio, 1997; Lannuzel et al., 2007; 2008) to glacial-interglacial changes in basin-scale export production (Martin, 1990; Moore et al., 2000; Watson et al., 2000). To understand how atmospheric Fe deposition affects phytoplankton production and the ocean-atmosphere carbon balance, it is important to quantify the Fe flux and solubility of mineral dust entering the surface ocean. Such information is of particular relevance for remote marine areas such as the Southern Ocean (SO), where it has been demonstrated that phytoplankton growth, photosynthesis and community composition are regulated by Fe availability (Martin et al., 1990; de Baar et al., 1995; Behrenfeld, 1996; Boyd et al., 2000; Johnson et al., 2010a; 2010b) but the contribution of dust relative to other Fe sources, such as upwelling and glacial melt, is poorly constrained (Sedwick et al., 2011; Boyd et al., 2008; 2002). 


\section{The bio-availability of aeolian iron}

From a biogeochemical and biological perspective, it is not the total amount of Fe supplied to the ocean that is important, but the amount that is bio-available i.e. the amount available for uptake and utilisation by living cells. However, a major difficulty in assessing the bioavailability of $\mathrm{Fe}$ in marine aerosols and dust, is the lack of consistency in the operational definition of soluble or bio-available Fe fractions, making inter-comparisons of Fe solubility difficult (Schroth et al., 2009). The most common approach to understanding the delivery of Fe-bearing dust to phytoplankton has been to quantify the solubility of Fe from dust and assume all Fe that is soluble is bio-available (e.g. Ozsoy and Saydam, 2001). Soluble Fe, while it principally influences bio-availability, can be absorbed and thus only a fraction of the Fe is potentially bio-available. In practice, a working definition of Fe bio-availability will be partly determined by the available methods. Such methods include Fe solubility experiments, Fe adsorption studies and numerical models. There are advantages and disadvantages of using Fe solubility as a measure of bio-availability. While solubility experiments do not determine if the $\mathrm{Fe}$ is taken up by phytoplankton, they are comparatively fast, inexpensive and reproducible when compared to more complex ecosystem studies. Boyd et al. (2010) proposes a scheme to merge Fe solubility and bio-availability estimates from the literature by estimating bio-availability from the amount of leached non-refractory Fe (i.e. within the mineral lattice) and the residence time of dust in surface waters. In this scheme, all Fe not present as refractory $\mathrm{Fe}$ is assumed to be potentially bio-available i.e. it is assumed Fe-limited biota in nature are as efficient in obtaining Fe from aeolian dust in seawater as that of the relatively more aggressive laboratory dust leaching experiments which estimate Fe solubility from dust particles. Therefore, whilst Fe solubility is not the same as Fe bio-availability, it is used in the present study to be consistent with literature. The terminology used here reflects that of the original author, as it is difficult to convert between soluble and bio-available Fe.

\subsubsection{The role of sea ice on supplying iron to the Ross Sea Region}

Sea ice represents one of the largest biomes on Earth, has significant impact on carbon cycling in the SO and thus global climate through the initiation of the thermohaline circulation and the thermal insulation of the Antarctic continent (e.g. Dieckmann and Hellmer, 2008). Sea ice also constitutes an important feeding ground for micro-organisms to 
mammals (e.g. Brierley and Thomas, 2002). When sea ice forms annually around Antarctica, it acts as a natural sediment trap by forming a platform for aeolian sand and dust (ASD) to accumulate over winter before being rapidly released into the ocean during the spring and summer melt. The solubility of this Fe-bearing ASD as well as the dissolved Fe (dFe) reservoir in sea ice regionally enhances the $\mathrm{dFe}$ concentration in the ocean compared to the Fe-depleted waters before its release (Grotti et al., 2005; Lannuzel et al, 2007; 2008; van der Merwe et al., 2009). Despite widespread Fe-limitation in the modern high nutrient low chlorophyll (HNLC) SO, intense phytoplankton blooms are mainly observed in the marginal sea ice zone (SIZ; Sullivan et al., 1993). Unusual accessibility of sea ice in McMurdo Sound made this region the ideal place to quantify the relationship between dust and $\mathrm{Fe}$ in more detail. The few previous studies related to ASD concentrations in surface snow on the sea ice in McMurdo Sound suggest locally derived ASD is in abundance (Atkins and Dunbar, 2009; Dunbar et al., 2009). This thesis explores the hypothesis that ASD accumulated on sea ice has the potential to be an importance source of Fe to the south-western (SW) Ross Sea.

\subsection{Research aims and objectives of this thesis}

\subsubsection{Description of research aims}

The overall aim of this project is to determine the biological significance of the temporal and spatial variability of bio-available Fe in McMurdo Sound by quantifying inputs of Fe relative to measured phytoplankton productivity. To achieve this, equipment, expertise and methodology have been developed to measure ASD accumulation rate and the composition and solubility of Fe. The present study is based on three shallow (upper $3 \mathrm{~m}$ ) firn cores from the northern margin of the McMurdo Ice Shelf (recovered by N. Bertler in 2008/09) and 70 surface snow samples collected from the McMurdo sea ice in November 2009 (Fig. 2.1 and Chapter 2.1.2). The shallow firn cores contain a record of variable ASD accumulation rates over the last $\sim 10$ years, including a major storm event in 2004 (Steinhoff et al., 2008; Dunbar et al., 2009) and surface snow samples contain a record of spatial variability of the ASD accumulation rate in McMurdo Sound. This is particularly important, as the SW Ross Sea is the only region in Antarctica with a significant local ASD source. 
To achieve this aim, this thesis focuses on two principal components of the biogeochemical cycle: i) analysis of the physical characteristics (mass and particle size) of ASD and its lateral dispersal and, ii) the analysis of geochemical characteristics of ASD, in particular its $\mathrm{Fe}$ content and solubility. The thesis then compares estimates of productivity derived from aeolian Fe obtained using a dust-biota model (Boyd et al., 2008) to independent estimates of chlorophyll- $a$, a proxy for phytoplankton stocks, by analysing SeaWiFS (Sea-viewing Wide Field-of-view Sensor) satellite imagery and methylsuphonate (MS), a proxy for primary productivity and open water areas in the Ross Sea (Rhodes et al., 2009).

\subsubsection{Objectives of this thesis}

Specific project objectives and the steps undertaken to achieve them are:

1. What is the spatial and temporal variability of ASD accumulation rate into McMurdo Sound over time?

a. Establish the chronology of the series of three firn cores $(\sim 3 \mathrm{~m})$ using $\delta^{18} \mathrm{O}$ stratigraphy.

b. Determine the quantity of ASD trapped in the three firn cores and 70 snow samples from McMurdo sea ice and develop an ASD dispersal model to estimate the spatial distribution of ASD in the McMurdo region.

c. Measure the particle size distribution of ASD in coastal firn cores and snow samples from McMurdo sea ice (only particles $<10 \mu \mathrm{m}$ are thought bio-available).

\section{How much soluble Fe is supplied to McMurdo Sound by ASD?}

a. Determine the total amount of Fe in ASD.

b. Estimate the proportion of Fe in ASD that is soluble.

\section{What is the link between ASD accumulation and phytoplankton blooms in} McMurdo Sound?

a. Develop a model of aeolian Fe flux into the SW Ross Sea.

b. Compare the relative importance of aeolian Fe from locally derived ASD with other sources of Fe to the SW Ross Sea.

c. Apply the dust-biota assessment tool of Boyd et al. (2010) to assess whether ASD deposited into the SW Ross Sea is a potential initiator of phytoplankton blooms. 
d. Independently estimate chlorophyll- $a$ concentration using SeaWiFS satellite data and correlate with MS concentrations from ice cores (proxy for primary production; e.g. Rhodes et al., 2009) and ASD accumulation on inter-annual to multi-annual timescales.

\subsection{Layout of the thesis}

This thesis is organised into the following sections:

\section{Introduction}

Chapter 1 describes the context of this research, the research aims and objectives, the layout of this thesis and outlines the analyses conducted.

\section{Sedimentology of ASD in McMurdo Sound}

Chapter 2 investigates the physical characteristics (mass and particle size distribution) of ASD on sea ice in McMurdo Sound. These data are then used to estimate i) dispersal patterns of the ASD (when combined with meteorological data) and, ii) and seafloor accumulation rates. The provenance of ASD is investigated using a combination of radiogenic isotopes $(\mathrm{Nd}$ and Sr), mineralogy and the physical ASD characteristics.

\section{Concentration of particulate and soluble Fe in ASD in McMurdo Sound}

Chapter 3 outlines the development of an ultra-clean methodology for determining particulate and soluble Fe concentrations in glacial dust samples using an internationally recognised standard. The developed method is then applied to ASD samples from McMurdo Sound and the difference between soluble and bio-available Fe is discussed.

\section{The effect of ASD on phytoplankton blooms in McMurdo Sound}

Chapter 4 describes an aeolian Fe flux model for McMurdo Sound and compares the aeolian Fe flux estimate to a Fe budget for the Ross Sea. It incorporates the data from Chapters 2 and 3 into a dust-biota assessment model (Boyd et al., 2010) to determine if enough ASD is supplied to McMurdo Sound to overcome the Fe limitation in surface waters to trigger a phytoplankton bloom. Here, the relationship between ASD accumulation rate and primary productivity is also investigated over the past decade using chlorophyll- $a$ data and a MS record from the Gawn Ice Piedmont Glacier. 


\section{Conclusion}

Chapter 5 states conclusions of this thesis and suggests an outline for future work.

\subsection{Analyses conducted in this thesis}

In completion of this thesis, the following work was conducted:

- Seventy surface snow samples were collected on sea ice in McMurdo Sound, using ultra clean sampling techniques as part of Dr Tim Haskell's Antarctic Field Party K0131.

- Three shallow firn cores were processed at the GNS Ice Core Research Laboratory for stable isotope data and ASD concentration.

- Seventy surface snow samples and 180 firn core samples were filtered and weighed.

- Seventy surface snow and 180 firn core samples were analysed for particle size distribution on a Beckman-Coulter laser particle size analyser.

- A visit to the Glaciology Laboratory, University of Milano-Bicooca, Italy to develop a modified Coulter-Counter methodology for ASD in coastal snow and ice samples with Dr Barbara Delmonte.

- The composition of the ASD samples was determined using scanning electron microscope (SEM) and energy dispersive spectrum (EDS) analysis for calcite particles, and petrographic analysis for marine components.

- A method was developed for analysing particulate (acid digestion) and soluble (ASD leaching) Fe concentrations in glacial dust using Arizona test dust. This method was sequentially used to prepare and analyse nine ASD samples for geochemical analysis using inductively coupled plasma mass spectrometry (ICP-MS) analysis at the VUW Geochemistry Laboratory with assistance by Dr Marc-Alban Millet.

- Chlorophyll- $a$ data for the SW Ross Sea was downloaded from SeaWiFS for 19972010 (http://gdata/sci.nasa.gov). 


\section{Chapter 2 \\ The sedimentology of aeolian sand and dust in McMurdo Sound}

\subsection{Background}

\subsubsection{Introduction}

Until recently, the main focus of research on sediment in Antarctic ice has been on investigations of ice cores from the Antarctic interior or the polar plateau such as European Project for Ice Coring in Antarctica (EPICA) Dome C and Vostok. These deep ice cores cover geological time spans of several hundred thousand years and dust extracted from them represents globally derived material that has travelled over long distances, as most of Antarctica is covered with ice (e.g. Grousset et al., 1992; Grousset and Biscaye, 2005; Basile et al., 1997; Delmonte et al., 2002; 2003; 2004a; 2004b, 2008; 2010a; 2010b). However, small pockets of exposed rock and sediment exist, of which the McMurdo Dry Valleys (MDV) are the largest area. Therefore, the MDV are potential sources of sediment deposited on the nearby glaciers, ice shelves and sea ice in addition to globally derived dust found in ice cores on the polar plateau. The proximity of local sources of sediment suggests that the accumulation of such material on the sea ice and ice shelf in McMurdo Sound is likely to be strongly affected by local aeolian processes and regional meteorological regimes (e.g. Ayling and McGowan 2006; Bertler et al., 2004; 2005). This, coupled with the coastal Antarctic region having particularly high snow accumulation, allowing sub-annual sample resolution, makes this location potentially the most important source of aeolian sand and dust (ASD) in Antarctica.

Although not the main focus of this thesis, studies of geological drill cores (e.g. Naish et al., 2009), which are tightly clustered in McMurdo Sound, have pondered the significance of ASD as a contributor to the sedimentary record, in particular as a source of the well sorted fine sand found in many seafloor samples (e.g. Barrett et al., 1983; Atkins and Dunbar, 2009). The initial suggestion of Barrett et al. (1983) has been used by subsequent authors (e.g. Anderson et al., 1984; Dunbar et al., 1985) who have questioned the significance of ASD in the broader Ross Sea. Understanding the trends in annual sediment accumulation rate and particle size distribution of ASD to the water column is not only important for quantifying 
this material in terms of global dust flux estimates (which has implications for the associated nutrients it provides to the surface waters) but also, little is known about present day sedimentation, although marine sedimentation in McMurdo Sound during past climates is well documented (Barrett et al., 1984; Collier et al., 2000; Naish et al., 2009). This is particularly important for interpreting the sedimentary record preserved in sediment cores in the region such as Antarctic Geological Drilling (ANDRILL; Naish et al., 2009). It has long been thought that the contribution of aeolian sand is important in McMurdo Sound but has never been quantified (Barrett et al., 1983). This chapter investigates potential sediment sources and the role of wind and ice in sediment transport in McMurdo Sound, SW Ross Sea, Antarctica, with the aim of determining the spatial and temporal variability of the ASD accumulation rate into McMurdo Sound. 


\subsubsection{Regional setting}

\subsubsection{Site location}

McMurdo Sound is located at the northwest McMurdo Ice Shelf edge, bounded by the Transantarctic Mountains (TAM) to the west, Ross Island and the Ross Sea to the east and Minna Bluff, White Island and Black Island to the south (Fig. 2.1). The MDV in Southern Victoria Land (SVL) are the largest area of ice-free land in Antarctica. The ablation zone of the McMurdo ice Shelf comprises a large area of exposed unconsolidated sediment known as the 'dirty ice' or 'debris bands' (Fig. 2.1), where sediment is lifted from the sea floor by anchor ice and frozen into the base of the ice shelf and eventually exposed by surface ablation (Debenham, 1920; Kellogg et al., 1990; Denton and Marchant, 2000). Two large bays on the western coast of Southern McMurdo Sound, New Harbour and Granite Harbour, are extensions of the outlet Ferrar and Mackay outlet glaciers respectively. Windless Bight $\left(77.723^{\circ} \mathrm{S}, 167.692{ }^{\circ} \mathrm{E}\right)$, McMurdo Ice Shelf, located on Ross Island's southern coast (Fig. $2.1)$, is an area of low elevation and has a high snow accumulation $\left(\sim 18 \mathrm{~cm} \mathrm{yr}^{-1}\right.$; McCrae, 1984; Arcone et al., 1994; Robinson, 2004; Knuth, 2007). Continuous meteorological measurements and automatic weather stations (AWS) have been deployed in the vicinity of Ross Island since the 1970s (Stearns and Savage 1981; O'Connor and Bromwich, 1988). 


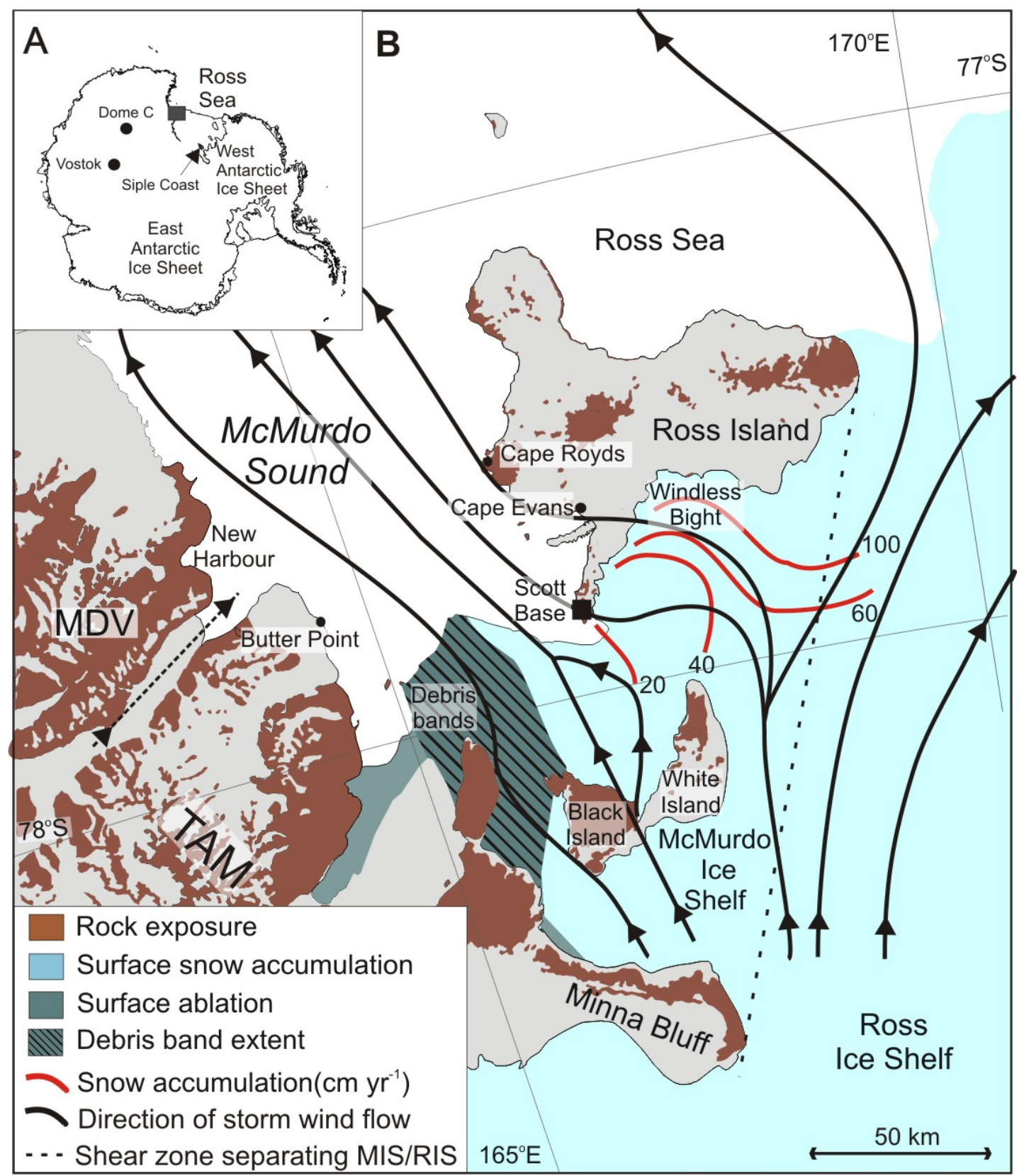

Fig. 2.1: Map of McMurdo Sound. Panel A: Location of panel B, in Antarctica. Black dots: Polar ice cores. Panel B: Map of the McMurdo Ice Shelf and sea ice region showing snow accumulation in Windless Bight (data from McCrea, 1984) and prevailing wind flow (data from Sinclair, 1982). Black dashed line: Katabatic winds from valley glaciers (King and Turner, 1997). Black Box: Location of Scott Base and McMurdo Station. MDV: McMurdo Dry Valleys; TAM: Transantarctic Mountains; MIS McMurdo Ice Shelf; RIS: Ross Ice Shelf.

\subsubsection{Geology}

The McMurdo Sound region is the most extensive ice-free area of Antarctica and consequently has potential as an ASD source region. Rocks exposed in the TAM, along the western coast of McMurdo Sound, can be divided into four main lithologies, each with distinguishing mineralogical characteristics that aid provenance identification (Fig. 2.2): 
1. The Basement Complex consists of the Ross Supergroup (Pre-Cambrian and early paleozoic metasedimentary sequence of calc-silicate schist, metapelitic rock and genesis; Warren, 1970) and the Koettlitz Group which contains mainly marbles, schists and granofelses (Gunn and Warren, 1962) and is distinguished by the occurrence of calcite.

2. The Granite Harbor Intrusives comprise all the Lower Palaeozoic intrusives in Victoria Land (Campbell and Claridge, 1987). These rocks range in composition from diorite, granodiorite to alkaline granite (Gunn and Warren, 1962). Characteristic particles are sub-angular to angular quartz, sodic plagioclase and potassium feldspar, mica, hornblende and basement-derived lithic fragments (Gunn and Warren, 1962; Wilson, 2003).

3. The Beacon Supergroup includes quartz and feldspathic sandstones, siltstones, glacial beds and carbonaceous shales (Barrett et al., 1972). Beacon sandstone is characterised by well rounded quartz particles (Gunn and Warren, 1962; Warren, 1970).

4. The Ferrar Supergroup consists of massive intrusions of tholeitic quartz dolerite (Warren, 1970). Indicative minerals include pyroxenes and calcic plagioclases as well as doleritic lithic fragments (Gunn and Warren, 1962; Wilson, 2003).

An additional unit is the McMurdo Volcanic Group (MVG) which stretches the eastern and southern margins of McMurdo Sound (Fig. 2.2), and includes Ross Island Volcanoes (Mt Erebus, Mt Terra and Mt Terra Nova), Mt Discovery, Minna Bluff and also small, volumetrically insignificant scattered cones on the western side of the sound from the Koettlitz neve to Taylor Valley (Fig. 2.2; McCraw, 1962; Blank et al., 1963; Haskell et al., 1965). The rocks from the MVG are mainly alkali-rich volcanic rocks, including olivine basalt, trachyte, phonolite and kenyte. Typical particles comprise of volcanic lithic fragments and vesicular volcanic glass with rare occurrences of olivine (Warren, 1970; Kyle and Cole, 1974; Campbell and Claridge, 1987; Wilson, 2003). 
The superficial deposits shown in Fig. 2.2 are a mixture of the above rock assemblages i.e. the late Cenozoic debris from rock weathering and glaciers covering slopes and valley floors.

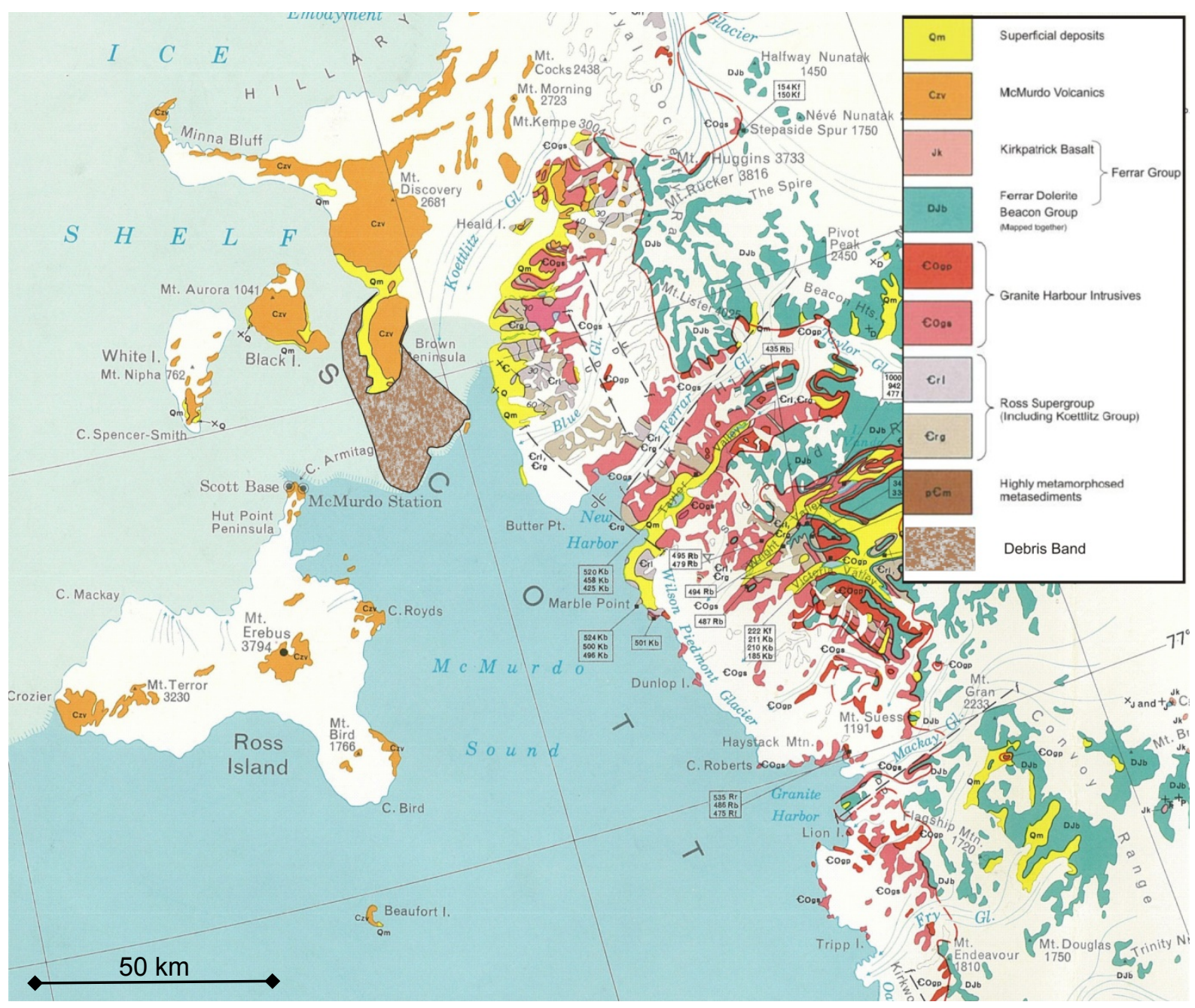

Fig. 2.2: Geological map of Southern McMurdo Sound illustrating the location and composition of exposed rock and sediment including the "debris band" of surficial sediments on McMurdo Ice Shelf (Kellogg et al, 1990). Modified after: Warren (1970).

\subsubsection{Potential aeolian sediment source areas in the McMurdo Sound region}

The present day distribution of unconsolidated sediment, shown in Figs. 2.2 and 2.3 as "superficial deposits" and "debris bands" suggests potential source regions of aeolian sediment in McMurdo Sound can be divided in two: i) a southern source, dominated by basaltic volcanic rocks of the MVG and ii) a western source, the MDV, dominated by granitic and metamorphic rocks, quartzose sedimentary rocks and dolerite (Fig. 2.2; Gunn and Warren, 1962; Goldich et al., 1975; LeMasurier and Thomson, 1990). 


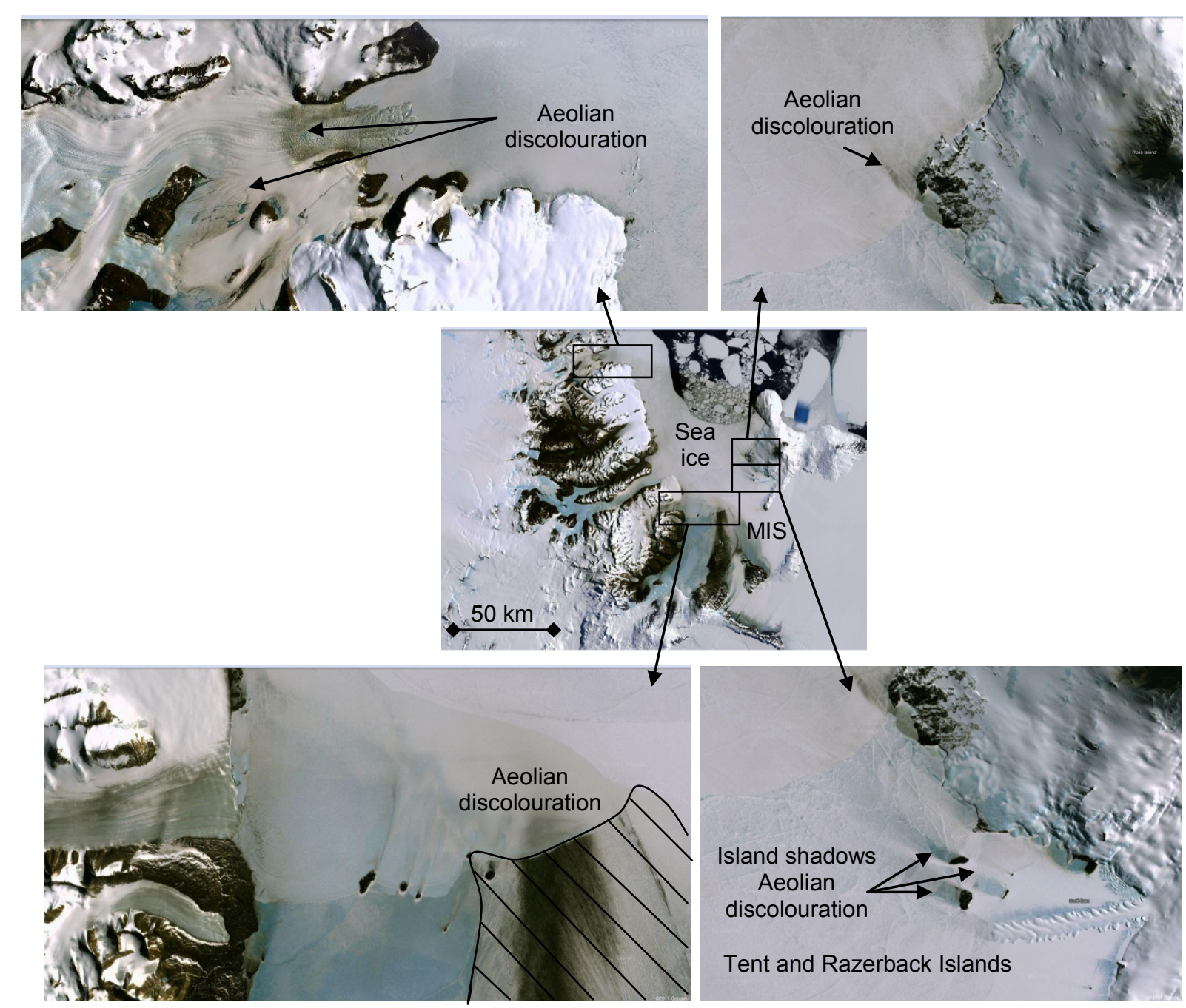

Fig. 2.3: Aeolian sediment on sea ice in western and Southern McMurdo Sound during the summer. Images: Landsat Image Mosaic of Antarctica (LIMA). Hashed area: Debris band as defined in Denton and Marchant (2000). MIS: McMurdo Ice Shelf.

Of particular note is the large volume of unconsolidated sediment lying on the surface of the McMurdo Ice Shelf, known as the 'debris bands' or 'dirty ice' downwind from Minna Bluff and Black Island (Figs. 2.2 and 2.3; Kellogg et al., 1990; Atkins and Dunbar, 2009). The debris bands are a consequence of basal freezing the McMurdo Ice Shelf, which incorporates sea floor sediment into the base of the ice shelf, and surface ablation which removes ice but leaves sediment behind. By this mechanism sediment is continuously brought to the surface, providing an extensive area of unconsolidated, extremely poorly sorted sediment (Debenham, 1920; Denton and Marchant, 2000; Kellogg et al., 1990; Atkins and Dunbar, 2009).

Unconsolidated surface sediments also occur in the snow and ice-free regions of the MDV which consist of heavily physically weathered exposed bedrock, aeolian deposits and glacial 
and glacio-fluvial sediments. The abrasion of exposed rock by windblown particulates is thought to be the primary source of sand-sized sediment as described by Alying and McGowan (2006) in the Victoria Valley. However, long exposure to katabatic winds has winnowed the surface sediment, resulting in a lack of dust and very fine sand-sized material over most of the valley floor (e.g. Selby et al., 1974), leaving behind relatively well sorted fine - medium sand deposits.

Bentley (1979) interpreted the mineralogy and pattern of fine-medium well-sorted sand accumulation on sea ice in Explorer's Cover, northern New Harbour, as a reflection of material being blown eastwards by katabatic winds from Taylor Valley, although the influence of this source was much less pronounced in samples collected near the Strand Moraines.

Further north, Alying and McGowan (2006) found in Victoria Valley that the highest sand transport rates occur during strong surface westerlies promoting the release of frozen sand particles from the surface and their subsequent entrainment by the airstream. Fine and medium sand is clearly blown onto Victoria Lower Glacier (VLG; Schuck, 2009) as is evident in satellite images, although the extent to which material from the MDV's is transported over the Wilson Piedmont Glacier to McMurdo Sound is an open question (Fig. 2.4).

\subsubsection{Wind regimes}

Wind is a primary ASD transport mechanism of interest in the present study. Topography plays a major role in determining surface wind in McMurdo Sound. Due to radiative cooling which creates high inversion layers (King and Turner, 1997), the air column is stable with the coldest (densest) air near the surface. Therefore, the surface wind flows around rather than over topographic obstacles and the most frequent wind directions are parallel to the coastline (Fig. 2.1; Sinclair, 1982).

Although wind velocities in the area average less than $5 \mathrm{~ms}^{-1}$, maximum wind speeds can exceed $55 \mathrm{~ms}^{-1}$ (198 $\left.\mathrm{km} \mathrm{hr}^{-1}\right)$ during serve storm events (Fig. 2.4; O’Connor and Bromwich, 1981; Stearns, 1997), most recently in 2004 (Steinhoff et al., 2008). These events are 
common in the region where $\sim 6$ events with wind gusts $>15 \mathrm{~ms}^{-1}$ occur per year at Marble Point (Marble Point AWS, University of Wisconsin).

Meteorological data have been captured by a number of AWS over the past $\sim 30$ years (Fig. 2.4) and suggest that wind fields in McMurdo Sound region can be grouped into three local patterns:

\section{Southern McMurdo Sound}

Southern McMurdo Sound is influenced by both katabatic and cyclonic winds. Continental katabatic outflows originate from the West Antarctic Ice Sheet (WAIS) and from major outlet glaciers in the region. Synoptic-scale cyclones typically track from Siple Coast, Marie Bryd Land (location in Fig. 2.1A) propagating across the Ross Ice Shelf. This circulation pattern is known as the Ross Ice Shelf airstream (RAS; Steinhoff et al., 2008; 2009). These systems are forced eastward towards the TAM where the elevated topography causes strengthened barrier winds along the Victoria Land Coast (Sinclair, 1982). Moisture entrained in these systems is attenuated, causing a precipitation gradient along the TAM: from high snow accumulation south of the ice shelf margin to significantly less snow accumulation to the north (Sinclair et al., 2010). Locally, the topography of Southern McMurdo Sound increases the southerly wind strength as winds are funnelled between Ross Island and the coast, which can extend up to $100 \mathrm{~km}$ downstream of Ross Island (Schwerdtfeger, 1984). Other topographic features in McMurdo Sound have a strong control on surface wind direction, notably the deflection of moderate strength southerlies around Minna Bluff, and Black and White Islands (Fig. 2.1; Sinclair, 1982). Mesoscale cyclogenesis focused over the western Ross Ice Shelf further contributes to southerly flow within the McMurdo Sound region (Carrasco and Bromwich, 1994) and snow accumulation in the region (Sinclair et al., 2010).

At Scott Base, Willie Field and Pegasus North the wind is bimodal in direction with the most frequent winds from the north and northwest but storms (wind speeds $>15 \mathrm{~ms}^{-1}$ ) are only associated with southerly winds (Fig. 2.4). Western McMurdo Sound is less windy than eastern McMurdo Sound and strong westerly katabatic winds from the MDV have only a localised effect on the western areas of McMurdo Sound adjacent to the Victoria Land Coast (Sinclair, 1982). Further seaward in McMurdo Sound southerly winds also dominate (Keys, 1980). Field observations of the orientation of sastrugi during November 2009 on Southern 
McMurdo Sound sea ice also indicate that that the prevailing surface wind, of sufficient speed to move snow, was from the southeast.

\section{McMurdo Dry Valleys}

Wind flow of the MDV is different to that observed in McMurdo Sound and is typically bidirectional, switching between katabatic easterlies (winter) and westerly marine breezes (summer; Keys, 1980; Doran et al., 2002; Bertler et al., 2004). Westerly winds reach speeds up to $37 \mathrm{~ms}^{-1}$ (Nylen et al., 2004), whereas easterly winds tend to have lower velocities (Doran et al., 2002). Figs. 2.1 and 2.4 illustrate the katabatic surges out of Ferrar and Mackay valley glaciers.

\section{Windless Bight}

Although Ross Island is subjected to a strong southerly mountain-parallel wind regime, Windless Bight on the island's southern coast is a region of anomalous calm (O'Conner and Broomwich, 1988). Here, a stagnation zone results from the strong stability of boundary layer air encountering the high steep topography of Ross Island, resulting in calm conditions at Windless Bight (O’Conner and Broomwich, 1988). 


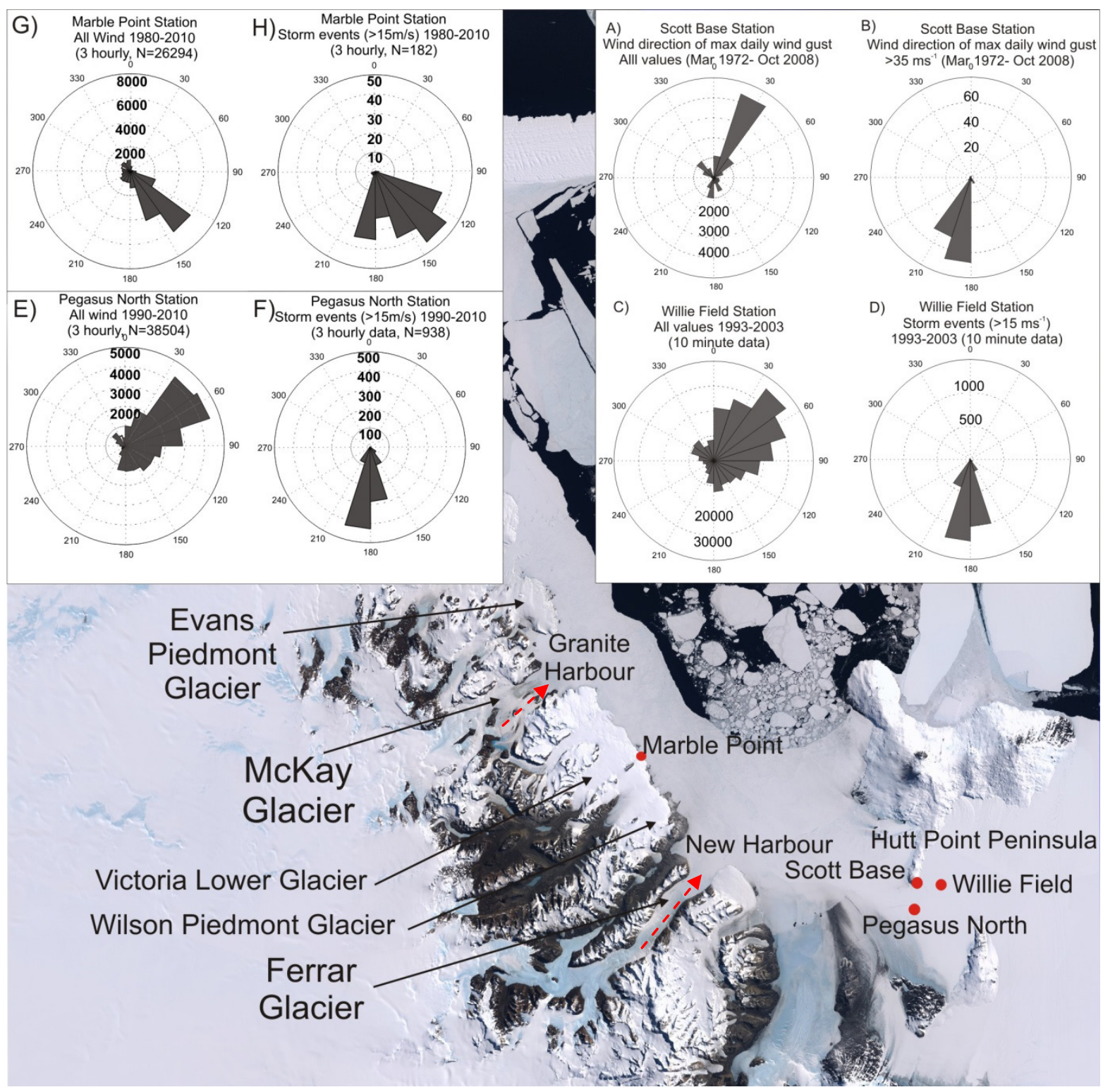

Fig. 2.4: Wind roses illustrating the dominant southerly wind direction for the strongest storms. Data is from AWS at Scott Base March 1972-October 2008, Willie Field 1993-2003, Pegasus North 1990-2010 and Marble Point 1980-2010. Red arrows: Katabatic winds. Scott Base and Willie Field data from Dunbar et al. (2009) and Pegasus North and Marble Point data acquired from http://amrc.ssec.wisc.edu/ on the 18/11/2010.

\section{A case study: the 2004 wind storm, McMurdo Sound}

On the 15-16 May 2004 a southerly, "condition 1" storm damaged buildings and meteorological instruments at McMurdo Station, Antarctica. Wind speeds of $71 \mathrm{~ms}^{-1}(255 \mathrm{~km}$ $\mathrm{h}^{-1}$ ) were measured at McMurdo Station. Data from nearby AWSs at Ross Island (Arrival Heights, Observation Hill, Crater Hill) and Black Island, which lies $34 \mathrm{~km}$ to the south of McMurdo Station, were used to reconstruct the pathway and characteristics of the storm (Steinhoff et al., 2008). Sustained wind speeds over $44 \mathrm{~ms}^{-1}\left(158 \mathrm{~km} \mathrm{~h}^{-1}\right)$ and gusts exceeding 
$53 \mathrm{~ms}^{-1}\left(190 \mathrm{~km} \mathrm{~h}^{-1}\right)$ were modelled by Powers et al. (2007). This storm provides an opportunity to quantify the effect of ASD transport and the subsequent phytoplankton bloom caused by a dateable, reconstructable, and quantifiable ASD accumulation record (Chapters 2.3 and 4.4).

\subsubsection{Temperature}

Fig. 2.5 illustrates monthly, mean annual and inter-annual air temperature variability in McMurdo Sound over the period of the firn core records used in this study. The mean annual temperature at Scott Base is $-19.9{ }^{\circ} \mathrm{C}$ with a seasonal range of -5 to $-35{ }^{\circ} \mathrm{C}$.
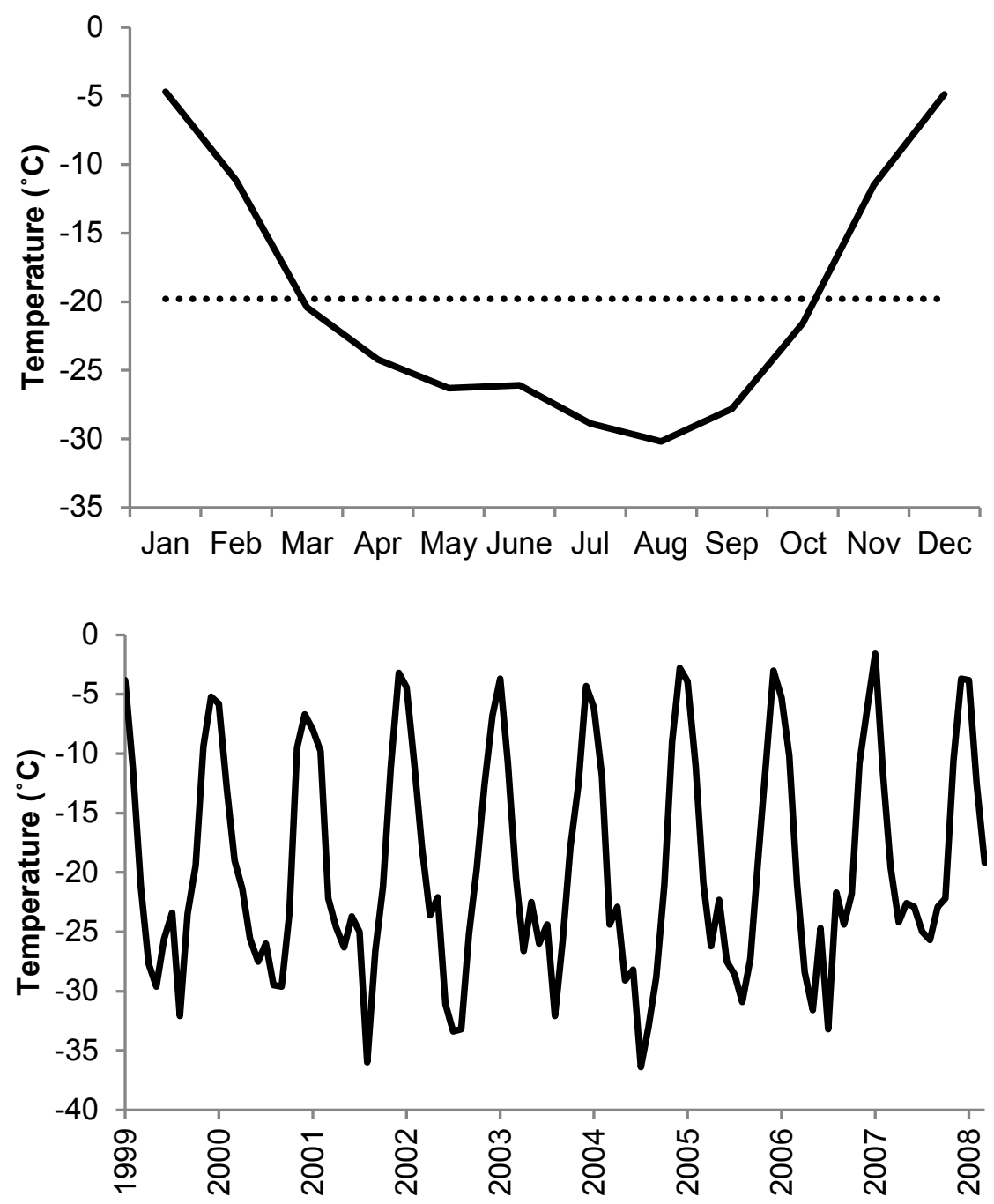

Fig. 2.5: Mean annual temperature (1957-2008) and inter-annual (1999-2008) variability from Scott Base AWS. Dotted line is the mean annual temperature. Data acquired from: htpp://cliflo-niwa.niwa.co.nz on the $1 / 7 / 2011$. 


\subsubsection{Precipitation}

Cold temperatures in McMurdo Sound cause precipitation to fall in the form of snow. The accumulation rate of snow varies markedly within this region and as a consequence so does the temporal resolution and length of firn cores. The source of atmospheric water precipitated over Antarctica is the Southern Ocean (SO; King and Turner, 1997). There is very little precipitation in Antarctica because the saturation vapor pressure of water is very low as a consequence of the low mean temperatures there. The Antarctic coastline receives significantly higher precipitation than the Antarctic plateau due to the greater influence of cyclonic activity and orographic lifting of marine air masses and higher air temperatures (Lettau, 1969; Schwerdtfeger, 1984; Connolley and Cattle, 1994). Snow accumulation at a given site is affected by horizontal transport of blowing snow, often due to katabatic winds. Not only spatially but also seasonally there are variations in the amount of precipitation in Antarctica. This is largely due to the amount of cyclonic activity that reaches the coast of the continent from the ocean. Overall, coastal locations receive above average snow precipitation during spring and autumn, due to an increase in cyclonic frequency during these seasons (King and Turner, 1997).

Published accumulation rates from snow stake measurements carried out in Windless Bight range from 16 to $127 \mathrm{~cm} \mathrm{yr}^{-1}$, with some years experiencing no snow accumulation (McCrae, 1984). In addition, a large spatial mass balance gradient exists on the McMurdo Ice Shelf. A shallow firn core from Windless Bight indicates an accumulation rate of $53 \pm 20 \mathrm{~cm} \mathrm{yr}^{-1}$ (Dunbar et al., 2009), whilst north of Black and White Islands the McMurdo Ice Shelf experiences a negative mass balance with the occurrence of an ablation zone (Fig. 2.1; McCrea, 1984). Snow accumulation measurements carried out during the 2009/10 field season on McMurdo first-year sea ice in November 2009 rendered values from $0 \mathrm{~cm} \mathrm{yr}^{-1}$ around Razerback and Tent Islands at Cape Evans to $>100 \mathrm{~cm} \mathrm{yr}^{-1}$ at Cape Evans to 5 - 10 $\mathrm{cm} \mathrm{yr}^{-1}$ on first-year sea ice between Ross Island and Butter Point. 


\subsubsection{Sea ice extent}

The presence of broad areas of sea ice potentially greatly extends the distance of which aeolian sediment can be distributed. The ice is a low friction, flat surface that facilitates transport of sand-sized particles that are unable to be suspended in the air column for long periods of time in particular, but also enhances transport of finer particle particles by acting as a temporary repository of this material between suspension events. The timing of sea ice growth, decay and extent is therefore critical to this thesis. Annual sea ice in Southern McMurdo Sound begins to form in autumn, when thin, transient films of new ice appear. As temperatures continue to decline sea ice becomes progressively thicker through winter, reaching maximum thickness and extent in spring or early summer (Fig. 2.6; Falconer and Pyne, 2004). Complete sea ice cover is usually prevented by three active polynya systems (Terra Nova Bay, McMurdo Sound, Ross Sea) maintained by strong westerly katabatic winds draining the TAMs (Fig. 2.7; Kurtz and Bromwich, 1983; Bromwich and Kurtz, 1984; Jacobs and Comiso, 1989; Bromwich and Parish, 1998; Goosse and Fichefet, 1999; Arrigo et al., 2000; 2004; Maqueda et al., 2004).

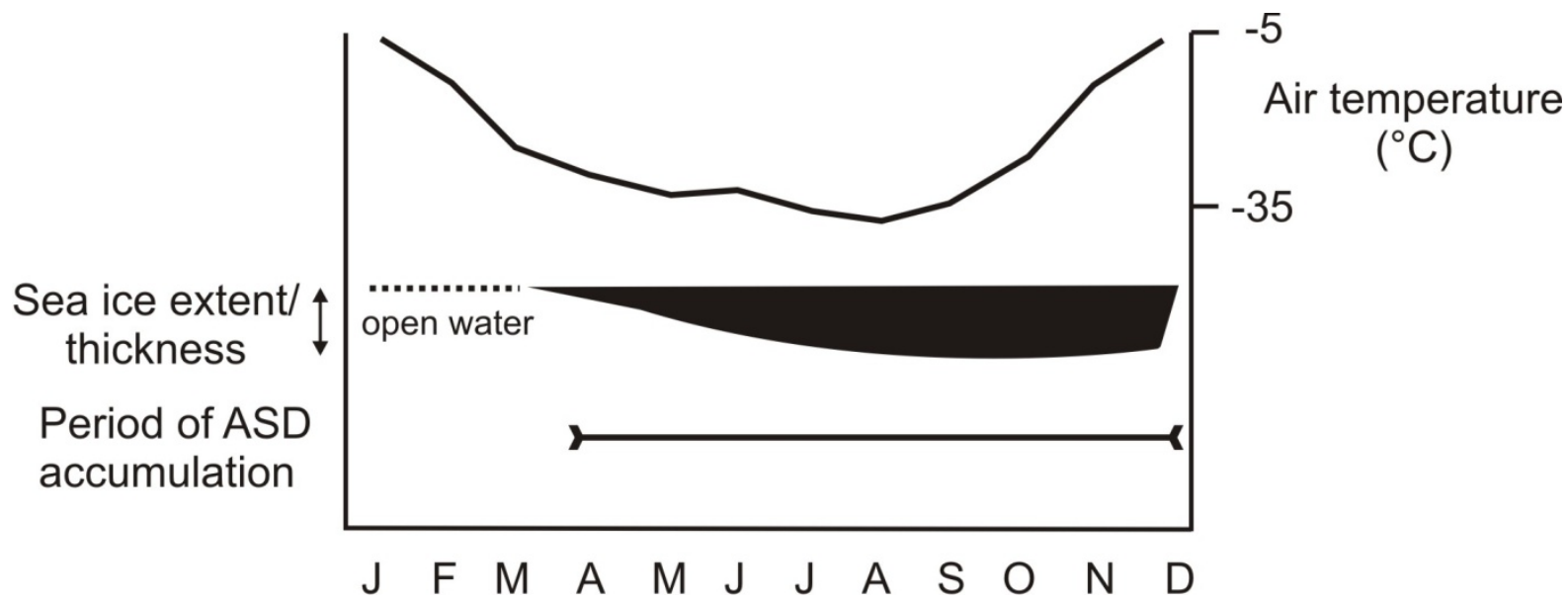

Fig. 2.6: Schematic of the timing of annual sea ice breakout, temperature and aeolian sand and dust (ASD) accumulation on the surface snow of the sea ice in McMurdo Sound. In some places the formation of sea ice is prevented by strong offshore winds which create a local "polynya". Temperature data derived from: Scott Base AWS (1957-2008) htpp://cliflo-niwa.niwa.co.nz on the 1/7/2011. 

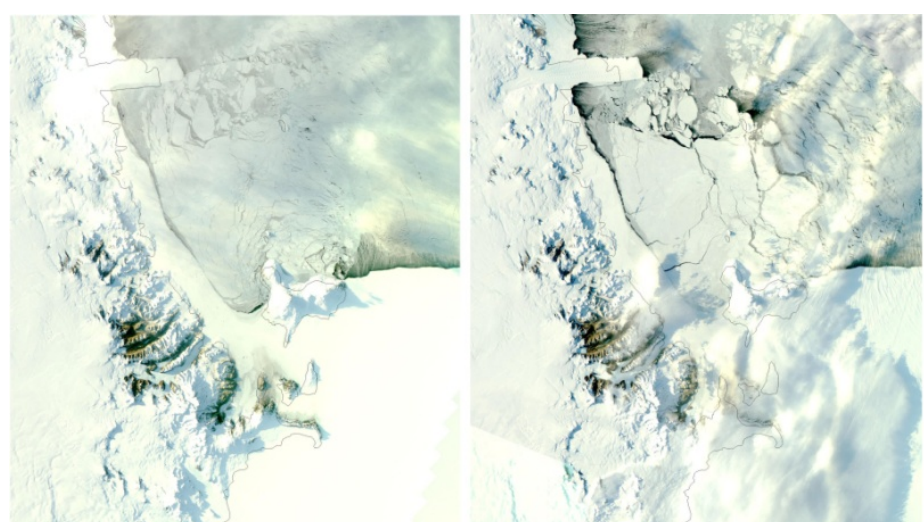

09/19/09

09/29/09
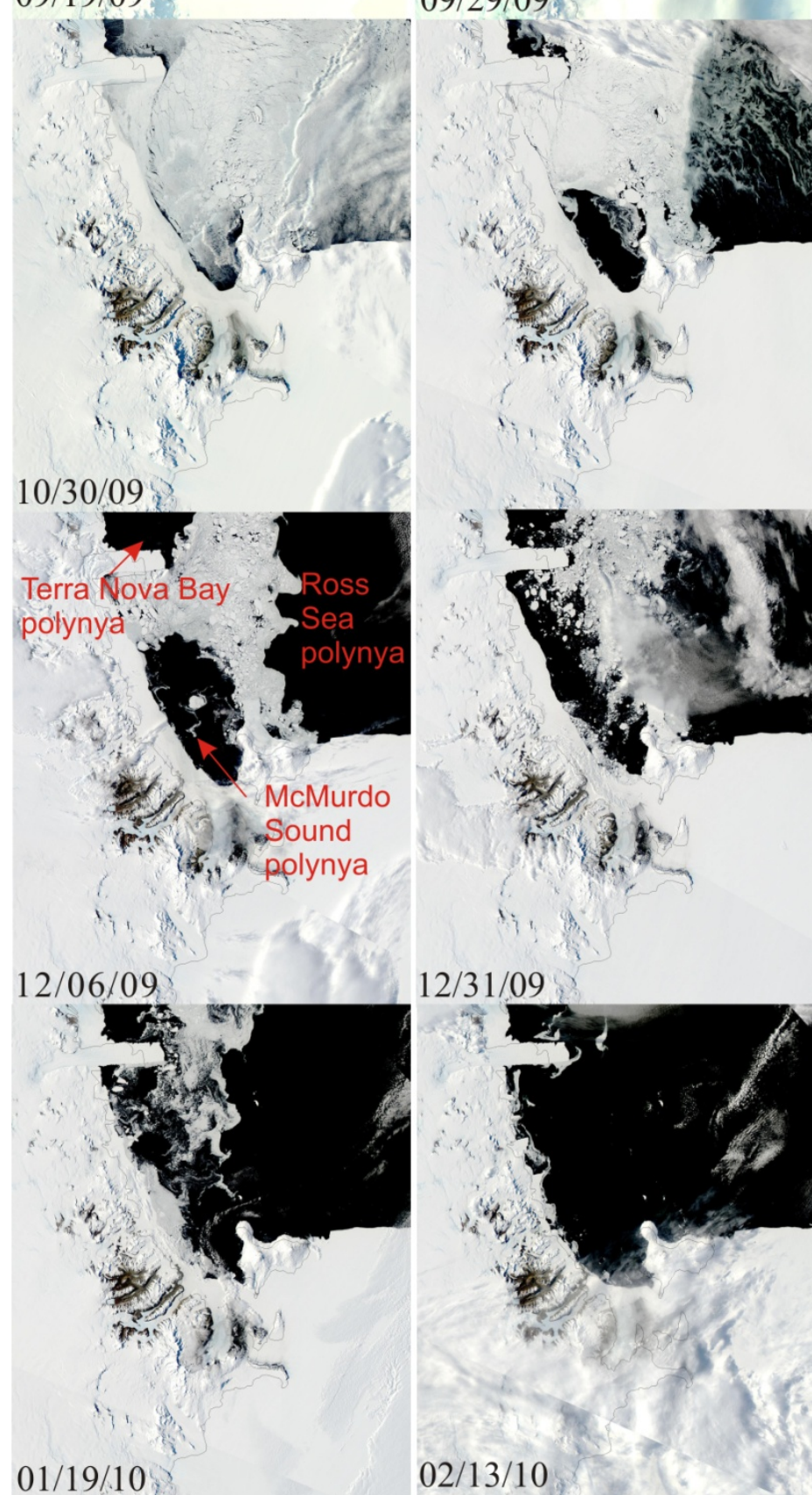

$01 / 19 / 10$

$02 / 13 / 10$

Fig. 2.7: Sea ice break up sequence in McMurdo Sound 2009-2010. The three polynyas in the SW Ross Sea are illustrated. True colour satellite images retrieved on 22/01/2011 from: http://rapidfire.sci.gsfc.nasa.gov/subsets/?project=antarctica\&subset=McMurdoSound. 
Fig. 2.7 illustrates the sequence of sea ice formation and break up in Southern McMurdo Sound in 2009/2010. The annual sea ice break out begins in September and during January and February most of the sea ice has left Southern McMurdo Sound, except for sheltered areas such as New Harbour and Granite Harbour. During the winter, it is replaced rapidly by newly formed ice (Arrigo et al., 1998a), reaching a maximum thickness of $1.5-2.0 \mathrm{~m}$ (Dunbar et al., 1989). Sediment therefore accumulates on first-year sea ice in Southern McMurdo Sound between May and January and has been accumulating on multi-year sea ice, which broke out for the first time in 13 years at the beginning of 2011 (Fig. 2.8).

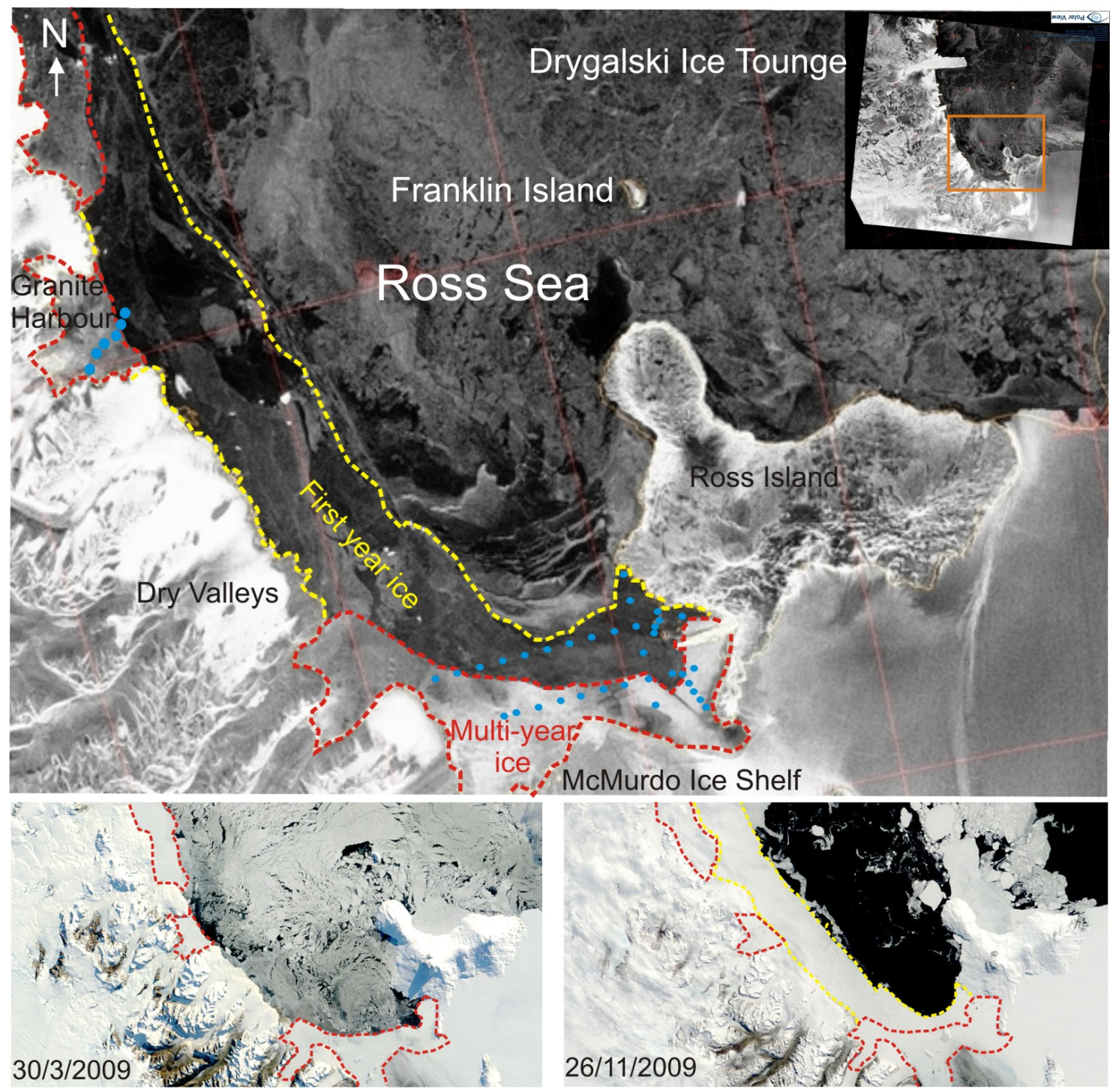

Fig 2.8: Top: Sea ice extent in McMurdo Sound during sampling in November 2009. Insert: Location of Southern McMurdo Sound. Red: Area of multi-year sea ice. Yellow: area of first-year sea ice. Blue dotes: surface snow sampling locations. Satellite image derived from: PolarView High Resolution Sea Ice Monitoring Service on the 19/10/2009. Bottom: MODIS satellite image showing multi-year sea ice on the $30 / 3 / 2009$ and the growth of fast year ice on the 26/11/2009. Satellite images derived from: http://rapidfire.sci.gsfc.nasa.gov/subsets/?subset=RossSea.2009330\&altdates on the 10/6/2011. 
The breakout of sea ice during the summer months is important in controlling the distribution of aeolian sediment to the sea floor in the Ross Sea. Whilst sand sized material will sink rapidly to the sea floor, once it enters the ocean, it may travel tens or hundreds of kilometres on the surface of drifting sea ice before melting releases it. Icebergs from McMurdo Sound travel north to the Drygalski Ice Tongue and then eastwards towards Franklin Island (Fig. 2.8; A. Pyne personal comm., 2010), and it is expected that flows derived from the breakup of sea ice would follow the same pattern, although there are insufficient data to consider this phenomena further. 


\subsubsection{Transport of sediment by wind}

Wind is an essential ingredient for dispersal of sediment on sea ice. Particle entrainment, dispersion and deposition processes are all influenced by the properties of low-level airflow and the surface over which air moves.

\subsubsection{Particle entrainment}

The threshold velocity is defined as the friction velocity above which particles begin to move under the influence of a moving fluid and is a function of surface roughness and whether airflow is laminar or turbulent, as well as particle size and density relative to the transporting fluid. The entrainment of particles by fluid flow can be described by Sheild's criterion, which is equivalent to Bagnold's (1941) "fluid threshold" and is given by:

$$
V_{* t}=A \sqrt{\frac{\sigma-\rho}{\rho} g d}
$$

where $V_{*}$ is threshold wind velocity for movement of particles of diameter $d$; $\sigma$ : density of the particle typically assumed to be $2.65 \mathrm{~g} \mathrm{~cm}^{-3}\left(\mathrm{~g} \mathrm{~cm}^{-3}\right) ; \rho$ : density of air $\left(\mathrm{g} \mathrm{cm}^{-3}\right) ; g$ : acceleration due to gravity $\left(\mathrm{cm} \mathrm{s}^{-2}\right) ; d$ : particle diameter $(\mathrm{cm}) ; A$ : empirical coefficient equal to 0.1 for particle friction Reynolds numbers which describes whether fluid flow is laminar or turbulent. Turbulent flow conditions $\left(\operatorname{Re}_{\mathrm{p}}>3.5\right)$ generally prevail when the transporting fluid is wind.

As wind velocity increases logarithmically with height above the bed (as friction between the air column and the surface diminishes with height) equation (1) can be modified to calculate the fluid threshold velocity for a given particle size for wind measured at any height above the surface $(z)$ as long as the surface roughness $(k)$ and relative particle density are known, hence equation (2) is a practical extension of equation (1) (Bagnold, 1941):

$$
v_{t}=5.75 A \sqrt{\frac{\sigma-\rho}{\rho} g d} \log \frac{z}{k}
$$

where $v_{t}$ is fluid threshold velocity; $z$ : height above surface; $k$ : surface roughness. 


\section{Particle size}

A further modification of equation (1) is the concept of 'impact threshold' which takes into account particle movement initiated by the impact on the bed of previously moving particles as well as stresses applied directly by wind. For sand-sized sediment moving by saltation the impact of a moving particle on the bed causes the ejection of new particles and also disrupts aggregates and dislodges them from the surface. This produces fine particles $(<10-20 \mu \mathrm{m}$ diameter) which can be lifted high into the atmosphere (e.g. Gillette, 1979; Gomes et al., 1990; Shao et al., 1993). Thus a reduced shear velocity (i.e. impact velocity) is sufficient for maintenance of particle movement once established (Fig. 2.9).

Threshold velocity as described by equations (1) and (2) indicates that larger particles require higher wind velocities to initiate movement. Particles $>50 \mu \mathrm{m}$ are infrequently suspended into the vertical flux and typically remain in the horizontal saltation flux (Greeley and Iversen, 1985). For small particles $<50 \mu \mathrm{m}$ equations (1) and (2) may not adequately describe the threshold velocity due to strong particle cohesion forces (Fig. 2.9; Bagnold, 1941). Instead, where inter-particle cohesive forces are important the threshold velocity for entrainment increases with decreasing particle size. These two effects lead to an optimum particle size of $\sim 80 \mu \mathrm{m}$ for which the threshold velocity is a minimum (Bagnold, 1941; Pye, 1989). 


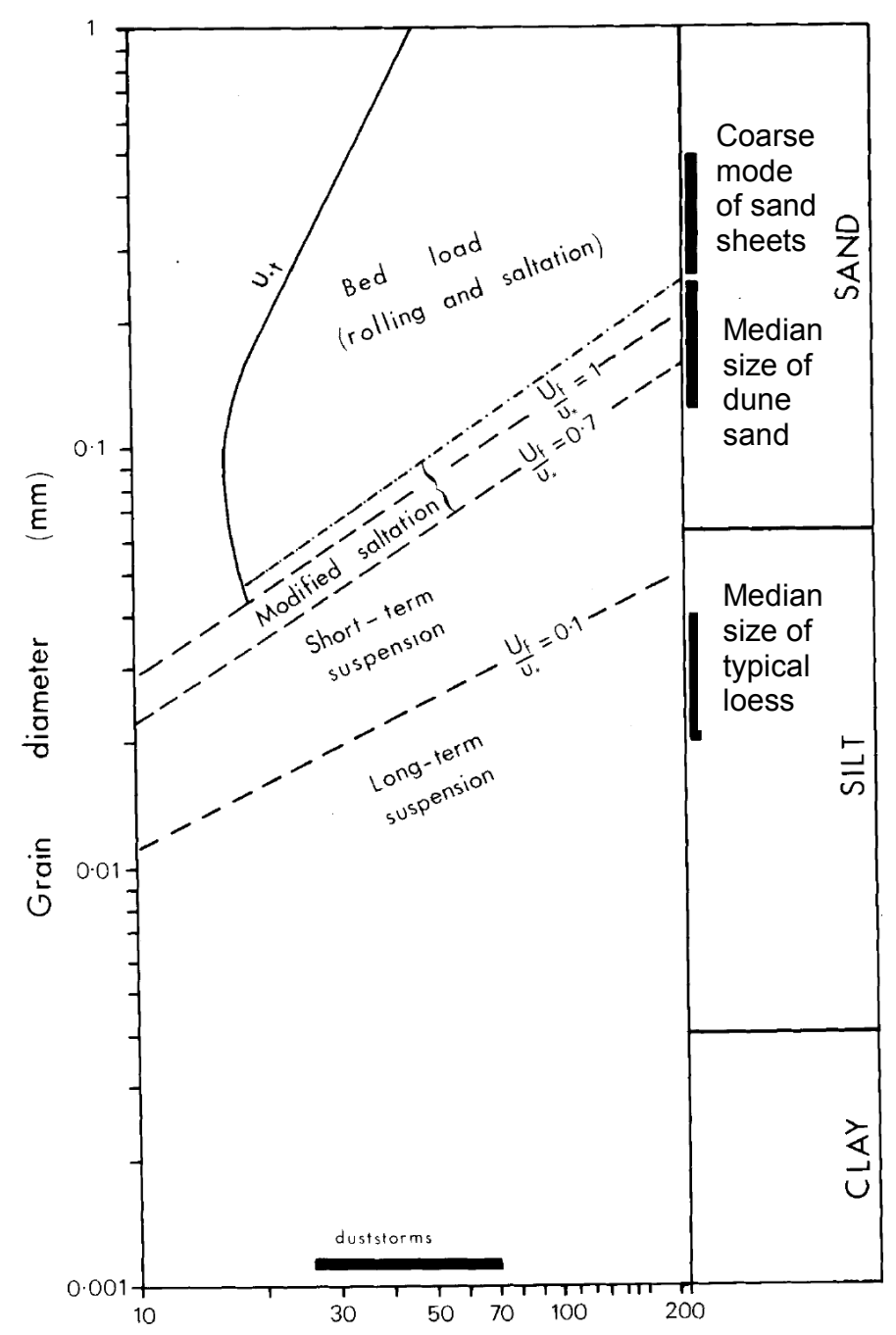

Fig. 2.9: Modes of transport of quartz spheres at different wind shear velocities $\left(u_{*}\right)$. Source: Tsoar and Pye (1987).

\section{Surface roughness}

After particle size, surface roughness is an important factor for particle entrainment. Surface roughness $(k)$ is defined as the height below which there is zero fluid velocity. Surface roughness depends on the properties of the surface above which the flow occurs and is approximately equal to $1 / 30$ of the diameter of the roughness elements on the surface but can be determined directly where wind velocity is measured at two (or more) heights above the bed (Bagnold, 1941). Roughness may be determined by the particle size of the bed, but also by bedform height (Bagnold, 1941). High surface roughness causes turbulence and exerts friction on the overflowing air mass (Pye, 1989). The shear stress acting on the erodible particles is reduced and thus less erosion occurs. There is a critical point at which erosion ceases even at a maintained wind velocity. This is known as bed armouring and is seen in old 
deserts in the MDV where the surface is covered with non-erodible lag gravel which protects the underlying finer sediments from being eroded (Selby et al., 1974).

\subsubsection{Dispersion}

Dispersion of aeolian sediment describes how particles move. The three mechanisms which by wind transports sediment are: i) surface creep (sliding and rolling), ii) saltation, and iii) suspension (Fig. 2.10; Pye, 1989). The mechanism by which a particle is transported depends on particle size and shape, or more specifically the relationship between particle settling velocity and fluid turbulence (air flow is almost always turbulent because of its low viscosity c.f. water; Bagnold, 1941; Pye, 1989). If the particle settling velocity exceeds the vertical velocity component of the wind, the particle will return to the surface a short distance downwind of the ejection point i.e. it will travel mainly by saltation or surface creep. Conversely, if the vertical velocity component of the wind exceeds the settling velocity, it will remain in suspension (Kalinske, 1943). The settling velocity of a particle depends on its mass and shape. Settling velocities can be calculated according to Stokes's Law for fine particle sizes.

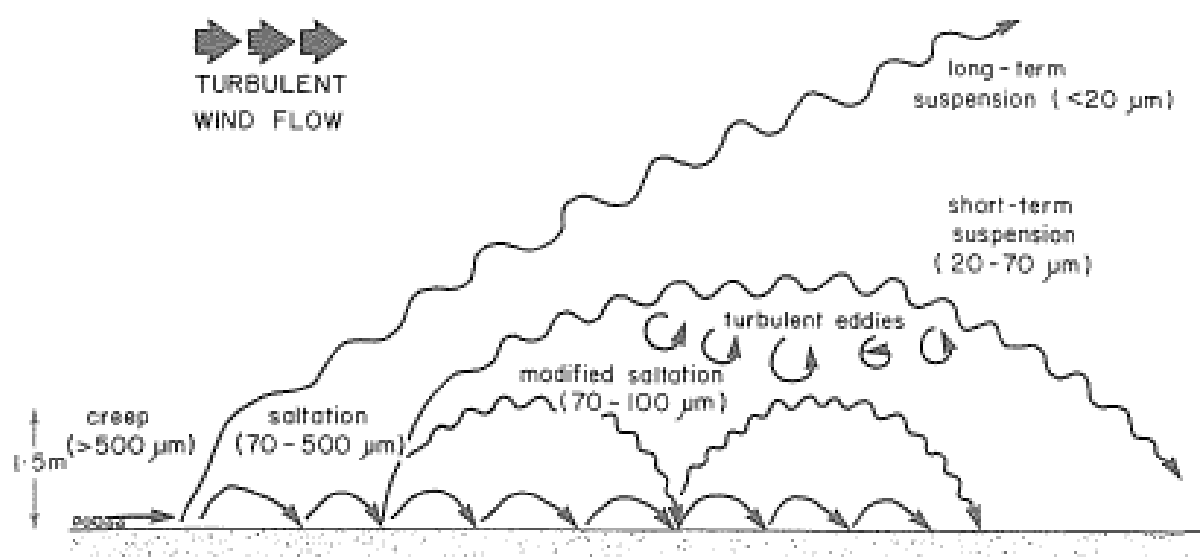

Fig. 2.10: Modes of particle transport by wind. Indicated particle-size ranges in different transport modes are those typically found during moderate windstorms $\left(E=10^{4}-10^{5} \mathrm{~cm}^{2} \mathrm{~s}^{-1}\right)$. Source: Pye (1989). 


\section{Surface creep}

Particles with a diameter $>500 \mu \mathrm{m}$ are transported mainly along the surface by surface creep. They remain on the surface because settling velocity is much greater than upward motion of turbulence, unless salting particles eject them to greater heights in the airflow (Fig. 2.10).

\section{Saltation}

Saltation transports particles between $70-500 \mu \mathrm{m}$ in size. Particles enter the air stream, are transported on the order of meters to tens of meters, and then impact the bed leading to other particles being ejected from the surface. A particle will remain in suspension if vertical fluctuating velocity is greater than the settling velocity (Fig. 2.10; Pye et al., 1989).

\section{Suspension}

Suspension of particles for up to several weeks occurs at particle sizes $<20 \mu \mathrm{m}$ (Pye, 1989), where their low settling velocity can be overcome by air turbulences for long periods of time. Dust records from polar ice cores show that dust originating from South America and Australia travels thousands of kilometres (Pye, 1989) to reach Antarctica, for example over 3 - 4 weeks for dust from Patagonia (Fig. 2.10; Betzer et al., 1988; Delmonte et al., 2002; 2004b; Petit and Delmonte, 2011).

\section{The rate of sediment transport}

Bagnold (1941) established an empirical relationship between wind speed and the rate of sand transport, given by:

$$
q=\alpha \mathrm{C} \sqrt{\frac{d}{\mathrm{D}}} \frac{\rho}{g}\left(v-\mathrm{V}_{i}\right)^{3}
$$

where $\mathrm{q}$ is rate of sediment transport, $\mathrm{V}_{i}$ is velocity above the fluid threshold velocity $v$; $\mathrm{g}$ is the acceleration of gravity and $p$ is density. The critical feature of this relationship is that the rate of sand transport is proportional to the cube of the excess wind velocity over the threshold velocity at which entrainment begins. 


\subsubsection{The deposition of aeolian sand and dust in Antarctica and the Southern Ocean}

Given the size of Antarctica and the potential importance of aeolian sediment as a source of trace metals for the surrounding marginal seas and oceans, it is surprising that there are few actual measurements of the accumulation rate of such material at high southern latitudes. Data on ASD accumulation rates come from ice cores (e.g. Grousset et al., 1992; Basile et al., 1997; Delmonte et al., 2002; 2004a; 2004b; Dunbar et al., 2009), sediment traps and marine cores in the Ross Sea (e.g. Collier et al., 2000), aerosol capture and modelling studies in the SO (summarised in Table 2.1, Figs. 2.11 and 2.12; Arimoto and Duce, 1986) and show that aeolian sediment can be usefully divided into two broad categories:

i) "global" dust, characterised by accumulation rates of 0.001 to $0.02 \mathrm{~g} \mathrm{~m}^{-2} \mathrm{yr}^{-1}$ (Fig. 2.11; e.g. Mahowald et al., 2005), a modal particle size $<5 \mu \mathrm{m}$, and a geochemical fingerprint showing it is derived from arid regions in Patagonia and/or Australia (Chapter 2.1.5; e.g. Delmonte et al., 2004a). Modelling of the present day flux of dust from these, and other, sources suggest this forms the "background" dust flux.

ii) "local" ASD characterised by accumulation rates 3 to 4 orders of magnitude higher (up to $25 \mathrm{~g} \mathrm{~m}^{-2} \mathrm{yr}^{-1}$ ), a modal particle size in the fine sand range and a geochemical affinity with local rocks (Fig. 2.1; Atkins and Dunbar, 2009).

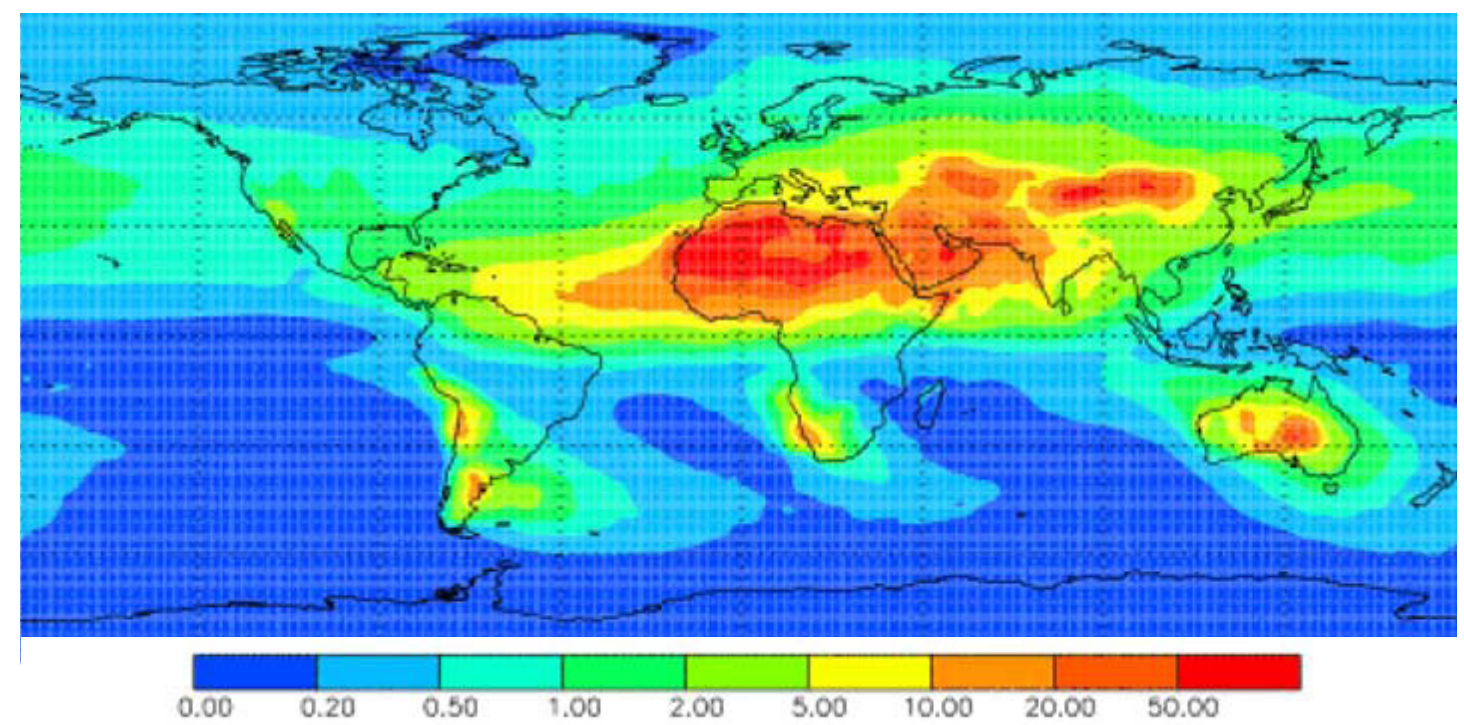

Fig. 2.11: Average dust deposition $\left(\mathrm{g} \mathrm{m}^{-2} \mathrm{yr}^{-1}\right)$ estimated from reanalysis based models simulated for $10+$ years (Luo et al., 2003; Ginoux et al., 2004; Tegen et al., 2004), and representing the best estimate of dust deposition. Models compare well to available sediment trap data (Kohfeld and Harrison, 2001) and satellite observations. Source: Mahowald (2005). 
Table 2.1: Aeolian sand and dust mass accumulation rates at various sites in Antarctica.

\begin{tabular}{|c|c|c|c|}
\hline Site & $\begin{array}{l}\text { Dust accumulation } \\
\text { rate }\left(\mathrm{g} \mathrm{m}^{-2} \mathrm{yr}^{-1}\right)\end{array}$ & $\begin{array}{l}\text { Primary modal } \\
\text { size }(\mu \mathrm{m})\end{array}$ & Reference \\
\hline "Global dust" & $0.001-0.2$ & $<5$ & $\begin{array}{l}\text { Duce et al, 1991; Marticorena and Bergametti, 1995; } \\
\text { Gao et al., 2001; Tegan, 2003; Mahowald et al., } \\
\text { 2005; Wagener et al., 2008; Basile et al., 1997; } \\
\text { Smith et al., 2003; Delmonte et al., 2004a; 2010a; } \\
\text { 2010b; Revel-Rolland et al., 2006; DeDecker et al., } \\
\text { 2010; Vallengona et al., 2002; 2005; } 2010\end{array}$ \\
\hline Taylor Dome, EAIS & 0.009 & & Hinkley and Matsomoto, 2001 \\
\hline Bryd Station, WAIS & 0.001 & & Windom, 1969 \\
\hline Vostok, EAIS & Holocene - 0.004 & 1 & $\begin{array}{c}\text { Delmonte et al., 2004a; Kohfeld and } \\
\text { Harrison, } 2001\end{array}$ \\
\hline Talos Dome, EAIS & Holocene -0.0013 & & Delmonte et al., 2010a \\
\hline Dome C, EAIS & Holocene -0.004 & 1 & $\begin{array}{c}\text { Delmonte et al., 2004a; Kohfeld and } \\
\text { Harrison, 2001 }\end{array}$ \\
\hline $\begin{array}{l}\text { Southern Ocean: } \\
\text { - Dust distribution models } \\
\text { - Aerosol sampling }\end{array}$ & $\begin{array}{c}0.001-0.2 \\
0.0002-0.004\end{array}$ & $\begin{array}{l}<5 \\
<5\end{array}$ & $\begin{array}{l}\text { Mahowald et al., } 2005 \\
\text { Wagener et al. } 2008\end{array}$ \\
\hline "Local dust" & $<25$ & $\sim 40-250$ & $\begin{array}{l}\text { Atkins and Dunbar, 2009; Dunbar et al., } \\
2009\end{array}$ \\
\hline Windless Bight, MIS & Modern - 0.8 & $40-100$ & Dunbar et al., 2009 \\
\hline $\begin{array}{l}\text { Southern McMurdo } \\
\text { Sound } \\
\end{array}$ & Modern - 7.8-24 & $76-130$ & Atkins and Dunbar, 2009 \\
\hline $\begin{array}{l}\text { Granite Harbour, } \\
\text { McMurdo Sound }\end{array}$ & 2.3 & & Macpherson, 1987 \\
\hline $\begin{array}{l}\text { Victoria Lower Glacier, } \\
\text { Southern Victoria Land }\end{array}$ & $\begin{array}{l}\text { Modern - } 24.9 \\
\text { Modern summer - } \\
5.4 \mathrm{~kg} \mathrm{ha}^{-1} \text { month }^{-1}\end{array}$ & $63-200$ & $\begin{array}{l}\text { Schuck, 2009; } \\
\text { Alying, } 2001\end{array}$ \\
\hline
\end{tabular}

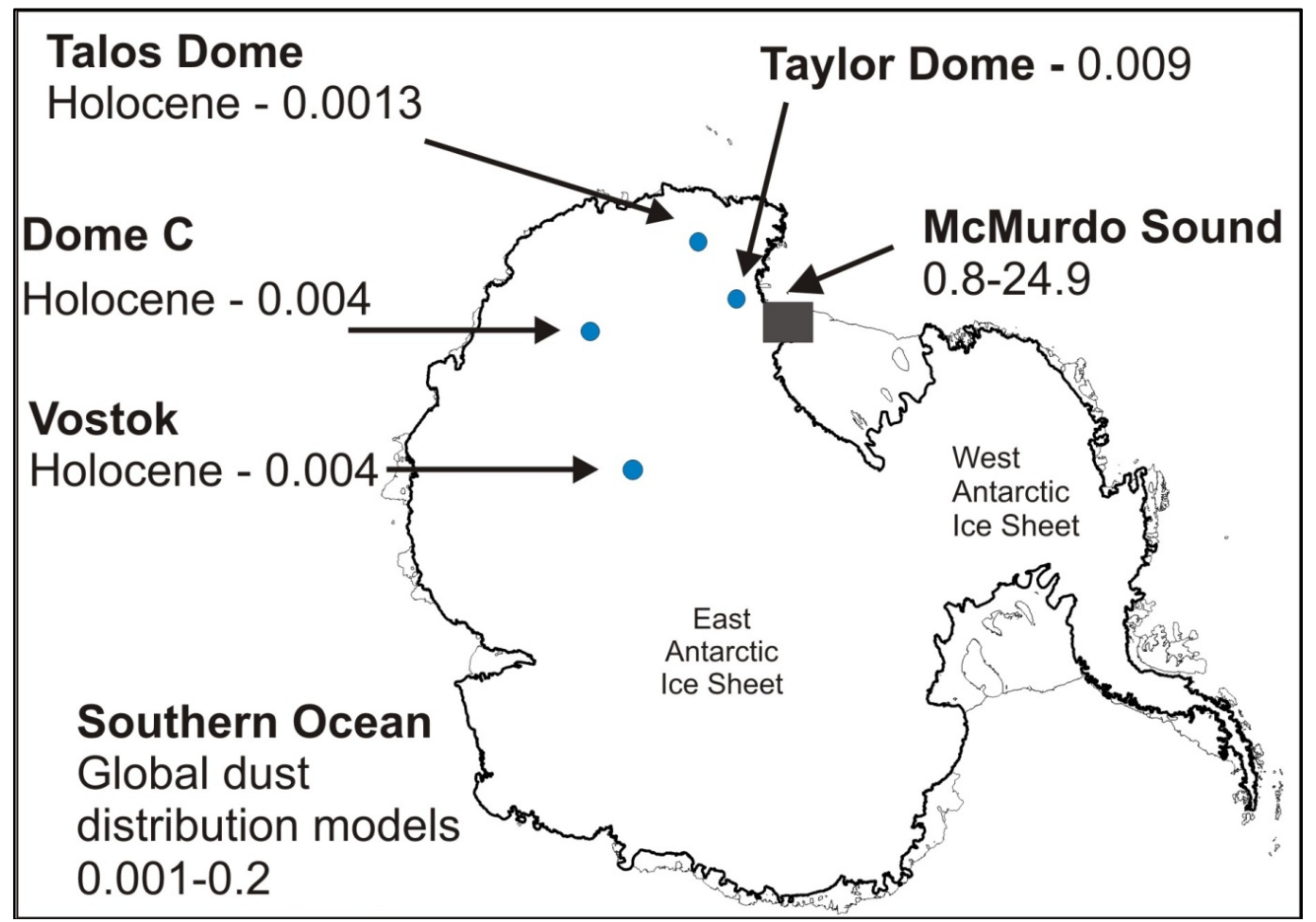

Fig. 2.12: Aeolian sand and dust mass accumulation $\left(\mathrm{g} \mathrm{m}^{-2} \mathrm{yr}^{-1}\right)$ at various sites in Antarctica highlighting the differences between McMurdo Sound and sites dominated by the accumulation of ASD from distal sources. Data from: Delmonte et al. (2004a; 2010a), Mahowald (2005) and Atkins and Dunbar (2009). 


\subsubsection{Aeolian sand and dust distribution on sea ice in McMurdo Sound}

Previous studies of ASD accumulation within McMurdo Sound show substantial spatial variability (Fig. 2.13), although all are considerably above the global background level defined above. Atkins and Dunbar (2009) considered the McMurdo Ice Shelf debris bands to be an effectively unlimited source of aeolian sediment. Their sampling sites, between 5 and $20 \mathrm{~km}$ north of the debris band in Southern McMurdo Sound record ASD mass accumulation rates between 7.8 and $24 \mathrm{~g} \mathrm{~m}^{-2} \mathrm{yr}^{-1}$, characterised by a progressive decrease in both accumulation rate and modal size from 76 to $130 \mu \mathrm{m}$ with distance from the debris band. Xray fluorescence (XRF) major element analysis confirms the elemental composition of this sediment is consistent with derivation from the debris bands.

In an earlier study, Bentley (1979) noted the presence of a west-east oriented aeolian sediment plume in New Harbour where westerly katabatic winds carry sediment from Taylor Valley onto the sea ice and estimated an average accumulation rate of $265 \mathrm{~g} \mathrm{~m}^{-2} \mathrm{yr}^{-1}$ there based on a uniform sediment covering of $1 \mathrm{~mm}$ across an area of $40 \mathrm{~km}^{2}$ with a density of $2.65 \mathrm{~g} \mathrm{~cm}^{-3}$. Unlike Southern McMurdo Sound samples, the sediments become increasingly well sorted, and less coarsely skewed with distance from source, such that sediment on the sea ice is well-sorted and uni-modal fine sand (Bentley, 1979) although this effect could have been enhanced by the method of determining particle size whereby melted snow was decanted, possibly removing the fine fraction with the snow melt (A. Pyne, personal comm., 2010).

It should be noted that both studies targeted areas where aeolian sediment was observed to accumulate and it was acknowledged by Atkins and Dunbar (2009) that as a consequence these results were almost certainly atypical of most of McMurdo Sound.

\subsubsection{Aeolian sand and dust distribution on McMurdo Ice Shelf}

A single $19.8 \mathrm{~m}$ firn core from Windless Bight, McMurdo Ice Shelf produced a 35 year ASD record which showed a general correlation between wind speed and ASD concentration, with higher accumulation rates occurring in winter when storms were more frequent (Dunbar et al., 2009). 


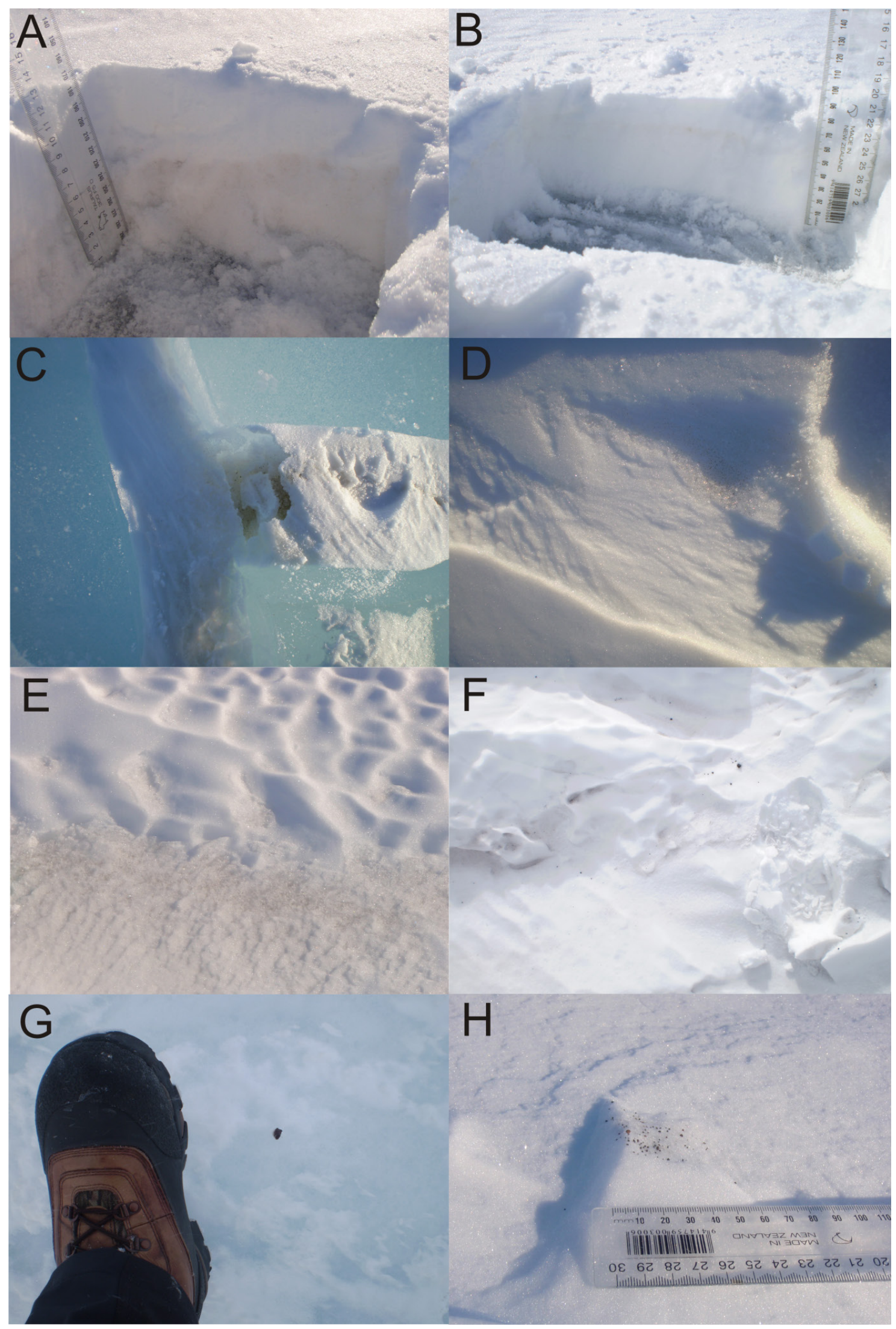

Fig. 2.13: Aeolian sand and dust trapped in surface snow Southern McMurdo Sound sea ice, November 2009. A-B) ASD layers in snow pack, C-D) ASD trapped due to undulations on snow cover, E) patch of ASD in dust plume north of debris band, F-G) coarse particles near Hut Point Peninsula, H) ASD on sustrgui. 


\subsubsection{The origin of aeolian dust in Antarctica}

\subsubsection{Methods for determining aeolian sediment provenance}

The connection between sediment source and sinks can be made by several means, for example numerical model simulations of dust erosion, deposition and air circulation, satellite imagery, archived meteorological data to calculate air mass trajectories from a point of sediment deposition upwind to its potential source area (PSA) and chemical fingerprints such as elemental concentrations and/or ratios and mineralogy between the PSA and sample (Grousset and Biscaye, 2005). In some cases particle size frequency distributions might reflect a given origin (e.g. McLaren 1981; 1985; le Roux, 1994; Barrett et al., 1983). Organic tracers, for example pollen, diatoms, and other biomarkers, can be used to indicate continental or marine origin, but are unlikely to reveal the precise source of the sediment. Trace element concentrations are often not unique to a single area or region, hence their discrimination power is limited. Therefore, this thesis places greater emphasis on isotopic ratios within elements as a method of unambiguously determining sediment provenance.

\section{Isotopic ratios}

The isotopic ratios of dust and aerosols reveal significant variability in different geographic provinces. Common radiogenic isotopes that are used to determine the provenance of aeolian sediment are ${ }^{87} \mathrm{Sr} /{ }^{86} \mathrm{Sr}, \varepsilon_{\mathrm{Nd}}(\mathrm{o}){ }^{*}$ and ${ }^{206} \mathrm{~Pb} /{ }^{204} \mathrm{~Pb}$. The combination of different isotopic signatures are useful in tracing PSAs because different geographic provenances can be discriminated by variations in radiogenic isotopes of mantle derived (basaltic rocks, tephra and soils derived from them, weathered and eroded mafic particles) or crustal (soils, sediments) derived sediments (Grousset and Biscaye, 2005). For example, Antarctica is one of the four principle domains on a worldwide scale and represented by particles derived from very old, crustal Antarctic rocks and exposed sand dunes (Fig. 2.14). These are characterised by a wide range of ${ }^{87} \mathrm{Sr} /{ }^{86} \mathrm{Sr}$ isotopic ratios $(0.713-0.77)$ and rather non-radiogenic $\mathrm{Nd}$ isotope ratios $\left(-25 \varepsilon_{\mathrm{Nd}}(\mathrm{o})>-47\right.$; Grousset and Biscaye, 2005). 


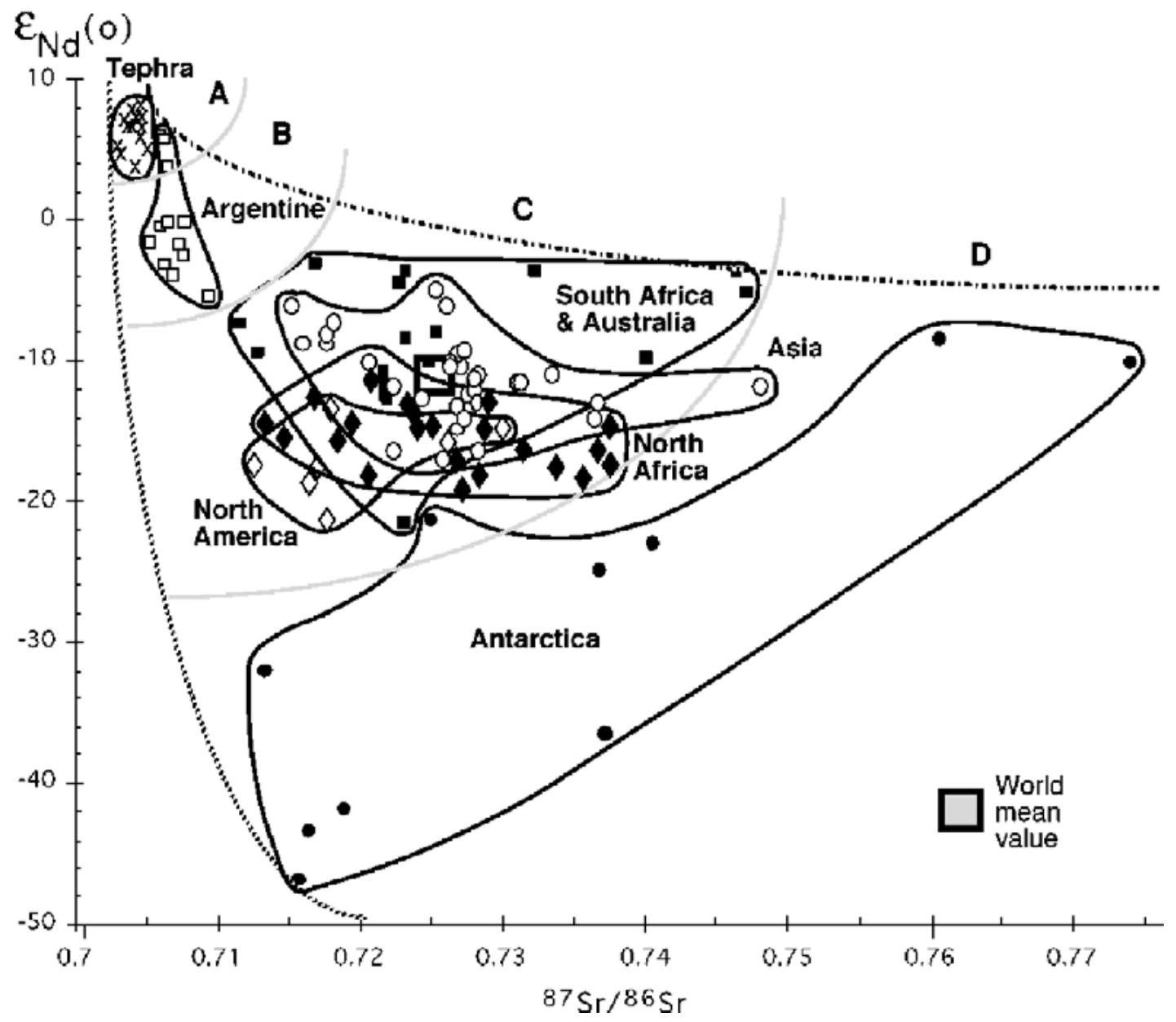

Fig. 2.14: Sr and Nd isotopic composition of worldwide aerosols, loess and sand deposits from Antarctica (closed circles), Asia (open circles), southern Africa and Australia (closed squares), North America (open diamonds), North Africa (closed diamonds), Argentina (open squares), tephra (crosses). Source: Grousset and Biscaye (2005).

*Rocks in the Earth are extremely variable in their isotopic composition and a comparison can be made only if samples are of the same age. In order to make the samples comparable, the ${ }^{143} \mathrm{Nd} /{ }^{144} \mathrm{Nd}$ ratios are normalized to the chondritic model of Earth composition (CHUR), that evolves over time. For this reason, DePaolo and Wasserburg (1976) introduced the Epsilon ( $\varepsilon$ ) parameter, normalizing the isotopic ratio of ${ }^{143} \mathrm{Nd} /{ }^{144} \mathrm{Nd}$ measured in the rocks at present time $\left({ }^{143} \mathrm{Nd} /{ }^{144} \mathrm{Nd}\right)_{\text {measured }}$ to the value of CHUR at the same time:

$\varepsilon \mathrm{Nd}(0)=\left(\left({ }^{143} \mathrm{Nd} /{ }^{144} \mathrm{Nd}\right)_{\text {measured }}\left({ }^{143} \mathrm{Nd} /{ }^{144} \mathrm{Nd}\right)_{\mathrm{CHUR}}{ }^{-1}\right) \times 10^{4}$

with present time $\left({ }^{143} \mathrm{Nd} /{ }^{144} \mathrm{Nd}\right)_{\mathrm{CHUR}}=0.512638$ (Jacobsen and Wasserburg, 1980). A positive $\varepsilon \mathrm{Nd}$ value indicates that rocks were derived from sources that were depleted in large ion lithophile (LIL) elements, while negative $\varepsilon \mathrm{Nd}$ values indicate that the rocks were derived from sources enriched in LIL elements, i.e. that the rocks derived or assimilated old crustal rocks whose $\mathrm{Sm} / \mathrm{Nd}$ ratio had been originally lowered when they separated from the uniform reservoir. The Sm and $\mathrm{Nd}$ isotopic system is not altered by geological processes in the crust such as metamorphism, sedimentation, erosion. 


\section{${ }^{87} \mathrm{Sr} /{ }^{86} \mathrm{Sr}$ and $\varepsilon_{\mathrm{Nd}}(0)$ isotopic composition as tracer for aeolian sediment provenance}

The use of ${ }^{87} \mathrm{Sr} /{ }^{86} \mathrm{Sr}$ versus $\varepsilon_{\mathrm{Nd}}(\mathrm{o})$ isotopic composition has a wide application for the identification of sediment provenance. The use of naturally occurring stable and radiogenic isotopes as dust tracers had first applications in oceanography (e.g. Grousset et al., 1988) and was subsequently used to investigate the origin of dust in Antarctica (Grousset et al., 1992; Basile et al., 1997, Biscaye et al., 1997, Bory et al., 2002).

In this thesis ${ }^{87} \mathrm{Sr} /{ }^{86} \mathrm{Sr}$ verse $\varepsilon_{\mathrm{Nd}}(\mathrm{o})$ isotopic ratios are used as tracers for identifying PSA. The rationale here is that sediments keep the $\mathrm{Sr}$ and $\mathrm{Nd}$ isotopic imprint of rocks from which they derive, which depends on lithology and geologic age (Biscaye et al., 1997). The significance of the isotopic signature of mixed sediments is primarily geographical and not necessarily geological since different types of rocks can have contributed to the formation of the detrital sediments in PSA (Delmonte et al., 2004a).

\subsubsection{Local potential source areas for McMurdo Sound aeolian sand and dust}

A PSA sample can either be i) a primary source of aeolian sediment derived directly from mechanical and/ or chemical alteration of the parent material (e.g. moraines) or ii) a secondary source of aeolian sediment, that is a mixture of particles already subjected to a phase of aeolian and or liquid (e.g. fluvio-glacial sediments) transport (Delmonte et al., 2010a).

\section{Distal sources}

South Africa, Australia and East Antarctica (Terre Adelie and Northern Victoria Land $(\mathrm{NVL}))$ have low radiogenic $\mathrm{Nd}\left(\mathrm{e}_{\mathrm{Nd}}(0)\right.$ values <-7), and high radiogenic $\mathrm{Sr}\left({ }^{87} \mathrm{Sr} /{ }^{86} \mathrm{Sr}\right.$ $>0.717$; Fig. 2.14; Delmonte et al., 2004a). This signature is typical of crustal rocks and is consistent with the geological history of East Antarctica. Whereas, the signatures of New Zealand, the MDV, Antarctic and southern South America partly superpose each other due to their similar tectonic context (all are young orogenic environments marked by andesitic volcanic activity). 


\section{Local sources}

The $\mathrm{Sr}$ and $\mathrm{Nd}$ isotopic signature of the different geographical source regions for McMurdo Sound are illustrated in Fig. 2.15. Low-altitude sources along the Victoria Land Coastline, for example, Holocene raised beaches at Terra Nova Bay, can be excluded as a major dust source for atmospheric transport of maritime air masses from the Pacific/Ross Sea region (Delmonte et al., 2010a). Delmonte et al. (2004a) groups PSA samples from the MVG and SVL into the MDV group (Fig. 2.15). These PSA samples include glacial sediments that are a mixture of crustal and volcanic rocks maintaining the imprint of the volcanism that began in the late Miocene and continues to the present day. Because of this grouping, $\mathrm{e}_{\mathrm{Nd}}(0)$ spans a wide interval $(-12.5<\varepsilon \mathrm{Nd}(0)<5.7)$. However, $\mathrm{Sr}$ isotopes are restricted to a narrow interval $\left(0.703<{ }^{87} \mathrm{Sr} /{ }^{86} \mathrm{Sr}<0.722\right)$. A volcanic imprint is particularly evident in samples from McMurdo Sound (Erebus volcanic area) and Cape Crozier, including rocks derived from magma sources that are residual solids after withdrawal of a partial melt from the undifferentiated mantle. Because of the isotopic differences of volcanic versus crustal PSAs within McMurdo Sound, in the context of this thesis, McMurdo Sound has been divided into two categories of PSAs: "SVL or MDV" and "MVG"; Chapter 2.1.2.3).

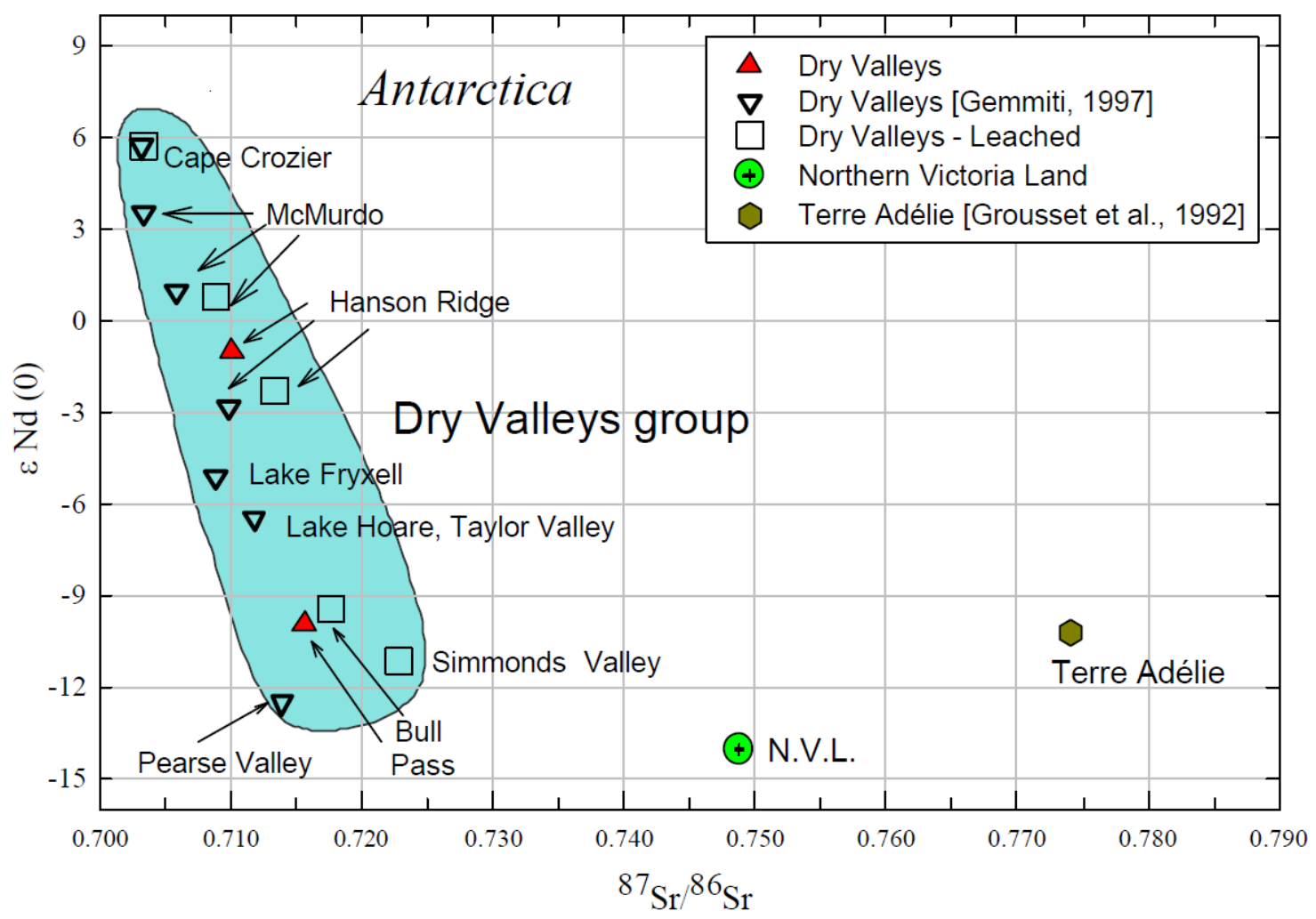

Fig. 2.15: Isotopic signature of non-glaciated areas in McMurdo Sound. Source: Delmonte (2003). 


\subsection{Spatial distribution of aeolian sand and dust in McMurdo Sound}

\subsubsection{Methodology}

To investigate the spatial distribution of sediment on the ice shelf and sea ice in McMurdo Sound, surface snow samples and shallow firn cores were collected and analysed for sediment mass and particle size distribution. The sampling locations are shown in Fig. 2.16 and Appendix 1. Surface snow samples were collected on sea ice as they represent ASD accumulation over the time period in which the sea ice was present and are added to the ocean each year as the sea ice melts. Furthermore, due to logistical constraints sampling locations in McMurdo Sound are proximal to Scott Base. Sampling took place in November 2009 in conjunction with the K131 event (Principle Investigator, Prof. Tim Haskell). K131 had predetermined transects established across McMurdo Sound sea ice which were also used to collect snow samples.

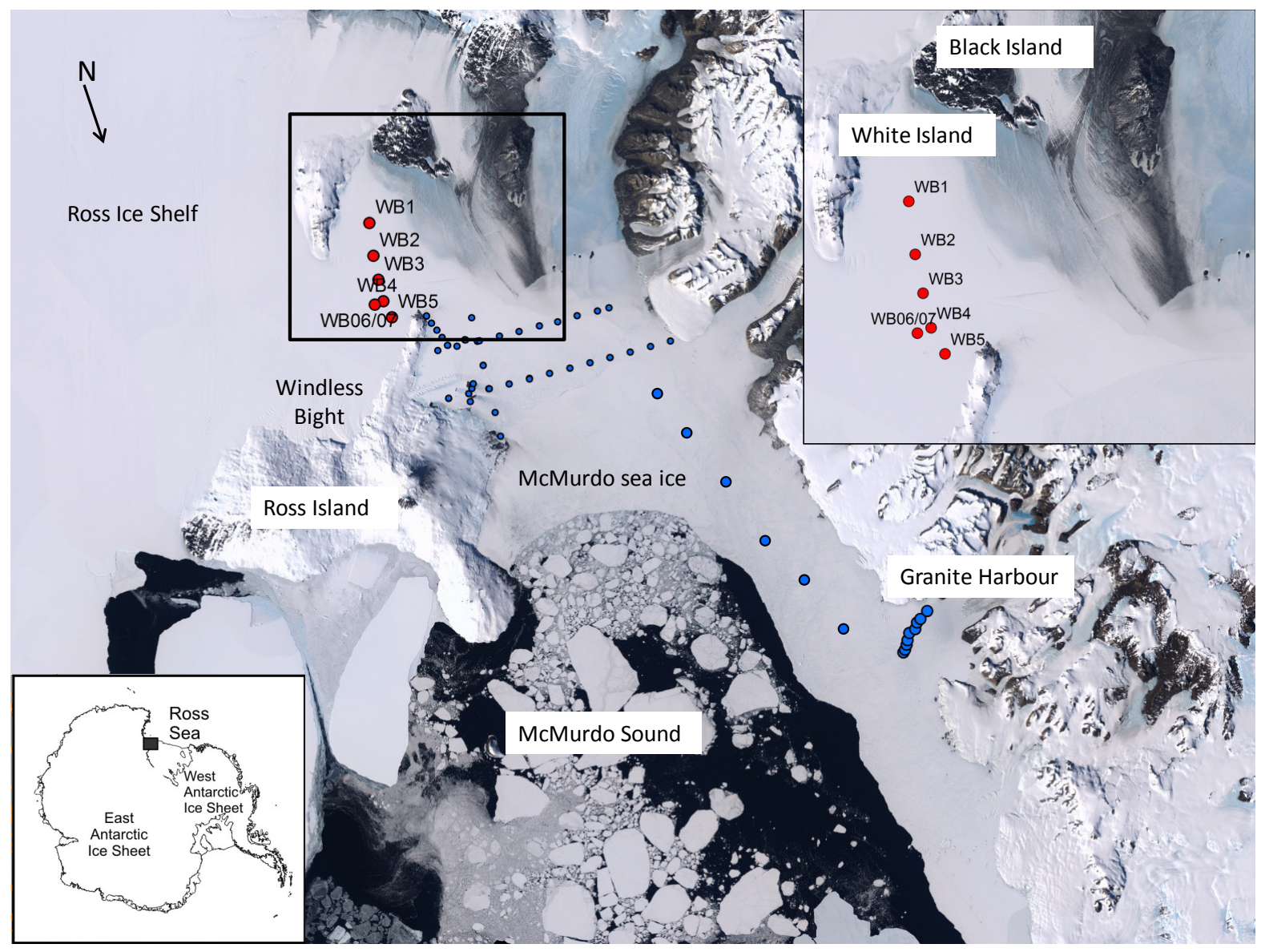

Fig. 2.16: LIMA satellite image of McMurdo Sound showing the location of samples in the present study. Blue dots: Surface snow samples. Red dots: Firn cores. Inset bottom left: Map of Antarctica showing location of McMurdo Sound. Top right: Insert of black box illustrating transect of firn cores. 


\subsubsection{Sample collection}

\subsection{Surface snow samples - sample collection, equipment and preparation}

To ensure samples were recovered in a manner to permit for trace element analysis they were collected in acid-washed low density polyethylene (LDPE) Nalgene screw cap bottles of either 500 or $1000 \mathrm{ml}$ capacity, depending on snow depth at each sample site. The acid cleaning procedure includes triple rinsing the bottles in certified Milli-Q (MQ) water with a resistivity of $18.2 \mathrm{M} \Omega$ to remove particulates, soaking the bottles in heated $5 \mathrm{wt} \% \mathrm{HNO}_{3}$ analytical grade (AR) for 48 hours, triple rinsing and soaking the bottles in MQ water for a further 48 hours and a final triple MQ water rinse. Sampling equipment (ceramic knives and plastic shovels) were triple rinsed, cleaned with methanol, soaked in MQ water for 48 hours and then triple rinsed in MQ water. Personnel wore Tyvek clean suits and polyethylene gloves to prevent contamination during sampling (Fig. 2.17).
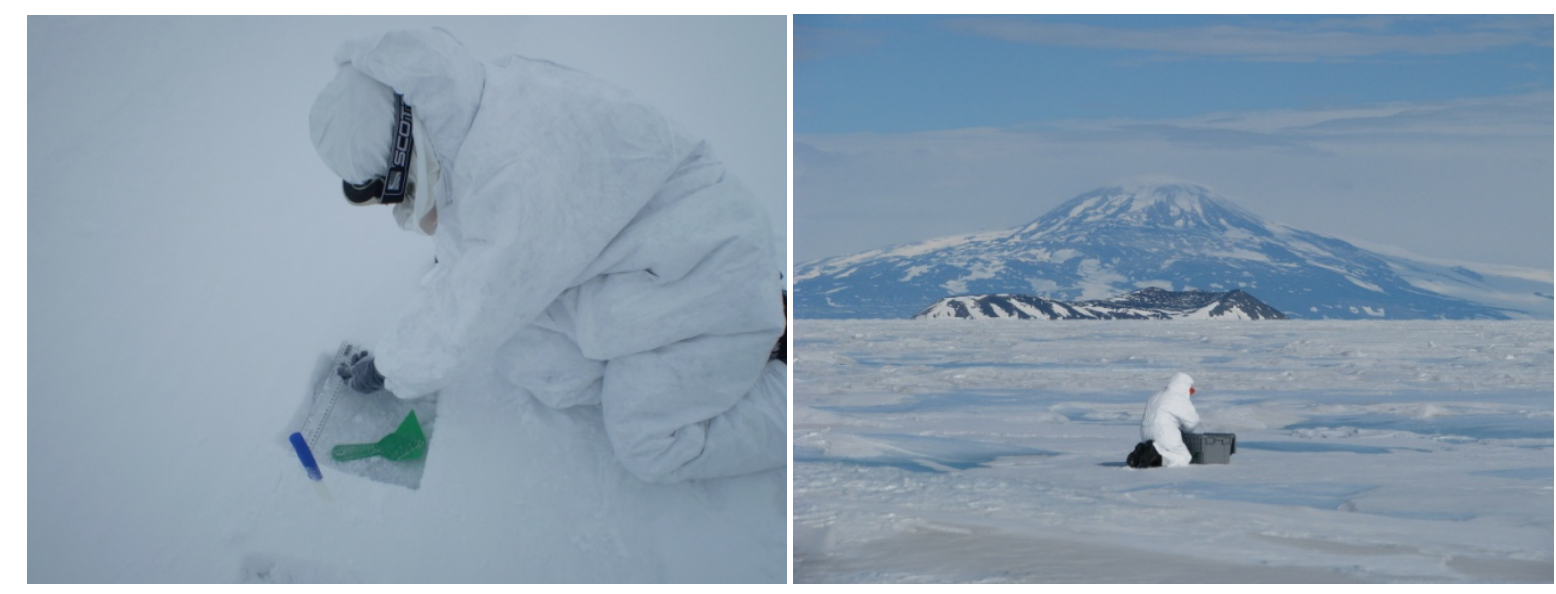

Fig. 2.17: Snow sampling procedure for bag samples, sampling downwind from Black Island and Minna Bluff, Southern McMurdo Sound, November 2009.

\subsection{Field sampling}

Seventy snow samples were collected in three transects (Fig. 2.16) across sea ice along the southern margin of McMurdo Sound which comprised both multi-year and first-year sea ice and these are labelled McMurdo sea ice (MSI). An additional ten samples were collected by Dr Ana Aguilar-Islas from Granite Harbour $120 \mathrm{~km}$ to the north (Fig. 2.16), and seven samples collected by Dr Cliff Atkins in November 2010 between these two areas. Two types of snow samples were collected at each site in order to obtain; i) an ultra clean sample for Fe 
geochemistry and ii) a large sample for the ASD accumulation rate and particle size. The first sample was collected, in pre-acid-washed Nalgene bottles and was taken from the snow profile from the surface to $2 \mathrm{~cm}$ above the sea ice to avoid saline snow-contaminated sea ice which would adversely affect the trace element chemistry of particular elements in these samples. Following sampling, each Nalgene container was sealed in a zip lock plastic bag. The second sample, collected in large bags $(60 \times 45 \mathrm{~cm})$, captured the complete snow profile down to the sea ice. Duplicate samples were taken at every second site to evaluate local variability. At each site snow thickness was measured and geographic coordinates determined by Global Positioning System (GPS). A visual assessment of the sediment concentration in the snow was then made, and highly localised concentrations of sediment were avoided in an effort to ensure each sample was representative of a broader area (Fig. 2.13). In the field, both the bottle and bag samples were kept frozen in ice core boxes. Freezing temperatures $\left(-20{ }^{\circ} \mathrm{C}\right)$ were maintained during transport to and subsequent storage at the National Ice Core Facility, Geological and Nuclear Sciences (GNS), New Zealand.

\subsubsection{Aeolian sand and dust analysis - the analytical technique}

The two methods for particle size distribution analysis were used in the present study electro-resistance using a Beckman Coulter Counter Multi-Sizer 3 (MS3; Appendix 2) and laser light scattering (LLS) using a Beckman-Coulter LS13-320 Particle Size Analyser. Similarly, two methods of quantifying particulate mass were used. First, by filtration and weighing on high precision balance (as described in Dunbar et al., 2009), and second by calculation from the particle size distribution and the number of particles present in each sample using an assumed particle density of $2.65 \mathrm{~g} \mathrm{~cm}^{-3}$, a method commonly used on snow samples with very low (ppb) concentrations of particulates (e.g. Bader, 1965; Steffenson, 1997; Delmonte et al., 2002; 2004a; 2004b; Ruth et al., 2002; Lambert, 2008. LLS e.g. Ram and Illing, 1997; Ruth et al., 2002). The perceived advantage of the Coulter Counter MS3 method where the particle size and mass are determined simultaneously meant this method was attempted first, however, the wide range of particle sizes in these samples meant that a modified Coulter Counter MS3 method would need to be established and this proved difficult for measuring particles $>100 \mu \mathrm{m}$ in size. Consequently subsequent measurements were made using the laser LS13-320 which has a much wider $(0.4-2000 \mu \mathrm{m})$ measurement range than the Coulter Counter MS3. 


\subsection{Beckman-Coulter LS 13320 Particle Size Analyser}

The LLS measures the particle size distribution by measuring the pattern of light scattered by particles suspended in water. Following Mie theory of light scattering, the finer the particle size the greater the angle of scatter. The resulting light scatter pattern is then calculated in terms of particle size distribution, assuming all particles are spherical and of uniform refractive index, largely unavoidable assumptions when measuring geological samples. While the advantage of this method is that it can measure a wide particle size range in a single measurement, it is also difficult to control sample concentration in snow and ice samples. Very low concentrations, such as in snow and ice samples from Antarctica, affect the obscuration (light blockage) and hence the accuracy of the results. The manufacture recommends an obscuration of $8-12 \%$ although in practice valid measurements can be made across a much broader range of obscuration values $(2-20 \%)$. In the present study it was not possible to alter the amount of ASD in each sample, thus the Aqueous Liquid Module (ALM) and Micro Liquid Module (MLM) were used depending on each sample's obscuration.

\subsection{Adopted method}

\section{Aeolian sand and dust mass by manual sample filtration}

At the National Ice Core Research Laboratory, GNS Science, the weight of ASD in the large bags of snow was measured using the following procedure. Each sample was melted and drained through pre-weighed and dried $0.4 \mu \mathrm{m}$ pore diameter polycarbonate filter papers using a Millipore vacuum filtration apparatus (Fig. 2.18). The filter papers showed consistent dry weights of $0.0154 \mathrm{~g} \pm 0.0022 \mathrm{~g}$. These filter papers were kept in labelled Petri dishes in desiccators before use to avoid any weight change due to water absorption after Schuck (2009) found unacceptable weight differences occurred when filter papers were left outside the desiccator. Prior to filtering all glass equipment was cleaned with MQ water and sonicated for 2 minutes between samples to remove any adhering particles. The polycarbonate filter paper was then placed on the frit (fine, porous glass surface which liquid can pass through) and water sucked through the filter by vacuum. All particulates $>0.4 \mu \mathrm{m}$ in size were trapped on the filter, which was then removed, dried at room temperature for 48 hours in a desiccator and reweighed to calculate sample mass by weight difference. 

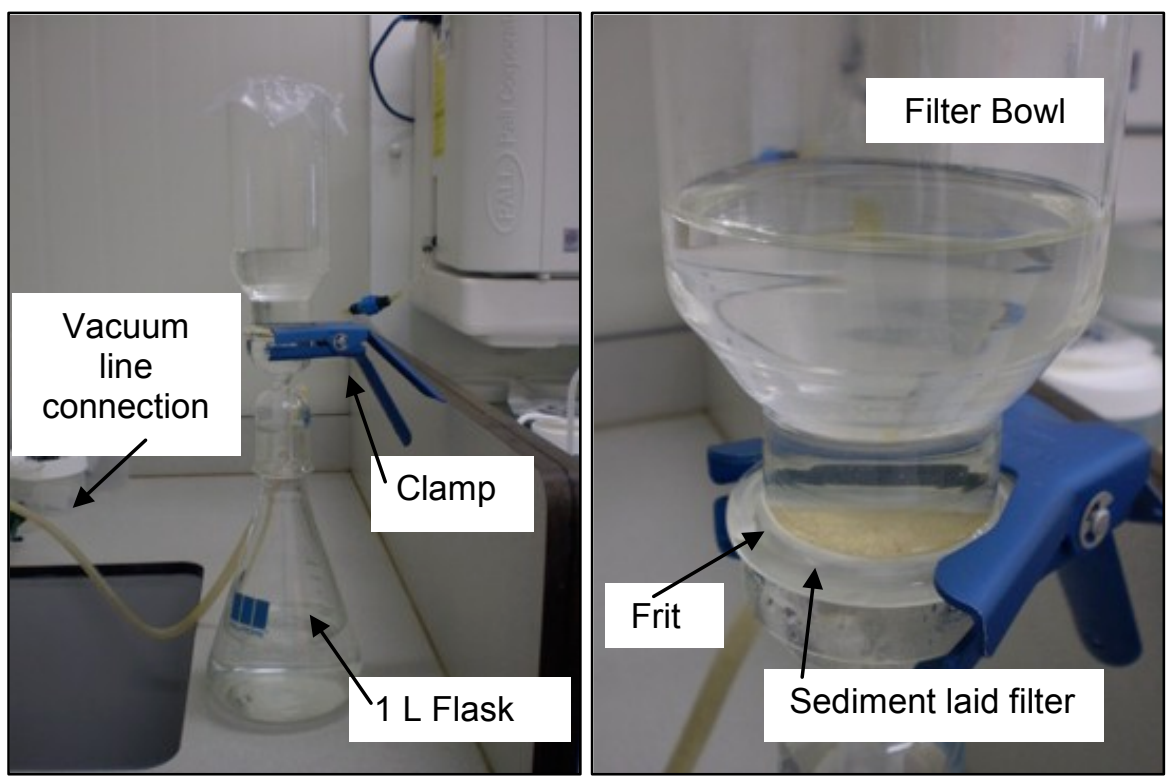

Fig. 2.18: Millipore filtration apparatus.

\section{Siphoning}

Some surface snow samples contained very high concentrations of ASD which rapidly clogged the polycarbonate filter paper. Initially this was addressed by dividing the sample into several aliquots and filtering each separately. However, this was extremely time consuming ( 2 days for the most concentrated sample). Therefore, for samples MSI 21, 22, 32, 44, 55 a different method was used. Bags were suspended overnight at room temperature to allow snow to melt and to allow fine particulates to settle to the bottom as illustrated in Fig. 2.19. Excess water was then removed from the bag with a siphon. The remaining particulates and water were poured into a pre-weighed beaker and dried at $40{ }^{\circ} \mathrm{C}$ for $>48$ hours before reweighing to calculate the weight of each sample. 

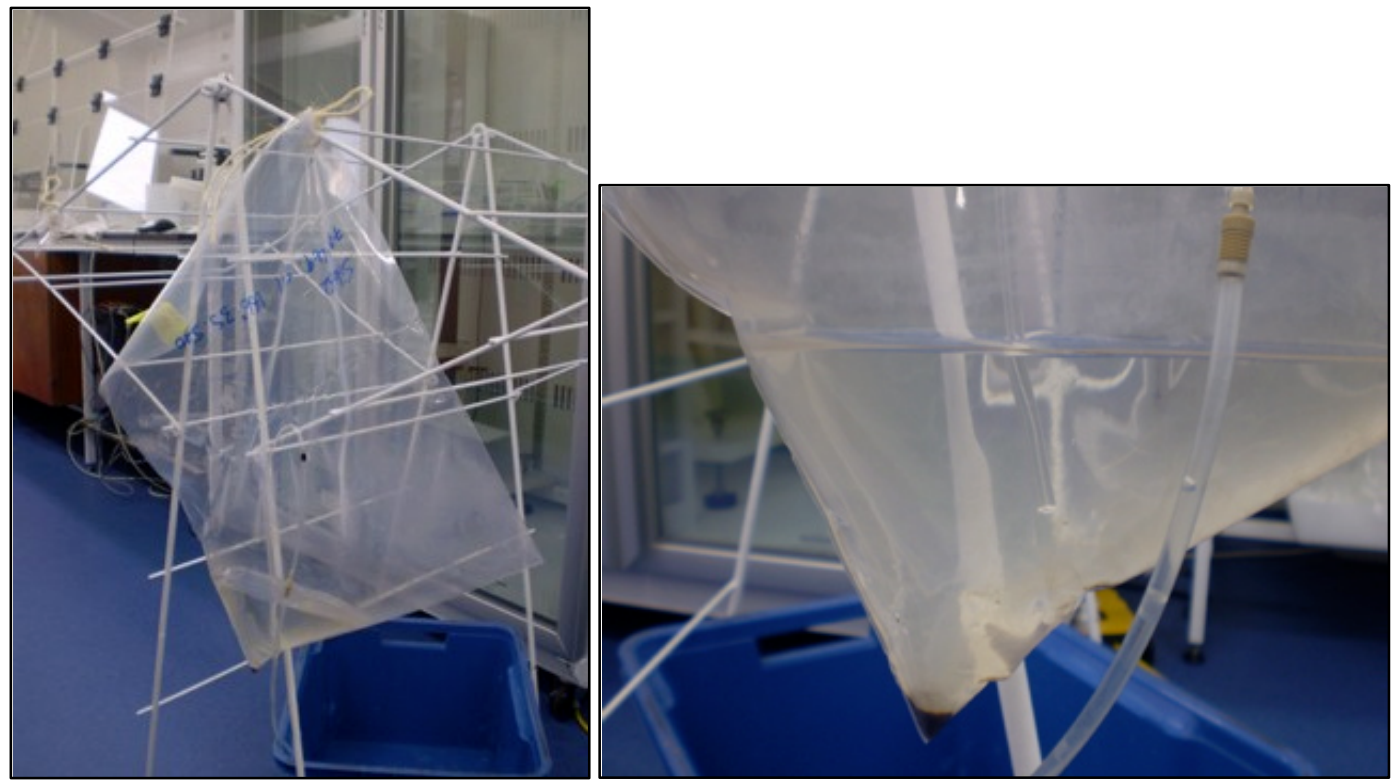

Fig. 2.19: Siphoning dusty samples in the clean room, National Ice Core Facility, GNS Science.

\section{Particle size distribution by LLS}

A Beckman-Coulter Laser Diffraction Particle Analyser (LS 13320) was used to measure the particle size distribution of the recovered ASD from filtering. Glass beads of known diameter were measured to test the reproducibility of the method. Fig. 2.20 illustrates the high reproducibility of $36 \mu \mathrm{m}$ and $125 \mu \mathrm{m}$ glass beads compared to test runs 6 months previously by Schuck (2009). A standard of $36 \mu \mathrm{m}, 86 \mu \mathrm{m}$ and $125 \mu \mathrm{m}$ were run at the beginning of every day to test for reproducibility. For every sample, an alignment and background (measured for 60 seconds) were measured.

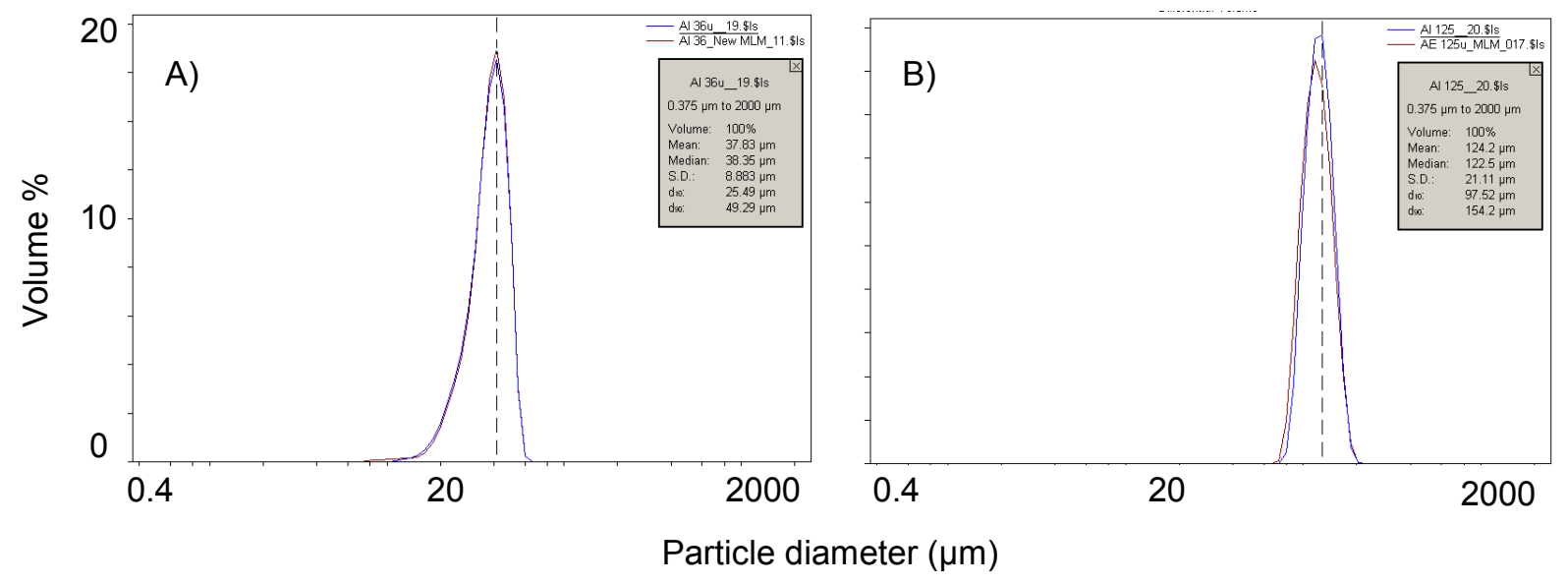

Fig. 2.20: Reproducibility tests of A) $36 \mu \mathrm{m}$ and B) $125 \mu \mathrm{m}$ glass bead. Blue: our test, Red: Schuk's (2009) test. 


\section{Aqueous Liquid Module}

The Aqueous Liquid Module (ALM), LS 13320 was used for samples with a calculated obscuration between 3 - $20 \%$. Sediment-laid filter papers were transferred to pre-rinsed beakers where the ASD was removed using a deionised water and a clean brush and then sonicated for 2 minutes. 'Clean' filter papers were dried and re-weighed showing that $95 \pm 2$ $\%$ of the ASD was recovered. $0.1 \mathrm{~g}$ Calgon (sodium hexametaphosphate) was added to the sample, sonicated and stirred for 30 minutes to disaggregate particles. The sample was the poured into the ALM and analysed for particle size on the LS 13320. The ALM was rinsed with deionised water between samples.

\section{Micro Liquid Module}

Samples that had a calculated obscuration $>20 \%$ were diluted with deionised water to obtain an obscuration $\sim 10 \%$. Particles were disaggregated as described above and measured in the Micro Liquid Module (MLM), LS 13320. Three measurements were obtained from different aliquots and then averaged. Fig. 2.21 illustrates the reproducibility of various aliquots of the diluted sample which is most precise at the fine end of the spectrum. The Micro Liquid Cell was thoroughly rinsed with deionised water between each sample run. An internal magnetic stirrer keeps particles in suspension.

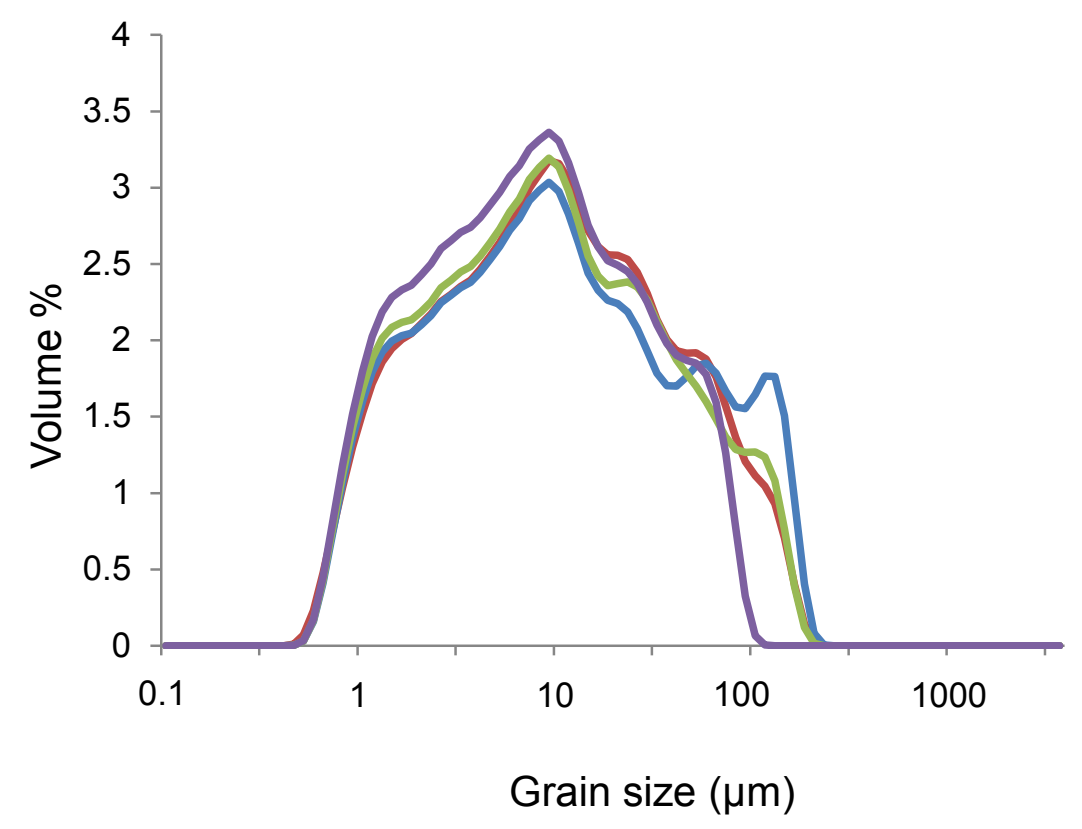

Fig. 2.21: WB1-25 example of the reproducibility from diluting concentrated samples for particle size analysis. 


\subsection{Spatial interpolation of aeolian sand and dust accumulation rate}

The accumulation rate of ASD from surface snow samples was calculated for each sampling site assuming it represented one year's accumulation on sea ice. Spatial interpolation in ArcGIS was used to estimate values of ASD accumulation at unsampled sites and create a contour map of the accumulation rates in the study area. The type of spatial interpolation used was kriging which is often used in soil science and geology to estimate unobserved values from observations of the same parameter at nearby locations (Burrough et al., 1986; Royle et al., 1981; Oliver 1990).

\subsubsection{Aeolian sand and dust provenance}

Provenance of ASD was analysed using a combination of radiogenic isotopic ratios and physical (mass and particle size) and mineralogical characteristics. The methodology for each analysis is described below.

\subsection{Radiogenic isotopic composition}

A selection of surface snow samples were shipped frozen to the University of MilanoBicocca, Italy for Coulter Counter MS3 analysis and three of these samples were selected for isotopic composition. These samples were selected as they cover a large spatial area and their isotopic signature is thought to be representative of Southern McMurdo Sound (samples MSI4 and MSI23) and Granite Harbour (GH9; Fig. 2.22). 

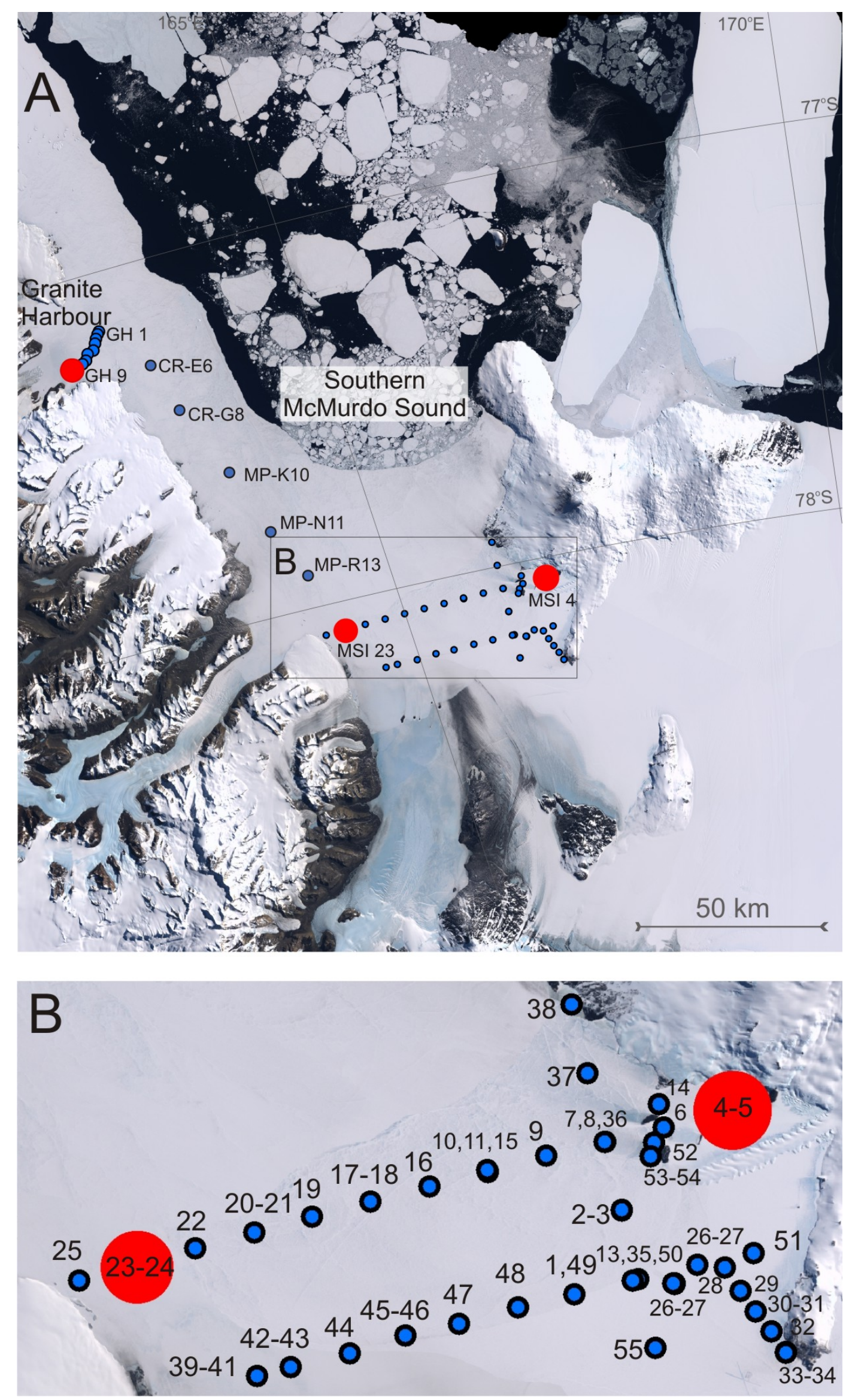

Fig. 2.22: A) Location of samples (red circles) for ASD provenance analysis using Sr and Nd isotopic ratios. B) Insert: MSI sample names. 


\section{Sr and Nd extraction from aeolian sand and dust}

An aliquot of MIS4 and MIS23 and the bulk GH9 sample had masses of 50, 43 and $29 \mathrm{ng}$ of $\mathrm{Nd}$ and 795, 518 and $228 \mathrm{ng}$ of $\mathrm{Sr}$ assuming an average upper continental crust (UCC) concentration for both elements of 350 and $26 \mathrm{ppm}$ respectively (Taylor and McLennan, 1985). Sediment-laid filters were sealed with parafilm in Petri dishes and transported to the Laboratory for Isotope Geology (LIG) at the Swedish Museum of Natural History, Stockholm where all radiogenic isotopic analyses was carried out by Dr Barbara Delmonte and Professor Per Anderson. Each filter was put into one pre-cleaned Corning tube filled with $\sim 10 \mathrm{ml} \mathrm{MQ}$ water, and micro-particles were removed from the filter through sonication. The liquid was then evaporated in a clean hood dedicated to chemical preparation of samples for $\mathrm{Rb}$ and $\mathrm{Sr}$, and $\mathrm{Sm}$ and $\mathrm{Nd}$ analyses.

At LIG a procedure for the chemical dissolution of dilute samples and the subsequent element separation of $\mathrm{Rb}-\mathrm{Sr}$ and $\mathrm{Sm}-\mathrm{Nd}$ using ion-exchange chromatography was previously developed and applied to mineral dust in ice cores (Delmonte et al., 2008; 2010a; 2010b). All dissolution and chemistry was carried out in Savillex Teflon beakers. The beakers were cleaned using $\mathrm{HCl}$ and $\mathrm{HNO}_{3}$ followed by rinsing with MQ water. The acids used for dissolution and chemistry were all Seastar (SS) high purity solutions. All laboratory materials and resins used were thoroughly cleaned and tested for blank levels before use. The samples were digested and purified following the protocol in Delmonte et al. (2008). Briefly, dissolution of ASD was made though an acid digestion ( $\sim 1.5 \mathrm{ml}$ acid mixture of $\mathrm{HNO}_{3}(\mathrm{SS})$ and $\mathrm{HF}(\mathrm{SS})$ and $\left.\mathrm{HClO}_{4}\right)$ and heated $\left(60^{\circ} \mathrm{C}\right)$ in closed vessels for 24 hours. The solution was evaporated to complete dryness on a hot plate and the residue dissolved in $4 \mathrm{ml} 6 \mathrm{M} \mathrm{HCl}$ (SS). Potential interfering elements ( $\mathrm{Fe}, \mathrm{Ba}, \mathrm{Rb}, \mathrm{Sm}, \mathrm{Ce}$, and $\mathrm{Pr}$ ) were separated through ionic chromatographic columns. $\mathrm{Sr}$ and $\mathrm{Nd}$ fractions were eluted and the total column yield for the Nd and Sr separation was $>95 \%$.

\section{Measurement of $\mathrm{Sr}$ and $\mathrm{Nd}$ isotopic ratios}

Isolated $\mathrm{Nd}$ and $\mathrm{Sr}$ fractions were spiked with ${ }^{150} \mathrm{Nd}$ and ${ }^{84} \mathrm{Sr}$ for isotope dilution determination of their concentrations and analysed on a thermal ionisation mass spectrometer (TIMS; TRITONC, Thermo Scientific Corp.) Neodymium was loaded mixed with Alfa Aesar graphite on double rhenium filaments and run as metal in static mode using rotating 
gain compensation. The isotopic ratio data was reduced assuming exponential fractionation. Calculated ${ }^{143} \mathrm{Nd} /{ }^{144} \mathrm{Nd}$ ratios were normalised to ${ }^{146} \mathrm{Nd} /{ }^{144} \mathrm{Nd}=0.7219$. Neodymium ratios are defined as:

$$
\mathrm{e}_{\mathrm{Nd}}(0)=\left[\left({ }^{143} \mathrm{Nd} /{ }^{144} \mathrm{Nd}\right)_{\text {sample }} /\left({ }^{143} \mathrm{Nd} /{ }^{144} \mathrm{Nd}\right)_{\mathrm{CHUR}}{ }^{-1}\right] \times 10^{4}
$$

using the present day chondritic uniform reservoir (CHUR) value for ${ }^{143} \mathrm{Nd} /{ }^{144} \mathrm{Nd}=0.512638$ (Jacobson and Wasserbury, 1980). The external precision for ${ }^{143} \mathrm{Nd} /{ }^{144} \mathrm{Nd}$ as determined from values for $15 \mathrm{ng}$ loads of a calibration standard was $30 \mathrm{ppm}$. Accuracy correction was not necessary as the mean ${ }^{143} \mathrm{Nd} /{ }^{144} \mathrm{Nd}$ ratio for $\mathrm{nNd} \beta$ standard was $0.511895 \pm 22(\mathrm{n}=20)$.

Strontium was analysed using a load of purified sample mixed with tantalum activator on a single rhenium filament. Two hundred 8 second integrations were recorded in multi-collector static mode, applying rotating gain compensation. Measured ${ }^{87} \mathrm{Sr}$ intensities were corrected for $\mathrm{Rb}$ interference using ${ }^{87} \mathrm{Rb} /{ }^{85} \mathrm{Rb}=0.38600$ and ratios were reduced using the exponential fractionation law and ${ }^{88} \mathrm{Sr} /{ }^{86} \mathrm{Sr}=8.375209$. The external precision for ${ }^{87} \mathrm{Sr} /{ }^{86} \mathrm{Sr}$ as determined from running $200 \mathrm{ng}$ loads of 987 standard was $8 \mathrm{ppm}(\mathrm{n}=12)$, while repeated measurements of prepared certified CIT \#39 sea water standard (100 ng loads) gave a reproducibility of \pm 0.0000083 or $12 \mathrm{ppm}(\mathrm{n}=14)$ which is taken to be the best estimate of the external precision. The ${ }^{87} \mathrm{Sr} /{ }^{86} \mathrm{Sr}$ ratio of $0.710245 \pm 06(\mathrm{n}=12)$ was corrected to the certified National Bureau of Standards (NBS) NBS 987. The total blank for Nd is $<10 \mathrm{pg}$ and for $\mathrm{Sr}<80 \mathrm{pg}$.

\subsubsection{Mineral composition}

Mineral composition, in particular the presence or absence of volcanic glass, was assessed by examining smear slides under a polarised light microscope. 


\subsubsection{Results - spatial trends in aeolian sand and dust accumulation rate and texture}

The location of sampling sites was specifically chosen to examine spatial trends in accumulation rate and texture as a guide to i) sediment source, ii) sediment dispersal mechanisms, and iii) to enable a calculation to be made of the mass of ASD entering the ocean on an annual basis. For that reason, changes in particle size distribution are considered in terms of north-south and east-west trends.

\subsubsection{Aeolian sand and dust accumulation rate}

Fig. 2.23A illustrates the spatial distribution of aeolian sediment in surface snow on the sea ice in Southern McMurdo Sound in 2009. ASD accumulation rates are available in Appendix 1. Elevated ASD concentrations are evident downwind of exposed unconsolidated sediment, for example ASD plumes are visible downwind of the debris band. There are two regions where surface snow contains particularly high ASD concentrations. The first is the ASD plume just north of the debris band (e.g. Kellogg et al., 1990) where the ASD accumulation rate lies between $26.00-83.24 \mathrm{~g} \mathrm{~m}^{-2} \mathrm{yr}^{-1}$ (Fig. 2.23A) and decreases exponentially to $0.70 \mathrm{~g}$ $\mathrm{m}^{-2} \mathrm{yr}^{-1}$ at Granite Harbour, $120 \mathrm{~km}$ to the north, and also to the east and west of the central axis of the ASD plume. 


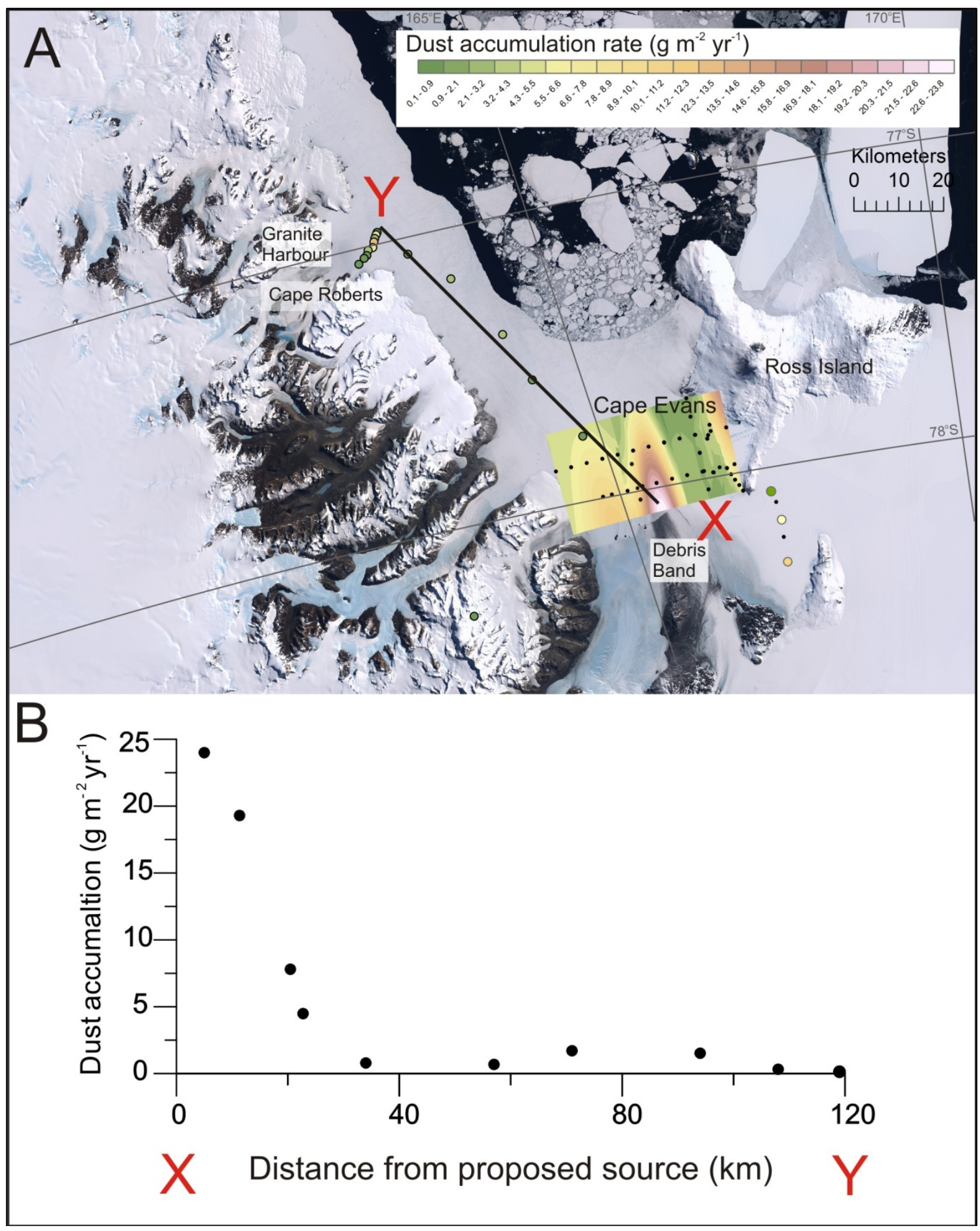

Fig. 2.23: Aeolian sand and dust accumulation in McMurdo Sound. A) Spatial distribution of total ASD accumulation rate $\left(\mathrm{g} \mathrm{m}^{-2} \mathrm{yr}^{-1}\right)$ on sea ice in McMurdo Sound in 2009 (surface samples, the present study) combined with measurements made in 2008 (core samples, the present study), data collected in 2008 from Atkins and Dunbar (2009). B) ASD accumulation rates with distance north from the debris bands (transect XY). Black dots: Snow sampling sites, other sites colour coded by mass accumulation rate. 
A second area of high ASD accumulation occurs on the sea ice at Cape Evans. Where 5 samples (MSI 2, 6, 8, 11 and 12) were dominated by a pale white coloured sediment and 9 samples contained a number of prominent whitish coloured particles (MSI 3, 9, 17, 20, 25, 31, 34, 43 and GH4), including a sample as far west as Granite Harbour. More detailed SEM (Fig. 2.24) and EDS (Fig. 2.25) analysis of MSI6 suggests the bulk of this material is calcite as it has high concentrations of $\mathrm{Ca}$ (Fig. 2.25); it also has high birefringence under polarised light (Fig. 2.24) and reacts with dilute $\mathrm{HCl}$.
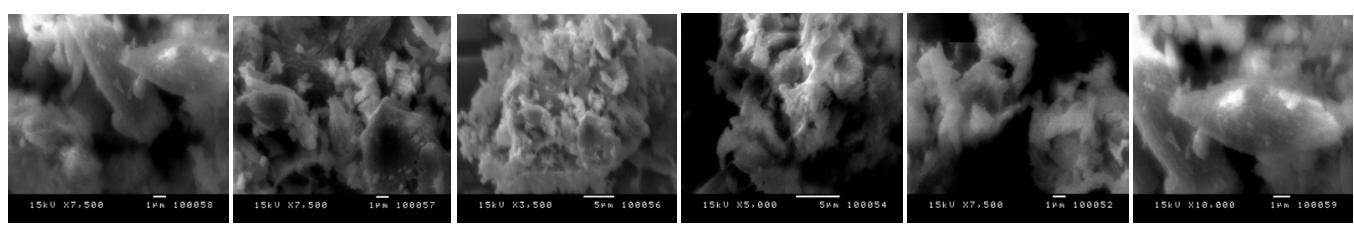

Fig. 2.24: Scanning Electron Microscope images of calcite particles from MSI6 found in surface snow at Cape Evans.

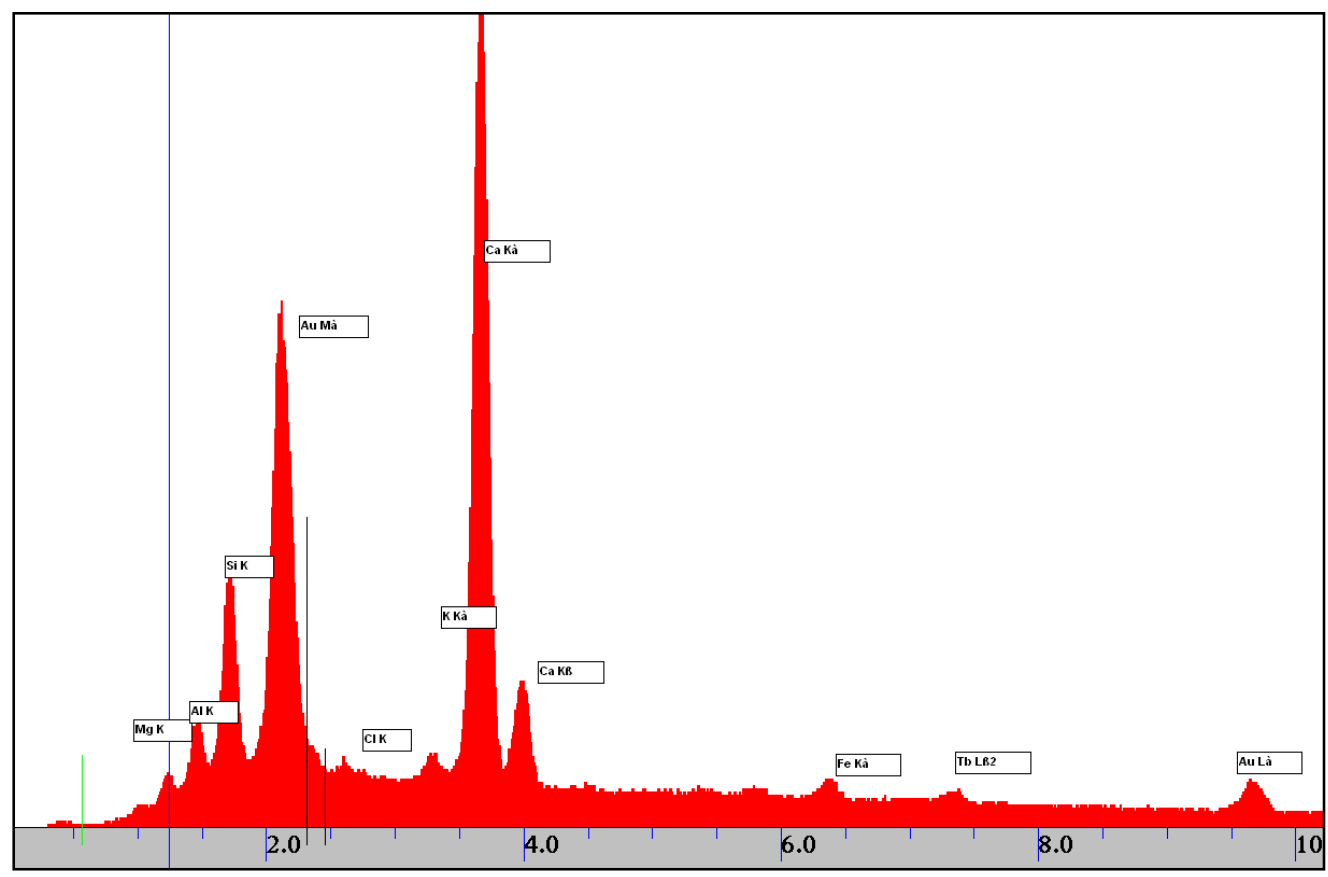

Fig. 2.25: Energy dispersive spectrum of elements in sample MS16 from Cape Evans. The high counts of Ca suggest the bulk of this material is calcite with a few aluminosilicate particles ( $\mathrm{Si}, \mathrm{Al}, \mathrm{Fe}$ ). Au counts are from the gold coating required for SEM analysis.

Keys (1980) reports the occurrence of calcite precipitates to be widespread but not in high concentrations on the sea ice at Cape Evans. Claridge and Campbell (1977) also report calcite veneers at Hutt Point Peninsula associated with carbonate-rich dust. The possible sources of this material are: i) it has been eroded from $\mathrm{CaCO}_{3}$ rocks in the MDV by normal weathering 
processes, such as in eastern Taylor Valley near the Koettlitz Glacier, Victoria Valley and Wright Lower Valley which contain outcrops of Skelton Group meta-sediments that are rich in marble; ii) it is a surface salt deposit associated with weathering of igneous rocks on Ross Island, of which are its main substrate; or iii) it is derived from fossiliferous marine sediments (Keys, 1980). The presence of euhedral crystals of calcite (Fig. 2.24) in high concentrations far from potential detrital sources suggests this material is probably derived from a local precipitate, although its particle size distribution (Chapter 2.2.2.2) and the presence of other minerals also suggest it has been deposited by wind. Therefore the mass of this material is included in the total ASD accumulation rate estimated for sediment blowing onto the sea ice McMurdo Sound.

Fig. 2.26 illustrates the accumulation rate of ASD $<10 \mu \mathrm{m}$ with distance from the McMurdo Ice Shelf debris band. 


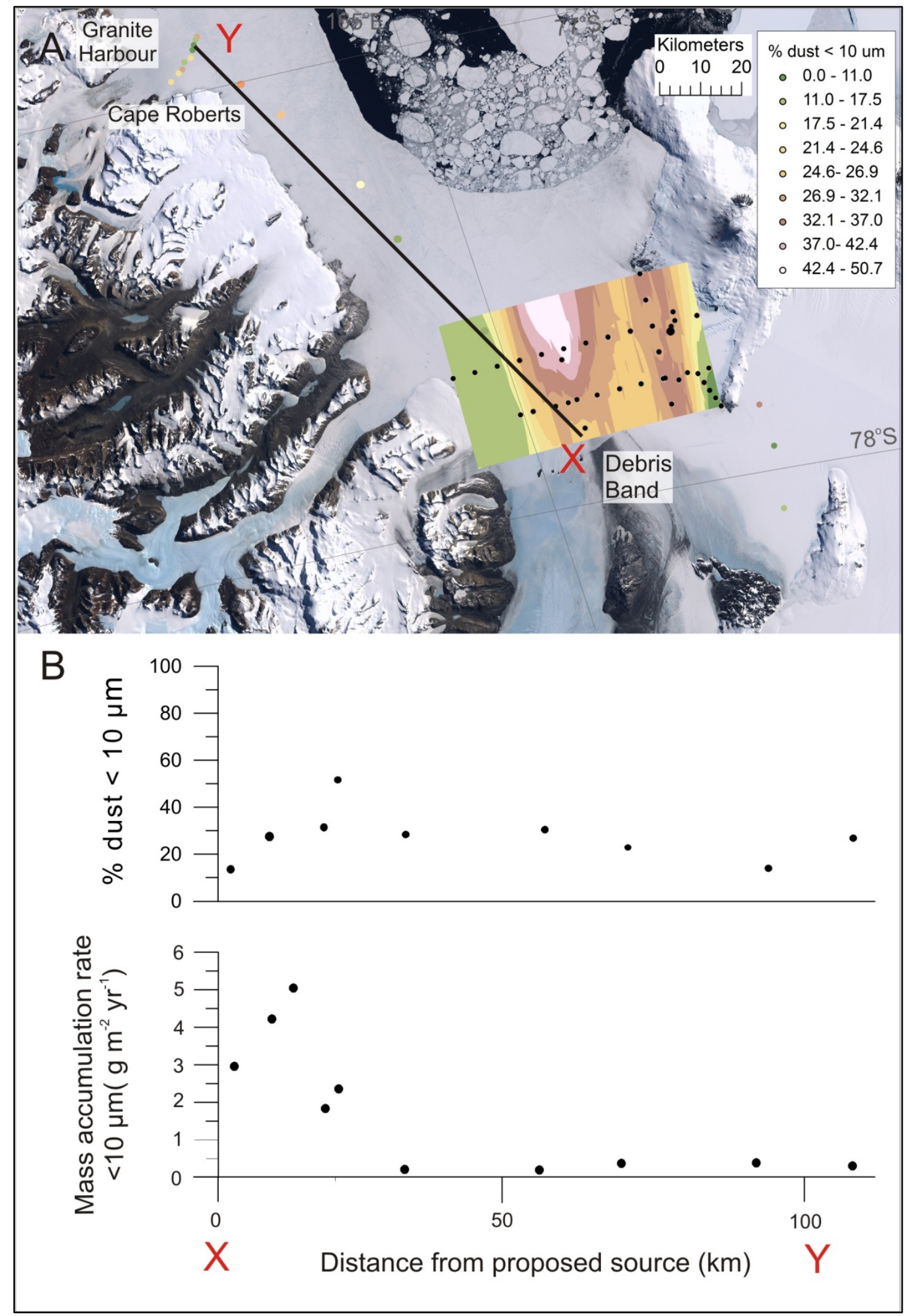

Fig. 2.26: Mass accumulation rate for the fraction of dust $<10 \mu \mathrm{m}$ in McMurdo Sound. A) Spatial distribution of the percentage of dust $<\mathbf{1 0} \mu \mathrm{m}$ on sea ice in McMurdo Sound in 2009 (surface samples, the present study) combined with measurements made in 2008 (core samples, the present study), data collected in 2008 from Atkins and Dunbar (2009). B) Distribution of the fraction $<10 \mu m$ and mass accumulation rate $<\mathbf{1 0} \boldsymbol{\mu m}$ of ASD with distance north from the debris bands (transect XY). Black dots: Snow sampling sites, other sites colour coded by mass accumulation rate. 


\subsubsection{Spatial results - sediment texture}

\subsection{General features}

All samples are mixtures of silt and sand-sized material with little $(<5 \%)$ clay and no gravel (Fig. 2.27). Following the classification of Folk (1980) samples fall between silty sand, sandy silt and silt and are poorly sorted to extremely poorly sorted. Often the distribution is bi- or poly-modal with a predominant very fine sand or coarse silt primary mode $(30-185 \mu \mathrm{m})$ and a much more poorly defined secondary mode of fine medium silt size in the fine-particled 'tail' of the distribution (Fig. 2.27). These observations are consistent with sediment textures reported by Atkins and Dunbar (2009) for ASD accumulating north of the debris band, but contain significantly more silt-sized material when compared to textural data reported for ASD on sea ice in New Harbour by Bentley (1979). Particle size data and statistics are available in Appendix 3A-D.

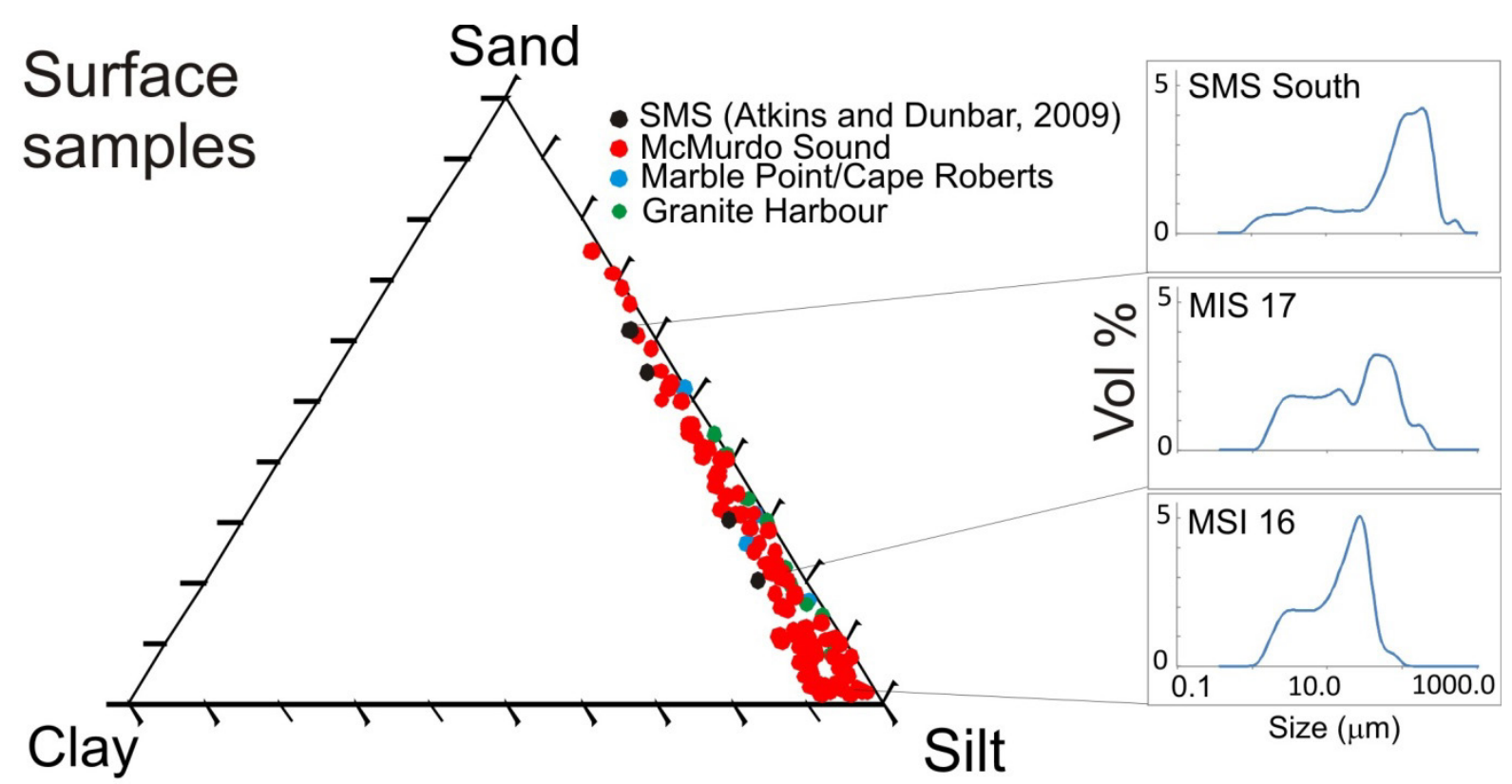

Fig. 2.27: Ternary plots of ASD from surface snow samples showing the proportion of sand, silt and clay. Top right: Representative histograms of particle size frequency for selected samples downwind from the debris bands, including data from Atkins and Dunbar (2009).

\subsection{Spatial trends}

Changes in particle size distribution are considered in terms of north-south (transect XY) and east-west (ZZ', WW' and YY') trends (Figs. 2.28-2.31). 


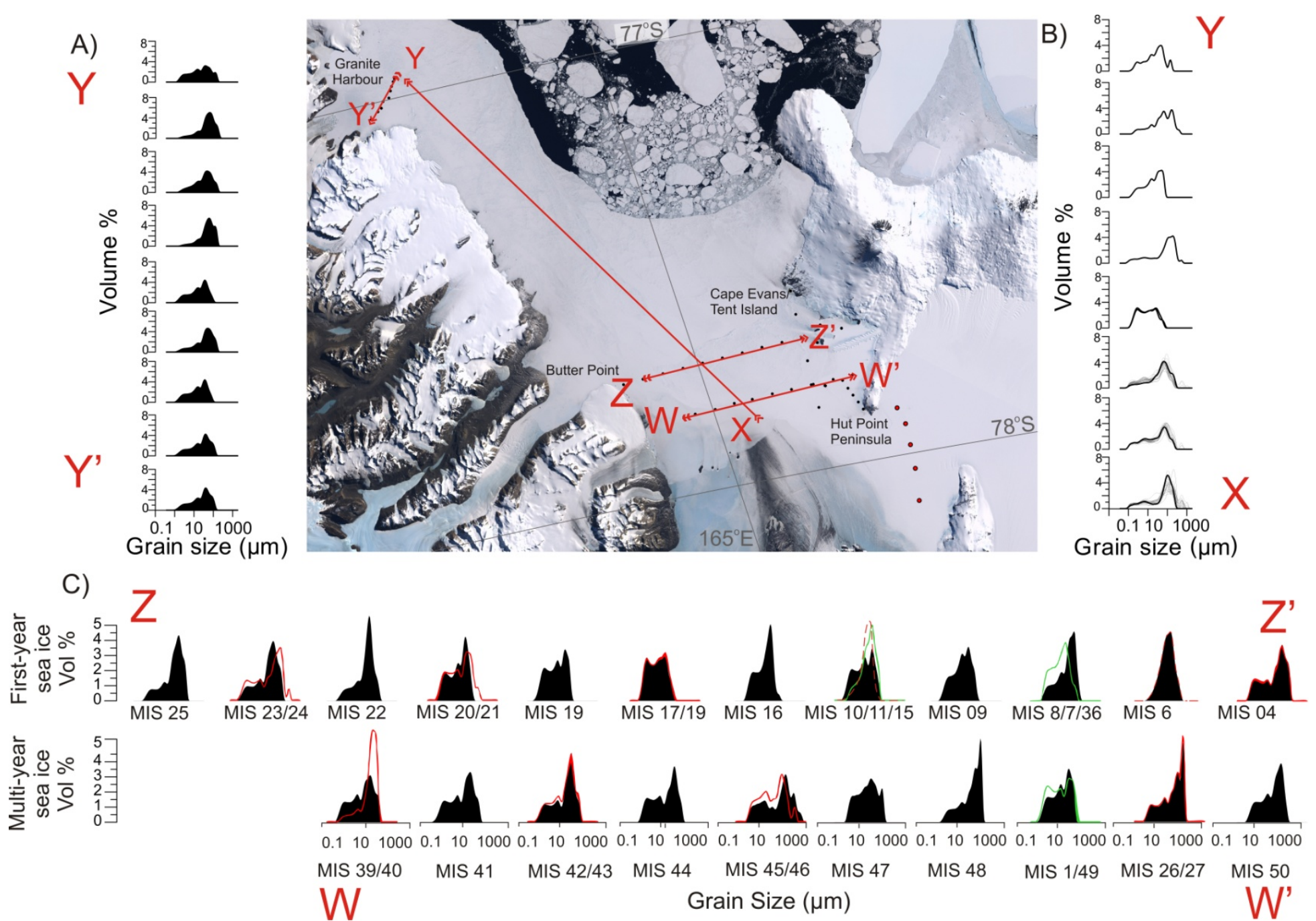

Fig. 2.28: Percentage frequency curves of particle size distribution of ASD in surface snow on McMurdo Sound sea ice in 2009. A) Granite Harbour transect YY'. B) Downwind transect XY from debris band to Granite Harbour, with data from Atkins and Dunbar (2009). C) West-east transect ZZ' of first-year sea ice and multiyear sea ice transect ZZ". Red curves: duplicate sample same day, green curve: duplicate sample second day, red dashed: calcite samples (duplicate from same day sampling). Refer to Fig. 2.22 for sample names. 


\section{North-south trends in size - McMurdo Sound to Granite Harbour}

The frequency curves downwind (i.e. to the north) of the dirty ice along transect XY (Fig. 2.28B) show a distinctive bimodal size frequency, with a well defined fine to very fine sand mode and a broad silt mode. The size of the primary (sand) mode deceases sharply from $\sim 130$ $\mu \mathrm{m}$ to $76 \mu \mathrm{m} 5$ and $18 \mathrm{~km}$ north of the debris band respectively (Atkins and Dunbar, 2009) and thereafter oscillates between $25-69 \mu \mathrm{m}$ between 20 and $150 \mathrm{~km}$ north of the debris bands, while the silt mode remains relatively constant at $2.7 \mu \mathrm{m}$ (Fig. 2.29). Simultaneously the percentage of sand also decreases from south to north (Fig. 2.29). Furthermore, the percentage of particles $<10 \mu \mathrm{m}$ increases from $12 \%$ to $26 \%$ to $30 \%$ within $18 \mathrm{~km}$ north of the debris bands (Atkins and Dunbar, 2009). From Marble Point to Granite Harbour the percentage of particles $<10 \mu \mathrm{m}$ fluctuates between 25 to $29 \%$ (Fig. 2.29).

\section{East-west trends in size - across Granite Harbour}

Granite Harbour samples are sandy silts (Fig. 2.27) that are very poorly sorted and strongly fine skewed with a prominent primary mode between $27-63 \mu \mathrm{m}$ and often a poorly defined secondary silt mode at $15 \mu \mathrm{m}$ (Fig. 2.28A). The samples become better sorted seaward of Granite Harbour. The primary silt mode increases from $40 \mu \mathrm{m}$ in the west of the YY' transect (Figs. 2.28A and 2.30) towards the sound until GH4, in the middle of transect YY', where the mode reaches a maximum at $58 \mu \mathrm{m}$ and then decreases further east towards the sound to 27 $\mu \mathrm{m}$. The samples containing the greatest mass accumulation rate of ASD in the centre of the Granite Harbour transect also contain the coarsest ASD (Fig. 2.28A).

\section{East-west trends in size - across McMurdo Sound}

Southern McMurdo Sound samples are silt to silty sand in texture and are poorly to extremely poorly sorted, with a strong fine skew (Fig. 2.28C). The coarsest sand mode along the WW' and $\mathrm{ZZ}$ ' transects is $185 \mu \mathrm{m}$ (at MIS 45) decreasing to $\sim 35 \mu \mathrm{m}$ in easterly and $120 \mu \mathrm{m}$ in westerly directions from this location (Fig. 2.31). The primary sand mode decreases in a south or south westerly direction from the transect WW' to the transect ZZ' (Figs. 2.28C and 2.31). Around the islands west of Cape Evans and also east of MDV there is a local concentration of sand sized particles with coarse sand modes. The size distribution of insoluble salts is significantly different to the other Southern McMurdo Sound samples. Here calcite particles dominate samples MIS 6, 8 and 11 and are well sorted, strongly fine skewed silt. They have a single silt mode of $23 \mu \mathrm{m}$ (Fig. 2.28C). 


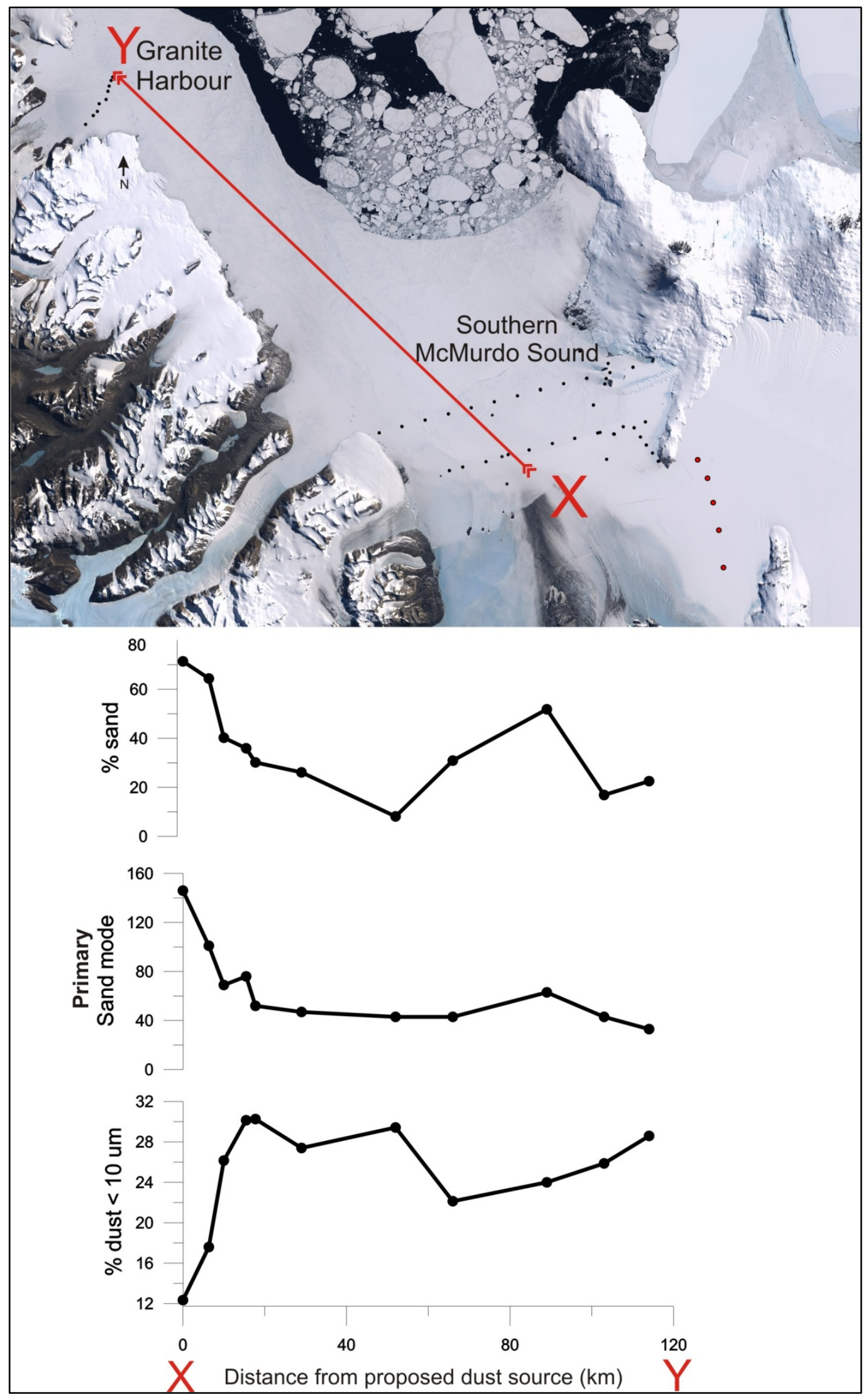

Fig. 2.29: North-south transect $X Y$ of particle size trends in the percentage of sand, the primary mode and the percentage of ASD $<10 \mu \mathrm{m}$. 


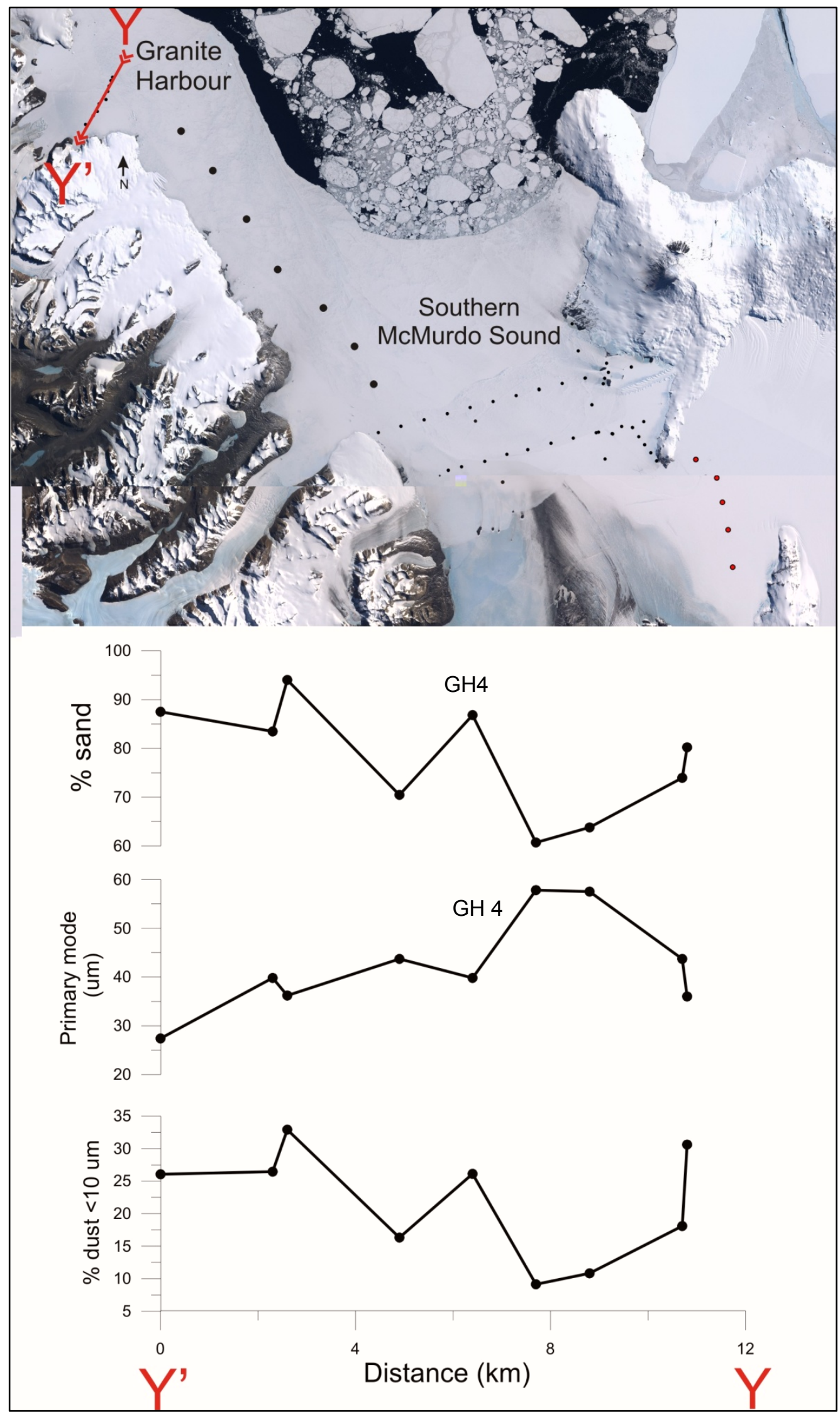

Fig. 2.30: Granite Harbour west-east transect $Y Y^{\prime}$ of particle size trends in the percentage of sand, the primary mode and the percentage of ASD $<10 \mu \mathrm{m}$. 


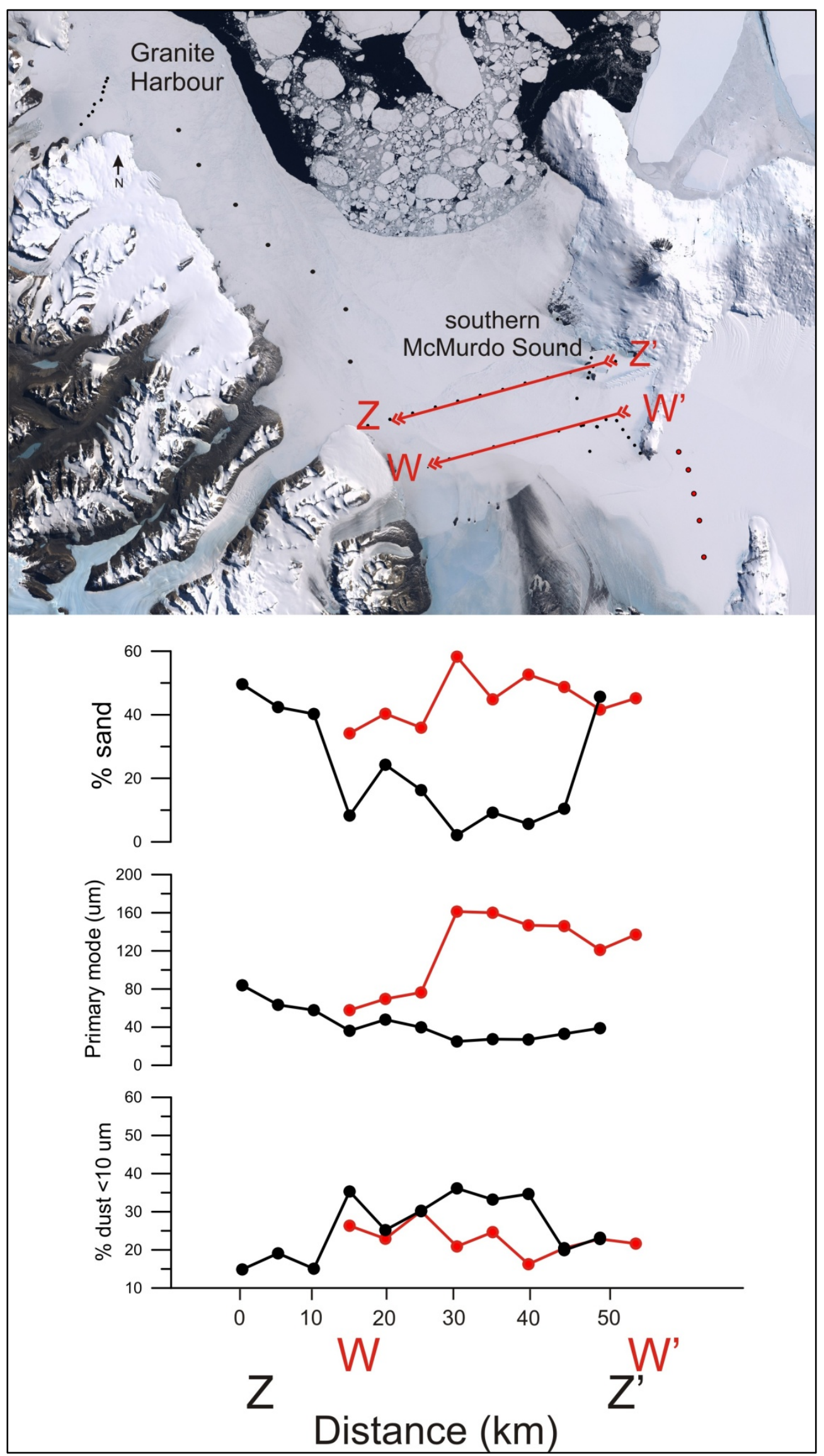

Fig. 2.31: Southern McMurdo Sound transects ZZ' (red) and WW' (black) of particle size trends in the percentage of sand, the primary mode and the percentage of ASD $<10 \mu \mathrm{m}$. 


\subsubsection{Radiogenic isotopic signature of McMurdo Sound aeolian sand and dust}

The $\mathrm{Sr}$ and $\mathrm{Nd}$ isotopic ratios of ASD in McMurdo Sound measured in the present study are reported in Table 2.2 and Fig. 2.32, and compared with previous PSA samples from Delmonte et al. (2004a; 2010a). Due to the large particle size and high ASD accumulation rate found within the samples the transport distance must be limited, therefore only local PSAs are considered. The two samples from Southern McMurdo Sound show a clear volcanic fingerprint of the MVG without marked differences between them however, the sample from Granite Harbour conversely is more similar to the crustal signature of SVL (Fig. 2.32).

A)

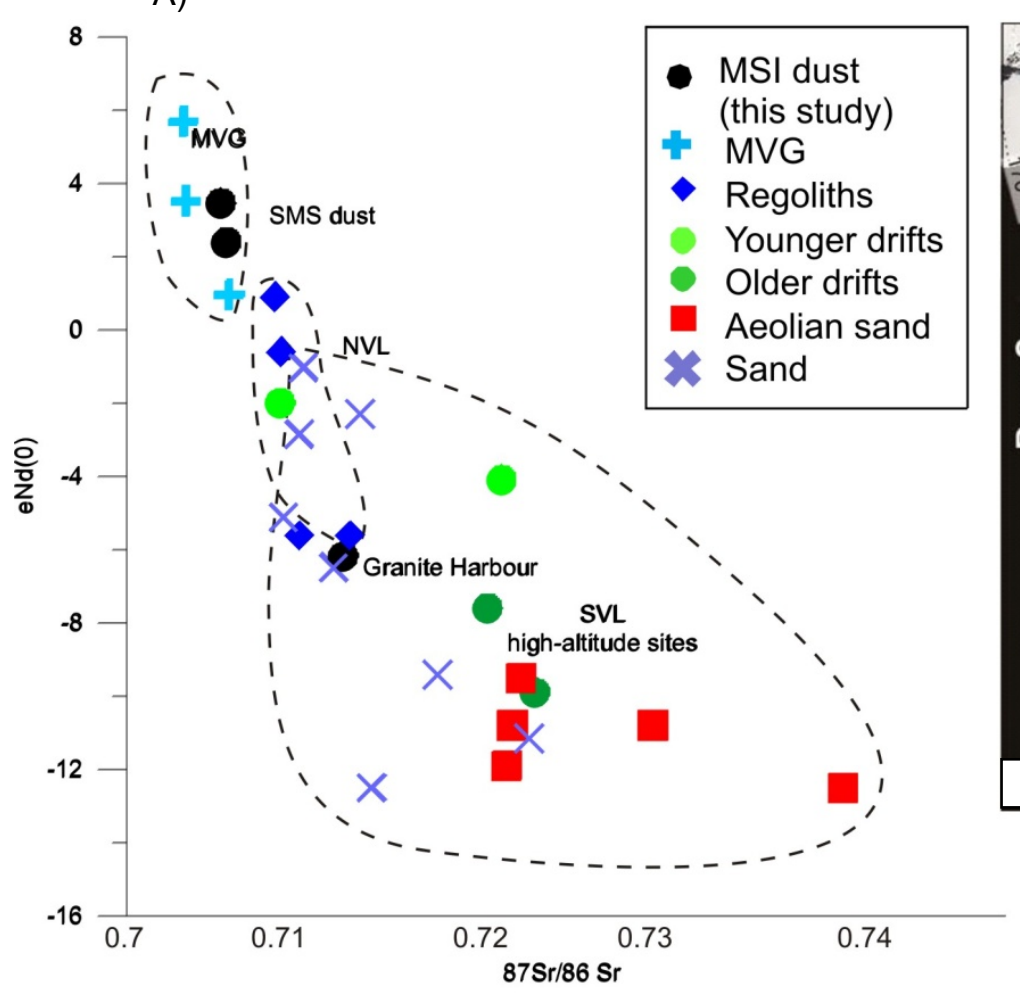

B)

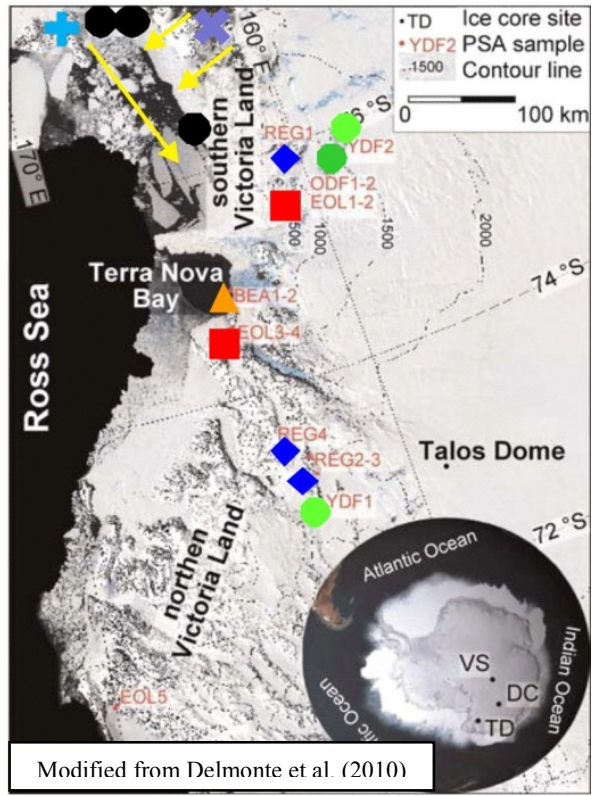

Fig 2.32: A) Isotopic composition of samples in the present study (black circles) compared with PSA samples from the literature (Delmonte et al., 2004a; 2010a). MVG: McMurdo Volcanic Group, NVL: northern Victoria Land, SVL: southern Victoria Land. B) Location of PSA samples in the Victoria Land coastline. Descriptions of PSA samples in Delmonte et al. (2004a; 2010a). Yellow arrows: proposed ASD transport path in McMurdo Sound. 
Table 2.2: Sr and Nd isotopic ratios for McMurdo Sound aeolian sand and dust.

\begin{tabular}{|c|c|c|c|c|c|c|c|c|c|c|c|c|c|c|c|c|}
\hline $\begin{array}{c}\text { Sample } \\
\text { Snow on } \\
\text { sea ice }\end{array}$ & $\begin{array}{l}\mathrm{C}_{\mathrm{Nd}} \\
\mathrm{ppm}\end{array}$ & $\begin{array}{c}\text { Max } \\
\text { load } \\
\text { Nd } \\
(n g)\end{array}$ & $\begin{array}{l}{ }^{143} \mathrm{Nd} / \\
{ }^{144} \mathrm{Nd}\end{array}$ & & $2 s_{\text {mean }}$ & $\mathbf{e}_{1}(0)$ & & $2 s^{2}$ & $\begin{array}{l}{ }^{87} \mathrm{Sr} / \\
{ }^{87} \mathrm{Sr}\end{array}$ & & $\begin{array}{c}2 \mathbf{s}_{\text {mea }} \\
n\end{array}$ & $\begin{array}{c}{ }^{87} \mathrm{Sr} / \\
{ }^{86} \mathrm{Sr} \\
\text { correct } \\
\text { ed }^{4}\end{array}$ & $2 s^{3}$ & $\begin{array}{c}\mathrm{C}_{\mathrm{Sr}} \\
\mathrm{ppm}\end{array}$ & $\begin{array}{c}\text { Max } \\
\text { load } \\
\text { Sr } \\
(n g)\end{array}$ & $\begin{array}{l}{ }^{85} \mathbf{R b} / \\
{ }^{86} \mathrm{Sr}\end{array}$ \\
\hline $\begin{array}{l}\text { GH 99 } \\
\text { (bulk) }\end{array}$ & 36.5 & 29 & $\begin{array}{c}0.5123 \\
21\end{array}$ & \pm & $\begin{array}{c}0.0000 \\
05\end{array}$ & -6.18 & \pm & $\begin{array}{c}0.3 \\
0\end{array}$ & $\begin{array}{c}0.71 \\
2260\end{array}$ & \pm & $\begin{array}{r}0.00 \\
0005\end{array}$ & $\begin{array}{c}0.7122 \\
88\end{array}$ & $\begin{array}{l}0.00 \\
0008\end{array}$ & 291 & 228 & $\begin{array}{c}0.000 \\
001\end{array}$ \\
\hline $\mathrm{MSI} 23 \mathrm{H}$ & 55.5 & 43 & $\begin{array}{c}0.5127 \\
60\end{array}$ & \pm & $\begin{array}{c}0.0000 \\
06\end{array}$ & 2.38 & \pm & $\begin{array}{c}0.3 \\
0\end{array}$ & $\begin{array}{l}0.70 \\
5608\end{array}$ & \pm & $\begin{array}{l}0.00 \\
0005\end{array}$ & $\begin{array}{c}0.7056 \\
36\end{array}$ & $\begin{array}{l}0.00 \\
0008\end{array}$ & 672 & 518 & $\begin{array}{c}0.000 \\
001\end{array}$ \\
\hline MSI4H & 51.4 & 50 & $\begin{array}{c}0.5128 \\
15\end{array}$ & \pm & $\begin{array}{c}0.0000 \\
04\end{array}$ & 3.45 & \pm & $\begin{array}{c}0.3 \\
0\end{array}$ & $\begin{array}{l}0.70 \\
5303\end{array}$ & \pm & $\begin{array}{l}0.00 \\
0005\end{array}$ & $\begin{array}{c}0.7053 \\
31\end{array}$ & $\begin{array}{c}0.00 \\
0008\end{array}$ & 818 & 795 & $\begin{array}{c}0.000 \\
001\end{array}$ \\
\hline
\end{tabular}

${ }^{1)} \mathrm{e}_{\mathrm{Nd}}(0)=\left[\left({ }^{143} \mathrm{Nd} /{ }^{144} \mathrm{Nd}\right)_{\text {sample }} /\left({ }^{143} \mathrm{Nd} /{ }^{144} \mathrm{Nd}\right)_{\mathrm{CHUR}}-1\right] \times 10^{4} ; \quad$ CHUR, chondritic uniform reservoir with ${ }^{143} \mathrm{Nd} /{ }^{144} \mathrm{Nd}=0.512638$.

${ }^{2)}$ The external precision for ${ }^{143} \mathrm{Nd} /{ }^{144} \mathrm{Nd}$ as judged from values for $15 \mathrm{ng}$ loads of a calibration standard was 30 ppm.

${ }^{3)}$ The external precision for ${ }^{87} \mathrm{Sr} /{ }^{86} \mathrm{Sr}$ as judged from running $200 \mathrm{ng}$ loads of 987 standard was 8 ppm $(\mathrm{n}=12)$, while repeated measurements of prepared CIT \#39 sea water (100 ng loads) gave a reproducibility of \pm 0.0000083 or $12 \mathrm{ppm}(\mathrm{n}=14)$ which is taken to be the best estimate of the external precision.

${ }^{4)}$ Corrected to a NBS $987{ }^{87} \mathrm{Sr} /{ }^{86} \mathrm{Sr}$ ratio of 0.710245 . 


\subsection{Temporal distribution of aeolian sand and dust in Windless Bight from firn cores}

\subsubsection{Methodology}

\subsubsection{Sample collection - firn core drilling}

The series of three Windless Bight firn cores of approximately $3 \mathrm{~m}$ in length were recovered from the northern margin of the McMurdo Ice Shelf in November 2008 along a transect perpendicular to the local snow accumulation gradient (Figs. 2.1 and 2.16). Based on historical data, snow accumulation rates at these sites were expected to be $\sim 30 \mathrm{~cm}$ per year (McCrea, 1984) yielding a decade long record. Once collected the cores were cut into one meter lengths and sealed in layflat plastic sleeves and stored and monitored at $-30{ }^{\circ} \mathrm{C}$ until they were transported to the New Zealand Ice Core Research Laboratory, at GNS Science, New Zealand where they are stored at $-35^{\circ} \mathrm{C}$.

\subsubsection{Sample preparation for aeolian sand and dust analyses}

Sampling of the cores Windless Bight (WB) core 1 (WB1), core 3 (WB3) and core 5 (WB5) (Fig. 2.16) was carried out in the processing freezer of the New Zealand Ice Core Research Facility. Personnel wore protective clothing to avoid contaminating the cores. Each core was then visually logged on an illuminated light table to identify snow crystal structures, cracks, ASD layers, melt layers and hoar layers. To determine the chronology, WB1 and WB3 were subsequently cut into consecutive $5 \mathrm{~cm}$ pieces and WB5 into $2.5 \mathrm{~cm}$ lengths using an ultraclean bandsaw (Fig. 2.33), yielding a total of 58, 56 and 115 samples respectively. This sample resolution was chosen to ensure a minimum of $\sim 6$ samples per year to resolve the annual cycle of $\delta^{18} \mathrm{O}$ variability used for dating the core. The stratigraphy error associated with each sample is $<2 \mathrm{~cm}$ which arises from the difficulty of cutting the core where cracks are present.

WB3 was used for methodology development. Each $5 \mathrm{~cm}$ sample from this core was split into four co-registered aliquots for i) $\delta^{18} \mathrm{O}$ and $\delta \mathrm{D}$ isotopes, ii) particle size distribution of lithogenic material (Beckman-Coulter LS13320), iii) sediment weight, and iv) archiving. Subsequently each $5 \mathrm{~cm}$ sample from WB5 and WB1 were split into two aliquots for i) $\delta^{18} \mathrm{O}$ 
and $\delta \mathrm{D}$ isotopes, ii) sediment weight and particle size distribution. Each aliquot was weighed on an electronic balance and heat-sealed in pre-weighed and labelled sterile plastic bags. Samples were melted over night at room temperature except for archived aliquots which are kept frozen.
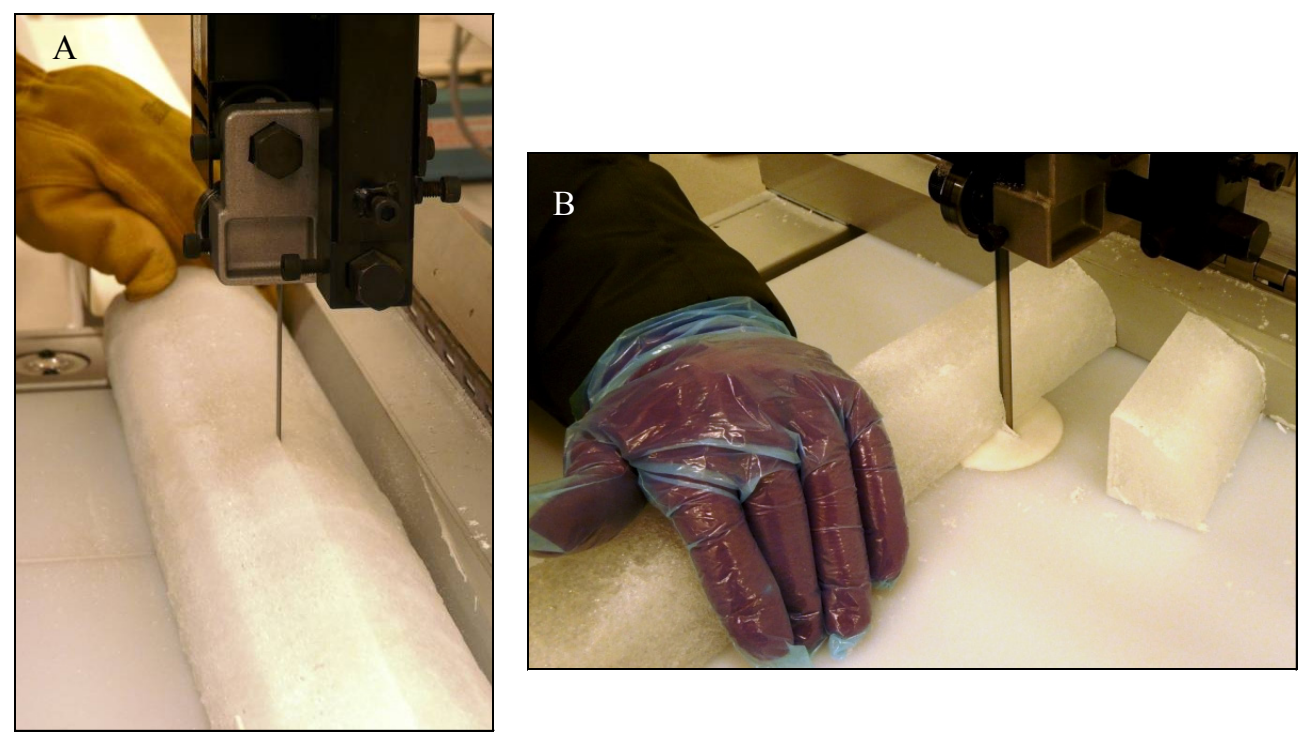

Fig. 2.33: A) Firn core sub-sampling in the cold room at the New Zealand Ice Core Facility, GNS Science. B) Cutting the core into $5 \mathrm{~cm}$ aliquots e.g. WB3. Source: Schuck (2009).

\subsubsection{Sample filtration}

The weight of ASD in each melted aliquot of the core was measured by draining meltwater through pre-weighed and dried $0.2 \mu \mathrm{m}$ polycarbonate filters using a Millipore vacuum filtration system (Chapter 2.2.1.2.2). The filters showed consistent dry weights of $0.0151 \mathrm{~g} \pm$ 0.0002 g. A few samples were contaminated with fibres, most likely from core handling in the field, and these were carefully removed from the dried sediment-laid filter paper using tweezers.

\subsubsection{Particle size distribution}

\section{Windless Bight core 3}

Centrifuge tubes were rinsed three times with MQ water in the clean room. Each melted sample from one concurrent aliquot were carefully poured into centrifuge tubes. The edge of 
the sample bag was cut to act as a funnel to rinse any particles remaining on the inside of the bag. The samples were centrifuged at $4000 \mathrm{rpm}$ for 4 minutes. Because the MLM has a volume of $12 \mathrm{ml}$ and headspace is required for rinsing the centrifuge tube, excess water was removed with a cleaned pipette under a fume hood, leaving $5 \mathrm{ml}$ of water and ASD in the base of the centrifuge tube. The excess water was checked under the microscope for particles that might have been pipetted, but no particles were found, indicating that the centrifuge velocity and time was sufficient to concentrate efficiently all particles in the bottom of the vials. The excess snow melt was then disposed off according to Ministry of Agriculture and Forestry (MAF) biosecurity regulations.

Particle size analysis was determined using the MLM described in Chapter 2.2.1.2.2. The centrifuged samples were carefully poured into the MLM cell (after a background of MQ water is measured) and the centrifuge tube was flushed with MQ water to obtain any remaining particles. During the first measurements, it was apparent that the samples were still contaminated with fibres (most likely from core handling in the field) and these were biasing the particle size distribution towards coarser particles. Particles size analysis from a nearby ice core at Windless Bight (WB06/07) showed that particles were $<250 \mu \mathrm{m}$ (Dunbar et al., 2009) and to remove this extraneous material it was therefore necessary to sieve the samples using a $250 \mu \mathrm{m}$ mesh.

\section{Windless Bight cores 1 and 5}

Polycarbonate filters were treated using the same method as for WB1 and WB5 and measured using the MLM. The methodology study using WB3 showed that low laser obscuration in dilute samples produced biased particle size data leaving gaps in the record. Therefore, it was sometimes necessary for consecutive aliquots downcore to be grouped together to gain an obscuration $>3 \%$ (Chapter 2.2.1.2.1).

\subsubsection{Stable Isotope Analyses}

The melted samples were measured for $\delta^{18} \mathrm{O}$ and $\delta \mathrm{D}$ isotope analysis in the Stable Isotope Laboratory, GNS Science in order to date the cores. WB3 and WB5 were analysed using traditional mass spectrometry, while WB1 and WB5 (re-analysed) were analysed using the more efficient technique laser absorption spectroscopy. 


\section{Stable isotope mass spectrometry}

Oxygen stable isotopes are analysed on a GVI AquaPrep attached to a GVI IsoPrime mass spectrometer by the classical equilibration method whereby $400 \mu 1$ of the melted sample is equilibrated with $3 \mathrm{ml}$ of headspace flushed with $\mathrm{CO}_{2}$ for 24 hours at $25.5{ }^{\circ} \mathrm{C}$. The $\mathrm{CO}_{2}$ is then extracted and analysed by dual inlet on the IsoPrime mass spectrometer. All oxygen results are reported with respect to VSMOW, normalized to the internal standards: INS11, INS9 and MM1 with reported values of $-0.3 \%$, $-17.3 \%$, and $-29.4 \%$ respectively. The analytical precision for these measurements is $0.1 \%$.

Hydrogen stable isotopes are analysed on a GVI PyrOH attached to a GVI IsoPrime mass spectrometer by direct injection over hot chromium. Five $\mu l$ of melted sample was injected into a helium stream through a quartz reactor filled with chromium granules and quartz chips held at $1050{ }^{\circ} \mathrm{C}$, where it is reduced to $\mathrm{H}_{2}$ gas. The $\mathrm{H}_{2}$ gas is then analysed by continuous flow mode on the IsoPrime mass spectrometer. All hydrogen results are reported with respect to VSMOW, normalized to the internal standards: INS11, INS9 and MM1 with reported values of $-3.3 \%,-136.5 \%$, and $-231.5 \%$ respectively. The analytical precision for these measurements is $1.0 \%$.

In addition, the deuterium excess ( $d$ excess) values were calculated from the $\delta^{18} \mathrm{O}$ and $\delta \mathrm{D}$ values. The precision of the $d$ excess is $1.004 \%$, calculated using the equation:

$$
\text { precisiond } d_{\text {excess }}=\sqrt{\left(\text { precision } d^{18} O\right)^{2}+(\text { precisiond } d)^{2}}
$$

\section{Laser Absorption Spectroscopy}

WB5 and WB1 were analysed using a Los Gatos Research (LGR) Liquid-Water Isotope Analyser (www.lgrinc.com). The LGR Liquid Water Isotope Analyser works on the basis of laser absorption spectroscopy whereby $\delta^{18} \mathrm{O}$ and $\delta \mathrm{D}$ are measured simultaneously by a nearinfrared laser system which quantifies spectral features of molecules in a gas passed through an optical measurement cavity. $\delta^{18} \mathrm{O}$ and $\delta \mathrm{D}$ are reported with respect to VSMOW and normalized to three internal standards described above. For each aliquot of the firn core, six injections were taken, the first two are disregarded due to memory effects and the remainder averaged. The analytical precision for this instrument is $<0.2 \%$ for $\delta^{18} \mathrm{O}$ and $0.6 \%$ for $\delta \mathrm{D}$ resulting in an uncertainty of $\pm 0.82 \%$ on $d$ excess. Fig. 2.34 illustrates the agreement 
between the two instruments for WB5 and the slightly higher precision of the liquid water isotope analyser.

Rhodes (2011) tested the comparability of isotope ratio measurements conducted on the two instruments and identified an offset between the datasets measured in the different instruments, most notably for $\delta \mathrm{D}$. Measurements of $\delta \mathrm{D}$ and $\delta^{18} \mathrm{O}$ on the two instruments produced a significant offset between the calculated values of $d$ excess for samples from this study (Fig. 2.34). The $\delta \mathrm{D}$ and $\delta^{18} \mathrm{O}$ values on the LGR liquid water isotope analyser were corrected to make them comparable to the mass spectrometer values using the linear relationship established by analysing samples on both instruments (Fig. 2.34). Deuterium excess values were then calculated from the corrected $\delta \mathrm{D}$ and $\delta^{18} \mathrm{O}$ datasets. A revised analytical uncertainty for $d$ excess, which takes into account the errors on both instruments, is calculated as: $\sqrt{1.04^{2}+0.82^{2}}=1.32 \%$. This value is applied when the entire record encompassing stable isotope ratios measured by both instruments is considered.

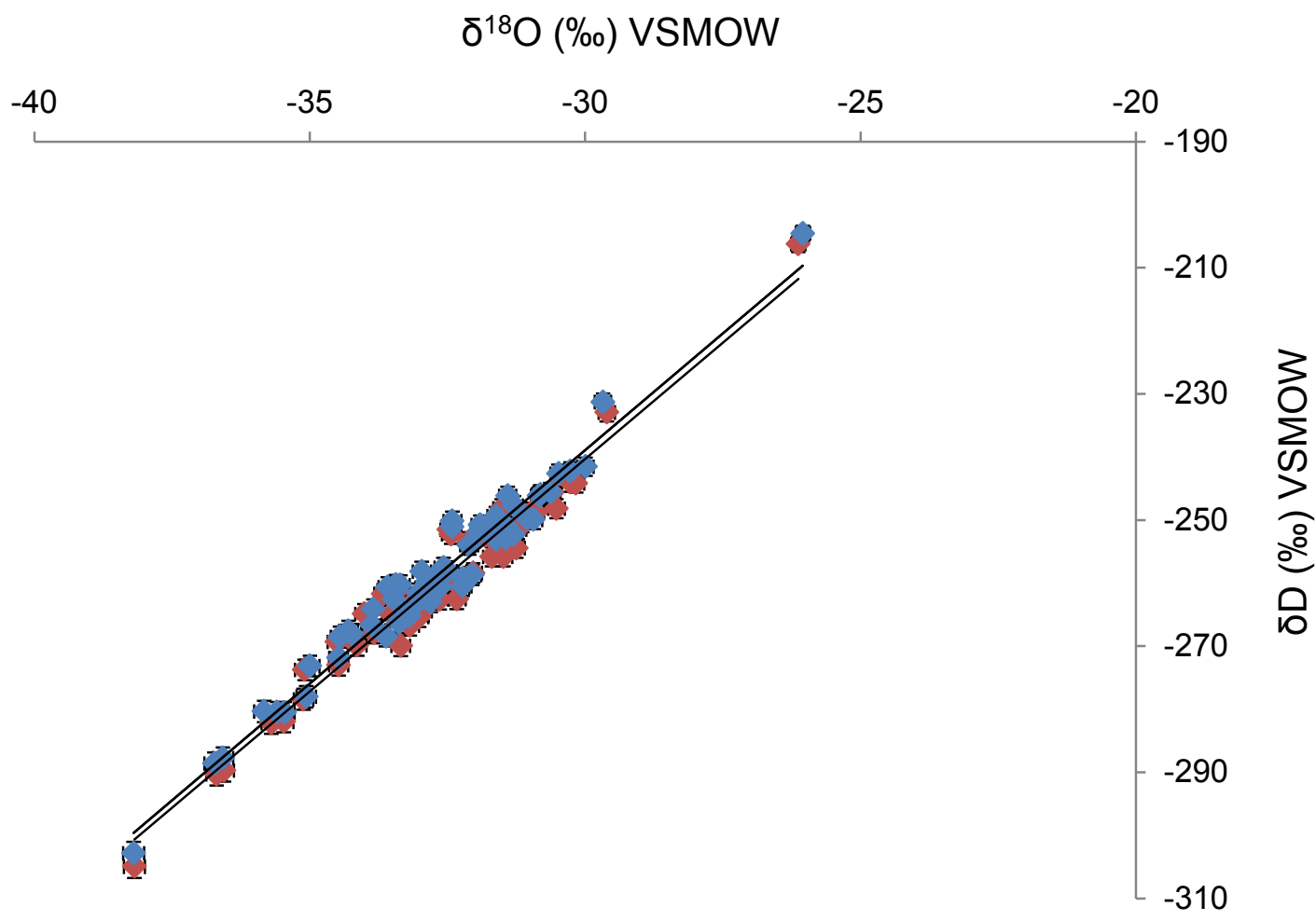

Fig. 2.34: Relationship between $\delta^{18} O$ and $\delta D$ for WB1 comparing results from laser absorption spectroscopy (blue) verse mass spectrometry (red). The small offset in $\delta \mathrm{D}$ is due to the higher precision of $\delta D$ measured by laser absorption spectroscopy. Instrumental errors are described above. 


\subsubsection{Results - temporal trends in aeolian sand and dust accumulation rate and texture}

An almost decade-long record of ASD accumulation rate and particle size was obtained from three firn cores from the McMurdo Ice Shelf. Stable isotope data and ASD mass accumulation rates for the firn cores can be found in Appendix 4, while the particle size data can be found in Appendix 3E-G.

\subsubsection{Temporal results - stable isotope data}

\subsection{Age model of the record}

The $\delta^{18} \mathrm{O}$ measurements reflect seasonal cycles in temperature, which were used to determine the age model of the core (Fig. 2.35). Winter precipitation $\left(\delta^{18} \mathrm{O}\right.$ values -32 to $-38 \%$ ) contrasts with summer values $\left(\delta^{18} \mathrm{O}\right.$ values -24 to $-30 \%$ ). Annual $\delta^{18} \mathrm{O}$ layer counting shows the firn cores span a 9 year period from 2000 to 2008 for WB1, an 8 year period from 2001 to 2008 for WB3 and a 7.5 year period from 2001 to 2008 for WB5. To evaluate the cumulative effect of dating errors (due to the difficulty of resolving every year based on $\delta^{18} \mathrm{O}$ values), two successive high ASD content layers the in firn cores were compared to the occurrence of what is assumed to be a coeval ASD rich year (2004) observed in a nearby well-dated ice core (WB06/07) from the McMurdo Ice Shelf by Dunbar et al. (2009; Fig. 2.16). The age models derived from $\delta^{18} \mathrm{O}$ for the WB cores independently places this ASD rich layer in 2004, confirming the validity of the overall age model (Fig. 2.35). Even though WB5 was sampled at higher resolution and similar seasonal variations in $\delta^{18} \mathrm{O}$ measurements are observed, a conservative dating error of \pm 1 year is assumed because one year of snow could have been lost through sublimation, and since two small peaks could have been counted as two years that may actually represent two events in one year. 


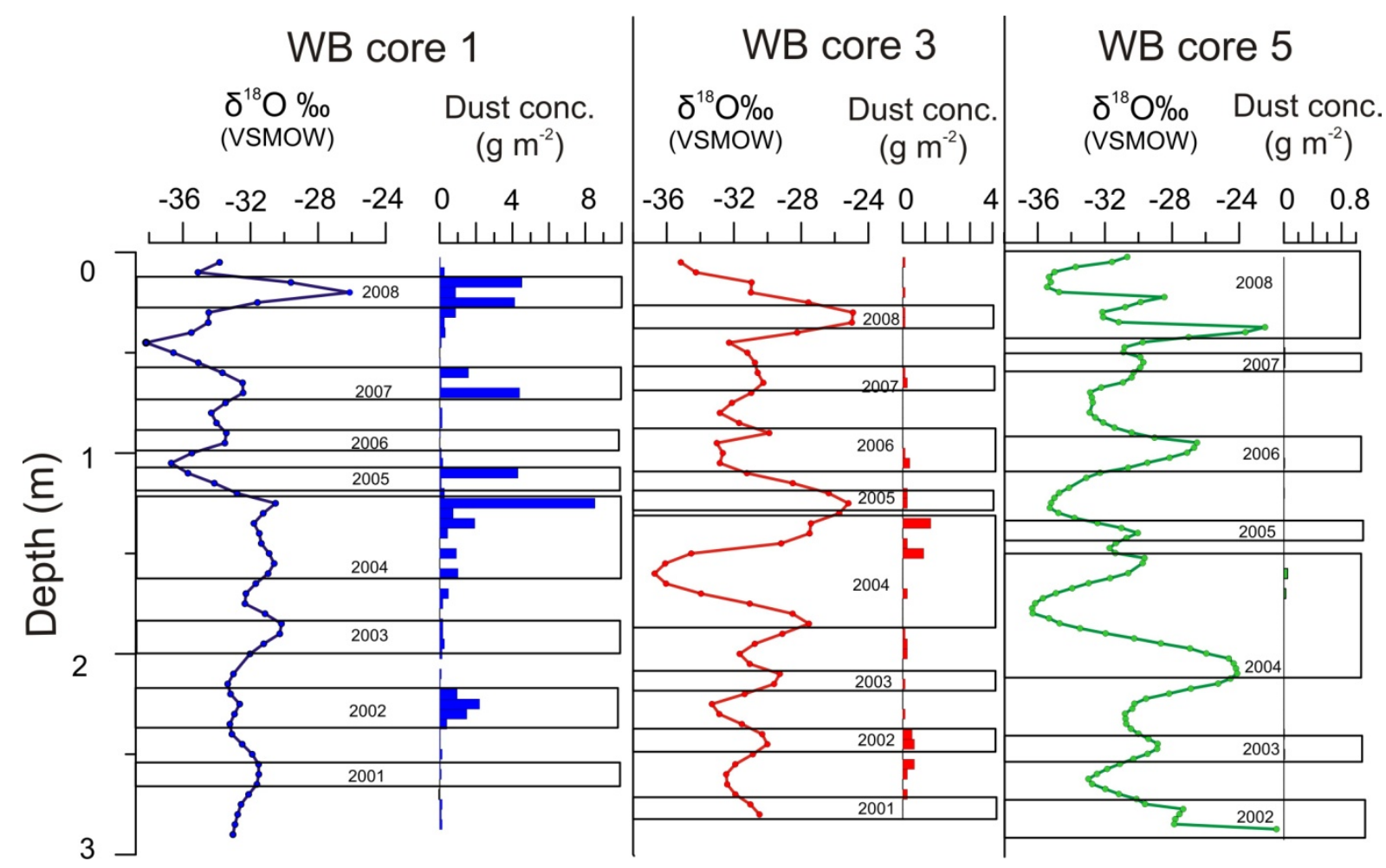

Fig. 2.35: Age model of Windless Bight firn cores showing downcore profiles of $\delta^{18} \mathrm{O}$ and ASD concentration. Boxes indicate summer seasons. Core locations are shown in Fig. 2.16.

\subsection{Snow accumulation rates}

The resulting age model was used to calculate annual snow accumulation rates. Snow accumulation increases downwind from Black Island toward Hutt Point Peninsula (Figs. 2.1 and 2.35). The distinct 2004 ASD layer occurs at greater depth near Hut Point Peninsula due to the increase in snow accumulation there. Snow accumulation ranges from $31.3 \mathrm{~cm} \mathrm{yr}^{-1}$ at WB1 to $37.8 \mathrm{~cm} \mathrm{yr}^{-1}$ at WB3 to $40.8 \mathrm{~cm} \mathrm{yr}^{-1}$ at WB5. The calculated snow accumulation rates in the present study lie within the observed variability from stake measurements for the McMurdo Ice Shelf reported in McCrae (1984) and snow accumulation rates from WB06/07 (53 $\pm 20 \mathrm{~cm} \mathrm{yr}^{-1}$; Dunbar et al., 2009), showing that seasonal layer count is a viable dating method for these cores.

\subsection{Climate history recorded in the firn cores}

The $\delta^{18} \mathrm{O}$ values for the whole core averages $-32.7 \%$ (WB1), $-30.81 \%$ (WB3) and $-30.71 \%$ (WB5) which are values typical of coastal Antarctic precipitation (Dansgaard, 1954; 1964; Johnson et al., 1972). As well as a seasonal cycle, all cores contain inter-annual variability in 
$\delta^{18} \mathrm{O}$ values which can either reflect true changes in seasonality with particularly warm summers in 2008 and 2005 and cold winters in 2004 and more moderate conditions during 2007, 2006, 2000-2003 (Fig. 2.5), or alternatively it could indicate that snow has partially been removed during windy conditions. For example, in 2004 the amplitude of the $\delta^{18} \mathrm{O}$ curve increases downwind from Black Island suggesting strongest downhill winds eroded the snow layer closer to Black Island. Partial removal of either summer precipitation or winter precipitation would shift the recorded annual average temperature to cooler or warmer signatures respectively.

To identify the source region of precipitation through time, the $d$ excess (which varies geographically and temporally) was calculated (Chapter 2.3.1.5). Three extremely negative values of $d$ excess in cores WB3 and WB5 lie outside two standard deviations. These values coincide with core breaks in the record that could reflect post-collection fractionation and have therefore been excluded. The calculated averaged $d$ excess value for the whole core is 1.39 for WB1, 5.56 for WB3, and -0.63 for WB5 which is characteristic of isotopically depleted waters in cold regions, such as coastal regions at high latitudes. There is a distinct seasonal pattern in $d$ excess that reflects the different air mass and moisture sources affecting the McMurdo region during the summer and winter seasons respectively. This confirms earlier studies (Patterson et al., 2005; Bertler, 2006; Rhodes et al., 2009; Sinclair et al., 2010) suggesting that the region experiences mass input from the Ross Sea during summer and from the Ross Ice Shelf during winter.

\subsubsection{Temporal results - aeolian sand and dust accumulation over time}

\subsection{Downcore sediment record}

WB1, WB3 and WB5 cores contain an average of $0.56 \mathrm{~g} \mathrm{~m}^{-2} \mathrm{yr}^{-1}, 0.12 \mathrm{~g} \mathrm{~m}^{-2} \mathrm{yr}^{-1}$ and $0.002 \mathrm{~g}$ $\mathrm{m}^{-2} \mathrm{yr}^{-1}$ of ASD respectively. A distinct decrease in ASD concentration downwind from Black and White Island towards Scott Base is evident in Fig. 2.36. 


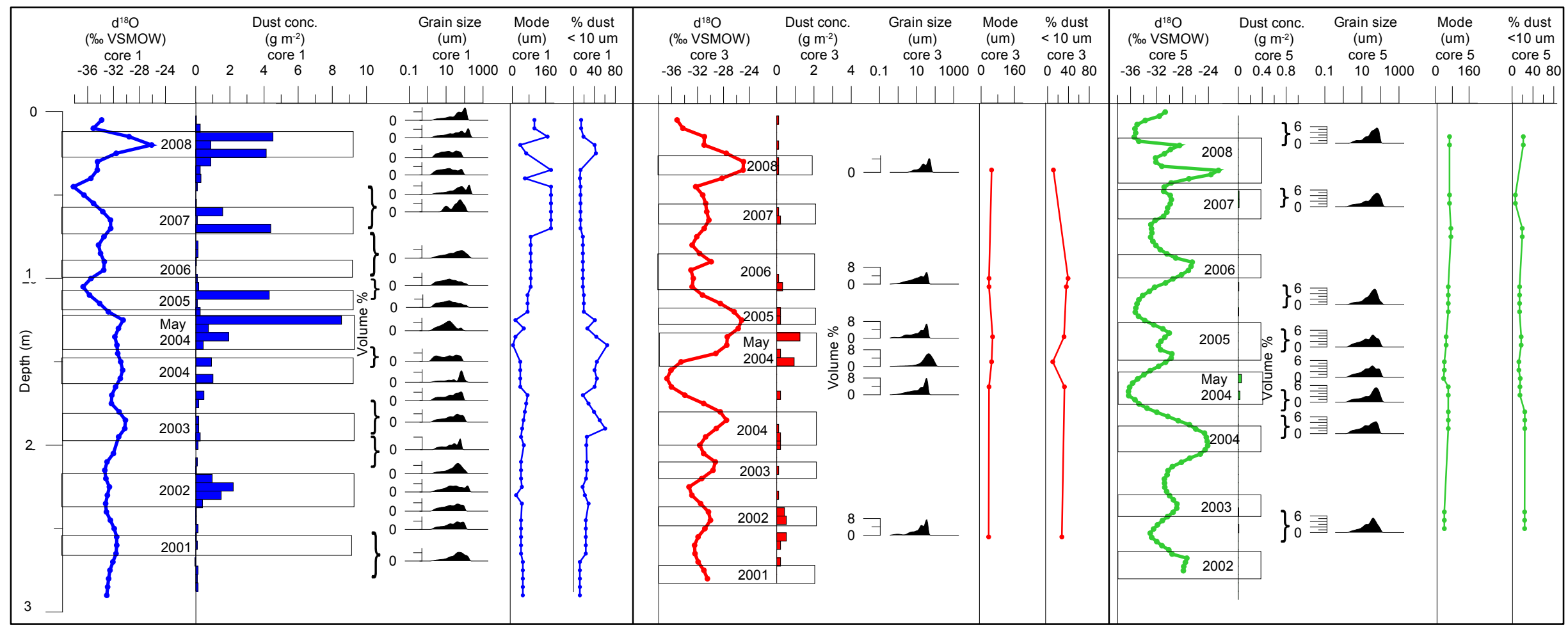

Fig. 2.36: Downcore sediment record in Windless Bight (WB) cores 1 (blue), 3 (red) and 5 (green) showing $\delta^{18} \mathrm{O}$, dust concentration, particle size distribution, particle mode and the percentage of ASD $<10 \mu \mathrm{m}$ for each core. Brackets indicate consecutive samples that were combined for grain size measurement due to low dust concentrations. 
All cores show a clear seasonal trend in ASD concentration with peaks coinciding with less negative $\delta^{18} \mathrm{O}$ values which reflect either warmer temperatures or that snow has been partially removed due to windy conditions. Particularly high ASD content years occur in 2002, 2004 and 2007 while relatively low ASD content years occur in 2001, 2003 and 2006. ASD peaks in 2002 and 2004 also occur in the WB06/07 core adjacent to WB3 (Dunbar et al., 2009). The ASD peaks WB06/07 coincide with winter rather than summer seasons and show a strong correlation to storminess (measured by peak wind speeds $>15 \mathrm{~ms}^{-1}$ ). Given that the most intense storms occur in winter at McMurdo Sound (King and Turner, 1997) and that as the rate of ASD transport increases with the cube of wind speed (Chapter 2.1.3.2; Pye et al., 1989) the $\delta^{18} \mathrm{O}$ values may reflect snow erosion. The similar seasonal pattern in cores WB1, WB3 and WB5 to Dunbar et al. (2009) is illustrated in Fig. 2.37.

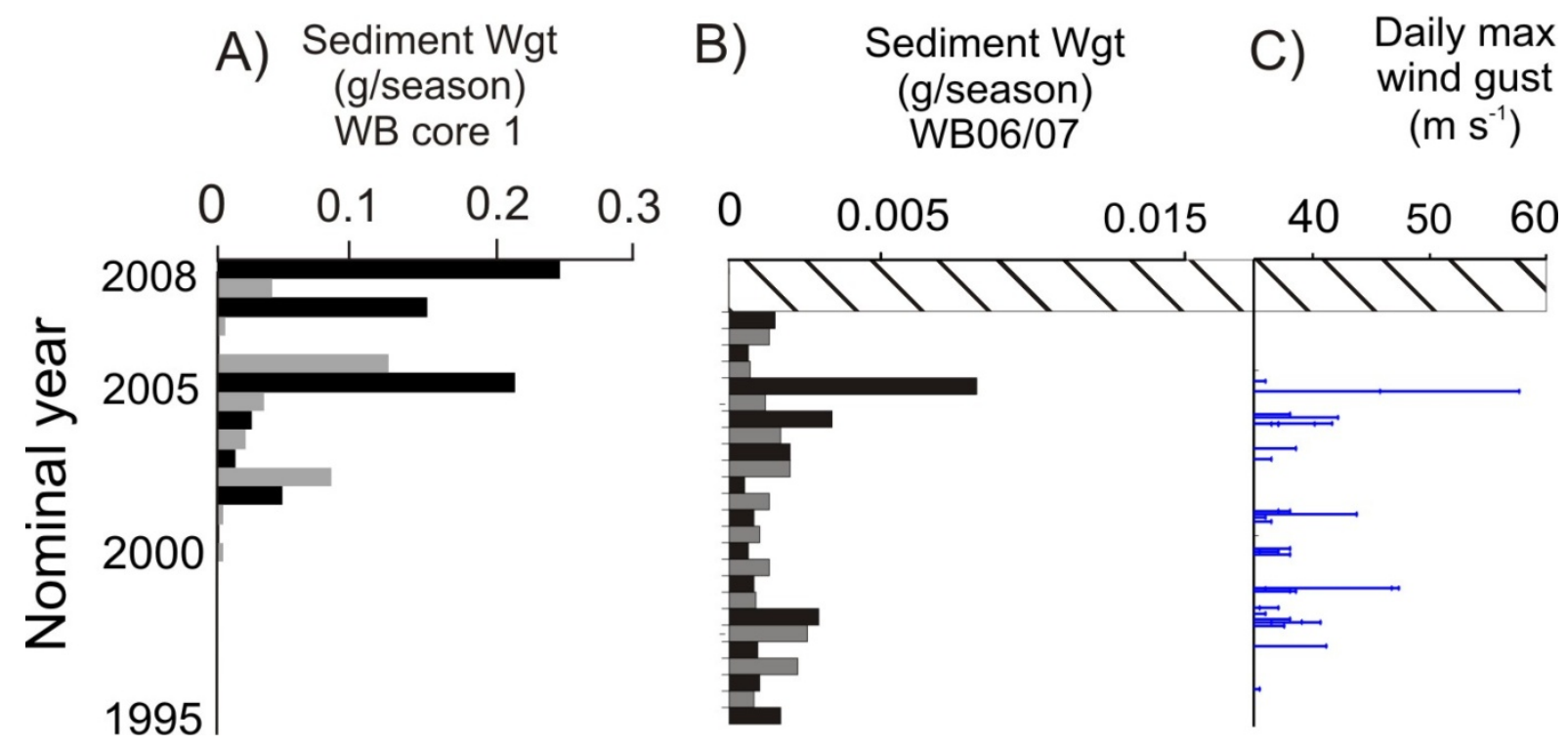

Fig. 2.37: Extending the dust record of McMurdo Sound. Comparison of WB1 with WB06/07. A) ASD accumulation rate concentration in WB1. B) Sediment weight per summer and winter season (data from Dunbar et al., 2009). C) Stem chart showing occurrence and magnitude of daily maximum wind gusts that exceeded $35 \mathrm{~m} \mathrm{~s}^{-1}$ for the period March 1990 to October 2008 at Scott Base AWS (\#6327; data from Dunbar et al., 2009). Hashed line: no data available from 2006 onwards for WB06/07.

Seasonal ASD peaks are evident in all three cores, but the ASD accumulation rate decreases with distance from Black Island. WB1 has the highest background (background=average excluding 2004) aeolian accumulation rate of $3.61 \mathrm{~g} \mathrm{~m}^{-2} \mathrm{yr}^{-1}$ which increases to $5.00 \mathrm{~g} \mathrm{~m}^{-2} \mathrm{yr}^{-1}$ when the ASD peak in 2004 is included. WB5 has the lowest background ASD accumulation rate of $0.007 \mathrm{~g} \mathrm{~m}^{-2} \mathrm{yr}^{-1}$ which increases to $0.017 \mathrm{~g} \mathrm{~m}^{-2} \mathrm{yr}^{-1}$ when the ASD peak in 2004 is 
included. The ASD accumulation rate for the fraction $<10 \mu \mathrm{m}$ also decreases with distance from Black Island. This background accumulation rate decreases from 0.81 to 0.38 to $0.00085 \mathrm{~g} \mathrm{~m}^{-2} \mathrm{yr}^{-1}$ for cores WB1, WB3, WB5 respectively and to $1.39,0.10,0.0025 \mathrm{~g} \mathrm{~m}^{-2} \mathrm{yr}^{-}$

${ }^{1}$ for the inclusion of the 2004 storm event. The ASD accumulation rate associated with a major storm event in 2004 (see Chapter 2.1.2.4) decreases dramatically over $22 \mathrm{~km}$ from 12.85 to 2.56 to $0.079 \mathrm{~g} \mathrm{~m}^{-2} \mathrm{yr}^{-1}$ in WB1, WB3, WB5 cores respectively and from 5.21 to 0.49 to $0.012 \mathrm{~g} \mathrm{~m}^{-2} \mathrm{yr}^{-1}$ for the ASD accumulation rate of sediment $<10 \mu \mathrm{m}$ in size. The 2004 storm event was 4.5 times greater than background ASD levels in WB1, which is consistent with the value reported by Dunbar et al. (2009) for WB06/07.

\subsection{Sediment texture}

Variability in particle size distribution decreases and samples become better sorted downwind from Black Island (Figs. 2.36 and 2.38). The size of the primary silt-fine sand mode clearly increases in 2004 in all cores compared to the background (Fig. 2.36). With the exception of 2004, the mode is fairly consistent downcore in WB3 and WB5, but WB1 shows seasonal variability where high ASD concentration correlates to coarser modes (Fig. 2.36). It is difficult to see a seasonal trend in the particle size distribution and particle size statistics because there was often insufficient ASD in each sample to make a measurement, thus samples were chronologically grouped together to gain enough ASD for measurement (Chapter 2.3.1.4). In particular particle size distributions measured from WB3 and WB5 are biased towards the season with greatest ASD content and hence greatest wind speed.

The percentage of ASD $<10 \mu \mathrm{m}$ increases downwind from Back Island (Fig. 2.36), which is similar to the trend downwind from the debris bands (see Chapter 2.2.2.1). In particularly 'dusty' years the percentage of ASD $<10 \mu \mathrm{m}$ decreases in 2002 and 2004 in all cores as the overall particle size becomes coarser (although the total amount of ASD $<10 \mu \mathrm{m}$ is still much greater than in background years), but actually increases in 2008 in WB5 (Fig. 2.36). 


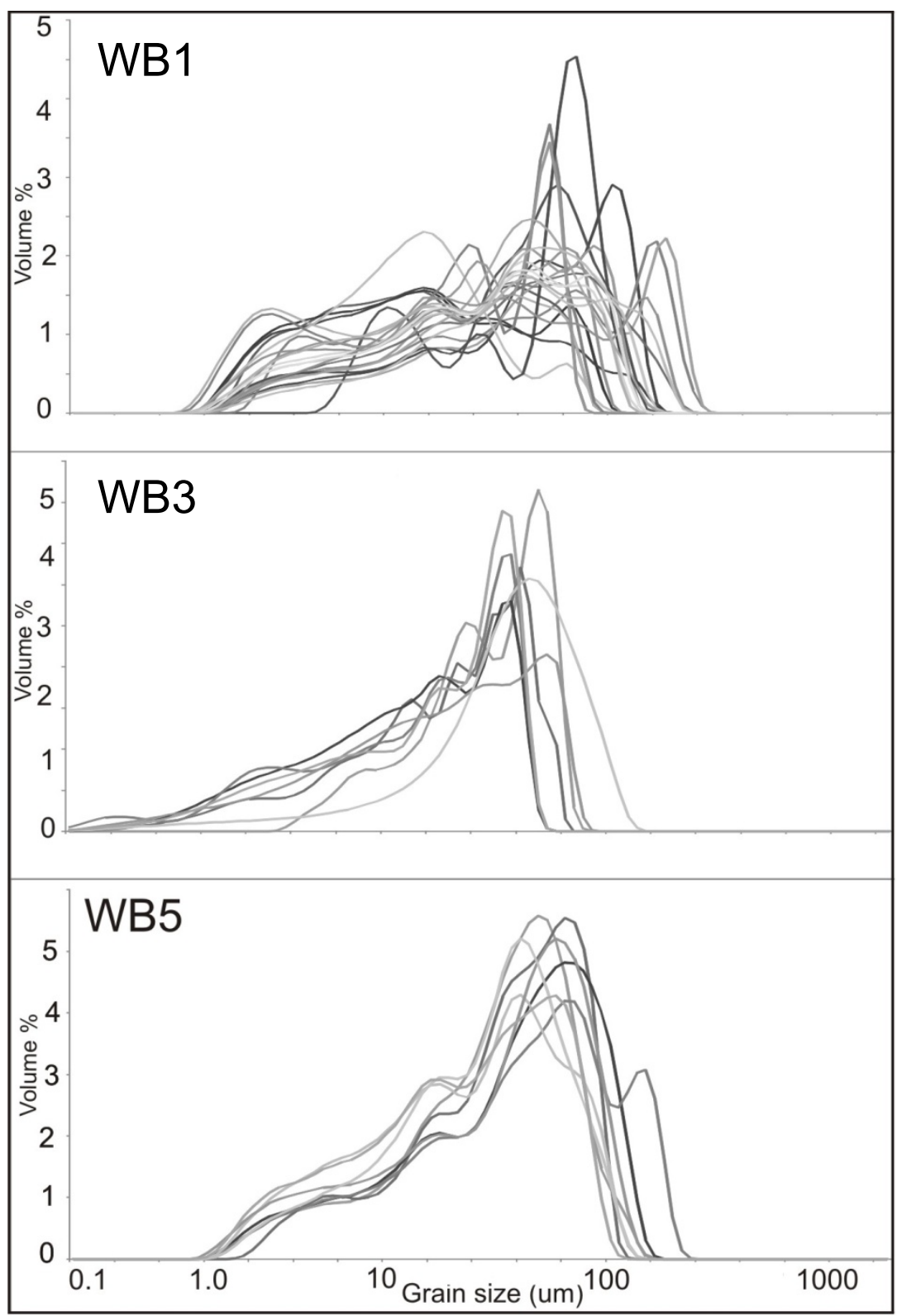

Fig. 2.38: Particle size distribution in firn cores along transect downwind from Black Island, McMurdo Ice Shelf.

\section{Windless Bight core 1}

Texturally, ASD in WB1 is variable mixtures of fine sand and silt with little clay and no coarse sand or gravel that are indistinguishable from surficial samples (Figs. 2.38 and 2.39). The core is dominated by sandy silt, which is characterised by a primary modal size ranging between 43 - $101 \mu \mathrm{m}$ and a finer tail, sometimes containing a poorly defined secondary mode. The ASD peaks in 2002, 2004, 2007 and 2008 with secondary sand modes. Coarser silt occurs in both 2008 and 2004, corresponding to high ASD concentrations and relatively less negative $\delta^{18} \mathrm{O}$ values. In particular, four samples from the 2004 storm have primary modes of 
fine-sand size (peaking at $73 \mu \mathrm{m}$ ) but retain a silty 'tail' meaning they are extremely poorly sorted and strongly fine skewed, except for 2008 where the samples are very poorly sorted. There is also considerable seasonal variability in the particle size distribution in WB1.

\section{Windless Bight core 3}

This core is comprised entirely of silt-sized sediment (primary mode $\sim 33 \mu \mathrm{m}$ ) with the exception of two samples of sandy silt composition (primary mode 42 and $53 \mu \mathrm{m}$ ) that correspond to the May 2004 storm (Figs. 2.38 and 2.29). As for surficial samples, all are characterised by a fine silty tail meaning they are extremely poorly sorted and fine skewed.

\section{Windless Bight core 5}

Sediment texture throughout the core is very similar to that described for WB3 and comprises extremely poorly sorted, strongly fine skewed, sandy silt. Throughout the core the silt mode ranges from $40 \mu \mathrm{m}$ to $66 \mu \mathrm{m}$ (Figs. 2.28 and 2.29). The highest ASD concentration in 2004 has a primary sand mode at $146 \mu \mathrm{m}$ and secondary sand mode at $63 \mu \mathrm{m}$.

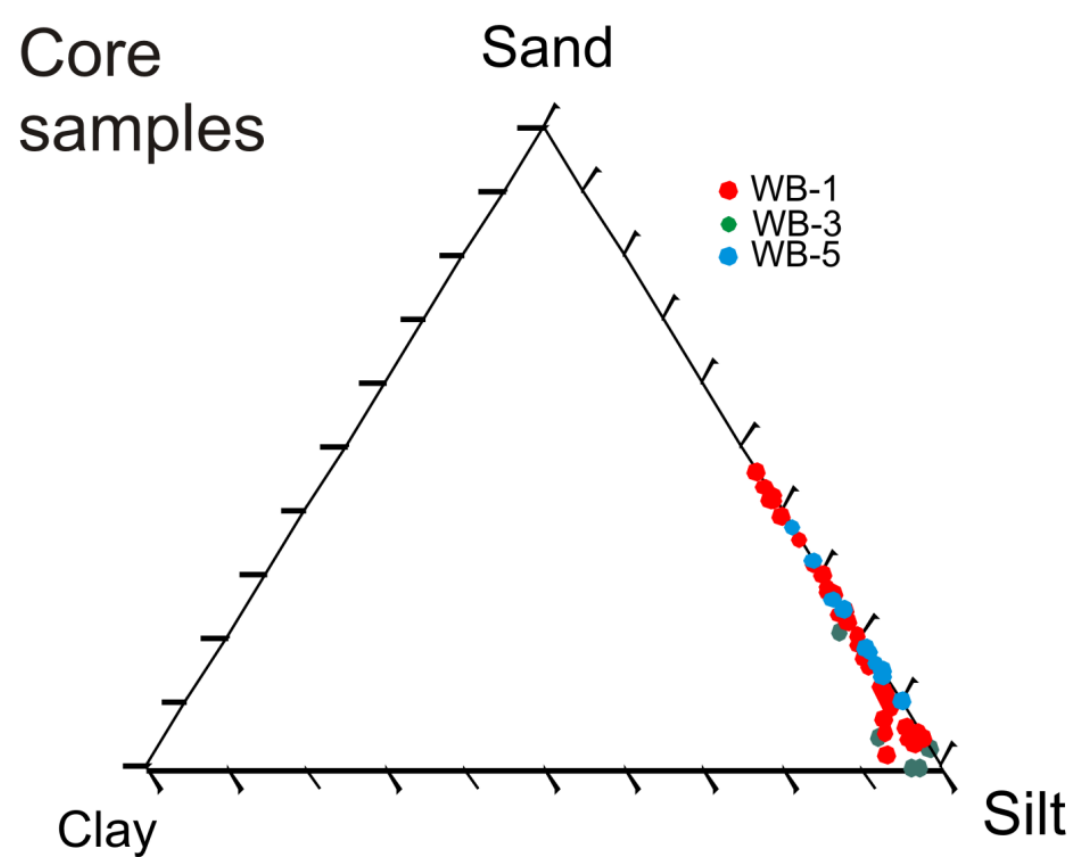

Fig. 2.39: Ternary plots of ASD from firn cores showing the proportion of sand, silt and clay. 


\subsubsection{Summary}

When considered together, the WB firn core records show that i) sediment concentrations are consistently (by a factor of $\sim 4$ ) higher and of coarser texture at all sites for the 2004 storm event; ii) the annual accumulation rate of sediment decreases downwind from Black and White Islands; iii) the percentage of ASD $<10 \mu \mathrm{m}$ increases and particle size distributions become better sorted downwind from Black and White Islands. These observations are consistent with the relationship between storm force $\left(>35 \mathrm{~ms}^{-1}\right)$, southerly winds and increased ASD accumulation reported in Dunbar et al. (2009). 


\subsection{Discussion - sedimentology and provenance of aeolian sand and dust}

This section estimates annual ASD dispersal over sea ice in Southern McMurdo Sound, discusses how this ASD is transported using a simple physical model, what contribution it makes to seafloor sedimentation rates and to the water column during storm events. In addition, a number of lines of evidence based on the sedimentological, petrological and geochemical characteristics of the ASD are presented which support the debris bands as the predominant source of ASD dispersed over much of the sea ice in McMurdo Sound.

\subsubsection{Annual aeolian sand and dust accumulation rate patterns in McMurdo Sound}

Although more than 70 locations were sampled for this study, spatial coverage remains sparse, particularly for sea ice sites $>20 \mathrm{~km}$ from the debris bands. Therefore a simple physical model was considered useful for estimating i) mass accumulation rates over the broader McMurdo Sound region and ii) for providing insight into the sedimentological processes at work (see Chapter 2.4.3). The critical assumptions for defining this model are that ASD is derived from a single (point), infinite (in terms of supply) source of ASD and that the dispersal of ASD is constrained by the topography of McMurdo Sound. The validity of these assumptions is examined below.

\subsubsection{Evidence for a single dominant source of aeolian sand and dust}

There are two broad lines of evidence suggesting the provenance of the ASD is dominantly derived locally from the debris band on the McMurdo Ice Shelf, which are both consistent with the local meteorology, are summarised as follows:

1) Sedimentological evidence:

a. The mass accumulation rate decreases downwind from the debris band, which is most easily accounted for by sediment eroding from an effectively infinite source, spreading laterally and also with a constant proportion being retained in the snow with distance from source.

b. The particle size becomes finer downwind from the debris band due to selective deposition from the source. 
2) Geochemical and petrographic evidence:

a. Fresh volcanic glass, of which the only known source are MVG sediments present in the Southern McMurdo Sound, which occurs in all samples.

b. $\mathrm{Sr}$ and $\mathrm{Nd}$ isotopic ratios matching the local PSA rocks.

\subsubsection{Sedimentological evidence}

\section{Southern McMurdo Sound}

The exponential decrease in accumulation rate and particle size (notably the primary mode and percent sand) both to the north (Figs. 2.23 and 2.28) as well as east and west of the debris bands (Fig. 2.31) are indicators of net transport away from the debris bands, ignoring high accumulation rates associated with chemical precipitates in the vicinity of Cape Evans (Chapter 2.2.2.1). The nature of the debris bands is also important in this regard; the continuously rejuvenated supply of wide range of particle sizes due to continual basal freezing and surface ablation prevents formation of armoured surface lag (c.f. MDV) and effectively exposes an infinite source of silt and fine sand-sized sediment on the surface consistent with the model (see Chapter 2.4.3). In addition, the dispersal northwards from the debris bands is also consistent with the local meteorology whereby the highest wind speeds, i.e. those most competent with respect to entraining silt and very fine sand, are always from the south (Fig. 2.4).

In a hemispheric context, the presence of relatively large size particles (coarse silt to fine sand) in all samples examined in the present study is also strong evidence this material is sourced locally (Fig. 2.40). This is due to the high settling velocities associated with such particles, which would preclude transport in suspension (section 2.1.3.1), the only viable mechanism for transporting ASD hundreds of kilometres across the ocean as is proposed for the "global" dust sourced from Australia or Patagonia found in ice cores on the Antarctic plateau. 


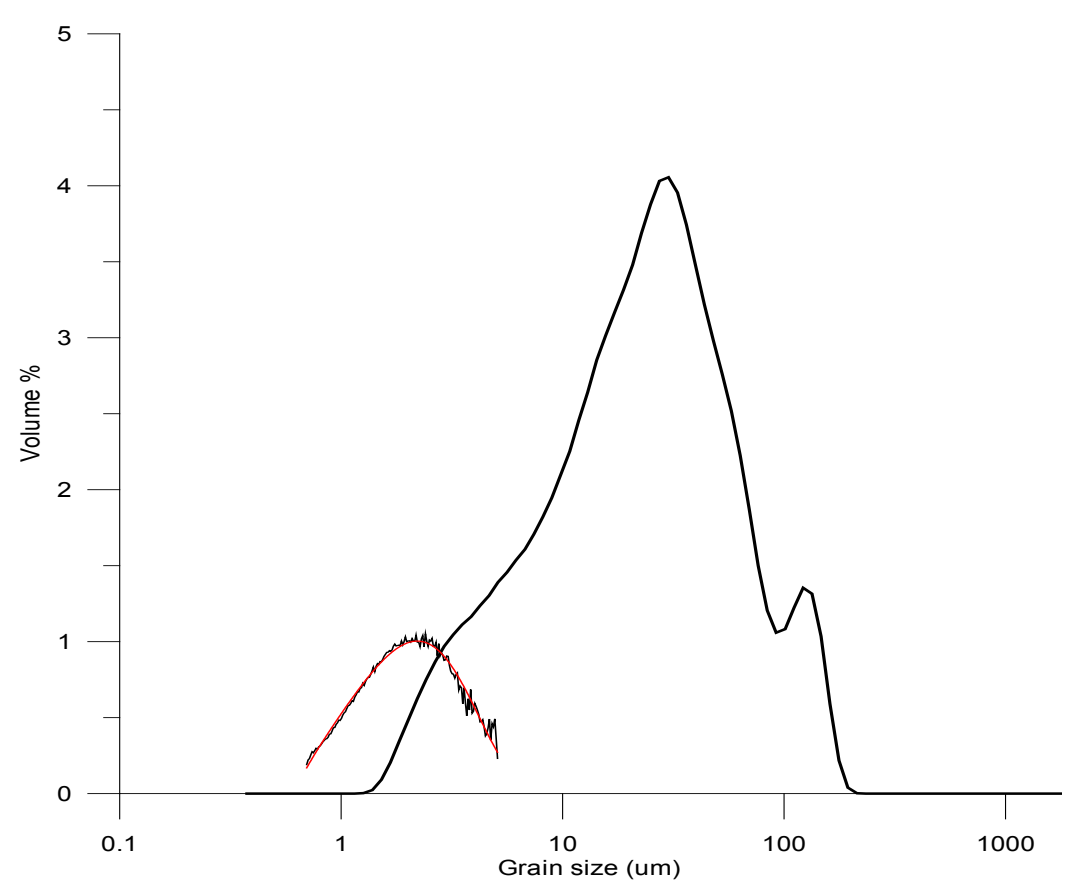

Fig. 2.40: Size distribution of Antarctic background ASD versus McMurdo Sound (eastern most sample at Granite Harbour (GH1). Antarctic background is typical Holocene dust at Dome $C$ and red line is Weibull fitting curve (Dome $C$ data from Delmonte et al., 2002).

Whilst suggesting that the contribution of global dust to ASD accumulating on the sea ice in McMurdo Sound is negligible, that contributed by sediment from the MDV is more difficult to assess. Personal observation, satellite images and the study of Bentley (1979) all suggest ASD plumes sourced from sediment blowing out of the MDVs occur on the sea ice in both New and Granite Harbours. There are three reasons for suggesting that while there might be considerable transport of sediment by wind in the MDV, most of this material is largely confined to the valleys themselves and adjacent coastal embayments. First, the particle size distribution of ASD accumulating on the ice is texturally different from that found in McMurdo Sound. It is typically coarser (fine-medium sand) and much better sorted, lacking a fine silty "tail" (Ayling and McGowan, 2006; Shuck, 2009). Second, ASD mass accumulation rates do not increase dramatically adjacent to New Harbour (Fig. 2.23). Third, while $\mathrm{Sr}$ and $\mathrm{Nd}$ isotopic ratios (see Chapter 2.2.2.3) of a sample from Granite Harbour have a distinctive signature of crustal MDV material (Fig. 2.32), the trend and magnitude of ASD accumulation seawards from the Granite Harbour embayment does not suggest a large supply of ASD accumulates on the sea ice from a westerly MDV source (Fig. 2.23). The relatively fine particle size and low accumulation rates found in the firn cores from Windless Bight are consistent with their location upwind from the debris bands (location Fig. 2.16). However, 
unconsolidated sediment is exposed around Black Island and Minna Bluff, to the south of the core sites, is a viable ASD source.

When considering the dispersal of ASD from the debris bands it is worth noting that ASD derived from there does not appear to enhance the accumulation rate at the relatively close by Taylor Dome (Hinkley and Matsomoto, 2001; Table 2.1), suggesting the presence of the TAMs is a significant constraint on the transport of this relatively coarse sediment to the west. The eastern boundary is much less well constrained but is considered to be the western part of Ross Island, given the observed blocking effect of the Island on southerly winds (and thus the occurrence of Windless Bight).

\section{Granite Harbour ASD source - ASD input to McMurdo Sound from the MDVs?}

Granite Harbour samples are used to evaluate the contribution of ASD from MDV by the dominant wind direction and hence the source of ASD material, as it is largely sheltered from southerly storms and instead is influenced by two dominant wind directions: westerly katabatic winds associated with cold dry air drainage from the MDV and moist easterly winds from the Ross Sea (Bertler et al., 2004). Thus air masses associated with easterly and westerly flow are derived from considerably different environments, and as such snow precipitation and ASD deposited on the sea ice at Granite Harbour will reflect the relative dominance of these source regions.

Petrographic analysis of ASD from the north-south (transect XY) and Granite Harbour transects (transect YY'; Chapter 2.2.2.1) reveals both biogenic and lithogenic components occur in sediments on the sea ice there. Importantly, the lithogenic component includes mica (Fig. 2.41C) and fresh volcanic glass (Fig. 2.41B) where the fresh volcanic glass can only be sourced from the MVG which outcrop at Minna Bluff, Black Island, Mount Discovery, Ross Island and physically weathered volcanic debris from the debris bands, well to the south of Granite Harbour (Fig. 2.2). Small Volcanic glass is also present in all surface snow samples along transect XY from Southern McMurdo Sound to Granite Harbour. Consistent with this, Macpherson (1987) reports basaltic sand particles (21\% of the sediment accumulation) at Granite Harbour $120 \mathrm{~km}$ north of the debris bands. Qualitative analysis in the present study shows there is an additional component of mica at Granite Harbour (Fig. 2.41C), that is more 
common in samples at Granite Harbour than Southern McMurdo Sound. Mica particles are sourced from westerly katabatic winds from the Mackay Glacier, MVD (location Fig 2.4) and deposited on the sea ice at Granite Harbour. Both of these lithogenic components suggest the prominent transport direction is south-north, as does the sea ice diatoms (Fig. 2.41F-O), sponge spicules (Fig. 2.41E) and foraminifera (Fig. 2.41D), but there may be a relatively minor contribution of ASD from New Harbour and Granite Harbour into McMurdo Sound via katabatic winds. Furthermore, easterly winds may blow ASD from the sea ice into these harbours under the right weather conditions, for example the ASD plume downwind from Cape Roberts evident in Figs. 2.28A and 2.30.

In summary, all samples along the north-south transect contain biogenic material, mostly seaice diatoms, but this becomes relatively more important further from the debris bands as there is less dilution from lithogenic material. The presence of a marine biogenic component and the volcanic glass is a useful tracer that shows ASD is dispersed across the sea ice and onto harbours and coastal piedmont glaciers (c.f. Bull, 2009). The dominant ASD source is the debris bands and ASD deposited in McMurdo Sound may travel $120 \mathrm{~km}$ north in a single year into the SW Ross Sea. 


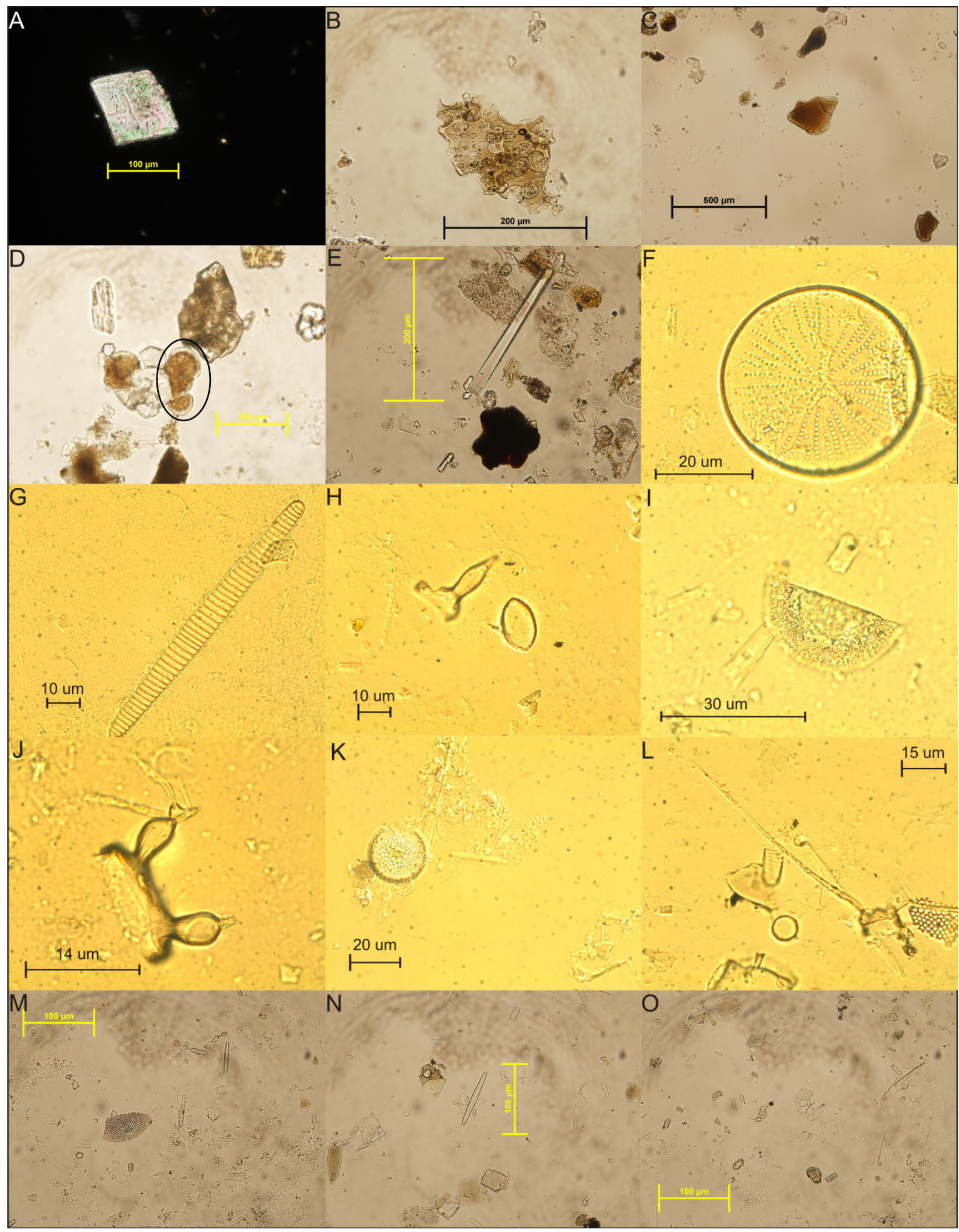

Fig. 2.41: Granite Harbour ASD composition. A) Calcite particle (GH4), B) fresh volcanic glass (GH4), C) Mica particle (GH4), D) Foraminifera (GH4), E) Sponge spicule (GH4), F-O) Diatoms: F) Actinocyclus actinochilus (GH8), G) Fragilariopsis obliquecostata (GH8), H) Fragilariopsis separanda (GH8), I) Porosira psudoentocclata (GH8), J) Bulbosa (GH8), K) Thalassiosira gracilis (GH8), L) Chaetoceros dicheata (GH8), M) Coscinodscus cf. oculus iridis (GH8), N) F. cf. Kerguelensis (GH8), O) Asteromphalus cf. Heptactis and Fragilariopsis c.f. curta (GH8). 


\subsubsection{Geochemical composition of particles and provenance}

\subsection{Volcanic glass}

As previously discussed volcanic glass was found in all ASD samples examined by smear slides from Southern McMurdo Sound up to Granite Harbour. Schuck (2009) measured a 20 $\%$ contribution of volcanic glass in samples of aeolian sediment immediately downwind of the debris band, but much less $(<2 \%)$ in aeolian sediment samples from the MDV. Qualitatively this suggests; i) that ASD derived from the MVG rocks and the debris bands containing a significant amount of fresh volcanic glass is transported at least $120 \mathrm{~km}$ to Granite Harbour from source each year (but only trace amounts into the MDV) and; ii) that the prominence of volcanic glass in smear slide samples ( $20 \%)$ seaward of Granite Harbour suggests that inputs of ASD from other sources (MDV outlet glaciers in particular) are not contributing substantial amounts of ASD to the sea ice beyond their immediate termini.

\subsubsection{Radiogenic isotopic signature of McMurdo Sound aeolian sand and dust}

\section{A locally-derived aeolian sand and dust source}

While it was only possible to measure three samples for isotopes of $\mathrm{Sr}$ and $\mathrm{Nd}$, they confirm ASD accumulating on the sea ice is locally derived. The two samples from Southern McMurdo Sound have a clear volcanic fingerprint and match the PSA samples from the MVG, while the sample from Granite Harbour has an isotopic signature of crustal material from SVL (Fig. 2.32). In particular, this signature is typical to the signature of PSA samples from regoliths (REG1) at the northern margin of The Mitten, Prince Albert Mountains and Pearse Valley, Lake Hoar and Lake Fryxell in Southern McMurdo Sound (Fig. 2.32). Fig. 2.32 illustrates the proposed transport by katabatic drainage down valley glaciers to the sea ice in the embayment and subsequent transport north along the SVL coastline. The isotopic composition of GH9 is important as it shows the influence of MDV and katabatic winds to ASD deposition on the sea ice within the embayment, although isotopic analysis of a sample further west of Granite Harbour is needed to confirm how far the MDV material is transported out of the embayment. 


\section{Dominant aeolian dust and sediment source: MVG or SVL?}

Sedimentological evidence points to Southern McMurdo Sound as the most important source of ASD in the McMurdo Sound region. However, the relative contribution of other sources can be investigated by considering the samples as mixtures between two end members. The samples from Southern McMurdo Sound consist of glacial sediments derived from the old East Antarctic continental shield (i.e. TAM lithologies; Chapter 2.1.2.2) mixed with young volcanic rocks (MVG rocks; Chapter 2.1.2.2). A mixing line can be used to determine the dominant local ASD source: a southern source from the MVG or a western source from the MDV. Overall, the Sr isotopic ratios for McMurdo Sound range between $0.703<{ }^{87} \mathrm{Sr} /{ }^{86} \mathrm{Sr}$ $<0.714$ while $\varepsilon \mathrm{Nd}(0)$ spans a relatively wider interval $(6<\varepsilon \mathrm{Nd}(0)>-13)$. Assuming these two end members (MVG and TAM), it can be observed that almost all PSA data are included within the mixing field. Fig. 2.42 illustrates the mixing line between these two isotopically distinct end member samples, assuming GH9 is representative of SVL and MIS4 is representative of MVG. This assumption is justified because isotopic ratios in the literature from both sources match these samples (Figs. 2.16 and 2.42). Furthermore, MIS23, located near Butter Point on the west side of Southern McMurdo Sound lies on the mixing line (Figs. 2.1 and 2.42). It would be expected that this sample contain mostly volcanic material with a small contribution of crustal MDV material via katabatic winds funnelling through valleys such as the Taylor Valley. Using the mixing line approach, this sample contains $86 \%$ of the MVG composition. This is consistent with the exponential decrease in ASD accumulation rate rate downwind from the debris band and the local meteorology. It confirms that the dominant ASD source for McMurdo Sound is from the dirty ice, comprised of MVG material, with minor additions of material from the MDV that is mostly contained within harbours themselves. 


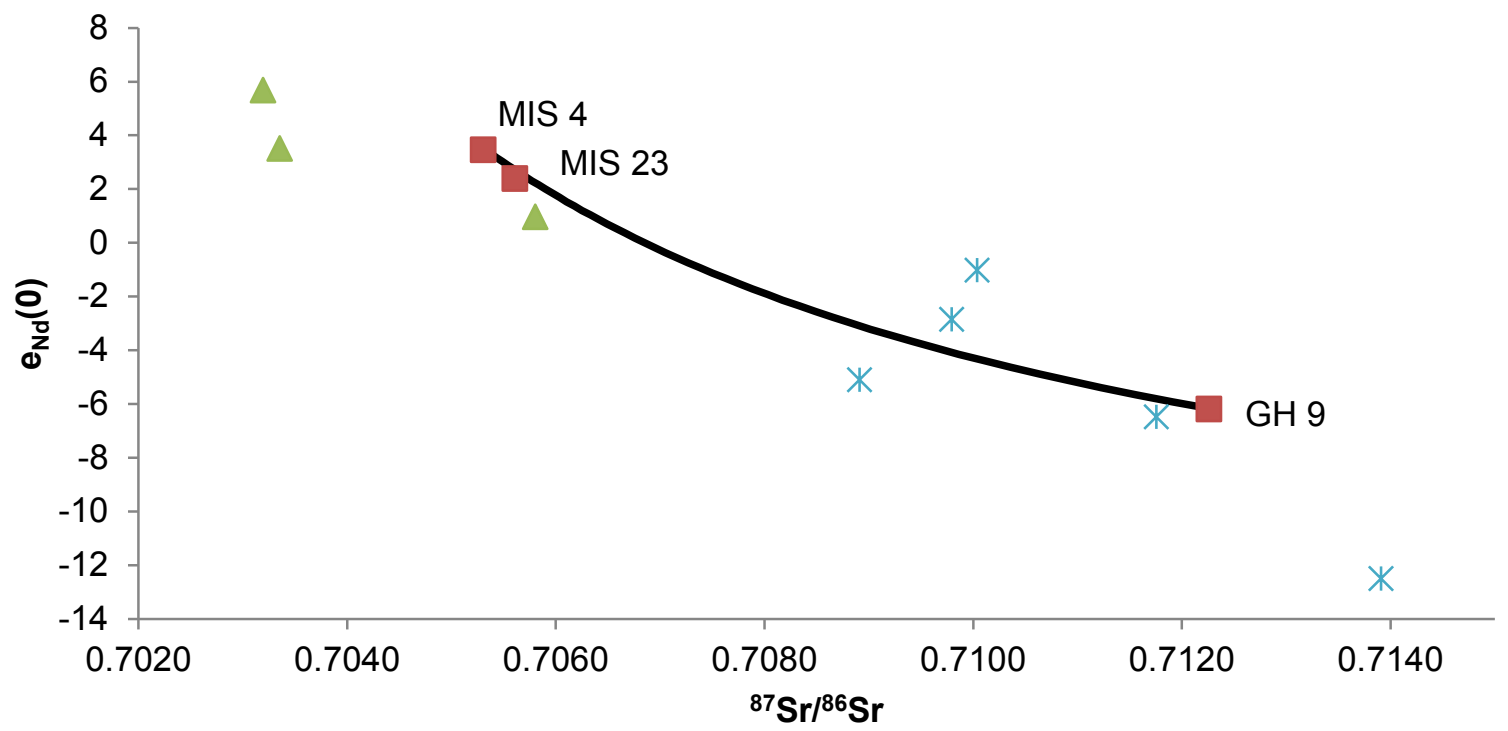

Fig. 2.42: Mixing line between two end member ASD sources in McMurdo Sound: MVG and the MDV, assuming sample GH9 is representative of material transported down valley glaciers onto sea ice in McMurdo Sound and MIS4 is representative sample of the MVG. The black line illustrates the estimated isotopic ratios of ASD between Southern McMurdo Sound and Granite Harbour formed by 100/0, 98/2, 96/4, 94/6, 94/6, 92/8, 90/10 etc to 0/100\% of the MVG-SVL mixtures. Red squares are the three samples analysed in the present study. Green triangles are isotopic ratios of the MVG PSA samples and blues asterisks are the isotopic ratios of SVL PSA samples from Delmonte et al. (2004a; 2010a). 


\subsubsection{Processes of aeolian sand and dust transport and particle size trends}

\subsubsection{Particle size and threshold velocity in McMurdo Sound}

The bimodal particle size nature of all the samples suggests that both suspension and saltation processes are important in transporting ASD onto the sea ice as the relatively high settling velocity of sand-sized sediment favours transportation by saltation whilst fine silt is more readily transported in suspension (Fig. 2.10).

In order to further examine the relationship between particle size and the prevailing meteorological conditions the threshold velocity for particle entrainment has been calculated using equation (2) in Chapter 2.1.3.1 and used to compare ASD transported under background conditions to the 2004 storm conditions. Use of this equation requires wind speed and density, particle size and surface roughness of which the wind is flowing to be known or estimated.

In particular, high surface roughness causes turbulence and exerts friction on the overflowing air mass (Pye, 1989). There are a number of measurements of surface bed roughness of sea ice by satellites in the literature which vary from 0.02 to $5.26 \mathrm{~cm}$ (e.g. Drinkwater et al., 1989; Patterson et al., 1999; Farmer et al., 1991; Kwok et al., 2004; Patterson et al., 2008; Manninen et al., 1997; Bank et al., 1980; Untersteiner 1965; Kim et al., 1985). In the present study, $5.26 \mathrm{~cm}$ is used to represent McMurdo Sound based on field observations of dune forms on surface snow. The particle entrainment threshold velocity was then calculated for wind measured at a height of $13 \mathrm{~m}$ above the bed as this is the height of the AWS at Pegasus North, the nearest site to the Southern McMurdo Sound transects (Fig. 2.4). An air density of $0.001437 \mathrm{~g} \mathrm{~m}^{-3}$ was calculated by Schuck (2009) for an annual average air temperature of 27.4 ${ }^{\circ} \mathrm{C}$ at Lake Vida (1995-2000; Doran et al., 2002).

The calculated wind velocity needed to transport particles of various sizes present in snow samples in the present study are shown in Table 2.3. The average wind velocity in Southern McMurdo Sound is $\sim 5 \mathrm{~ms}^{-1}$ (Stearns, 1997; Monaghan et al., 2005), which is barely sufficient to move sand-sized particles. The calculated values for $\mathrm{v}_{\mathrm{t}}$ for the mean annual temperature at Lake Vida indicate that sand $(>63 \mu \mathrm{m})$ will only move when winds exceed the entrainment threshold velocity of $4.50 \mathrm{~ms}^{-1}$. By contrast, southerly storm conditions in winter 
often exceed this entrainment threshold velocity (Stearns, 1997; Alying and McGlowen, 2006) for fine to medium sand-sized particles (Fig. 2.4). Modelling by Steinhoff et al. (2008) highlights that wind speeds ranged from $25-35 \mathrm{~ms}^{-1}$ at McMurdo Station during the May 2004 storm, greatly exceeding the threshold velocity for all particle sizes found in samples from the sea ice in the present study (Table 2.3). A further consideration when relating wind speed to sediment transport is that the rate of sediment movement is proportional to the wind speed to the third power (Chapter 2.1.3.1; Bagnold, 1941), reinforcing the importance of storm force winds as the most important distributor of ASD in McMurdo Sound and probable reason for the high ASD concentrations and greater modal size for ASD contained in the 2004 peak in the firn cores.

Table 2.3: Threshold velocities for several particle sizes in McMurdo Sound with mean temperature of $-27.4{ }^{\circ} \mathrm{C}\left(0.001437 \mathrm{~g} \mathrm{~cm}^{-3}\right)$ at Lake Vida (1995-2000; Doran et al., 2002).

\begin{tabular}{cc}
\hline $\begin{array}{c}\text { Particle size } \\
(\boldsymbol{\mu m})\end{array}$ & $\begin{array}{c}\mathbf{v t} \\
\left(\mathbf{m s}^{-\mathbf{1}}\right)\end{array}$ \\
\hline 63 & 4.50 \\
125 & 6.34 \\
250 & 9.00 \\
500 & 13.00 \\
\hline
\end{tabular}

\subsubsection{Dispersal of aeolian sand and dust in McMurdo Sound}

Therefore, the results presented here are consistent with southerly storms dispersing ASD from the debris bands north and northwest along the Victoria Land coast. Dispersal further west is limited by the presence of the TAM but transport to the east, into the SW Ross Sea remains an open question. Nonetheless, the basic assumptions of ASD dispersed from a single source by southerly storms appears valid. A simple ASD dispersal model (Fig. 2.43) based on these assumptions is given below.

The model is expressed in terms of two physical parameters:

1. The amount (\%) of ASD trapped in snow with each unit distance travelled from source. Without this parameter ASD transported north from the debris bands will 
move as a series of "pulses" of high accumulation zones associated with each storm event.

2. Lateral spreading of the ASD concentration at the source with distance (ratio of the distance spreading to angle).

Which are expressed as:

$\mathrm{y}=(158 / \mathrm{x}) \exp (-\mathrm{x} / 46)$

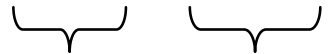

spreading trapping

where $\mathrm{y}$ is the ASD accumulation rate $\left(\mathrm{g} \mathrm{m}^{-2} \mathrm{yr}^{-1}\right)$ and $\mathrm{x}$ is the distance from source.

The model is constrained by these two parameters and this conceptual model has been fitted to the observed data.

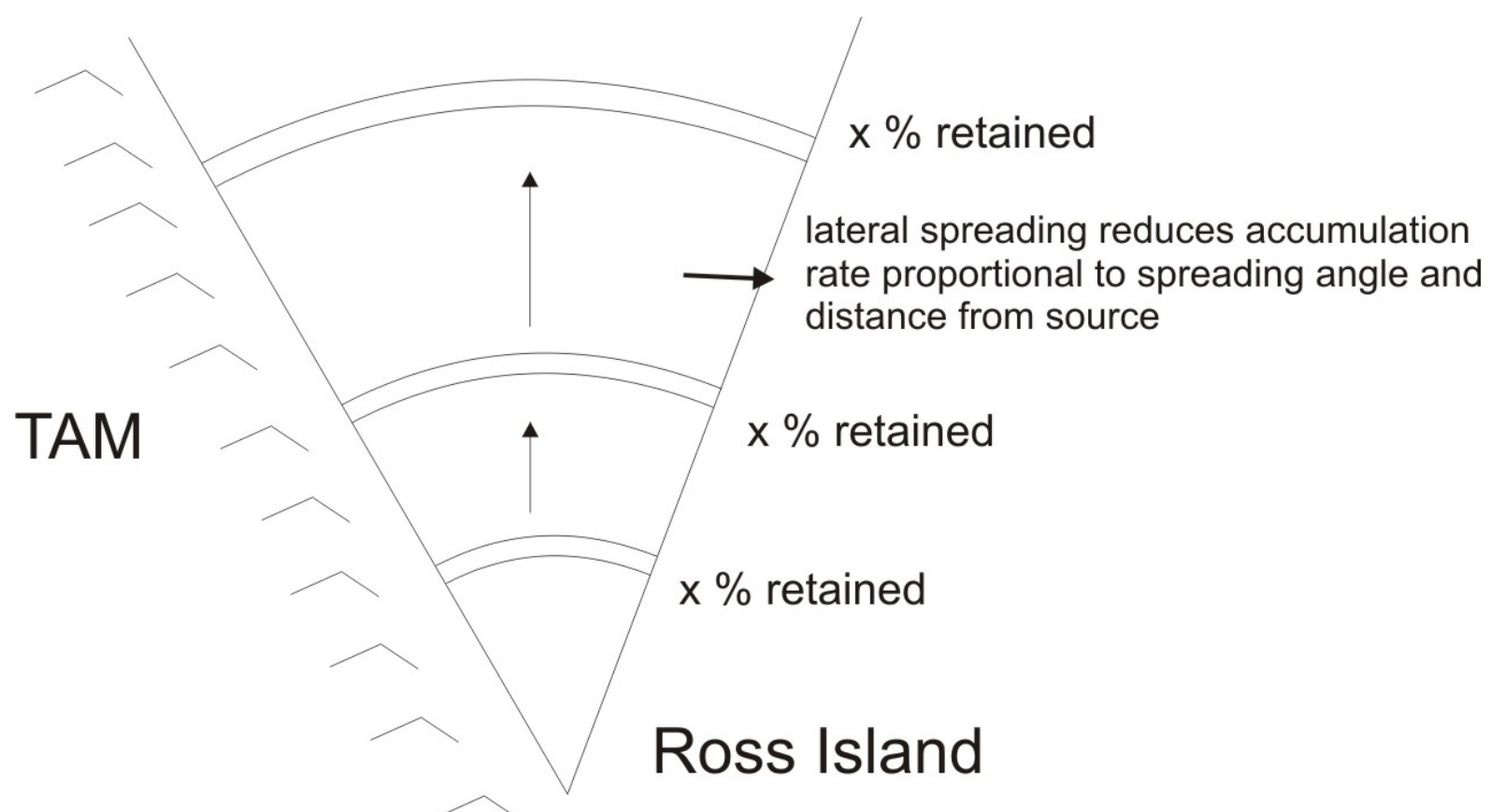

Fig. 2.43: Schematic of aeolian sand and dust dispersal in McMurdo Sound. As formulated " $x$ " has a value of 37 \%. TAM: Transantarctic Mountains 
By minimising the error in the fit between the observed accumulation rate and distance from the source, the formulated model predicts that $37 \%$ of the ASD is captured in the snow for every $46 \mathrm{~km}$ it is blown from the source and dispersed laterally across McMurdo Sound (Fig. 2.44. Assuming this accumulation rate on the sea ice also characterises areas $120 \mathrm{~km}$ from the source, that could not be sampled due to logistical reasons, these accumulation rates are characteristic of $7400 \mathrm{~km}^{2}$ of McMurdo Sound (Fig. 2.44).

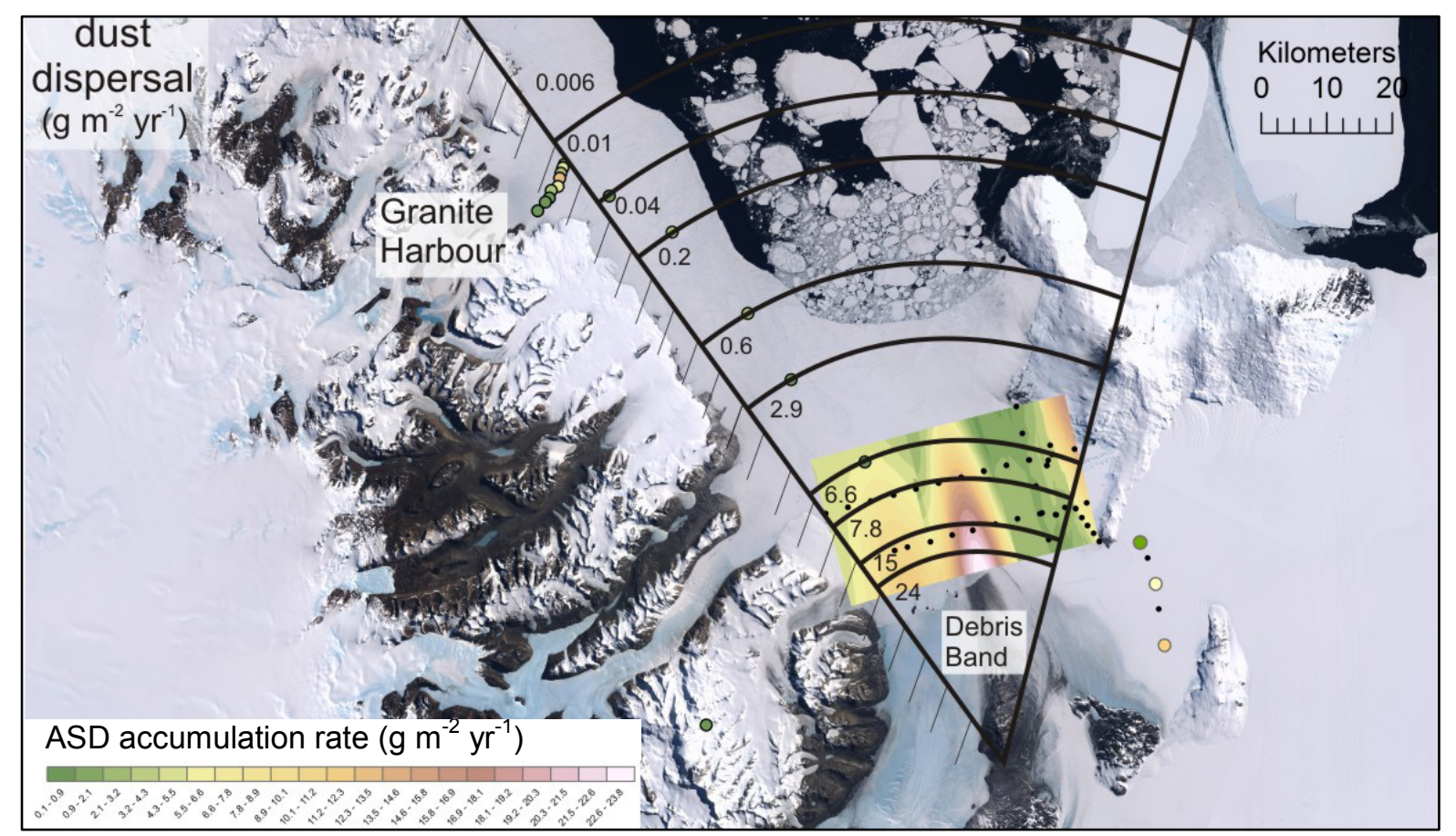

Fig. 2.44: Sediment dispersal model for McMurdo Sound superimposed on observed accumulation rate data.

The exponential downwind decrease in ASD mass from Southern McMurdo Sound to the most seaward sample at Granite Harbour (Figs. 2.43 and 2.44), suggests a minimum annual accumulation rate into McMurdo Sound of $\sim 0.70 \mathrm{~g} \mathrm{~m}^{-2} \mathrm{yr}^{-1}$ and a minimum annual accumulation rate of ASD $<10 \mu \mathrm{m}$ of $0.08 \mathrm{~g} \mathrm{~m}^{-2} \mathrm{yr}^{-1}$ which characterises accumulation between 30 and $120 \mathrm{~km}$ from the debris band.

These accumulation rates can be compared against global models and aerosols concentrations for dust accumulation in the $\mathrm{SO}$, thousands of kilometres from source, which suggest a "background" dust fallout rate for the SO of $0.002 \mathrm{~g} \mathrm{~m}^{-2} \mathrm{yr}^{-1}$ (Mahowald et al., 2005) and $0.0002-0.0004 \mathrm{~g} \mathrm{~m}^{-2} \mathrm{yr}^{-1}$ (Wagener et al., 2008) respectively. The annual supply of ASD 
over much of McMurdo Sound is therefore $\sim 3$ orders of magnitude greater for bulk sediment and 2 orders of magnitude greater for sediment $<10 \mu \mathrm{m}$ than the most recent estimate for the SO, a large ocean basin, by Wagener et al. (2008; see Table 2.1). Notably these values are also considerably higher than those reported from ice cores on the EAIS and WAIS where dust is primarily sourced from distal sources (see Table 2.1). Therefore, the dispersal of ASD from local sources in Southern McMurdo Sound contributes greatly to the input of ASD in McMurdo Sound, i.e. the model predicts ASD is transported up to $500 \mathrm{~km}$, and possibly further, as the model is very sensitive to dust mass, before it reaches the global background level.

\section{Decimetre and kilometre sample variability}

The particle size distribution and ASD accumulation rate of duplicate samples, sampled $\sim 20$ $\mathrm{m}$ apart, is consistent for 4 pairs of duplicate samples. However there is variability in samples MIS 45 and 46 where there is a significant difference in ASD concentration $\left(60 \mathrm{~g} \mathrm{~m}^{-2} \mathrm{yr}^{-1}\right)$ at the same site, a result supported by field observations which show local ASD variability within centimetres to meters (Figs. 2.7 and 2.28) and the difference in particle size distribution and ASD concentration highlights this. Variability of this magnitude was also reported by Atkins and Dunbar (2009) for one sample in a grid of 25, $500 \mathrm{~m}$ spaced samples. However, considering the spatial patterns observed in Fig. 2.23, it appears spatial variability on meter to decimetre scale does not mask regional trends although it contributes to the uncertainty in predicating accumulation rate at any given site.

\section{Temporal variability}

The elevated ASD concentration observed in the firn cores for 2004 (by a factor of 4) corresponds with the major windstorm at this time (Steinhoff et al, 2008) and suggest there is also considerable intra-annual variability in the supply of ASD into McMurdo Sound. Although ASD from this event was only sampled at three sites in the present study, a radar reflective layer associated with the 2004 ASD deposition is found throughout the McMurdo region (N. Kruetzmann, personal comm., 2009). The consistency of these findings with those of Dunbar et al. (2009), Kruetzmann's radar measurements and Steinhoff et al.'s (2008) modelling, suggest the May 2004 storm is a mesoscale phenomena and such storms this magnitude are potentially important mechanisms of providing enhanced ASD (and associated Fe) to McMurdo Sound. 


\subsubsection{Contribution of aeolian sand and dust to seafloor accumulation rate}

Past workers, noting the prevalence of well-sorted fine sand on the seafloor of McMurdo Sound suggested that aeolian sediments could represent an important part of the sedimentary record, both there and across the broader Ross Sea (Bentley, 1979; Barrett et al., 1983; Anderson et al., 1984; Dunbar, 1985). Barrett et al. (1983) suggests that the survival of textual modes from entrainment to deposition in near-shore marine environments is unusual but has come about in McMurdo Sound due to the passive transport of sediment by floating ice and the lack of textual modification by waves or by bottom currents. Furthermore, Bentley (1979) estimated a sedimentation rate of the seafloor at New Harbour of $20 \mathrm{~cm} \mathrm{ky}^{-1}$ based on these observations of aeolian sand accumulating on the local sea ice, a rate $2-3$ times higher than reported for lithogenic sediments accumulating on the sea floor in Windless Bight (Mackay et al., 2008). However, Atkins and Dunbar (2009) estimated that ASD on the sea ice north of the debris band could account for a linear sedimentation rate on the sea floor of only 1.53 to $0.49 \mathrm{~cm} \mathrm{ky}^{-1}$, assuming a wet bulk density of $1.6 \mathrm{~g} \mathrm{~cm}^{-3}$ and a particle density of $2.65 \mathrm{~g} \mathrm{~cm}^{-3}$ and that all sediment on the sea ice falls directly to the seafloor.

Using the same approach as Atkins and Dunbar (2009), the accumulation rates on sea ice measured here suggest sea floor sedimentation rates of up to 0.42 to $5.20 \mathrm{~cm} \mathrm{ky}^{-1}$ for the ASD plume extending north from the debris band in McMurdo Sound, but $20 \mathrm{~km}$ to the west and $15 \mathrm{~km}$ to the east of the plume the calculated sedimentation rate decreases to 0.16 and 0.14 $\mathrm{cm} \mathrm{ky}^{-1}$ respectively. Similarly estimates based on annual accumulation rates in firn cores yield sea floor sedimentation rates of $3.5 \mathrm{~cm} \mathrm{ky}^{-1}, 0.19 \mathrm{~cm} \mathrm{ky}^{-1}$ and $0.0007 \mathrm{~cm} \mathrm{ky}^{-1}$ for WB1, WB3 and WB5 respectively. Taking a more conservative approach and assuming most silt and clay-sized sediment is advected northwards by ocean currents and only well-sorted fine sand settles to the sea floor in the vicinity of the sampling sites, a linear sedimentation rate using the average sand percentage of each sample decreases the average linear sedimentation rate to $0.17 \mathrm{~cm} \mathrm{ky}^{-1}$ and $3.03 \mathrm{~cm} \mathrm{ky}^{-1}$ for Southern McMurdo Sound samples directly beneath the plume. Twenty kilometers to the west the sedimentation rate decreases to $0.08 \mathrm{~cm} \mathrm{ky}^{-1}$ and 15 kilometres to the east it decreases to $0.005 \mathrm{~cm} \mathrm{ky}^{-1}$, which is more likely to characterise greater McMurdo Sound. Sedimentation rates for WB1, WB3 and WB5 cores respectively are: $1.5 \mathrm{~cm} \mathrm{ky}^{-1}, 0.17 \mathrm{~cm} \mathrm{ky}^{-1}, 0.000003 \mathrm{~cm} \mathrm{ky}^{-1}$. 
Considered together these results suggest that ASD is likely an important direct contributor to sea floor accumulation only very close $(\sim 20 \mathrm{~km})$ to source. The linear sedimentation rates estimated here for the broader McMurdo Sound region (i.e. distal from source) are several orders of magnitude less than the $\sim 5-130 \mathrm{~cm} \mathrm{ky}^{-1}$ Holocene sedimentation rates reported by Domack et al. (1999) for sites across the Ross Sea which are dominated by biosiliceous ooze, further reinforcing the idea that ASD is volumetrically an insignificant source of sediment to the seafloor over most of the Ross Sea. 


\subsubsection{Aeolian sand and dust transport during 2004 storm event}

Detailed measurements of meteorological conditions during the May 2004 storm allow the use of air mass forward and back trajectories as a method for reconstructing atmospheric circulation during this prominent ASD transport event (e.g. Sinclair et al., 2010). Back trajectories describe the most probable hypothetical upwind path taken by an air mass (and associated particulate material) for a given location. Forward trajectories were generated to give an indication of the hypothetical depositional path of ASD during this event and help evaluate its significance for transporting ASD across the broader SW Ross Sea region.

\subsubsection{Air mass trajectories}

Air mass trajectories were initiated using the Hybrid Single-Particle Lagrangian Integrated Trajectory (HYsplit) model (e.g. Sinclair et al., 2010). The NOAA global reanalysis meteorological data used in this model were obtained from NCEP/NCAR (National Centre for Environmental Prediction/National Centre for Atmospheric Research). Sinclair et al. (2010) highlighted that lower trajectories, that is at $500 \mathrm{~m}$ above the surface are influenced by underlying terrain, obscuring regional circulation patterns whereas trajectories at $1000 \mathrm{~m}$ appear to minimise disturbance from underlying topography while still being sufficiently close to terrain to be dynamically linked to surface wind field. Here, daily trajectories were initiated at heights $500 \mathrm{~m}$ above the surface so that air mass trajectories could be directly related to the likely sediment transport direction at the surface.

The back trajectories (Fig. 2.45A) illustrate that the air mass associated with the windstorm on 15 May 2004 originated from Siple Coast propagating across the Ross Ice Shelf towards McMurdo Station (location in Fig 2.1A). This track agrees with the modelling by Steinhoff et al. (2008) and Powers et al. (2007) using the Antarctic Mesoscale Prediction System (AMPS) and Weather and Research Forecasting (WRF) models respectively and AWS data from the Ross Ice Shelf and McMurdo region for this storm. 
A)

NOAA HYSPLIT MODEL

Backward trajectories ending at 0000 UTC 15 May 04 CDC1 Meteorological Data

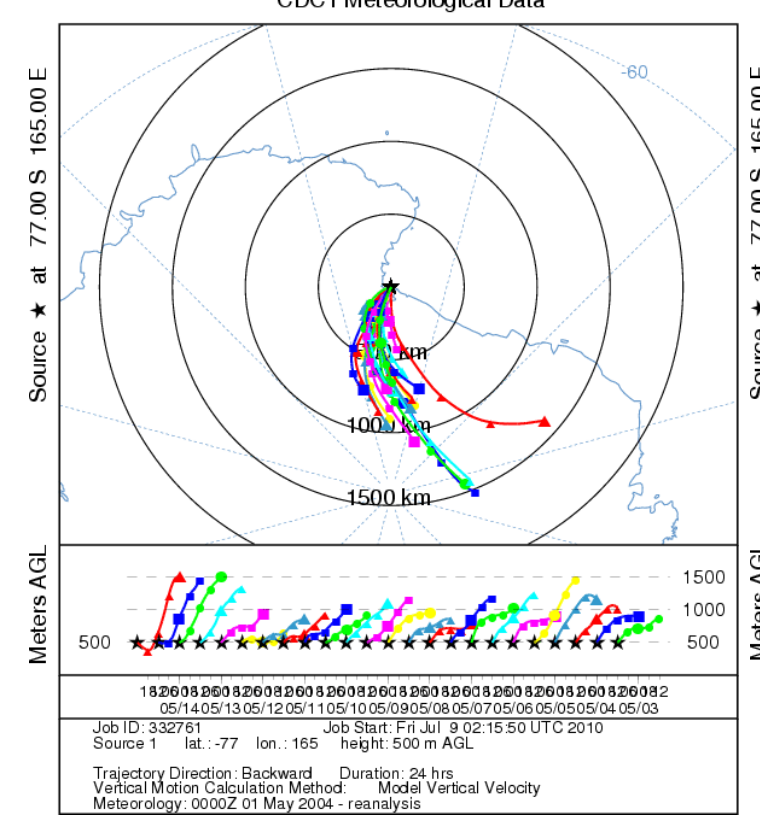

B)

NOAA HYSPLIT MODEL

Forward trajectories starting at 0000 UTC 15 May 04 CDC1 Meteorological Data

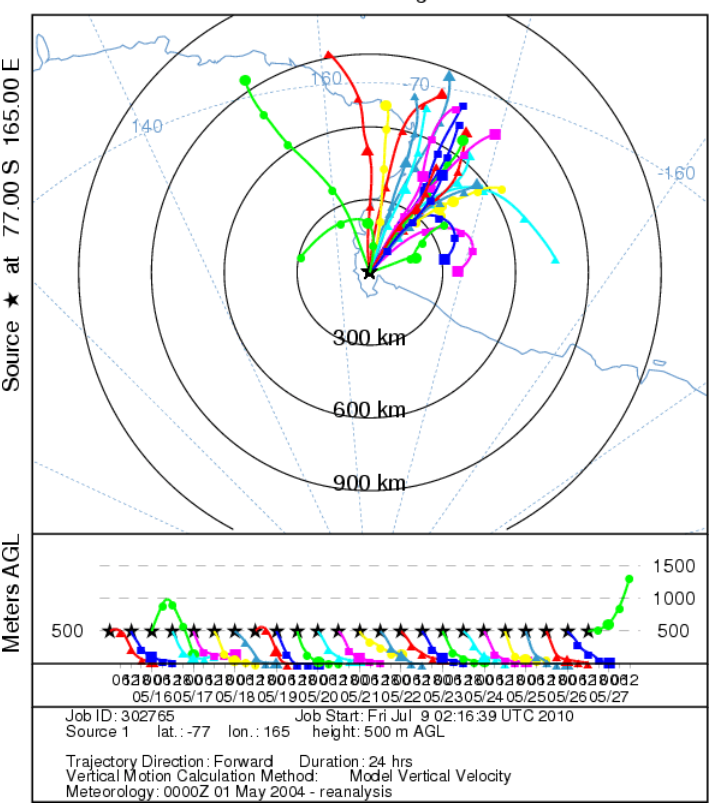

Fig. 2.45: Back A) and forward B) air mass trajectories for the 24 hour period 0000 UTC 15 May initiated at $500 \mathrm{~m}$ above sea level. Circles indicate distance from McMurdo Station. Trajectories derived from: http://ready.arl.noaa.gov/HYSPLIT.php

Fig. 2.45 illustrates that as the cyclone reaches McMurdo Sound the TAM block the cyclone from propagating further west and channel the airflow northwards as a "barrier wind" along the Victoria Land coastline. Barrier winds, with associated wind speeds of $25-35 \mathrm{~ms}^{-1}$, are important for development of windstorms at McMurdo, because they have sufficient kinetic energy to traverse Minna Bluff and Black Island. Acceleration of wind down the lee slopes of Minna Bluff and Black Island indicates a downslope windstorm which produces strong winds in the McMurdo area. The maximum wind speed extends $\sim 30 \mathrm{~km}$ downstream of Black Island (Steinhoff et al., 2008). 


\subsubsection{Implications for aeolian sand and dust transport and Fe deposition}

Visual observations of ASD associated with the 2004 storm event contained in three shallow firn cores show a trend of decreasing ASD concentration from Black Island to Hutt Point Peninsula possibly reflecting both a decreasing wind speed from Black Island to the north and the progressive retention of sediment in surfical snow along the transport pathway (Fig. 2.46). The wind vectors in Fig. 2.46 show that ASD entrained in the downslope windstorms of ice free Black Island and from unconsolidated sediment fringing Minna Bluff would have been transported into two regions of the SW Ross Sea. Where the airflow splits downwind of Black Island and Minna Bluff, it follows two pathways to the SW Ross Sea (Powers et al., 2007). The first pathway flows east around Windless Bight and Ross Island and the second between Ross Island and the MDV into McMurdo Sound. Furthermore, sediment exposed on the debris bands and entrained in the downslope windstorm, will be deposited in McMurdo Sound in the western and even perhaps eastern areas of open water around Ross Island where phytoplankton regularly bloom shown by chlorophyll- $a$ concentration in Fig 2.46. Blooms are defined as chlorophyll concentrations $>1 \mathrm{ng} \mathrm{m}^{-3}$ (Balch, 1991). Furthermore, forward trajectories (Fig. 2.45B) highlight airflow over the SW Ross Sea, which flows adjacent to the Victoria Land Coastline and a few trajectories have a cyclonic flow into the Ross Sea. Iron contained in ASD transported by this airflow could well stimulate both blooms over a broad region of the SW Ross Sea. Powers et al., (2007) suggest that downslope windstorms along the lee slopes of Minna Bluff, Black Island and White Island are common in the region where there are southerly winds greater than $15 \mathrm{~ms}^{-1}$ and when low level conditions are present. Therefore, locally derived ASD may regularly be transported to the east and west of Ross Island into the SW Ross Sea. 


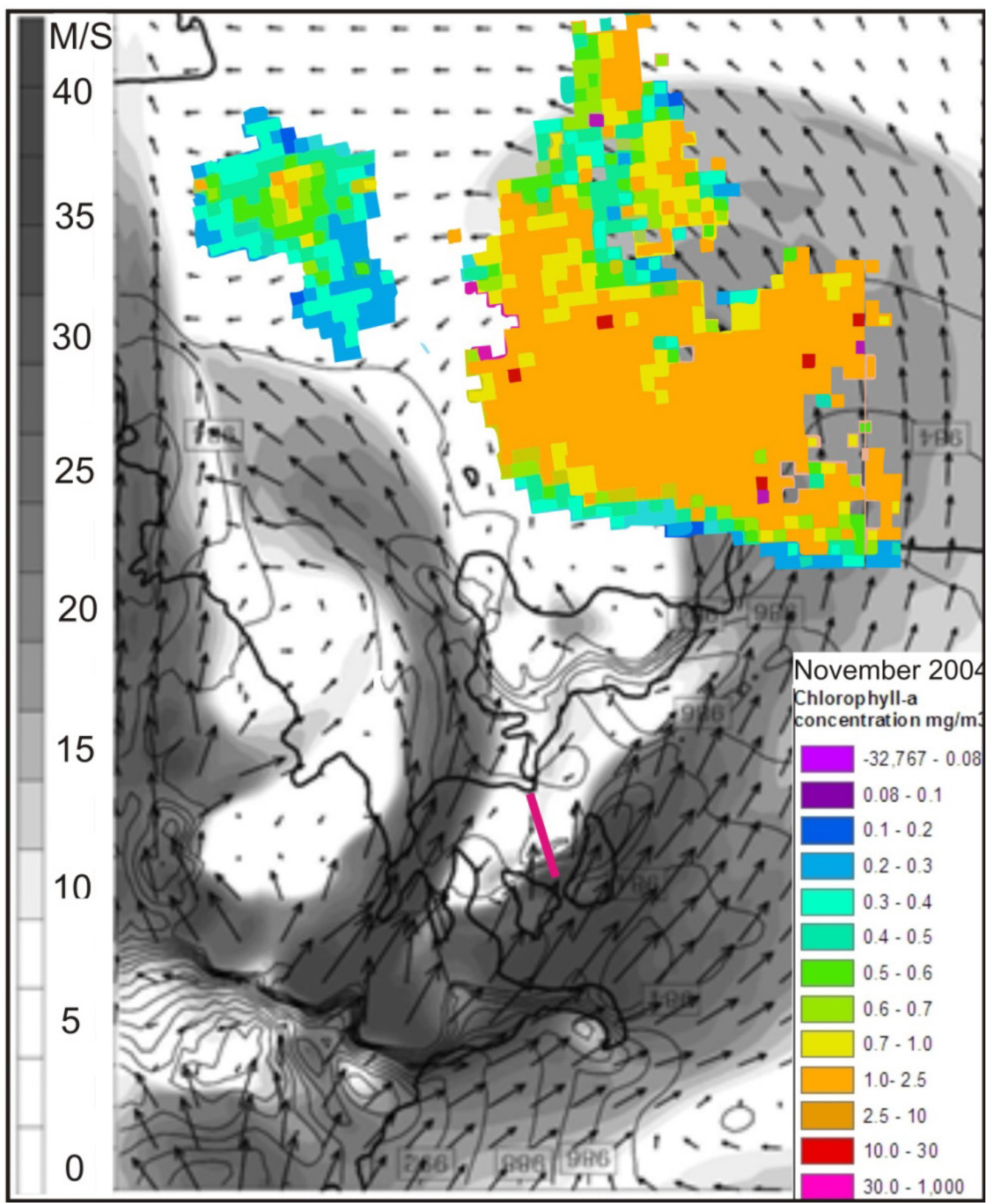

Fig. 2.46: Chlorophyll-a concentration (colour scale on right) in McMurdo Sound during sea ice break up in November 2004 superimposed on surface winds for 2300 UTC 15 May 2004. Wind speed (ms $\left.{ }^{-1}\right)$ shaded $^{-1}$ (scale at left). Arrows indicate wind directions and magnitudes are $22 \mathrm{~ms}^{-1}$ (vector length interval). Red line indicates transect of shallow firn cores on McMurdo Ice Shelf. Modified from Powers et al. (2007). Chlorophyll- $a$ data derived from https://gdata/sci.c.nasa.gov. 


\subsubsection{Conclusions and implications for Fe-fertilisation}

The mass accumulation rate and particle size distribution of ASD samples on sea ice in McMurdo Sound suggest that over the $\sim 2$ month period when sea ice breaks up, approximately $0.70 \mathrm{~g} \mathrm{~m}^{-2} \mathrm{yr}^{-1}$ (bulk) or $0.08 \mathrm{mg} \mathrm{m}^{-2} \mathrm{yr}^{-1}$ of sediment finer than $10 \mu \mathrm{m}$ (i.e. that most likely to become bio-available) is deposited in the ocean. These values form the basis of the calculation of the input of aeolian Fe into McMurdo Sound and are discussed further in Chapter 3.

Much greater ASD mass accumulation rates are confined to areas close to the debris bands and in localised hotspots were sediment is also sourced from chemical precipitates. Although the mass accumulation rate decreases rapidly to the north, petrographic analysis of ASD seaward of Granite Harbour shows it contains fresh volcanic glass, derived from the MVG rocks and sediment $120 \mathrm{~km}$ to the south, specifically the debris bands. This in combination with the local meteorology suggests southerly storms distribute sediment over considerable distances along the western margin of the SW Ross Sea. Radiogenic isotopes of $\mathrm{Sr}$ and $\mathrm{Nd}$ also trace ASD to local PSA rocks in the region. Furthermore, the relatively coarse particle size and relatively small increase in the mass accumulation rate at Granite Harbour along transect XY suggests that ASD derived from the MDV appears to be largely confined to the valleys themselves and adjacent coastal embayments.

Particle size and ASD accumulation rate exhibit a seasonal trend where southerly winter storms (wind speeds greater than $15 \mathrm{~ms}^{-1}$ ), e.g. May 2004, are the most important for transporting locally derived ASD. The bimodal particle size nature of all samples examined in the present study suggests that both suspension and saltation processes are important in transporting material onto the sea ice. The calculations of entrainment threshold show that sand will only move when winds exceed the threshold velocity of $4.50 \mathrm{~ms}^{-1}$. Given that southerly storm conditions in winter often exceed this threshold velocity, local winds are strong enough to move sand-size particles. In particular the severe storm in May 2004 was responsible for transporting a vast quantity of coarse ASD over the sea ice.

Sedimentation rates are greatest directly under the ASD plume on the sea ice just north of the McMurdo Ice Shelf debris bands and decreases in all directions from the plume. Here, the sand fraction will fall directly to the sea floor when the sea ice melts each summer, while the 
$<10 \mu \mathrm{m}$ fraction is likely to be deposited directly into the ocean as the sea ice breaks up and is advected along the Victoria Land coastline as indicated by forward air mass trajectories. 


\section{Chapter 3 \\ The iron geochemistry of aeolian sand and dust in McMurdo Sound}

\subsection{Iron in the Ross Sea, Southern Ocean}

\subsubsection{Introduction}

The co-variation of dust flux, $\mathrm{Fe}$ concentration, atmospheric $\mathrm{CO}_{2}$ concentrations and temperature in Antarctic ice cores suggested that Fe-fertilisation from dust might stimulate phytoplankton growth in high nutrient low chlorophyll (HNLC) waters and hence increase the magnitude of the ocean's "biological carbon pump" during glacial periods (Fig. 3.1B), drawing down atmospheric $\mathrm{CO}_{2}$ into the ocean and initiating global temperature changes (Fig. 3.2; Martin, 1990). Evidence from Fe-fertilisation experiments now confirms aspects of Martin's (1990) "Fe hypothesis", whereby the rate of supply of Fe to Fe-limited waters control rates of primary productivity in HNLC waters, including the Southern Ocean (SO), which comprises $\sim 30 \%$ of the world ocean (de Baar et al., 1990; 2005; Martin et al., 1991; 1994; Liss and Tindale, 1994; Blain et al., 2001; Hutchins and Bruland, 1998; Boyd et al., 2000; 2001; 2002; 2007; de Baar and Boyd, 2000; Hutchins et al., 2002; Coal et al., 2004; 2005; Frew et al., 2006). Phytoplankton require Fe to carry out photosynthesis, respiration and nitrogen fixation. Although $\mathrm{Fe}$ is abundant in the Earth's crust (3.5\%; Taylor and McLennan, 1995), it is only present at trace concentrations in seawater (e.g. Boyd et al., 2002; 2007). This is because Fe has a low solubility, high particle reactivity and high biological demand (Martin et al., 1994; Liu and Millero, 2002).

Current literature suggests mineral dust is an important, but not the only external source of $\mathrm{Fe}$ to remote waters (Duce and Tindale, 1991; Boyd and Ellwood, 2010). Other potential Fe sources include; upwelling of deep ocean water (with possible enrichment due to contact with sediments at depth), mineral dust associated with continental (Elrod et al., 2004; Jickells et al., 2005) and extra-terrestrial (Johnson, 2001) inputs, sediment resuspension and lateral advection (Moore and Braucher, 2008), sea ice melting (Smith and Nelson, 1986; Edwards and Sedwick, 2001; Lannuzel et al., 2007; 2008; 2010), and iceberg melting (Smith et al., 2007; Raiswell et al., 2008a; 2008b). In particular, Fe budgets for the SO point to regional 
differences in the relative contributions of oceanic and atmospheric Fe supply (e.g. Fung et al., 2000; Lancelot et al., 2009). Fe in the subarctic Pacific is supplied mainly by dust deposition (Martin et al., 1989; Boyd et al., 1998). In contrast, current literature suggests the SO is mainly supplied with Fe by oceanic sources (de Baar et al., 1995; Edwards and Sedwick, 2001; Measures and Vink, 2001; Lannuzel, 2008; Lancelot et al. 2009). These large scale variations in the importance of Fe sources are played out on a smaller scale within the Ross Sea, with both dust and ocean upwelling being proposed as the primary source of Fe in the region (Lancelot et al., 2009; Fung et al., 2000), but the present data in the literature does not allow any source of Fe to be excluded (Sedwick et al., 2011).

This section reviews both the evidence for the importance of aeolian dust as a source of Fe in the SO in general and more specifically the Ross Sea, by examining i) the sources Fe and the significant delivery mechanisms to the ocean and ii) discussing the bio-availability of aeolian Fe.

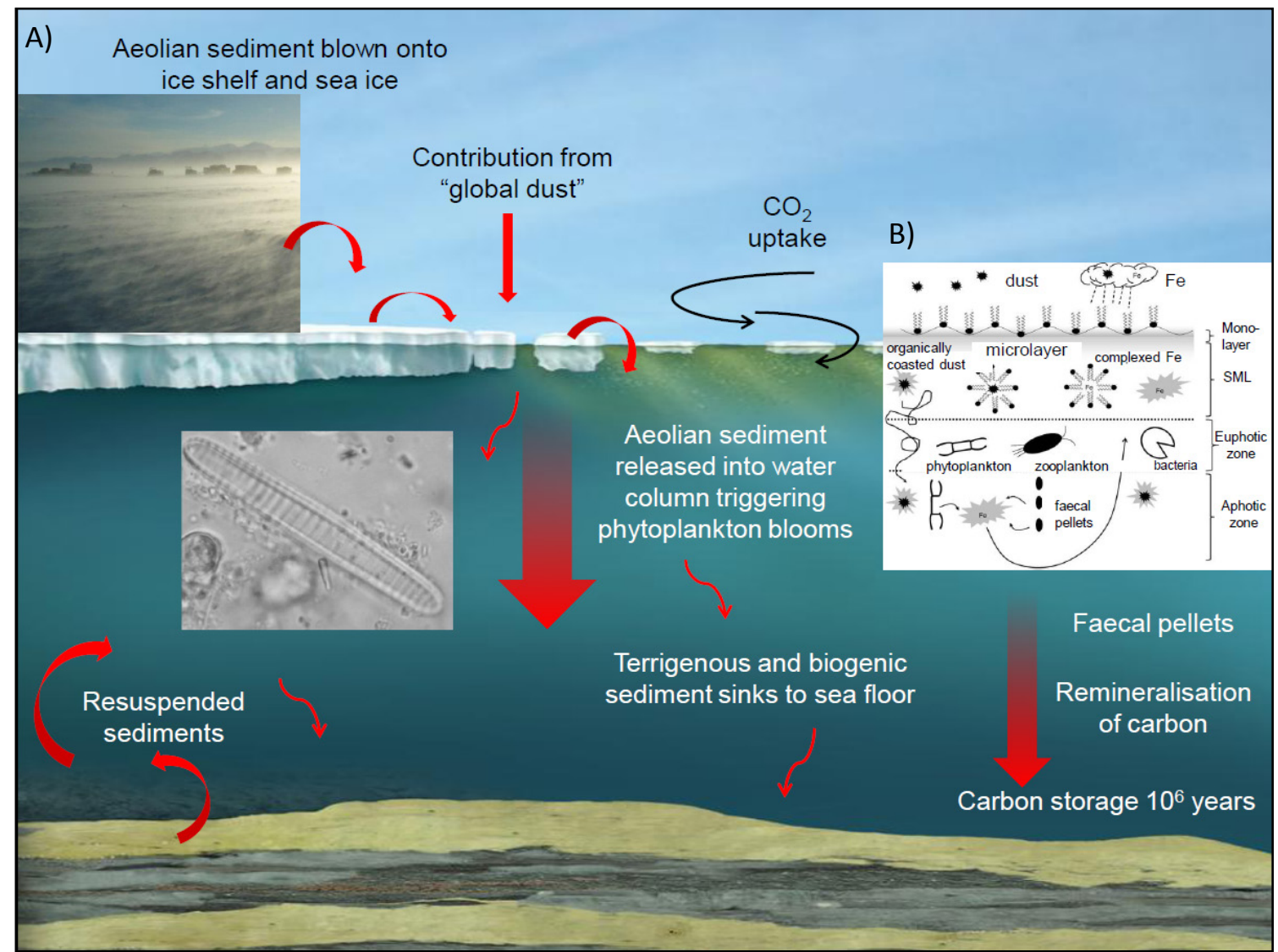

Fig. 3.1: Schematic illustrating A) Fe supply, cycling and B) the 'biological carbon pump' in Antarctic waters and the processing and fate of aerosol $\mathrm{Fe}$ in the ocean. Fe sources include: local and global dust, sea ice and ice bergs, resuspended shelf sediments and upwelling. B) Three modes of Fe supply (atmospheric, oceanic and the interface between the ocean and atmosphere). Source for insert: Boyd et al. (2010). 


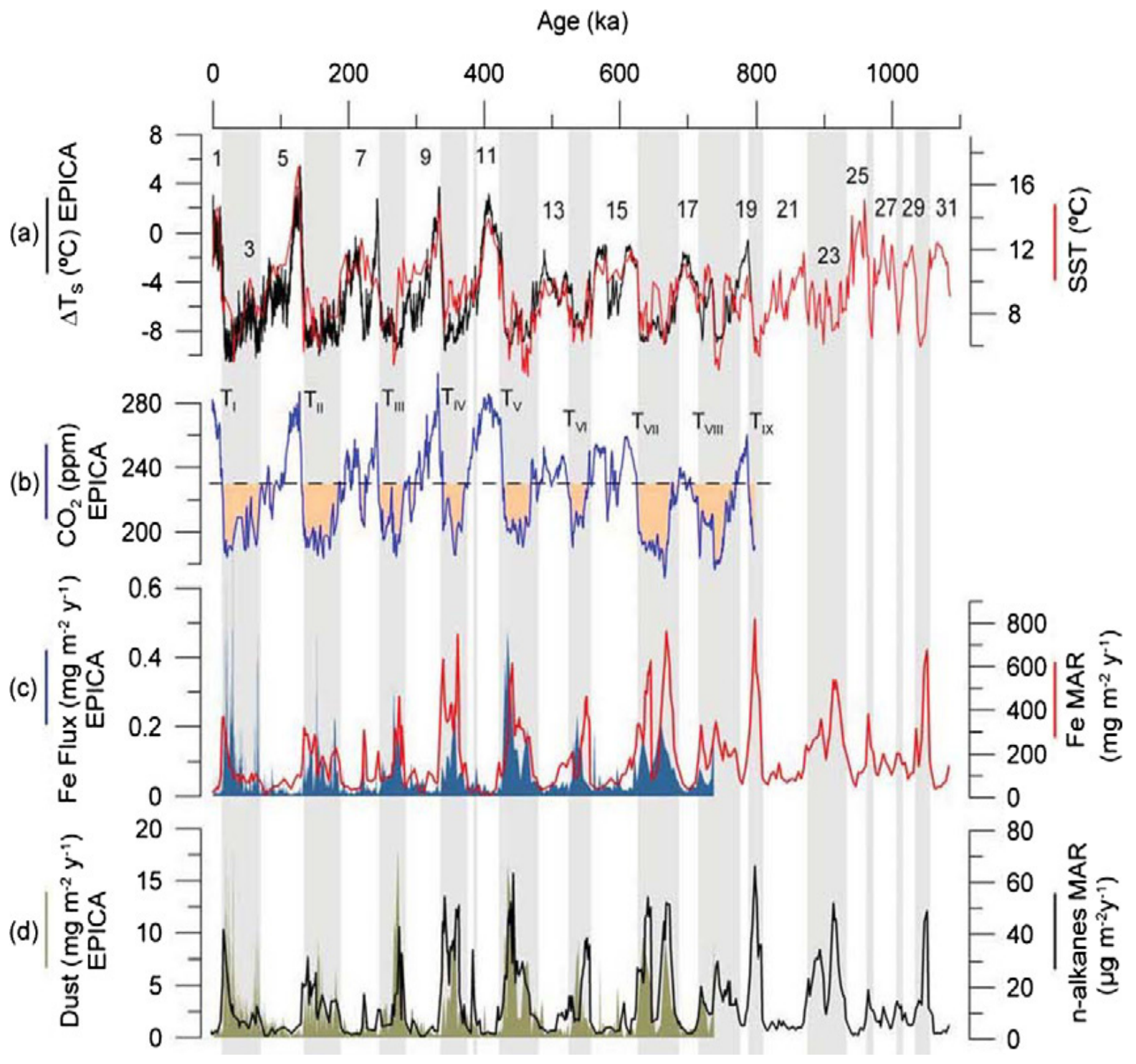

Fig. 3.2: Comparison of marine and terrestrial records over the last 1.1 Ma. (a) Temperature reconstruction from EPICA ice cores (black; Jouzel et al., 2007) and alkenone-based SST (red). Marine isotope stages (MIS) are shown for reference. (b) Atmospheric $\mathrm{CO}_{2}$ concentrations from the EPICA ice cores (Luthi et al., 2008; Petit et al., 1999; Siegenthaler et al., 2005). Dashed line indicates the $\mathrm{CO}_{2}$ level when productivity starts to increase above the average interglacial value. Filled area illustrates $\mathrm{CO}_{2}$ concentrations below 230 ppmv. Glacial terminations are shown for reference. (c) EPICA Fe flux (blue) and Fe mass accumulation rate flux (red). (d) EPICA insoluble dust (light brown) and long-chain odd carbon-numbered n-alkanes $(\mathrm{C23}$ - 33) mass accumulation rate (blue). (e) alkenones mass accumulation rate (green) and TOC mass accumulation rate (black; Diekmann and Kuhn, 2002). Shaded areas highlight the high-productivity intervals when alkenones mass accumulation rate are three times higher than the average interglacial value. Source: Martínez-Garcia et al. (2009). 


\subsubsection{Sources of iron in the Southern Ocean}

\subsubsection{The distribution of dissolved iron concentrations in Southern Ocean}

Dissolved Fe concentrations in the SO are variable (De Baar and De Jong, 2001). Typical Antarctic open ocean waters contain low dissolved Fe concentrations of $0.16 \mathrm{nM}$ (Martin et al., 1990b). In the Ross Sea, dissolved Fe values of $0.1-0.2 \mu \mathrm{mol} \mathrm{m}^{-3}$ at $\sim 200 \mathrm{~m}$ depth (the base of the ocean's upper mixed layer) have been observed in summer (Coal et al., 2005). Fe concentrations are three times as great in the southern Ross Sea than the northern Ross Sea (Fitzwater et al., 2000). In contrast, Sedwick et al. (2008) show that dissolved Fe concentrations in the Australian sector of the SO range from $0.76 \mathrm{nM}$ (subtropical front) to $<0.1 \mathrm{nM}$ (between polar front and Antarctic continental shelf). This is consistent with Bowie et al. (2009) who found that south of Tasmania dissolved Fe concentrations in the mixed layer range from $\sim 0.1-0.7 \mathrm{nmol} \mathrm{L}^{-1}$ (Bowie et al., 2009). Dissolved Fe in the Kerguelen Islands $(0.41-0.71 \mathrm{nM})$ is greater than typical values of the SO (Bucciarelli et al., 2001), because this coastal zone is supplied by the input of riverine Fe, soil leaching by rain waters and aeolian dust inputs (Bucciarelli et al., 2001).

\subsubsection{Sources of iron to the Southern Ocean}

Sources of $\mathrm{Fe}$ in Antarctic waters can be divided into three main categories; i) aeolian sediment, either via direct input from atmospheric fallout, or following storage and release from sea ice; ii) oceanic sources, including resuspension of bottom sediment followed by upwelling or advection and regeneration of organic material within the water column and; iii) sediment derived from melting icebergs (Fig. 3.1). The relative importance of each source changes both geographically and temporally (Boyd et al., 2004; Cassar et al, 2007; 2008; Boyd and Mackie, 2008; Lannuzel et al., 2010; de Baar et al., 1995; Blain et al., 2007; Sedwick et al., 2007; Boyd and Ellwood, 2010). Appendix 5 and 6 illustrates estimates of Fe concentrations from various sources for the SO. 


\section{Iron from aeolian dust}

The supply of Fe from aeolian sediment into the Ross Sea and SO has only been measured at a few locations (e.g. Measures and Vink, 2000; Edwards and Sedwick, 2001) but has also been estimated from models of global dust deposition (Duce and Tindall, 1991; Tegan and Fung 1995; Luo et al., 2003; Mahowald et al. 2005; Fan et al., 2006; Li et al., 2008; Wagner et al., 2008). Modelling studies, measured dust accumulation rates and aerosol samples (i.e. from samples collected in the air column) provide estimates of the aeolian Fe flux to the SO. In general, the atmospheric dust load in the SO is known to be among the lowest in the world (Prospero et al., 1981; 1990; Duce and Tindale, 1991; Wagner et al., 2008). Based on aerosol samples the dust flux is estimated as $0.37 \pm 0.18 \mathrm{mg} \mathrm{m}^{-2} \mathrm{~d}^{-1}$ (Bowie et al., 2009) for samples collected south of Tasmania or $12 \pm 7 \mathrm{ng} \mathrm{m}^{-3}$ representative of the broader SO. Similarly, the modelling study of Duce et al. (1991) suggests the SO has a dust flux of $0.27 \mathrm{mg} \mathrm{m}^{-2} \mathrm{~d}^{-1}$. The most recent study found aerosol concentrations to be two orders of magnitude lower than these estimates for the SO (Figs. 2.11 and 2.12; Wagner et al., 2008). Models also suggest the dust flux is lower for the SO: $1.12 \mathrm{mg} \mathrm{m}^{-2} \mathrm{~d}^{-1}$ (Mahowald et al., 2005); and $2.47 \mathrm{mg} \mathrm{m}^{-2} \mathrm{~d}^{-}$ ${ }^{1}$ (Luo et al., 2003).

Edwards and Sedwick (2001) directly measured particulate Fe deposited from the atmosphere in snow samples from Prydz Bay, Dumont d'Durville Sea, the Ross Sea and Princesses Elizabeth Land, East Antarctica. After leaching their samples for $>3$ months following acidification, they report total dissolvable $\mathrm{Fe}$ concentrations (soluble at $\mathrm{pH} \sim 2$ ) ranging from 20 - $2950 \mathrm{pg} \mathrm{g}^{-1}$ (Appendix 6); an atmospheric Fe deposition flux of $0.017-0.11 \mathrm{mg} \mathrm{m}^{-2} \mathrm{yr}^{-1}$; and soluble $\mathrm{Fe}$ as a percentage of total $\mathrm{Fe}$ in filtered snow ranging from $\sim 10-90 \%$. They assume these results are representative of atmospheric deposition over seasonally ice-covered, high nutrient Antarctic waters and calculate that atmospheric Fe potentially supports annual phytoplankton production of $1.1 \times 10^{12}$ mole C in Antarctic seasonal sea ice zone (SSIZ), which is less than $5 \%$ of the estimated total annual primary production in this ocean region (Edwards and Sedwick, 2001). Sedwick et al. (2000) report values of $12.9-19.2 \mathrm{nM}$ total dissolved Fe from snow samples collected on Ross Sea pack ice.

The importance of aeolian Fe to the SO is debated (Loscher et al., 1997; Visser et al., 2003; Cassar et al., 2007; 2008; Boyd and Mackie, 2008). Fe dust supply from Australia is thought to stimulate regional SO phytoplankton blooms (Cassar et al., 2007; Mackie et al., 2008; 
Boyd et al., 2010). Cassar et al. (2007) compared patterns of primary production with model estimates of dissolved Fe added to the surface ocean by aerosols and found that production is proportional to modelled input of soluble Fe in aerosols.

Although Cassar et al. (2008) disagrees, Boyd and Mackie (2008) argue that the productivity and simulated aerosol Fe deposition data by Cassar et al. (2008) from the northern hemispheric model is not appropriate for use in the SO. The most recent study of aerosol Fe deposition to the SO shows that dust and Fe deposition fluxes are up to two orders of magnitude lower the previous predictions $\left(38 \mu \mathrm{g} \mathrm{m}^{-2} \mathrm{~d}^{-1}\right)$, confirming that dust deposition is not the dominant source of Fe to the HNLC areas of the SO (Wagner et al., 2008). Similarly, Johnson et al. (2011) show that summer episodic dust fluxes from Patagonia are not likely to be a major source of bio-available Fe to the South Atlantic Ocean, which is characterised by high primary productivity, but they argue that even if Patagonian dust plumes may not cause visible phytoplankton blooms, they could still influence background chlorophyll- $a$ in the South Atlantic sector of the SO. Furthermore, Boyd et al. (2010) use a simple dust-biota assessment tool for both the contemporary and paleooceanographic ocean to suggest that dust deposition can easily be mistaken as a primary cause of enhanced biological activity and that due to the slow dissolution rate of Fe, dust-mediated phytoplankton blooms are likely to be rare in the modern ocean. Furthermore, direct atmospheric dust deposition as a primary Fe source was ruled out as the main source of Fe to the SO by Martin et al. (1990) because of low surface water dissolved Fe $\left(0.2 \mathrm{nmol} \mathrm{kg}{ }^{-1}\right)$ concentrations in offshore Drake Passage waters. Fitzwater et al. (2000) suggest that aeolian dust deposition may contribute at most, about $0.1 \mathrm{nM}$ of Fe to the mixed layer when considered over winter particulate $\mathrm{Fe}$ in snow found on seasonal ice.

During 1997-2001 episodic elevated chlorophyll- $a$ (a proxy for primary productivity stocks) occurred in subantarctic waters southeast of NZ. Boyd et al. (2004) found no evidence that these events were mediated by atmospheric Fe supply from Australia, nor mediated by winddriven lateral advection or vertical mixing. However, during this period dust storms sent plumes of sediment over subtropical waters and subantarctic waters in early spring when phytoplankton are likely to be limited by light rather than Fe (Boyd et al., 2004). Moreover, only two air mass trajectories were found over HNLC waters, each of which may have had a low Fe load and also cloud contamination prevented remote sensing of ocean colour, hence the relationship between dust and chlorophyll was inconclusive (Boyd et al., 2004). 


\section{Iron from melting sea ice}

Sea ice is not a new source of Fe. Instead it can act as a significant repository of trace nutrients derived from either the atmosphere in the form of particulates (Jickells et al., 2005; Lannuzel et al., 2007), or incorporated from the underlying ocean during ice formation in the form of i) 'regenerated' Fe associated with organic matter from the preceding summer season, ii) via upwelling/ vertical diffusion (e.g. de Baar and Je Jong, 2001), iii) and lateral advection of resuspended sediments from the Antarctic continental shelf (de Baar et al., 1995; Croot and Hunter, 1998; Schoemann et al., 1998; Johnson et al., 1999; Fitzwater et al. 2000; Sedwick et al., 2000). The seasonal growth and melt of sea ice places important constraints on the timing and magnitude of Fe accumulation and release to Antarctic surface waters. Sea ice releases nutrients when conditions, such as 24 hour summer sunlight, are coincidently right for phytoplankton blooms. Lannuzel et al. (2010) reports a decrease in dissolved Fe concentrations in sea ice from winter to summer and then a replenishment of $\mathrm{Fe}$ in winter due to percolation processes from ice melting and biological uptake.

Trace element data for Antarctic sea ice in general is scarce and the various processes leading to Fe accumulation in sea ice have not been studied in detail. Published observations report Fe concentrations up to two orders of magnitude higher in sea ice than in the adjacent or underlying seawater (Grotti et al., 2005; Lannuzel et al., 2006; 2007; 2008; Aguilar-Islas et al., 2008; Vancoppenolle et al., 2009). Furthermore, fast ice attached to the continental margin has been found to contain up to two orders of magnitude greater particulate Fe than pack ice (Boye et al., 2001; de Baar and de Jong, 2001; Grotti et al., 2005; Lannuzel et al., 2007; 2008; 2010; van der Merwe et al., 2009), an observation attributed to greater incorporation of sediment resuspended from relatively shallow waters in winter through deep vertical mixing and lateral advection from the Antarctic continent (Sedwick et al., 2000; Lannuzel et al., 2010). However, as most ( $>85 \%$ ) of the Fe supplied by sediment resuspension is associated with the particle phase (bio-genic or particulate), it is not readily available for phytoplankton uptake. Yet, gradual solution of resuspended sediment during the lateral transport of coastal waters to the open ocean is a more bio-valuable source of Fe to the Antarctic ice pack (Lam et al., 2006). 
Modelling studies also suggest that the largest changes of Fe capture and storage by sea ice, during its formation, occur close to the coast (Lancelot et al., 2009). For example, in the western Ross Sea, ice forms each year leading to an annual mean Fe uptake rate in sea ice from the ocean of 9 pmol Fe m $\mathrm{m}^{-2}$. Fitzwater et al. (2000) suggests that melting sea ice adds $3.25 \mathrm{nM} \mathrm{Fe}$ to the Ross Sea. During melting of sea ice in the Ross Sea in 1995-1996 the significance of ice-derived Fe was observed, where surface Fe concentrations decreased from $2.25 \mathrm{nM}$ to $0.16-0.17 \mathrm{nM}$ seventeen days later, simultaneously chlorophyll increased three fold (Sedwick and DiTullio, 1997). Sedwick and DiTullio (1997) attribute this biological activity to the release of $\mathrm{Fe}$ from the melting sea ice with subsequent reduction of dissolved Fe due to biological removal. In the Ross Sea, as with other regions in the SO, the release of Fe from sea ice induces an increase in chlorophyll- $a$ observable by satellite (Arrigo et al., 2004).

\section{Iron from Icebergs}

Icebergs, glaciers, ice sheets and ice shelves are other sources of Fe to the ocean during melting (Löscher et al., 1997; Fitzwater et al., 2000; Sedwick et al., 2000; Smith et al., 2007). The iceberg-derived Fe pattern mimics iceberg migration (e.g. Raiswell et al., 2006). Raiswell et al. (2006) found floating glacial ice collected from the ocean in the wake of an iceberg had an acid-soluble Fe concentration of 20.4 nM. Raiswell et al. (2008a) estimates (using iceberg samples melted and filtered through a $0.2 \mu \mathrm{m}$ filter) the flux of iceberg hosted

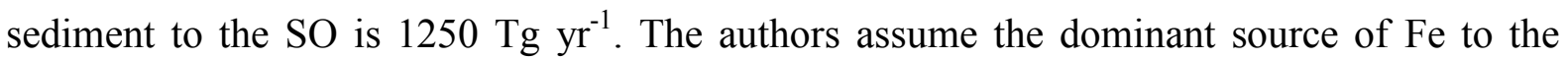
continental shelf is from aeolian sediment and the main source to the open ocean is from icebergs. Sediment from Antarctica trapped in glaciers and from maritime Antarctic Islands show low concentrations of Fe, present as (oxyhydr)oxides (Raiswell et al., 2006). Lancelot et al. (2009) shows that the release of dissolved Fe from iceberg calving and melting contributes up to $255 \mathrm{mg} \mathrm{m}^{-3}$ of chlorophyll- $a$ in areas influenced by icebergs.

Most recently, a special issue of Deep-Sea Research II (vol. 58, 2011) on free-drifting icebergs in the SO examines the impact of icebergs on the chemical and biological characteristics of the surrounding waters when compared to more peripheral waters in the Scotia and Weddell Seas. Free drifting icebergs as a source of dissolved and particulate Fe can influence i) phytoplankton biomass and composition (e.g. diatom abundance; Lin et al., 2011; Murray et al., 2011); ii) higher grazing pressure on enhanced stocks of phytoplankton 
by zooplankton within $2 \mathrm{~km}$ of icebergs (Vernet et al., 2011); iii) algal communities attached to icebergs comprising mainly of diatoms (Robinson et al., 2011); iv) macrozooplankton and microplankton communities around icebergs that are qualitatively and quantitatively different reflecting enhanced biological activity adjacent to ice free waters (Kaufmann et al., 2011); v) and higher sea bird densities and different composition within a few hundred meters of icebergs (Ruhl et al., 2011).

\subsubsection{An iron budget for the Southern Ocean}

Considering these various sources, several biogeochemical Fe budgets have been attempted for the SO (Bowie et al., 2001; Edwards and Sedwick, 2001; Boyd et al., 2005; Lannuzel et al., 2007; Tagliabue et al., 2009) but further work is required due to insufficient knowledge of oceanic Fe concentrations (de Baar and De Jong, 2001; Sedwick et al., 2011) and aerosol Fe deposition rates (Jickells and Spookes, 2001). Furthermore, the relative importance of episodic Fe supply events such as dust storms compared to more sustained Fe supply, such as vertical diffusivity (Law et al., 2003), is difficult to assess. Edwards and Sedwick (2001) and Lannuzel et al. (2007) suggest that upwelling is the most important Fe source, while a modelling study by Lancelot et al., (2009) excludes upwelling as a Fe source in their modelling study and suggests continental sediments are the primary source of Fe in the Ross and Weddell Seas (Table 3.1).

The spatial distribution of export production in the SO reflects indirectly the importance of local (regions of high dust deposition adjacent to Australia and Patagonia) and non-local sources of Fe from both sediments and the atmosphere (Moore and Braucher, 2008; Tagliabue et al., 2009). Bowie et al. (2009) reports biogeochemical Fe budgets for three sites south of Australia. They found that distinct regional environments were responsible for differences in the source of Fe supply mechanisms, with higher Fe supply and fluxes observed in surface northern subantarctic waters. Subsurface waters southeast of Tasmania were also enriched with particulate $\mathrm{Fe}, \mathrm{Mn}$ and $\mathrm{Al}$, indicative of a strong lateral advective source from shelf sediments. They suggest that phytoplankton blooms are therefore driven by both seasonal Fe supply from southward advection of subtropical waters and by dust deposition. 
Table 3.1: Annual Fe sources for the SO south of $60^{\circ} \mathrm{S}$ from modelled estimates by Lancelot et al. (2009).

\begin{tabular}{ccc}
\hline Source & $\begin{array}{c}\text { Fe flux } \\
\left(\mathbf{1 0} \mathbf{~ m o l ~ F e ~} \mathbf{~ y r}^{-\mathbf{1}}\right)\end{array}$ & Total (\%) \\
\hline Dust & 0.014 & 3 \\
Continental sediment & 0.28 & 90 \\
Iceberg calving/ melting & 0.028 & 7 \\
\hline
\end{tabular}

\subsubsection{Atmospheric iron and phytoplankton productivity}

\subsubsection{The measurement of soluble iron}

Reported values of the percentage of Fe in dust that is soluble range from 0.01 to $90 \%$ (e.g. Mahowald et al., 2005). This variation in Fe solubility values is due to differences in the properties of dust particles (e.g. source and mineralogy) but also due to the inconsistency in dust leaching experiments. Leaching methods involve the extraction of soluble Fe from dust via exposure to a particular leaching solution. Table 3.2 shows the variations in the type and volume of the leaching solution as well as the length of dust leaching in different leaching methods. The time of leaching varies from instantaneous (Ooki et al., 2009) to three month periods (Edwards and Sedwick, 2000). The result of this variation could be considerable given that solubility of Fe is expected to vary as a function of time (e.g. Aguilar-Islas et al., 2010). Short exposure (5 - 10 seconds) of aeolian dust to leaching solutions of MQ water or seawater are likely to mimic the instantaneous dissolution processes affecting aeolian dust that occurs during dry deposition to the ocean.

Table 3.2: Examples of iron leaching methods employed in published research accounts.

\begin{tabular}{cccc}
\hline Author & Leaching solution & Volume (ml) & Time \\
\hline Aguilar-Islas et al. (2010) & UVSW $^{\mathrm{a}}$; UVSW $; \mathrm{MQ}^{\mathrm{c}}$ & $3-4 \mathrm{x} 40$ & $<1,30 \& 90 \mathrm{~min}$ \\
Bonnet et al. (2004) & & & $<5,20 \& 60 \mathrm{~min}$ \\
Buck et al. (2006; 2010b) & Filtered seawater & 500 & $24 \mathrm{~h} ; 7 \mathrm{days}$ \\
Cwiertny et al. (2008a) & MQ water; filtered seawater & 100 & $10 \mathrm{~s}$ \\
Journet et al. (2008) & Acidified MQ water & 40 & $120 \mathrm{~h}$ \\
Mendez et al. (2010) & Acidified MQ water & 250 & $60 \mathrm{~min}$ \\
Ooki et al. (2009) & Filtered seawater & 1000 & 35 days \\
Sarthou et al. (2003) & Filtered seawater & $1100-2100$ & Instantaneous \\
Scroth et al. (2009) & Ammonium acetate buffer & 25 & $1-2$ hours \\
Trapp et al. (2010) & MQ water & $20 \mathrm{x} 250$ & $40 \mathrm{~s}$ \\
Edwards and Sedwick (2001) & $1 \mathrm{M} \mathrm{NaCl} \mathrm{solution} \mathrm{acidified} \mathrm{to} \mathrm{pH} \mathrm{2}$ & $25-50$ & $300 \mathrm{~s}$ \\
Howe (2009) & Acidified (HCl) snow melt & & 3 months \\
\hline
\end{tabular}

${ }^{\mathrm{a}} \mathrm{UVSW}$ is seawater which has been exposed to ultraviolet radiation to remove natural Fe-binding ligands.

${ }^{\mathrm{b}}$ Desferal is a terrestrial siderophore used as an artificial Fe-binding ligand to mimic natural Fe-binding ligands.

${ }^{\mathrm{c}} \mathrm{MQ}$ water is ultrapure deionised water produced by a Milli-Q system from Millipore, USA. 
The heterogeneous chemistry of seawater and aeolian dust are also likely to contribute to the large variation in reported Fe solubility estimates in the literature. The obvious means to determine aerosol Fe solubility is the direct exposure of a dust sample to an aliquot of surface seawater. However, seawater aliquots taken from year to year are heterogeneous, making it difficult to reproduce results from leaching experiments (Baker and Croot, 2010). The difficulty of interpreting these experiments in the wider biogeochemical context lead Baker and Croot (2010) to suggest the use of standardised aerosol materials and a standardised artificial seawater solution in leaching experiments. A MQ water leaching solution allows for easy comparison of Fe solubility measurements among different research groups and standardisation of results from different dust source regions.

The amount of Fe that can be leached from dust may be significantly lower using seawater than MQ water is used as a leachate. Buck et al. (2006) found that soluble aerosol concentrations of Fe in seawater and MQ water showed a significant correlation $\left(\mathrm{r}^{2}=0.93\right)$. Aguliar-Islas et al. (2010) found that higher or equal Fe solubility was obtained when using seawater as the leaching solution versus using MQ water. Because MQ water is not a buffered solution, protocols that use MQ water as the leaching solution are likely to reach lower $\mathrm{pH}$ values and possibly overestimate the percentage dissolution of aerosol $\mathrm{Fe}$ in seawater. Experiments usually do not use any strongly binding Fe ligands thought responsible for the enhancements of Fe solubility in seawater (except Aguilar-Islas et al., 2010). Furthermore, differences in filter pore size $(0.45,0.2,0.02 \mu \mathrm{m})$ used to separate solid and aqueous phases after leaching could also strongly influence the fraction of Fe defined as soluble. Aguilar-Islas et al. (2010) quantified the influence of experimental artefacts upon Fe solubility by pairing data sets collected with leaching methods, differing in only one set variable, suggesting that the leaching method alone is not sufficient to account for the wide variety of Fe solubility reported in the literature.

\subsubsection{Modes of aeolian iron supply}

Aside from complexities in measuring soluble Fe there are a number of environmental controls that have a substantial impact on the magnitude of this value and the complexity of the controls on Fe solubility further contribute to the wide range of aerosol Fe solubility in the literature. The following section is divided into two; the first discusses processes that 
occur in the atmosphere that are known to influence Fe solubility and the second discusses those processes that occur in the ocean (Figs. 3.1 and 3.5).

\subsection{Atmospheric controls on Fe solubility}

\section{Atmospheric residence time of dust}

The solubility of $\mathrm{Fe}$ is controlled by many complex interactions and processes that can take place during the atmospheric transport of aeolian dust. Laboratory studies and field experiments have shown that Fe in soil is generally less soluble than in atmospheric aerosols (Zhung et al., 1990; Baker and Jickells, 2006; Buck et al., 2006) and these results have been interpreted to suggest that atmospheric transport and processing (e.g. chemical weathering) provide mechanisms in which Fe from mineral dust becomes increasingly soluble with transit time (Jickells and Spokes, 2001; Mahowald et al., 2005). Iron solubility in dust will be maximal when the rate of deposition for a given storm event is minimal i.e. where dust has travelled the furthest (fallout of large particles and low mass loading) and has been exposed to light (photochemistry) and cloud processing for the longest time period. For example, Gaspari et al. (2006) found that $55 \%$ of Fe from the EPICA Dome C ice core, EAIS was dissolved from particulates contained in Antarctic snow. Global dust samples in Antarctica with high Fe solubility (Barbante et al., 1997; Edwards and Sedwick, 2001; Gaspari et al., 2006) are transported thousands of kilometres from the source (e.g. Delmonte et al., 2004a; 2004b) to Antarctica, whereas Saharan soil samples taken from their source region have a considerably lower Fe solubility (0.001 \%; Guieu and Thomas, 1996).

\section{Particle concentration effect}

A number of studies have observed that Fe solubility is a function of aerosol particle concentration with higher Fe solubility observed at lower suspended particle concentration, known as the "particle concentration effect" (Fig. 3.3; Zhung et al., 1992; Spokes and Jickells, 1996; Bonnet and Guieu, 2004; Chen and Siefer, 2004; Baker and Jickells, 2006; Trapp et al., 2010). However, Baker et al. (2006) observes that this effect on Fe solubility is comparatively small in nature and instead suggest that a major factor controlling the observed differences in Fe solubility is the source of the dust (see section 3.3.1.5). 


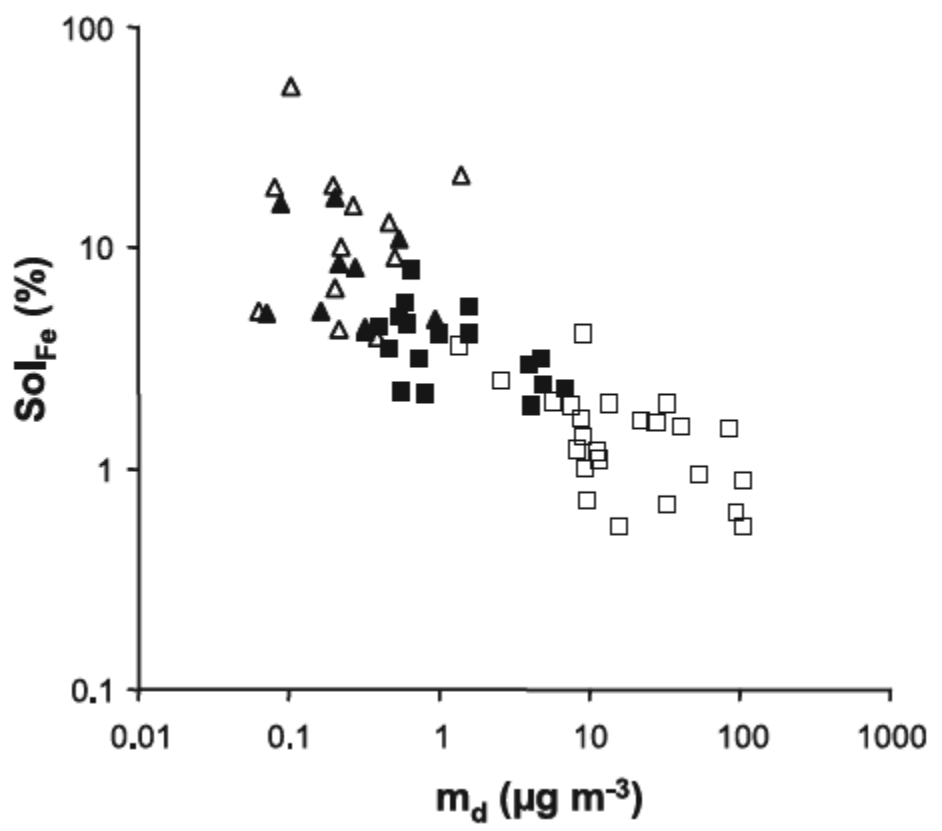

Fig. 3.3: The percentage of soluble $\mathrm{Fe}\left(\mathrm{Sol}_{\mathrm{Fe}}\right)$ in aerosol collected over the Atlantic Ocean as a function of mineral aerosol atmospheric mass loading $\left(m_{d}\right)$. Samples collected from the northern hemisphere are indicated by open symbols, those collected from southern hemisphere air by filled symbols. Squares indicate tropical/subtropical samples; triangles indicate temperate samples. Source: Baker and Jickells (2006).

\section{Grain size}

Baker and Jickells (2006) proposed a physical control on aerosol Fe solubility: the preferential removal via settling of larger particles (low surface area to volume) during longrange transport. Thus, at lower dust concentrations far from source the modal particle size of the dust population is smaller and the dust population has a correspondingly larger surface area to volume ratio (Fig. 3.4) and hence a greater proportion of its Fe content is at the particle surface making it more soluble. By contrast, the particle size distribution of mineral dust over the SO is rather uniform with a mass median diameter of approximately $5 \mu \mathrm{m}$ (e.g. Delmonte et al., 2002). 


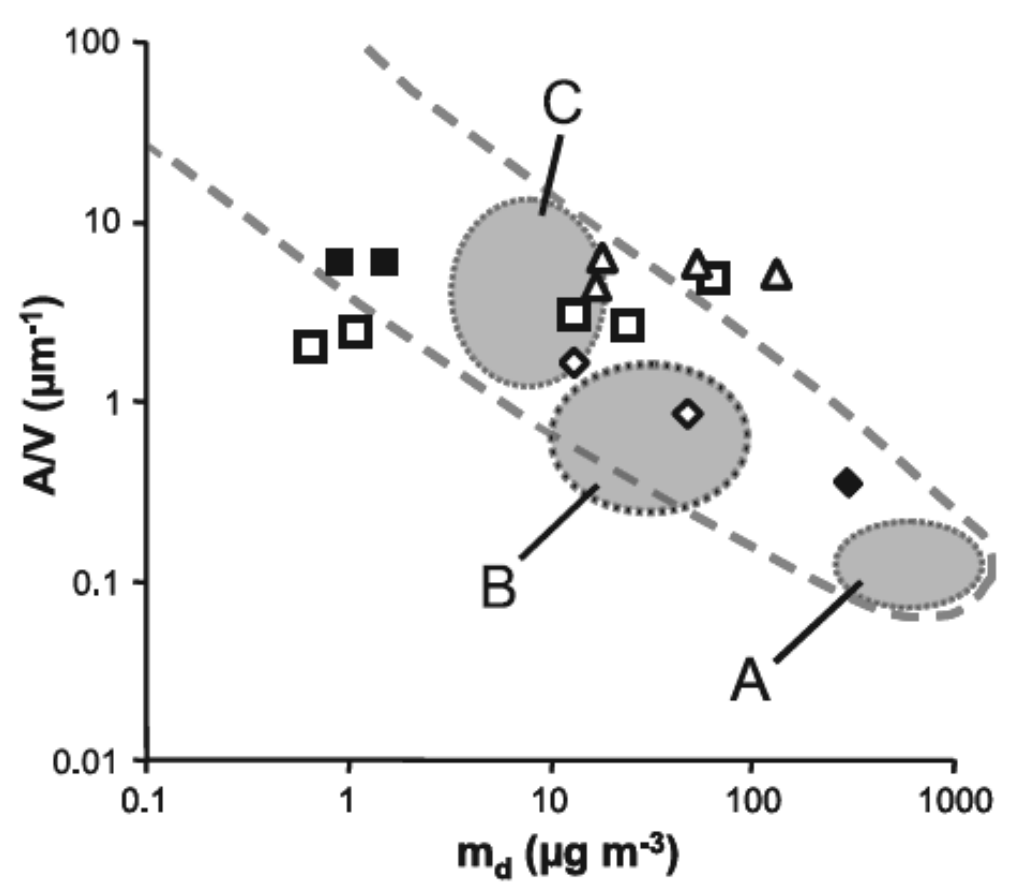

Fig. 3.4: The variation in surface area to volume ratio (A/V) of a simple spherical particle with atmospheric concentration $\left(\mathrm{m}_{\mathrm{d}}\right)$ calculated for observed mineral particle sizes and mass loadings for near-source mineral dust (A), dust sampled off the coast of West Africa (B), and Saharan dust observed over the Caribbean (C). Also shown is the general form of the $A / V$ versus $m_{d}$ relationship extrapolated to lower dust concentrations. Source: Baker and Jickells (2006).

Models of atmospheric dust and its role in the marine Fe cycle have incorporated a range of particle sizes into simulations (e.g. Fung et al., 2000; Gao et al., 2003; Fan et al., 2006). Furthermore, studies of Fe solubility use different particle size classes to differentiate the operationally defined fine and coarse dust fraction (e.g. Luo et al., 2005; 2008; Chen and Siefert, 2004; Johansen and Hoffman, 2003; Buck et al., 2010a). Some studies have found Fe solubility to be higher in fine grained particles than in coarse grained particles (Duce et al., 1991; Baker and Jickells, 2006; Siefert et al., 1999; Johansen et al., 2000; Chen and Siefert 2004; Hand et al., 2004). However, other modelling and field sampling studies have not been able to confirm these effects (Baker et al., 2006; Buck et al., 2006; Luo et al., 2005). Importantly, it appears that the solubility of $\mathrm{Fe}$ is not a simple function of surface area to volume ratio and particle size. Baker and Croot (2010) suggest that some other transport related process may contribute to the observed trends in the Fe solubility. Trapp et al. (2010) suggests that the increase in Fe solubility with decreasing modal particle size could be a result of a shift in mineralogy of the dust load during transport. This could cause rapid loss of larger particles (e.g. quartz and other resistant minerals with potentially lower Fe content than 
finer particles). Furthermore, clay size minerals are smaller and have high fractional solubility (Glaccum and Propsero, 1980; Journet et al., 2008; Mackie et al., 2008).

\section{Acidity controls / Acid processing}

Internal mixing (chemical composition of each particle in the aerosol population is the same as the bulk composition of the aerosol population) of the acidic and mineral dust components of an aerosol population enhances Fe fractional solubility (Zhu et al., 1992; Spokes et al, 1994; Jickells and Spokes, 2001; Jickells et al., 2005; Mahowald et al., 2005). This has been demonstrated in laboratory experiments that simulate changes in aerosol $\mathrm{pH}$ (Spokes et al., 1994; Spokes and Jickells, 1996; Mackie et al., 2005). The solubility of Fe in rainwater is often reported to be higher than $\mathrm{Fe}$ in aerosols which could be due to the low $\mathrm{pH}$ of rainwater (Jickells and Spookes, 2001).

Furthermore, repeated wetting of particle surfaces during cloud cycling under acidic conditions is expected to solubilise the Fe on the outer surfaces of the particles as well as enhancing the reduction of Fe(III) (Zhu et al., 1992; 1993). For example, Saharan dust transported over the Atlantic Ocean is entrained into air that already contains anthropogenic acids emitted from southern Europe and North Africa, so that acid species are likely already established in the aerosol population before dust entrainment (Sholkovitz et al., 2009). Another example is where Asian dust transported over regions of intense industrial emissions before its passage over the Pacific. In this case the dust supply likely provides a significant surface area for direct uptake of acid species from the gas phase. Furthermore, biomass burning and fossil fuel combustion have been suggested to be potential sources of aerosol Fe (Luo et al., 2008) with higher Fe solubility than mineral dust (Spokes et al., 1994; Sedwick et al., 2007).

However, Buck et al. (2010b) found that there was no correlation between the concentration of acidic species and Fe solubility $\left(\mathrm{R}^{2}=0.04\right)$, which questions the significance of acid processing as a control on Fe solubility in the environment. No relationship between $\mathrm{Fe}$ solubility and the concentrations of acid species (non-sea salt $\mathrm{SO}_{4}{ }^{2}, \mathrm{NO}_{3}{ }^{-}$) nor the net acidity of the aerosol were found by Baker et al. (2006), nor were significant relationships were found in Spokes and Jickells (1995) or Hand et al. (2000). 


\section{Photochemical reactions in the atmosphere}

During dust transport in the atmosphere, photolysis can further increase the amount of soluble Fe. It has been suggested that exposing insoluble Fe(III) to solar radiation can convert Fe(III) species to more soluble forms of Fe(II) during atmospheric transport (Zhu et al., 1997; Siefert et al. 1999; Luo et al., 2005). Photolysis experiments conducted under light and dark conditions show that both total dissolved Fe and dissolved Fe(II) increase with temperature and upon light exposure (Fu et al., 2010; Rubansinshege et al., 2010).

\section{Source, mineral composition, speciation and weathering}

Fe solubility is highly dependent on the mineral composition of the dust (Baker et al., 2006; Sedwick et al., 2007; Schroth et al., 2009; Aguilar-Islas et al., 2010). Aguilar-Islas et al. (2010) found that different types of aerosols contribute more variability in aerosol $\mathrm{Fe}$ solubility than do different leaching protocols. The study found that the percent of $\mathrm{Fe}$ solubility obtained from aerosols collected in two urban regions (1\%) was lower that the solubility from samples collected in the open ocean (6-13\%). The authors attribute the low solubility resulting from the mixture of silicate (less soluble) and carbonaceous (more soluble) particles. Raiswell et al. (2008b) shows the presence of potentially bio-available Fe as ferrihydrite and goethite in nanoparticulate clusters in sediments collected from icebergs in the SO and glaciers on the Antarctic landmass.

Fe speciation (oxidation state and bonding environment) can vary considerably among PSAs. Speciation in dust source appears to be an important driver of Fe solubility (Schroth et al., 2009; Trapp et al., 2010). Schroth et al. (2009) showed that speciation data from arid soil samples indicate that Fe species in the dust source are primarily partitioned as secondary ferric weathering products produced during pedogenenesis on oxidating soil environments. Low solubility of $\mathrm{Fe}$ in these dusts is associated with the insolubility of these ferric precipitates. The relative abundances of Fe(II) bearing phases in loess compared to African dust will be influenced by the environmental factors related to surface soil formation (such as weathering regime and soil parent material composition), which will produce spatial variability in the speciation and solubility of Fe in arid dust storms (Schroth et al., 2009). 


\section{Glacial weathering}

In glacial dust, $\mathrm{Fe}$ species primarily reflect the Fe mineral composition of mechanically weathered bedrock of the glacial catchment, rather than ferric alteration products in soils that have been exposed to chemical processes for extended time periods (Schroth et al., 2009). Fe in glacial flour of the Wrangell Volcanic Field (Kuskulana Glacier) was considerably more soluble than Fe sourced in the metasedimentary and igneous rocks of the Chugach Mountains (Matanuska Glacier; Schroth et al., 2009). Furthermore, chemical weathering is likely to dissolve Fe from minerals in glacial debris either before transport or in subglacial environments where water is present and during iceberg transport, where melting sea ice can interact with iceberg-hosted sediment (Raiswell et al., 2008a).

\subsection{Oceanic controls of iron solubility}

There are a number of oceanic controls of Fe solubility (Boyd et al., 2010) which are illustrated in Figs. 3.1B and Fig. 3.5 and discussed below.

\section{Ocean and ocean-atmosphere interface}

The concentration of Fe(III) in natural waters is under strong $\mathrm{pH}$ control with a minimum around pH8 (Jolivet et al., 2004). This minimum in Fe concentration is a major reason why dissolved $\mathrm{Fe}$ is found in such low concentrations in the ocean compared to other natural waters. $\mathrm{Fe}(\mathrm{II})$ is the reduced form of $\mathrm{Fe}$, which is more soluble than $\mathrm{Fe}(\mathrm{III})$ and is rapidly oxidised in seconds to minutes in oxygenated seawater by $\mathrm{O}_{2}$ or $\mathrm{H}_{2} \mathrm{O}$ (Millero et al., 1987; Millero and Sotolongo, 1989; Kuma et al., 1996; Millero, 1998; Gonzalez-Davila et al., 2006). This oxidation is also strongly temperature-dependent with longer half lives (hours to days) in cold polar waters (Croot and Laan, 2002). Post-depositional processes can also solubilise Fe from recently deposited dust and aerosols in the surface waters. These processes include: residence time, photochemistry, grazing, scavenging and organic complexation. 


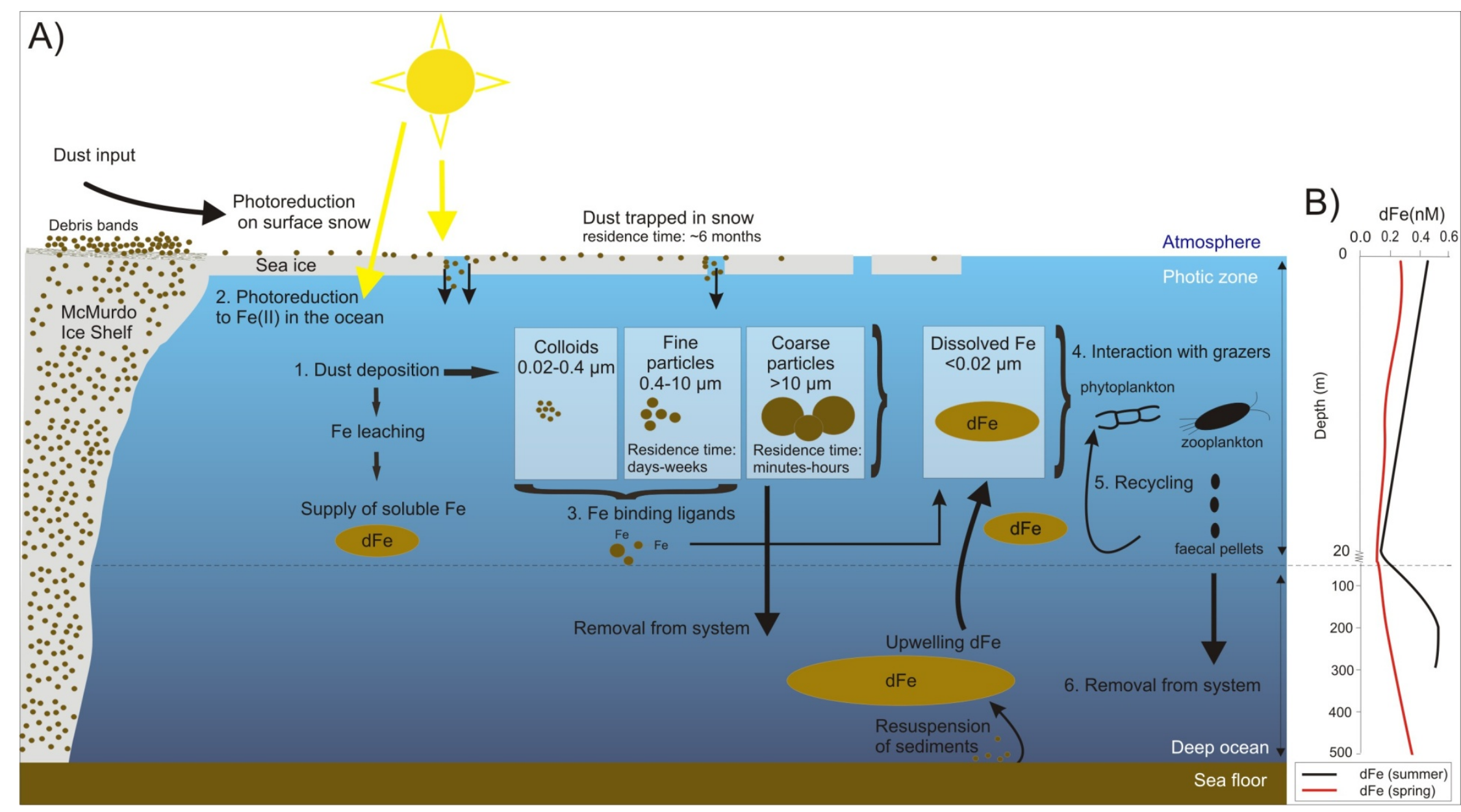

Fig. 3.5: Processing and fate of aeolian Fe in the ocean. A) Upon deposition of this aeolian dust into the ocean (1) instantaneous leaching of Fe ( $\sim 9 \%)$ is followed by longer term (days to weeks) leaching and coupled with photochemical mechanisms which enhance its solubility (2). During leaching most Fe probably enters the colloidal pool where it interacts with ligands (3), although particulate Fe can directly enter the soluble pool. Coarse particles settle out after a residence time of days to months. Soluble, colloidal and particulate Fe is potentially available for biota (4). After uptake of Fe by biota, Fe is recycled through the photic zone (5) and some Fe is removed from the system (6). The solubility of aeolian Fe on the snow surface could be enhanced by photo-reduction. Dissolved Fe (dFe) is sourced not only from the atmosphere by aeolian dust but also from the ocean by upwelling and the resuspension of sediments on the seafloor. B) These two sources result in a dFe profile for the SW Ross Sea with maximum dFe concentrations at the surface and the bottom of the ocean. The profiles shows the depletion in summer of surface dFe due to grazing. Source of dFe data: Sedwick et al. (2011). 


\section{Residence time in the upper ocean}

The residence time of different Fe species is important for assessing the relevance of each process for supplying and removing soluble $\mathrm{Fe}$. Residence times for dissolved $\mathrm{Fe}$ in surface waters are typically on the order of a year (Jickells, 1995; Croot et al., 2004). However, estimates are considerably shorter for i) Fe(III) solubility controlled by the concentration of inorganic Fe species on the order of 2 - 6 hours (Bowie et al., 2009), ii) particles in high flux lithogenic regions such as in surface waters under the Saharan dust plume which have a residence time of six days (Croot et al., 2004), and iii) at different strata of the water column such as the surface layer which has a residence time of particulate Fe of around 33 - 100 days (Boyd et al., 2010).

\section{Bio-availability of colloids and nanoparticles}

The larger surface area to volume ratio of Fe colloids and nanoparticles allows a greater interaction with the leaching solution and increases Fe solubility (Fig. 3.5). There is evidence that colloidal Fe is bio-available (Wells and Lewis, 1983; Rich and Morel 1990; Barbeau and Moffett, 1998; Nordwell and Price, 2001; Chen et al., 2003). Wu et al. (2001) suggested that aeolian deposition of Fe was mainly released into the colloidal pool. Aguliar-Islas et al. (2010) suggests that the dissolution of aerosol Fe in seawater is dominated by the colloidal fraction (0.02 - $0.4 \mu \mathrm{m}$, compared to dissolved $\mathrm{Fe}<0.02 \mu \mathrm{m})$. This pool consists of $\mathrm{Fe}$ associated with colloidal ligands, colloidal size particles leached from aerosols, or formed in situ during the aerosol dissolution processes.

Nanoparticles have also been suggested as potentially bio-available. Raiswell et al. (2008b) found that suspended glacial sediments contain Fe (oxyhydr)oxide nanoparticles typically $\sim 5$ $\mathrm{nM}$ which occur as single particles or aggregates that may by isolated or attached to sediment particles. The dissolved Fe concentrations of surface waters in the wake of melting icebergs in the Polar Frontal region of Antarctica (1 - $9 \mathrm{nM})$ appear to be enhanced over local seawater concentrations (1 - 2 nM; Raiswell et al., 2008a). 


\section{Photochemical reactions in the ocean}

Photochemical reactions are important for maintaining bio-available $\mathrm{Fe}$ in the mixed layer and also influence Fe solubility when the surface ocean is sunlit (Fig. 3.5). Fe-fertilisation experiments in the SO show that photo-reduction extends the lifetime of Fe(II) (Johnson et al., 1994; Croot et al., 2001; 2005).

\section{Grazing}

There are a number of ways in which grazing by phytoplankton, zooplankton and bacteria recycles Fe in the water column (Fig. 3.5). Iron contained in colloids and particulates may be ingested by zooplankton. The action of digestive enzymes results in part of the Fe becoming soluble. Grazing of phytoplankton by zooplankton may also release Fe-binding ligands, further increasing Fe solubility (Sato et al., 2007). Furthermore, the excretion of Fe as faeces from zooplankton may subsequently be taken up by phytoplankton (Figs. 3.1B and 3.5; Hutchins et al., 1993; 1995; Barbeau et al., 1996).

\section{Scavenging of dissolved Fe by particles}

Scavenging of soluble and colloidal $\mathrm{Fe}$ by particles is a major sink of dissolved $\mathrm{Fe}$ in seawater (Fig. 3.5). Scavenging appears to be the least understood process affecting aerosol dissolution in the ocean (Baker and Croot, 2010; Boyd and Ellwood, 2010).

\section{Organic complexation}

The concentration of particulate Fe solubility in seawater is controlled by the presence of strong Fe-binding ligands (Fig. 3.5; Kraemer, 2004; Kraemer et al., 2005). Complexation of $\mathrm{Fe}(\mathrm{III})$ by organic ligands such as formate, acetate and oxalate promotes the photo-production of more soluble Fe(II). When Fe(III) complexes form at particle surfaces, photo-reduction results in the release of $\mathrm{Fe}(\mathrm{II})$ into solution. Siderophores, both marine and terrestrial, have been identified in marine waters (e.g. Mucha et al., 1999; Gledhill et al., 2004). However, not

all Fe-binding ligands need to be siderophores (Hutchins et al., 1999; Gledhill, 2007; Walters and Hedges, 1998). 


\subsubsection{Other limiting nutrients in the surface water and initial concentration in surface} waters

In addition to the solubility of Fe from mineral dust, a number of other biologically important elements, including $\mathrm{Al}, \mathrm{Mn}, \mathrm{P}, \mathrm{Zn}, \mathrm{Co}$, are also supplied to the water column by dust deposition (e.g. Baker et al., 2006). The solubility of these often limiting nutrients also remains uncertain. Irrespective of the dust leaching process, the maximum amount of Fe that is rendered soluble is determined by the maximum concentration of soluble of $\mathrm{Fe}$ in seawater (assuming that dust has the potential to supply this amount of Fe). Fig. 3.6 illustrates that in areas of low deposition, high aerosol Fe solubility would be expected as in the case of the SO (Baker and Croot, 2010).

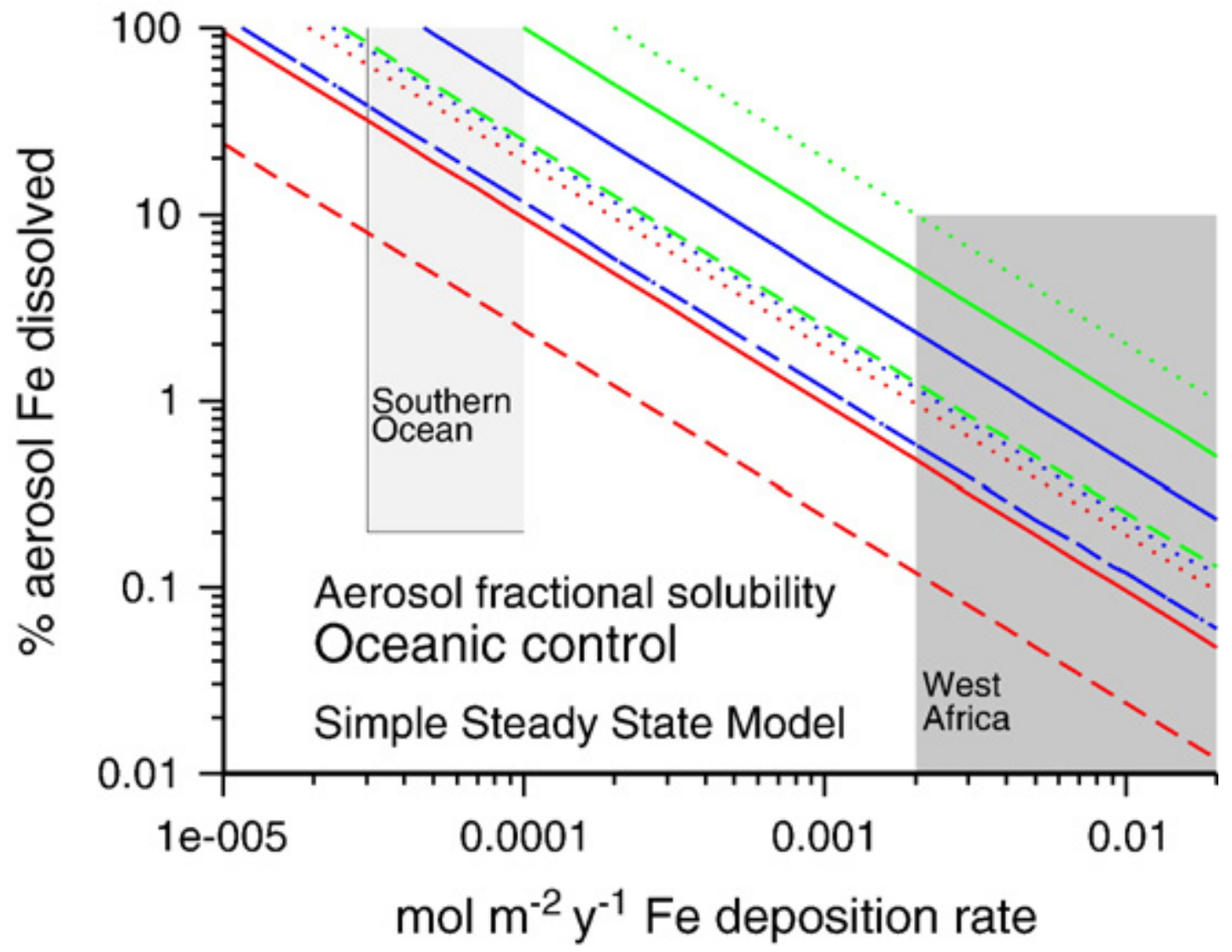

Fig. 3.6. Simple model of steady state aerosol Fe fractional solubility under oceanic control of Fe dissolution assuming a constant deposition rate. The diagonal lines represent isolines for the product of residence time and mixed layer depth. The red lines indicate values where only inorganic solubility in tropical waters $\left(25^{\circ} \mathrm{C}\right)$ is considered, the blue lines are for inorganic solubility in polar waters $\left(4^{\circ} \mathrm{C}\right)$ and the green lines for Fe solubility controlled by $1 \mathrm{nM} \mathrm{L}^{-1}$. Source: Baker and Croot (2010). 


\subsection{Development of a method for leaching iron from sediment contained in snow and ice samples}

\subsubsection{Introduction}

Ongoing research into the amount of soluble $\mathrm{Fe}$ in dust is hindered due to the lack of a universally accepted leaching method that replicates the process of dust dissolution in seawater (see Chapter 3.1.3). It has been postulated that much of the variation in the reported values of Fe solubility in atmospheric dust is attributable to these inconsistencies (Table 3.2) (Baker et al., 2006; Wu et al., 2007; Baker and Croot, 2010; Buck et al., 2010b). Although, Aguilar-Islas et al. (2010) quantified the influence of experimental artefacts upon $\mathrm{Fe}$ solubility and concluded that different types and sources of aerosols produce more variability in Fe solubility estimates than different leaching methods, there is a clear need for a reproducible method between laboratories. In addition, there appears to be no established method available for leaching Fe from sand and dust contained in snow and ice samples. Therefore, an investigation was conducted into the release of Fe from ultra-fine and medium particle-sized Arizona test dust (ATD) when leached in certified Milli-Q (MQ) ultrapure deionised water, with a resistivity of $18.2 \mathrm{M} \Omega$, to mimic snow melt during summer sea ice melting and subsequent deposition into seawater. Leaching scheme variables were altered systematically between experiments to determine the effect of changing these variables upon the solubility of Fe from the ATD.

A common assertion throughout the literature is the need for an experimental scheme representative of natural systems, which can also provide reproducible results. Because of the need to have a method which is analogous to the process occurring in the nature, seawater would be the logical leaching solution. However, it is advantageous to have a method which can be replicated across different laboratories to yield comparable results. Reproducibility is commonly used as the argument in support of selecting a more uniform matrix of MQ water, over seawater (e.g. Schroth et al., 2009).

Furthermore, the design of an experiment, to specifically address the effect of altering leaching scheme parameters on the solubility of $\mathrm{Fe}$ in dust particles, requires a dust matrix, homogenous in nature, to act as a certified reference material. This would act to reduce the influence of sample heterogeneity upon the observed Fe solubility results. ATD has been 
used by Howe (2009), Cwiertny et al. (2008a; 2008b) and Shi et al. (2009). For this reason, ATD was also used in the present study.

\subsubsection{Methods, materials and dust leaching tests}

\subsubsection{Characterisation of Arizona test dust}

Mineralogical and particle size properties can potentially influence the way in which $\mathrm{Fe}$ is released during leaching (Chapter 3.1.3.3.1). The medium and ultra-fine ATD particle size fractions were characterised by particle size and major elements, including $\mathrm{Fe}$, to help in the interpretation of the leaching experiment results. An accurate measurement of the total $\mathrm{Fe}$ content of the dust is particularly important as the standard procedure for reporting the results of leaching experiments is the fraction of the total dust-derived Fe that has moved into the soluble phase during leaching.

\section{Major elements}

Both ultra-fine and medium particle-sized ATD were analysed for major elements by X-Ray Fluorescence (XRF) at SpectramChem Analytical, Lower Hutt, New Zealand (http://www.crl.co.nz/spectrachem/default.htm). Major element results are illustrated in Table 3.3 .

Table 3.3: Triplicate major element analysis of ultra-fine and medium particle-sized Arizona test dust by $\mathrm{XRF}$ analysis. $\mathrm{Fe}_{2} \mathrm{O}_{3}$ content is highlighted.

\begin{tabular}{|c|c|c|c|c|c|c|c|c|}
\hline Sample & $\begin{array}{c}\text { ATD } \\
\text { medium } \\
1\end{array}$ & $\begin{array}{c}\text { ATD } \\
\text { medium } \\
2 \\
\end{array}$ & $\begin{array}{c}\text { ATD } \\
\text { medium } \\
3 \\
\end{array}$ & Sd & $\begin{array}{l}\text { ATD } \\
\text { ultra- } \\
\text { fine } 4 \\
\end{array}$ & $\begin{array}{l}\text { ATD } \\
\text { ultra- } \\
\text { fine } 5\end{array}$ & $\begin{array}{l}\text { ATD } \\
\text { ultra- } \\
\text { fine } 6 \\
\end{array}$ & Sd \\
\hline $\mathrm{SiO}_{2}$ & 81.11 & 81.15 & 80.88 & 0.15 & 76.16 & 76.15 & 76.27 & 0.07 \\
\hline $\mathrm{Al}_{2} \mathrm{O}_{3}$ & 7.90 & 7.93 & 7.88 & 0.03 & 9.68 & 9.69 & 9.69 & 0.01 \\
\hline $\mathrm{Fe}_{2} \mathrm{O}_{3}$ & 1.79 & 1.79 & 1.79 & 0.00 & 2.50 & 2.51 & 2.50 & 0.01 \\
\hline $\mathrm{MnO}$ & 0.04 & 0.04 & 0.04 & 0.00 & 0.07 & 0.07 & 0.07 & 0 \\
\hline $\mathrm{MgO}$ & 0.67 & 0.66 & 0.66 & 0.01 & 1.06 & 1.06 & 1.05 & 0.01 \\
\hline $\mathrm{CaO}$ & 2.10 & 2.10 & 2.10 & 0.00 & 2.38 & 2.37 & 2.38 & 0.01 \\
\hline $\mathrm{Na}_{2} \mathrm{O}$ & 1.63 & 1.60 & 1.58 & 0.03 & 1.45 & 1.47 & 1.47 & 0.01 \\
\hline $\mathrm{K}_{2} \mathrm{O}$ & 2.06 & 2.07 & 2.05 & 0.01 & 2.39 & 2.39 & 2.39 & 0 \\
\hline $\mathrm{TiO}_{2}$ & 0.30 & 0.30 & 0.30 & 0.00 & 0.33 & 0.34 & 0.34 & 0.01 \\
\hline $\mathrm{P}_{2} \mathrm{O}_{5}$ & 0.08 & 0.08 & 0.08 & 0.00 & 0.11 & 0.11 & 0.11 & 0 \\
\hline LOI & 2.21 & 2.17 & 2.31 & 0.07 & 3.52 & 3.47 & 3.46 & 0.03 \\
\hline SUM & 99.89 & 99.89 & 99.67 & 0.13 & 99.65 & 99.63 & 99.73 & 0.05 \\
\hline
\end{tabular}




\section{Particle size}

Particle size distribution for both ultra-fine and medium particle-sized ATD was analysed on a Beckman-Coulter Laser Diffraction Particle Analyser (LS 13320; see Chapter 2.2.1.2.2). Particle size distribution curves are illustrated in Fig. 3.7.

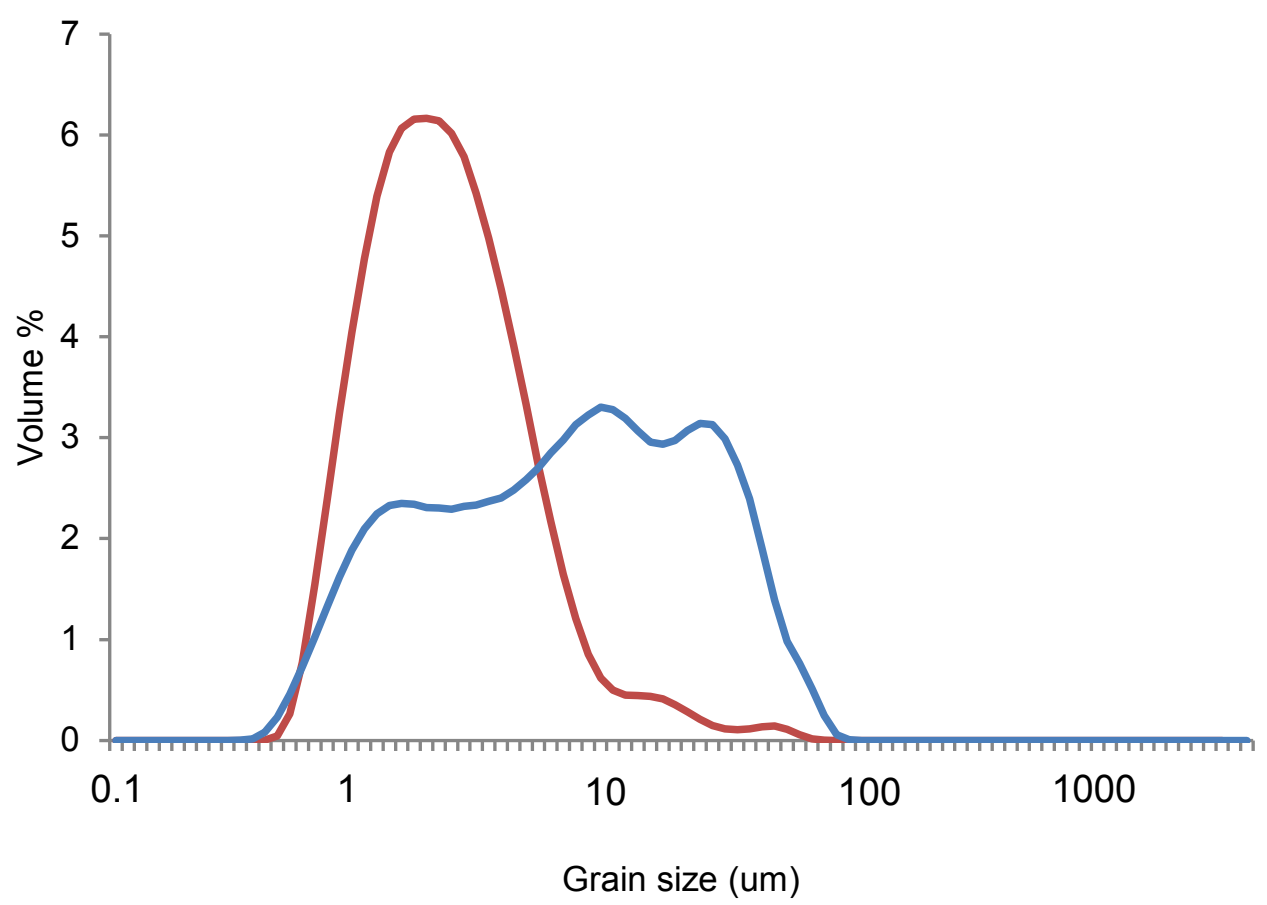

Fig. 3.7: Particle size distribution (percentage volume per particle diameter) for ultra-fine (red) and medium (blue) Arizona test dust.

\section{Ultra-fine particle-sized Arizona Test Dust}

A small difference in the particle size mode of ultra-fine particle-sized ATD between that provided by the supplier and that measured in the present study (Fig. 3.7) was observed (the mode for the ultra-fine particle-sized ADT is reported at $5 \mu \mathrm{m}$ by the manufacturer however, while laser diffraction analysis yielded a mode of $3.5 \mu \mathrm{m}$ ), because each method measures a different physical property of the dust.

Reported measurements by XRF analysis yielded a total mass of Fe measured as $\mathrm{Fe}_{2} \mathrm{O}_{3}$ of 2.24 wt \% (Howe, 2009); $2.2 \pm 0.1$ wt \% (Vlasenko et al., 2005); 2.44 wt \% (Desboeufs et al., 2005). These are consistent with the findings of the present study of $2.5 \mathrm{wt} \%$ (Table 3.3 ). Information gained about the mineralogy and nature of the Fe-bearing phases within the dust particles is likely to assist in data interpretation. Further work could be directed towards X- 
Ray diffraction (XRD) and Scanning Electron Microscope (SEM) analysis to establish the Fe-bearing phase.

\section{Medium particle-sized Arizona Test Dust}

The medium particle-sized ATD has a primary mode of $14.3 \mu \mathrm{m}$ (Fig. 3.7) and covers the broad range of particle sizes found in samples from Southern McMurdo Sound.

The total $\mathrm{Fe}$ mass measured as $\mathrm{Fe}_{2} \mathrm{O}_{3}$ by XRF analysis is reported as $1.71 \mathrm{wt} \%$ by Howe (2009), which is consistent with finding in the present study of $1.79 \mathrm{wt} \%$ (Table 3.3).

\subsubsection{Leaching experiments}

All leaching experiments were conducted in the class 100 clean room at the National Ice Core Facility, GNS Science, at room temperature. All work was carried out wearing LabServ gloves and Tyveck clean suits. Certified MQ water was used throughout the course of the experimental work. The purity of reagents used are defined as the following: certified analytical reagent grade (AR) $\mathrm{HNO}_{3}$; sub boiled (SB) $\mathrm{HNO}_{3}$, which is distilled $\mathrm{HNO}_{3}$ (AR) in the Geochemical Laboratory, VUW; and high purity certified Seastar (SS) $\mathrm{HNO}_{3}$ with metal concentrations $<10$ ppt. All acid washed equipment was cleaned in the class 100 ultraclean room of the Geochemistry Laboratory, VUW and washed three times with MQ water prior to use. Filter membranes used were $47 \mathrm{~mm}$ polycarbonate membranes with a $0.4 \mu \mathrm{m}$ or $10.0 \mu \mathrm{m}$ pore size which had been soaked in $5 \mathrm{wt} \% \mathrm{HNO}_{3}$ (AR) for at least 48 hours under a hot plate and rinsed thoroughly with MQ water. Leachates were collected in low density polyethylene (LDPE) $250 \mathrm{ml}$ Nalegne screw top bottles pre-rinsed three times with MQ water.

All dust leaching experiments were conducted using an all Teflon flow-through reactor (Fig. 3.8; Wu et al., 2007; Aguilar-Islas et al., 2010) consisting of a two stage Savillex filter holder with $0.4 \mu \mathrm{m}$ and $10 \mu \mathrm{m}$ acid washed filter membranes (Fig. 2.8) mounted upon and a Teflon valve for controlling solution flow. The flow-through reactor was attached to a Savillex Teflon jar which was used as a vacuum chamber and a place to hold $250 \mathrm{ml}$ Nalagene bottles used in leachate collection. The reactor was initially acid washed by soaking in $5 \mathrm{wt} \% \mathrm{HNO}_{3}$ (AR) for two weeks and rinsed three times with MQ water. The filtration apparatus was 
soaked in MQ water while not in use and thoroughly rinsed with MQ water before and after leaching experiments. Prior to each leaching experiment the device was cleaned by passing $0.1 \mathrm{wt} \% \mathrm{HCl}(\mathrm{SS})$ through the device and rinsing with MQ water. The leaching solution used in these experiments was MQ water.

A)

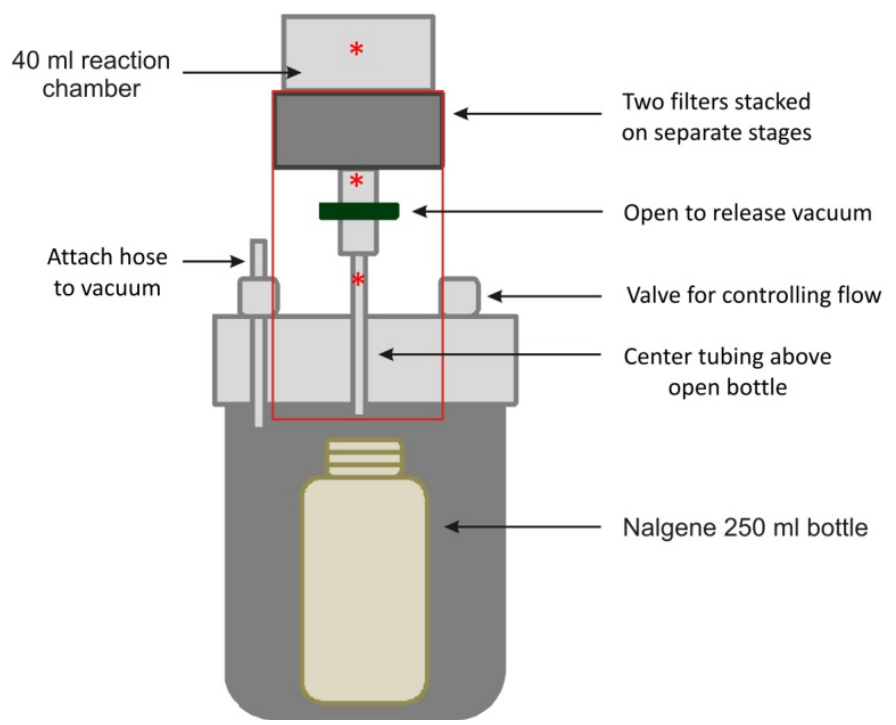

B)

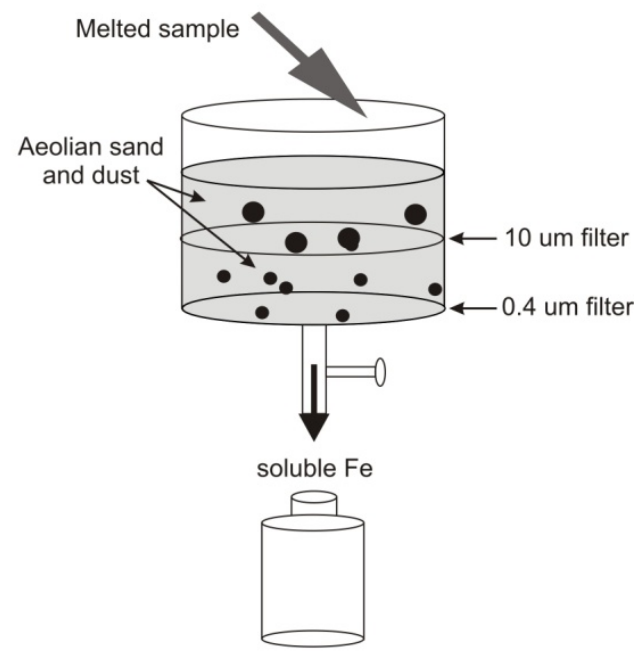

Fig. 3.8: Dust leaching apparatus attached to a Teflon jar. A) Teflon flow-through reactor for aerosol Fe solubility measurements. Red asterisks are acid washed components. B) Insert from red box in A) showing the how the bulk sand and dust is spilt into fine and coarse size fractions on two stacked filter stages in the reaction chamber.

\subsubsection{Arizona test dust leaching tests}

Leaching scheme variables were controlled between experiments to investigate the influence of the variable upon the resulting percentage of soluble Fe. These variables included the mass of dust, the dust leaching time and the volume of the leaching solution. The ultra-fine particle-sized ATD was used for the majority of leaching experiments (except leaches 10a10d) as the finer particle size is most likely to be representative of the size fraction that is transported in suspension to the open ocean during i) dry deposition and ii) sea ice melt and advection (Chapter 2.1.3.2).

\section{Time dependency}

In order to determine if $\mathrm{Fe}$ solubility is time dependent, numerous aliquots of $10 \pm 0.098 \mathrm{mg}$ of ultra-fine and medium particle-sized ATD, weighed out precisely on a micro balance, were leached with $180 \mathrm{ml}$ of MQ water allowing it to sit for a predetermined amount of time (5 
min, 10, min, $20 \mathrm{~min}, 40 \mathrm{~min}, 60 \mathrm{~min}, 90 \mathrm{~min}, 180 \mathrm{~min}, 2$ months) in a acid washed Nalgene jar at room temperature. The leaching solution and ATD was sonicated in an ultra-sonic bath for $<20$ minutes, included within the leaching time, for all samples which is necessary to disaggregate particles for particle size separation on the $0.4 \mu \mathrm{m}$ and $10 \mu \mathrm{m}$ filter membranes during filtering. Clean filter membranes were placed in the reaction chamber and after the pre-determined length of time the leaching solution and ATD was poured into the reaction chamber and the leachate collected in $250 \mathrm{ml}$ bottles (Fig. 3.8). The ADT was recovered on the $0.4 \mu \mathrm{m}$ and $10 \mu \mathrm{m}$ filter membranes and dried for 48 hours in a desiccator at room temperature.

\section{Mass dependency}

Similar tests were conducted on variable weights of ATD $(2 \mathrm{mg}, 8 \mathrm{mg}, 10 \mathrm{mg}, 12 \mathrm{mg}$ and 20 mg of ultra-fine ATD) to approximate the range of sand and dust weights in McMurdo Sound surface snow samples in order to assess if Fe solubility is mass dependent. The variable dust mass aliquots were leached for 20 minutes in $180 \mathrm{ml}$ of MQ water following the procedure above.

\section{Successive MQ water leaches}

In order to determine how the number of cumulative leaches affects Fe solubility, an ultrafine particle-sized ATD aliquot was leached multiple times with MQ water. ATD was initially leached for 20 minutes in an acid washed Nalgene jar (to mimic the snow melt in the sample bottle). After a pre-determined length of time the leaching solution and ATD was poured into the reaction chamber containing clean filter membranes on two separate stages (Fig. 3.8), and the leachate was collected in a $250 \mathrm{ml}$ Nalgene bottles. From here, the ATD aliquot on separate stages was leached with successive aliquots of $40 \mathrm{ml}$ (maximum volume of the chamber) of leaching solution in the reaction chamber at room temperature for 20 minutes. At the end of the leaching time the valve was opened and the leachate collected in a $250 \mathrm{ml}$ bottle. The valve was then closed and a new aliquot of $40 \mathrm{ml}$ of leaching solution was added and allowed to sit for 20 minutes. This procedure was repeated five times.

The conditions used for each of the different leaching experiments are shown in Table 3.4. 
Table 3.4: Values of controlled parameters for Arizona test dust leaching experiments. Leaching solution was MQ water. Experiments are grouped according to variable parameters, from top to bottom: leaching time, dust mass, ultra fine or medium Arizona test dust, subsequent leaches and particle size leached.

\begin{tabular}{|c|c|c|c|c|c|c|c|}
\hline $\begin{array}{c}\text { Type of } \\
\text { test }\end{array}$ & $\begin{array}{c}\text { Leach } \\
\text { number }\end{array}$ & $\begin{array}{c}\text { Mass of } \\
\text { dust } \\
\text { (mg) }\end{array}$ & $\begin{array}{c}\text { Size } \\
\text { fraction } \\
\text { leached } \\
\end{array}$ & $\begin{array}{l}\text { Leaching } \\
\text { time (min) }\end{array}$ & $\begin{array}{c}\text { Number } \\
\text { of } \\
\text { leaches }\end{array}$ & $\begin{array}{c}\text { Volume of } \\
\text { leaching } \\
\text { solution (ml) }\end{array}$ & $\begin{array}{c}\text { ADT } \\
\text { particle } \\
\text { size } \\
\end{array}$ \\
\hline Time & $1-5$ & 10 & Bulk & 5 & 1 & $\sim 180$ & Ultra-fine \\
\hline \multirow{6}{*}{ dependent } & $6-10$ & 10 & Bulk & 10 & 1 & $\sim 180$ & Ultra-fine \\
\hline & $11-15$ & 10 & Bulk & 20 & 1 & $\sim 180$ & Ultra-fine \\
\hline & $16-20$ & 10 & Bulk & 40 & 1 & $\sim 180$ & Ultra-fine \\
\hline & $21-25$ & 10 & Bulk & 90 & 1 & $\sim 180$ & Ultra-fine \\
\hline & $57-60$ & 10 & Bulk & 180 & 1 & $\sim 180$ & Ultra-fine \\
\hline & $31-32$ & 10 & Bulk & 2 months & 1 & $\sim 180$ & Ultra-fine \\
\hline Mass & $33-37$ & 12 & Bulk & 20 & 1 & $\sim 180$ & Ultra-fine \\
\hline \multirow[t]{3}{*}{ dependent } & $40-44$ & 8 & Bulk & 20 & 1 & $\sim 180$ & Ultra-fine \\
\hline & $54-56$ & 2 & Bulk & 20 & 1 & $\sim 180$ & Ultra-fine \\
\hline & $72-74$ & 20 & Bulk & 20 & 1 & $\sim 180$ & Ultra-fine \\
\hline $\begin{array}{c}\text { Dust } \\
\text { recovery }\end{array}$ & Test $10 \mathrm{a}-10 \mathrm{~d}$ & $\sim 10$ & Bulk & 20 & 1 & $\sim 200$ & Medium \\
\hline Successive & 26 & 10 & Bulk & 20 & 1 & $\sim 180$ & Ultra-fine \\
\hline \multirow[t]{3}{*}{ leaches } & $26 a-26 e$ & 10 & Bulk & 20 & 5 & $\sim 40$ & Ultra-fine \\
\hline & 28 & 10 & Bulk & 20 & 1 & $\sim 180$ & Ultra-fine \\
\hline & $28 \mathrm{a}-28 \mathrm{e}$ & 10 & Bulk & 20 & 5 & $\sim 40$ & Ultra-fine \\
\hline
\end{tabular}

The operationally defined size fractions of Fe are defined as follows: Fe that passed through $0.4 \mu \mathrm{m}$ filter membrane is defined as soluble $\mathrm{Fe} ; \mathrm{Fe}>0.4 \mu \mathrm{m}$ is defined as particulate. Total Fe is the sum of soluble and particulate Fe. The percentage of soluble Fe is calculated by:

$\%$ of soluble $\mathrm{Fe}=$ soluble $\mathrm{Fe} \times 100$ total $\mathrm{Fe}$

where the total Fe content of the ultra-fine particle-sized ATD is calculated from Table 3.3.

\subsubsection{Filter blanks}

Filter procedural blanks were sequentially leached with multiple aliquots of $180 \mathrm{ml}$ of MQ water by placing a 0.4 and $10 \mu \mathrm{m}$ filter membrane in the reaction chamber and adding the leaching solution to the holder and filtering the leachate into $250 \mathrm{ml}$ Nalgene bottles. The precision (2 SE\%) from the replicate blanks is illustrated in Table 3.5. 
Table 3.5: Reproducibility of the Fe concentration in blank solutions when the filtration apparatus is stored dry, in $1 \mathrm{wt} \% \mathrm{HNO}_{3}$ and in MQ water.

\begin{tabular}{|c|c|c|c|c|c|}
\hline $\begin{array}{l}\text { Apparatus } \\
\text { storage }\end{array}$ & $\begin{array}{c}\text { Mean } \\
\left(\text { nmol L }^{-1}\right)\end{array}$ & 2 SE & $\begin{array}{c}\text { Precision } \\
2 \text { SE } \% \\
\end{array}$ & $\begin{array}{c}\text { Detection limit } \\
(3 \sigma \text { blanks })\end{array}$ & $\mathbf{n}$ \\
\hline Dry & 1.18 & 0.91 & 76.79 & 3.33 & 6 \\
\hline $1 \mathrm{wt} \% \mathrm{HNO}_{3}$ & 1.57 & 1.12 & 70.97 & 4.10 & 6 \\
\hline MQ water & 0.08 & 0.41 & 534.39 & 1.06 & 6 \\
\hline
\end{tabular}

\subsubsection{Measurement of iron by inductively coupled plasma mass spectrometry}

Leachate solutions were acidified using $1 \mathrm{wt} \% \mathrm{HNO}_{3}$ (SS) in a class 100 clean room prior to analysis on an Agilent 7500 cs series inductively coupled plasma mass spectrometry (ICP-MS) at the Geochemistry Laboratory. An analytical blank made of 1 wt \% $\mathrm{HNO}_{3}$ (SS) was measured immediately before each sample. Counts recorded on those blank measurements were subtracted from sample counts to correct for instrumental background. A hydrogen flushed collision cell was used for measurement of two isotopes of $\mathrm{Fe},{ }^{54} \mathrm{Fe}$ and ${ }^{56} \mathrm{Fe}$, which is necessary because of the difficulty of measuring $\mathrm{Fe}$ in dilute solutions where gas based interferences from other elements within samples and oxidization of the argon carrier gas occur. In the collision cell ions collide and react with hydrogen gas, so that polyatomic inferring ions (e.g. ${ }^{40} \mathrm{Ar}^{16} \mathrm{O}$ on ${ }^{56} \mathrm{Fe}$ and ${ }^{40} \mathrm{Ar}{ }^{14} \mathrm{~N}$ on ${ }^{54} \mathrm{Fe}$ ) will be converted to non interfering species. Two isotopes are measured which allows detection of any residual interferences present and perturbing the measurements. Furthermore, the use of secondary standards also allows for correction of interferences.

Standard solutions (0.16 ppb Fe, $1.80 \mathrm{ppb} \mathrm{Fe}, 3.04$ ppb Fe, 4.50 ppb Fe, 8.22 ppb Fe) were made in $1 \mathrm{wt} \% \mathrm{HNO}_{3}$ (SS) from a certified mono-elemental $1000 \mathrm{ppm} \mathrm{Fe} \mathrm{standard.} \mathrm{Initial}$ calibration standards showed a linear relationship between elemental concentration and ICPMS signal intensity. Therefore, a single calibration standard (3.04 ppb Fe) was analysed for a test analysis with elemental concentrations of the standard approximating mean values expected in leachate samples. However, a $5 \%$ inaccuracy resulted from enhanced interferences during measurement and an empirical calibration curve proved to be more accurate (Table 3.6). The calibration standards were run as bracketing standards every fourfive samples to correct for instrumental drift. 
Table 3.6: Accuracy and precision (reported as 2 significant figures) of repeat ICP-MS measurement of Fe standard solutions.

\begin{tabular}{ccccc}
\hline $\begin{array}{c}\text { Fe Standard } \\
\text { ppb }\end{array}$ & Mean & 2 SE & $\begin{array}{c}\text { Precision } \\
\text { 2 SE\% }\end{array}$ & n \\
\hline 0.16 & 0.18 & 0.03 & 16.28 & 10 \\
1.80 & 1.92 & 0.12 & 6.20 & 5 \\
4.50 & 4.59 & 0.16 & 3.38 & 5 \\
8.22 & 8.23 & 0.07 & 0.79 & 9 \\
\hline
\end{tabular}




\subsubsection{Results - Arizona test dust leaching tests}

\subsubsection{Method development}

A high standard of cleanliness and a meticulous acid cleaning process for all equipment to eliminate contamination was developed. However, precipitation of Fe from solution with subsequent absorption to container walls occurred during storage in LDPE Nalgene bottles before measurement on ICP-MS. Fig. 3.9 illustrates the substantial decrease in percentage of soluble Fe following a repeated measurement of 5 leachate samples 10 days after the original measurement.

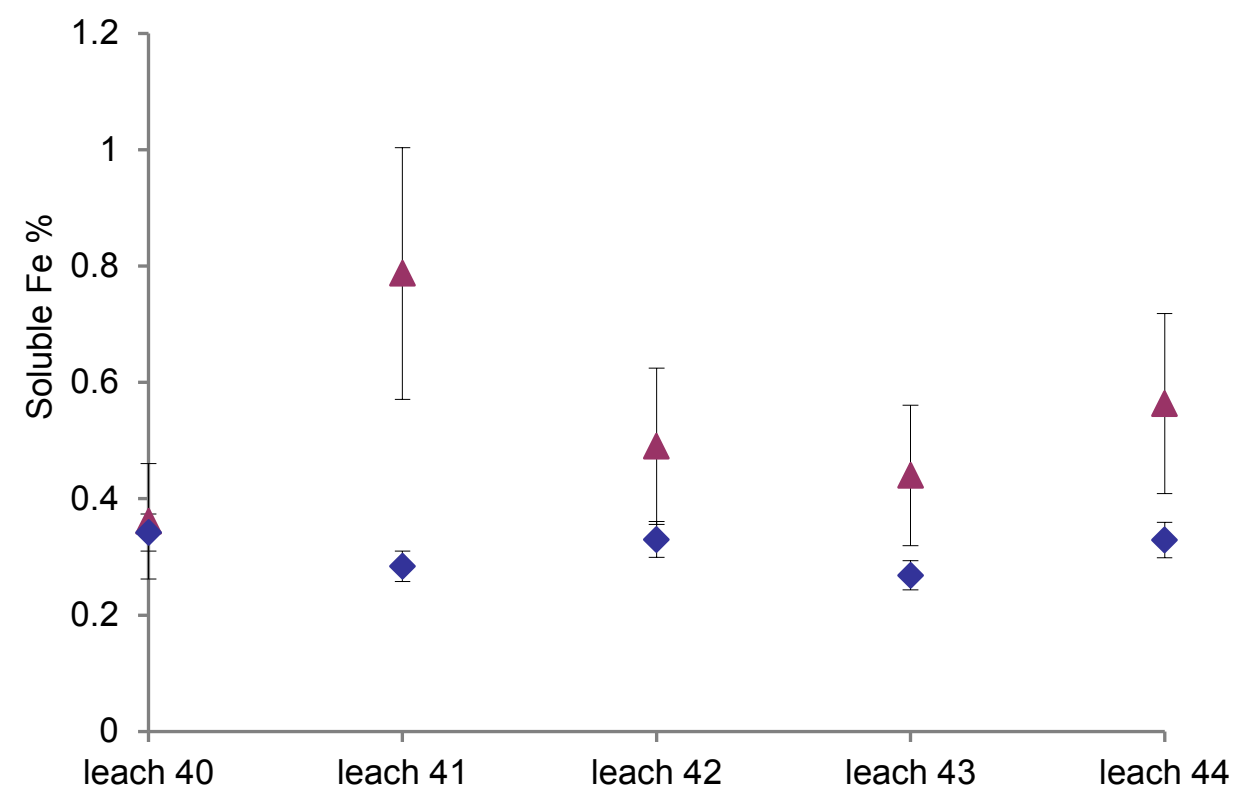

Fig. 3.9: Repeat measurement of the percentage of soluble Fe (blue diamonds) 10 days after initial measurement (red triangles) for leaches 40-44. Details of the leaches are found in Table 3.4.

\subsubsection{Blank Fe concentrations}

The procedural blank level of Fe which is commonly reported in the literature is $0.1 \mathrm{nmol} \mathrm{L}^{-1}$. This represents a conventionally accepted blank level for Fe concentration. The initial blank Fe levels in the present study are on average $1.18 \pm 0.91 \mathrm{nmol} \mathrm{L}^{-1}(\mathrm{n}=6)$, which is higher than the acceptable level reported in the literature. Table 3.3 shows the variability of the blank Fe concentrations, often an order of magnitude lower than the acceptable level and also an order of magnitude greater than the acceptable level. This resulted in the adoption of a more rigorous acid cleaning procedure between leaching tests involving soaking the leaching 
apparatus in $1 \mathrm{wt} \% \mathrm{HNO}_{3}(\mathrm{AR})$ while not in use, resulting in a new blank level average of $1.57 \mathrm{nmol} \mathrm{L}^{-1}(\mathrm{n}=6)$ which is still above the acceptable level. The filtration apparatus was then soaked in MQ water while not in use and this resulted in a new low blank level averaging $0.08 \mathrm{nmol} \mathrm{L}^{-1}(\mathrm{n}=6)$ which is less than the acceptable concentration. In addition, the variability of the measurements is often used as a gauge of the limit of detection, normally reported as $3 \sigma$, giving a value of $1.06 \mathrm{nmol} \mathrm{L}^{-1}$.

\subsubsection{Dust recovery}

The medium particle-sized ATD was used in dust recovery experiments as it is most representative of the range of particle sizes typical of samples from Southern McMurdo Sound. The filtration apparatus recovered $95 \pm 11 \%$ of the original dust material after it was found necessary to sonicate samples. Qualitative petrographic analysis showed no dust was captured on the $<10 \mu \mathrm{m}$ on the $10 \mu \mathrm{m}$ filter membrane and no dust $>10 \mu \mathrm{m}$ on the $0.4 \mu \mathrm{m}$ filter membrane (Fig. 3.10).
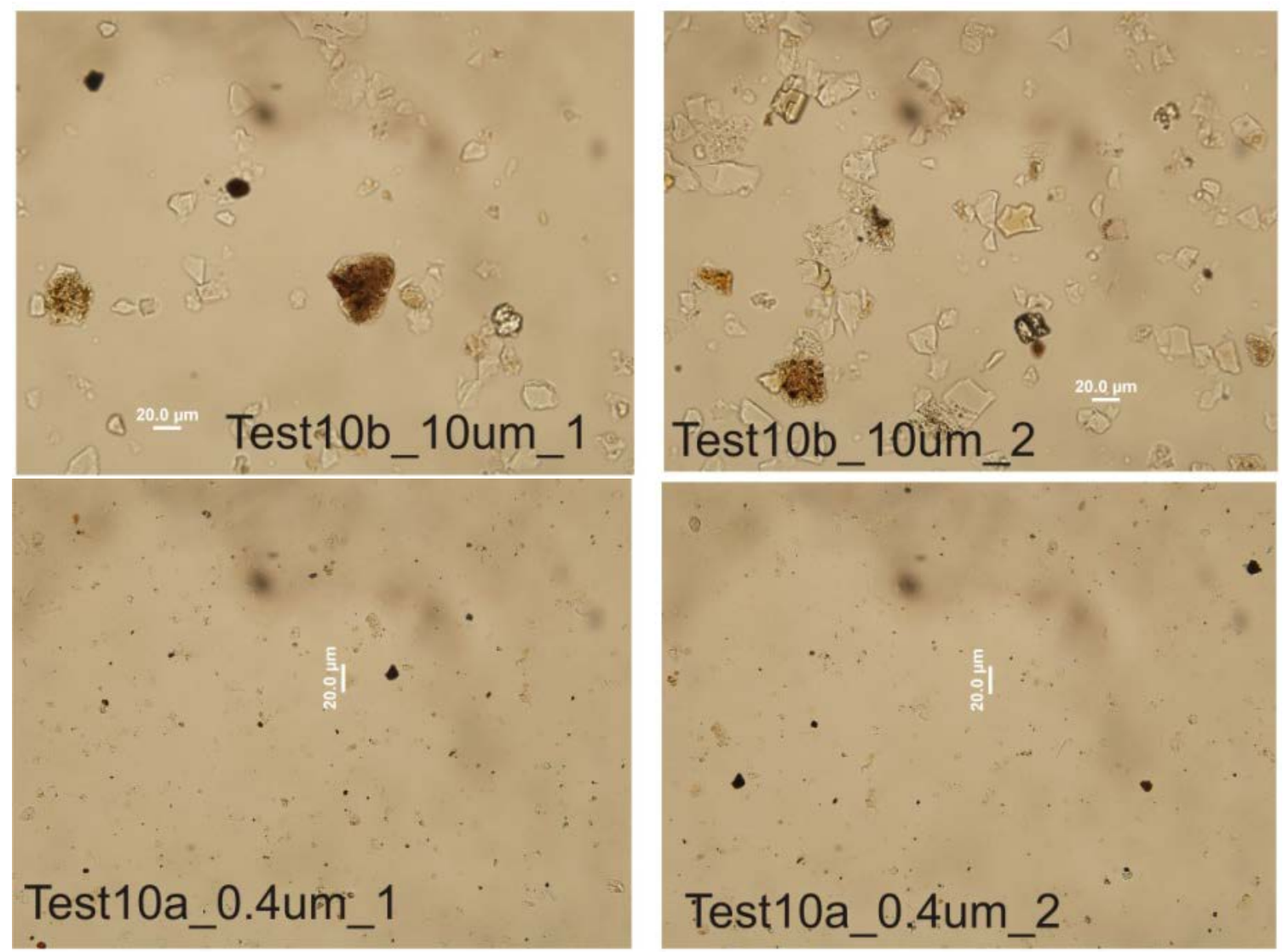

Fig. 3.10: Microscopic images of the particle size of Arizona test dust on $10 \mu \mathrm{m}$ (top) and $0.4 \mathrm{\mu m}$ (bottom) polycarbonate filter membranes. 


\subsubsection{Iron leaching experiments}

Following completion of the method described in Chapter 3.2.2.2, results of the three different experiments are described below.

\section{Time dependency}

The maximum Fe leached in one hour is $4.91 \mathrm{ppb}$ or $87.61 \mathrm{nmol} \mathrm{L}^{-1}$. The Fe supplied from dust leaching for different time intervals appears to be an initial rapid release of the percentage of soluble $\mathrm{Fe}$ in the first 5 minutes, followed by a slow and sustained release between $40-120$ minutes of $0.4-0.5 \%$, followed by another substantial increase after 2 months (Fig. 3.11). This time dependent pattern is consistent with Aguilar-Islas et al. (2010) during the shorter leaching times. Greater leaching times between 40 - 180 minutes show no discernable differences in percentage of soluble Fe, indicating that kinetic factors control the release of Fe. Fig. 3.11 also illustrates that variability in the percentage of soluble $\mathrm{Fe}$ decreases, or reproducibility of the leaching method increases, with leaching times between $40-120$ minutes.

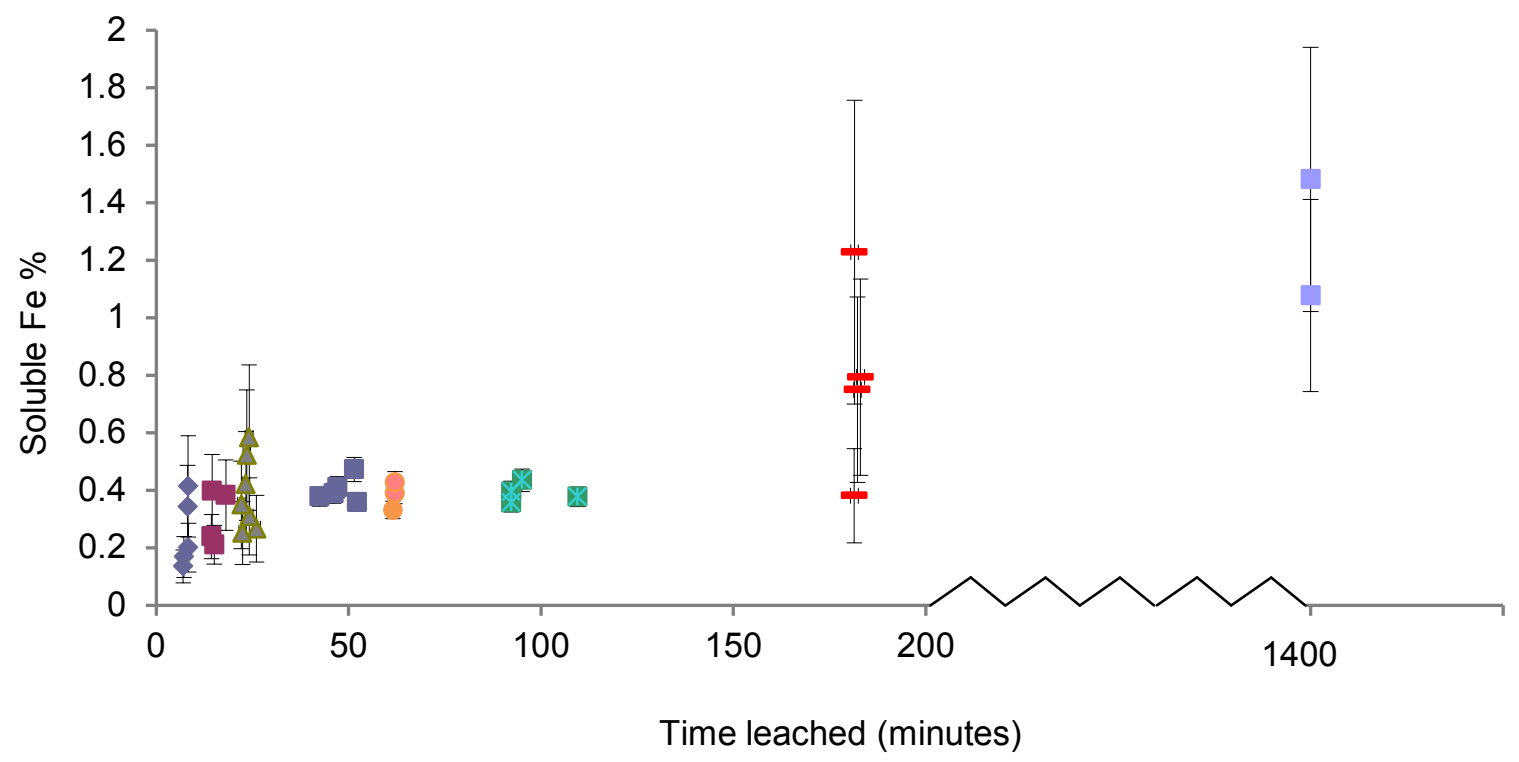

Fig. 3.11: The percentage of soluble Fe derived from $10 \mathrm{mg}$ aliquots of ultra-fine particle-sized ATD leached each in $180 \mathrm{ml}$ of MQ water at various time intervals.

To investigate the cause of the variability in the percentage of soluble Fe with leaching time, the percentage of soluble $\mathrm{Fe}$ is plotted against dust recovery and the length of time the leachate sat in the bottle before ICP-MS measurement. Fig. 3.12 illustrates no apparent 
relationship between the percentage of soluble Fe, dust recovery and the number of days before ICP-MS measurement. This highlights the difficulty in reproducing soluble Fe concentrations during short leaching times and indicates that samples need to be leached between 40-120 minutes.
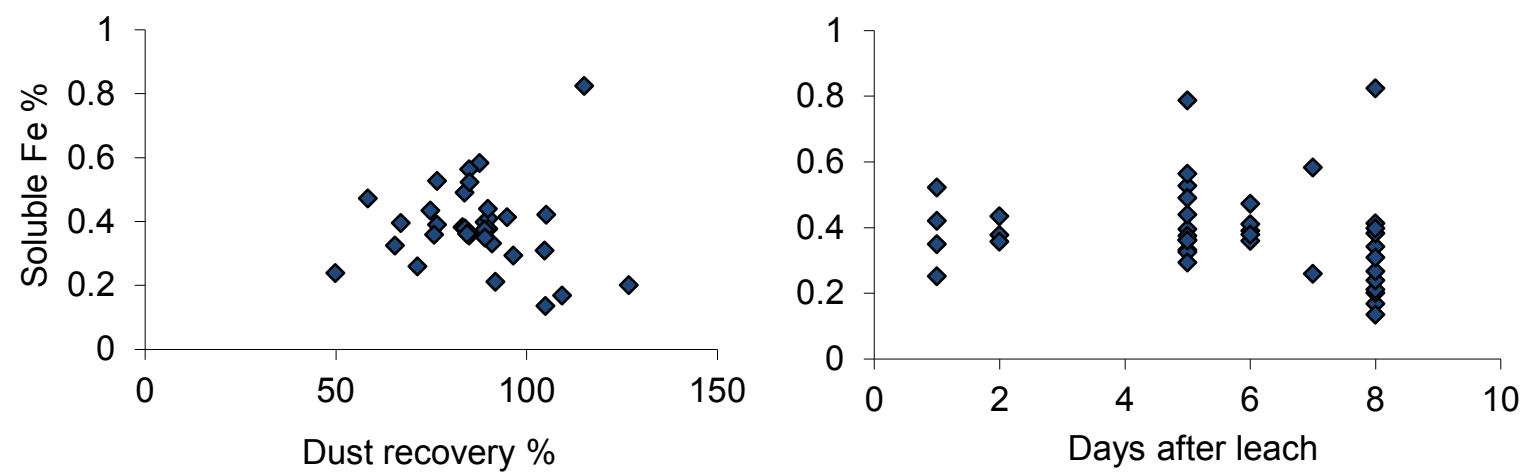

Fig. 3.12: The percentage of soluble Fe verse dust recovery (left) and days between leaching and dust ICP-MS measurement (right).

\section{Dust mass dependency}

The percentage of soluble Fe is not dependent on the mass of dust between $2-20 \mathrm{mg}$ (Fig. 3.13). Replicate analysis of ATD leaches for each dust mass during this test also produced variability in the percentage of soluble Fe.

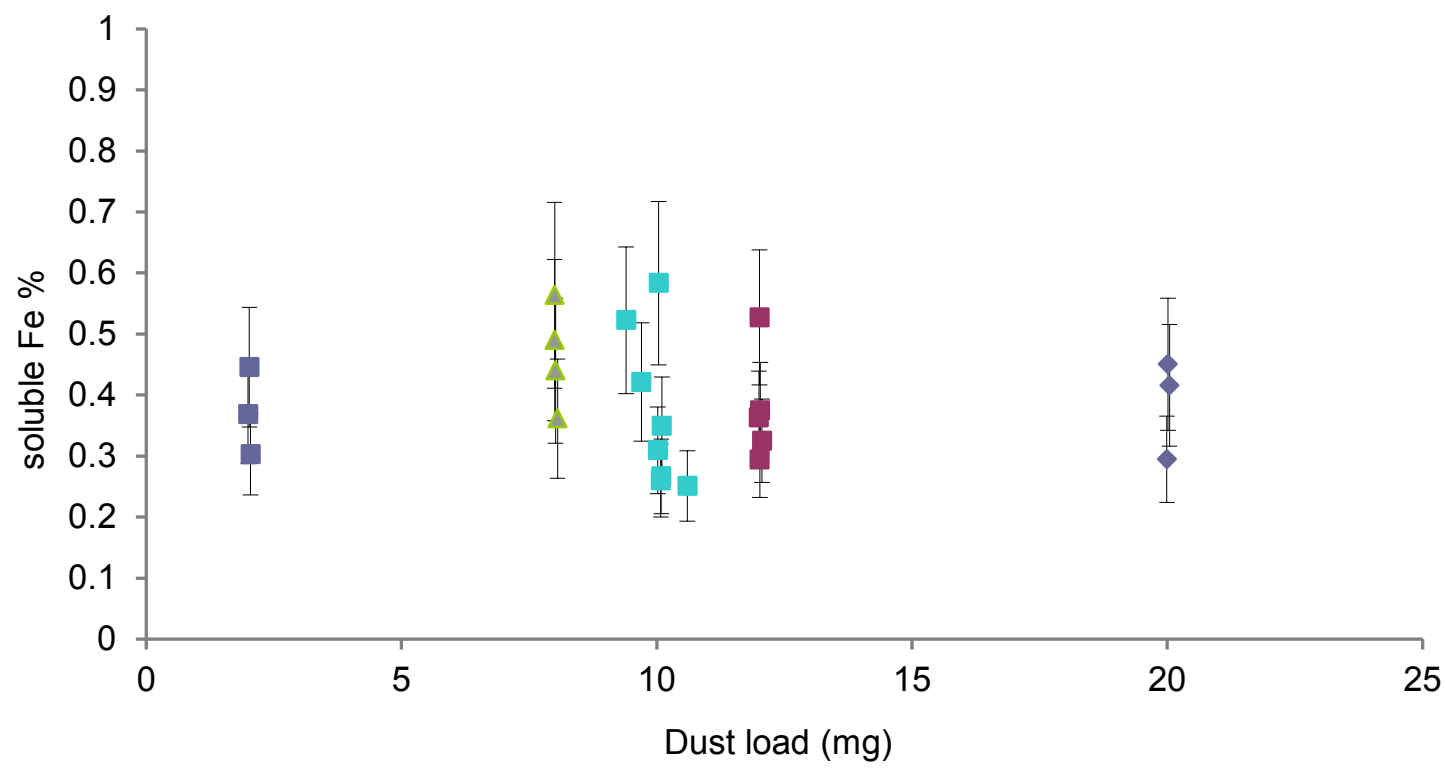

Fig. 3.13: Arizona test dust mass versus the percentage of soluble Fe for 20 minute leaches in $180 \mathrm{ml}$ of MQ water. 


\section{Leaching volume dependency}

ATD was also leached with successive aliquots of MQ water. These tests show an initial decrease in percentage of soluble Fe after the first leach, following this the percentage of soluble Fe plateaus to an average of $0.056 \pm 0.027$ \% (Fig. 3.14). This experiment was repeated rendering a lower percentage of soluble Fe plateauing to an average of $0.012 \pm$ $0.003 \%$.

The Fe concentration of the first leach is $5.24 \mathrm{ppb} \mathrm{Fe}$ or $93.54 \mathrm{nmol} \mathrm{L}^{-1}$ which represents the solubility-controlled maximum for Fe solubility in MQ water over 20 minutes. Successive 20 minute leaches yielded Fe concentrations in the vicinity of 1 ppb Fe or $0.3 \mathrm{nmol} \mathrm{L}^{-1} \mathrm{Fe}$.

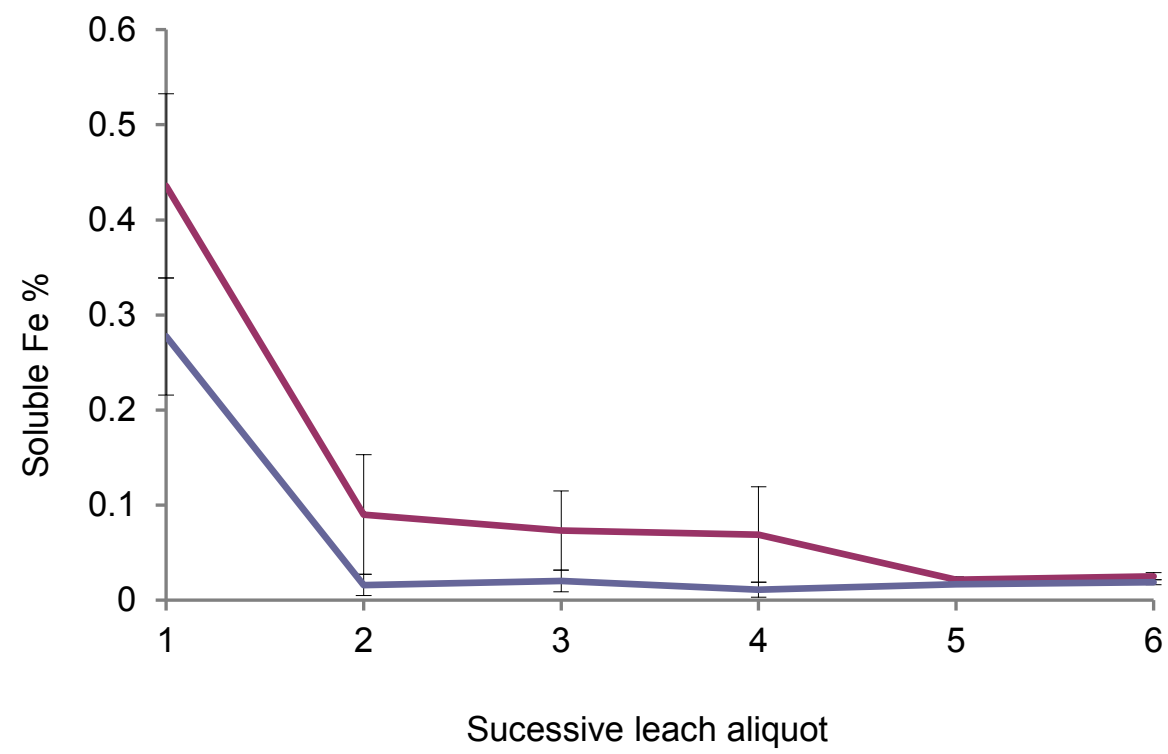

Fig. 3.14: The Fe solubility of ultra fine Arizona test dust determined for 2 repeat experiments of successive aliquots of MQ water.

\subsubsection{Analysis of other trace metals}

ICP-MS analysis of $\mathrm{Zn}, \mathrm{Co}, \mathrm{Mg}$ on leachate samples are in the ppb concentration range and below the detection limit, indicating that these elements are effectively insoluble under the leaching conditions in Table 3.4. Thus these results are not included in this thesis. 


\subsubsection{Reproducibility of the method}

Leaches 16-25, 57-60, 29 and 30 represent a ATD standard Fe solubility reference value of $0.49 \%$ (for $\mathrm{t}=40-180 \mathrm{~min}$; ultra-fine particle-sized ATD=10 mg; MQ water leach=180 ml) against which all comparisons of McMurdo Sound ASD samples can be made. The $2 \mathrm{SE} \%$ of $11.09 \%$ highlights the reproducibility of the method. All measurements agreed within analytical error. Whilst this agreement is a desirable result it indicates only the precision of the technique and not the accuracy. Accuracy can only be determined by measuring the percentage of soluble Fe of a certified reference material, such as ATD, but currently there is no reference value for ultra-fine ATD in the literature. Such values would aid in the validation of this method as a suitable technique for the analysis of low concentrations of soluble Fe (Croot and Johansson, 2000).

The limit of the reproducibility of the method could arise from the following factors:

\section{Physical and chemical heterogeneity of Arizona test dust}

While, the Fe bearing phase of the ATD is not discerned conclusively, as the form of Fe held in the ATD measured by XRF is a product of the combustion process, Fe could be present within a purely Fe-bearing phase such as $\mathrm{Fe}_{2} \mathrm{O}_{3}$ and/or Fe could be is substituted within the dominant alumniosilicate phase. Iron being present in both of these phases has consequences for the use of low mass dust samples, such as $2 \mathrm{mg}$, in these experiments. If the dust is not sufficiently homogenous in composition, an aliquot of ATD with a high proportion of the more purely Fe-bearing phase, could produce variability in the results from the dust leaching experiments. This suggestion is supported by results from SEM and EDX of fine-particle sized ATD particles by Cwiertny et al. (2008). They found that within aggregates there appears to be single particles rich in Fe but depleted in Al and Si supporting the existence of $\mathrm{Fe}_{2} \mathrm{O}_{3}$ particles. They also found particles with $\mathrm{Fe}$, along with $\mathrm{Si}$ and $\mathrm{Al}$ dispersed throughout, thus being consistent with Fe substitution into the alumniosilicate matrix. Whilst these findings are of the fine particle-sized ATD which is likely to differ to the ultra-fine and medium particle-sized ATD used in the present study, the three size fractions are expected to be similar given they originate from the same parent material. Any differences in composition are likely to arise from fractionation of the different mineral phase when the particles are segregated upon the basis of size. The XRF analysis in the present study shows the finer particle-sized ATD has a higher Fe content than the coarse particle-sized ATD (Table 3.3), 
and depending on the aliquot of ATD, the proportion of particle sizes in two different aliquots of ATD could result in a different percentage of soluble Fe and hence contribute to the variability in percentage of soluble Fe in these the tests in the present study (Figs. 3.10-3.13).

\section{Experimental artefacts}

The presence of residual acid following cleaning could have been caused by insufficient MQ water rinsing of the filtration apparatus. Furthermore, contamination of airborne dust particles could have arisen when the dust sample was transferred from the manufactures container to a $2 \mathrm{ml}$ (pre-washed with MQ water and dried) sealed vial on the microbalance at VUW and from this vial to the filtration apparatus at GNS science. As the weighing and filtering took place in a class 100 clean room and there were no large anomalous peaks in the concentration of soluble Fe, this potential source of contamination is disregarded.

\section{Residual water on filter papers}

The leaching process was not the only time when an ATD aliquot was in contact with MQ water. The amount of time ATD spent in contact with any residual water on the filter paper between leaches could contribute to the variability the percentage of soluble Fe. Howe (2009) conducted an experiment where each leach was separated by time intervals ranging from several minutes to an hour and concluded that the length of time the ATD spent exposed on the filter membrane following dust leaching did not significantly alter the percentage of soluble Fe. This is an important conclusion as it eliminates the possibility of wetting and drying of the dust on the filter membrane inducing differences in observed Fe solubility (Chapter 3.1.3.3.1). 


\subsubsection{Discussion}

\section{Reference iron solubility value for Arizona test dust}

A reference value for ultra-fine particle-sized ADT Fe solubility of $0.49 \% \pm 11.09 \%(\mathrm{t}=40$ 180 hour, ultra-fine particle-sized ADT=10 mg, MQ water leaching solution=180 ml) has been established as reference material for future experiments.

\section{Comparison to other Fe solubility estimates}

Compared to Fe solubility values reported in the literature which most commonly range from $1-10 \%$, the low percentage of soluble Fe derived from ATD is likely to arise from the dust not having undergone atmospheric transport, weathering and other natural processes that enhance the soluble nature of the Fe on the dust surface (Chapter 3.1.3.3.1). However, the low percentage of soluble $\mathrm{Fe}$ in the present study appears feasible in the context of other studies. The ATD reference value from the present study is consistent with Howe (2009), who used a similar method (where $\sim 3 \mathrm{mg}$ ultra-fine particle-sized ATD was leached with 250 $\mathrm{ml}$ MQ water) and reports the percentage of soluble Fe to range between $0.40-0.62 \%$. The values in the present study are lower than i) Desboeufs et al. (2005), who report a value $1.5 \%$, but use a different method to attain their value (where $20 \mathrm{mg}$ of dust was leached with $4 \mathrm{~L}$ of MQ water acidified with $\mathrm{H}_{2} \mathrm{SO}_{4}$ to $\mathrm{pH} 4.7$ for 120 minutes), and ii) Cwiertny et al. (2008b) who report a considerably higher Fe solubility for ATD of $12.59 \pm 0.08 \%$ also use a different method (where ATD was leached at $\mathrm{pH} 1$ for 24 hours; the leaching reaction was $12.5 \mathrm{~g} \mathrm{~L}^{-1}$ and an electrolyte of $0.1 \mathrm{~m} \mathrm{NaCl}$ ). Given that the same dust substrate was used, the difference in the values is likely due to variations in the variables of the leaching method, such as $\mathrm{pH}$ of the leaching solution. In both these experiments, leaching solutions are used that have a considerably lower $\mathrm{pH}$ than seawater $(\mathrm{pH}$ 7.5) and although the $\mathrm{pH}$ of MQ water also differs from that of seawater, the argument of using MQ water as a reproducible leaching solution has been justified (Chapter 3.2.1). It has been shown here that leaching time and dust load have little effect on the observed percentage of soluble Fe so these parameters are excluded as reasons for the variability.

Buck et al. (2006) observes that for dust particles collected on filter papers at sea, over $95 \%$ of the soluble Fe is released in the first $100 \mathrm{ml} \mathrm{MQ}$ water leach. Only $78 \%$ of soluble Fe was 
released in the first leach during 20 minute leaches and $70 \%$ during one hour leaches. The lower percentage in the present study can also be attributed to differences between natural dust samples and ATD, e.g. the absence of atmospheric processing of the ATD taken directly from the manufactures container.

\section{The influence of leaching variables on the percentage of soluble iron}

Variations in the leach time, between $2-120$ minutes, had little influence on percentage of soluble Fe (Fig. 3.11). Furthermore, percentage of soluble Fe remained constant with variations in the dust load between 2 - $20 \mathrm{mg}$ (Fig. 3.13). The literature describes a "particle concentration effect" applicable to dust masses over several orders of magnitude (Fig. 3.3), which is not applicable to the considerably smaller range of dust masses used in these tests or in samples from McMurdo Sound samples. Similarly, Baker et al. (2006) observes only a comparatively small effect on aerosol Fe solubility with variable dust mass and suggests that the major factor controlling the observed differences in Fe solubility is the character and origin of the aerosols themselves. The lack of variability of dust load on Fe solubility cannot be attributed to aggregation of the dust particles loaded onto the filter as the first leach is conducted in an ultra-sonic bath following dust recovery tests that found aggregated particles were not separated properly onto the different pore sized filter membranes.

\section{Implications for methodology}

The results of these tests have important implications for the design of a method specifically for ASD contained in snow and ice samples. All samples in the tests in the present study attain their maximal soluble Fe concentration within the first leach, which contains particulate $\mathrm{Fe}<0.4 \mu \mathrm{m}$ and $\mathrm{Fe}$ leached from the dust surface. This rapid release is particularly significant for dust leaching conducted over extended periods of time (as in the case of melting snow over 3 - 4 hours for $500 \mathrm{ml}$ bottle samples) because if the solubility maximum is reached within an hour as maybe occurring in this case, longer leaching times are unlikely to yield any more information. Although more Fe may leach from the dust surface, it could be expected to precipitate back out of solution (Fig. 3.9). Subsequent leaches, which render lower soluble Fe percentages, also contain soluble Fe from the dust surface but possibly the Fe is released at a slower rate from the dust surface. This result is important as it suggests previously published methods which rely on only a single leach (e.g. Buck et al., 2006) or those methods which use leaching times as short as 40 seconds (Table 3.2; e.g. Buck et al., 
2006; Schroth et al., 2009), which result in significant variability in the percentage of soluble Fe (Figs. 3.10 and 3.12), may significantly underestimate the potential percentage of soluble Fe from dust particles. Moreover, this confirms the need to carry out subsequent leaches on the McMurdo Sound ASD after an initial snow melt leach.

With respect to the design of a leaching method, the lack of variability in the percentage of soluble Fe with varying dust load indicates that it is not necessary to compensate this effect for dust concentrations between $2-20 \mathrm{mg}$ as it would be particularly difficult to control the amount of ASD in each snow samples due to the variable nature of ASD in McMurdo Sound.

Future leaching experiments using ATD could involve the study of the Fe species on the surface of dust particles which are involved in the dissolution phase. This may provide further information about the heterogeneity of ATD and how this influences the percentage of soluble Fe of the dust and hence the reproducibility using ATD as a uniform matrix for these leaching experiments. Furthermore, using an artificial certified seawater standard as the leaching solution to mimic the natural environment would be advantageous, however a previous account of this with ATD could not be found in the literature. If artificial seawater was made to strict specifications (uniform in nature to produce comparable results) it would provide a close imitation of seawater.

The use of a standard leaching method across all laboratories would improve the ability to compare leaching experiments conducted by different research groups and enhance the understanding of the natural process of dust solubility. This would improve the characterisation of $\mathrm{Fe}$ solubility in different dust sources and provide more accurate values of this parameter for use in modelling programs which would enhance the understanding of how the Earth system may respond to the effects of global change.

A successful method for determining low soluble Fe concentrations in ASD contained in snow and ice samples has been established using trace metal clean techniques (Chapter 3.2.5). 


\subsubsection{Method arising from Arizona test dust leaching tests}

A successful method for determining low soluble Fe concentrations has been established and described in Chapter 3.3, briefly:

\section{Cleaning protocol and blank solutions}

Soak filtration apparatus in MQ water when not in use and thoroughly rinse with MQ water before and after leaching experiments. Rinse filtration apparatus with $0.1 \mathrm{wt} \% \mathrm{HCl}$ (SS) between samples, and rinse thoroughly with MQ water. At the beginning of each day filter two blank solutions of $180 \mathrm{ml} \mathrm{MQ}$ water through the filtration system on pre-acid washed filter papers and collect supernatant in two separate pre-washed (3x rinse MQ water) $250 \mathrm{ml}$ screw top Naglene bottles.

\section{Snow leach}

Melt each surface snow sample from McMurdo Sound at room temperature (3 - 4 hours) in the $500 \mathrm{ml}$ bottle it was sampled in (Chapter 2.2.1.1.1). As soon as each sample is melted, filter with acid washed and pre-weighed $0.4 \mu \mathrm{m}$ and $10 \mu \mathrm{m}$ polycarbonate filter papers mounted on separate stages. Collected filtered snow melt in pre-washed (3x rinse MQ water) $250 \mathrm{ml}$ screw top Naglene bottles. This leachate is termed the instantaneous soluble Fe.

\section{Subsequent leaches}

Assuming the mixed layer is between 5 and $20 \mathrm{~m}$ in McMurdo Sound (Arrigo et al., 2010), coarse particles will reside in the mixed layer for a minimum of $0.5-2$ hours respectively (if $90 \%$ of the particles are finer than $75 \mu \mathrm{m}$ which have a settling velocity of $0.31 \mathrm{~cm} \mathrm{~s}^{-1}$; Gibbs et al., 1971) and fine particles will reside for 1-4 days respectively (if $25 \%$ of the particles are $<10$ um which have a settling velocity of $0.006 \mathrm{~cm} \mathrm{~s}^{-1}$; Gibbs et al., 1971).

Due to the high settling velocity of particles greater than $10 \mu \mathrm{m}$ in the ocean, and that the majority of total and soluble Fe is found on particles larger than $1 \mu \mathrm{m}$ (Buck et al., 2010a), it is justifiable to leach only the $0.4-10 \mu \mathrm{m}$ size fraction in the McMurdo Sound samples after the initial snow melt leach. 
As the ASD is laid on the two different pore size filter papers, the stage containing the $10 \mu \mathrm{m}$ filter can be removed using acid washed ceramic tweezers. Dry this fraction in a desiccator for 48 hours. Leach ASD contained on the $0.4 \mu \mathrm{m}$ filter with $40 \mathrm{ml}$ MQ water for 1 hour and collect the supernatant in pre-washed (3x rinse MQ water) $250 \mathrm{ml}$ screw top Naglene bottles. Repeat this 1 hour leach 3 times using fresh aliquots of MQ water, collecting the supernatant in separate bottles. The experiment takes $\sim 7$ hours ( 4 hours to melt sample and 3 hours for $3 \mathrm{x}$ fine fraction leaches) for each individual sample and can be completed in a single day. Fe concentrations are analysed by hydrogen flushed collision cell ICP-MS analysis the same day.

\section{Weighing sample ASD}

Carefully transfer sediment laid filter papers using acid washed ceramic tweezers to petri dishes in desiccators and dry for 48 hours. Weigh each sample on balance three times and take average weight $(2 \mathrm{Sd}<0.0005 \mathrm{~g})$. The filter papers were later acid digested for total $\mathrm{Fe}$ content of the ASD. 


\subsubsection{Conclusions}

Varying the leaching time, dust load and leaching volume in dust leaching tests has provided useful information for the design of a leaching method specifically for ASD samples contained in snow and ice samples. In conclusion:

1) it is necessary to measure the Fe concentration in the leachate the same or following day to avoid Fe precipitation and subsequent absorption into the bottle walls;

2) to gain reproducible results using a MQ water leaching solution, ASD needs to be leached with MQ water between 40 - 180 minutes;

3) varying the dust load between 2 - $20 \mathrm{mg}$ has little effect on the percentage of soluble $\mathrm{Fe}$;

4) during the first leach, Fe is leached predominately from finer sized-particles (0.4 - 10 $\mu \mathrm{m})$ due to the greater surface area to volume ratio, and a second successive leach will start to leach Fe from the surface of coarser particles $(<10 \mu \mathrm{m})$. The $\mathrm{Fe}$ concentration exponentially decreases during successive leaches. For this reason it is necessary to carry out at least three successive leaches to leach the maximum Fe concentration from the $\mathrm{Fe}$ on the dust surface.

McMurdo Sound is an ideal location for investigating the Fe solubility of natural ASD, as opposed to anthropogenic emissions to the atmosphere, which are often acidic in nature and considered as a source of readily available Fe to the ocean (Sholkovitz et al., 2009). McMurdo Sound offers a unique study area where these anthropogenic emissions are insignificant as a source of $\mathrm{Fe}$, where locally derived ASD dominates the deposition of dust to the ocean, and where the global background dust flux is at least an order of magnitude lower than locally derived ASD (Chapter 2.4.3). 


\subsection{Particulate and soluble iron methodology for Antarctic aeolian sand and dust}

\subsubsection{Samples}

Nine samples, collected using ultra-clean methodology (see Chapter 2.2), were chosen for soluble and total Fe analysis because they represent a broad area of McMurdo Sound (Fig. 3.15 and Table 3.7). An ASD layer from the 2004 storm was also investigated as this storm contained $\sim 4$ times more ASD than the background ASD accumulation rate (see Chapter 2.3.2.2).

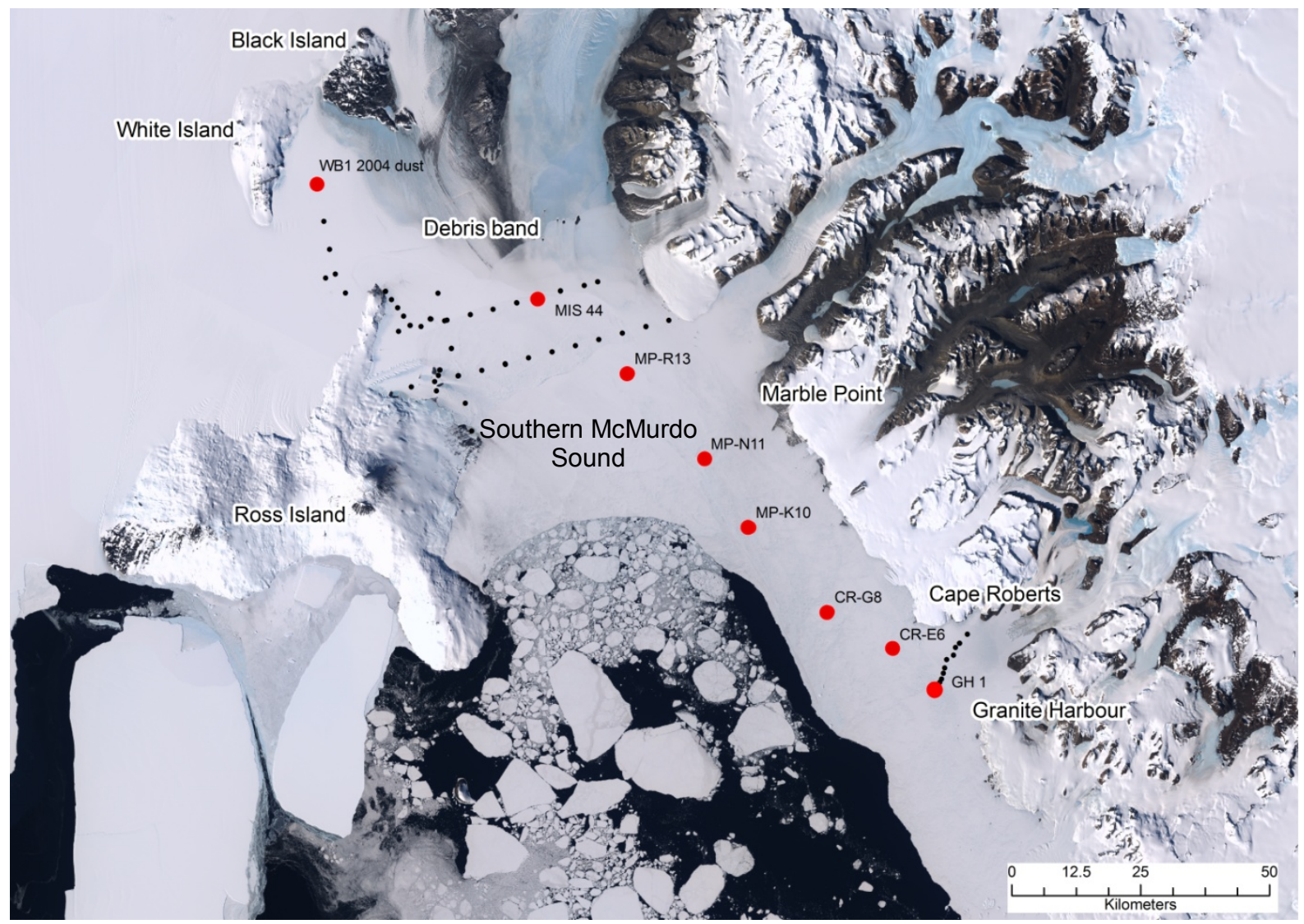

Fig. 3.15: Location of samples for soluble and total Fe analyses (red dots). 
Table 3.7: McMurdo Sound samples, collected using ultra-clean methodology, used in ASD leaching analyses. WB: Windless Bight; MIS: McMurdo sea ice; MP: Marble Point; CR: Cape Roberts; GH: Granite Harbour.

\begin{tabular}{|c|c|c|c|c|}
\hline Sample & Sampling date & $\begin{array}{l}\text { Area of } \\
\text { snow } \\
\left(\mathrm{cm}^{2}\right)\end{array}$ & $\begin{array}{l}\text { Mass of } \\
\text { ASD } \\
\text { (mg) }\end{array}$ & $\begin{array}{c}\text { ASD accumulation } \\
\text { rate } \\
\left(\mathrm{g} \mathrm{m}^{-2} \mathrm{yr}^{-1}\right)\end{array}$ \\
\hline WB1 2004 ASD layer & November 2008 & 22.8 & 64.3 & 28.3 \\
\hline MIS 44 & November 2009 & 33.2 & 97.9 & 29.5 \\
\hline MPR13-5 & November 2010 & 33.2 & 5.4 & 1.6 \\
\hline MPN11-5 & November 2010 & 33.2 & 24.8 & 7.5 \\
\hline MPK10 & November 2010 & 33.2 & 2.7 & 0.9 \\
\hline CREG8-5 & November 2010 & 33.2 & 6.5 & 2.0 \\
\hline CRE6-5 & November 2010 & 33.2 & 1.1 & 0.3 \\
\hline GH1a & November 2009 & 33.2 & 0.4 & 0.1 \\
\hline GH1b & November 2009 & 33.2 & 51.1 & 15.4 \\
\hline
\end{tabular}

\subsubsection{Soluble iron}

\subsubsection{Methods and materials}

All dust leaching experiment is described in Chapter 3.2.2.2. The leaching procedure for one sample could be completed in a single day as each sample took approximately $\sim 7$ hours (4 hours to melt sample and 3 hours for $3 \times 0.4 \mu \mathrm{m}$ leaches).

\subsubsection{Filter blanks and blank solutions}

Prior to each leaching experiment two filter paper blanks were leached with $180 \mathrm{ml}$ of MQ water by placing a $0.4 \mu \mathrm{m}$ and $10 \mu \mathrm{m}$ filter membrane in the reaction chamber and adding the leaching solution to the holder and filtering (Fig. 3.8). The leachate was collected in $250 \mathrm{ml}$ Nalagene bottles and blank filter papers stored frozen in Petri dishes. The precision (2 Sd\%) from the replicate blank solutions was within the conventionally acceptable range for $\mathrm{Fe}$ concentration (Table 3.3). The precision $(2 \mathrm{Sd} \%)$ and detection limits $(3 \sigma)$ for the filter papers is described in Chapter 3.3.3.7. 


\subsubsection{Snow leach}

Each surface snow sample was melted, taking $\sim 3-4$ hours, in the pre-acid washed $500 \mathrm{ml}$ bottle it was collected in the field at room temperature (see Chapter 2.2.1.1). As soon as the sample was melted, it was sonicated for 20 minutes in an ultra-sonic bath to break up aggregate particles to allow the sample to be adequately separated into the fine and coarse fractions during filtering (Fig. 3.8). Immediately after sonication the snow melt was weighed and filtered on clean (pre-acid washed and pre-weighed) $0.4 \mu \mathrm{m}$ and $10 \mu \mathrm{m}$ polycarbonate filter papers mounted on separate stages and placed in the reaction chamber. The filtered snow melt was collected in pre-washed (3x rinse MQ water) $250 \mathrm{ml}$ screw top Naglene bottles. The ASD was recovered on the 0.4 and $10 \mu \mathrm{m}$ filter membranes.

\subsubsection{Successive leaches}

Successive leaches were carried out on the $<10 \mu \mathrm{m}$ ASD fraction. After the initial snow melt leach the ASD from the sample is contained on the two different pore sized filter papers. The stage containing the $10 \mu \mathrm{m}$ filter was removed using acid washed ceramic tweezers and dried in a desiccator for 48 hours. The remaining ASD on the $0.4 \mu \mathrm{m}$ filter $(<10 \mu \mathrm{m})$ was leached with $40 \mathrm{ml}$ MQ water (maximum volume the reaction chamber can hold) for 1 hour in the reaction chamber. At the end of the leaching time the valve was opened and the leachate collected in pre-washed (3x rinse MQ water) $250 \mathrm{ml}$ screw top Naglene bottles. The valve was then closed and a new aliquot of $40 \mathrm{ml}$ leaching solution was added and allowed to sit for 1 hour. This procedure was repeated three times.

\subsubsection{Weighing aeolian sand and dust samples}

The sediment laid filter papers were carefully transferred, using acid washed ceramic tweezers, to Petri dishes in desiccators and dried for 48 hours. The weight of the ASD was determined gravimetrically by 3 repeat measurements on balance with 4 decimal places and an average weight calculated. These filter papers were later digested for total Fe content of the ASD (Chapter 3.3.3). 


\subsubsection{Inductively coupled plasma mass spectrometry}

Leachate solutions were transferred to the Geochemistry Laboratory, VUW (MAF Reg. No. 14541 ) and acidified using $1 \mathrm{wt} \% \mathrm{HNO}_{3}$ (SS) in a class 100 clean room prior to analysis on an Agilent 7500cs series ICP-MS. See Chapter 3.2.2.5 for Fe analysis, standard solutions and calibration.

\subsubsection{Particulate iron}

\subsubsection{Total digestion of McMurdo Sound sand and dust}

All analytical work was carried out in the class 100 clean room at the Geochemistry Laboratory, VUW. Savillex Teflon $23 \mathrm{ml}$ beakers with screw caps were acid washed using the procedure in Table 3.8. Dried sediment laid filter papers were transported to the Geochemistry Laboratory, VUW in sealed Petri dishes. Sediment laid filter papers were transferred to the cleaned Savillex beakers using acid washed (5 wt \% $\mathrm{HNO}_{3}(\mathrm{AR})$ ) ceramic tweezers (cleaned with methanol between each sample) and weighed to 6 decimal places on a high precision balance. The mass of the sediment was calculated by weight difference.

Table 3.8: Savilex beaker acid washing procedure for total Fe digestion of sediment.

\begin{tabular}{cc}
\hline Step & Procedure \\
\hline 1 & Wipe with methanol, rinsed with MQ water \\
2 & Acid bath of half concentrated $\mathrm{HCl}(\mathrm{AR})$ on hot plate for 24 hours, rinsed 3x MQ water \\
3 & Acid bath of half concentrated $\mathrm{HNO}_{3}(\mathrm{AR})$ on hot plate for 24 hours, rinsed 3 x MQ water \\
4 & Dry on hot plate \\
5 & Flux $\mathrm{HNO}_{3},(\mathrm{SB}) 24$ hours, rinse 3x MQ water \\
6 & Flux $7 \mathrm{M} \mathrm{HNO}_{3}(\mathrm{SS}) 24$ hours, rinse 3x MQ water \\
7 & Flux $7 \mathrm{M} \mathrm{HNO}_{3}(\mathrm{SS}) 24$ hours, rinse 3x MQ water \\
8 & Store in acid. Rinse and dry immediately prior to use
\end{tabular}




\subsubsection{Arizona test dust Fe digestion test}

Arizona test dust sediment-laid filter samples (both size fractions of leaches 31 and 32; Table 3.4) were tested for the total Fe digestion procedure, as digestion of polycarbonate filter membranes had not been previously carried out in the Geochemistry Laboratory.

A strong acid digestion procedure was used to determine total Fe. Sediment laid filter membranes were digested on a hotplate for 48 hours in 20 drops of $69 \mathrm{wt} \% \mathrm{HNO}_{3}$ (SS) and 35 drops of concentrated HF (29M) (SS) and then boiled to dryness to destroy organic matter, filter membrane and the silicate matrix of the mineral component of the dust. The residue was redissolved in $5.0 \mathrm{ml}$ of $69 \mathrm{wt} \% \mathrm{HNO}_{3}$ (SS) overnight on hotplate. Filter residue was still present so samples were evaporated until dryness and attacked again with $3.0 \mathrm{ml}$ of $69 \mathrm{wt} \%$ $\mathrm{HNO}_{3}$ (SS) boiled to dryness overnight. Still filter residue was present, so samples were attacked with $3.0 \mathrm{ml}$ of $6-7 \mathrm{M} \mathrm{HCl}(\mathrm{SS}), 3.0 \mathrm{ml}$ of MQ water and $1.5 \mathrm{ml}$ of $69 \mathrm{wt} \% \mathrm{HNO}_{3}$ (SS) reflux overnight for the residue to be taken up in solution. Samples were dried down and the filter residue was redissolved with $9.0 \mathrm{ml}$ of $1 \mathrm{M} \mathrm{HNO}_{3}$ (SS) on hotplate for 24 hours. Due to the presence of solid matter from filter residue, the samples were centrifuged for 3 minutes at $1500 \mathrm{rpm}$. From here a $1 \mathrm{ml}$ aliquot of each sample was pipetted into acid cleaned $10 \mathrm{ml}$ centrifuge tubes and this sample was diluted with $9.0 \mathrm{ml}$ of $1 \mathrm{wt} \% \mathrm{HNO}_{3}$ (SS) for ICP-MS analysis.

\subsubsection{Inductively coupled plasma mass spectrometry analysis for ATD total Fe}

Acidified solutions were analysed on an Agilent 7500cs series ICP-MS at the Geochemistry Laboratory, VUW. A blank made of $1 \mathrm{wt} \% \mathrm{HNO}_{3}$ (SS) was measured immediately before each sample. Counts recorded on those background measurements were subtracted from the counts measured on the sample to correct for background. A hydrogen flushed collision cell was used for measurement of ${ }^{54} \mathrm{Fe},{ }^{57} \mathrm{Fe},{ }^{27} \mathrm{Al},{ }^{43} \mathrm{Ca},{ }^{25} \mathrm{Mg}{ }^{47} \mathrm{Ti}$ and ${ }^{49} \mathrm{Ti}$. Two digestions of a rock standard, basalt powder (BJ2) from the Oshima volcano, Tokyo, with a known Fe content of 100,200 $\mathrm{g} \mathrm{g} \mathrm{g}^{-1}$ (Korotev, 1996) were also digested by the same method above and measured by ICP-MS. The first digestion of JB2 was used as a calibration standard, and the second was used as a check standard. Calibrated Fe concentrations were corrected for a dilution factor. 
A minor isotope of $\mathrm{Ca}$ was used as an internal standard to account for the potential loss of sample during preparation. The ${ }^{43} \mathrm{Ca}$ concentration is constrained for both JB2 (Korotev, 1996) and ATD tests and used as an internal standard. Incomplete filter dissolution made little difference to the results (Fig. 3.16). Table 3.9 illustrates the excellent precision of the Fe digestion method, and therefore an acceptable method for use on actual McMurdo Sound ASD samples.

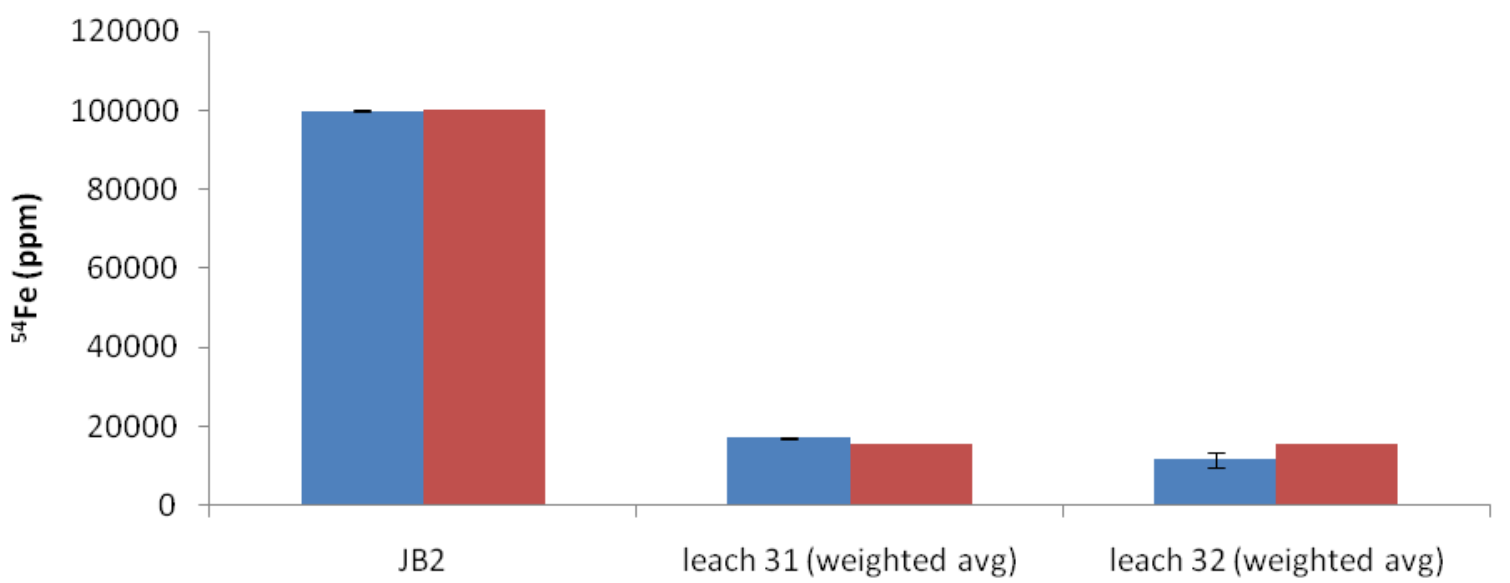

Fig. 3.16: Analysis of ultra-fine Arizona test dust filters and JB2 standard (blue) compared to the known Fe concentration (red) for that sample. Error bars are $2 \mathrm{Sd} \%$.

Table 3.9: Precision of the Fe digestion method for ultra-fine Arizona test dust leaches 31 and 32.

\begin{tabular}{cccc}
\hline JB21 & $\begin{array}{c}\text { Measured Fe54 } \\
\left(\boldsymbol{\mu g ~ \mathbf { ~ } ^ { - 1 } )}\right.\end{array}$ & $\begin{array}{c}\text { Precision } \\
(\mathbf{2 ~ S d \% )}\end{array}$ & $\begin{array}{c}\text { Accuracy } \\
(\% \text { difference })\end{array}$ \\
\hline Leach 31 (weighted avg) ${ }^{2}$ & 99839.66 & 0.51 & 0.36 \\
Leach 32 (weighted avg) & 17126.25 & 2.93 & 2.06 \\
\hline
\end{tabular}

${ }^{1}$ Actual Fe concentration $100200 \mu \mathrm{g} \mathrm{g}^{-1}$ (Korotev, 1996)

${ }^{2}$ Actual Fe concentration $17486 \mu \mathrm{g} \mathrm{g}^{-1}$ calculated from XRF measurements of the ultra fine ATD of $2.5 \mathrm{wt} \%$ assuming all $\mathrm{Fe}$ is $\mathrm{Fe}_{2} \mathrm{O}_{3}$. 


\subsubsection{Total digestion of particulate material in McMurdo Sound ASD}

A strong acid digestion procedure, similar to the ATD test above, was used to determine total Fe in McMurdo Sound samples. In acid washed Teflon $23 \mathrm{ml}$ screw cap beakers, sediment laid filter membranes, blank filter membranes and JB2 rock standards were spiked with 100 ppm Indium (In) as an internal standard. Indium is used as an internal standard as this element is virtually absent in crustal rocks and because samples have an unknown $\mathrm{Ca}$ concentration. These were digested on a $120^{\circ} \mathrm{C}$ hotplate for $>48$ hours in 15 drops of $69 \mathrm{wt} \%$ $\mathrm{HNO}_{3}$ (SS) and 40 drops of HF (29M) (SS) and then boiled to dryness to destroy organic matter, filter membrane and the silicate matrix of the mineral component of the ASD. The tests in Fig. 3.15 show that potentially incomplete filter dissolution makes little difference to the results. The residue was redissolved in $5 \mathrm{ml}$ of $69 \mathrm{wt} \% \mathrm{HNO}_{3}$ (SS) 24 hours on a hotplate at $120{ }^{\circ} \mathrm{C}$ and then repeated. Samples were then redissolved in $5.0 \mathrm{ml}$ of $6-7 \mathrm{M} \mathrm{HCl}(\mathrm{SS})$ on a hot plate for 24 hours and dried down. $1.0 \mathrm{ml}$ of $69 \mathrm{wt} \% \mathrm{HNO}_{3}$ (SS) was added to the samples and dried down immediately. Samples were then taken up in $1 \mathrm{M} \mathrm{HNO}_{3}$ (SS) for 24 hours and centrifuged for 3 minutes at $1500 \mathrm{rpm}$.

\subsubsection{ICP-MS analysis of McMurdo Sound ASD samples}

Samples were diluted and acidified with 1 wt $\% \quad \mathrm{HNO}_{3}$ (SS) for total ${ }^{54} \mathrm{Fe},{ }^{57} \mathrm{Fe},{ }^{24} \mathrm{Mg},{ }^{25} \mathrm{Mg},{ }^{27} \mathrm{Al},{ }^{43} \mathrm{Ca},{ }^{47} \mathrm{Ti},{ }^{49} \mathrm{Ti}$ and ${ }^{115} \mathrm{In}$ measurement using the hydrogen flushed collision cell on ICP-MS described in Chapter 3.3.3.3.

\subsubsection{Calibration and standards}

Samples were spiked with Indium (In) as an internal standard. Three JB2 rock standards were digested using the same technique as the McMurdo Sound samples for use as an external standard. Initial investigation of calibration standard, JB2-C, showed a linear relationship between elemental concentration and ICP-MS signal intensity. Therefore, a single calibration standard, JB2-C, run as a bracketing standard analysed every 6 samples to correct for instrumental sensitivity, was measured with elemental concentrations of the standard approximating mean values expected in ASD samples. Accuracy was determined by measuring 2 additional less concentrated calibration standards, JB2-A and JB2-B, every 6 
samples in between the JB2-C calibration standards. Analytical precision was determined by 5 repeated measurements of JB2-C. The concentrations determined in the external reference material are within error of the certified values and shown in Table 3.10. Concentrations of two isotopes of $\mathrm{Fe}, \mathrm{Mg}$ and $\mathrm{Ti}$ isotopes are with $0.35 \%$ of each other. The ratio of measured In, measured on ${ }^{115} \mathrm{In}$, to actual In doped in each standard ranges from 0.97 to 1.08 illustrating precision of the analytical technique. Elemental concentrations are normalised to the In offset as discussed above. Both internal and external standards highlight the accuracy and precision of the technique.

Table 3.10: Element concentrations (ppm: parts per million) determined by repeated measurement of JB2 rock standard with precision and accuracy of measurements.

\begin{tabular}{ccccccccc}
\hline & ${ }^{24} \mathbf{M g}$ & ${ }^{25} \mathbf{M g}$ & ${ }^{27} \mathbf{A l}$ & ${ }^{43} \mathbf{C a}$ & ${ }^{47} \mathbf{T i}$ & ${ }^{49} \mathbf{T i}$ & ${ }^{54} \mathbf{F e}$ & ${ }^{57} \mathbf{F e}$ \\
\hline $\begin{array}{c}\text { Mean } \\
\text { (ppm) }\end{array}$ & 26804 & 26900 & 72536 & 68377 & 6667 & 6668 & 100568 & 100928 \\
$\begin{array}{c}\text { Accuracy } \\
\text { \% difference) }\end{array}$ & 3.79 & 3.45 & 5.48 & 6.33 & 4.67 & 4.65 & 0.37 & 0.73 \\
$\begin{array}{c}\text { Precision } \\
\text { (2 SD\%) }\end{array}$ & 5.96 & 6.60 & 8.10 & 6.89 & 8.92 & 8.76 & 5.24 & 5.01 \\
\hline
\end{tabular}

\subsubsection{Filter blanks}

For the analyses described above, blanks were determined by carrying out identical extraction procedures on filters that had been treated as for samples (acid cleaned filters which had undergone leaching experiments with a MQ water leaching solution which did not contain ASD) which were otherwise not used. Blank filter papers contained between $0-1.30 \mathrm{ppm} \mathrm{Fe}$ $(n=3$; Chapter 3.2.2.4.1), which is low enough to disregard correcting samples for blank Fe concentrations. Limits of detection (Table 3.11) are governed by uncertainties in the filter blank, rather than the instrumental detection limits for each analysis.

Table 3.11: Detection limit (3 $\sigma)$ of blank filter papers (ppb).

\begin{tabular}{cccccccc}
\hline${ }^{24} \mathbf{M g}$ & ${ }^{25} \mathbf{M g}$ & ${ }^{27} \mathbf{A l}$ & ${ }^{43} \mathbf{C a}$ & ${ }^{47} \mathbf{T i}$ & ${ }^{49} \mathbf{T i}$ & ${ }^{54} \mathbf{F e}$ & ${ }^{57} \mathbf{F e}$ \\
\hline 1.40 & 1.50 & 1.97 & 2.69 & 6.69 & 5.73 & 2.70 & 1.33 \\
\hline
\end{tabular}




\subsection{Results - particulate and soluble aeolian iron in McMurdo Sound}

\section{Terminology}

The operationally defined size fractions of Fe are defined as follows: Fe that passed through the $0.4 \mu \mathrm{m}$ filter membrane is defined as soluble Fe; Fe that passed through the $0.4 \mu \mathrm{m}$ filter membrane during the first snow melt leach is termed "instantaneous" soluble Fe; the cumulative sum of all the soluble Fe leaches is termed cumulative soluble $\mathrm{Fe} ; \mathrm{Fe}>0.4 \mu \mathrm{m}$ is defined as particulate $\mathrm{Fe}$; total $\mathrm{Fe}$ is the sum of soluble and particulate $\mathrm{Fe}$; the percentage of cumulative soluble $\mathrm{Fe}$ is the cumulative sum the percentage of soluble Fe from the four leaches; and the percentage of soluble Fe is described in Chapter 3.2.2.3.

As discussed in Chapter 3.3 instantaneous soluble Fe was leached from the bulk ASD sample, where soluble Fe was leached from the fine ASD fraction i.e. $0.4 \mu \mathrm{m}-10 \mu \mathrm{m}$. Particulate Fe was analysed on the fine $(0.4 \mu \mathrm{m}-10 \mu \mathrm{m})$ and coarse $(>10 \mu \mathrm{m})$ ASD fractions and bulk particulate Fe was calculated using a weighted average of the fine and coarse size fractions.

\subsubsection{Particulate iron concentration in lithogenic particles}

Particulate Fe concentrations in both the fine and coarse size fractions between Southern McMurdo Sound and Cape Roberts (Fig. 3.15) range from $2.39 \mathrm{wt} \%$ to $6.97 \mathrm{wt} \%$ (Fig. 3.17). The exception to the trend is sample MPK10 and CREG8 which have an unusually high Fe content in the fine fraction (12.57 and $5.40 \mathrm{wt} \%$ respectively). Granite Harbour ASD is particularly Fe-rich with up to $15 \mathrm{wt} \%$. With the exception of sample MPK10 and CREG8, particulate Fe concentration in McMurdo Sound ASD appears remarkably constant with an average of $3.84 \pm 1.99$ wt \% Fe. Fig. 3.17 plots the concentration of particulate Fe versus distance from the debris band, including the 2004 ASD layer from WB core 1. With the exception of MPK10 and CREG8 (highlighted with circles in Fig. 3.17) the fine and the coarse fractions exhibit similar particulate Fe content. 


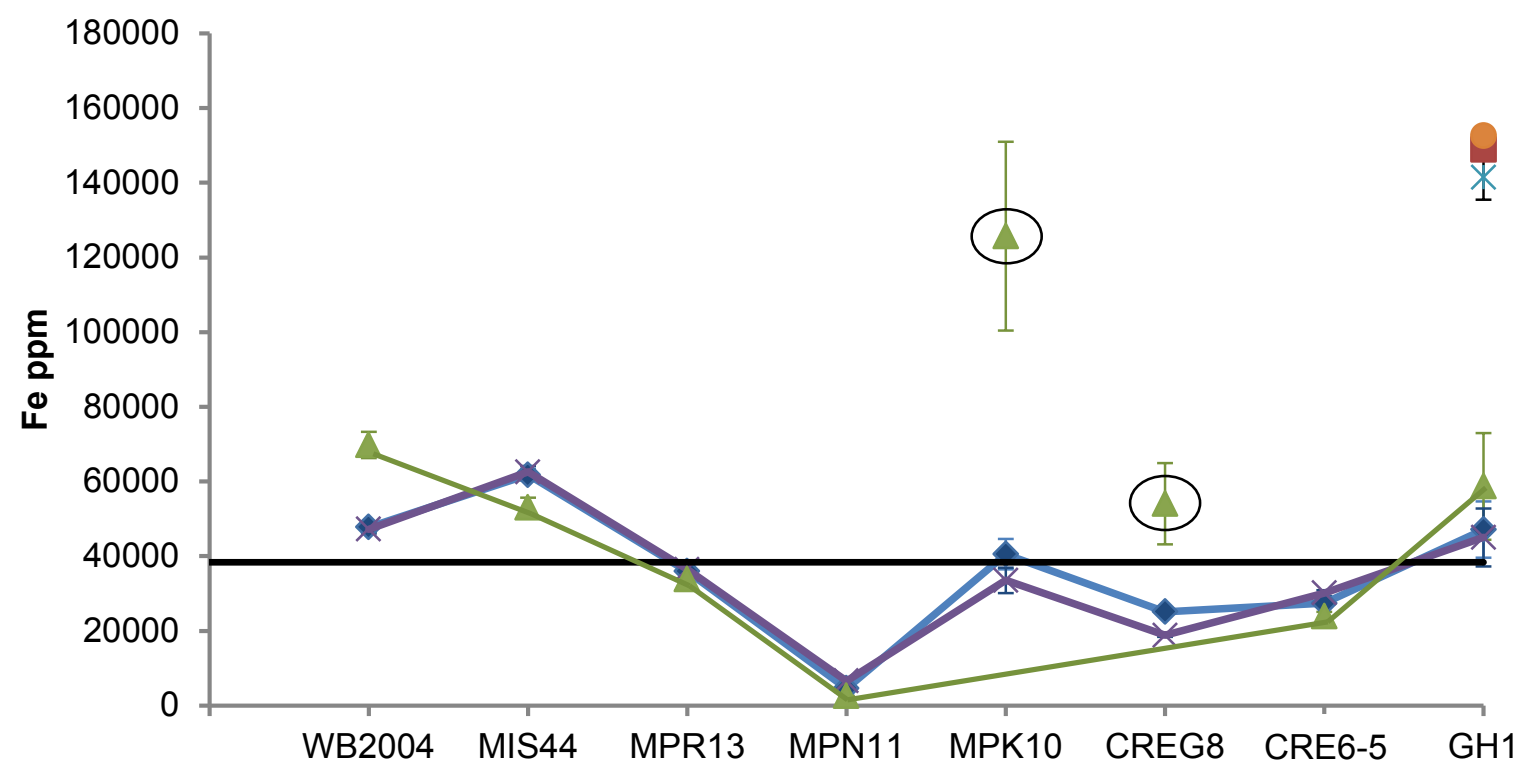

Fig. 3.17: Total digested Fe along transect XY. Green line: bulk sample; purple line: coarse fraction; blue line; fine fraction; red square: GH duplicate bulk; orange circle: GH duplicate coarse fraction; blue asterisk: GH duplicate fine fraction. Black line: average Fe ASD content in McMurdo Sound (excluding anomalously high MPK10 fine sample circled). Error bars are the precession (2 Sd\%) of the measurements.

\subsubsection{Soluble iron}

Fig. 3.18 illustrates the concentration of soluble Fe leached from ASD during successive leaches at each sampling site. Two major trends are observed:

1. For each sample the first leach, i.e. the instantaneous soluble Fe, yields the maximum concentration of soluble Fe ranging from 0.17 to $1.68 \mu \mathrm{mol} \mathrm{L}{ }^{-1}$ along transect XY. This instantaneous leach consistently yields $76 \pm 14 \%$ of the total cumulative soluble Fe. Subsequent leaches (leaches 2, 3 and 4) yield progressively less soluble Fe from the ASD.

2. For all four leaches a decreasing trend of soluble Fe with distance from the ASD source is observed (Fig. 3.17). The maximum amount of soluble Fe was rendered from sample MIS44 closest to the ASD source: after the final leach the cumulative soluble Fe concentration was $1.87 \mu \mathrm{mol} \mathrm{L} \mathrm{L}^{-1}$. While the minimum amount of soluble Fe was rendered from sample CRE6-5, which leached a cumulative soluble Fe concentration of $0.03 \mu \mathrm{mol} \mathrm{L}{ }^{-1} \mathrm{Fe}$. Samples in between this range follow the same 
trend as ASD mass accumulation rate, i.e. an exponential decrease in soluble Fe with distance from the source. The exception to this trend is the duplicate sample from Granite Harbour (GH1b), demonstrating there is a degree of local variability in ASD and Fe concentration in McMurdo Sound surface snow (Fig. 3.18). Sample GH1b was sampled from a 'dirty snow' patch and $94 \%$ more Fe was leached compared to sample GH1a which was sampled from 'clean snow,' in which GH1a follows the regional trend of decreasing soluble Fe from source. The sample from the storm event in 2004 also had elevated soluble Fe concentration: cumulative soluble Fe rendered a concentration of $0.71 \mu \mathrm{mol} \mathrm{L}{ }^{-1}$ from this ASD layer in WB1.

All Fe geochemical data for the ASD samples in this chapter can be found in Appendix 7.

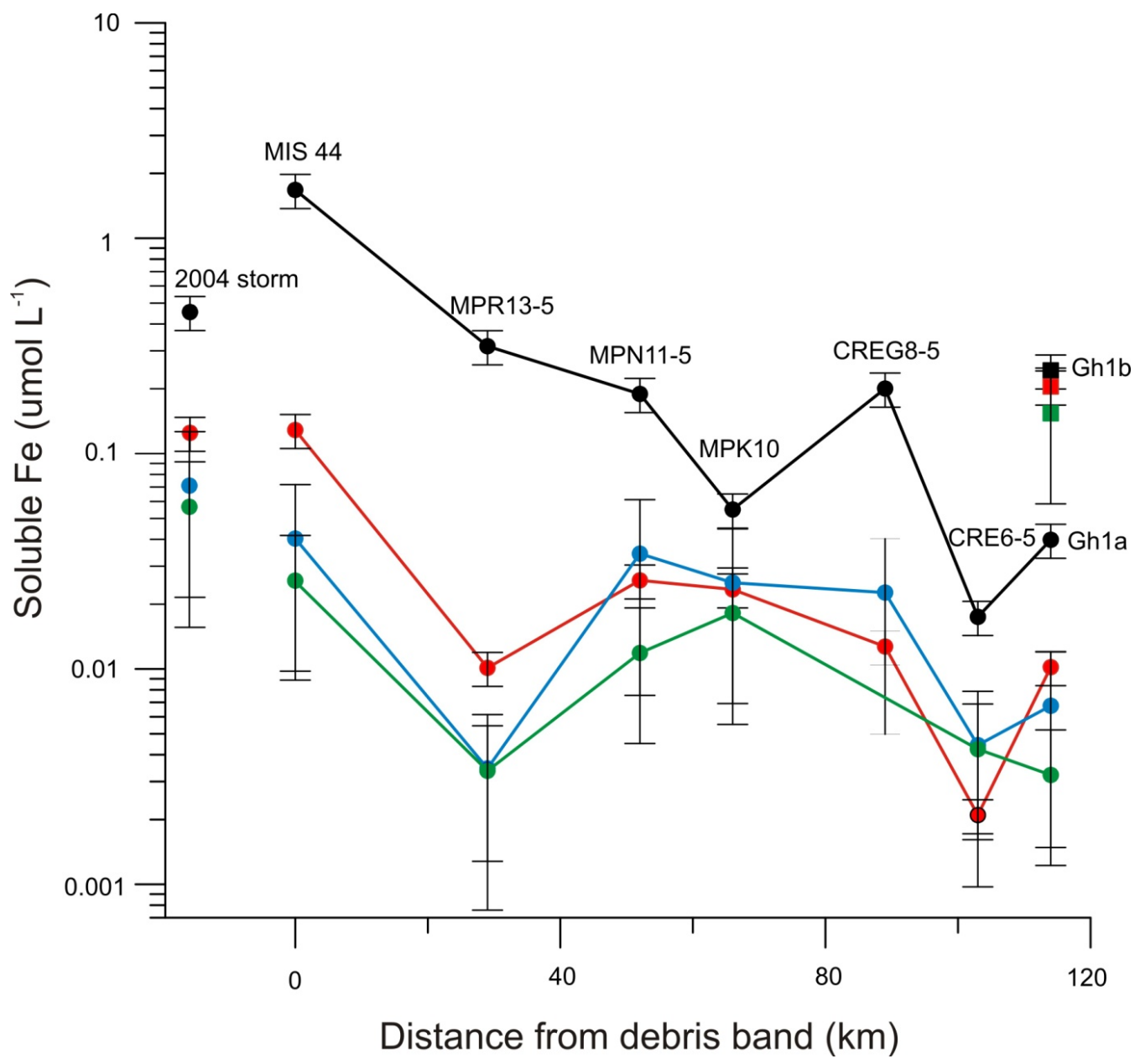

Fig. 3.18: Concentration of soluble leached from ASD samples along transect XY. Black: 'Instantaneous' snow melt leach. Red: MQ water leach 2. Blue: MQ water leach 3. Green: MQ water leach 4. Squares: duplicate sample (GH1b) at Granite Harbour. Error bars are associated with the procedural 2 SE\% calculated from replicate analysis of Arizona test dust successive leaching tests (see Chapter 3.2.3.6). 


\subsubsection{The percentage of soluble iron}

\subsubsection{Variability in the percentage of soluble Fe in successive leaches}

The percentage of soluble Fe in the three MQ water leaches rendered a greater percentage of Fe than the snow melt leach, on average $60 \%$ more. As shown in Fig. 3.19 the cumulative percentage of soluble Fe from all four leaches was mainly derived from Fe leached from the ASD surface of the $0.4-10 \mu \mathrm{m}$ ASD fraction during successive MQ water leaches (percentage of soluble $\mathrm{Fe}$ ) rather than the instantaneous bulk ASD leach (the percentage of instantaneous soluble $\mathrm{Fe}$ ).

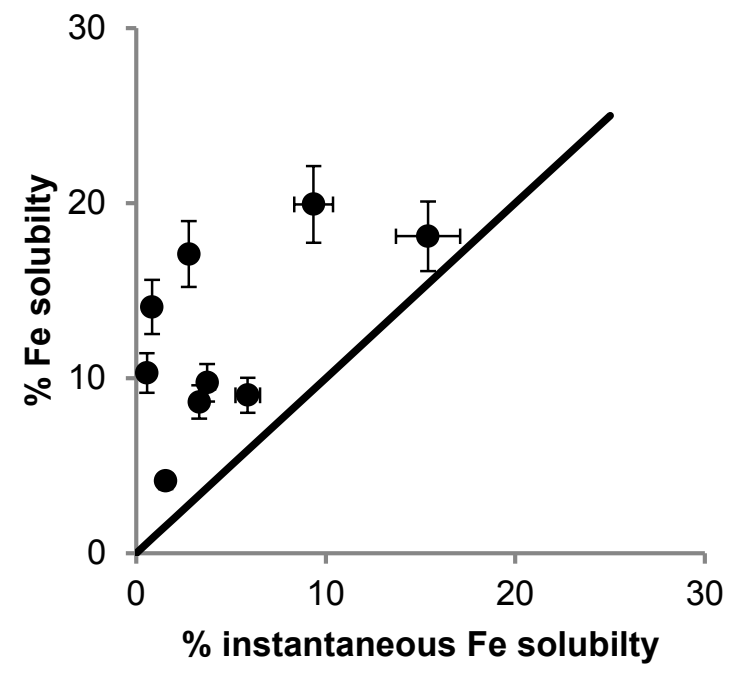

Fig. 3.19: Percentage of instantaneous soluble Fe (particles $<0.4 \mu \mathrm{m}$ ) as a function of the percentage of soluble Fe for samples from McMurdo Sound, and a 1:1 line.

The dissolution of Fe is largely complete after two leaches (2 hours) for Marble Point, Southern McMurdo Sound, Granite Harbour and ultra-fine particle-sized ATD (as shown by the percentage of cumulative soluble Fe plateauing after the second leach in Fig. 3.20). In contrast, dissolution continues in the natural samples of WB1 2004 storm layer and Cape Roberts ASD beyond the fourth leach (4 hours). It is interesting to note that ATD samples continued leaching Fe beyond 4 hours for at least a month and but at a slower rate (Fig. 3.21). 


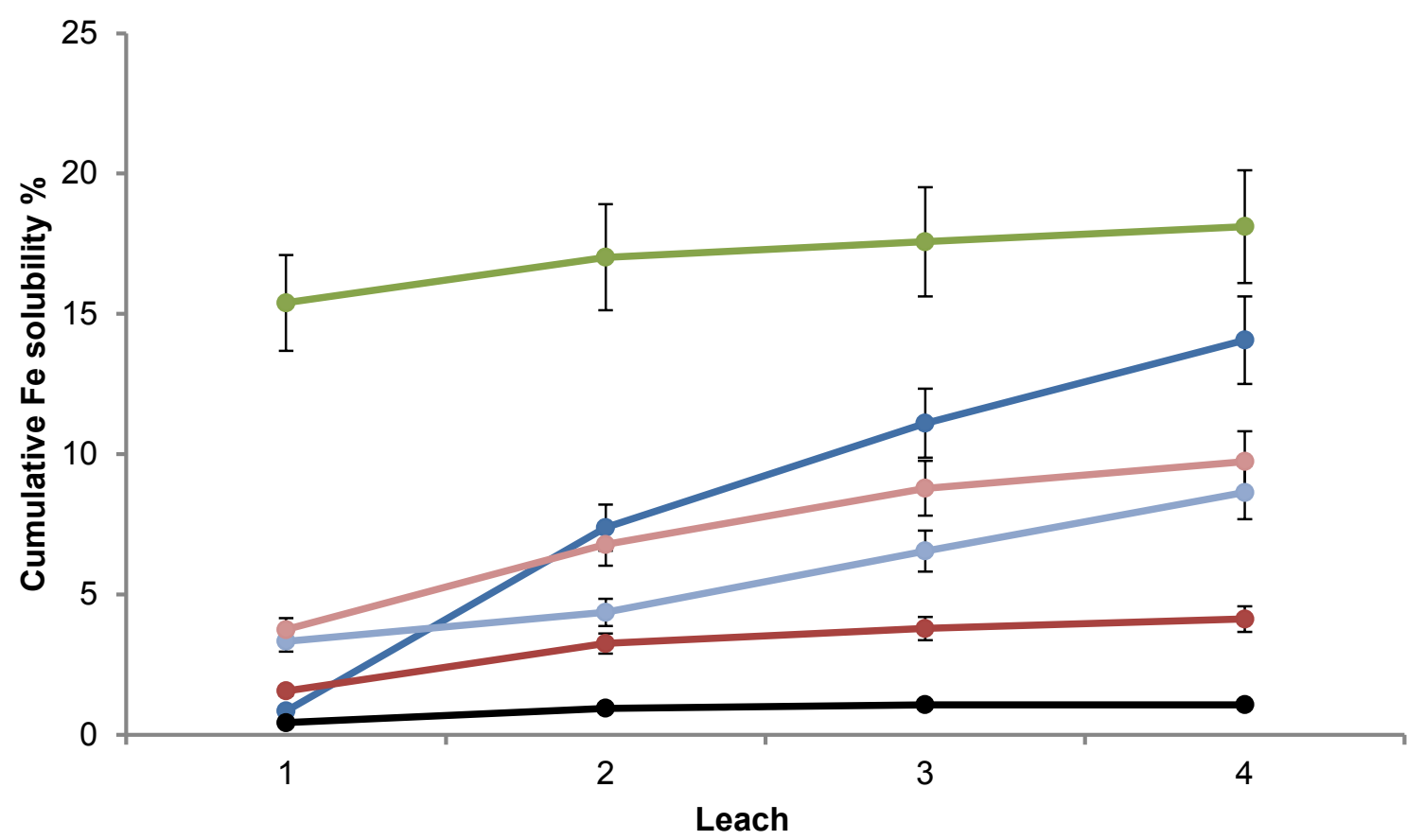

Fig. 3.20: The cumulative percentage of soluble Fe for successive leaches. Leach 1: snow melt leach for bulk sample; leaches 2-4: 1 hour MQ water leaches for fine ASD fraction. Green: Marble Point; Pink: Granite Harbour; Red: Southern McMurdo Sound; Dark blue: WB 2004; Light blue: Cape Roberts; Black ATD ultra-fine reference ( $t=1$ hour, MQ water leach). Error bars are $11.092 \mathrm{2SE} \%$ calculated from the reproducibility of ATD tests (Chapter 3.2.3.6). Location of samples in Fig. 3.15.

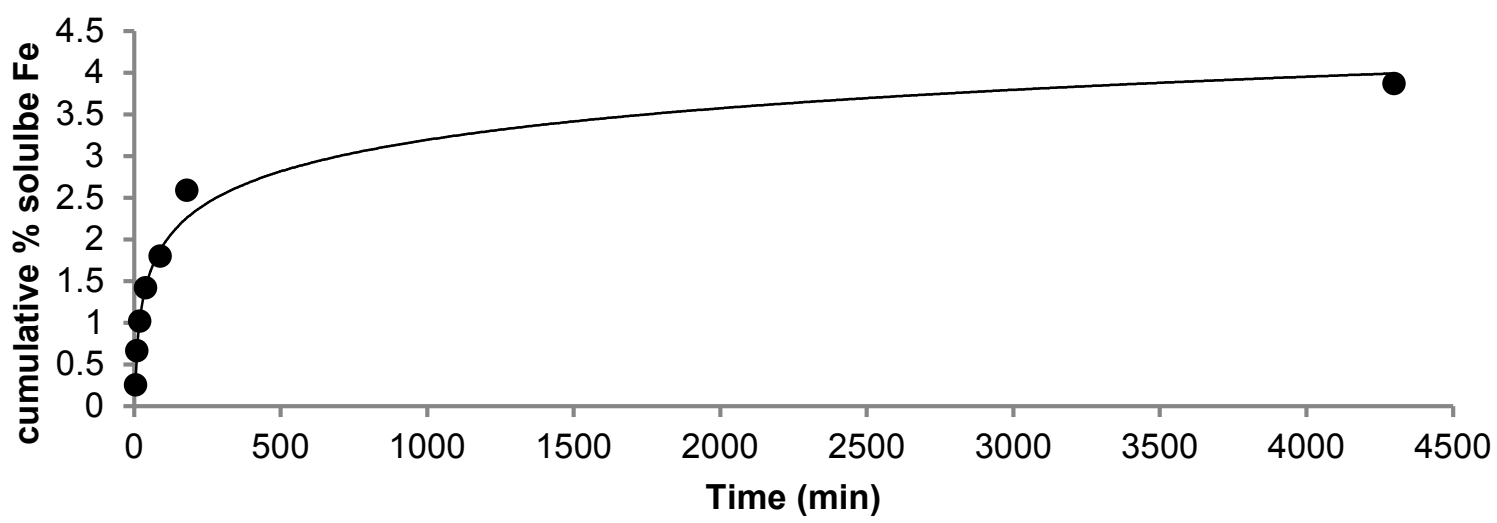

Fig. 3.21: The percentage of cumulative Fe solubility for Arizona test dust leaching at various time intervals.

\subsubsection{Homogeneity of the cumulative percentage of soluble Fe}

The cumulative percentage of soluble Fe in McMurdo Sound ASD ranges from 4.12 to $19.92 \%$ and averages $12.33 \%$ (Fig. 3.22). The duplicate samples collected at Granite Harbour yielded similar values of cumulative percentage of soluble Fe (9.73 and $10.29 \%$ ), which highlights 
the reproducibility of the method. In addition, the sample capturing the 2004 ASD storm has a high Fe solubility of $14.07 \%$. The percentage of soluble Fe is highest at Marble Point and Windless Bight (17.3 $\pm 1.43 \%$ and $14.07 \%$ respectively) and lowest directly under the ASD plume in Southern McMurdo Sound (4.12\%). Cape Roberts and Granite Harbour samples render the similar cumulative percentage of soluble $\mathrm{Fe}(9.27 \pm 0.7 \%)$. The particulate $\mathrm{Fe}$ concentration and the percentage of soluble Fe along transect XY shows limited variability compared to the cumulative soluble Fe concentration and ASD accumulation rate.

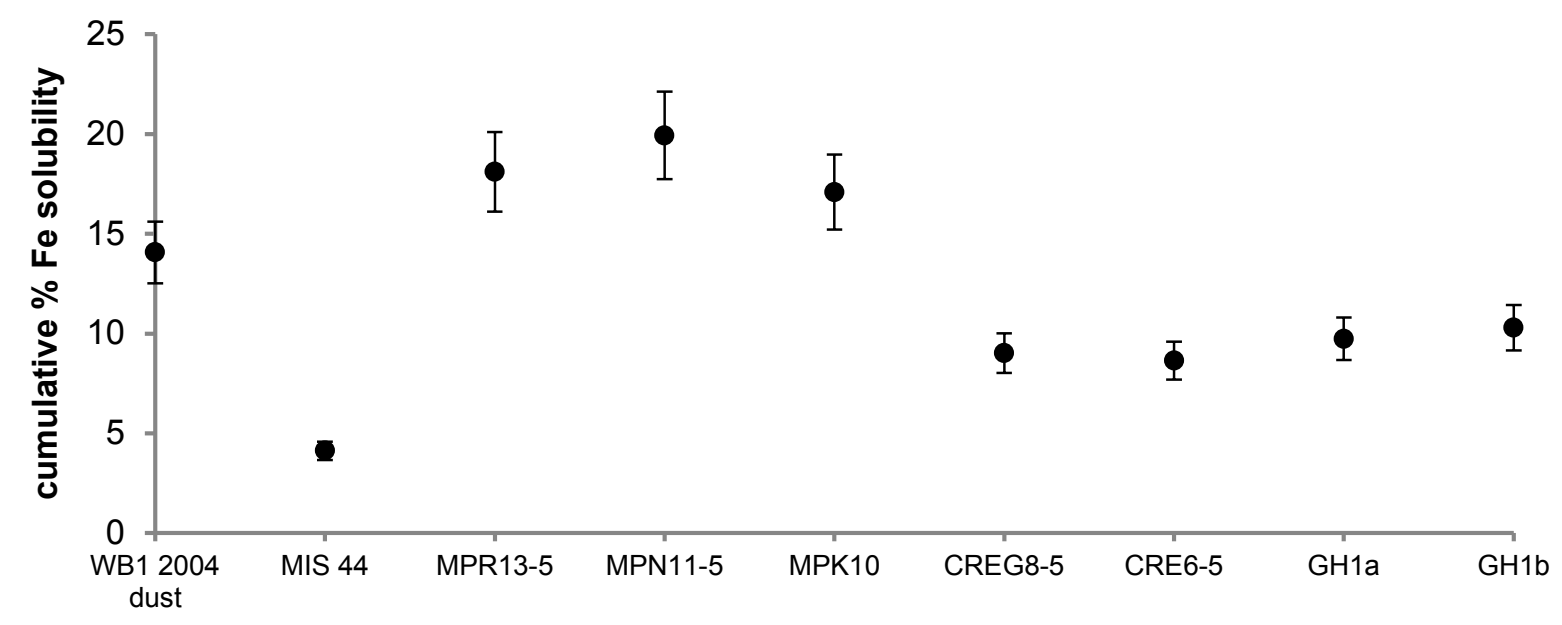

Fig. 3.22: Cumulative percentage of soluble Fe in McMurdo Sound ASD samples. Error bars are 11.09 2 SE\% calculated from the reproducibility of ATD tests (Chapter 3.2.3.6). Location of samples in Fig. 3.15.

Fig. 3.23 illustrates the percentage of instantaneous soluble Fe derived from the snow melt leach alone. Two samples collected at Marble Point have anomalously high Fe concentrations (circled in Fig. 3.23). Excluding these samples, the percentage of instantaneous soluble Fe averages $2.70 \%$ which is significantly less than the average percentage of cumulative soluble Fe (12.33\%). This highlights the significance of the subsequent MQ water leaches rendering a higher percentage of the potential soluble Fe from the ASD surface of the fine fraction. 


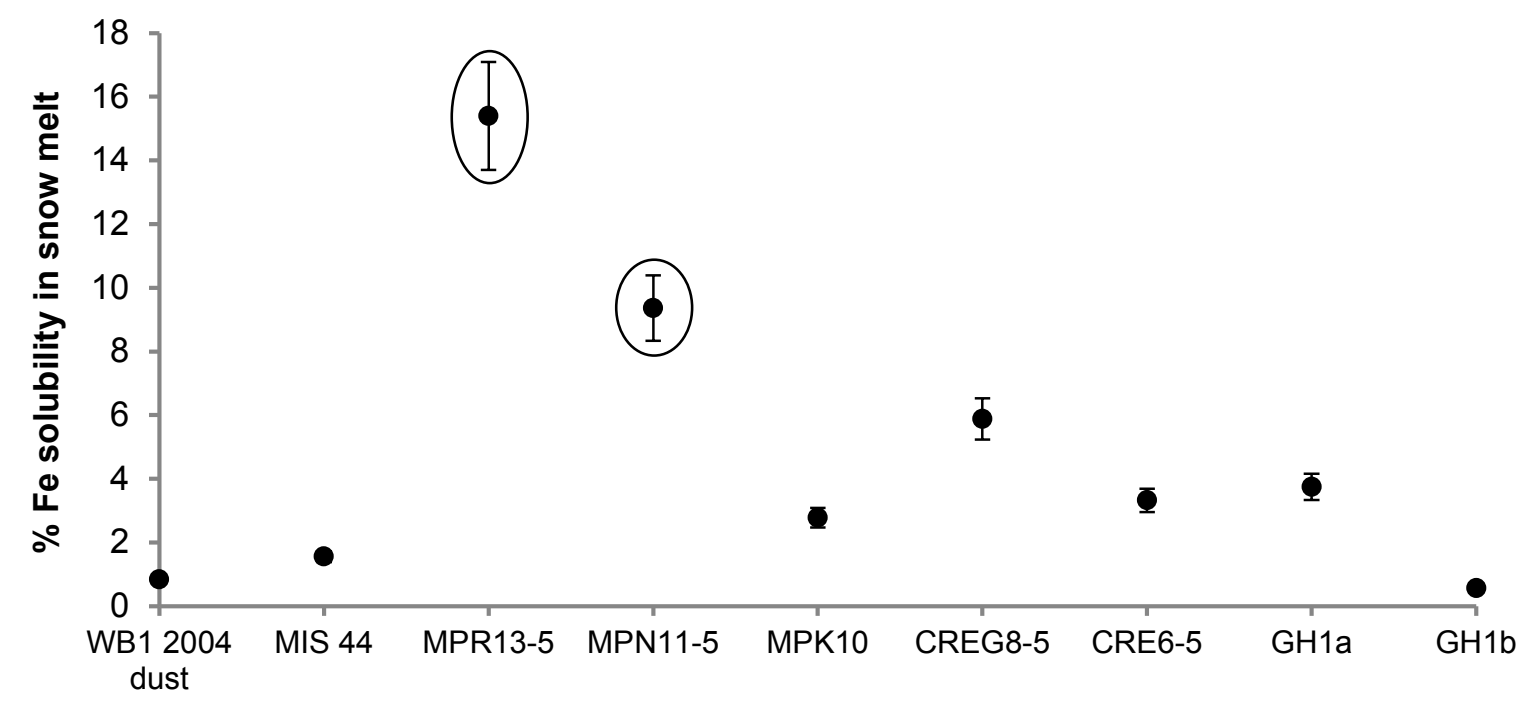

Fig. 3.23: Percentage of soluble Fe in snow melt (leaching the bulk ASD sample). Error bars are 11.09 2 SE\% calculated from the reproducibility of ATD tests (Chapter 3.2.3.6). Location of samples in Fig. 3.15. Circled samples have anomalously high percentage of soluble $\mathrm{Fe}$. 


\subsection{Discussion - particulate and soluble aeolian iron}

\subsubsection{Particulate iron concentration in lithogenic particles}

From the results above, it can be concluded that particulate Fe in McMurdo Sound ASD is relatively uniform in terms of i) the particulate $\mathrm{Fe}$ concentration with distance from the source, which is consistent with the ASD originating from a homogenous source (the debris band), and ii) the particulate Fe concentration of the fine and coarse size fractions (Fig. 3.17) in which $\mathrm{Fe}$ is homogenously distributed throughout both size fractions indicating that there is no fractionation by particle size during transport.

Samples immediately downwind of the debris band (MIS44) and ASD from the 2004 storm show maximal total Fe concentrations (6.97 and $5.81 \mathrm{wt} \%$ respectively) proximal to the debris bands, which are consistent with Atkins and Dunbar (2009) who report a total of 6.99 wt $\% \mathrm{Fe}$ for ASD in surface snow on sea ice close to the debris bands. From Marble Point to Granite Harbour particulate Fe concentration is constant and averages 3.84 wt \%. Thus ASD downwind from the debris band has a homogenous Fe composition associated with a mixture of mainly MVG rocks with a small contribution from crustal TAM and the uniformity along transect $\mathrm{XY}$ is consistent with a single provenance as shown by: i) the presence of volcanic glass in each sample, and ii) the local meteorology of the region (Chapter 2.4.2).

There are two exceptions to the homogenous trend of particulate Fe along transect XY:

1) MPK10 and CREG8 have anomalously high Fe content in the fine fraction, which is inconsistent with all other samples having similar Fe concentrations in both size fractions. However, it is interesting to note that the surrounding site at Marble Point experiences 'heavy traffic' due to the Marble Point Road and the field station located there. It is possible that this sample could be contaminated by anthropogenic sources. Recent studies have argued that aerosols derived from the combustion of organic material yield significantly higher Fe percent solubility than aerosols derived from aluminosilicate materials (e.g. Chuang et al., 2005; Sedwick et al., 2007).

2) The duplicate sample from Granite Harbour also has high Fe concentrations in both size fractions. Air mass back trajectory modelling in the present study (Fig. 2.45) and 
in others (e.g. Sinclair et al., 2010; Markle, 2011) as well as ASW data (Figs. 2.1 and 2.4) suggests that particulates captured in snow samples in Southern McMurdo Sound and Windless Bight encounter southerly local air masses during the strongest winds. In contrast to particulates recovered from Granite Harbour which have an additional ASD supply from westerly katabatic winds, transporting ASD originating in the TAM (confirmed by $\mathrm{Sr}$ and $\mathrm{Nd}$ isotopes from Granite Harbour matching PSA rocks from SVL; Chapter 2.4.2.2). TAM rocks however, have lower Fe content than rocks from the MVG, so the mixing of these two sources (Fig. 2.42) is an unlikely explanation for the high particulate Fe in the GH1b duplicate sample, and it is more likely explained by the small ASD mass $(0.4 \mathrm{mg})$ which is dependent on one or two single particles and not necessarily representative of the broader area. For this reason, the particulate Fe concentration might be somewhat biased by the representation of a few Fe-rich (e.g. hornblende) compared to Fe-poor (e.g. calcite) particles. The possibility of an additional particulate source causing the exceptionally high Fe concentration has been excluded (Chapter 2.4.2).

\subsubsection{Soluble iron}

\subsubsection{Soluble iron in snow melt and subsequent MQ water leaches}

The data collected in the present study suggest that instantaneous soluble $\mathrm{Fe}$ is an important mechanism for providing Fe to the surface waters. The leachates from all samples attain a maximal soluble Fe concentration within the first leach i.e. the snow melt leach (Fig. 3.18). Between $61 \%$ and $94 \%$ of the cumulative soluble Fe in each sample is released in the first leach, $10 \pm 8 \%$ in the second leach, $9 \pm 7 \%$ in the third leach and $6 \pm 7 \%$ in the fourth leach. This suggests that even coarse-sized particles (Chapter 2.2.2.2) with high settling velocities in water, although they will only reside in surface waters for a short period of time (for example, a particle with a diameter of $60 \mu \mathrm{m}$ has a settling velocity of $0.22 \mathrm{~cm} \mathrm{~s}^{-1}$ (Gibbs et al., 1971) i.e. will fall through the mixed layer in McMurdo Sound of $20 \mathrm{~m}$ in 2.5 hours) also contribute a significant amount of the soluble Fe portion from dust to the ocean. Furthermore, fine particles with a longer oceanic residence time will continue to leach Fe from the dust surface for at least 1 month (indicated by ATD leaching experiments in Fig. 3.21) and most likely longer (c.f. Edwards and Sedwick, 2001) in surface waters which is the length of time that 
McMurdo Sound and the SW Ross Sea have open water conditions, given that particles $<10$ $\mu \mathrm{m}$ have low settling velocities and are suspended in the water column. The residence time of Fe in the water column is estimated to be 0.1 - 1.3 years (Moore et al., 2004; Fan et al., 2006), so that it is likely that fine particles in the water column will continue to leach Fe at the constant rate past the duration of 2 - $4 \mathrm{MQ}$ water leaches (4 hours) and considerably longer.

The instantaneous soluble Fe concentration includes soluble Fe present in the snow pack, colloidal Fe, particles of $\mathrm{Fe}<0.4 \mu \mathrm{m}$ and Fe leached from the bulk ASD surface during snow melt. It is interesting to note that melted snow from an area in the Ross Sea distal to an ASD source (Roosevelt Island) rendered soluble Fe concentrations below the ICP-MS instrumental detection limit (i.e. $<10 \mathrm{ppt}$ ), indicating that background concentrations of $\mathrm{Fe}$ in snow are negligible for non-local (hemispherical or global) sources and do not contribute to the relatively high soluble Fe measured in the snow melt. This Fe must therefore be supplied by colloidal Fe, Fe particles $<0.4 \mu \mathrm{m}$ and Fe leached from the bulk ASD surface during snow melt. Further evidence for this comes from external measurements of soluble Fe in melted snow from Granite Harbour collected during the same season as the field work in the present study. These independent measurements also rendered relatively high Fe concentrations (Dr A. Aguilar-Islas, personal comm., 2011), implying there is a large mass of ASD within the snow pack contributing to the high Fe concentrations observed.

The lower Fe concentration exhibited by leachates following the instantaneous soluble $\mathrm{Fe}$ peak is most likely due to the slower release of Fe from the ASD surface (particles $0.4 \mu \mathrm{m}$ $10 \mu \mathrm{m})$ and the lack of colloidal and particulate $\mathrm{Fe}<0.4 \mu \mathrm{m}$. MQ water leaches following the snow melt leach in natural samples are expected to plateau around a constant concentration of $0.02-0.14 \mu \mathrm{mol} \mathrm{L}{ }^{-1}$ from the second leach onwards as Fe leaching tests using ATD (Fig. 3.14) show there is an initial exponential decrease in Fe leached in successive MQ water leaches but a plateau of soluble Fe concentration is reached by the second and third leach. This is supported by Boyd et al. (2010) who show that the result of aeolian Fe supplied to surface waters is an initial rapid release of Fe followed by a slow and sustained release of $\mathrm{Fe}$ while particles reside in the surface mixed layer. This may result in nature in small increases in the dissolved Fe mixed-layer in seawater. 


\subsubsection{Soluble iron along transect $X Y$}

As the mineral composition of each sample is similar (Fig. 3.17), the concentration of soluble Fe in the samples decreases from the debris bands (Fig. 3.18) due to a decrease in the mass of ASD leached $\left(\mathrm{R}^{2}=0.89\right.$; Fig. 3.24) i.e. the particulate Fe concentration and the cumulative percentage of soluble $\mathrm{Fe}$ along transect $\mathrm{XY}$ shows limited variability compared to the cumulative soluble Fe concentration and the ASD mass accumulation rate. The GH1b duplicate sample (sampled from a 'dirty' snow patch) deviates from the exponential decreasing trend in ASD mass. However, the high ASD accumulation rate for this sample also produces a proportionally high soluble Fe concentration, thus not masking the relationship between ASD mass and soluble Fe concentration. Therefore, the variability in cumulative soluble Fe is attributable to variations in ASD accumulation rate (Fig. 3.24), rather than the variability in particulate Fe concentrations.

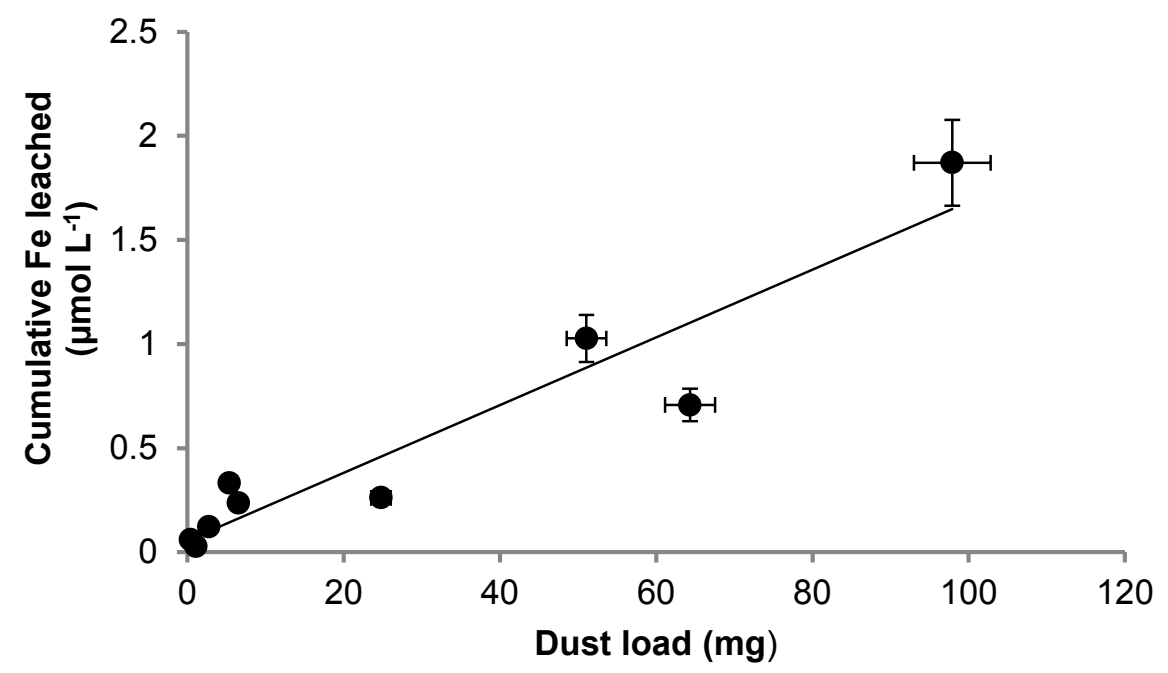

Fig. 3.24: Sand and dust load versus soluble Fe concentration leached from McMurdo Sound ASD samples $\left(\mathbf{R}^{2}=\mathbf{0 . 8 9}\right)$.

\subsubsection{The percentage of soluble iron}

The percentage of soluble Fe of samples from Cape Roberts and Granite Harbour exhibit the lowest variability and average $9.27 \pm 0.7 \%$ (Fig. 3.22). For this reason and also due to the possibility of contamination at Marble Point and the relatively high dust accumulation rate at Southern McMurdo Sound, this conservative average is take to represent sea ice on McMurdo Sound within the area $120 \mathrm{~km}$ north of the debris band. 
Determining soluble Fe from ASD leaching experiments is a complex process and while studies have compared Fe solubility for different leaching solutions (e.g. Aguliar-Islas et al., 2010), to date Fe solubility values have not been evaluated when leached with Ross Sea precipitation. It is therefore difficult to assess the relative importance of particles $<0.4 \mu \mathrm{m}$ or colloidal Fe versus soluble Fe derived from the surface of ASD during snow melt as the leaching behaviour and capability/strength of Antarctic precipitation is unknown in contributing to the soluble Fe values reported here.

\subsubsection{Comparison to published estimates of mineral dust iron solubility}

Cumulative Fe solubility percentages for McMurdo Sound ASD are relatively high (4 to $20 \%$ ) but are within the range of published mineral dust estimates, generally between $1-10 \%$ (e.g. Baker and Croot, 2010). While there are no published values for the percentage of soluble Fe from ASD in McMurdo Sound or the Ross Sea, a comparison can be made between McMurdo Sound ASD and values for dust transported to Antarctica from distal sources. It is believed that most trace metals in Antarctic snow and ice samples are rendered soluble under mildly acidic conditions (e.g. Ng and Patterson, 1981; Boutron and Patterson, 1983; Dick and Peel, 1985; Dick, 1991; Barbante et al., 1997). While Fe solubility estimates for Antarctic dust are scarce, Gaspari et al. (2006) suggests that the acid leachable fraction of $\mathrm{Fe}$ in dust was $60 \%$ of the total Fe mass in the glacial dust samples from the last Glacial Maximum (LGM), which is twice that found in Holocene samples. Edwards and Sedwick (2001) also report high Fe solubility ranging from 9 - $90 \%$ for present day dust leached in East Antarctic snow. Although the authors acidified the dust during leaching enhancing the Fe solubility, the percentage of cumulative soluble Fe in the present study is comparable to part of the lower range estimated for Antarctic dust by Edwards and Sedwick (2001). This reinforces the need for a standard leaching method to be used by all researchers. Furthermore, the cumulative percentage of soluble Fe values in the present study is within those modelled values for the SO ranging between $10-100 \%$ (Fig. 3.6) by Baker and Croot (2010). While it is difficult to compare local ASD from McMurdo Sound to dust that has travelled thousands of kilometres during which it undergoes significant atmospheric processing, there are similarities in the exposure of the dust depositional environment which could enhance its solubility and thus be explanations for the relatively high cumulative percentage of soluble $\mathrm{Fe}$ observed in the present study, in addition to the reasons derived from the leaching method. 


\subsubsection{Explanations for the relatively high percentage of soluble iron}

\section{Snow, light penetration and photo-chemical reactions}

It has been shown that high photo-reduction rates of $\mathrm{Fe}(\mathrm{III})$ are predominately a consequence of the physical environment, for example incident light, water column stratification and low temperature in the Terra Nova Bay and Ross Sea polynyas in the SW Ross Sea and these processes can greatly impact the rate of supply of bio-available Fe within surface waters (Tagliabue and Arrigo, 2006). It has also been demonstrated that light can penetrate into the porous snow pack and sea ice where diatoms grow both within the sea ice and at the sea iceocean interface in McMurdo Sound, SW Ross Sea (e.g. McMinn et al., 1999). Furthermore, modelling and laboratory light exposure experiments (Chapter 3.1.3.2.1; Fig. 3.5) indicate that long exposure of dust (within the snow pack) to photo-reductive processes could enhance the solubility of particulate Fe.

Given that the length of time ASD experiences photo-reduction while deposited on surface snow in Antarctica is much greater (dust can accumulate in snow for thousands of years; Delmonte et al., 2004) than non glaciated environments due to i) the 24 hour daylight during summer in Antarctica, and ii) the porous nature of snow allowing light to penetrate to great depth (Bunt and Wood, 1964) compared to a terrestrial surface, photo-reduction could be one mechanism that partly explains the relatively high Fe solubility of the ASD in the present study and other studies of Antarctic dust.

The samples in the present study were collected during the same time of the year. However the ASD layer from the 2004 storm resided in the snow pack for at least 4 years and thus encountered greater light exposure before it was sampled. This sample rendered a relatively high cumulative percent of soluble Fe compared to samples collected at Cape Roberts and Granite Harbour which only accumulated over $<1$ year on first-year sea ice. Further work, not achievable within the timeframe of this project, is needed to confirm if Fe solubility is enhanced in ASD that has been accumulating within snow, which could have implications on the Fe flux of local ASD to the SW Ross Sea. 


\section{History of the snow}

Measures and Vink (2001) suggest that Fe solubility might be affected by the process of dust deposition and accumulation on sea ice during the winter months potentially increasing the Fe flux from the source. Given that the composition and source of the ASD is homogenous, the relatively high percentage of soluble Fe could reflect the chemistry and interaction of ASD deposition and the history of the snow. For example, if the snow is wet versus dry, Fe on the surface of the ASD could start leaching before the ice breaks up and enters the ocean. Some leachable metals are likely to percolate into the sea ice as temperatures warm and some melting occurs. Furthermore, the crystal structure of the snow and the temperature of the snow could affect Fe solubility, but future work is needed to evaluate these possibilities.

\section{Methodology - MQ water versus seawater leaching solution}

Experimental artefacts such as i) the organic complexing capacity of the leaching solution (Wu et al., 2007), ii) the $\mathrm{pH}$ of the leaching solution (Spokes and Jickells, 1995; Mackie et al., 2005; Buck et al., 2006; Wu et al., 2007), iii) the adsorption of soluble Fe on container walls (Wu et al., 2007), and iv) the ratio between particle load and leaching solution (Spokes and Jickells, 1996; Bonnet and Guieu, 2004) have been shown to contribute to the variability in the estimates of ASD Fe solubility in seawater.

However, it has been argued that the heterogeneity of seawater does not provide a consistent and reproducible leaching solution, thus complicating comparison of Fe solubility data among different research groups (Buck et al., 2006; Sedwick et al., 2007). Upon contact with dust, the $\mathrm{pH}$ of the leaching solution can be lowered by the acidity of the dust particles. As MQ water is not a buffered solution, MQ water leaches are likely to reach lower $\mathrm{pH}$ values and possibility overestimate the percentage of soluble Fe of dust in seawater. Aguilar-Islas et al. (2010) observed an equal or higher percentage of soluble Fe when using seawater as the leaching solution compared to using MQ water. While the authors used same leaching apparatus as the present study (Fig. 3.8), they used a semi-continuous leaching method over time instead of successive leaches as conducted here. Sedwick et al. (2007) also suggested that solubility of aerosol Fe obtained with their instantaneous MQ water leach method underestimated the true solubility of aerosol Fe. 
These observations suggest that although MQ water has a lower $\mathrm{pH}$ than seawater, MQ water leaches are unlikely to overestimate Fe solubility in seawater (Aguilar-Islas et al., 2010). The present study shows that the MQ water leaches are more aggressive at leaching Fe from ASD than snow leaches, possibly due to the presence of ligands or the $\mathrm{pH}$ of the snow. Thus the validity of using MQ water remains an open question. Ideally a seawater standard would be used, but this adds to the complexities in Fe solubility that are not fully understood at present (see Chapter 3.1.3.1). Therefore, the percentage of soluble Fe obtained from a snow melt leach and subsequent MQ water leaches could indeed be greater than what could be expected in the natural environment of McMurdo Sound and the wider SW Ross Sea area, given the aggressive nature of MQ water as a leaching solution, the availability of ligands in the surface seawater not present in MQ water and the higher $\mathrm{pH}$ of seawater compared to MQ water.

\subsubsection{Factors effecting the percentage of soluble iron in McMurdo Sound}

The source material and atmospheric processing are responsible for the different 'characteristics' (particle size and composition) of ASD samples collected in surface snow. Natural differences in aeolian Fe solubility can exist due to i) particle size (Baker and Jickells, 2006), ii) the type of association between Fe and the solid matrix (Desboeufs et al., 2005); iii) the particle concentration (e.g. Jickells and Spookes, 2001), iv) mineralogy and speciation (e.g. Journet et al., 2008), and v) leaching methodology (see above). Fig. 3.25 illustrates the changes in some of these factors with distance from the ASD source.

\section{Aeolian sand and dust concentration in McMurdo Sound}

A number of studies have observed that Fe solubility is a function of particle concentration, with higher solubility at lower suspended particle concentration (Chapter 3.1.3.2.1). The relatively low percentage of soluble Fe for MIS44 (4.1\%) could be due the extremely high ASD accumulation rate at the site (an order of magnitude greater than samples between Marble Point and Granite Harbour) corresponding to reduced Fe solubility near the ASD source. As other samples have relatively constant ASD masses, the "particle concentration effect" is less applicable to these samples and some other factor must be controlling the variability in the percentage of soluble Fe. With the exception of MIS44, as discussed above, McMurdo Sound ASD does not vary as a function of particle mass (Fig. 3.25), which is also confirmed by ATD tests showing that varying the ASD load between $2-20 \mathrm{mg}$ did not affect the percentage of soluble Fe. 


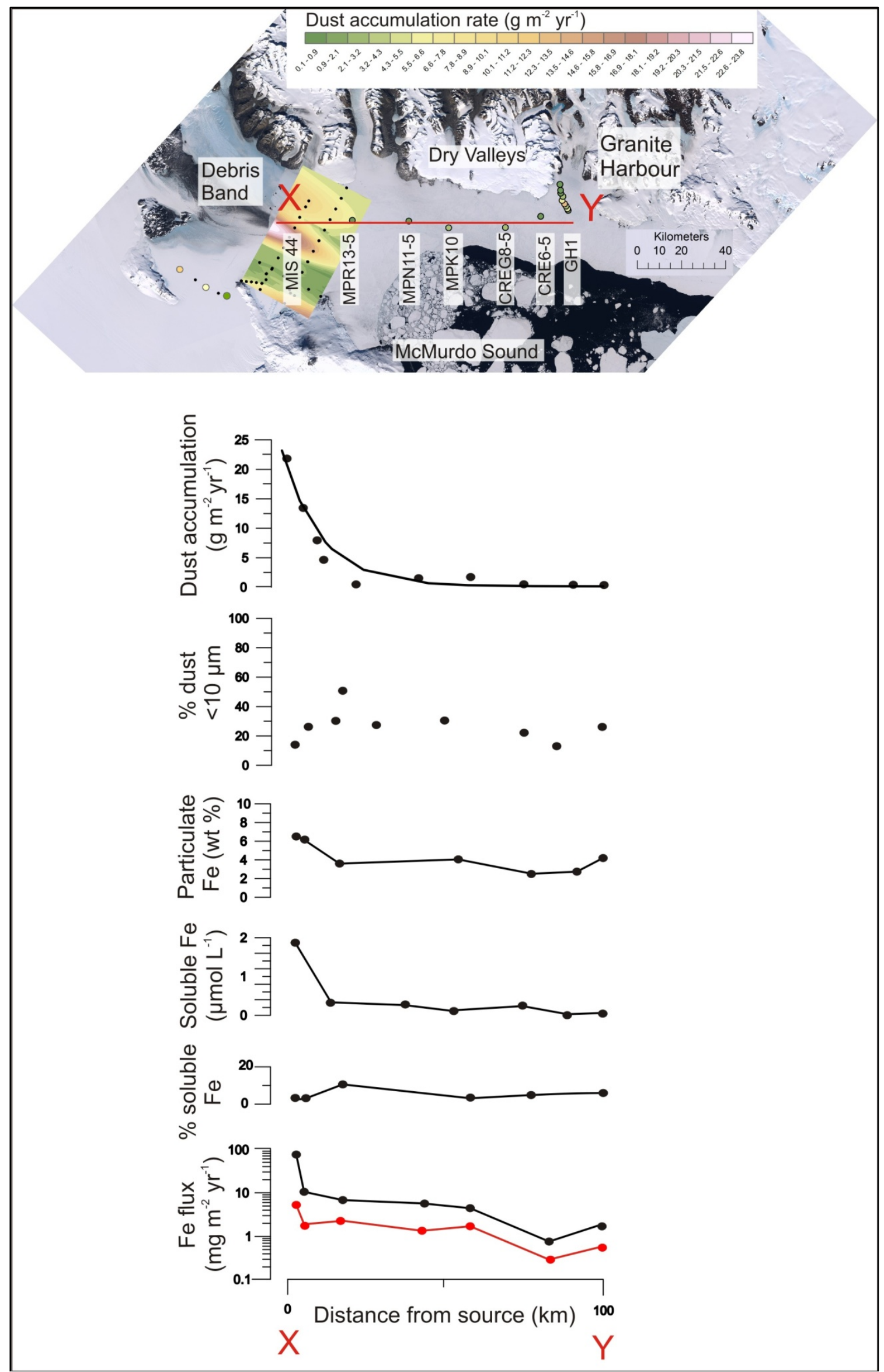

Fig. 3.25: Physical and chemical properties of McMurdo Sound ASD along transect XY from the debris band. Black: bulk ASD fraction, red: fine ASD fraction. 


\section{Particle size of ASD in McMurdo Sound}

The observed trend from field studies often indicates that fine particles have a high percentage of soluble Fe (Chapter 3.1.3.2.1; Chen and Siefert, 2004; Chuang et al., 2005; Baker et al., 2006; Buck et al., 2006; Baker and Jickells, 2006). Despite the lack of consensus in the literature, the percentage of soluble Fe in the present study is not dependent on particle size. It is interesting that Fe solubility was dominated by the soluble Fe in initial leach (Fig. 3.18), consisting of $\mathrm{Fe}$ associated with particles $<0.4 \mu \mathrm{m}$, colloidal size particles ( $\mathrm{Dr} \mathrm{A}$. Aguilar-Islas, personal comm., 2011), or Fe formed in situ from the dissolution process. As snow in the broader Ross Sea region contains Fe concentrations below the ICP-MS instrumental detection limit, this pool of $\mathrm{Fe}$ is likely to be dominated by the $<0.4 \mu \mathrm{m}$ fraction, which is consistent with the findings of Wu et al. (2001), Bergquist et al. (2007) and AguilarIslas et al. (2010), who report that the solubility of aeolian Fe was dominated by the colloidal fraction.

\section{Atmospheric mixing from dust source in McMurdo Sound}

High percentages of soluble Fe have often been associated with low dust concentrations (Chen and Siefert, 2004; Baker et al., 2006). Yet, dust concentrations decrease with transport distance, so that percentage of soluble Fe would appear to increase with particle age in the atmosphere (Fan et al., 2006). Modelling studies (e.g. Fan et al., 2006) and in situ high Fe solubility estimates of dust transported long distances to Antarctica (e.g. Barbante et al., 1997; Edwards and Sedwick, 2001; Gaspari et al., 2006) show the percentage of soluble Fe increases with distance from the source. Furthermore, aerosols collected from different air mass trajectories have been found to render considerably different soluble Fe concentrations (Baker et al., 2006). The different Fe solubility values for Windless Bight and Marble Point compared to Cape Roberts and Granite Harbour (Fig. 3.22) could be an indication of the degree of internal mixing of the two PSAs (MVG and SVL) in the ASD samples collected. However, transect $\mathrm{XY}$ is $120 \mathrm{~km}$ long and hence insufficient to identify significant changes due to atmospheric transport influences. For this reason, it is not possible to distinguish between these two possible sources in this dataset. 


\section{Mineralogy of aeolian sand and dust in McMurdo Sound}

As the ASD is derived from the same source of the debris bands (Chapter 2.4.2), the mineralogy is unlikely to influence the variability in the percentage of soluble Fe (Fig. 3.25). In contrast, the amount of calcium does vary along transect XY and is consistently greater in the bulk sample compared to the fine fraction (Fig. 3.26). The presence of $\mathrm{Ca}$ in the ASD samples is dominantly derived from calcite particles (Chapter 2.2.2.1), and these particles are unlikely to contribute to the relatively high percentage of Fe solubility in McMurdo Sound, but the greater concentration of $\mathrm{Ca}$ particles in a sample could be an indicator for the lower cumulative percentage of soluble $\mathrm{Fe}$, as a greater volume of the ASD mass contains relatively less Fe-bearing minerals and more non Fe-bearing minerals i.e. calcite.

Furthermore, high Fe solubility from anthropogenic aerosols of 3 - 16 \% (Buck et al., 2006; Desboeufs et al., 2005; Aguilar-Islas et al., 2010) has been attributed to anthropogenic carbonaceous particles being more soluble than silicate particles. As McMurdo Sound has regionally insignificant anthropogenic ASD sources, these are also unlikely to contribute to the high Fe solubility observed. Although Pb isotopes from deep central Antarctic ice cores confirm that anthropogenic aerosols are transported to Antarctica (Vallelonga et al., 2002), the mass of these aerosols is insignificant compared to the mass of locally derived ASD.

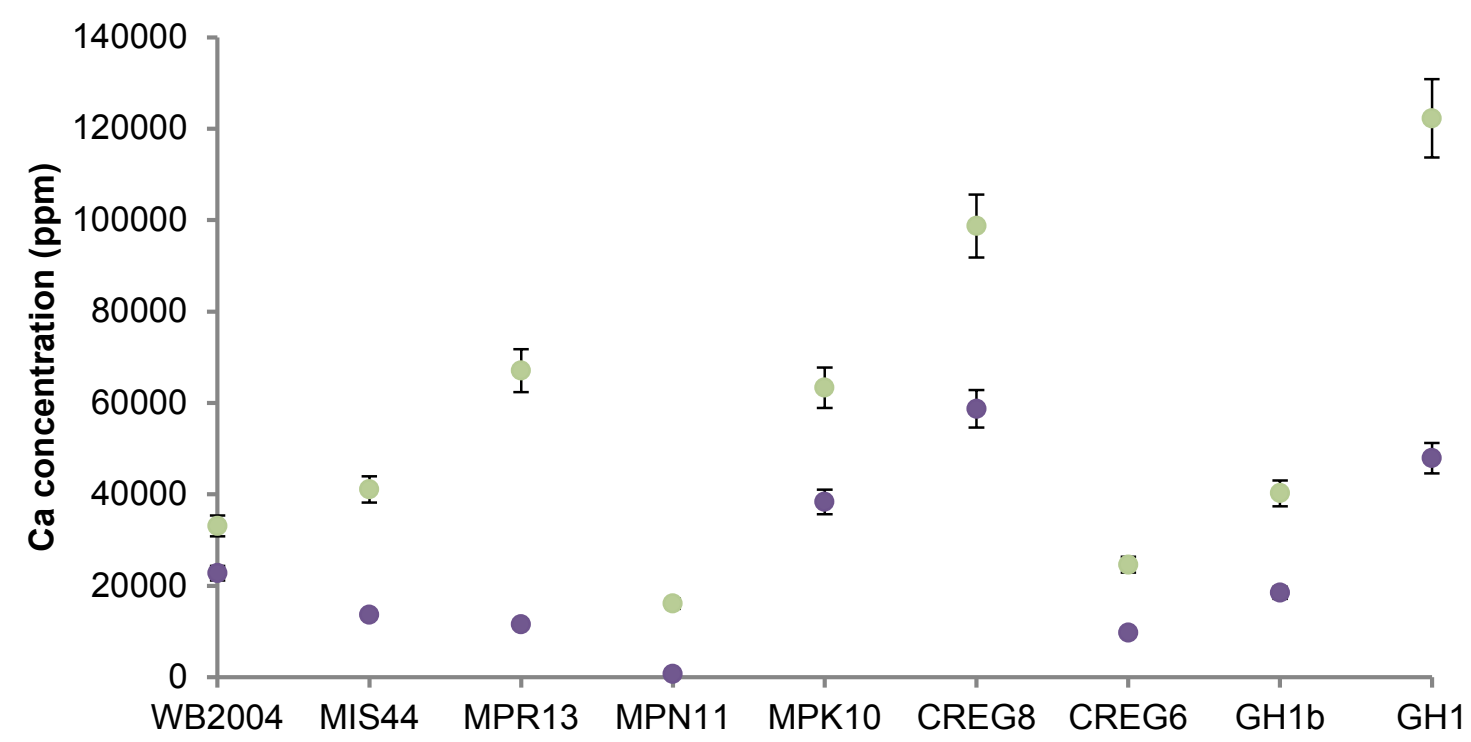

Fig. 3.26: Particulate Ca concentrations in McMurdo Sound ASD samples. Purple: bulk Ca; green: fine Ca. Error bars are the precision $2 \mathrm{Sd} \%$ of the measurements. Location of samples in Fig. 3.15. 


\section{Aeolian sand and dust provenance}

Changes in the aerosol and dust provenance produce large differences in percentage of soluble Fe and would appear to be the major factor controlling the reported differences in aerosol solubility within the literature (e.g. Baker et al., 2006; Scroth et al., 2009). Given the lack of anthropogenic emissions in McMurdo Sound and the negligible influence of particle size and ASD concentration on the percentage of cumulative soluble Fe, it is concluded that because of the homogenous mineralogy and single ASD source, provenance is the dominant factor controlling the homogenous cumulative percentage of soluble Fe of ASD in McMurdo Sound.

\subsubsection{Bio-availability of McMurdo Sound aeolian iron}

The primary interest in accurate estimates of aeolian Fe solubility is the assumption that the bio-availability of aeolian-derived $\mathrm{Fe}$ in the surface ocean is related to its solubility, thereby relating Fe solubility to the carbon cycle (Fig. 3.1B). Whether the soluble Fe in McMurdo Sound is dominated by particles $<10 \mu \mathrm{m}$ or leached from the ASD surface, has important consequences for its bio-availability and subsequent cycling in the water column. There is evidence that particulate Fe can be bio-available (Kraemer, 2005). Although the settling velocity of aggregates in surface waters can provide a pathway of removal of particulate $\mathrm{Fe}$, the instantaneous soluble Fe derived from the first leach provided $76 \pm 14 \%$ of the total soluble Fe indicating that these particles still provide a large proportion of the soluble Fe that is leached from the ASD. Furthermore, according to settling velocities of Gibbs et al. (1971) the fine particulates $(<10 \mu \mathrm{m})$ that reside in the water column for considerably longer periods of time (3-4 days in a mixed layer of $20 \mathrm{~m}$ ) than coarse particles (e.g. a $60 \mu \mathrm{m}$ particle will settle out of a $20 \mathrm{~m}$ mixed layer in only 2.5 hours) continue to leach soluble $\mathrm{Fe}$, although at a slower rate. This contribution of soluble $\mathrm{Fe}$ is on the same time order as other limiting conditions (e.g. light and open water area) available for phytoplankton growth in the Ross Sea. Clearly a better understanding of the biogeochemical cycle of fine Fe particles is required. 


\subsection{Conclusions}

The percentage of soluble Fe of McMurdo Sound ASD $(9.42 \pm 0.70 \%)$ is higher than typical values of mineral dust, but comparable to other estimates of Antarctic dust. The solubility of aeolian $\mathrm{Fe}$ in the leaching experiments was dominated by Fe particles $<0.4 \mu \mathrm{m}$, colloidal $\mathrm{Fe}$ and Fe leached from surface of ASD during snow melt. Successive MQ water leaches were aggressive in leaching Fe from the ASD, and these could overestimate the amount of soluble Fe in seawater.

The relatively high percentage of soluble Fe appears to be location-specific i.e. consistent with other estimates of Fe solubility from dust samples in polar environments, but also influenced by the leaching method. It has been speculated in the present study that photoreduction occurring on the surface of ASD on sea ice for long periods time (due to the 24 hour summer sunlight in Antarctica) which could influence or act as a mechanism to enhance Fe solubility of mineral ASD in Antarctica and other polar regions where the snow and ice is porous allowing sunlight to penetrate into the snow pack.

The single provenance of the ASD is the most likely driver of the constant percentage of soluble Fe (in terms of the difference in the percentage of cumulative soluble Fe being orders of magnitude less than the difference in ASD mass) with increasing distance from the source. 


\section{Chapter 4}

\section{Synthesis: The role of local dust-derived iron on setting the primary production of McMurdo Sound}

\subsection{Introduction}

This synthesis examines the contribution of local ASD to primary productivity in the SW Ross Sea through the following objectives:

1. What is the role of ASD as a source of Fe to the SW Ross Sea?

This is investigated by constructing an aeolian Fe flux model and Fe budget for the region.

2. Does locally-derived Fe stimulate significant primary productivity?

This is quantified by applying the estimates of soluble and particulate Fe and ASD accumulation in McMurdo Sound to the dust-biota model of Boyd et al. (2010).

3. Is there a quantifiable relationship between ASD and primary productivity in SW Ross Sea?

This is evaluated by comparing ASD, chlorophyll- $a$ and methylsuphonate (MS) data for the SW Ross Sea over the last decade.

\subsection{Background}

\subsubsection{Primary production in the Southern Ocean and Ross Sea (also see section 3.1.1)}

The SO is the largest high nutrient low chlorophyll (HNLC) region in the world. HNLC regions are characterised by an abundance of macro-nutrients, such as nitrate, phosphate and silicic acid (Fig. 4.1) and if these nutrients in the upper mixed layer were exhausted by primary productivity, atmospheric $\mathrm{CO}_{2}$ would be reduced by as much as $100 \mathrm{ppm}$ (Peng and Broecker, 1991). However, these macro-nutrients are not fully utilised, resulting in unusually low levels of primary productivity, and hence chlorophyll-a (Arrigo et al., 2008b). Despite generally low phytoplankton abundance and productivity in the SO (Sullivan et al., 1993), intense phytoplankton blooms do develop. $\mathrm{CO}_{2}$ drawdown within these blooms is sufficient 
to maintain a positive $\mathrm{CO}_{2}$ gradient between the ocean surface and the atmosphere, facilitating the influx of $\mathrm{CO}_{2}$ (Sweeney et al., 2000; Louanchi et al., 2001). The proposed environmental factors responsible for controlling the rates of phytoplankton production and incomplete utilisation of inorganic macronutrients include i) grazing (Banse, 1991), ii) temperature (Bunt and Wood, 1963), iii) light availability (Mitchell and Holm-Hansen, 1991; Mitchell et al., 1991), iv) trace metal availability (e.g. Fe; Martin et al., 1990; 1990b; Sedwick and DiTullio, 1997; Sedwick et al., 1997; 2000), or some combination of these (Smith and Nelson, 1986; Arrigo et al., 2000; Boyd, 2002).

A)

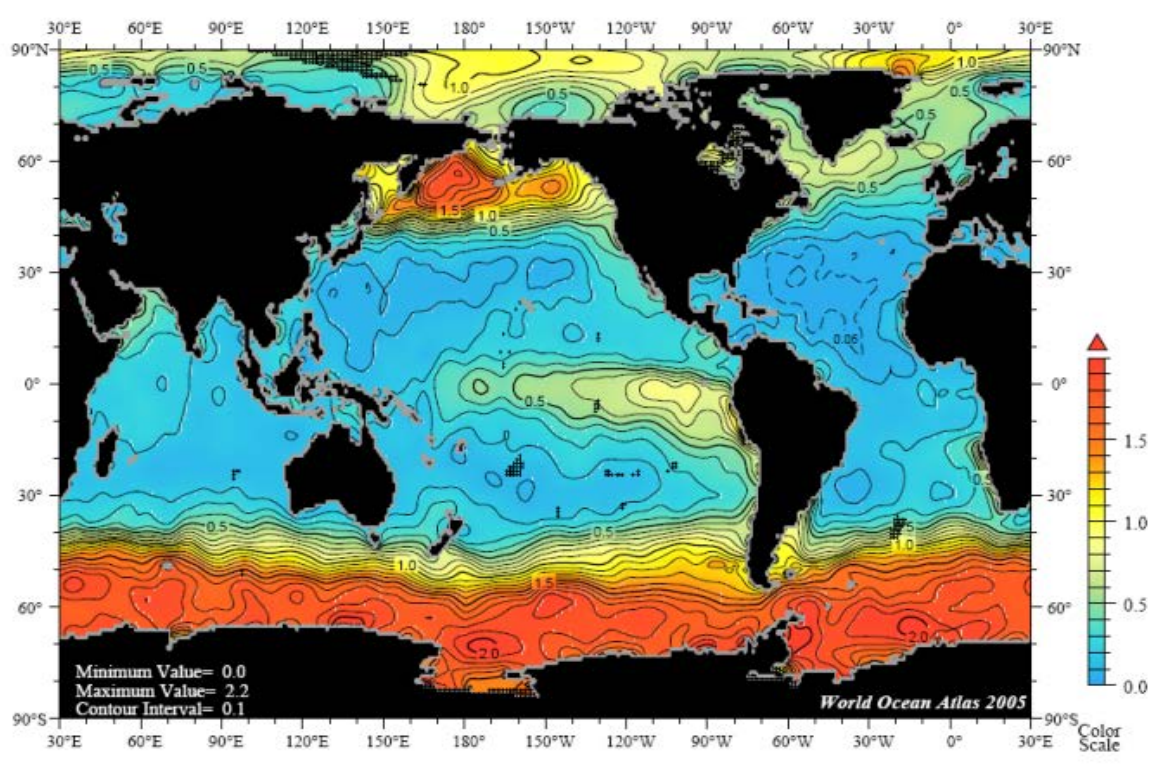

B)

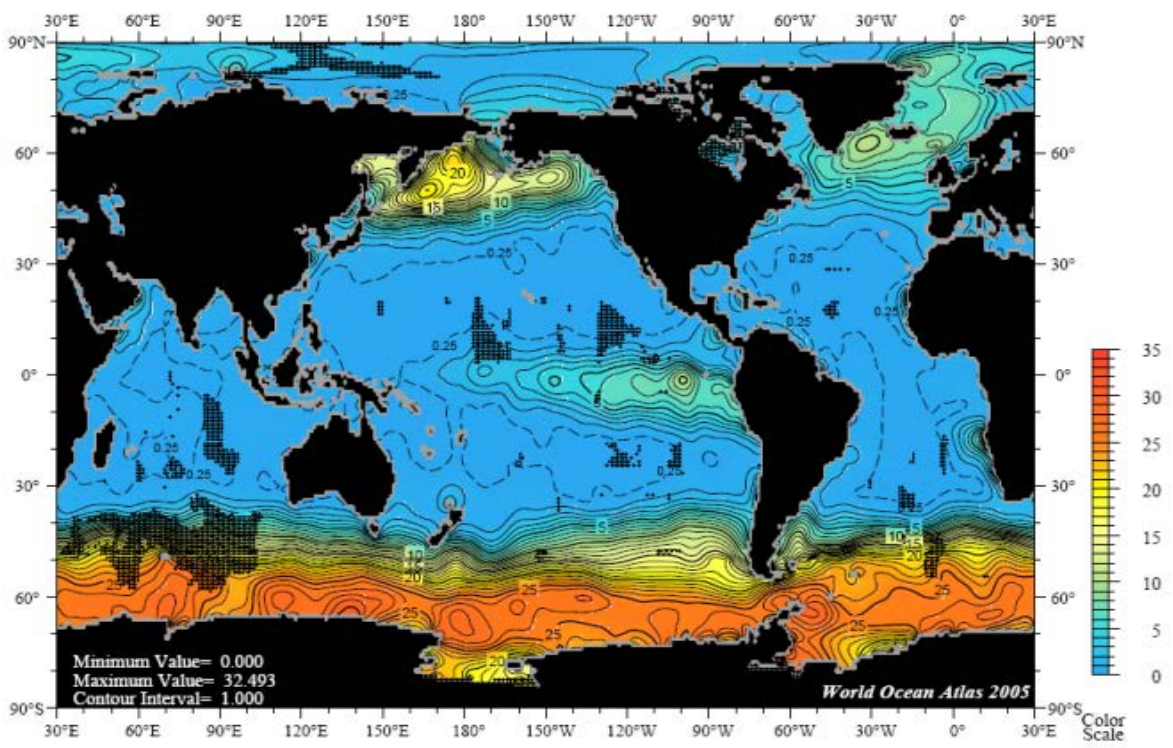

Fig. 4.1: Macro-nutrients in the surface waters of the world's ocean. A): Nitrate ( $\left.\mu \mathrm{mol} \mathrm{L}^{-1}\right)$. B): Phosphate $\left(\mu \mathrm{mol} \mathrm{L}{ }^{-1}\right)$. Source: World Ocean Atlas (2005). 
In contrast to the SO, the Ross Sea is the most biologically productive continental shelf region in Antarctica and is characterised by intense seasonal phytoplankton blooms evident in Fig. 4.2. Rates of primary production in the Ross Sea often exceed $2 \mathrm{~g} \mathrm{C} \mathrm{m}^{-2} \mathrm{~d}^{-1}$ (Arrigo et al., 2005). These high rates result in the accumulation of a large amount of particulate organic carbon and chlorophyll-a by early summer (Fig. 4.2) and a consequent marked reduction in the water column total dissolved inorganic carbon (Tagliabue and Arrigo et al., 2000). As a result surface $p \mathrm{CO}_{2}$ is as low as $120 \mu \mathrm{atm}$ during the peak of a midsummer phytoplankton bloom (Sweeny et al., 2000). Because of its capacity to support intense phytoplankton blooms, the Ross Sea is distinct from the HNLC waters of the SO (Tagliabue and Arrigo et al., 2005).

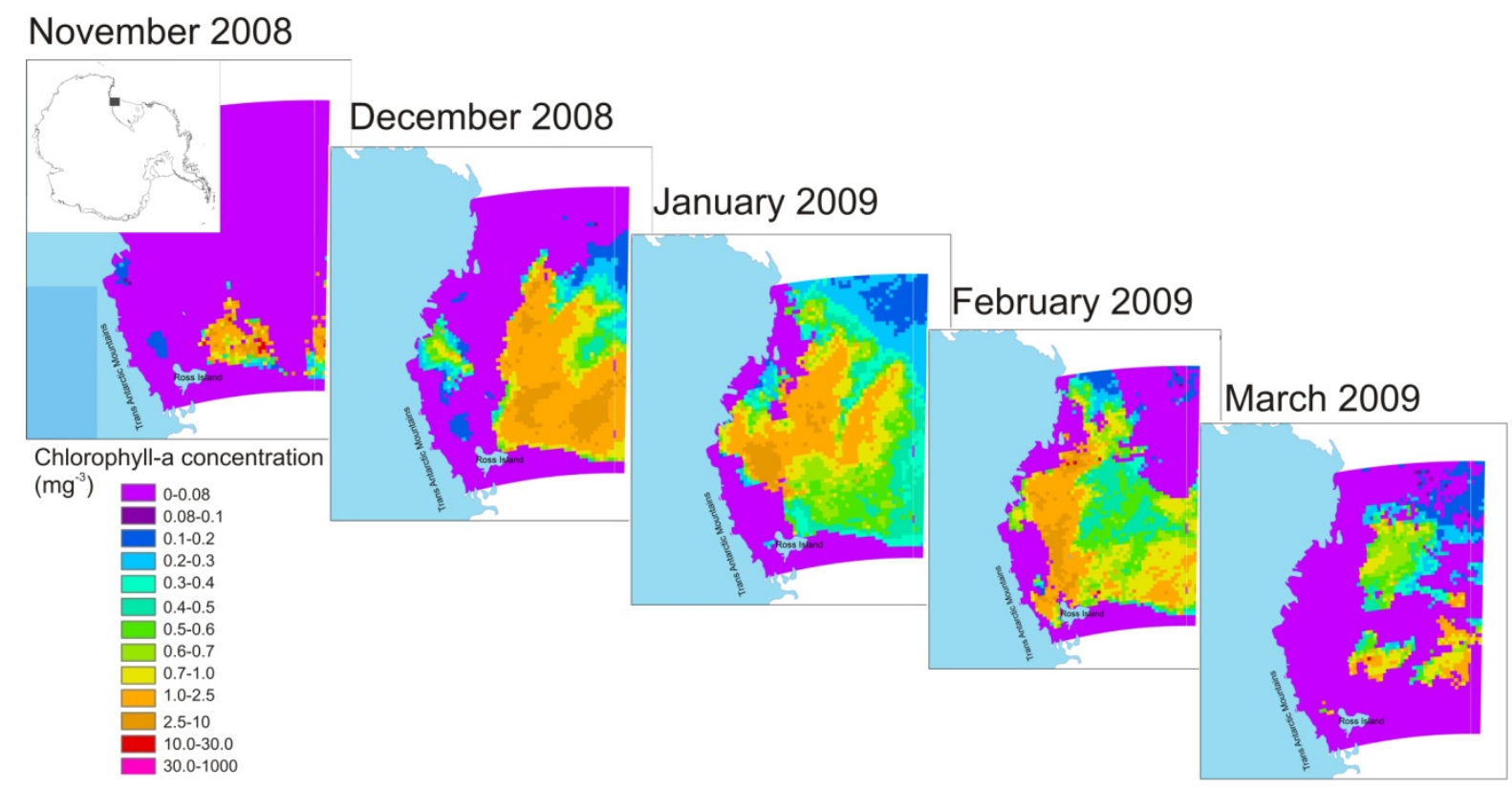

Fig. 4.2: Seasonal evolution of phytoplankton stocks indicated by chlorophyll-a concentration in the SW Ross Sea November 2008 - March 2009. Satellite data derived from SeaWiFS (https://gdata/sci.c.nasa.gov). Insert: location of SW Ross Sea in Antarctica.

Productivity in the Ross Sea is correlated with the areal extent of open water production and sea ice distribution: annual sea ice restricts the length of the phytoplankton growth season and thus limits production (Buesseler et al., 2003; Meguro et al., 2004). As the area of open water increases so does annual production at a rate of approximately 100 - $300 \mathrm{Tg} \mathrm{C}$ for every additional million $\mathrm{km}^{2}$ of open water (Arrigo and van Dijken, 2004). 


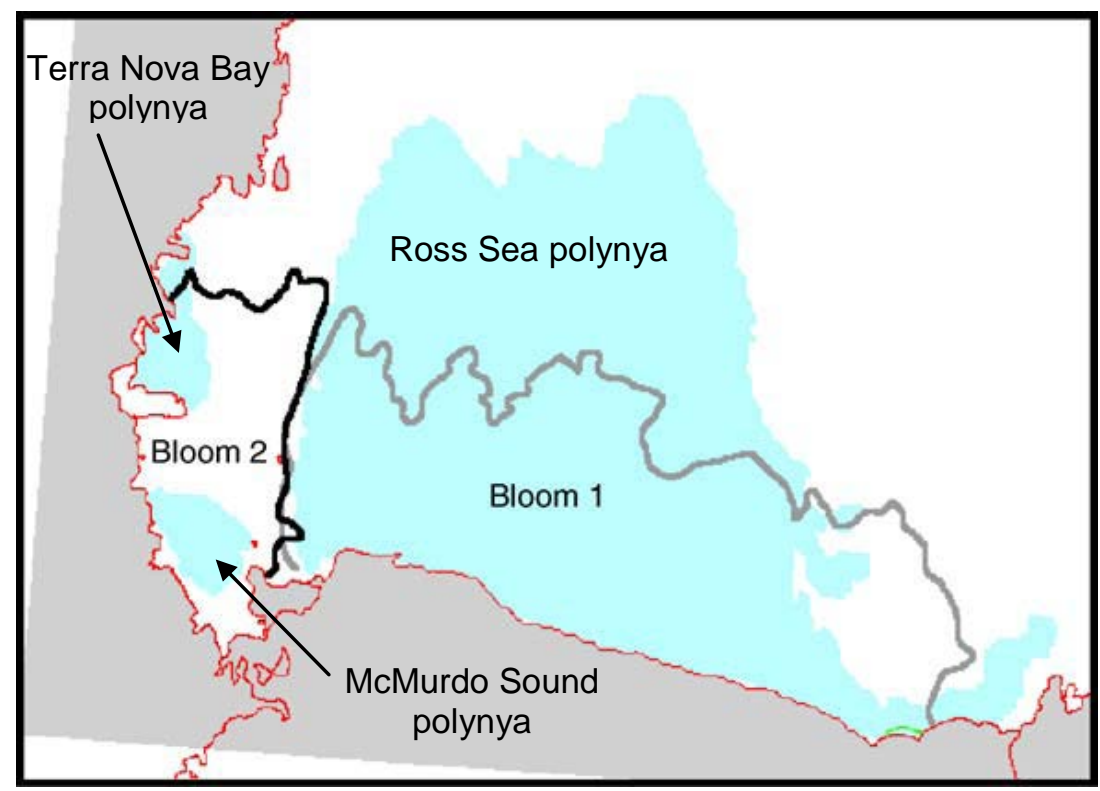

Fig. 4.3: Spatial relationship between the phytoplankton bloom that forms in the Ross Sea polynya (Bloom 1, denoted by gray outline), the later bloom that forms on the western shelf (Bloom 2, denoted by black outline), and the sea ice position at the start of the bloom (white area). Modified from: Arrigo and van Dijken (2004).

\subsubsection{Phytoplankton blooms in the SW Ross Sea}

Spring and summer phytoplankton blooms in the SW Ross Sea can be divided into two categories based on species composition, temporal evolution, hydrographic conditions and sea ice dynamics (Fig. 4.3; Arrigo et al., 2000). The largest blooms are dominated by the nonskeletal photosynthetic algae Phaeocystis antarctica and are located in relatively unstratified waters north of the Ross Ice Shelf (e.g. Bloom 1; Fig. 4.3; Arrigo et al., 2000). Waters associated with the Ross Sea polynya are only weakly stratified because most of the sea ice is advected northward out of the region rather than melting locally (Arrigo et al., 1998a; 2000). This is the first bloom of the annual cycle to develop and terminate, beginning in late October or early November, when sea ice is still abundant in the region and declining in midDecember (Arrigo and McClain, 1994; Arrigo et al., 1998a; Arrigo et al., 2000). The Ross Sea polynya is maintained by katabatic winds (Fig. 4.4; Bromwich et al., 1992) and these winds could also deliver locally derived ASD from outlet glaciers along the TAM to these areas of open water in Terra Nova Bay and McMurdo Sound.

The second bloom develops later in the year beginning in December and January in association with the highly stratified surface waters of the western Ross Sea, including Terra Nova Bay and McMurdo Sound and is dominated by diatom production (Bloom 2; Fig. 4.3; 
Arrigo et al., 2000; Goffart et al., 2000). The high degree of water column stratification is due to the melting of a large amount of sea ice and subsequent release of low-salinity (i.e. low density) water that accumulates along the western margin of the Ross Sea. The delay in the phytoplankton bloom in this region is due to the much stronger springtime winds in Terra Nova Bay (Fig. 4.4) that preclude stratification until January when solar insolation and sea ice meltwater fluxes have increased sufficiently to overcome turbulent mixing at the surface (Arrigo et al., 1998a). There appears to be little spatial or temporal overlap between $P$. antarctica and diatom blooms, suggesting that the observed patterns of species dominance are due to environmental conditions rather than seasonal succession (Arrigo et al., 2000).

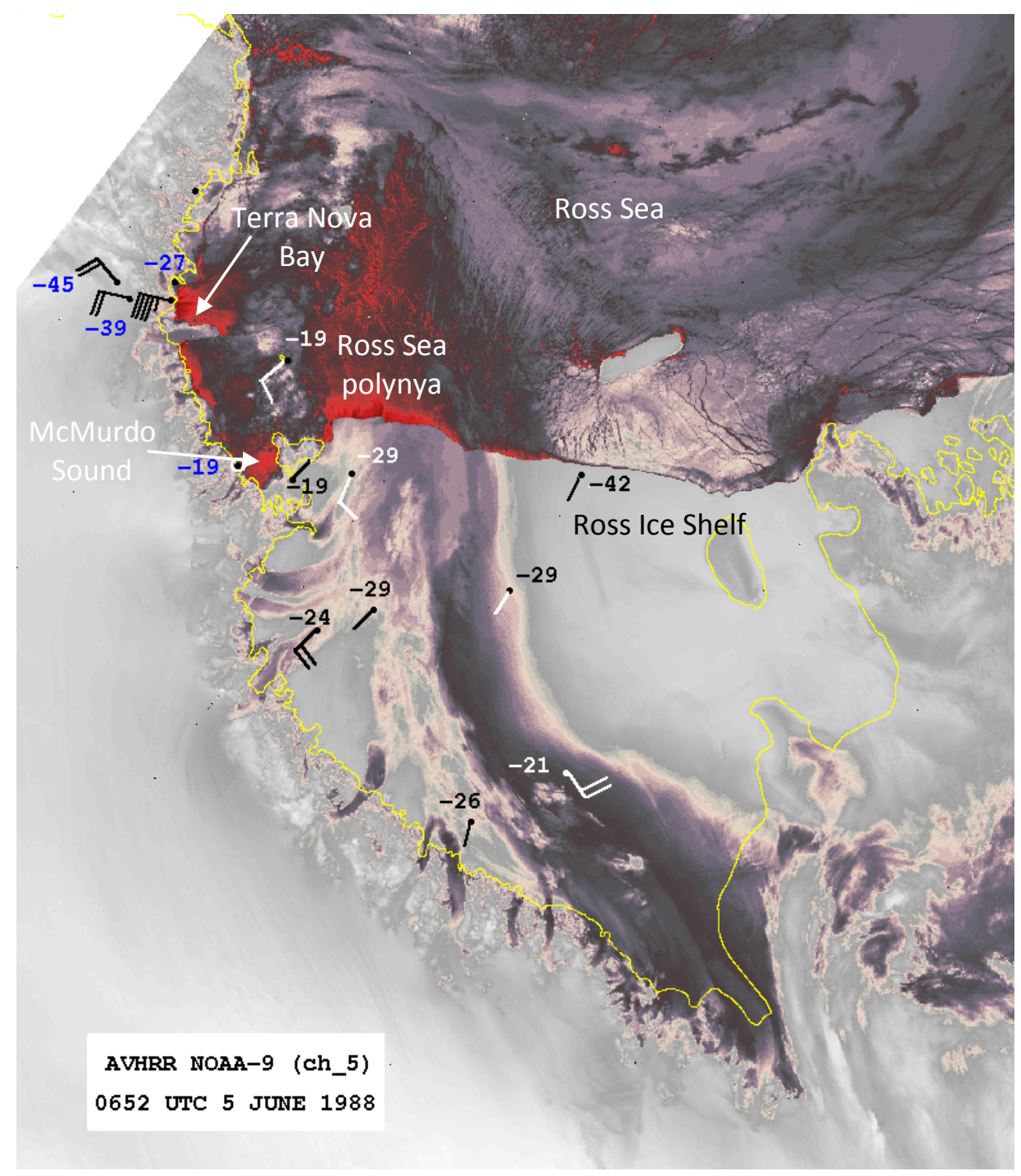

Fig. 4.4: Satellite image showing katabatic outflow of air (dark purple) from major outlet glaciers across the Ross Ice Shelf. Image derived from: http://www.bprc.mps.ohiostate.edu/PolarMet/imagery.html. 


\subsubsection{Environmental drivers of seasonal phytoplankton blooms}

Several environmental drivers of bloom generation each season during sea ice break up in the SW Ross Sea have been proposed:

1. Low salinity water from pack ice melting in spring and summer produces a stable layer at the surface of the ocean which allows phytoplankton to grow in a well illuminated environment (Smith and Nelson, 1986).

2. Ice edge upwelling increases water movement and replenishes nutrients depleted by phytoplankton growth (Arrigo et al., 2005).

3. A decrease in turbulence within the mixed layer is caused by reduced wind stress due to the presence of ice (Broomwich et al., 1992).

4. The release of algae from sea ice (e.g. the diatom Nitzschia curta) into surface waters from melting ice floes seeds productivity. $N$. curta has contributed to siliceous deposits for $\sim 18,000$ years (Tuesdale and Kellogg, 1979) in the western Ross Sea, suggesting that seasonal blooms have persisted throughout the late glacial and Holocene periods (Smith and Nelson, 1986).

More recently it has been suggested that dissolved Fe coupled with sea ice dynamics controls the magnitude of annual primary production within the Ross Sea (Sedwick and DiTullio, 1997; Arrigo et al., 2003; Arrigo and van Dijken, 2004; Sedwick et al., 2011). Evidence from both field and modelling studies shows that annual production in the Ross Sea is limited by Fe supply (Arrigo et al., 1998a; Lancelot et al., 2009; Tagliabue et al., 2009). Maximum dissolved Fe concentrations are found in surface waters of the SW Ross Sea in spring and summer and result from the release of Fe as sea ice melts (Tagliabue and Arrigo, 2006). Similarly high dissolved Fe concentrations are found near the sea floor due to resuspension of Fe-bearing sediments. Iron concentrations increase with depth, averaging $\sim 0.3-0.5 \mathrm{nM}$ at 500 m (Coale at al., 2004; Fizwater et al., 2000). In the Ross Sea polynya, because the sea ice is advected away rather than melting locally (Arrigo et al., 1998a), the primary Fe source is thought to be seasonal upwelling. However, in late December, dissolved Fe in the Ross Sea Polynya is nearly undetectable, leading to the decline of the phytoplankton bloom even though macro-nutrients have not been exhausted (Arrigo et al., 2003; Fitzwater et al., 2000). In Terra Nova Bay and the western shelf marginal ice zone (MIZ), macro-nutrients are still well above growth-limiting concentrations when diatom stocks begin to decline. Here, dissolved Fe concentrations are likely to be greater in the surface waters due to intense sea ice 
melt (Sedwick and DiTullio, 1997). Sedwick and DiTullio (1997) found that Fe derived from melting sea ice and snow can raise Fe concentrations in the upper $\sim 30 \mathrm{~m}$ up to $2 \mathrm{nM}$.

Processes such as dissolved Fe release from melting sea ice and photo-reduction of Fe(III) to Fe(II) may greatly influence the amount of bio-available Fe in surface waters and the role of "sea ice processes" is relatively more important in the MIZ near the Terra Nova Bay polynya compared to the Ross Sea polynya. Tagliabue and Arrigo (2006) suggest that photo-reduction of $\mathrm{Fe}(\mathrm{III})$ is the key process governing the supply of bio-available Fe to phytoplankton in the Terra Nova Bay and Ross Sea polynyas where there is a substantial increase in irradiance resulting from the loss of sea ice and increased stratification as winds subside in spring. The high Fe photo-reduction rates are therefore a consequence predominately of the physical environment (e.g. high light, water stratification, low oceantemperature) and not simply due to additional Fe input from the sea ice melting. 


\subsection{The role of aeolian sand and dust as a source of iron to the SW Ross Sea}

\subsubsection{Introduction}

To advance the understanding of the effect of aeolian Fe deposition on phytoplankton production, it is important to improve estimates of both the Fe flux and solubility of mineral dust entering the surface ocean. Estimates for the SO are few, and there are none for locallyderived dust from McMurdo Sound, nor has it been shown how much this source contributes to the total Fe budget of the Ross Sea. It has long been observed that the breakup of sea ice coincides with seasonal phytoplankton blooms and that the sea ice is an important source of Fe triggering these blooms (Smith and Nelson, 1986; Sedwick and D'Tullio, 1997; Arrigo and van Dijken, 2004). To determine the role of ASD as a source of Fe to the SW Ross Sea in the present study i) a Fe flux dispersal model has been developed for ASD sourced from McMurdo Sound and deposited into the SW Ross Sea, and ii) compared this local aeolian Fe flux to other sources of Fe in Antarctic waters.

\subsubsection{Aeolian Fe-dispersal model}

To quantify the contribution of aeolian Fe to McMurdo Sound sea ice, ASD mass accumulation rates, particulate and soluble Fe values (Chapters 2.2.2.2 and 3.4; Fig. 3.15) are used here. A model for aeolian Fe dispersal in McMurdo Sound is based on the combination of mass accumulation rate and Fe solubility, where a background atmospheric Fe deposition derived from ASD for each sampling site is calculated using the following equation:

Fe flux $=$ ASD accumulation rate $\left(\mathrm{mg} \mathrm{m}^{-2} \mathrm{yr}^{-1}\right) \times$ Fe content $(\mathrm{wt} \%) \times$ Fe solubility (\%)

As ASD in McMurdo Sound has a homogenous composition and the mass accumulation rate drives Fe solubility, the Fe flux to the ocean is largely determined by the supply of ASD. 


\subsubsection{Assumptions of annual ASD deposition into the SW Ross Sea}

Although the following processes may be important they have been ignored in the development of this model for reasons of i) simplicity and ii) because their importance has yet to be quantified:

- first year sea ice breaks up each summer and wind-driven currents advect sea ice some distance northward with melting away from McMurdo Sound i.e. ASD accumulating on sea ice in McMurdo Sound may enter the water column elsewhere and for the purposes of this model, this process has been ignored;

- not all ASD deposited on multi-year sea ice enters the water column each year as there are inter-decadal variations in multi-year sea ice break up in McMurdo Sound, but when considered on longer (decadal) timescales, the contribution of ASD from multi-year ice is assumed to be in proportion to the annual accumulation rate measured at the surface.

However, the present study assumes:

- a "background" ASD accumulation for McMurdo Sound, excluding the 2004 storm, of $0.70 \mathrm{~g} \mathrm{~m}^{-2} \mathrm{yr}^{-1}$ for the $>10 \mathrm{um}$ ASD fraction and $0.08 \mathrm{~g} \mathrm{~m}^{-2} \mathrm{yr}^{-1}$ for the $<10 \mu \mathrm{m}$ fraction for the broader McMurdo Sound area (7400 km²; Fig. 2.26B) and;

- that ASD on first year sea ice in McMurdo Sound is indicative of what is accumulating more broadly in the SW Ross Sea region at least $500 \mathrm{~km}$, and possibly further, from the debris band (Chapter 2.4.3.2) and;

- a decreasing trend in ASD accumulation away from the source i.e. parallel, not perpendicular, to the coast (Chapter 2.4.3.2 and Fig. 2.23).

For the purposes of the present study, the annual Fe flux into the ocean is calculated for an area between 30 and $120 \mathrm{~km}$ from the debris bands (Figs. 4.5 and 4.6), representing a total area of $7400 \mathrm{~km}^{2}$. This area was selected because i) there are observed values for both ASD accumulation rate and Fe content within it; ii) values for ASD and Fe content are relatively constant there (i.e. are likely to be representative of the region); and iii) it covers an area of known seasonal phytoplankton blooms (Fig. 4.5). The simple ASD dispersal model (Figs. 2.43 and 2.44) suggests ASD from the debris bands may still accumulate at rates above the global background up to $500 \mathrm{~km}$ north of McMurdo Sound. This model output is the basis for 
speculation as to the significance of McMurdo Sound ASD and hence Fe flux over the broader SW Ross Sea.

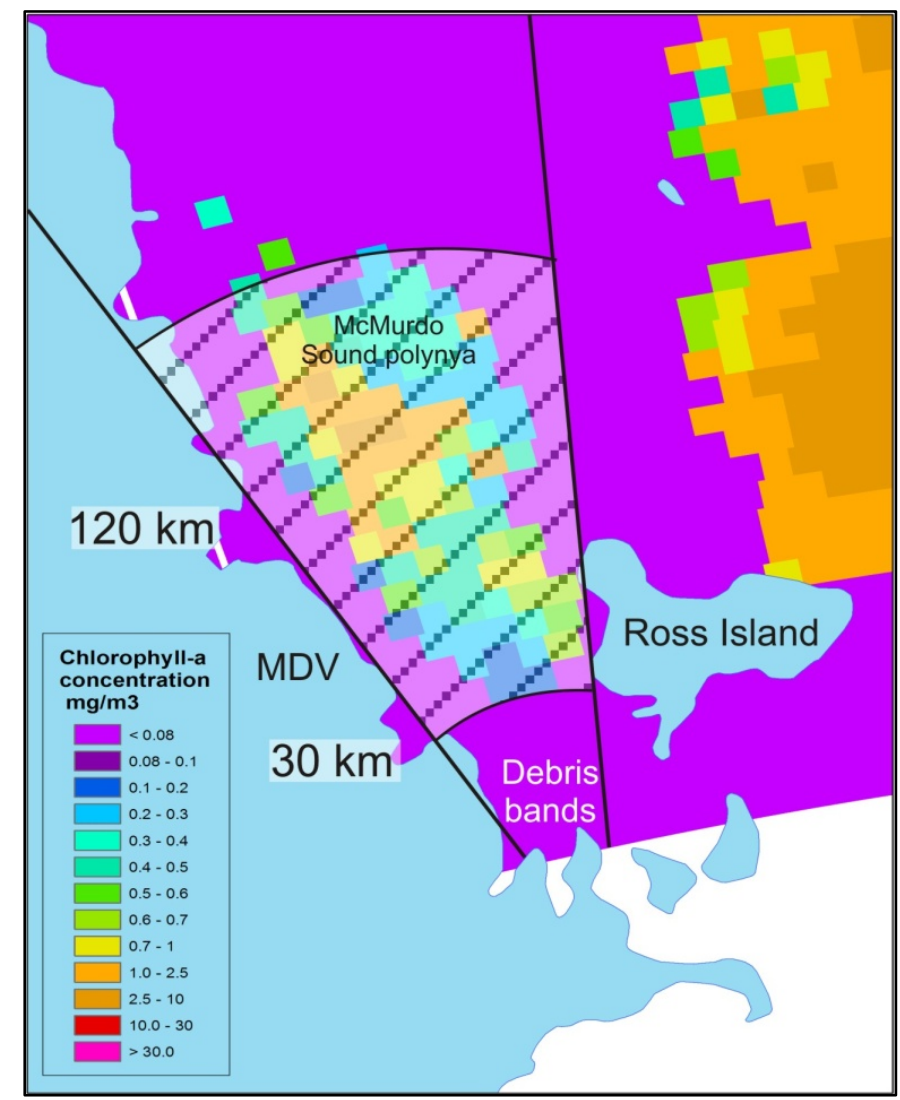

Fig. 4.5: Area defined as the "broader McMurdo Sound region" (shaded) which is constrained by the location of sampling sites up to $120 \mathrm{~km}$ from the debris band and inclusive of the seasonal phytoplankton bloom in the McMurdo Sound polynya. Chlorophyll-a concentration data are for December 2009 and derived from: SeaWiFS satellite data (https://gdata/sci.c.nasa.gov).

\subsubsection{Aeolian iron flux for McMurdo Sound}

Concentrations of cumulative soluble Fe from four leaches ranged from ranged from 0.79 to $0.03 \mu \mathrm{g} \mathrm{g}^{-1}$, with the lowest concentrations measured in snow from Granite Harbour (Fig. 3.18). Measurements of total Fe (particulate and soluble) in samples were homogenous and averaged $3.84 \pm 1.99 \mathrm{wt} \%$ (Fig. 3.17). The average percentage of soluble Fe is $9.42 \pm 0.7 \%$ for samples from Cape Roberts to Granite Harbour (Chapter 3.5.3) and is representative of the McMurdo Sound region (Fig. 3.22).

Assuming the snow accumulation at each sampling site on first year sea ice is representative of accumulation for that year, annual aeolian Fe fluxes into the ocean range between 6.61 $0.55 \mathrm{mg} \mathrm{m}^{-2} \mathrm{yr}^{-1}$ for the fine ASD fraction $(<10 \mu \mathrm{m})$, while the bulk aeolian Fe flux gradient 
ranges from 189.81 to $1.75 \mathrm{mg} \mathrm{m}^{-2} \mathrm{yr}^{-1}$ downwind of the debris band to Granite Harbour (Fig. 4.6). The Fe flux increases with increasing quantities of ASD per unit area per year $\left(\mathrm{R}^{2}=0.76\right)$, hence the trend of decreasing from the source (Fig. 4.6) predominately reflects the quantity of ASD available for leaching, rather than the Fe content (Fig. 3.19). Although, the peak Fe flux occurred from the 2004 storm event, along transect XY the greatest Fe flux of $75.19 \mathrm{mg} \mathrm{m}^{-2} \mathrm{yr}^{-1}$ was calculated for the sample MIS44 within the debris band dust plume. Therefore, as the aeolian Fe flux decreases exponentially to Marble Point where it is fairly constant between 30 - $120 \mathrm{~km}$ from the debris band, an average background Fe flux of 3.90 $\mathrm{mg} \mathrm{m}^{-2} \mathrm{yr}^{-1}$ for the bulk and $1.24 \mathrm{mg} \mathrm{m}^{-2} \mathrm{yr}^{-1}$ for the fine fraction is representative of this region in McMurdo Sound, a region where phytoplankton bloom each season in the McMurdo Sound polynya (Figs. 4.2 and 4.5).

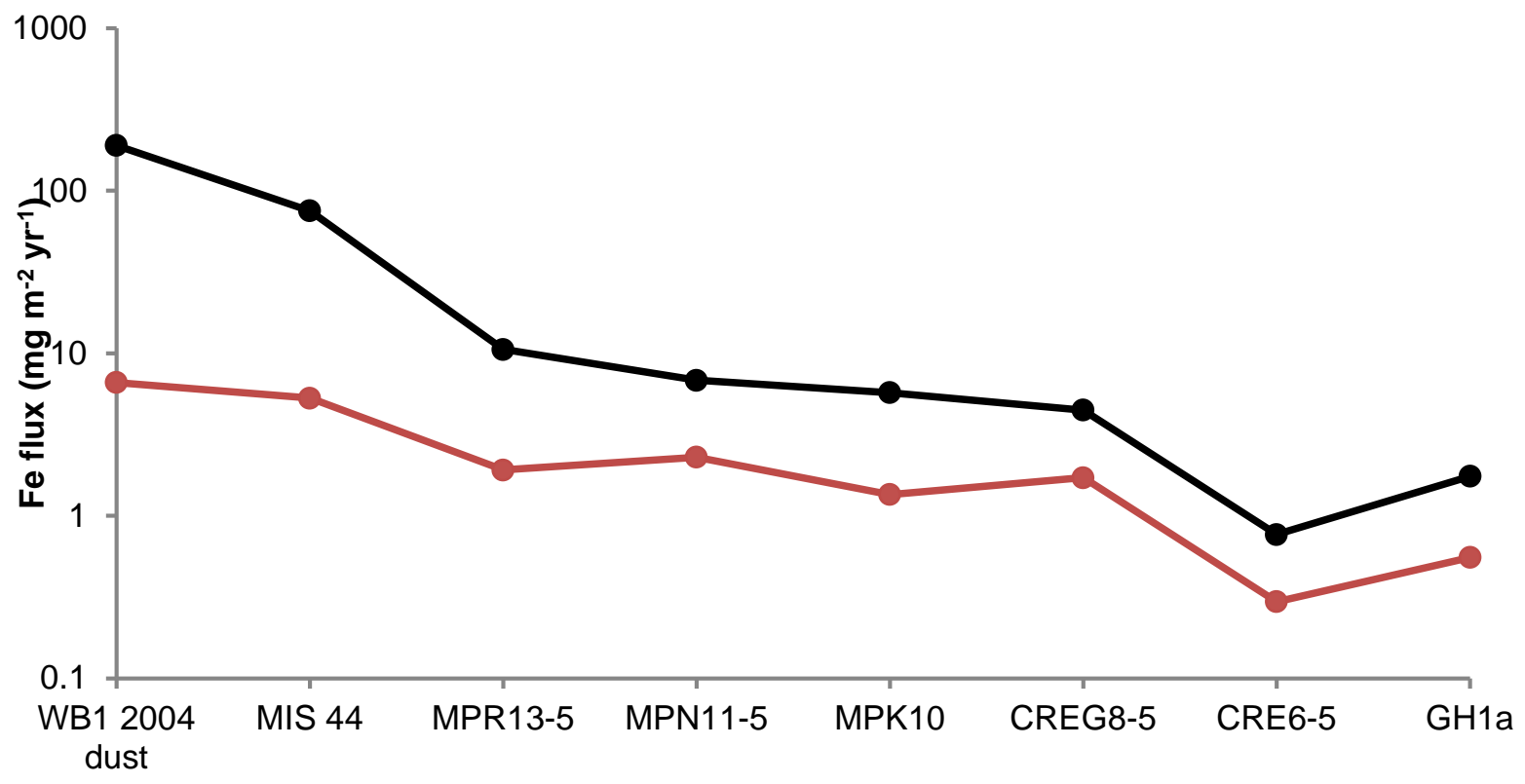

Fig. 4.6: Fe flux in McMurdo Sound. Black: bulk sample; red: $<10 \mu \mathrm{m}$ fraction i.e. the fraction that will reside in the water column for the longest period of time. Location of samples along transect XY in Fig. 3.15.

\subsubsection{Aeolian iron flux in the SW Ross Sea}

Given that the dominant mechanism for controlling Fe solubility is the mineral composition of the ASD which for the present study is shown to be relatively constant, and given that ASD is transported up to $500 \mathrm{~km}$ from the source, and possibly further depending on the dispersal model, before it reaches background levels (Chapter 2.4.3.2; Wagener et al., 2008), 
a regional background Fe Flux has been estimated for this region of ASD dispersal using measurements from Chapters 2.4 and 3.4 of a particulate Fe content of $3.84 \%$, a soluble Fe concentration of $0.10 \mu \mathrm{mol} \mathrm{L}{ }^{-1}$ and an ASD accumulation rate of $0.70 \mathrm{~g} \mathrm{~m}^{-2} \mathrm{yr}^{-1}$ for the $>10$ $\mu \mathrm{m}$ fraction ASD and $0.08 \mathrm{mg} \mathrm{m}^{-2} \mathrm{yr}^{-1}$ for the $<10 \mu \mathrm{m}$ fraction (Chapter 2.2.2.2). The extrapolation of the fine fraction Fe flux to $500 \mathrm{~km}$ from the source indicates that the flux is constant and thus the atmospheric Fe flux for the SW Ross Sea is $1.75 \mathrm{mg} \mathrm{m}^{-2} \mathrm{yr}^{-1}(31.23$ $\mu \mathrm{mol} \mathrm{m} \mathrm{yr}^{-1}$ ) for the bulk ASD and $0.55 \mathrm{mg} \mathrm{m}^{-2} \mathrm{yr}^{-1}\left(9.89 \mu \mathrm{mol} \mathrm{m}^{-2} \mathrm{yr}^{-1}\right)$ for the fine ADS fraction (Fig. 4.7). The presence of sand in all samples shows that ASD is transported along the sea ice by saltation and if sea ice continues northward, ASD and its Fe content can theoretically travel hundreds of kilometres from McMurdo Sound. Thus local ASD is regionally important and this value can be potentially used to characterise this broader region of productivity in the SW Ross Sea.

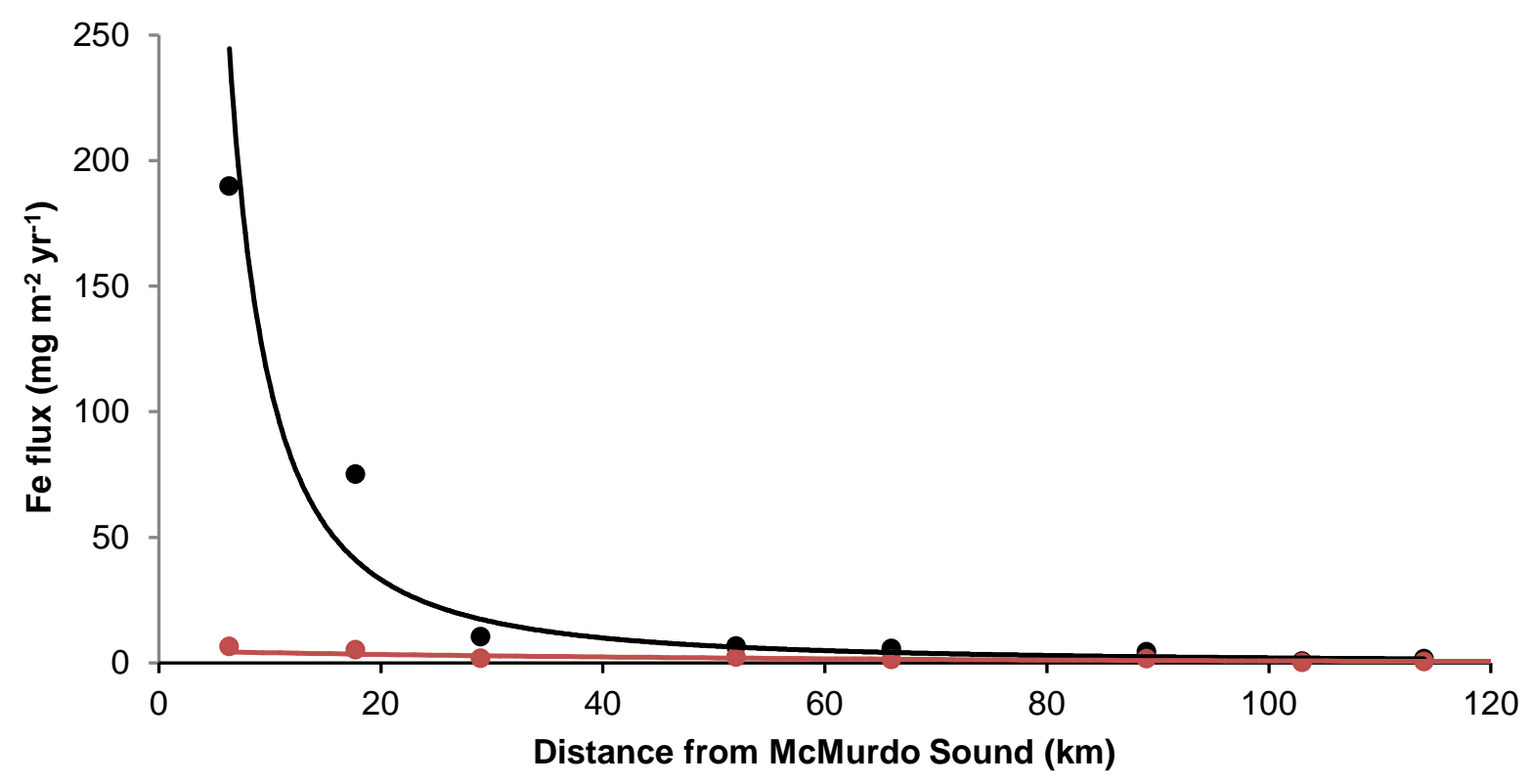

Fig. 4.7: Exponential decrease in the annual Fe flux into the ocean from the debris band to $120 \mathrm{~km}$ north of McMurdo Sound, estimated by sediment accumulation rates and soluble and particulate Fe content measurements in Chapters 2.4 and 3.4. Red: fine ASD fraction $(<10 \mu \mathrm{m})$; black: coarse ASD fraction ( $>10$ $\boldsymbol{\mu m})$.

No previous in situ Fe flux has been published for locally-derived ASD in the Ross Sea. The locally-derived Fe flux for McMurdo Sound is similar to Fe flux estimates derived from aerosol measurements and estimated $\mathrm{Al}$ concentrations in the SO surface waters in Table 4.1 and Fig. 4.8. The Fe deposition estimates in the present study are much greater than estimates from snow sampling in East Antarctica (Fig. 4.8), although it is important to note 
methodological differences in the generation of different datasets. Edwards and Sedwick (2001) estimate an atmospheric Fe flux from $0.3 \mu \mathrm{mol} \mathrm{m}^{-2} \mathrm{yr}^{-1}$ in the Dumont d'Urville Sea to $1.1 \mu \mathrm{mol} \mathrm{m} \mathrm{yr}^{-1}$ in Prydz Bay. Although, Edwards and Sedwick (2001) acidified their samples using $\mathrm{HCl}$ for 3 months to allow for dissolution of Fe-bearing minerals, the Fe flux calculated for local ASD in the present study is higher by at least an order of magnitude. In addition, the background Fe deposition estimate in the present study is 30 times higher than the estimated deposition of extraterrestrial Fe (Johnson, 2001), which is presumably included within this Fe flux. Not only is the Fe flux for the SW Ross Sea derived from local ASD more significant than other regions in Antarctica, it is also considerably higher than the estimate for the most recent Fe ASD deposition in the SO (Table 4.1 and Fig. 4.8; Wagener et al., 2008).

Estimates of Fe deposition in the present study are generally lower than older estimates of global Fe distribution models of Fung et al. (2000) and Maholwald et al. (1999). This may reflect that aerosol concentrations in the Southern Hemisphere are undersampled and these models overestimate the dust flux to the SO by up to 2 orders of magnitude (Wagener et al., 2008). Wagener et al. (2008) argue that their most recent Fe flux estimates of 0.073 and 1.168 $\mu \mathrm{mol} \mathrm{m} \mathrm{mr}^{-1}$ are representative of actual fluxes for the SO (Table 4.1). Although, incomplete dissolution of Fe-bearing ASD could have occurred in MQ water leaches in the present study, the fraction of Fe that is not rendered soluble after 4 leaches is probably small (Fig. 3.21) and thus cannot explain the order of magnitude difference.

Table 4.1: Present day atmospheric Fe fluxes in Antarctica. Al denotes aluminium.

\begin{tabular}{|c|c|c|c|}
\hline Sample type & Location & Fe flux $\left(\mu \mathrm{mol} \mathrm{m}^{2} \mathrm{yr}^{-1}\right)$ & Reference \\
\hline \multirow{2}{*}{$\begin{array}{c}\text { Aerosol } \\
\text { concentrations }\end{array}$} & Southern Ocean & $0.073-1.168$ & Wagener et al., 2008 \\
\hline & Southern Ocean & $1.8-18$ & $\begin{array}{c}\text { Duce and Tindale, 1991; } \\
\text { Donaghay et al., } 1991\end{array}$ \\
\hline \multirow[t]{2}{*}{ Snow sampling } & Dumont d'Urville Sea & 0.3 & Edwards and Sedwick, 2001 \\
\hline & Prydz Bay & 1.1 & Edwards and Sedwick, 2001 \\
\hline Al concentrations & Southern Ocean & $2.5-32$ & Measures and Vink, 2000 \\
\hline \multirow{4}{*}{$\begin{array}{l}\text { Global dust } \\
\text { transport model }\end{array}$} & Princess Elizabeth Land & $0-6.3$ & Mahowald et al., 1999 \\
\hline & Prydz Bay and Ross Sea & $3.4-12.5$ & Mahowald et al., 1999 \\
\hline & Dumout d'Urville & $12.5-125$ & Mahowald et al., 1999 \\
\hline & Antarctic waters & $\sim 10-200$ & Fung et al., 2000 \\
\hline Extra terrestrial & & 0.3 & Johnson, 2001 \\
\hline Local ASD & $\begin{array}{l}\text { McMurdo Sound, Ross } \\
\text { Sea (fine fraction) } \\
\text { (bulk fraction) }\end{array}$ & 31.23 & This study \\
\hline
\end{tabular}




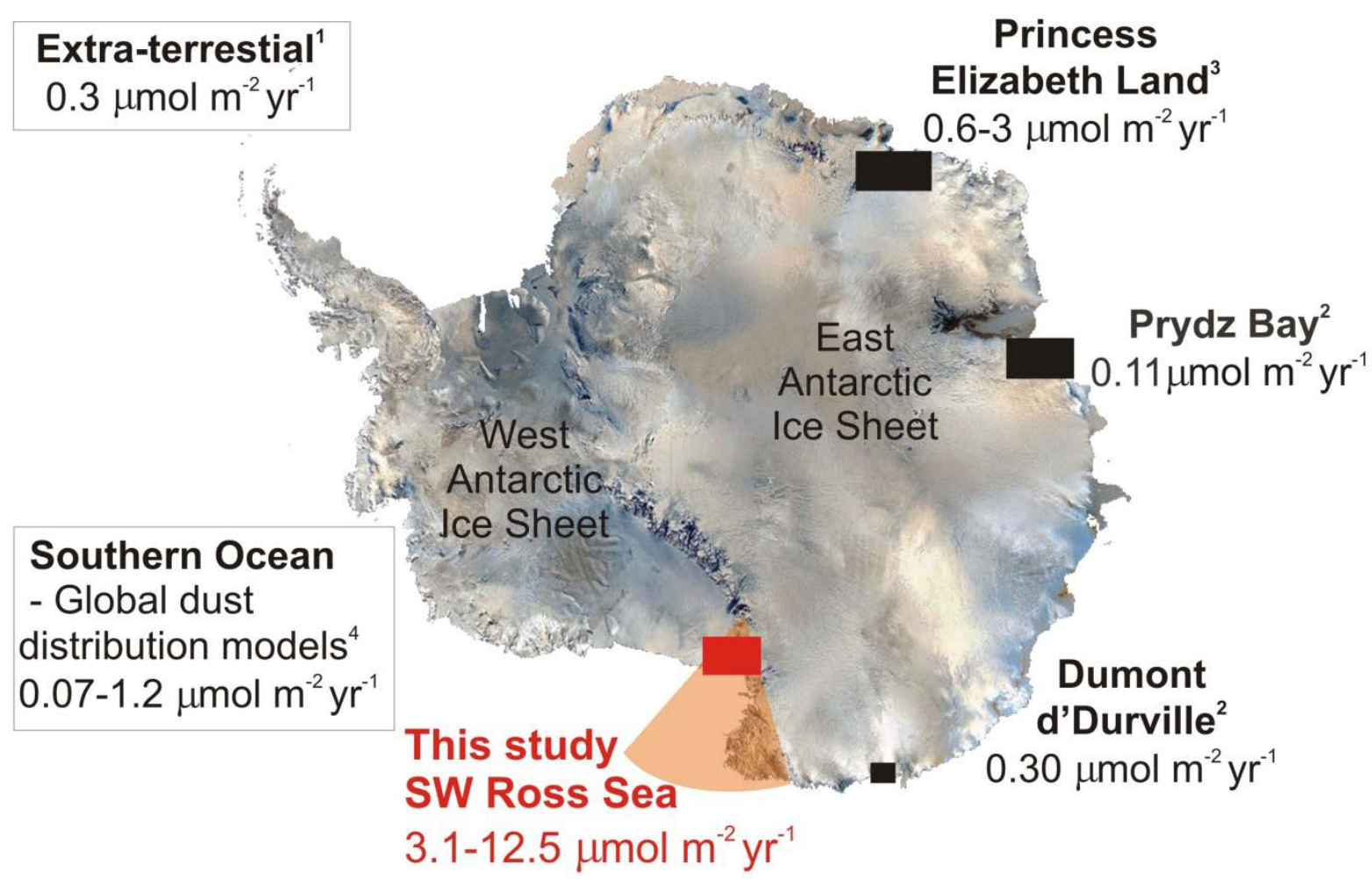

Fig. 4.8: Present day atmospheric Fe fluxes in Antarctica compared to local Fe derived from aeolian ASD in McMurdo Sound for the SW Ross Sea. ${ }^{1}$ Extra-terresteial Fe (Johnson et al., 2001) ${ }^{2}$ Snow sampling (Edwards and Sedwick, 2001); ${ }^{3}$ Model Fe deposition (Mahowald et al., 2005); ${ }^{4}$ Aerosol sampling (Wagener et al., 2008); ${ }^{5} \mathrm{Al}$ concentrations (Measures and Vink, 2001).

\subsubsection{Fe budget}

A simple sea ice Fe-budget for the Ross Sea, modified from Lannuzel et al. (2007) to include a locally derived ASD source is shown in Table 4.2. This budget assumes the sea ice in Wilkes Land, where the Lannuzel et al. (2007) study is based, has a similar Fe concentration to that of the SW Ross Sea, but acknowledges considerable uncertainty due to the variability in nutrient concentrations, water stratification, light availability and turbulence at different oceanic settings. Upwelling and sea ice melt are the dominant sources of Fe to the surface waters (Lannuzel et al., 2007) with and without a local ASD component (Table 4.2). Local ASD contributes $\sim 13 \%$ of the Fe to this sea ice budget, although this estimate is dependent on the rate at which ASD derived from the debris bands is dispersed into McMurdo Sound. Moreover, it could be underestimated if the percentage of soluble Fe increases as ASD is transported further from source and is thus exposed to greater levels of photo-reduction (Chapter 3.1.3.3; Fan et al., 2006). Although, the "global” aeolian Fe component is typically small in the Southern Hemisphere and is relatively unimportant in the SO (Boyd and Mackie, 
2008), local ASD cannot be excluded for the SW Ross Sea region, as it contributes an order of magnitude more Fe to this budget than the global dust component, highlighting the importance of McMurdo Sound in generating local Fe-bearing ASD at time coinciding with open water phytoplankton blooms.

Table 4.2: Fe budget for sea ice in the Ross Sea (modified from Lannuzel et al., 2007).

\begin{tabular}{|c|c|c|c|}
\hline Fe source & $\begin{array}{c}\text { Fe flux } \\
\left(\mu \mathrm{mol} / \mathrm{m}^{2} / \text { day }^{-1}\right)\end{array}$ & $\begin{array}{c}\text { Fe source } \\
\text { (no local dust) \% }\end{array}$ & $\begin{array}{c}\text { Fe source (with } \\
\text { local ASD) } \%\end{array}$ \\
\hline Southern Hemispheric dust & $0.0008^{1-4}$ & 0.14 & 0.12 \\
\hline Extra-terrestrial & $0.0008^{5}$ & 0.14 & 0.12 \\
\hline Vertical diffusion & $0.017^{6-9}$ & 2.98 & 2.56 \\
\hline Upwelling & $0.26^{6}$ & 44.94 & 39.15 \\
\hline Sea ice melting & $0.3^{7}$ & 54.83 & 45.17 \\
\hline Local ASD (fine) & $0.0270^{8}$ & & 12.88 \\
\hline
\end{tabular}

${ }^{1}$ Duce and Tindale, 1991; ${ }^{2}$ Wedephol, 1995; ${ }^{3}$ Baker and Jickells, 2006; ${ }^{4}$ Edwards and Sedwick, 2001; ${ }^{5}$ Johnson, 2001; ${ }^{6}$ de Baar et al., 1995; ${ }^{7}$ Law et al., 2003; ${ }^{8}$ Bowie et al., 2001; ${ }^{9}$ Boyd et al., 2005; ${ }^{7}$ Lannuzel et al., 2007; ${ }^{8}$ This study, assuming the Fe flux at Granite Harbour is representative of SW Ross Sea region.

However the lack of available Fe data for the Ross Sea (Sedwick et al., 2011) makes it difficult to a compare locally-derived dust source to other sources of Fe. To put the local ASD contribution of Fe into the context of Antarctic waters, the local aeolian Fe flux has been included in the Fe budget of Measures and Vink (2001) along the Antarctic Polar Front at $170^{\circ} \mathrm{W}$ (Table 4.3). Measures and Vink (2001) do not include an upwelling source in their budget because there is no data in this specific zone and they also argue that in general it is not possible to calculate the relative contribution of upwelling to the annual Fe budget of the surface waters. However, because of the large contribution upwelling makes to the dissolved Fe inventory in surface waters, in the present study the East Antarctic upwelling estimate of Lannuzel at al. (2007) has been included and is assumed to be similar for the SW Ross Sea. Also included is the ice rafted debris (IRD) estimate for the SO by Raiswell (2011) who estimates that ice berg hosted material provides approximately $2-20 \mathrm{mg} \mathrm{m}^{-2} \mathrm{yr}^{-1}$ of Fe to the SO. There are large uncertainties involved in this budget, due to meso-scale variability and advection patterns (Measures and Vink, 2001), in addition to this budget being applied to a different oceanographic setting. 
Table 4.3 shows that upwelling is the dominant Fe source to the surface waters with (68 \%) and without (72\%) a local ASD source. Similar to the sea ice Fe budget (Table 4.2), global aeolian Fe deposition fluxes to surface waters in this region therefore do not appear to play a significant role in the supply of Fe to surface waters. According to this crude budget in Table 4.3, local ASD however contributes $7 \%$ Fe to the ocean. This Fe includes ASD that has accumulated on sea ice over winter and its solubility could be affected by this process (Chapter 3.5.3.2), potentially increasing the contribution of soluble $\mathrm{Fe}$ flux from the McMurdo Sound debris band. Fe dispersal through ice melt occurs rapidly and is restricted to a shallow stratified layer because of its low density, thus Measures and Vink (2001) suggest the local effect of Fe addition of ASD might be greater than their calculations suggest.

Table 4.3: Fe budget for Antarctic waters including a local ASD source (modified from Measures and Vink, 2001).

\begin{tabular}{|c|c|c|c|}
\hline Fe source & 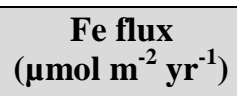 & $\begin{array}{l}\text { Fe source (no } \\
\text { local dust) \% }\end{array}$ & $\begin{array}{c}\text { Fe source (with } \\
\text { local ASD) } \%\end{array}$ \\
\hline Upwelling & $95^{1}$ & 73.22 & 68.03 \\
\hline Deep mixing inventory & $30-33^{2}$ & 24.31 & 22.58 \\
\hline Eddy diffusion & $0.47^{2}$ & 0.36 & 0.34 \\
\hline Ice melt & $0.23^{2}$ & 0.18 & 0.16 \\
\hline Aeolian deposition & $0.5-5^{2}$ & 1.93 & 1.79 \\
\hline IRD & $0.98^{3}$ & 0.75 & 0.70 \\
\hline Local ASD & $9.89^{4}$ & & 7.04 \\
\hline
\end{tabular}

${ }^{1}$ Lannuzel et al., 2007; ${ }^{2}$ Meausres and Vink, 2001; ${ }^{3}$ Raiswell, 2011; ${ }^{4}$ this study. 


\subsection{Is there enough aeolian iron in McMurdo Sound to stimulate phytoplankton blooms?}

\subsubsection{Introduction}

This section considers whether enough Fe derived from local ASD sources in McMurdo Sound is supplied to surface waters to overcome the Fe-limitation threshold and stimulate primary productivity in the SW Ross Sea. To do this data from Chapters 2 and 3 have been used in the Boyd et al. (2010) dust-biota assessment tool as a guide to the possible links between local ASD derived Fe and biological response during background ASD years and during elevated (by a factor of $\sim 4$; Chapter 2.3.2.2) ASD accumulation rates in storm years.

\subsubsection{Modelling ASD flux and phytoplankton blooms}

The dust-biota assessment tool of Boyd et al. (2010) assumes each dust event travels as a linear front and the fall-out zone is divided into strips of $100 \mathrm{~km}$. The concentration of dissolved non-biogenic Fe in the ocean's mixed layer on any given day is defined as the concentration of total dissolved Fe from the previous day plus the total supply of Fe for that day. Table 4.4 illustrates the parameter model inputs used in the present study for McMurdo Sound ASD. If the dust supplies sufficient Fe to overcome the Fe-limitation of phytoplankton growth in the model, i.e. above a threshold concentration of $0.2 \mathrm{nM}$, determined by the Southern Ocean Iron RElease Experiment (SOIREE; Boyd and Abraham, 2001), a phytoplankton bloom is assumed to occur.

The dust-biota model does not take into account the amount of ASD trapped in snow with each unit distance travelled from source like the ASD dispersal model in Figs. 4.43 and 4.44 and therefore may overestimate the distance over which the dust travels and supplies Fe to the ocean. For this reason in the case of McMurdo Sound it is assumed that sea ice break up and thus Fe supply is instantaneous. The model is nevertheless useful for determining whether enough ASD is supplied to the ocean to stimulate a phytoplankton bloom. 
Table 4.4: Parameters used in the dust-biota model of Boyd et al. (2010).

\begin{tabular}{c|c|c}
\hline Parameter & Value & Reference \\
\hline Background ASD accumulation rate & $0.70 \mathrm{~g} \mathrm{~m}^{-2} \mathrm{yr}^{-1}$ & This study, Chapter 2.4.3.2 \\
ASD accumulation rate 2004 storm (WB1) & $12.85 \mathrm{~g} \mathrm{~m}^{-2} \mathrm{yr}^{-1}$ & This study, Chapter 2.3.2.2 \\
Total iron content & $3.84 \%$ & This study, Chapter 3.4 \\
Soluble iron & $9.27 \%$ & This study, Chapter 3.4 \\
Initial seawater Fe concentration & $0.16 \mathrm{nM}$ & Sedwick and DiTullio, 1997 \\
& & Ross Sea after sea ice melt: 0.16-0.17 nM \\
Mixed layer depth & $10 \mathrm{~m}$ & Arrigo et al., 2010 \\
& & McMurdo Sound: 5-20 m \\
Latitude & -75.5 to $-77.8^{\circ} \mathrm{S}$ & \\
Longitude & 162 to $169^{\circ} \mathrm{E}$ & \\
\hline
\end{tabular}

\subsubsection{Fe supply in a background year}

The background ASD accumulation rate in McMurdo Sound of $0.70 \mathrm{~g} \mathrm{~m}^{-2} \mathrm{yr}^{-1}$ is sufficient to initiate a bloom during summer months up to $1200 \mathrm{~km}$ from the source (Fig. 4.9), assuming local ASD has an Fe content of $3.84 \%$, a solubility of $9.27 \%$, a mixed depth layer of $10 \mathrm{~m}$; and an initial seawater Fe concentration of $0.16 \mathrm{nM}$ (Sedwick and DiTullio, 1997). This distance spans the open water areas of the McMurdo Sound polynya, Terra Nova Bay polynya and parts of the Ross Sea polynya (Fig. 2.46). New Fe supplied to the surface water is $1.13 \mathrm{nM}$ at $400 \mathrm{~km}$ from the source, $0.6 \mathrm{nM}$ at $800 \mathrm{~km}$ from the source and $0.34 \mathrm{nM}$ at $1200 \mathrm{~km}$ from the source, up to four days after deposition (Fig. 4.9A). Hence this new Fe could potentially influence bloom 2 in Fig 4.3, an area where the phytoplankton bloom declines at a time when macro-nutrients are not exhausted (Fitzwater et al., 2000; Arrigo et al., 2003). 


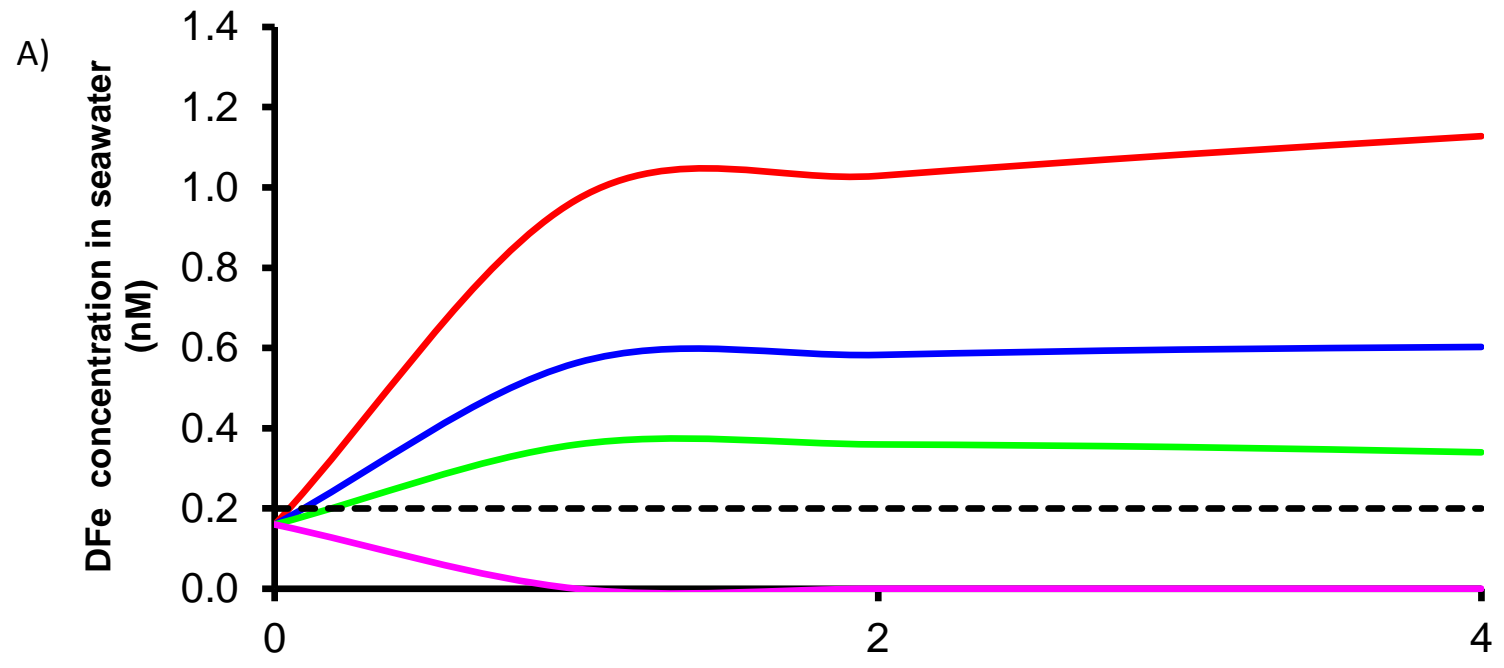

Days after storm event

B)

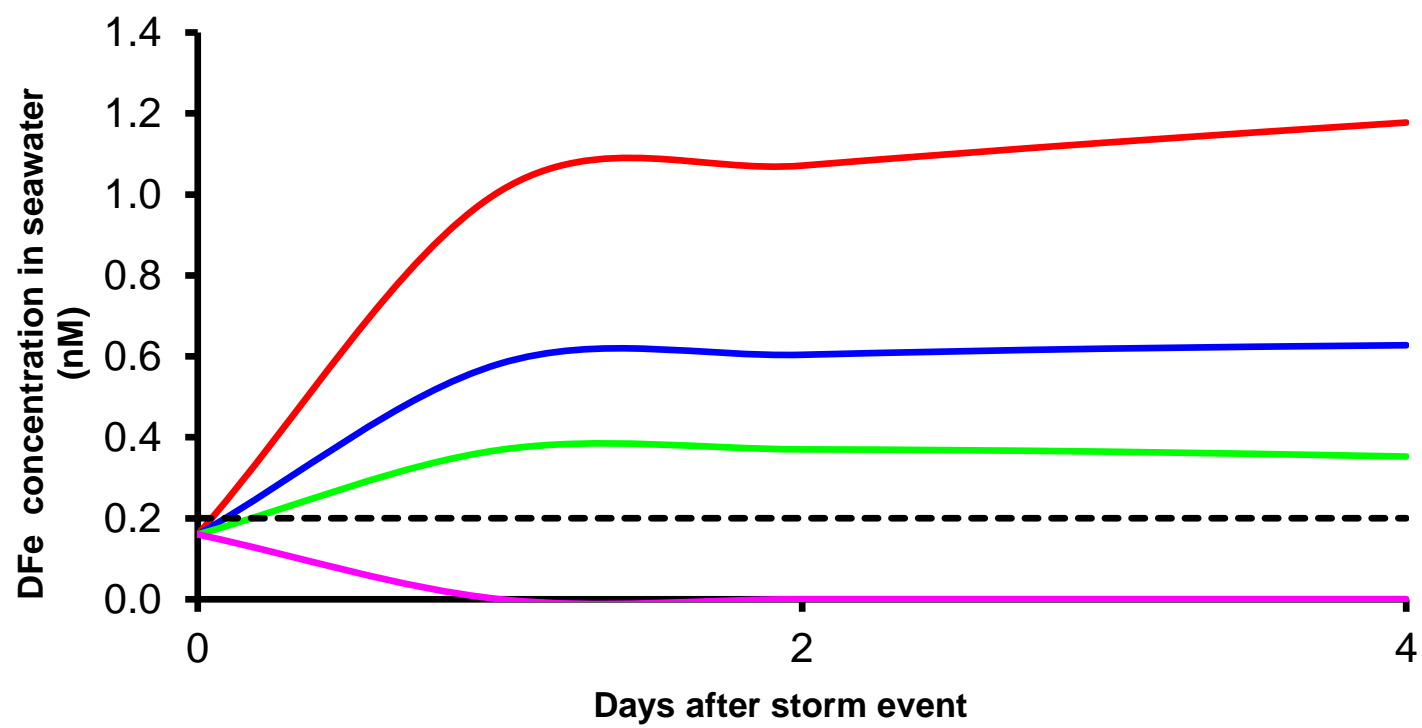

Fig. 4.9: The dissolved Fe (DFe) concentration versus time resulting from the deposition of local aeolian Fe in the SW Ross Sea for A) background ASD accumulation rate of $0.70 \mathrm{~g} \mathrm{~m}^{-2} \mathrm{yr}^{-1}$ when sea ice breaks up each summer and B) during a severe storm event in 2004 with a ASD accumulation rate of $12.85 \mathrm{~g} \mathrm{~m}^{-2}$ $\mathrm{yr}^{-1}$. When concentrations of Fe in the seawater exceed $0.2 \mathrm{nM}$ (dashed line), Fe is no longer limited and given favourable conditions, e.g. light and water stratification, a phytoplankton bloom is likely to occur. Coloured lines are distance from the source; red: $400 \mathrm{~km}$, blue: $800 \mathrm{~km}$, green: $1200 \mathrm{~km}$, pink: $1600 \mathrm{~km}$. This assumes an Fe content of $3.84 \%$, solubility of $9.27 \%$, mixed depth layer of $10 \mathrm{~m}$ and initial Fe concentration of $0.16 \mathrm{nM}$.

\subsubsection{Fe supply during storm years}

Extreme weather events can transport larger quantities of ASD onto the sea ice. In McMurdo Sound, the ASD accumulation rate associated with the severe 2004 storm event was significantly greater than the background, however the predicted Fe concentration associated 
with this ASD does not increase by the same proportion as the additional ASD generated by the storm (Fig. 4.9B; Chapter 4.4.6). The model predicts that new Fe supplied to the surface water is $1.18 \mathrm{nM}$ at $400 \mathrm{~km}$ from the source, $0.63 \mathrm{nM}$ at $800 \mathrm{~km}$ from the source and $0.35 \mathrm{nM}$ at $1200 \mathrm{~km}$ from the source (Fig. 4.9B). Back trajectory analysis (Chapter 2.4.5) indicates that the air mass associated with the 2004 storm travelled parallel to the SVL coastline at least $1200 \mathrm{~km}$ from McMurdo Sound. Although this storm occurred in May when Antarctica has 24 hours of darkness and extensive sea ice cover preventing photosynthesis of diatoms and P. antarctica, the output of the model indicates that more ASD accumulating on sea ice has the potential to fertilise the ocean the following summer during sea ice break out. Supporting evidence is illustrated in Fig. 4.10 of satellite images of chlorophyll- $a$ concentration for the following summer, which indicate that types I and II (see Fig. 4.3) phytoplankton blooms did occur, extending to at least 1200 km from McMurdo Sound.

A)

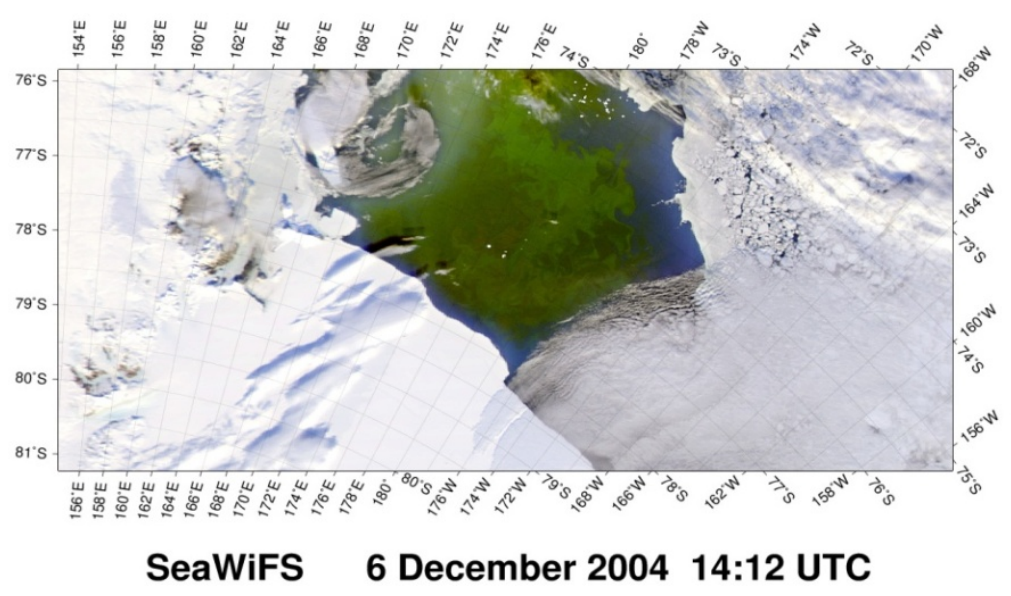

B)

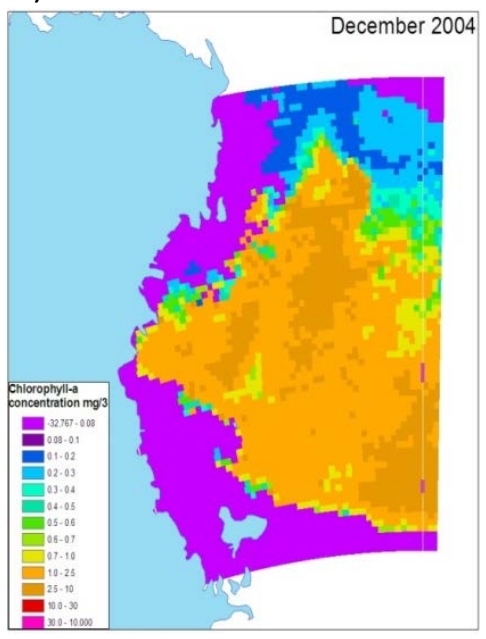

Fig. 4.10: SW Ross Sea polynya and ice cover during December 2004. A) Phytoplankton bloom on the surface of the ocean. White flecks: ice bergs; smooth white sheet of ice in left corner of image: Ross Ice Shelf. Source: SeaWiFS Sensor flying on the OrbView-2 satellite http://eoimages.gsfc.nasa.gov/images/imagerecords/5000/5065/RossSea_SEA_2004341_lrg.jpg B) Chlorophyll-a data downloaded from: https:/gdata/sci.c.nasa.gov. Blue: Antarctic continent; purple: sea ice.

\subsubsection{Inter-annual variability in aeolian Fe flux}

The background ASD accumulation rate in McMurdo Sound is at least two orders of magnitude higher than the global background. For this reason it is useful to investigate whether the McMurdo Sound ASD source could supply Fe to the surface waters driving inter- 
annual variations in Fe concentration, that is, whether greatly enhanced ASD deposition into the ocean associated with storms will have an additional effect on the dissolved $\mathrm{Fe}$ concentration in the surface waters. As the model predicts only small increases in dissolved Fe concentration supplied to the surface water during storm conditions (Fig. 4.9), it would appear that inter-annual variations are minor.

This is an unexpected outcome of the model. Therefore the sensitivity of the model to changes in dust mass was investigated. The results indicate that the occurrence of a bloom in the model is based on a critical threshold of dust being exceeded and that increases in the supply of dust beyond this threshold do not result in a proportional increase in primary productivity. However, the output from the model does suggest that relatively small contributions of dust (assuming $9.27 \%$ solubility, $3.84 \%$ total Fe and $0.70 \mathrm{~g} \mathrm{~m}^{2} \mathrm{yr}^{-1}$ ASD accumulation rate) are able to supply sufficient Fe for the stimulation of phytoplankton blooms. The implications of this finding are that; i) there is sufficient ASD in McMurdo Sound, even during low ASD accumulation years, so that Fe concentrations derived from ASD are above the threshold level in McMurdo Sound. Enhanced ASD supply during storms is therefore unimportant in this region; ii) the importance of storms in aeolian Fe supply therefore, could be due to the greater area over which dust is dispersed, that is, raising Fe levels above the critical threshold hundreds of kilometres from the source.

ASD dispersed from the debris bands may contribute to aeolian Fe supply over a broad although as yet undefined, region of the SW Ross Sea. However, more locally the high rate of ASD accumulation does not influence the Fe concentration, but during storms ASD may travel further from the source than during background years and inter-annual variations in Fe supply from local ASD may therefore depend on the location the dust is deposited within the SW Ross Sea.

Type I and II phytoplankton blooms occur each year in SW Ross Sea (Fig. 4.3; Arrigo et al., 2004), apparently independent of the quantity of ASD deposited beyond the background threshold, that is, during summer months Fe supply is always above the bloom threshold, however, the source of this $\mathrm{Fe}$ cannot be completely constrained by the existing data. Although, for $1200 \mathrm{~km}$ beyond McMurdo Sound, ASD supplied is sufficient on its own to stimulate a bloom (Fig. 4.9). Arrigo et al. (2003) argues that excess Fe supplied to the eastern 
sector of the Ross Sea is not utilised compared to the SW Ross Sea which is depleted each season. Given that Fe is the limiting nutrient for primary productivity, the present study however, cannot trace whether phytoplankton blooms in the SW Ross Sea are triggered by Fe from local ASD or a different Fe source, such as upwelling. Although Edwards and Sedwick (2001) suggest ice derived Fe only supports $<10 \%$ of regional net primary productivity, the Fe deposition rate in the present study is much greater suggesting that local ASD could support $>10 \%$ of regional net primary productivity as explained in Chapter 4.5. 


\subsection{Connections between aeolian sand and dust flux, chlorophyll-a concentrations and methylsuphonate concentrations}

\subsubsection{Introduction}

Several ice core studies have identified a statistical relationship between the sulphur species, methylsuphonate (MS) and sea ice extent (Welch et al., 1993; Pasteur et al., 2000; O’Dwyer et al., 2000; Meyerson et al., 2002; Curran et al., 2003; Junying et al., 2002; Abram et al., 2007). MS has a single source through the oxidation of dimethylsuphide (DMS; Saltzman et al., 1963). In turn, in the SO, DMS is a by-product of dimethylsulphoniopropionate (DMSO), produced by phytoplankton such as P. antarctica (DiTullio et al., 1995; 2003) and diatoms that are found in close association with sea ice (Bunt, 1963; Bunt and Lee, 1970; Bunt and Wood, 1983, DiTullio et al., 2003). The record of MS concentration over time extracted from a snow pit at Mt Erebus Saddle (location in Fig. 2.2) shows a strong positive linear correlation with changes in the area of open water $\left(\mathrm{R}^{2}=0.90, \mathrm{p}<0.05\right)$, and phytoplankton net primary production derived from satellite measurements of chlorophyll $\left(\mathrm{R}^{2}=0.93, \mathrm{p}<0.01\right)$ in the Ross Sea summer (Rhodes et al., 2009). Rhodes et al. (2009) therefore suggested there was a strong causal relationship between regional phytoplankton productivity and MS trapped in coastal ice (c.f. Wolff et al., 2006). Assuming therefore that MS in coastal ice is a proxy for regional phytoplankton productivity in the Ross Sea, the importance of ASD in stimulating blooms could be further examined by comparing variations in the concentration of ASD and MS over the same period of time.

In contrast, Wolff et al. (2006) found no relationship between dimethylsuphonate, dust and climate on glacial-interglacial timescales in the Dome $\mathrm{C}$ ice core record, although the temporal resolution used is much coarser than data in the present study, and the distance between potential source areas of dust and MS production and Dome C much greater. Potentially then only coastal ice cores proximal to the ocean may preserve an MS record that faithfully reflects regional productivity. Air mass trajectory analysis (Figs. 2.45) and the dustbiota model (Chapter 4.4) shows that ASD and Fe could be deposited $1200 \mathrm{~km}$ from McMurdo Sound into the SW Ross Sea. Furthermore, air mass back trajectories illustrate the MS record used in the present study are derived from the Ross Sea rather than a "SO 
average”, thus there is a clear basis for interpreting both the ASD and MS records as regional in extent, but not influenced by dust sourced from the SO or beyond (Fig. 4.11).

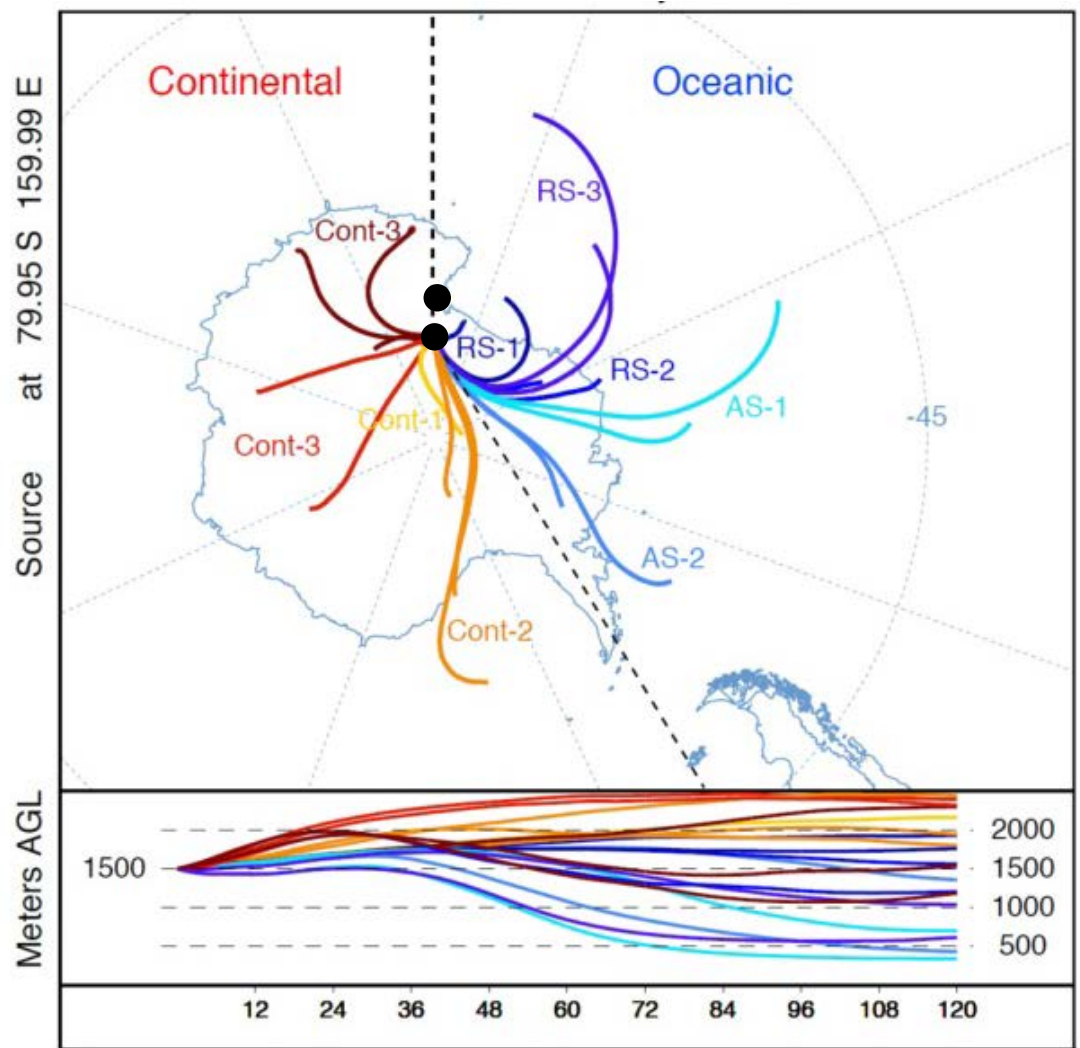

Fig. 4.11: Back trajectory analysis and cluster means for Gawn Ice Piedmont Glacier. Source: Markle (2011). Black circles: Location of the Mt Erebus Saddle (top) and Gawn Ice Piedmont Glacier (bottom) ice cores.

\subsubsection{Description of records used in the present study}

\subsubsection{Methylsuphonate record}

Two coastal MS records acting as a proxy for Ross Sea primary productivity were investigated.

1. The first record was recovered from a $12 \mathrm{~m}$ ice core from Gawn Ice Piedmont Glacier, located at the Byrd Glacier outlet at the boundary of the TAM and the Ross Ice Shelf (8005’58.15”'S, $159^{\circ} 16^{\prime} 37.60^{\prime \prime} E$; Fig. 4.11). At this site there is relatively high snow accumulation (1.12 m water equivalent per year) which allows for sub-seasonal resolution of the ice core record. The core spans a period from 2000 to 2008. See Markle (2011) for sampling and dating details. Cyclonic frequency at the site is 
highest in late summer and early spring entraining air masses that originate over the sea ice zone in the Ross Sea (Fig 4.11).

2. The second MS record is taken from a $2 \mathrm{~m}$ deep snow pit on Mt Erebus Saddle, Ross Island (77 30.090'S, $167^{\circ} 40.59^{\prime}$ E; Figs. 2.2 and 4.11) in 2004/2005. See Rhodes et al. (2009) for sampling and dating details. The high accumulation of $0.40 \mathrm{~m} \mathrm{yr}^{-1}$ allowed for sub-seasonal resolution of the 5 year record. These samples cover the period 1999 to 2004. The proximity of the snow pit to seasonally open water of the Ross Sea polynya and the predominant summer northeasterly wind direction that also originates over the sea ice zone, makes the record at the site highly sensitive to the oceanatmosphere interactions.

Both the ice core and snow pit samples were analysed at the Climate Change Institute, University of Maine for MS concentration by ion chromatography (see Bertler et al., 2004).

\subsubsection{Chlorophyll- $a$ archive}

Phytoplankton production in McMurdo Sound between 1997 and 2010 was quantified using satellite data of chlorophyll- $a$ concentration from the Sea-viewing Wide Field-of-view Sensor (SeaWiFS) obtained from the Goddard Earth Sciences (GES) Data and Information Services Center (DISC).

\section{Satellite image processing}

All chlorophyll- $a$ concentration data were acquired from the SeaWiFS R2009 sensor downloaded from the Giovanni Ocean Colour Online Visualisation (http://gdata1.sci.gsfc.nasa.gov/daac-bin/G3/gvi.cgi?instance-id=ocean-month) Goddard Earth Sciences Data and Information Services Centre, except for January 2008 where data were acquired from MODIS-Aqua sensor because of failure of the SeaWiFS sensor at this time. These satellite data were mapped onto a common geographic reference frame (south polar-stereographic projection) using ArcMap GIS. All analyses were performed within the geographical boundaries of the black box in Fig. 4.12. 


\section{Calibration and validation of chlorophyll-a data set}

Remote sensing at high-latitudes requires appropriate calibration and validation of the data set. Arrigo and van Dijken (2004) found a good agreement of chlorophyll-a concentrations measured by SeaWiFS and those measured in situ by two recent field programs in the Ross Sea: Research on Ocean-Atmosphere Variability and Ecosystem Response in the Ross Sea (ROAVERRS) and Antarctic Environment and Southern Ocean Process Study (AESOPS). The overlapping time period of the data in the present study with Arrigo and Dijken's (2004) suggests that this time series will also be in good agreement.
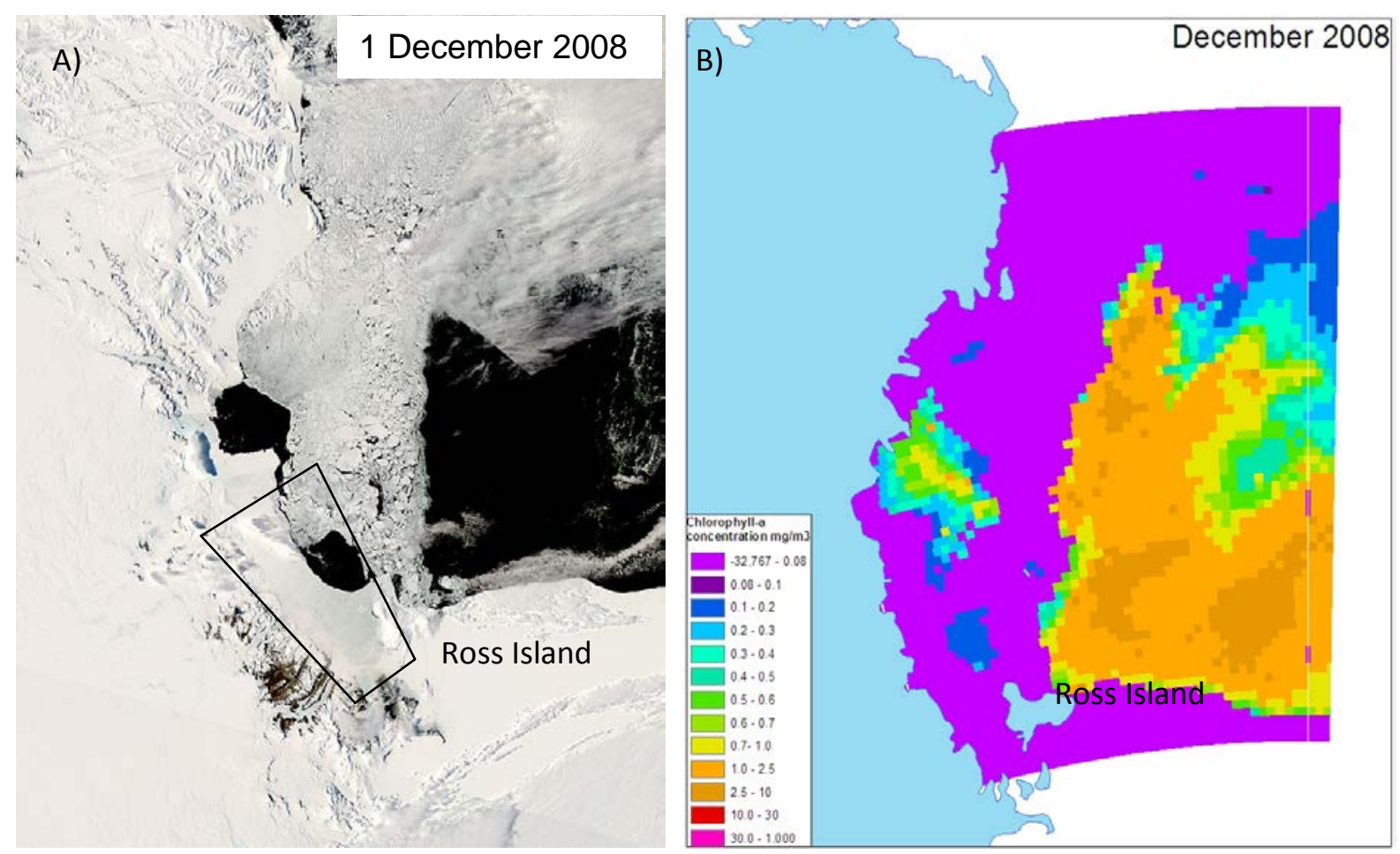

Fig. 4.12: Relationship between sea ice location and primary productivity in the SW Ross Sea. A) SW Ross Sea ice location on the 1/12/2008 MODIS-Aqua true colour satellite image (retrieved from: http://rapidfire.sci.gsfc.nasa.gov/subsets/?projectantarctica\&subset=Ross-Sea.2008337.aqua.1km on the 22/04/2010). B) Monthly average chlorophyll-a concentration for December 2008. Data derived from: SeaWiFS R2009 http://gdata1/sci.gsfc.nasa.gov/daac-bin/G3/gui.cgi?isntance_id=ocean_month.

\subsubsection{Aeolian sand and dust flux record}

The seasonally-resolved ASD record used in this comparison is derived from a shallow firn core (WB1) recovered from the McMurdo Ice Shelf (see Chapter 2.3 for accumulation rates, sampling and dating of this core). The core spans the period 2000-2008 and overlaps the MS record from the Gawn Ice Piedmont Glacier. An annual ASD accumulation rate for the record was calculated by summing the total ASD in the core from March to March, assuming that 
this period represents ASD accumulated on the sea ice surface over winter and subsequently deposited into the ocean that summer when the sea ice breaks up (Fig. 2.6).

The ASD and MS datasets were resampled at monthly resolution to place them on a common time scale with the chlorophyll- $a$ data. The age model for the Gawn Ice Piedmont Glacier ice core is taken from Markle (2011). The data for the three records can be found in Appendix 8.

\subsubsection{Methylsuphonate and chlorophyll-a concentration and ASD flux records}

\subsubsection{Methylsuphonate record}

The summer background MS concentration during 2000-2004 in the Gawn Ice Piedmont Glacier record is $\sim 30 \mathrm{ug} \mathrm{L^{-1 }}$. The MS record in the summer of 2005 peaks $\sim 4.5$ times above the background of the 8 year record and 2 times in the summer of 2007 (Fig. 4.13; Markle, 2011).

In the Mt Erebus Saddle record, austral summers that had increased open water areal extent (decreased ice cover) in the Ross Sea (e.g. 2001/2001) are associated with higher concentrations of MS in the snow pit record. An increase in open water areal extent leads to a proportional increase in MS concentration and vice versa (Rhodes et al., 2009). When MS concentration is low e.g. 2002/2003 the maximum rate of primary productivity was less than a quarter of that reached in 2001/2001 when the MS concentration peaks (Rhodes et al., 2009).

\subsubsection{Chlorophyll-a time series}

Many studies have found a clear spatial and seasonal correlation between chlorophyll- $a$ concentration and open water area in the Ross Sea, for example Fig. 4.12 (Arrigo and van Dijken, 2004; Rhodes et al., 2009). A seasonal cycle of phytoplankton blooms is evident between October and March each year (Fig. 4.13). The blooms generally peak in December and decline in January and February (Fig. 4.2; Arrigo and McClain, 1994). Chlorophyll- $a$ in McMurdo Sound and the SW Ross Sea largely co-vary (covariance=0.6016), however this suggests primary productivity in McMurdo Sound has additional influences when compared 
to the SW Ross Sea. These additional influences are considered to include sea ice break out patterns, water column stratification, light penetration, availability of micro-nutrients (e.g. $\mathrm{Mn}, \mathrm{Zn}, \mathrm{Co}$ ) and abundance of grazers. The annual bloom in McMurdo Sound averages $8 \%$ of the total chlorophyll- $a$ in the SW Ross Sea, attributable to the area small of open water in McMurdo Sound compared to the SW Ross Sea (Fig. 4.12).

Two distinct time series are discussed, which represent the influence of the El Nino Southern Oscillation (ENSO) on sea ice and the influence of a particularly large iceberg on sea ice extent.

\section{The influence of ENSO on sea ice: 1997-1998}

During El Nino years sea ice increases and therefore there are less extensive phytoplankton blooms. Arrigo and van Dijken (2004) found that in the Ross Sea both in 1997-1998 and 2000-2001 primary productivity was 40 \% lower than November 1998 and March 2000 when open water was greatest in spring/summer.

\section{The influence of the B15 ice berg on sea ice: 2000-2001}

The B15 ice berg trapped in the Ross Sea changed the normal advection pattern of sea ice out of the sound in 2000-2001. This increased sea ice extent and caused a delay in the onset of summer phytoplankton productivity. Chlorophyll-a peaked in February which is two months later than usual (Arrigo and Dijken, 2004) and reduced overall primary productivity during this time (Rhodes et al. 2009).

\subsubsection{Inter-annual variability in ASD, Methylsuphonate and chlorophyll-a concentrations}

The severe storm event that affected McMurdo Sound in May 2004 (Chapter 2.4.5) was responsible for ASD accumulation rates in the WB ice core $\sim 4$ times greater than occurred in the preceding decade at that site (Dunbar et al., 2009). The following summer (2004/2005) when ASD from this same event is deposited into the ocean an anomalously large peak in MS concentration is observed in the Gawn Ice Piedmont Glacier record. Fig. 4.13 illustrates the three records: Gawn Ice Piedmont MS concentration (a proxy for phytoplankton stocks; Rhodes, et al., 2009); chlorophyll- $a$ (an independent measure of primary productivity) and 
the ASD record from Windless Bight, here assumed to be a proxy for Fe supply to the surface ocean (Fig. 3.19). All three records show the expected seasonal cycle of summer maxima in chlorophyll- $a$ concentration, MS concentration, and ASD accumulation rates. The annual average of MS concentration and ASD shows a high degree of correlation $\left(\mathrm{R}^{2}=0.68\right.$, $\mathrm{p}<0.001$ ), although the magnitude of the peaks is not correlated, except for the two largest ASD and MS events in 2004/2005 and 2006/2007 which coincide in timing and show similar amplitudes.

Air mass back trajectory analysis for the large MS peaks in summer 2005 and summer 2007 strongly suggest MS is sourced from the Ross Sea region (dark blue trajectories RS1-3 in Fig. 4.11). The two largest peaks ASD accumulation and MS concentration coincide with large frequencies of these oceanic trajectories, but the anticipated correlation between chlorophyll$a$ and MS concentrations is not present $\left(\mathrm{R}^{2}=0.03\right)$. However, it is curious that ASD accumulation rates and MS co-vary for the whole length of the record including the two large storm years, but not during "background" years where the storm years are excluded $\left(\mathrm{R}^{2}=0.49\right.$; Chapter 2.3.2.2.1). One possible explanation is that there is a threshold value for the ASD accumulation rate (Fig. 4.13) above which sufficient local aeolian Fe is dispersed across a broad enough area of the Ross Sea to enhance regional productivity (such as in the case of storm years which are capable of transporting ASD greater distances enhancing phytoplankton blooms beyond the immediate local effect in McMurdo Sound; Chapter 4.4.6), while during average years the Fe supply from non ASD sources (upwelling, continental shelf sediments and storage release from sea ice melt) is sufficient to sustain a "background" chlorophyll- $a$ level, therefore the correlation is weak in these years. However, ASD accumulation rates are above the dust threshold level in Boyd et al.’s (2010) model during non-storm years sufficient to stimulate a phytoplankton bloom. Based on these findings, this study proposes that enhanced Fe-fertilisation associated with large ASD storms (e.g. dust storms in 2005 and 2007) has the potential to significantly increase primary productivity because wide dispersal of dust enables the threshold to be exceeded over a greater area, and hence chlorophyll- $a$ and MS concentration. However, longer time series are needed to achieve a statistically representative data set which includes more of these extreme storm events. 
Although ASD concentration was not measured directly in the Gawn Ice Piedmont Glacier ice core, aluminium (Al) in ice cores is a proxy for aeolian dust (Legrand and Mayewski, 1997; Bertler et al., 2005). Although there appears to be no seasonal cycle in $\mathrm{Al}$ in the Gawn Ice Piedmont Glacier record, the record contains large $\mathrm{Al}$ concentration peaks, in particular during summer 2005. These peaks are thought to arise from dust particles in the samples during measurement and not from soluble $\mathrm{Al}$ in the meltwater (Rhodes et al., 2011). It is interesting to note that the presence of dust particle(s) coincides during the elevated MS peak in 2005.

Chlorophyll-a concentrations for the SW Ross Sea are not anomalously high in 2005 suggesting that the areal extent of primary production represented by chlorophyll-a concentration (Black box Fig. 4.12) differs from that for MS concentration. The area is constrained largely by sea ice extent shown to be influenced by ENSO and iceberg persistence (Arrigo and van Dijken, 2004; Rhodes et al., 2009), but for example two years with a comparable areal extent are likely to respond more strongly to variability in other factors (e.g. micro and macro-nutrient limitations, light, water column stratification, grazing) that could lead to different total chlorophyll-a production. Furthermore, the transport of MS and how MS it is recorded in the ice core affects the MS record independently of MS production. Based on the trajectories to the Gawn Ice Piedoment Glacier (Fig. 4.11), two potential sources, the Ross Sea including the Terra Nova Bay and McMurdo Sound polynyas, and the Weddell Sea on the opposite side of the ice shelf, influence the MS concentration in the core differently and hence the MS record may not always be representative of the entire Ross Sea MS production during summer.

The disproportionate effect of ASD on surface chlorophyll- $a$ in McMurdo Sound may also reflect that in surface waters of McMurdo Sound and the SW Ross Sea that sustain large phytoplankton blooms in summer, the majority of Fe budget is likely delivered through oceanic (upwelling of deep water, resuspension of sediments, remineralisation of sinking material) and glacial (iceberg and glacial melt) pathways, as suggested in Table 4.2 and 4.3. Even if McMurdo Sound ASD may not cause proportionate increases in chlorophyll-a during major storm years, it could still influence the background chlorophyll-a concentration in McMurdo Sound and the SW Ross Sea. 
In contrast, the Mt Erebus Saddle record represents MS predominantly derived from the Ross Sea polnyna, even though the ASD record and MS data overlap only for 6 years. Annual mean MS concentration was regressed against chlorophyll- $a$. This analysis yielded a stronger correlation $\left(\mathrm{R}^{2}=0.76\right)$, which highlights the importance of understanding the air mass trajectories to an ice core site and confirms the potential of MS concentration and ASD accumulation rate as an indicator of primary productivity, whereby an increase in ASD accumulation rate leads to a proportional increases in both chlorophyll- $a$ and MS concentration as the areal extent of primary production is represented by both chlorophyll- $a$ and MS concentration.

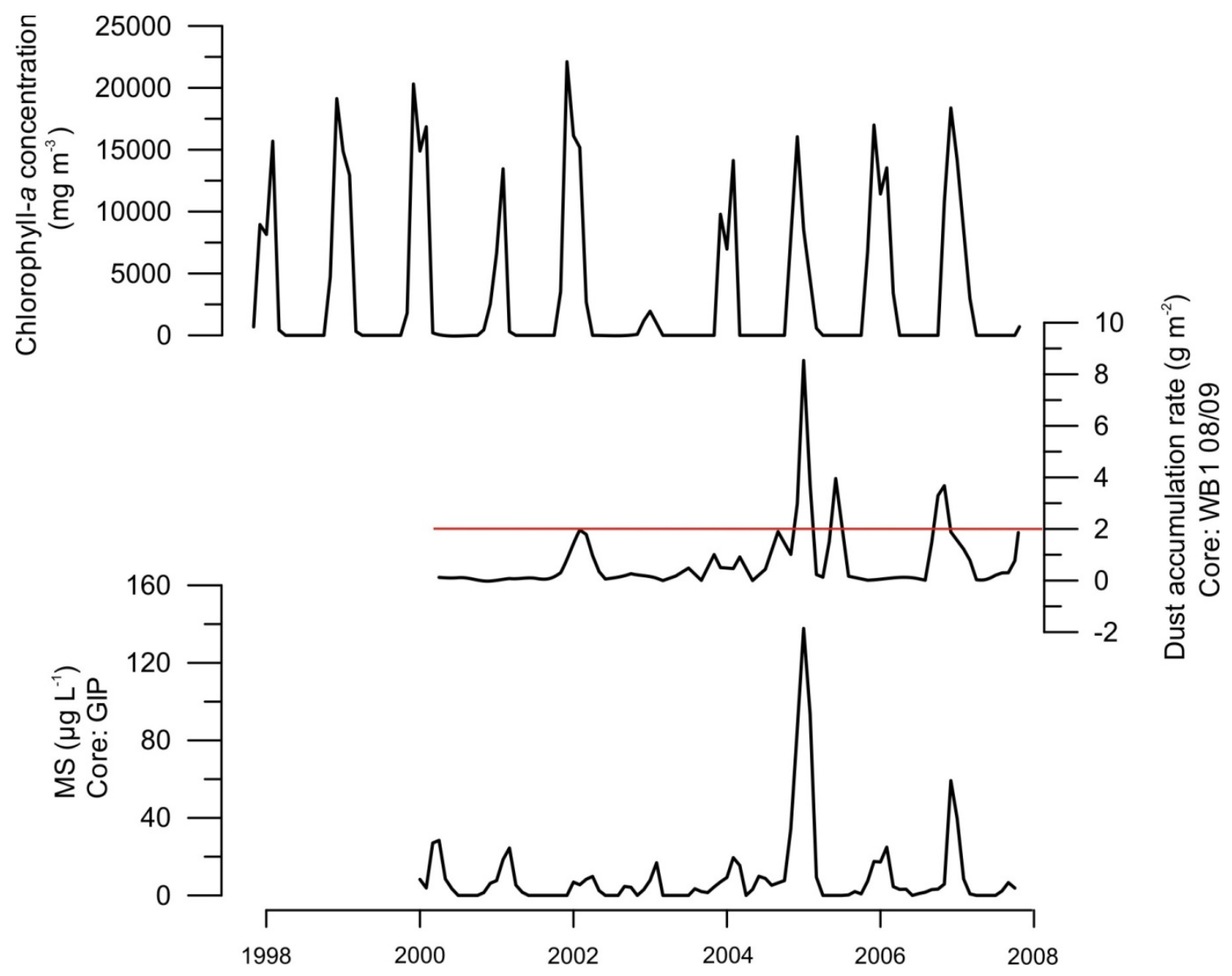

Fig. 4.13: Chlorophyll-a concentration, ASD accumulation rate and MS concentration seasonal records for the SW Ross Sea. Top: Chlorophyll-a concentration for the SW Ross Sea, acquired from SeaWiFS satellite data. Middle: ASD accumulation rate for the McMurdo Sound region during the past decade. Horizontal red line indicates an estimated threshold ASD accumulation rate needed to overcome Felimitation, via a locally derived aeolian dust source. Bottom: MS record (Markle, 2001) from Gawn Ice Piedmont Glacier, Ross Sea region, Antarctica. 


\subsubsection{Summary}

Although, Antarctic ice core climate records from the polar plateau consistently link low atmospheric $\mathrm{CO}_{2}$ levels with low air temperatures and high levels of Fe-rich global dust, the correlation between ASD accumulation rate and chlorophyll- $a$ concentrations in McMurdo Sound over the past 8 years are less significant in the records presented here. The understanding of the relationships between ASD, aeolian Fe supply, and primary productivity could be improved by investigating both the ASD and MS concentration records from numerous longer ice cores, that contain a history of thousands of years into the past, and a high resolution marine sediment record from McMurdo Sound to quantify the frequency and magnitude of storm events that are capable of triggering increased primary productivity.

Correlation does not necessarily equal causation. Hence, MS records from a new deep ice core from Roosevelt Island, eastern Ross Sea and also from a archived shallow firn core from Windless Bight could be used to test whether local MS data record the chlorophyll- $a$ concentrations representative of primary productivity over the entire Ross Sea and if so, to obtain the correlative function, which could be linear, threshold-driven, or some other relationship. Currently, the MS and chlorophyll- $a$ records in the present study and in Rhodes et al. (2009) are the only records of this kind for the SW Ross Sea area. Furthermore, the areal extent of chlorophyll-a, rather than the concentration over the total area, could be regressed with MS concentration and ASD. While this is only a short record (less than a decade), the discussed comparison shows potential for a causal relationship between MS concentration and ASD accumulation rate over longer records, especially during storm years, and the supply of aeolian Fe to the biogeochemical cycle in the SW Ross Sea. 


\subsection{Conclusion}

Local ASD investigated in the present study quantifies an exceptionally high Fe flux compared to recent estimates of global atmospheric Fe reaching Antarctica. An atmospheric Fe flux of $0.55 \mathrm{mg} \mathrm{m}^{-2} \mathrm{yr}^{-1}\left(9.89 \mu \mathrm{mol} \mathrm{m} \mathrm{mr}^{-2}\right)$ is estimated for the SW Ross Sea, highlighting the importance of locally-derived Fe ASD for the SW Ross Sea beyond the immediate local effect in McMurdo Sound. In the context of global change, as glaciers recede exposing greater areas of erodible ice-free substrate and wind speeds strengthen, the flux of locally derived aeolian Fe is also likely to change. Global change has the potential to have large impact not only on the flux of particulate Fe but also on the solubility of the Fe input to the ocean with implications for marine productivity and the carbon cycle.

The present study estimates that local ASD contributes up to $13 \%$ of Fe released by sea ice into the ocean. Locally-derived aeolian Fe is sufficient to stimulate blooms up to $1200 \mathrm{~km}$ from the source which includes all areas of open water in the SW Ross Sea. It is however difficult to trace the stimulation of seasonal phytoplankton blooms in the SW Ross Sea to local ASD or a different Fe source due to limited data for the Ross Sea. These results highlight the potentially important contribution of local ASD to the biogeochemical cycle in the SW Ross Sea marine ecosystem. They also reveal the importance of Fe deposition models taking local scale into consideration, when investigating large spatial areas such as the SO, as local ASD indicates that global estimates of aeolian Fe deposition are underestimated for the Ross Sea, an important region of $\mathrm{CO}_{2}$ sequestration and Antarctic Bottom Water formation. 


\section{Chapter 5}

\section{Conclusions and future work}

\subsection{Conclusions}

The overall aim of the present study was to determine the biological significance of the temporal and spatial variability of aeolian Fe deposition in McMurdo Sound, SW Ross Sea. To achieve this surface snow samples were collected from the sea ice in Southern McMurdo Sound during November 2009 as part of K131. In addition to these the following samples were used: three shallow firn cores recovered by Dr N. Bertler from the McMurdo Ice Shelf in November 2008, surface snow samples collected at Granite Harbour in November 2009 by Dr A. Aguilar Islas, and surface snow samples collected in McMurdo Sound in November 2010 by Dr C. Atkins. Equipment and methodology were developed to measure the physical (ASD accumulation rate, particle size) and geochemical (soluble and particulate Fe) properties of locally derived ASD. The conclusions to the objectives of this project are described below.

\section{What is the spatial and temporal variability of the ASD accumulation rate into McMurdo Sound over time?}

The mass and particle size of ASD from surface snow samples on the sea ice in McMurdo Sound show an exponential decrease in mass accumulation rate (from $26.07 \mathrm{~g} \mathrm{~m}^{-2} \mathrm{yr}^{-1}$ to 0.70 $\mathrm{g} \mathrm{m}^{-2} \mathrm{yr}^{-1}$ ) and a decrease in modal particle size (from 130 to $69 \mu \mathrm{m}$ ) over a distance of $120 \mathrm{~km}$ from Southern McMurdo Sound northwards to Granite Harbour. Both these trends are consistent with ASD being dispersed northwards across the sea ice by southerly storms from a single area of unconsolidated sediment known as the "debris band" in Southern McMurdo Sound. The Sr and Nd isotopic signature of ASD matching local source rocks and the presence of vesicular glass of Southern McMurdo Sound origin in all samples also point to the debris bands as the origin of ASD in McMurdo Sound.

Calculated threshold velocity values at the mean annual temperature indicate that sand will only be transported when winds exceed $4.50 \mathrm{~ms}^{-1}$. Southerly storm conditions in winter 
often exceed this threshold velocity and thus are strong enough to move sand-size particles in McMurdo Sound.

A simple physical ASD dispersal model suggests a background accumulation rate of $\sim 0.08$ $\mathrm{g} \mathrm{m}^{-2} \mathrm{yr}^{-1}$ (for particles $<10 \mu \mathrm{m}$ ) and $0.70 \mathrm{~g} \mathrm{~m}^{-2} \mathrm{yr}^{-1}$ (for the bulk ASD) is representative for the region at least $500 \mathrm{~km}$ from the debris band on the sea ice in McMurdo Sound. This equates to a linear sedimentation rate of $0.14 \mathrm{~cm} \mathrm{ky}^{-1}$ which suggests aeolian sand is not as important as previously thought for sedimentation in McMurdo Sound.

The ASD accumulation rate and particle size is also variable on inter-annual and interdecadal scales in McMurdo Sound, most notably in May 2004 when a severe storm deposited $\mathrm{ASD}$ at a rate $\sim 4$ times greater and significantly coarser in particle size than non stormy years.

2. How much bio-available Fe is supplied to McMurdo Sound by aeolian sand and dust?

McMurdo Sound offers a unique natural environment to examine the Fe solubility of locally derived ASD from a point source as it is largely free from anthropogenic aerosol emissions. Soluble Fe was analysed using a step-wise leaching method designed in the present study specifically for snow samples containing dust-rich material. The method development involved leaching a homogenous dust standard, Arizona test dust (ATD), with MQ water to test the influence of various parameters on the percentage of soluble $\mathrm{Fe}$ rendered from the dust. The outcome of a reproducible dust leaching method provided a baseline Fe solubility of $0.49 \%$ for ultra-fine particle-sized ATD ( $\mathrm{t}=1$ hour, leaching solution=180 ml MQ water) for comparison with McMurdo Sound ASD samples downwind from the debris bands.

Both the bulk and fine particle size fractions of ASD in McMurdo Sound are important for supplying soluble Fe. The bulk ASD although containing particles with a high settling velocity in the water column, renders the greatest soluble Fe concentration (dominated by Fe particles $<0.4 \mu \mathrm{m}$ and Fe leached from the surface of the ASD) during the first leach, while 
the fine fraction that resides in the surface waters in suspension for longer time periods, continues to leach Fe (from the surface of the ASD) during this time but at a slower rate.

Soluble Fe concentrations decreased exponentially from the debris band ranging from 0.79 to $0.03 \mu \mathrm{g} \mathrm{g}^{-1}$ with the lowest concentrations measured in samples from Granite Harbour, while particulate $\mathrm{Fe}$ concentrations were fairly homogenous and averaged 3.84 wt \%, consistent with a single ASD source of the debris bands. Excluding potentially contaminated samples, these values yield an average Fe solubility of $9.42 \pm 0.7 \%$ for McMurdo Sound dust. This is higher than typical values of mineral dust (estimated by a variety of leaching methods) and also the baseline value of ATD, but comparable to other estimates for Antarctic dust. Possible explanations for this high percentage of soluble Fe include the polar environment the ASD is exposed to in addition to the leaching method used. The homogenous percentage of soluble Fe with distance from the point source suggests provenance is the likely driver of $\mathrm{Fe}$ solubility in McMurdo Sound.

\section{What is the link between the aeolian sand and dust accumulation rate and phytoplankton blooms in the Southern McMurdo Sound?}

The background Fe flux derived from local ASD $(<10 \mu \mathrm{m})$ for an area $500 \mathrm{~km}$ north of the debris bands is $0.55 \mathrm{mg} \mathrm{m}^{-2} \mathrm{yr}^{-1}\left(9.89 \mu \mathrm{mol} \mathrm{m} \mathrm{yr}^{-1}\right)$. This is significantly greater, by at least an order of magnitude, than "global" atmospheric Fe reaching Antarctica. While the relative importance of locally-derived aeolian Fe, compared to other Fe sources, is constrained by the lack of available data for the Ross Sea, at the very least it contributes to the background Fe stock each season as the sea ice breaks up releasing the Fe-bearing dust to the ocean.

Locally-derived ASD is the most important aerosol source of Fe but is not necessarily the most important source of Fe to the SW Ross Sea (Table 4.2). A simple dust-biota model (Boyd et al., 2010) suggests that locally derived aeolian Fe during "background years" is sufficient overcome the Fe-limitation threshold in the SW Ross Sea and stimulate phytoplankton blooms. While the model suggests additional dust supplied to the water column during storm events, for example in 2004, does not produce sufficiently greater Fe 
concentrations in the surface waters, the importance of this additional Fe supply is over the greater distance storms are capable of transporting dust thereby extending the area of the SW Ross Sea that is supplied by aeolian Fe above the threshold value to stimulate phytoplankton blooms. These results highlight the potentially important contribution of ASD to the biogeochemical cycle beyond the immediate local effect in McMurdo Sound to the SW Ross Sea marine ecosystem.

Short, $\sim 10$ year long, firn core records of ASD mass accumulation and methylsuphonate concentration, a proxy for phytoplankton productivity in coastal regions, show a close correspondence between the two during particularly stormy years. Whilst not demonstrating a cause-and-effect relationship, this observation suggests deeper coastal ice cores may contain an important record of the interplay between climate, dust supply, Fe supply and phytoplankton productivity on decadal and longer timescales.

The primary interest in accurate estimates of aeolian Fe solubility is the assumption that the bio-availability of aeolian derived $\mathrm{Fe}$ in the surface ocean is related to its solubility, thereby relating Fe solubility to the carbon cycle. The present study indicates that locally derived ASD could be an important source of aeolian Fe for primary productivity in McMurdo Sound, SW Ross Sea region an important area of $\mathrm{CO}_{2}$ sequestration and Antarctic Bottom Water formation. 


\subsection{Suggestions for future work}

In addition to longer records of MS concentration and ASD accumulation rate for the Ross Sea region needed to better constrain the effect of local ASD on Fe-fertilisation, the following for future work is suggested to better understand the Fe solubility of ASD samples in McMurdo Sound:

- Determine how Fe speciation varies in McMurdo Sound ASD samples and how it influences Fe solubility (c.f. Lam et al., 2011; Schroth et al., 2009).

- Use $\mathrm{HCl}$ rather than $\mathrm{HNO}_{3}$ for all Fe solubility analytical work including the acid cleaning protocol and acidifying samples as this will render Fe in its most soluble form.

- Determine the percentage of soluble Fe from samples derived purely from MVG and SVL sources, as the relative influence of these two sources could help explain the variation in the percentage of soluble Fe in dust directly under the debris bands, at Marble Point and Granite Harbour sites. Time constraints prevented $\mathrm{Pb}$ isotopic analysis to determine precisely how the provenance of the ASD changes along the SVL coastline but this would also help determine if dust provenance drives the percentage of soluble Fe in McMurdo Sound.

- Photo-reduction experiments could be undertaken to determine if increased light exposure to ASD within a snow pack increases the percentage of soluble Fe on the dust surface. For example, ATD could be added to a seawater standard in a tank in a freezer laboratory to simulate a sea ice environment, and light exposure adjusted to replicate a polar versus non polar environment. Similar tanks have been used for albedo experiments in laboratory grown sea ice (e.g. Light et al., 2010). Furthermore, satellite irradiance data or analysis of inter-annual variations of ${ }^{10} \mathrm{Be}$ (c.f. Baroni et al., 2011) in Windless Bight firn cores could be investigated for the McMurdo Sound region and compared to ASD and Fe solubility measurements over the past decade to compare variations in irradiance with Fe solubility for those years. Fe solubility and irradiance could also be compared spatially from various geographic regions where daily light exposure times are variable, e.g. Antarctica, Australia, and the Sahara. 


\section{References}

ABRAM, N. J., MULVANEY, R., WOLFF, E. W. AND MUDELSEE, M. 2007. Ice core records as sea ice proxies: An evaluation from the Weddell Sea region of Antarctica. Journal of Geophysical Research, 112, D15101.

ACRONE, S., DELANEY, A. AND TOBIASSON, W. 1994. Subsurface radar investigations at the Pegasus GlacialIce Runway and Williams Field, McMurdo Station, Antarctica. CRREL report, 94-12.

AGUILAR-ISLAS, A. M., REMBER, R. D., MORDY, C. W. AND WU, J. 2008. Sea ice-derived dissolved iron and its potential influence on the spring algal bloom in the Bering Sea. Geophysical Research Letters, 35, L24601.

AGUILAR-ISLAS, A. M., WU, J., REMBER, R., JOHANSEN, A. M. AND SHANK, L. M. 2010. Dissolution of aerosol-derived iron in seawater: Leach solution chemistry, aerosol type, and colloidal iron fraction. Marine Chemistry, 120, 25-33.

ANDERSON, J. B., BRAKE, C. F. AND MYERS, N. C. 1984. Sedimentation on the Ross Sea continental shelf, Antarctica. Marine Geology, 57, 295-333.

ARCHER, D. E. AND JOHNSON, K. 2000. A model of the iron cycle in the ocean. Global Biogeochemical Cycles, $14,269-279$.

ARIMOTO, R. AND DUCE, R. A. 1986. Dry Deposition Models and the Air/Sea Exchange Of Trace Elements. Journal of Geophysical Research, 91, 2787-2792.

ARRIGO, K. R. AND MCCLAIN, C. R. 1994. Spring Phytoplankton Production in the Western Ross Sea. Science, 266, 261-263.

ARRIGO, K. R., WEISS, A. M. AND SMITH, W. O., JR. 1998a. Physical forcing of phytoplankton dynamics in the southwestern Ross Sea. Journal of Geophysical Research, 103, 1007-1021.

ARRIGO, K. R., WORTHEN, D., SCHNELL, A. AND LIZOTTE, M. P. 1998b. Primary production in Southern Ocean waters. Journal of Geophysical Research, 103, 15587-15600.

ARRIGO, K. R., DitUlliO, G. R., DUNBAR, R. B., ROBINSON, D. H., VANWOERT, M., WORTHEN, D. L. AND LIZOTTE, M. P. 2000. Phytoplankton taxonomic variability in nutrient utilization and primary production in the Ross Sea. Journal of Geophysical Research, 105, 8827-8846.

ARRIGO, K. R., DUNBAR, R. B., LIZOTTE, M. P. AND ROBINSON, D. H. 2002. Taxon-specific differences in C/P and N/P drawdown for phytoplankton in the Ross Sea, Antarctica. Geophysical Research Letters, 29, 1938.

ARRIGO, K. R., WORTHEN, D. L. AND ROBINSON, D. H. 2003. A coupled ocean-ecosystem model of the Ross Sea: 2. Iron regulation of phytoplankton taxonomic variability and primary production. Journal of Geophysical Research, 108, 3231.

ARRIGO, K. R. AND VAN DIJKEN, G. L. 2004. Annual changes in sea-ice, chlorophyll a, and primary production in the Ross Sea, Antarctica. Deep Sea Research Part II: Topical Studies in Oceanography, 51, 117-138.

ARRIGO, K. R. AND THOMAS, D. N. 2004. Large scale importance of sea ice biology in the Southern Ocean Antarctic Science 16, 471-486.

ARRIGO, K. R. AND VAN DIJKEN, G. L. 2007. Interannual variation in air-sea $\mathrm{CO}_{2}$ flux in the Ross Sea, Antarctica: A model analysis. Journal of Geophysical Research, 112, C03020. 
ARRIGO, K. R., VAN DIJKEN, G. AND LONG, M. 2008a. Coastal Southern Ocean: A strong anthropogenic CO2 sink. Geophysical Research Letters, 35, L21602.

ARRIGO, K. R., VAN DIJKEN, G. L. AND BUSHINSKY, S. 2008b. Primary production in the Southern Ocean, 1997-2006. Journal of Geophysical Research, 113, C08004.

ATKINS, C. B. AND DUNBAR, G. B. 2009. Aeolian sediment flux from sea ice into Southern McMurdo Sound, Antarctica. Global and Planetary Change, 69, 133-141.

AYLING, B. AND MCGOWAN, H. 2006. Niveo-eolian Sediment Deposits in Coastal South Victoria Land, Antarctica: Indicators of Regional Variability in Weather and Climate. Arctic, Antarctic, and Alpine Research, 38, 313-324.

BADAR, H., HAMILTION, W. AND BROWN 1965. Measurement of Natural particulate fallout onto high polar ice sheets. Part 1 Laboratory techniques and first results. Cold regions Research and Engineering Laboratory, Hanover.

BAGNOLD, R. 1941. The physics of blown sand and desert dunes, London, Methuen.

BAKER, A. R. AND JICKELLS, T. D. 2006. Mineral particle size as a control on aerosol iron solubility. Geophysical Research Letters, 33, L17608.

BAKER, A. R., JICKELLS, T. D., WITT, M. AND LINGE, K. L. 2006. Trends in the solubility of iron, aluminium, manganese and phosphorus in aerosol collected over the Atlantic Ocean. Marine Chemistry, 98, 43-58.

BAKER, A. R. AND CROOT, P. L. 2010. Atmospheric and marine controls on aerosol iron solubility in seawater. Marine Chemistry, 120, 4-13.

BAlCH, W. M., HOLligAN, P. M., ACKLESON, S. G. AND VOSS, K. J. 1991. Biological and Optical Properties of Mesoscale Coccolithophore Blooms in the Gulf of Maine. Limnology and Oceanography, 36, 629-643.

BANSE, K. 1991. Rates of Phytoplankton Cell Division in the Field and in Iron Enrichment Experiments. Limnology and Oceanography, 36, 1886-1898.

BARBANTE, C., BELlOMI, T., MEZZADRI, G., CESCON, P., SCARPONI, G., MOREL, C., JAY, S., VAN DE VELDE, K., FERRARI, C. AND BOUTRON, C. F. 1997. Direct Determination of Heavy Metals at Picogram per Gram Levels in Greenland and Antarctic Snow by Double Focusing Inductively Coupled Plasma Mass Spectrometry. Journal of Analytical Atomic Spectrometry, 12, 925-931.

BARBEAU, K., MOFFETT, J. W., CARON, D. A., CROOT, P. L. AND ERDNER, D. L. 1996. Role of protozoan grazing in relieving iron limitation of phytoplankton. Nature, 380, 61-64.

BARBEAU, K. A. AND MOFFETT, J. W. 1998. Dissolution of Iron Oxides by Phagotrophic Protist\$Jing a Novel Method to Quantify Reaction Rates. Environmental Science and Technology, 32, 2969-2975.

BARONI, M., BARD, E., PETIT, J.-R., MAGAND, O. AND BOURLES, D. 2011. Volcanic, Solar Activity, and Atmospheric Circulation Influences on Cosmogenic ${ }^{10} \mathrm{Be}$ Fallout at Vostok and Concordia (Antarctica) over the Last 60 Years. Mineralogical Magazine, 75, 3, 490.

BARRETT, P., GRINDLEY, G. AND WEBB, P. 1972. The Beacon Supergroup of East Antarctica. 319-312. In: ADIE, R. I. (ed.) Geology and Geophysics. Universitesforlaget, Oslo.

BARRETT, P. 1982. Immediate report of VUWAE 1981-82. Antarctic Research centre Wellington, Victoria University of Wellington. 
BARRETT, P., PYNE, A. AND WARD, B. 1983. Modern sedimentation in McMurdo Sound, Antarctica In: OLIVER, R.L., JAMES, P.R., JAGO, J.B. (ed.). Antarctic Earth Science. Canberra: Australian Academy of Sciences.

BARRETT, P.J., STOFFERS, P., GLASBY, G.P. AND PLUGER, W.L., 1984. Texture, mineralogy and composition of four sediment cores from Granite Harbour, southern Victoria Land, Antarctica. New Zealand Journal of Geology and Geophysics, 27:477-485.

BARRETT, P. 1986. Antarctic Cenozoic history from the MSSTS-1 drillhole, McMurdo Sound. In: Science information publishing editor (ed.) Bulletin 237. Wellington: Department of Scientific and Industrial Research.

BASILE, I., GROUSSET, F. E., REVEL, M., PETIT, J. R., BISCAYE, P. E. AND BARKOV, N. I. 1997. Patagonian origin of glacial dust deposited in East Antarctica (Vostok and Dome C) during glacial stages 2, 4 and 6. Earth and Planetary Science Letters, 146, 573-589.

BEHRENFELD, M. J., BALE, A. J., KOLBER, Z. S., AIKEN, J. AND FALKOWSKI, P. G. 1996. Confirmation of iron limitation of phytoplankton photosynthesis in the equatorial Pacific Ocean. Nature, 383, 508-511.

BENTLEY, P. 1979. Characteristics and distribution of wind blown sediments, Western McMurdo Sound, Antarctica. BSc Honours thesis, Victoria University of Wellington.

BERGQUIST, B. A., WU, J. AND BOYLE, E. A. 2007. Variability in oceanic dissolved iron is dominated by the colloidal fraction. Geochimica et Cosmochimica Acta, 71, 2960-2974.

BERTLER, N. A. N., MAYEWSKI, P. A., SNEED, S. B., NAISH, T. R., MORGENSTERN, U. AND BARRETT, P. J. 2005. Solar forcing recorded by aerosol concentrations in coastal Antarctic glacier ice, McMurdo Dry Valleys. Annals of Glaciology, 41, 52-56.

BERTLER, N. A. N., MAYEWSKI, P. A., BARRETT, P. J., SNEED, S. B., HANDLEY, M. J. AND KREUTZ, K. J. 2004. Monsoonal circulation of the McMurdo Dry Valleys, Ross Sea region, Antarctica: signal from the snow chemistry. Annals of Glaciology, 39, 139-145.

BETZER, P. R., CARDER, K. L., DUCE, R. A., MERRILl, J. T., TINDALE, N. W., UEMATSU, M., COSTELLO, D. K., YOUNG, R. W., FEELY, R. A., BRELAND, J. A., BERNSTEIN, R. E. AND GRECO, A. M. 1988. Longrange transport of giant mineral aerosol particles. Nature, 336, 568-571.

BISCAYE, P. E., GROUSSET, F. E., REVEL, M., VAN DER GAAST, S., ZIELINSKI, G. A., VAARS, A. AND KUKLA, G. 1997. Asian provenance of glacial dust (stage 2) in the Greenland Ice Sheet Project 2 Ice Core, Summit, Greenland. Journal of Geophysical Research, 102, 26765-26781.

BLAIN, S., QUEGUINER, B., ARMAND, L., BELVISO, S., BOMBLED, B., BOPP, L., BOWIE, A., BRUNET, C., BRUSSAARD, C., CARLOTTI, F., CHRISTAKI, U., CORBIERE, A., DURAND, I., EBERSBACH, F., FUDA, J.L., GARCIA, N., GERRINGA, L., GRIFFITHS, B., GUIGUE, C., GUILLERM, C., JACQUET, S., JEANDEL, C., LAAN, P., LEFEVRE, D., LO MONACO, C., MALITS, A., MOSSERI, J., OBERNOSTERER, I., PARK, Y.-H., PICHERAL, M., PONDAVEN, P., REMENYI, T., SANDRONI, V., SARTHOU, G., SAVOYE, N., SCOUARNEC, L., SOUHAUT, M., THUILLER, D., TIMMERMANS, K., TRULL, T., UITZ, J., VAN BEEK, P., VELDHUIS, M., VINCENT, D., VIOLLIER, E., VONG, L. AND WAGENER, T. 2007. Effect of natural iron fertilization on carbon sequestration in the Southern Ocean. Nature, 446, 1070-1074.

BLAIN, S., TREGUER, P., BELVISO, S., BUCCIARELlI, E., DENIS, M., DESABRE, S., FIALA, M., MARTINJEZEQUEL, V., LE FEVRE, J. AND MAYZAUD, P. 2001. A biogeochemical study of the island mass effect in the context of the iron hypothesis: Kerguelen Islands, Southern Ocean. Deep-Sea Research, 48, 163 - 187.

BLANK, H., COOPER, A., WHEELER, R. AND WILLIS, I. 1963. Geology of the Koettlitz-Blue Glacier Region, south Victoria Land, Antarctica, Trans. Roy. Soc. NZ. Geology, 2, 79-100. 
BONNET, S. AND GUIEU, C. 2004. Dissolution of atmospheric iron in seawater. Geophysical Research Letters, 31, L03303.

BORY, A. J. M., BISCAYE, P. E., SVENSSON, A. AND GROUSSET, F. E. 2002. Seasonal variability in the origin of recent atmospheric mineral dust at North GRIP, Greenland. Earth and Planetary Science Letters, 196, 123134.

BOUTRON, C. F. AND PATTERSON, C. C. 1983. The occurrence of lead in Antarctic recent snow, firn deposited over the last two centuries and prehistoric ice. Geochimica et Cosmochimica Acta, 47, 1355-1368.

BOWIE, A. R., LANNUZEL, D., REMENYI, T. A., WAGENER, T., LAM, P. J., BOYD, P. W., GUIEU, C., TOWNSEND, A. T. AND TRULL, T. W. 2009. Biogeochemical iron budgets of the Southern Ocean south of Australia: Decoupling of iron and nutrient cycles in the subantarctic zone by the summertime supply. Global Biogeochemical Cycles, 23, GB4034.

BOWIE, A. R., MALDONADO, M. T., FREW, R. D., CROOT, P. L., ACHTERBERG, E. P., MANTOURA, R. F. C., WORSFOLD, P. J., LAW, C. S. AND BOYD, P. W. 2001. The fate of added iron during a mesoscale fertilisation experiment in the Southern Ocean. Deep Sea Research Part II: Topical Studies in Oceanography, 48, 2703-2743.

BOYD, P. W. AND ELLWOOD, M. J. 2010. The biogeochemical cycle of iron in the ocean. Nature Geoscience, 3, 675-682.

BOYD, P. W., MACKIE, D. S. AND HUNTER, K. A. 2010. Aerosol iron deposition to the surface ocean -- Modes of iron supply and biological responses. Marine Chemistry, 120, 128-143.

BOYD, P. AND MACKIE, D. 2008. Comment on 'The Southern Ocean biological response to aeolian iron deposition'. Science, 319, 159.

BOYD, P. W., JICKELLS, T., LAW, C. S., BLAIN, S., BOYLE, E. A., BUESSELER, K. O., COALE, K. H., CULLEN, J. J., DE BAAR, H. J. W., FOLLOWS, M., HARVEY, M., LANCELOT, C., LEVASSEUR, M., OWENS, N. P. J., POLLARD, R., RIVKIN, R. B., SARMIENTO, J., SCHOEMANN, V., SMETACEK, V., TAKEDA, S., TSUDA, A., TURNER, S. AND WATSON, A. J. 2007. Mesoscale Iron Enrichment Experiments 1993-2005: Synthesis and Future Directions. Science, 315, 612-617.

BOYD, P. W., LAW, C. S., HUTCHINS, D. A., ABRAHAM, E. R., CROOT, P. L., ELLWOOD, M., FREW, R. D., HADFIELD, M., HALL, J., HANDY, S., HARE, C., HIGGINS, J., HILL, P., HUNTER, K. A., LEBLANC, K., MALDONADO, M. T., MCKAY, R. M., MIONI, C., OLIVER, M., PICKMERE, S., PINKERTON, M., SAFI, K., SANDER, S., SANUDO-WILHELMY, S. A., SMITH, M., STRZEPEK, R., TOVAR-SANCHEZ, A. AND WILHELM, S. W. 2005. FeCycle: Attempting an iron biogeochemical budget from a mesoscale SF6 tracer experiment in unperturbed low iron waters. Global Biogeochemical Cycles, 19, GB4S20.

BOYD, P. W., MCTAINSH, G., SHERLOCK, V., RICHARDSON, K., NICHOL, S., ELLWOOD, M. AND FREW, R. 2004. Episodic enhancement of phytoplankton stocks in New Zealand subantarctic waters: Contribution of atmospheric and oceanic iron supply. Global Biogeochemical Cycles, 18, GB1029.

BOYD, P. W. 2002. The role of iron in the biogeochemistry of the Southern Ocean and equatorial Pacific: a comparison of in situ iron enrichments. Deep Sea Research Part II: Topical Studies in Oceanography, 49, 18031821.

BOYD, P. W. \& ABRAHAM, E. R. 2001. Iron-mediated changes in phytoplankton photosynthetic competence during SOIREE. Deep Sea Research Part II: Topical Studies in Oceanography, 48, 2529-2550.

BOYD, P. W. AND LAW, C. S. 2001. The Southern Ocean Iron RElease Experiment (SOIREE)--introduction and summary. Deep Sea Research Part II: Topical Studies in Oceanography, 48, 2425-2438. 
BOYD, P. W., WATSON, A. J., LAW, C. S., ABRAHAM, E. R., TRULL, T., MURDOCH, R., BAKKER, D. C. E., BOWIE, A. R., BUESSELER, K. O., CHANG, H., CHARETTE, M., CROOT, P., DOWNING, K., FREW, R., GALL, M., HADFIELD, M., HALL, J., HARVEY, M., JAMESON, G., LAROCHE, J., LIDDICOAT, M., LING, R., MALDONADO, M. T., MCKAY, R. M., NODDER, S., PICKMERE, S., PRIDMORE, R., RINTOUL, S., SAFI, K., SUTTON, P., STRZEPEK, R., TANNEBERGER, K., TURNER, S., WAITE, A. AND ZELDIS, J. 2000. A mesoscale phytoplankton bloom in the polar Southern Ocean stimulated by iron fertilization. Nature, 407, 695-702.

BOYD, P. W., WONG, C. S., MERRILL, J., WHITNEY, F., SNOW, J., HARRISON, P. J. AND GOWER, J. 1998. Atmospheric iron supply and enhanced vertical carbon flux in the NE subarctic Pacific: Is there a connection? Global Biogeochemical Cycles, 12, 429-441.

BOYE, M., VAN DEN BERG, C. M. G., DE JONG, J. T. M., LEACH, H., CROOT, P. AND DE BAAR, H. J. W. 2001. Organic complexation of iron in the Southern Ocean. Deep Sea Research Part I: Oceanographic Research Papers, 48, 1477-1497.

BRIERLEY, A. S. \& THOMAS, D. N. 2002. Ecology of Southern Ocean pack ice. Advances in Marine Biology. Academic Press, 43, 171-IN4.

BROECKER, W. S. \& HENDERSON, G. M. 1998. The Sequence of Events Surrounding Termination II and their Implications for the Cause of Glacial-Interglacial CO2 Changes. Paleoceanography, 13, 352-364.

BROMWICH, D. AND PARISH, T. 1998. Meteorology of the Antarctic. Meteorological Monographs, 49, 175-200.

BROMWICH, D. H., CARRASCO, J. F. AND STEARNS, C. R. 1992. Satellite Observations of Katabatic-Wind Propagation for Great Distances across the Ross Ice Shelf. Monthly Weather Review, 120, 1940-1949.

BROMWICH, D. H. AND KURTZ, D. D. 1984. KATABATIC WIND FORCING OF THE TERRA NOVA BAY POLYNYA. Journal of Geophysical Research, 89, 3561-3572.

BUCCIARELLI, E., BLAIN, S. AND TRÉGUER, P. 2001. Iron and manganese in the wake of the Kerguelen Islands (Southern Ocean). Marine Chemistry, 73, 21-36.

BUCK, C. S., LANDING, W. M. AND RESING, J. A. 2010a. Particle size and aerosol iron solubility: A highresolution analysis of Atlantic aerosols. Marine Chemistry, 120, 14-24.

BUCK, C. S., LANDING, W. M., RESING, J. A. AND MEASURES, C. I. 2010b. The solubility and deposition of aerosol $\mathrm{Fe}$ and other trace elements in the North Atlantic Ocean: Observations from the A16N CLIVAR/CO2 repeat hydrography section. Marine Chemistry, 120, 57-70.

BUCK, C. S., LANDING, W. M., RESING, J. A. AND LEBON, G. T. 2006. Aerosol iron and aluminium solubility in the northwest Pacific Ocean: Results from the 2002 IOC cruise. Geochemistry, Geophysics, Geosystems, 7 , Q04M07.

BUESSELER, K. O., BARBER, R. T., DICKSON, M.-L., HISCOCK, M. R., MOORE, J. K. AND SAMBROTTO, R. 2003. The effect of marginal ice-edge dynamics on production and export in the Southern Ocean along $170^{\circ} \mathrm{W}$. Deep Sea Research Part II: Topical Studies in Oceanography, 50, 579-603.

BULL, J. 2009. Stable isotope, major and trace element chemistry of modern snow from Evans Piedmont Glacier, Antarctica: insights into potential source regions and relationship of glaciochemistry to atmospheric circulation and vigour. MSc thesis, Victoria University of Wellington.

BUNT, J. S. 1963. Microbiology of Antarctic Sea-ice: Diatoms of Antarctic Sea-ice as Agents of Primary Production. Nature, 199, 1255-1257.

BUNT, J. S. AND WOOD, E. J. F. 1963. Microbiology of Antarctic Sea-ice: Microalgae and Antarctic Sea-ice. Nature, 199, 1254-1255. 
BUNT, J. S. AND LEE, C. 1970. Seasonal primary production in Antarctic sea ice at McMurdo Sound. Journal of Marine Research, 28, 304-320.

BURROUGH, P. 1986. Principles of Geographical Information Systems for Land Resources Assessment, New York, Oxford University Press.

CAMPBELL, J. AND CLARIDGE, G. 1987. Antarctica: Soils, Weathering Processes and Environment. Developments in Soil Science 16, Elsevier.

CARRASCO, J. F., BROMWICH, D. H. AND MONAGHAN, A. J. 2003. Distribution and Characteristics of Mesoscale Cyclones in the Antarctic: Ross Sea Eastward to the Weddell Sea. Monthly Weather Review, 131, 289301.

CASSAR, N., BENDER, M. L., BARNETT, B. A., FAN, S., MOXIM, W. J., LEVY, H. AND TILBROOK, B. 2008. Response to Comment on "The Southern Ocean Biological Response to Aeolian Iron Deposition". Science, $319,159$.

CASSAR, N., BENDER, M. L., BARNETT, B. A., FAN, S., MOXIM, W. J., LEVY, H. AND TILBROOK, B. 2007. The Southern Ocean Biological Response to Aeolian Iron Deposition. Science, 317, 1067-1070.

CHEN, Y. AND SIEFERT, R. L. 2004. Seasonal and spatial distributions and dry deposition fluxes of atmospheric total and labile iron over the tropical and subtropical North Atlantic Ocean. Journal of Geophysical Research, 109, D09305.

CHEN, Y. AND SIEFERT, R. L. 2003. Determination of various types of labile atmospheric iron over remote oceans. Journal of Geophysical Research, 108, 4774.

CHEN, M., DEI, R., WANG, W.-X. AND GUO, L. 2003. Marine diatom uptake of iron bound with natural colloids of different origins. Marine Chemistry, 81, 177 - 189.CLARIDGE, G. G. C., AND I. B. CAMPBELL, 1977. The salts in Antarctic soils, their distribution and relationship to soil processes, Soil Science, 123, 377- 384.

CHUANG, P. Y., DUVALL, R. M., SHAFER, M. M. \& SCHAUER, J. J. 2005. The origin of water soluble particulate iron in the Asian atmospheric outflow, Journal of Geophysical Research, 32, L07813.

COALE, K. H., MICHAEL GORDON, R. AND WANG, X. 2005. The distribution and behavior of dissolved and particulate iron and zinc in the Ross Sea and Antarctic circumpolar current along $170^{\circ} \mathrm{W}$. Deep Sea Research Part I: Oceanographic Research Papers, 52, 295-318.

COALE, K. H., JOHNSON, K. S., CHAVEZ, F. P., BUESSELER, K. O., BARBER, R. T., BRZEZINSKI, M. A., COCHLAN, W. P., MILLERO, F. J., FALKOWSKI, P. G., BAUER, J. E., WANNINKHOF, R. H., KUDELA, R. M., ALTABET, M. A., HALES, B. E., TAKAHASHI, T., LANDRY, M. R., BIDIGARE, R. R., WANG, X., CHASE, Z., STRUTTON, P. G., FRIEDERICH, G. E., GORBUNOV, M. Y., LANCE, V. P., HILTING, A. K., HISCOCK, M. R., DEMAREST, M., HISCOCK, W. T., SULLIVAN, K. F., TANNER, S. J., GORDON, R. M., HUNTER, C. N., ELROD, V. A., FITZWATER, S. E., JONES, J. L., TOZZI, S., KOBLIZEK, M., ROBERTS, A. E., HERNDON, J., BREWSTER, J., LADIZINSKY, N., SMITH, G., COOPER, D., TIMOTHY, D., BROWN, S. L., SELPH, K. E., SHERIDAN, C. C., TWINING, B. S. AND JOHNSON, Z. I. 2004. Southern Ocean Iron Enrichment Experiment: Carbon Cycling in High- and Low-Si Waters. Science, 304, 408-414.

COLliER, R., DYMOND, J., HONJO, S., MANGANINI, S., FRANCOIS, R. AND DUNBAR, R. 2000. The vertical flux of biogenic and lithogenic material in the Ross Sea: moored sediment trap observations 1996-1998. Deep Sea Research Part II: Topical Studies in Oceanography, 47, 3491-3520.

CONNOLLEY, W. AND CATTLE, H. 1994. Antarctic Climate of the UKMO Unified Model. Antarctic Science, 6, 115-122. 
CROOT, P. L., LAAN, P., NISHIOKA, J., STRASS, V., CISEWSKI, B., BOYE, M., TIMMERMANS, K. R., BELlERBY, R. G., GOLDSON, L., NIGHTINGALE, P. AND DE BAAR, H. J. W. 2005. Spatial and temporal distribution of $\mathrm{Fe}(\mathrm{II})$ and $\mathrm{H} 2 \mathrm{O} 2$ during EisenEx, an open ocean mescoscale iron enrichment. Marine Chemistry, 95, 65-88.

CROOT, P. L., STREU, P. AND BAKER, A. R. 2004. Short residence time for iron in surface seawater impacted by atmospheric dry deposition from Saharan dust events. Geophysical Research Letters, 31, L23S08.

CROOT, P. L. AND LAAN, P. 2002. Continuous shipboard determination of Fe(II) in polar waters using flow injection analysis with chemiluminescence detection. Analytica Chimica Acta, 466, 261-273.

CROOT, P. L., BOWIE, A. R., FREW, R. D., MALDONADO, M. T., HALL, J. A., SAFI, K. A., LA ROCHE, J.,

BOYD, P. W. AND LAW, C. S. 2001. Retention of dissolved iron and Fe II in an iron induced Southern Ocean phytoplankton bloom. Geophysical Research Letters, 28, 3425-3428.

CROOT, P. L. AND HUNTER, K. A. 1998. Trace metal distributions across the continental shelf near Otago Peninsula, New Zealand. Marine Chemistry, 62, 185-201.

CURRAN, M. A. J., VAN OMMEN, T. D., MORGAN, V. I., PHILlIPS, K. L. AND PALMER, A. S. 2003. Ice Core Evidence for Antarctic Sea Ice Decline Since the 1950s. Science, 302, 1203-1206.

CWIERTNY, D. M., BALTRUSAITIS, J., HUNTER, G. J., LASKIN, A., SCHERER, M. M. AND GRASSIAN, V. H. 2008a. Characterization and acid-mobilization study of iron-containing mineral dust source materials. Journal of Geophysical Research, 113, D05202.

CWIERTNY, D. M., YOUNG, M. A. AND GRASSIAN, V. H. 2008b. Chemistry and Photochemistry of Mineral Dust Aerosol. Annual Review of Physical Chemistry, 59, 27-51.

DAMIANI, D. AND GIORGETTI, G. 2008. Provenance of glacial-marine sediments under the McMurdo/Ross Ice Shelf (Windless Bight, Antarctica): Heavy minerals and geochemical data. Palaeogeography, Palaeoclimatology, Palaeoecology, 260, 262-283.

DANSGAARD, W. 1954. The ${ }^{18} \mathrm{O}$ abundance in fresh water. Geochimica et Cosmochimica Acta 6, 241-260.

DE BAAR, H. J. W., BOYD, P. W., COALE, K. H., LANDRY, M. R., TSUDA, A., ASSMY, P., BAKKER, D. C. E., BOZEC, Y., BARBER, R. T., BRZEZINSKI, M. A., BUESSELER, K. O., BOYÉ, M., CROOT, P. L., GERVAIS, F., GORBUNOV, M. Y., HARRISON, P. J., HISCOCK, W. T., LAAN, P., LANCELOT, C., LAW, C. S., LEVASSEUR, M., MARCHETTI, A., MILLERO, F. J., NISHIOKA, J., NOJIRI, Y., VAN OIJEN, T., RIEBESELL, U., RIJKENBERG, M. J. A., SAITO, H., TAKEDA, S., TIMMERMANS, K. R., VELDHUIS, M. J. W., WAITE, A. M. AND WONG, C.-S. 2005. Synthesis of iron fertilization experiments: From the Iron Age in the Age of Enlightenment. Journal of Geophysical Research, 110, C09S16.

DE BAAR, H. AND DE JONG, J. 2001. Distribution, sources and sinks of iron in seawater. The Biogeochemistry of Iron in Seawater, 123 - 253.

DE BAAR, H. J. W. AND BOYD, P. 2000. The role of iron in plankton ecology and carbon dioxide transfer of the global oceans, in The Dynamic Ocean Carbon Cycle: A Midterm Synthesis of the Joint Global Ocean Flux Study, New York, Cambridge Univ. Press.

DE BAAR, H. J. W., DE JONG, J. T. M., BAKKER, D. C. E., LOSCHER, B. M., VETH, C., BATHMANN, U. AND SMETACEK, V. 1995. Importance of iron for plankton blooms and carbon dioxide drawdown in the Southern Ocean. Nature, 373, 412-415.

DE BAAR, H., BUMA, A., NOLTIN, R., CADEE, G., JAQUES, G. AND TREGUER, P. 1990. On iron limitation of the Southern Ocean: experimental observations in the Weddell and Scotia seas. Marine Ecology Progress Series, $65,105-122$. 
DEBENHAM, F., 1920. A new mode of transportation by ice: The raised marine muds of South Victoria Land. Quarterly Journal of the Geological Society of London, 75, 51-76.

DeDECKKER, P., NORMAN, M., GOODWIN, I. D., WAIN, A. AND GINGELE, F. X. 2010. Lead isotopic evidence for an Australian source of aeolian dust to Antarctica at times over the last 170,000 years. Palaeogeography, Palaeoclimatology, Palaeoecology, 285, 205-223.

DELMONTE, B., BARONI, C., ANDERSSON, P. S., SCHOBERG, H., HANSSON, M., ACIEGO, S., PETIT, J.R., ALBANI, S., MAZZOLA, C., MAGGI, V. AND FREZZOTTI, M. 2010a. Aeolian dust in the Talos Dome ice core (East Antarctica, Pacific/Ross Sea sector): Victoria Land versus remote sources over the last two climate cycles. Journal of Quaternary Science, 25, 1327-1337.

DELMONTE, B., ANDERSSON, P. S., SCHÖBERG, H., HANSSON, M., PETIT, J. R., DELMAS, R., GAIERO, D. M., MAGGI, V. AND FREZZOTTI, M. 2010b. Geographic provenance of aeolian dust in East Antarctica during Pleistocene glaciations: preliminary results from Talos Dome and comparison with East Antarctic and new Andean ice core data. Quaternary Science Reviews, 29, 256-264.

DELMONTE, B., ANDERSSON, P. S., HANSSON, M., SCHÖBERG, H., PETIT, J. R., BASILE-DOELSCH, I. AND MAGGI, V. 2008. Aeolian dust in East Antarctica (EPICA-Dome C and Vostok): Provenance during glacial ages over the last 800 kyr. Geophysical Research Letters, 35, L07703.

DELMONTE, B., BASILE-DOELSCH, I., PETIT, J. R., MAGGI, V., REVEL-ROLLAND, M., MICHARD, A., JAGOUTZ, E. AND GROUSSET, F. 2004a. Comparing the Epica and Vostok dust records during the last 220,000 years: stratigraphical correlation and provenance in glacial periods. Earth-Science Reviews, 66, 63-87.

DELMONTE, B., PETIT, J. R., ANDERSEN, K. K., BASILE-DOELSCH, I., MAGGI, V. AND YA LIPENKOV, V. 2004b. Dust size evidence for opposite regional atmospheric circulation changes over east Antarctica during the last climatic transition. Climate Dynamics, 23, 427-438.

DELMONTE, B. 2003. Quaternary variations and origin of continental dust in East Antarctica PhD thesis, Università degli Studi di Milano-Bicocca.

DELMONTE, PETIT AND MAGGI 2002. Glacial to Holocene implications of the new 27000-year dust record from the EPICA Dome C (East Antarctica) ice core. Climate Dynamics, 18, 647-660.

DENTON, G. H. AND MARCHANT, D. R. 2000. The Geologic Basis for a Reconstruction of a Grounded Ice Sheet in McMurdo Sound, Antarctica, at the Last Glacial Maximum. Geografiska Annaler: Series A, Physical Geography, 82, 167-211.

DEPAOLO, D. J. AND WASSERBURG, G. J. 1976. Inferences about magma sources and mantle structure from variations of 143Nd/144Nd. Geophysical Research Letters, 3, 743-746.

DESBOEUFS, K. V., SOFIKITIS, A., LOSNO, R., COLIN, J. L. \& AUSSET, P. 2005. Dissolution and solubility of trace metals from natural and anthropogenic aerosol particulate matter. Chemosphere, 58, 195-203.

DICK, A. L. 1991. Concentrations and sources of metals in the Antarctic Peninsula aerosol. Geochimica et Cosmochimica Acta, 55, 1827-1836.

DICK, A. L. AND PEEL, A. 1985. Trace Elements in Antarctic Air And Snowfall. Annals of Glaciology, 7, 12-19.

DIECKMANN, G. S. \& HELLMER, H. H. 2008. The Importance of Sea Ice: An Overview. Sea Ice. Blackwell Science Ltd.

Ditullio, G. R., Geesey, M. E., JONES, D. R., DAly, K. L., CAMPBell, L. \& JR, W. O. S. 2003. Phytoplankton assemblage structure and primary productivity along $170^{\circ} \mathrm{W}$ in the South Pacific Ocean. Marine Ecology Progress Series, 255, 55-80. 
DiTULLIO, G. R. \& SMITH JR, W. O. 1995. Relationship between dimethylsulfide and phytoplankton pigment concentrations in the Ross Sea, Antarctica. Deep Sea Research Part I: Oceanographic Research Papers, 42, 873892.

DiTULLIO, G. R. AND LAWS, E. A. 1991. Impact of an atmospheric-oceanic disturbance on phytoplankton community dynamics in the North Pacific Central Gyre. Deep Sea Research Part A. Oceanographic Research Papers, 38, 1305-1329.

DOMACK, E. W., JACOBSON, E. A., SHIPP, S. AND ANDERSON, J. B. 1999. Late Pleistocene-Holocene retreat of the West Antarctic Ice-Sheet system in the Ross Sea: Part 2-Sedimentologic and stratigraphic signature. Geological Society of America Bulletin, 111, 1517-1536.

DONAGHAY, P., LISS, P., DUCE, R. A., KESTER, D., HANSON, A., VILLAREAL, T., TINDALE, N. W. AND GIFFORD, D. 1991. The role of episodic atmospheric nutrient input in the chemical and biological dynamics of ocean ice ecosystems. Oceanography, 4, 62-70.

DORAN, P. T., MCKAY, C. P., CLOW, G. D., DANA, G. L., FOUNTAIN, A. G., NYLEN, T. AND LYONS, W. B. 2002. Valley floor climate observations from the McMurdo Dry Valleys, Antarctica, 1986-2000. Journal of Geophysical Research, 107, 4772.

DRINKWATER, M. R. 1989. LIMEX '87 ice surface characteristics: implications for band SAR backscatter signatures. Geoscience and Remote Sensing, 27, 501-513.

DUCE, R. AND TINDALE, N. 1991. The atmospheric transport of iron and its deposition in the ocean. Limnology and Oceanography, 36, 1715 - 1726.

DUCE, R. A., LISS, P. S., MERRILL, J. T., ATLAS, E. L., BUAT-MENARD, P., HICKS, B. B., MILLER, J. M., PROSPERO, J. M., ARIMOTO, R., CHURCH, T. M., ELLIS, W., GALLOWAY, J. N., HANSEN, L., JICKELLS, T. D., KNAP, A. H., REINHARDT, K. H., SCHNEIDER, B., SOUDINE, A., TOKOS, J. J., TSUNOGAI, S., WOLLAST, R. AND ZHOU, M. 1991. The atmospheric input of trace species to the world ocean. Global Biogeochemical Cycles, 5, 193-259.

DUNBAR, G. B., BERTLER, N. A. N. AND MCKAY, R. M. 2009. Sediment flux through the McMurdo Ice Shelf in Windless Bight, Antarctica. Global and Planetary Change, 69, 87-93.

DUNBAR, R. B., LEVENTER, A. R. AND STOCKTON, W. L. 1989. Biogenic sedimentation in McMurdo Sound, Antarctica. Marine Geology, 85, 155-179.

DUNBAR, R., ANDERSON, J. AND DOMACK, E. 1985. Oceanographic influences on sedimentation along the Antarctic continental shelf. Antarctic Journal of the United States, 19, 71-73.

EDWARDS, R. AND SEDWICK, P. 2001. Iron in East Antarctic snow: Implications for atmospheric iron deposition and algal production in Antarctic waters. Geophysical Research Letters, 28, 3907-3910.

ELROD, V., BERELSON, W., COALE, K. AND JOHNSON, K. 2004. The flux of iron from continental shelf sediments: a missing source for global budgets. Geophysical Research Letters, 31, L12307.

FALCONER, T. AND PYNE, A. 2004. Sea ice breakout history in southern McMurdo Sound, Antarctica (19882002). Antarctic Data Series 27.

FAN, S.-M., MOXIM, W. AND LEVY, H. 2006. Aeolian input of bioavailable iron to the ocean. Geophysical Research Letters, 33, L07602.

FITZWATER, S. E., JOHNSON, K. S., GORDON, R. M., COALE, K. H. AND SMITH, W. O. 2000. Trace metal concentrations in the Ross Sea and their relationship with nutrients and phytoplankton growth. Deep Sea Research Part II: Topical Studies in Oceanography, 47, 3159-3179. 
FOLK, R. L. 1980. Petrology of sedimentary rocks. Austin, Texas, Hemphill Pub. Co.

FREW, R. D., HUTCHINS, D. A., NODDER, S., SANUDO-WILHELMY, S., TOVAR-SANCHEZ, A., LEBLANC, K., HARE, C. E. AND BOYD, P. W. 2006. Particulate iron dynamics during FeCycle in subantarctic waters southeast of New Zealand. Global Biogeochemical Cycles, 20, GB1S93.

FU, H., CWIERTNY, D. M., CARMICHAEL, G. R., SCHERER, M. M. AND GRASSIAN, V. H. 2010. Photoreductive dissolution of Fe-containing mineral dust particles in acidic media. Journal of Geophysical Research, 115, D11304.

FUNG, I., MEYN, S., TEGAN, I., DONEY, S., JOHN, J. AND BISHOP, J. 2000. Iron supply and demand in the upper ocean. Global Biogeochemical Cycles, 14, 281 - 295.

GAO, Y., FAN, S.-M. AND SARMIENTO, J. L. 2003. Aeolian iron input to the ocean through precipitation scavenging: A modeling perspective and its implication for natural iron fertilization in the ocean. Journal of Geophysical Research, 108, 4221.

GAO, Y., KAUFMAN, Y. J., TANRÉ, D., KOLBER, D. AND FALKOWSKI, P. G. 2001. Seasonal distributions of aeolian iron fluxes to the global ocean. Geophysical Research Letters, 28, 29-32.

GASPARI, V., BARBANTE, C., COZZI, G., CESCON, P., BOUTRON, C. F., GABRIELLI, P., CAPODAGLIO, G., FERRARI, C., PETIT, J. R. AND DELMONTE, B. 2006. Atmospheric iron fluxes over the last deglaciation: Climatic implications. Geophysical Research Letters, 33, L03704.

GIBBS,R., MATHEWS, M. AND UNK, D. 1971. The relationship between sphere size and settling velocity. Journal of Sedimentary Petrology, 41, 1, 7-18.

GILLETTE, D. 1979. Environmental factors affecting dust emission by wind erosion, in Saharan Dust, Hoboken, John Wiley.

GINOUX, P., PROSPERO, J. M., TORRES, O. AND CHIN, M. 2004. Long-term simulation of global dust distribution with the GOCART model: correlation with North Atlantic Oscillation. Environmental Modelling and Software, 19, 113-128.

GLACCUM, R. A. AND PROSPERO, J. M. 1980. Saharan aerosols over the tropical North Atlantic - Mineralogy. Marine Geology, 37, 295-321.

GLEDHILL, M. 2007. The determination of heme b in marine phyto- and bacterioplankton. Marine Chemistry, 103, 393-403.

GLEDHILl, M., MCCORMACK, P., USSHER, S., ACHTERBERG, E. P., MANTOURA, R. F. C. AND WORSFOLD, P. J. 2004. Production of siderophore type chelates by mixed bacterioplankton populations in nutrient enriched seawater incubations. Marine Chemistry, 88, 75-83.

GOFFART, A., CATALANO, G. AND HECQ, J. H. 2000. Factors controlling the distribution of diatoms and Phaeocystis in the Ross Sea. Journal of Marine Systems, 27, 161-175.

GOLDICH, S. S., TREVES, S. B., SUHR, N. H. AND STUCKLESS, J. S. 1975. Geochemistry of the Cenozoic Volcanic Rocks of Ross Island and Vicinity, Antarctica. The Journal of Geology, 83, 415-435.

GOMES, L., BERGAMETTI, G., COUDÉ-GAUSSEN, G. AND ROGNON, P. 1990. Submicron Desert Dusts: A Sandblasting Process. Journal of Geophysical Research, 95, 13927-13935.

GONZÁLEZ-DÁVILA, M., SANTANA-CASIANO, J. AND MILLERO, F. 2006. Competition Between $\mathrm{O}_{2}$ and $\mathrm{H}_{2} \mathrm{O}_{2}$ in the Oxidation of Fe(II) in Natural Waters. Journal of Solution Chemistry, 35, 95-111. 
GOOSSE, H. AND FICHEFET, T. 1999. Importance of ice-ocean interactions for the global ocean circulation: A model study. Journal of Geophysical Research, 104, 23337-23355.

GREELEY, R., AND J. D. IVERSEN, 1985. Wind as a Geological Process, 333 pp., Cambridge Univ. Press, New York.

GROTTI, M., SOGGIA, F., IANNI, C. AND FRACHE, R. 2005. Trace metals distribution in coastal sea ice of Terra Nova Bay, Ross Sea, Antarctica. Antarctic Science, 17, 289-300.

GROUSSET, F. E. AND BISCAYE, P. E. 2005. Tracing dust sources and transport patterns using $\mathrm{Sr}, \mathrm{Nd}$ and $\mathrm{Pb}$ isotopes. Chemical Geology, 222, 149-167.

GROUSSET, F. E., BISCAYE, P. E., REVEL, M., PETIT, J.-R., PYE, K., JOUSSAUME, S. AND JOUZEL, J. 1992. Antarctic (Dome C) ice-core dust at 18 k.y. B.P.: Isotopic constraints on origins. Earth and Planetary Science Letters, 111, 175-182.

GROUSSET, F. E., BISCAYE, P. E., ZINDLER, A., PROSPERO, J. \& CHESTER, R. 1988. Neodymium isotopes as tracers in marine sediments and aerosols: North Atlantic. Earth and Planetary Science Letters, 87, $367-378$.

GUIEU, C. AND THOMAS, A. 1996. Saharan aerosols: from the soil to the ocean. Environmental Science and Technology Library, 11, 207-216.

GUNN, B. AND WARREN, G. 1962. Geology of the Victoria Land between the Mawson and Mulock Glaciers, Antarctica. New Zealand Geological Survey Bulletin, 71, pp157.

HAND, J. L., MAHOWALD, N. M., CHEN, Y., SIEFERT, R. L., LUO, C., SUBRAMANIAM, A. AND FUNG, I. 2004. Estimates of atmospheric-processed soluble iron from observations and a global mineral aerosol model: Biogeochemical implications. Journal of Geophysical Research, 109, D17205.

HASKELL, Z., KENNETT, S., PREBBLE, W., SMYTH, G. AND WILLIS, I. 1965. The Geology of the middle and lower Taylor Valley of South Victoria Land. Transactions of the Royal Society of New Zealand, 2, 169-186.

HINKLEY, T. K. AND MATSUMOTO, A. 2001. Atmospheric regime of dust and salt through 75,000 years of Taylor Dome ice core: Refinement by measurement of major, minor, and trace metal suites. Journal of Geophysical Research, 106, 18487-18493.

HOWE, J. 2009. Towards a standard method for leaching iron from dust. Honours, University of Otago.

HUTCHINS, D. A., HARE, C. E., WEAVER, R. S., ZHANG, Y., FIRME, G. F., DITULLIO, G. R., ALM, M. B., RISEMAN, S. F., MAUCHER, J. M., GEESEY, M. E., TRICK, C. G., SMITH, G. J., RUE, E. L., CONN, J. AND BRULAND, K. W. 2002. Phytoplankton Iron Limitation in the Humboldt Current and Peru Upwelling. Limnology and Oceanography, 47, 997-1011.

HUTCHINS, D., WITTER, A., BUTLER, A. AND LUTHER, G. 1999. Competition among marine phytoplankton for different iron species. Nature, 400, 858 - 861.

HUTCHINS, D. A. \& BRULAND, K. W. 1998. Iron-limited diatom growth and Si:N uptake ratios in a coastal upwelling regime. Nature, 393, 561-564.

HUTCHINS, D. A., WANG, W.-X. AND FISHER, N. S. 1995. Copepod Grazing and the Biogeochemical Fate of Diatom Iron. Limnology and Oceanography, 40, 989-994.

HUTCHINS, D. A., DITUlLIO, G. R. AND BRULAND, K. W. 1993. Iron and Regenerated Production: Evidence for Biological Iron Recycling in Two Marine Environments. Limnology and Oceanography, 38, $1242-1255$. 
JACOBS, S. S. AND COMISO, J. C. 1989. Sea Ice and Oceanic Processes on the Ross Sea Continental Shelf. Journal of Geophysical Research, 94, 18195-18211.

JACOBSEN, S. B. AND WASSERBURG, G. J. 1980. Sm-Nd isotopic evolution of chondrites. Earth and Planetary Science Letters, 50, 139-155.

JICKELlS, T., AN, Z., ANDERSON, K., BAKER, A., BERGAMETTI, G., BROOKS, N., CAO, J., BOYD, P., DUCE, R., HUNTER, K., KAWAAHATA, H., KUBILAY, N., LAROCHE, J., LISS, P., MAHOWALD, N., PROSPERO, J., RIDGEWELL, A., TEGEN, I. AND TORRES, R. 2005. Global iron connections between desert dust, ocean biogeochemistry, and climate. Science, 308, 67 - 73.

JICKELLS, T. AND SPOKES, L. 2001. Atmospheric inputs to the ocean. The Biogeochemistry of Iron in Seawater, $85-121$.

JICKELLS, T. 1995. Atmospheric inputs of metals and nutrients to the oceans: their magnitude and effects. Marine Chemistry, 48, 199-214.

JOHANSEN, A. M. AND HOFFMANN, M. R. 2003. Chemical characterization of ambient aerosol collected during the northeast monsoon season over the Arabian Sea: Labile-Fe(II) and other trace metals. Journal of Geophysical Research, 108, 4408.

JOHANSEN, A. M., SIEFERT, R. L. \& HOFFMANN, M. R. 2000. Chemical composition of aerosols collected over the tropical North Atlantic Ocean. Journal of Geophysical Research, 105, 15277-15312.

JOHNSON, K. S. 2001. Iron supply and demand in the upper ocean: Is extraterrestrial dust a significant source of bioavailable iron? Global Biogeochemical Cycles, 15, 61-63.

JOHNSON, K., CHAVEZ, F. AND FRIEDERICH, G. 1999. Continental-shelf sediment as a primary source of iron for coastal phytoplankton. Nature, 398, $697-700$.

JOHNSON, K., COALE, K., ELROD, V. AND TINDALE, W. 1994. Iron photochemistry in seawater from the equatorial Pacific. Marine Chemistry, 46, 319 - 334.

JOHNSON, M. S., MESKHIDZE, N., KILIYANPILAKKIL, V. P. AND GASSÓ, S. 2011. Understanding the transport of Patagonian dust and its influence on marine biological activity in the South Atlantic Ocean. Atmospheric Chemistry and Physics, 11, 2487-2502.

JOHNSON, M. S., MESKHIDZE, N., SOLMON, F., GASSÓ, S., CHUANG, P. Y., GAIERO, D. M., YANTOSCA, R. M., WU, S., WANG, Y. \& CAROUGE, C. 2010. Modeling dust and soluble iron deposition to the South Atlantic Ocean. Journal of Geophysical Research, 115, D15202

JOHNSEN, S. J., DANSGAARD, W., CLAUSEN, H. B. AND LANGWAY, C. C. 1972. Oxygen Isotope Profiles through the Antarctic and Greenland Ice Sheets. Nature, 235, 429-434.

JOURNET, E., DESBOEUFS, K. V., CAQUINEAU, S. AND COLIN, J.-L. 2008. Mineralogy as a critical factor of dust iron solubility. Geophysical Research Letters, 35, L07805.

JUNYING, S., JIAWEN, R. AND DAHE, Q. 2002. 60 years record of biogenic sulfur from Lambert Glacier basin firn core, East Antarctica. Annals of Glaciology, 35, 362-367.

KELlOGG, T. B., KELLOGG, D. AND STUIVER, M. 1990. Late Quaternary History of the Southwestern Ross Sea: Evidence from Debris Bands on the McMurdo Ice Shelf, Antarctica. Antarctic Research Series. (AGU), 50, 2556.

KEYS, J. 1980. Air temperature, wind, precipitation and atmospheric humidity in the McMurdo region. Antarctic Data Series, 17, pp 57. 
KING, J. AND TURNER, J. 1997. Antarctic Meteorology and Climatology, Cambridge, Cambridge University Press.

KNUTH, S. 2007. Estimation of snow accumulation in Antarctica using automated acoustic depth gauge measurements. Master of Science, University of Wisconsin-Madison.

KOHFELD, K. E. AND HARRISON, S. P. 2001. DIRTMAP: the geological record of dust. Earth-Science Reviews, $54,81-114$.

KOROTEV, R. L. 1996. A SELF-CONSISTENT COMPILATION OF ELEMENTAL CONCENTRATION DATA FOR 93 GEOCHEMICAL REFERENCE SAMPLES. Geostandards Newsletter, 20, 217-245.

KRAEMER, S. M., BUTLER, A., BORER, P. AND CERVINI-SILVA, J. 2005. Siderophores and the Dissolution of Iron-Bearing Minerals in Marine Systems. Reviews in Mineralogy and Geochemistry, 59, 53-84.

KRAEMER, S. 2004. Iron oxide dissolution and solubility in the presence of siderophores. Aquatic Sciences Research Across Boundaries, 66, 3-18.

KUMA, K., NISHIOKA, J. AND MATSUNAGA, K. 1996. Controls on Iron(III) Hydroxide Solubility in Seawater: The Influence of pH and Natural Organic Chelators. Limnology and Oceanography, 41, 396-407.

KURTZ, D. D. AND BROMWICH, D. H. 1983. Satellite Observed Behavior of the Terra Nova Bay Polynya. Journal of Geophysical Research, 88, 9717-9722.

KYLE, P. AND COLE, J. 1974. Structural control of volcanism in the McMurdo Volcanic Group, Antarctica. Bulletin of Volcanology, 38, 16-25.

LAM, P. J., OHNEMUS, D. C. AND MARCUS, M. A. 2011. The Speciation of Marine Particulate Iron Adjacent to Active and Passive Continental Margins. Mineralogical Magazine, 75, 3, 1267.

LAM, P., BISHOP, J., HENNING, C., MARCUS, M., WAYCHYNAS, G. AND FUNG, I. 2006. Wintertime phytoplankton bloom in the subarctic Pacific supported by continental margin iron. Global Biogeochemical Cycles, 20, GB1006.

LAMBert, F., Delmonte, B., Petit, J. R., BIGLER, M., KAUfMANN, P. R., HUTTERli, M. A., STOCKER, T. F., RUTH, U., STEFFENSEN, J. P. AND MAGGI, V. 2008. Dust-climate couplings over the past 800,000 years from the EPICA Dome C ice core. Nature, 452, 616-619.

LANCELOT, C., DE MONTETY, A., GOOSSE, H., BECQUEVORT, S., SCHOEMANN, V., PASQUER, B. AND VANCOPPENOLLE, M. 2009. Spatial distribution of the iron supply to phytoplankton in the Southern Ocean: a model study. Biogeosciences, 6, 2861-2878.

LANNUZEL, D., SCHOEMANN, V., DE JONG, J., PASQUER, B., VAN DER MERWE, P., MASSON, F., TISON, J.-L. \& BOWIE, A. 2010. Distribution of dissolved iron in Antarctic sea ice: Spatial, seasonal, and interannual variability. Journal of Geophysical Research, 115, G03022.

LANNUZEL, D., SCHOEMANN, V., DE JONG, J., CHOU, L., DELILlE, B., BECQUEVORT, S. AND TISON, J.-L. 2008. Iron study during a time series in the western Weddell pack ice. Marine Chemistry, 108, 85-95.

LANNUZEL, D., SCHOEMANN, V., DE JONG, J., TISON, J.-L. AND CHOU, L. 2007. Distribution and biogeochemical behaviour of iron in the East Antarctic sea ice. Marine Chemistry, 106, 18-32.

LAW, C. S., ABRAHAM, E. R., WATSON, A. J. \& LIDDICOAT, M. I. 2003. Vertical eddy diffusion and nutrient supply to the surface mixed layer of the Antarctic Circumpolar Current. Journal of Geophysical Research, 108, 3272. 
LE ROUX, J. P. 1994. An alternative approach to the identification of net sediment transport paths based on grainsize trends. Sedimentary Geology, 94, 97-107.

LEFÈVRE, N. AND WATSON, A. J. 1999. Modeling the geochemical cycle of iron in the oceans and its impact on atmospheric CO2 concentrations. Global Biogeochemical Cycles, 13, 727-736.

LEGRAND, M. AND MAYEWSKI, P. 1997. Glaciochemistry of polar ice cores: A review. Reviews of Geophysics, $35,219-243$.

LeMASURIER, W. E. AND THOMSON, J. 1990. Volcanoes of the Antarctic plate and southern oceans. Antarctic Research Series, 48, 80-145.

LETTAU, B. 1969. The transport of moisture into the Antarctic interior. Tellus, 21, 331-340.

LI, F., GINOUX, P. AND RAMASWAMY, V. 2008. Distribution, transport, and deposition of mineral dust in the Southern Ocean and Antarctica: Contribution of major sources. Journal of Geophysical Research, 113, D10207.

LIGHT. B, WARREN, S. AND BRANDT, R. 2010. A technique for albedo measurements of laboratory-grown sea ice in extreme climates. International symposiums on sea ice in the physical and biogeochemical systems. International Glaciological Society. Tormso, Norway

LISS, P. AND TINDALE, N. W. 1994. Testing the iron hypothesis in ecosystems of the equatorial Pacific Ocean. Nature, 371, 123-129.

LIU, X. AND MILLERO, F. J. 2002. The solubility of iron in seawater. Marine Chemistry, 77, 43-54.

LOSCHER, B. M., DE BAAR, H. J. W., DE JONG, J. T. M., VETH, C. AND DEHAIRS, F. 1997. The distribution of Fe in the antarctic circumpolar current. Deep Sea Research Part II: Topical Studies in Oceanography, 44, 143187.

LOUANCHI, F., RUIZ-PINO, D. P., JEANDEL, C., BRUNET, C., SCHAUER, B., MASSON, A., FIALA, M. AND POISSON, A. 2001. Dissolved inorganic carbon, alkalinity, nutrient and oxygen seasonal and interannual variations at the Antarctic Ocean JGOFS-KERFIX site. Deep Sea Research Part I: Oceanographic Research Papers, 48, 1581-1603.

LUGMAIR, G. W. AND MARTI, K. 1978. Lunar initial 143Nd/144Nd: Differential evolution of the lunar crust and mantle. Earth and Planetary Science Letters, 39, 349-357.

LUO, C., MAHOWALD, N., BOND, T., ChUANG, P. Y., ARTAXO, P., SIEFERT, R., CHEN, Y. AND SCHAUER, J. 2008. Combustion iron distribution and deposition. Global Biogeochemical Cycles, 22, GB1012.

LUO, C., MAHOWALD, N. M., MESKHIDZE, N., CHEN, Y., SIEFERT, R. L., BAKER, A. R. AND JOHANSEN, A. M. 2005. Estimation of iron solubility from observations and a global aerosol model. Journal of Geophysical Research, 110, D23307.

LUO, C., MAHOWALD, N. M. AND DEL CORRAL, J. 2003. Sensitivity study of meteorological parameters on mineral aerosol mobilization, transport, and distribution. Journal of Geophysical Research, 108, 4447.

MACKIE, D. S., BOYD, P. W., MCTAINSH, G. H., TINDALE, N. W., WESTBERRY, T. K. AND HUNTER, K. A. 2008. Biogeochemistry of iron in Australian dust: From eolian uplift to marine uptake. Geochemistry, Geophysics, Geosystems, 9, Q03Q08.

MACKIE, D. S., BOYD, P. W., HUNTER, K. A. AND MCTAINSH, G. H. 2005. Simulating the cloud processing of iron in Australian dust: $\mathrm{pH}$ and dust concentration. Geophysical Research Letters, 32, L06809.

MACPHERSON, A. 1987. The MacKay Glacier/Granite Harbour system (Ross Dependency, Antarctica). A study in nearshore glacial marine sedimentation. Thesis, Victoria University of Wellington. 
MAHOWALD, N. M., BAKER, A. R., BERGAMETTI, G., BROOKS, N., DUCE, R. A., JICKELlS, T. D., KUBILAY, N., PROSPERO, J. M. AND TEGEN, I. 2005. Atmospheric global dust cycle and iron inputs to the ocean. Global Biogeochemical Cycles, 19, GB4025.

MAHOWALD, N., KOHFELD, K., HANSSON, M., BALKANSKI, Y., HARRISON, S. P., PRENTICE, I. C., SCHULZ, M. AND RODHE, H. 1999. Dust sources and deposition during the last glacial maximum and current climate: A comparison of model results with paleodata from ice cores and marine sediments. Journal of Geophysical Research, 104, 15895-15916.

MANNINEN, A. T. 1997. Surface roughness of Baltic sea ice. Journal of Geophysical Research, 102, 1119-1139.

MAQUEDA, M. A., WILlmOTT, A. J. \& BIGGS, N. R. T. 2004. Polynya Dynamics: a Review of Observations and Modeling. Reviews of Geophysics., 42, RG1004.

MARKLE, B. 2010. Dominant synoptic controls and influence of decadal climate oscillations in the Ross Sea region, Antarctica. MSc thesis, Victoria University of Wellington.

MARTICORENA, B. AND BERGAMETTI, G. 1995. Modeling the atmospheric dust cycle: 1. Design of a soilderived dust emission scheme. Journal of Geophysical Research, 100, 16415-16430.

MARTin, J. H., COALE, K. H., JOHNSON, K. S., FITZWATER, S. E., GORDON, R. M., TANNER, S. J., HUNTER, C. N., ELROD, V. A., NOWICKI, J. L., COLEY, T. L., BARBER, R. T., LINDLEY, S., WATSON, A. J., VAN SCOY, K., LAW, C. S., LIDDICOAT, M. I., LING, R., STANTON, T., STOCKEL, J., COLLINS, C., ANDERSON, A., BIDIGARE, R., ONDRUSEK, M., LATASA, M., MILLERO, F. J., LEE, K., YAO, W., ZHANG, J. Z., FRIEDERICH, G., SAKAMOTO, C., CHAVEZ, F., BUCK, K., KOLBER, Z., GREENE, R., FALKOWSKI, P., CHISHOLM, S. W., HOGE, F., SWIFT, R., YUNGEL, J., TURNER, S., NIGHTINGALE, P., HATTON, A.,

MARTIN, J. H., GORDON, R. M. AND FITZWATER, S. E. 1991. The Case for Iron. Limnology and Oceanography, 36, 1793-1802.

MARTIN, J. 1990. Glacial-interglacial $\mathrm{CO}_{2}$ change: the iron hypothesis. Paleoceanography, 5, 1 - 11.

MARTIN, J. H., GORDON, R. M. AND FITZWATER, S. E. 1990. Iron in Antarctic waters. Nature, 345, 156-158.

MARTIN, J. H., GORDON, R. M., FITZWATER, S. AND BROENKOW, W. W. 1989. Vertex: phytoplankton/iron studies in the Gulf of Alaska. Deep Sea Research Part A. Oceanographic Research Papers, 36, 649-680.

MARTÍNEZ-GARCIA, A., ROSELL-MELÉ, A., GEIBERT, W., GERSONDE, R., MASQUÉ, P., GASPARI, V. AND BARBANTE, C. 2009. Links between iron supply, marine productivity, sea surface temperature, and $\mathrm{CO} 2$ over the last 1.1 Ma. Paleoceanography, 24, PA1207.

McCRAW, J. 1962. Volcanic detritus in Taylor, Victoria Land, Antarctica. New Zealand Journal of Geology and Geophysics, 5, 30.

McCREA, I. 1984. A summary of glaciological measurements made between 1960 and 1984 on the McMurdo Ice Shelf Antarctica. University of Auckland, School of Engineering.

McCULLOCH, M. T. AND BLACK, L. P. 1984. Sm Nd isotopic systematics of Enderby Land granulites and evidence for the redistribution of Sm and Nd during metamorphism. Earth and Planetary Science Letters, 71, 46-58.

McKAY, R. M., DUNBAR, G. B., NAISH, T. R., BARRETT, P. J., CARTER, L. AND HARPER, M. 2008. Retreat history of the Ross Ice Sheet (Shelf) since the Last Glacial Maximum from deep-basin sediment cores around Ross Island. Palaeogeography, Palaeoclimatology, Palaeoecology, 260, 245-261.

McKENNA NEUMAN, C. 1999. Recent investigations of airflow and sediment transport over desert dunes. Chichester, Wiley. 
McKENNA NEUMAN, C. 1998. Particle transport and adjustments of the boundary layer over rough surfaces with an unrestricted, upwind supply of sediment. Geomorphology, 25, 1-17.

McKENNA NEUMAN, C. 1993. A review of aeolian transport processes in cold environments. Progress in Physical Geography, 17, 137-155.

McLAREN, P. AND BOWLES, D. 1985. The effects of sediment transport on grain-size distributions. Journal of Sedimentary Research, 55, 457-470.

McLAREN, P. 1981. An interpretation of trends in grain size measures. Journal of Sedimentary Research, 51, 611624.

McMINN, A., ASHWORTH, C. \& RYAN, K. 1999. Growth and Productivity of Antarctic Sea Ice Algae under PAR and UV Irradiances. Botanica Marina, 42, 401-407.

MEASURES, C. I. AND VINK, S. 2001. Dissolved Fe in the upper waters of the Pacific sector of the Southern Ocean. Deep Sea Research Part II: Topical Studies in Oceanography, 48, 3913-3941.

MEGURO, H., TOBA, Y., MURAKAMI, H. AND KIMURA, N. 2004. Simultaneous remote sensing of chlorophyll, sea ice and sea surface temperature in the Antarctic waters with special reference to the primary production from ice algae. Advanced Space Research, 33, 116 - 1172.

MENDEZ, J., GUIEU, C. AND ADKINS, J. 2010. Atmospheric input of manganese and iron to the ocean: Seawater dissolution experiments with Saharan and North American dusts. Marine Chemistry, 120, 34-43.

MEYERSON, E. A., MAYEWSKI, P. A., KREUTZ, K. J., MEEKER, L. D., WHITLOW, S. I. AND TWICKLER, M. S. 2002. The polar expression of ENSO and sea-ice variability as recorded in a South Pole ice core. Annals of Glaciology, 35, 430-436.

MILLERO, F. J. 1998. Solubility of Fe(III) in seawater. Earth and Planetary Science Letters, 154, 323-329.

MILlERO, F. J. AND SOTOLONGO, S. 1989. The oxidation of Fe(II) with $\mathrm{H} 2 \mathrm{O} 2$ in seawater. Geochimica et Cosmochimica Acta, 53, 1867-1873.

MILlERO, F. J., SOTOLONGO, S. AND IZAGUIRRE, M. 1987. The oxidation kinetics of Fe(II) in seawater. Geochimica et Cosmochimica Acta, 51, 793-801.

MITCHELL, B. G., BRODY, E. A., HOLM-HANSEN, O., MCCLAIN, C. \& BISHOP, J. 1991. Light limitation of phytoplankton biomass and macronutrient utilization in the Southern Ocean. Limnology and Oceanography; 36, 8, 1662-1677.

MONAGHAN, A. J., BROMWICH, D. H., POWERS, J. G. AND MANNING, K. W. 2005. The Climate of the McMurdo, Antarctica, Region as Represented by One Year of Forecasts from the Antarctic Mesoscale Prediction System. Journal of Climate, 18, 1174-1189.

MOORE, J. K. \& BRAUCHER, O. 2008. Sedimentary and mineral dust sources of dissolved iron to the world ocean. Biogeosciences, 5, 631-656.

MOORE, J. K., DONEY, S. C. AND LINDSAY, K. 2004. Upper ocean ecosystem dynamics and iron cycling in a global three-dimensional model. Global Biogeochemical Cycles, 18, GB4028.

MOORE, J. K., ABBOTT, M. R., RICHMAN, J. G. \& NELSON, D. M. 2000. The southern ocean at the Last Glacial Maximum: A strong sink for atmospheric carbon dioxide. Global Biogeochemical Cycles, 14, 455-475.

MORALES MAQUEDA, M. A., WILlMOTT, A. J. AND BIGGS, N. R. T. 2004. Polynya Dynamics: a Review of Observations and Modeling. Reviews of Geophysics, 42, RG1004. 
MUCHA, P., REKOWSKI, P., KOSAKOWSKA, A. AND KUPRYSZEWSKI, G. 1999. Separation of siderophores by capillary electrophoresis. Journal of Chromatography A, 830, 183-189.

NAISH, T., POWELL, R., LEVY, R., WILSON, G., SCHERER, R., TALARICO, F., KRISSEK, L., NIESSEN, F., POMPILIO, M., WILSON, T., CARTER, L., DECONTO, R., HUYBERS, P., MCKAY, R., POLLARD, D., ROSS, J., WINTER, D., BARRETT, P., BROWNE, G., CODY, R., COWAN, E., CRAMPTON, J., DUNBAR, G., DUNBAR, N., FLORINDO, F., GEBHARDT, C., GRAHAM, I., HANNAH, M., HANSARAJ, D., HARWOOD, D., HELLING, D., HENRYS, S., HINNOV, L., KUHN, G., KYLE, P., LAUFER, A., MAFFIOLI, P., MAGENS, D., MANDERNACK, K., MCINTOSH, W., MILLAN, C., MORIN, R., OHNEISER, C., PAULSEN, T., PERSICO, D., RAINE, I., REED, J., RIESSELMAN, C., SAGNOTTI, L., SCHMITT, D., SJUNNESKOG, C., STRONG, P., TAVIANI, M., VOGEL, S., WILCH, T. AND WILLIAMS, T. 2009. Obliquity-paced Pliocene West Antarctic ice sheet oscillations. Nature, $458,322-328$.

NAKABAYASHI, S., KUMA, K., SASAOKA, K., SAITOH, S., MOCHIZUKI, M., SHIGA, N. AND KUSAKABE, M. 2002. Variation in Iron(III) Solubility and Iron Concentration in the Northwestern North Pacific Ocean. Limnology and Oceanography, 47, 885-892.

NG, A. AND PATTERSON, C. 1981. Natural concentrations of lead in ancient Arctic and Antarctic ice. Geochimica et Cosmochimica Acta, 45, 2109-2121.

NODWELL, L. M. AND PRICE, N. M. 2001. Direct Use of Inorganic Colloidal Iron by Marine Mixotrophic Phytoplankton. Limnology and Oceanography, 46, 765-777.

O'CONNOR, W. P. AND BROMWICH, D. H. 1988. Surface airflow around Windless Bight, Ross Island, Antarctica. Quarterly Journal of the Royal Meteorological Society, 114, 917-938.

O'DWYER, J., ISAKSSON, E., VINJE, T., JAUHIAINEN, T., MOORE, J., POHJOLA, V., VAIKMÄE, R. AND VAN DE WAL, R. S. W. 2000. Methanesulfonic acid in a Svalbard Ice Core as an indicator of ocean climate. Geophysical Research Letters, 27, 1159-1162.

OLIVER, M. 1990. Kriging: A Method of Interpolation for Geographical Information Systems. International Journal of Geographic Information Systems, 4, 313-332.

OOKI, A., NISHIOKA, J., ONO, T. \& NORIKI, S. 2009. Size dependence of iron solubility of Asian mineral dust particles. Journal of Geophysical Research, 114, D03202.

O'NIONS, R. K., HAMILTON, P. J. AND EVENSEN, N. M. 1977. Variations in ${ }^{143} \mathrm{Nd} /{ }^{144} \mathrm{Nd}$ and ${ }^{87} \mathrm{Sr} /{ }^{86} \mathrm{Sr}$ ratios in oceanic basalts. Earth and Planetary Science Letters, 34, 13-22.

ÖZSOY, T. AND SAYDAM, A. C. 2001. Iron Speciation in Precipitation in the North-Eastern Mediterranean and Its Relationship with Sahara Dust. Journal of Atmospheric Chemistry, 40, 41-76.

PASTEUR, E. C. AND MULVANEY, R. 2000. Migration of methane sulphonate in Antarctic firn and ice. Journal of Geophysical Research, 105, 11525-11534.

PATTERSON, N. G., BERTLER, N. A. N., NAISH, T. R. AND MORGENSTERN, U. 2005. ENSO variability in the deuterium-excess record of a coastal Antarctic ice core from the McMurdo Dry Valleys, Victoria Land. Annals of Glaciology, 41, 140-146.PENG, T. H. \& BROECKER, W. S. 1991. Dynamical limitations on the Antarctic iron fertilization strategy. Nature, 349, 227-229.

PETIT, J. R. AND DELMONTE, B. 2011. Glacial Interglacial Aerosol Input over Antarctica and the Global Hydrological Cycle. Mineralogical Magazine, 75, 3, 1629.

POWERS, J. G. 2007. Numerical Prediction of an Antarctic Severe Wind Event with the Weather Research and Forecasting (WRF) Model. Monthly Weather Review, 135, 3134-3157. 
PROSPERO, J. M. 1996. The atmospheric transport of particles to the ocean, in Particle Flux in the Ocean. Hoboken, N. J, John Wiley.

PROSPERO, J. M., 1981. Aeolian transport to the World Ocean, in The Sea, vol. 7, The Oceanic Lithosphere, edited by C. Emiliani, pp. 801-974, Wiley Interscience, New York.

PYE, K. 1989. Aeolian dust and dust deposits, Academic Press. Harcourt Brace Jovanovich, Publishers.

RAISWELL, R. 2011. Iron Transport from the Continents to the Open Ocean: The Aging-Rejuvenation Cycle. Elements, 7, 101-106.

RAISWELL, R., BENNING, L., TRANTER, M. AND TULACZYK, S. 2008a. Bioavailable iron in the Southern Ocean: the significance of the iceberg conveyor belt. Geochemical Transactions, 9, 7, doi:10.1186/1467-4866-9-7.

RAISWELL, R., BENNING, L. G., DAVIDSON, L. AND TRANTER, M. 2008b. Nanoparticulate bioavailable iron minerals in icebergs and glaciers. Mineral Magazine, 72, 345-348.

RAISWELL, R., TRANTER, M., BENNING, L., SIEGERT, M., DE'ATH, R., HUYBRECHTS, P. AND PAYNE, T. 2006. Contributions from glacially derived sediment to the global iron (oxyhydr)oxide cycle: implications for iron delivery to the oceans. Geochim Cosmochim Acta, 70, 2765 - 2780.

RAISWELL, R. 1984. Chemical models of solute acquisition in glacial meltwaters. Journal of Glaciology, 30, 49 57.

RAM, M. AND ILLING, M. 1994. Polar ice stratigraphy from laser-light scattering: Scattering from meltwater, Journal of Glaciology, 40, 136, 504-508.

REVEl-ROlland, M., DE DECKKER, P., DELMONTE, B., HESSE, P. P., MAGEE, J. W., BASILEDOELSCH, I., GROUSSET, F. AND BOSCH, D. 2006. Eastern Australia: A possible source of dust in East Antarctica interglacial ice. Earth and Planetary Science Letters, 249, 1-13.

RHODES, R. H., BAKER, J. A., MILLET, M.-A. AND BERTLER, N. A. N. 2011. Experimental investigation of the effects of mineral dust on the reproducibility and accuracy of ice core trace element analysis. Chemical Geology, 286, 3-4, 207-221.

RHODES, R. H. 2011. Insights into Late Holocene climate of the Ross Sea region, Antarctica, from high resolution ice core chemistry. $\mathrm{PhD}$ thesis, Victoria University of Wellington.

RHODES, R. H., BERTLER, N. A. N., BAKER, J. A., SNEED, S. B., OERTER, H. AND ARRIGO, K. R. 2009. Sea ice variability and primary productivity in the Ross Sea, Antarctica, from methylsulphonate snow record. Geophysical Research Letters, 36, L10704.

RIBIC, C., AINLEY, D. G. AND FRASER, W. R. 1991. Habitat selection by marine mammals in the marginal ice zone. Antarctic Science, 3, 181-186.

RICH, H. W. AND MOREL, F. M. M. 1990. Availability of Well-Defined Iron Colloids to the Marine Diatom Thalassiosira weissflogii. Limnology and Oceanography, 35, 652-662.

ROBINSON, N. 2004. An oceanographic study of the cavity beneath the McMurdo Ice Shelf. MSc thesis, Victoria University of Wellington.

ROYLE, A., F., C. AND FREDERIKSEN, P. 1981. Practical Universal Kriging and Automatic Contouring. Geoprocessing, 1, 377-394. 
RUBASINGHEGE, G., LENTZ, R. W., SCHERER, M. M. AND GRASSIAN, V. H. 2010. Simulated atmospheric processing of iron oxyhydroxide minerals at low $\mathrm{pH}$ : Roles of particle size and acid anion in iron dissolution. Proceedings of the National Academy of Sciences, 107, 6628-6633.

RUHL, H. A., ELLENA, J. A., WILSON, R. C. AND HELLY, J. 2011. Seabird aggregation around free-drifting icebergs in the northwest Weddell and Scotia Seas. Deep Sea Research Part II: Topical Studies in Oceanography, $58,1497-1504$.

RUTH, U. 2002. Concentration and size distribution of microparticles in the NGRIP Ice Core during the Last Glacial period. Dissertation, University Bremen.

SALTZMAN, E., SAVOIE, R., ZIKA, R. AND PROSPERO, J. M. 1983. Methane sulfonic acid in the marine troposphere. Journal of Geophysical Research, 88, 10897-10902.

SARThOU, G., BAKer, A. R., BlAin, S., ACHTERBERG, E. P., BOYE, M., BOWIE, A. R., CROOT, P., LAAN, P., DE BAAR, H. J. W., JICKELLS, T. D. AND WORSFOLD, P. J. 2003. Atmospheric iron deposition and sea-surface dissolved iron concentrations in the eastern Atlantic Ocean. Deep Sea Research Part I: Oceanographic Research Papers, 50, 1339-1352.

SATO, M., TAKEDA, S. AND FURUYA, K. 2007. Iron regeneration and organic iron(III)-binding ligand production during in situ zooplankton grazing experiment. Marine Chemistry, 106, 471-488.

SCHOEMANN, V., BAAR, H. J. W. D., JONG, J. T. M. D. AND LANCELOT, C. 1998. Effects of Phytoplankton Blooms on the Cycling of Manganese and Iron in Coastal Waters. Limnology and Oceanography, 43, 1427-1441.

SCHROTH, A. W., CRUSIUS, J., SHOLKOVITZ, E. R. AND BOSTICK, B. C. 2009. Iron solubility driven by speciation in dust sources to the ocean. Nature Geoscience, 2, 337-340.

SCHUCK, I. 2009. Mineralogical characterisation and geographic provenance of atmospheric particles in Coastal ice cores - Indicators of past climate variability. BSc Honours thesis, University Karlsruhe.

SCHWERDTFEGER, W. 1984. Weather and climate of the Antarctic. Amsterdam, Elsevier.

SEDWICK, P. N., MARSAY, C. M., SOHST, B. M., AGUILAR-ISLA, A. M., LOHAN, M. C., LONG, M. C., ARRIGO, K. A., DUNBAR, R. B., SAITO, M. A., SMITH, W. O., DiTULLIO. 2011. Early-season depletion of dissolved iron in the Ross sea polynya: Implications of iron dynamics on the Antarctic continental shelf. Journal of Geophysical Research. doi:10.1029/2010JC006553, in press.

SEDWICK, P. N., SHOLKOVITZ, E. R. AND CHURCH, T. M. 2007. Impact of anthropogenic combustion emissions on the fractional solubility of aerosol iron: Evidence from the Sargasso Sea. Geochemistry, Geophysics, Geosystems, 8, Q10Q06.

SEDWICK, P. N., DiTULLIO, G. R. AND MACKEY, D. J. 2000. Iron and manganese in the Ross Sea, Antarctica: Seasonal iron limitation in Antarctic shelf waters. Journal of Geophysical Research, 105, 11321-11336.

SEDWICK, P. N., EDWARDS, P. R., MACKEY, D. J., GRIFFITHS, F. B. AND PARSLOW, J. S. 1997. Iron and manganese in surface waters of the Australian subantarctic region. Deep Sea Research Part I: Oceanographic Research Papers, 44, 1239-1253.

SEDWICK, P. N. AND DiTULLIO, G. R. 1997. Regulation of algal blooms in Antarctic Shelf Waters by the release of iron from melting sea ice. Geophysical Research Letters, 24, 2515-2518.

SELBY, M., RAINS, J. AND PALMER, R. 1974. Eolian deposits of the ice free Victoria Valley, Southern Victoria Land, Antarctica. New Zealand Journal of Geology and Geophysics, 17, 543-562. 
SHAO, Y., RAUPACH, M. R. AND FINDLATER, P. A. 1993. Effect of Saltation Bombardment on the Entrainment of Dust by Wind. Journal of Geophysical Research, 98, 12719-12726.

SHAW, T. J., SMITH JR, K. L., HEXEL, C. R., DUDGEON, R., SHERMAN, A. D., VERNET, M. AND KAUFMANN, R. S. 2011. ${ }^{234}$ Th-Based Carbon Export around Free-Drifting Icebergs in the Southern Ocean. Deep Sea Research Part II: Topical Studies in Oceanography, 58, 1384-1391.

SHI, Z., BONNEVILLE, S., KROM, M. D., CARSLAW, K. S., JICKELLS, T. D., BAKER, A. R. \& BENNING, L. G. 2011. Iron dissolution kinetics of mineral dust at low $\mathrm{pH}$ during simulated atmospheric processing. Atmospheric Chemistry and Physics, 11, 995-1007.

SHOLKOVITZ, E. R., SEDWICK, P. N. AND CHURCH, T. M. 2009. Influence of anthropogenic combustion emissions on the deposition of soluble aerosol iron to the ocean: Empirical estimates for island sites in the North Atlantic. Geochimica et Cosmochimica Acta, 73, 3981-4003.

SIEFERT, R. L., JOHANSEN, A. M. AND HOFFMANN, M. R. 1999. Chemical characterization of ambient aerosol collected during the southwest monsoon and intermonsoon seasons over the Arabian Sea: Labile-Fe(II) and other trace metals. Journal of Geophysical Research, 104, 3511-3526.

SINCLAIR, K. E., BERTLER, N. A. N. AND TROMPETTER, W. J. 2010. Synoptic controls on precipitation pathways and snow delivery to high-accumulation ice core sites in the Ross Sea region, Antarctica. Journal of Geophysical Research, 115, D22112.

SINCLAIR, M. 1982. Weather Observations in the Ross Sea Area, Antarctica. New Zealand Meteorological Office. SLOMP, C., MALSCHAERT, J., LOHSE, L. AND VAN RAAPHORST, W. 1997. Iron and manganese cycling in different sedimentary environments on the North Sea continental margin. Continental Shelf Research, 17,1083 1117.

SLOTTEN, H. R. AND STEARNS, C. R. 1987. Observations of the Dynamics and Kinematics of the Atmospheric Surface Layer on the Ross Ice Shelf, Antarctica. Journal of Climate and Applied Meteorology, 26, 1731-1743.

SMITH, J., VANCE, D., KEMP, R. A., ARCHER, C., TOMS, P., KING, M. AND ZÁRATE, M. 2003. Isotopic constraints on the source of Argentinian loess - with implications for atmospheric circulation and the provenance of Antarctic dust during recent glacial maxima. Earth and Planetary Science Letters, 212, 181-196.

SMITH, K. L., ROBISON, B. H., HELLY, J. J., KAUFMANN, R. S., RUHL, H. A., SHAW, T. J., TWINING, B. S. AND VERNET, M. 2007. Free-Drifting Icebergs: Hot Spots of Chemical and Biological Enrichment in the Weddell Sea. Science, 317, 478-482.

SMITH, W. O., JR. AND GORDON, L. I. 1997. Hyperproductivity of the Ross Sea (Antarctica) polynya during austral spring. Geophysical Research Letters, 24, 233-236.

SMITH, W. O., JR. AND NELSON, D. M. 1986. Importance of Ice Edge Phytoplankton Production in the Southern Ocean. BioScience, 36, 251-257.

SPOKES, L. J. \& JICKELLS, T. D. 1995. Factors controlling the solubility of aerosol trace metals in the atmosphere and on mixing into seawater. Aquatic Geochemistry, 1, 355-374.

SPOKES, L. J., JICKELLS, T. D. AND LIM, B. 1994. Solubilisation of aerosol trace metals by cloud processing: A laboratory study. Geochimica et Cosmochimica Acta, 58, 3281-3287.

STEARNS, C. R. 1997. Ross Island area wind field. Antarctic Journal of the United States of America, 33, $181-182$.

STEARNS, C. R. AND M, SAVAGE. 1981. Automatic weather stations, 1980-1981. ibid., 16, 190-192. 
STEFFENSEN, J. P. 1997. The size distribution of microparticles from selected segments of the Greenland Ice Core Project ice core representing different climatic periods. Journal of Geophysical Research, 102, 26755-26763.

STEINHOFF, D. F., CHAUDHURI, S. AND BROMWICH, D. H. 2009. A Case Study of a Ross Ice Shelf Airstream Event: A New Perspective. Monthly Weather Review, 137, 4030-4046.

STEINHOFF, D. F., BROMWICH, D. H., LAMBERTSON, M., KNUTH, S. L. AND LAZZARA, M. A. 2008. A Dynamical Investigation of the May 2004 McMurdo Antarctica Severe Wind Event Using AMPS. Monthly Weather Review, 136, 7-26.

SUlLIVAN, C. W., ARRIGO, K. R., MCCLAIN, C. R., COMISO, J. C. AND FIRESTONE, J. 1993. Distributions of Phytoplankton Blooms in the Southern Ocean. Science, 262, 1832-1837.

SWEENEY, C., HANSEll, D. A., CARlSON, C. A., CODISPOTI, L. A., GORDON, L. I., MARRA, J., MILLERO, F. J., SMITH, W. O. AND TAKAHASHI, T. 2000. Biogeochemical regimes, net community production and carbon export in the Ross Sea, Antarctica. Deep Sea Research Part II: Topical Studies in Oceanography, 47, 3369-3394.

TAGLIABUE, A., BOPP, L. AND AUMONT, O. 2009. Evaluating the importance of atmospheric and sedimentary iron sources to Southern Ocean biogeochemistry. Geophysical Research Letters, 36, L13601.

TAGLIABUE, A. AND ARRIGO, K. R. 2006. Processes governing the supply of iron to phytoplankton in stratified seas. Journal of Geophysical Research, 111, C06019.

TAGLIABUE, A. AND ARRIGO, K. R. 2005. Iron in the Ross Sea: 1. Impact on $\mathrm{CO}_{2}$ fluxes via variation in phytoplankton functional group and non-Redfield stoichiometry. Journal of Geophysical Research, 110, C03009.

TAYLOR, L. AND GLIOZZI, J. 1964. Distribution of particulate matter in a firn core from Eights Station, Antarctica. American Geophysical Union, Washington DC.

TAYLOR, S. R. AND MCLENNAN, S. M. 1995. The geochemical evolution of the continental crust. Reviews of Geophysics, 33, 241-265.

TAYLOR, S. R. AND MCLENNAN, S. M. 1985. The continental crust: Its composition and evolution. Blackwell Scientific Pub., Palo Alto.

TEGEN, I., WERNER, M., HARRISON, S. P. AND KOHFELD, K. E. 2004. Relative importance of climate and land use in determining present and future global soil dust emission. Geophysical Research Letters, 31, L05105.

TEGEN, I. 2003. Modeling the mineral dust aerosol cycle in the climate system. Quaternary Science Reviews, 22, 1821-1834.

TEGEN, I. AND FUNG, I. 1995. Contribution to the atmospheric mineral aerosol load from land surface modification. Journal of Geophysical Research, 100, 18707-18726.

THOMPSON, L. G. AND MOSLEY-THOMPSON, E. 1981. Temporal variability of microparticle properties in polar ice sheets. Journal of Volcanology and Geothermal Research, 11, 11-27.

TIAN, Z., OLIVER, P., VERON, A. AND CHURCH, T. M. 2008. Atmospheric Fe deposition modes at Bermuda and the adjacent Sargasso Sea. Geochemistry, Geophysics, Geosystems, 9, 8, doi:10.1029/2007GC001868.

TRAPP, J. M., MILLERO, F. J. AND PROSPERO, J. M. 2010. Trends in the solubility of iron in dust-dominated aerosols in the equatorial Atlantic trade winds: Importance of iron speciation and sources. Geochemistry, Geophysics, Geosystems, 11, Q03014. 
TRUESDALE, R. S. AND KELlOGG, T. B. 1979. Ross Sea diatoms: Modern assemblage distributions and their relationship to ecologic, oceanographic, and sedimentary conditions. Marine Micropaleontology, 4, 13-31.

TSOAR AND PYE, K. 1987. Dust transport and the question of desert loess formation. Sedimentology, 34, 139-153.

VAllelonga, P., Gabrielli, P., BAlliana, E., Wegner, A., Delmonte, B., Turetta, C., BURTON, G., VANHAECKE, F., ROSMAN, K. J. R., HONG, S., BOUTRON, C. F., CESCON, P. AND BARBANTE, C. 2010. Lead isotopic compositions in the EPICA Dome C ice core and Southern Hemisphere Potential Source Areas. Quaternary Science Reviews, 29, 247-255.

VALLELONGA, P., GABRIELli, P., ROSMAN, K. J. R., BARBANTE, C. AND BOUTRON, C. F. 2005. A 220 kyr record of $\mathrm{Pb}$ isotopes at Dome $\mathrm{C}$ Antarctica from analyses of the EPICA ice core. Geophysical Research Letters, 32, L01706.

VALlELONGA, P., VAN DE VELDE, K., CANDELONE, J. P., MORGAN, V. I., BOUTRON, C. F. AND ROSMAN, K. J. R. 2002. The lead pollution history of Law Dome, Antarctica, from isotopic measurements on ice cores: 1500 AD to 1989 AD. Earth and Planetary Science Letters, 204, 291-306.

VAN DER MERWE, P., LANNUZEL, D., BOWIE, A. R., MANCUSO NICHOLS, C. A. AND MEINERS, K. M. 2011. Iron fractionation in pack and fast ice in East Antarctica: Temporal decoupling between the release of dissolved and particulate iron during spring melt. Deep Sea Research Part II: Topical Studies in Oceanography, 58, $1222-1236$.

VAN DER MERWE, P., LANNUZEL, D., NICHOLS, C. A. M., MEINERS, K., HEIL, P., NORMAN, L., THOMAS, D. N. AND BOWIE, A. R. 2009. Biogeochemical observations during the winter-spring transition in East Antarctic sea ice: Evidence of iron and exopolysaccharide controls. Marine Chemistry, 115, 163-175.

VANCOPPENOLLE, M., FICHEFET, T., GOOSSE, H., BOUILLON, S., MADEC, G. AND MAQUEDA, M. A. M. 2009. Simulating the mass balance and salinity of Arctic and Antarctic sea ice. 1. Model description and validation. Ocean Modelling, 27, 33-53.

VISSER, F., GERRINGA, L. J. A., VAN DER GAAST, S. J., DE BAAR, H. J. W. AND TIMMERMANS, K. R. 2003. The Role Of The Reactivity And Content Of Iron Of Aerosol Dust On Growth Rates Of Two Antarctic Diatom Species1. Journal of Phycology, 39, 1085-1094.

VLASENKO, A., SJ, OUML, GREN, S., WEINGARTNER, E., AUML, GGELER, H. AND AMMANN, M. Generation of Submicron Arizona Test Dust Aerosol: Chemical and Hygroscopic Properties. Aerosol Science and Technology, 39, 452-460.

WAGENER, T., GUIEU, C., LOSNO, R., BONNET, S. AND MAHOWALD, N. 2008. Revisiting atmospheric dust export to the Southern Hemisphere ocean: Biogeochemical implications. Global Biogeochemical Cycles, 22, GB2006.

WALTERS, J. AND HEDGES, J. 1988. Simultaneous determination of uronic acids and aldoses in plankton, plant tissues, and sediment by capillary gas-chromatography of Nhexylaldonamide and alditol acetates. Analytical Chemistry, 60, 988-994.

WARREN, G. 1970. Geology of the Terra Nova Bay-McMurdo Sound Area, Victoria Land, Antarctica. Plate XIII, Fiolio 12- Geology, Antarctic Map Filio Series.

WATSON, A. J., BAKKER, D. C. E., RIDGWELL, A. J., BOYD, P. W. \& LAW, C. S. 2000. Effect of iron supply on Southern Ocean CO2 uptake and implications for glacial atmospheric CO2. Nature, 407, 730-733.

WEDEPOHL, K. 1995. The composition of the continental crust. Geochimica et Cosmochimica Acta, 59, 12171232. 
WELCH, K. A., MAYEWSKI, P. A. AND WHITLOW, S. I. 1993. Methanesulfonic acid in coastal Antarctic snow related to sea ice extent. Geophysical Research Letters, 20, 443-446.

WELLS, M. AND MAYER, L. 1991. The photoconversion of colloidal iron oxyhydroxides in seawater. Deep-Sea Research, 38, 1379 - 1395.

WELLS, M. L., PRICE, N. M. AND BRULAND, K. W. 1995. Iron chemistry in seawater and its relationship to phytoplankton: a workshop report. Marine Chemistry, 48, 157-182.

WELLS, M., MAYER, L., OLIVIER, F., DE SOUZE SIERRA, M. AND ACKELSON, S. 1991. The photolysis of colloidal iron in the oceans. Nature, 353, $248-260$.

WELLS, M., ZORKIN, N. AND LEWIS, A. 1983. The role of colloidal chemistry in providing a source of iron to phytoplankton. Journal of Marine Research, 41, 731 - 746.

WILSON, N. 2003. The movement and origin of the sand dunes in the Victoria Valley, Antarctica. BSc Honours thesis, Victoria University of Wellington.

WINDOM, H. L. 1969. Atmospheric Dust Records in Permanent Snowfields: Implications to Marine Sedimentation. Geological Society of America Bulletin, 80, 761-782.

WOLFF, E. W., FISCHER, H., FUNDEL, F., RUTH, U., TWARLOH, B., LITTOT, G. C., MULVANEY, R., RÖTHLISBERGER, R., DE ANGELIS, M., BOUTRON, C. F., HANSSON, M., JONSELL, U., HUTTERLI, M. A., LAMBERT, F., KAUFMANN, P., STAUFFER, B., STOCKER, T. F., STEFFENSEN, J. P., BIGLER, M., SIGGAARD-ANDERSEN, M. L., UDISTI, R., BECAGLI, S., CASTELLANO, E., SEVERI, M., WAGENBACH, D., BARBANTE, C., GABRIELLI, P. AND GASPARI, V. 2006. Southern Ocean sea-ice extent, productivity and iron flux over the past eight glacial cycles. Nature, 440, 491-496.

WU, J., REMBER, R. AND CAHILL, C. 2007. Dissolution of aerosol iron in the surface waters of the North Pacific and North Atlantic oceans as determined by a semicontinuous flow-through reactor method. Global Biogeochemical Cycles, 21, GB4010.

WU, J., BOYLE, E., SUNDA, W. AND WEN, L.-S. 2001. Soluble and Colloidal Iron in the Oligotrophic North Atlantic and North Pacific. Science, 293, 847-849.

YOUNG, G. M. AND NESBITT, H. W. 1998. Processes controlling the distribution of Ti and Al in weathering profiles, siliciclastic sediments and sedimentary rocks. Journal of Sedimentary Research, 68, 448-455.

ZHU, X. R., PROSPERO, J. M. \& MILLERO, F. J. 1997. Diel variability of soluble Fe(II) and soluble total Fe in North African dust in the trade winds at Barbados. Journal of Geophysical Research, 102, 21297-21305.

ZHU, X., PROSPERO, J. M., SAVOIE, D. L., MILlerO, F. J., ZIKA, R. G. AND SALTZMAN, E. S. 1993. Photoreduction of Iron(III) in Marine Mineral Aerosol Solutions. Journal of Geophysical Research, 98, 9039-9046.

ZHU, X., PROSPERO, J. M., MILLERO, F. J., SAVOIE, D. L. AND BRASS, G. W. 1992. The solubility of ferric ion in marine mineral aerosol solutions at ambient relative humidities. Marine Chemistry, 38, 91-107.

ZHUANG, G., YI, Z., DUCE, R. A. AND BROWN, P. R. 1992. Chemistry of iron in marine aerosols. Global Biogeochemical Cycles, 6, 161-173.

ZHUANG, G., DUCE, R. A. AND KESTER, D. R. 1990. The Dissolution of Atmospheric Iron in Surface Seawater of the Open Ocean. Journal of Geophysical Research, 95, 16207-16216. 
Appendix 1: Field sampling, dust mass and grain size statistics for dust samples from southern McMurdo Sound.

\begin{tabular}{|c|c|c|c|c|c|c|c|c|c|c|c|c|c|c|c|c|c|c|c|c|c|c|c|c|c|c|c|c|}
\hline & $\begin{array}{l}\text { Field } \\
\text { notes }\end{array}$ & & & & & & & $\begin{array}{l}\text { Dust } \\
\text { mass }\end{array}$ & & & $\begin{array}{c}\text { Grain } \\
\text { size } \\
\text { statistics }\end{array}$ & & & & & & & & & & & & & & & & & \\
\hline $\begin{array}{c}\text { Sam- } \\
\text { ple }\end{array}$ & Latitude & $\begin{array}{l}\text { Long- } \\
\text { titude }\end{array}$ & $\begin{array}{c}\text { Date } \\
\text { sampled }\end{array}$ & $\begin{array}{l}\text { Snow } \\
\text { depth } \\
\text { (cm) }\end{array}$ & $\begin{array}{c}\text { Length } \\
(\mathrm{cm})\end{array}$ & $\begin{array}{l}\text { Width } \\
(\mathrm{cm})\end{array}$ & $\begin{array}{c}\text { Dupli- } \\
\text { cate } \\
\text { taken }\end{array}$ & $\begin{array}{c}\text { Snow } \\
(\mathbf{k g})\end{array}$ & $\begin{array}{c}\text { Dust } \\
\text { weigh } \\
t(g)\end{array}$ & $\begin{array}{l}\text { Dust } \\
\text { conc. } \\
\left(\mathrm{g} \mathrm{m}^{2}\right)\end{array}$ & $\begin{array}{c}\text { Grain } \\
\text { size } \\
\text { Module }\end{array}$ & Mode & $\begin{array}{c}\text { Med- } \\
\text { ian }\end{array}$ & $\begin{array}{l}\text { Obscu } \\
\text { ration }\end{array}$ & $\begin{array}{c}\text { Skew- } \\
\text { ness } \\
\text { (right) }\end{array}$ & $\begin{array}{c}\text { Kurt- } \\
\text { osis }\end{array}$ & $\begin{array}{l}\text { Stdev } \\
(\mu \mathrm{m})\end{array}$ & $\begin{array}{l}\% \\
<10 \\
\mu \mathrm{m}\end{array}$ & $\begin{array}{c}\% \\
\text { gravel }\end{array}$ & $\begin{array}{l}\% \mathrm{mu} \\
\mathrm{d}(0- \\
62.5 \\
\mu \mathrm{m})\end{array}$ & $\begin{array}{c}\% \\
\text { sand } \\
(>62 . \\
5 \mu \mathrm{m})\end{array}$ & $\begin{array}{c}\text { sand: } \\
\text { mud }\end{array}$ & $\begin{array}{c}\% \\
\text { clay } \\
(<2 \\
\mu \mathrm{m})\end{array}$ & $\begin{array}{c}\% \text { silt } \\
(2-62.5 \\
\mu \mathrm{m})\end{array}$ & $\begin{array}{l}\text { clay: } \\
\text { silt }\end{array}$ & $\begin{array}{l}\text { Descr- } \\
\text { iption }\end{array}$ & Sorting & Skewed \\
\hline 1 & $\begin{array}{c}77^{\circ} \\
46.020 \mathrm{~S}\end{array}$ & $\begin{array}{c}166^{\circ} \\
12.043 \mathrm{E}\end{array}$ & $11 / 11 / 09$ & 10 & 30 & 30 & $\begin{array}{c}\text { Yes - } \\
\text { sample } 3\end{array}$ & 1.425 & 0.051 & 0.575 & MLM & 41.68 & 21.17 & 22,20 & 1.238 & 1.059 & 30.13 & 33 & 0 & 84 & 15 & 0.180 & 4 & 79 & 0.062 & $\begin{array}{c}\text { Sandy } \\
\text { silt }\end{array}$ & $\begin{array}{l}\text { extremel } \\
\text { y poorly }\end{array}$ & $\begin{array}{l}\text { strongly } \\
\text { fine } \\
\text { tepwe }\end{array}$ \\
\hline 2 & $\begin{array}{c}77^{\circ} \\
43.004 \mathrm{~S}\end{array}$ & $\begin{array}{c}166^{\circ} 12 . \\
099 \mathrm{E}\end{array}$ & $11 / 11 / 09$ & 20 & 6 & 40 & Yes & 1.445 & 0.352 & 14.67 & MLM & 223.4 & 74.27 & 9 & 0.496 & -1.191 & 89.22 & 11 & 0 & 47 & 52 & 1.126 & 1 & 46 & 0.023 & $\begin{array}{l}\text { Silty } \\
\text { Sand }\end{array}$ & $\begin{array}{c}\text { Sorted } \\
\text { very } \\
\text { poorly } \\
\text { sorted }\end{array}$ & $\begin{array}{l}\begin{array}{c}\text { skewed } \\
\text { fine } \\
\text { skewed }\end{array}\end{array}$ \\
\hline 3 & $\begin{array}{c}77^{\circ} \\
42.981 \mathrm{~S}\end{array}$ & $\begin{array}{c}166^{\circ} 12 . \\
131 \mathrm{E}\end{array}$ & $11 / 11 / 09$ & 15 & 4 & 10 & $\begin{array}{c}\text { Yes - } \\
\text { sample } 5\end{array}$ & 1.425 & 0.038 & 0.955 & MLM & 34.58 & 17.18 & 25,20 & 1.496 & 2.097 & 24.86 & 36 & 0 & 90 & 9 & 0.102 & 6 & 85 & 0.069 & Silt & $\begin{array}{l}\text { extremel } \\
\text { y poorly } \\
\text { sorted }\end{array}$ & $\begin{array}{c}\text { strongly } \\
\text { fine } \\
\text { skewed }\end{array}$ \\
\hline 4 & $\begin{array}{c}77^{\circ} \\
40.028 \mathrm{~S}\end{array}$ & $\begin{array}{c}166^{\circ} \\
35.965 \mathrm{E}\end{array}$ & $12 / 11 / 09$ & $0-10$ & 20 & 40 & Yes & 1.255 & 0.464 & 5.81 & ALM & 87.9 & 54.61 & 15 & 1.044 & 0.401 & 68.6 & 23 & 0 & 54 & 45 & 0.840 & 2 & 52 & 0.046 & $\begin{array}{l}\text { Sandy } \\
\text { silt }\end{array}$ & $\begin{array}{l}\text { very } \\
\text { poorly } \\
\text { sorted }\end{array}$ & $\begin{array}{l}\text { strongly } \\
\text { fine } \\
\text { skewed }\end{array}$ \\
\hline 5 & $\begin{array}{c}77^{\circ} \\
40.0441 \mathrm{~S}\end{array}$ & $\begin{array}{c}166^{\circ} 35 \\
940 \mathrm{E}\end{array}$ & $12 / 11 / 09$ & 20 & 10 & 20 & No & 1.645 & 0.664 & 41.54 & ALM & 87.9 & 50.69 & 12 & 1.066 & 8.436 & 67.04 & 25 & 0 & 56 & 43 & 0.781 & 3 & 53 & 0.055 & $\begin{array}{l}\text { Sandy } \\
\text { silt }\end{array}$ & $\begin{array}{l}\text { very } \\
\text { poorly } \\
\text { sorted }\end{array}$ & $\begin{array}{l}\text { strongly } \\
\text { fine } \\
\text { skewed }\end{array}$ \\
\hline 6 & $\begin{array}{c}77^{\circ} \\
40.014 \mathrm{~S}\end{array}$ & $\begin{array}{c}166^{\circ} \\
24.072 \mathrm{E}\end{array}$ & $12 / 11 / 09$ & 10 & 50 & 10 & $\begin{array}{c}\text { Yes - } \\
\text { sample } 8\end{array}$ & 1.795 & 0.675 & 13.5 & MLM & 16.4 & 17.36 & 5,11 & 1.53 & 2.673 & 21.49 & 31 & 0 & 94 & 5 & 0.057 & 2 & 92 & 0.026 & Silt & $\begin{array}{l}\text { extremel } \\
\text { y poorly } \\
\text { sorted }\end{array}$ & $\begin{array}{c}\text { strongly } \\
\text { fine } \\
\text { stewed }\end{array}$ \\
\hline 7 & $\begin{array}{c}77^{\circ} 40 . \\
013 \mathrm{~S}\end{array}$ & $\begin{array}{c}166^{\circ} \\
12.043 \mathrm{E}\end{array}$ & 12/11/09 & 9 & 21 & 22 & Yes & 1.11 & 0.002 & 0.049 & MLM & 37.97 & 19.76 & $\begin{array}{c}7,17,1 \\
8\end{array}$ & 1.504 & 2.699 & 27.8 & 19 & 0 & 68 & 31 & 0.453 & 2 & 67 & 0.023 & Silt & $\begin{array}{l}\text { extremel } \\
\text { y poorly } \\
\text { sorted }\end{array}$ & $\begin{array}{l}\text { strongly } \\
\text { fine } \\
\text { skewed }\end{array}$ \\
\hline 8 & $\begin{array}{c}77^{\circ} \\
40.017 \mathrm{~S}\end{array}$ & $\begin{array}{c}166^{\circ} 12 \\
036 \mathrm{E}\end{array}$ & $12 / 11 / 09$ & 3 & 33 & 39 & No & 1.155 & 0.145 & 1.126 & MLM & 34.58 & 25.98 & 9,15 & 1.255 & 1.652 & 23.98 & 19 & 0 & 89 & 10 & 0.116 & 2 & 87 & 0.025 & Silt & $\begin{array}{l}\text { extremel } \\
\text { y poorly } \\
\text { sorted }\end{array}$ & $\begin{array}{l}\text { strongly } \\
\text { fine } \\
\text { skewed }\end{array}$ \\
\hline 9 & $\begin{array}{l}77^{\circ} 39 \\
981 \mathrm{~S}\end{array}$ & $\begin{array}{c}166^{\circ} 00 . \\
070 \mathrm{E}\end{array}$ & $12 / 11 / 09$ & 10 & 30 & 32 & $\begin{array}{c}\text { Yes- } \\
\text { sample } \\
11\end{array}$ & 1.475 & 0.021 & 0.228 & MLM & 34.58 & 18.49 & 15,14 & 1.293 & 1.319 & 23.93 & 33 & 0 & 90 & 9 & 0.103 & 5 & 86 & 0.052 & $\begin{array}{l}\text { Sandy } \\
\text { silt }\end{array}$ & $\begin{array}{l}\text { extremel } \\
\text { y poorly } \\
\text { sorted }\end{array}$ & $\begin{array}{c}\text { strongly } \\
\text { fine } \\
\text { skewed }\end{array}$ \\
\hline 10 & $\begin{array}{c}77^{\circ} 40 . \\
018 \mathrm{~S}\end{array}$ & $\begin{array}{c}166^{\circ} \\
48.003 \mathrm{E}\end{array}$ & $12 / 11 / 09$ & 7 & 15 & 7 & Yes & 1.68 & 0.042 & 4.038 & MLM & 37.97 & 14.53 & 20,21 & 1.244 & 0.992 & 21.13 & 41 & 0 & 94 & 5 & 0.063 & 7 & 87 & 0.080 & Silt & $\begin{array}{l}\text { extremel } \\
\text { y poorly } \\
\text { sorted }\end{array}$ & $\begin{array}{c}\text { strongly } \\
\text { fine } \\
\text { skewed }\end{array}$ \\
\hline 11 & $\begin{array}{c}77^{\circ} \\
40.041 \mathrm{~S}\end{array}$ & $\begin{array}{c}165^{\circ} \\
47.995 \mathrm{E}\end{array}$ & $12 / 11 / 09$ & 3 & 60 & 40 & $\begin{array}{c}\text { Yes - } \\
\text { sample } \\
13\end{array}$ & 1.155 & 0.461 & 1.923 & MLM & 26.14 & 19.43 & 17,8 & 1.301 & 2.994 & 13.85 & 23 & 0 & 98 & 2 & 0.020 & 2 & 96 & 0.018 & Silt & $\begin{array}{l}\text { extremel } \\
\text { y poorly } \\
\text { sorted }\end{array}$ & $\begin{array}{c}\text { strongly } \\
\text { fine } \\
\text { skewed }\end{array}$ \\
\hline 12 & $\begin{array}{c}77^{\circ} \\
46.556 \mathrm{~S}\end{array}$ & $\begin{array}{c}166^{\circ} \\
18.876 \mathrm{E}\end{array}$ & $15 / 11 / 09$ & 5 & 27 & 27 & Yes & 1.44 & 0.051 & 0.709 & MLM & 41.68 & 16.39 & $\begin{array}{c}19,13 \\
19\end{array}$ & 1.137 & 0.51 & 25.71 & 38 & 0 & 88 & 11 & 0.127 & 5 & 84 & 0.062 & $\begin{array}{c}\text { Sandy } \\
\text { silt }\end{array}$ & $\begin{array}{l}\text { extremel } \\
\text { y poorly } \\
\text { sorted }\end{array}$ & $\begin{array}{l}\text { strongly } \\
\text { fine } \\
\text { skewed }\end{array}$ \\
\hline 13 & $\begin{array}{c}77^{\circ} \\
46.572 \mathrm{~S}\end{array}$ & $\begin{array}{c}166.18 .8 \\
48 \mathrm{E}\end{array}$ & $15 / 11 / 09$ & 12 & 25 & 25 & No & 1.425 & 0.013 & 0.216 & MLM & 41.68 & 17.64 & 11 & 0.89 & -0.194 & 24.54 & 37 & 0 & 89 & 11 & 0.120 & 6 & 83 & 0.072 & $\begin{array}{c}\text { Sandy } \\
\text { silt }\end{array}$ & $\begin{array}{l}\text { extremel } \\
\text { y poorly } \\
\text { sorted }\end{array}$ & $\begin{array}{c}\text { strongly } \\
\text { fine } \\
\text { skewed }\end{array}$ \\
\hline 14 & $\begin{array}{c}77^{\circ} \\
39.007 \mathrm{~S}\end{array}$ & $\begin{array}{c}166^{\circ} \\
24.263 \mathrm{E}\end{array}$ & 15 -Nov & $\sim 50$ & 10 & 16 & No & 1.735 & 0 & 0.05 & & & & & & & 25.32 & 32 & 0 & 90 & 10 & 0.116 & 2 & 87 & 0.028 & $\begin{array}{c}\text { Sandy } \\
\text { silt }\end{array}$ & & \\
\hline 15 & $\begin{array}{c}77^{\circ} \\
39.974 \mathrm{~S}\end{array}$ & $\begin{array}{c}165^{\circ} \\
48.011 \mathrm{E}\end{array}$ & $16 / 11 / 09$ & 6 & 26 & 39 & No & - & 0.001 & 0.011 & & & & & & & 21.74 & 19 & 0 & 90 & 10 & 0.106 & 0.8 & 90 & 0.009 & Silt & $\begin{array}{l}\text { extremel } \\
\text { y poorly } \\
\text { sorted }\end{array}$ & $\begin{array}{l}\text { strongly } \\
\text { fine } \\
\text { skewed }\end{array}$ \\
\hline 16 & $\begin{array}{c}77^{\circ} \\
40.033 \mathrm{~S}\end{array}$ & $\begin{array}{c}165^{\circ} \\
36.009 \mathrm{E}\end{array}$ & $16 / 11 / 09$ & 4.5 & 30 & 38 & Yes & 1.265 & 0.042 & 0.376 & MLM & 28.7 & 16.99 & $\begin{array}{c}15,12 \\
15\end{array}$ & 1.483 & 3.571 & 16.26 & 36 & 0 & 98 & 2 & 0.021 & 4 & 94 & $\begin{array}{l}0.044 \\
74165\end{array}$ & Silt & $\begin{array}{l}\text { extremel } \\
\text { y poorly } \\
\text { sorted }\end{array}$ & $\begin{array}{c}\text { strongly } \\
\text { fine } \\
\text { skewed }\end{array}$ \\
\hline 17 & $\begin{array}{c}77^{\circ} \\
40.030 \mathrm{~S}\end{array}$ & $\begin{array}{c}165^{\circ} \\
28.977 \mathrm{E}\end{array}$ & $16 / 11 / 09$ & 9 & 14 & 32 & Yes & 1.48 & 0.103 & 8.062 & MLM & 26.14 & 10.49 & 21,18 & 1.84 & 3.676 & 18.64 & 50 & 0 & 96 & 4 & 0.039 & 7.7 & 88 & 0.087 & Silt & $\begin{array}{l}\text { extremel } \\
\text { y poorly } \\
\text { sorted }\end{array}$ & $\begin{array}{l}\text { strongly } \\
\text { fine } \\
\text { skewed }\end{array}$ \\
\hline 18 & $\begin{array}{c}77^{\circ} \\
40.030 \mathrm{~S}\end{array}$ & $\begin{array}{c}165^{\circ} \\
24.003 \mathrm{E}\end{array}$ & $16 / 11 / 09$ & 11 & 21 & 29 & No & 1.33 & 0.055 & 0.904 & MLM & 28.7 & 17.07 & 21,20 & 1.471 & 2.007 & 16.41 & 49. & 0 & 98 & 2 & 0.021 & 6.7 & 91 & 0.072 & Silt & $\begin{array}{l}\text { extremel } \\
\text { y poorly } \\
\text { sorted }\end{array}$ & $\begin{array}{l}\text { strongly } \\
\text { fine } \\
\text { skewed }\end{array}$ \\
\hline
\end{tabular}




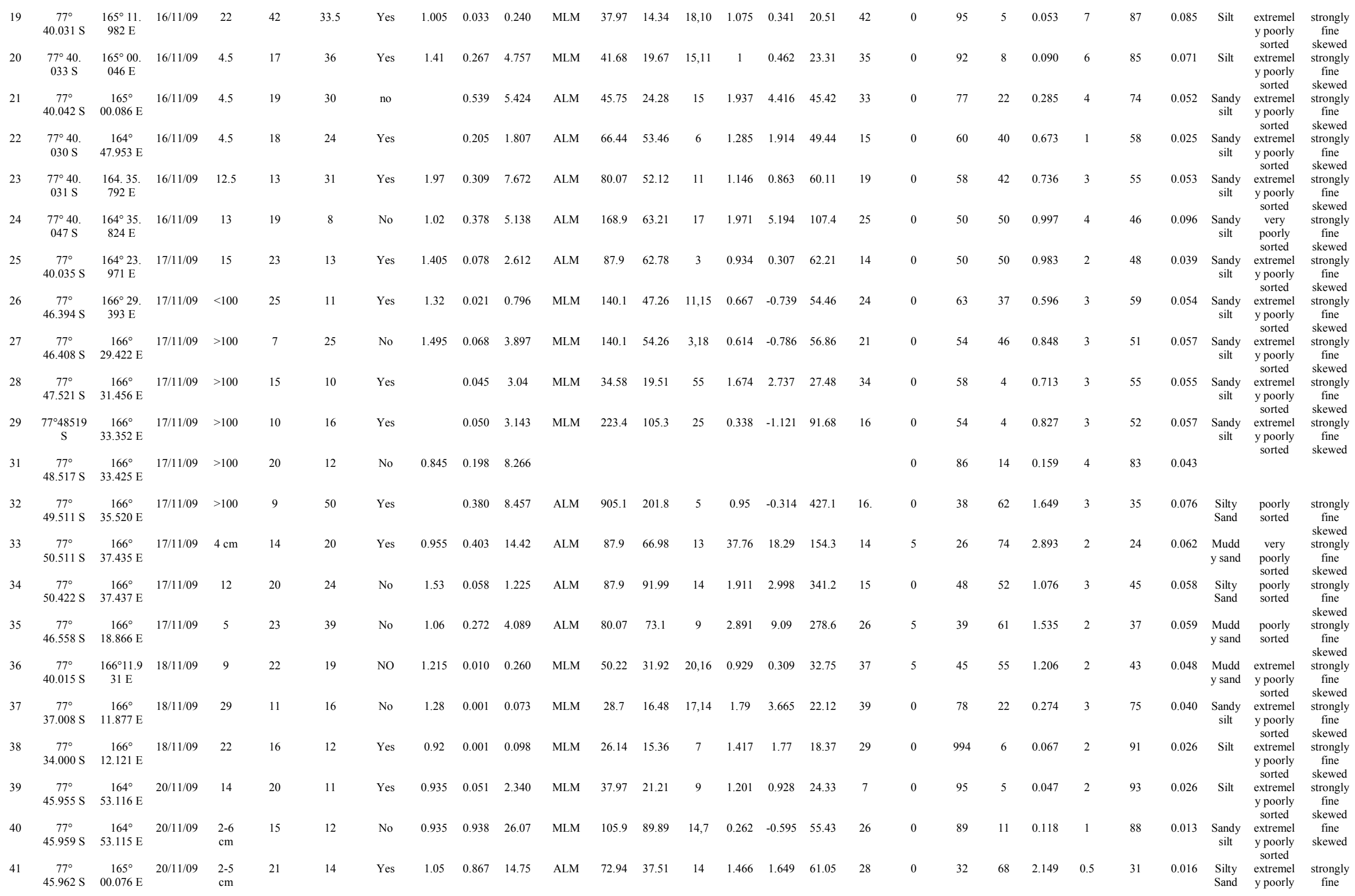




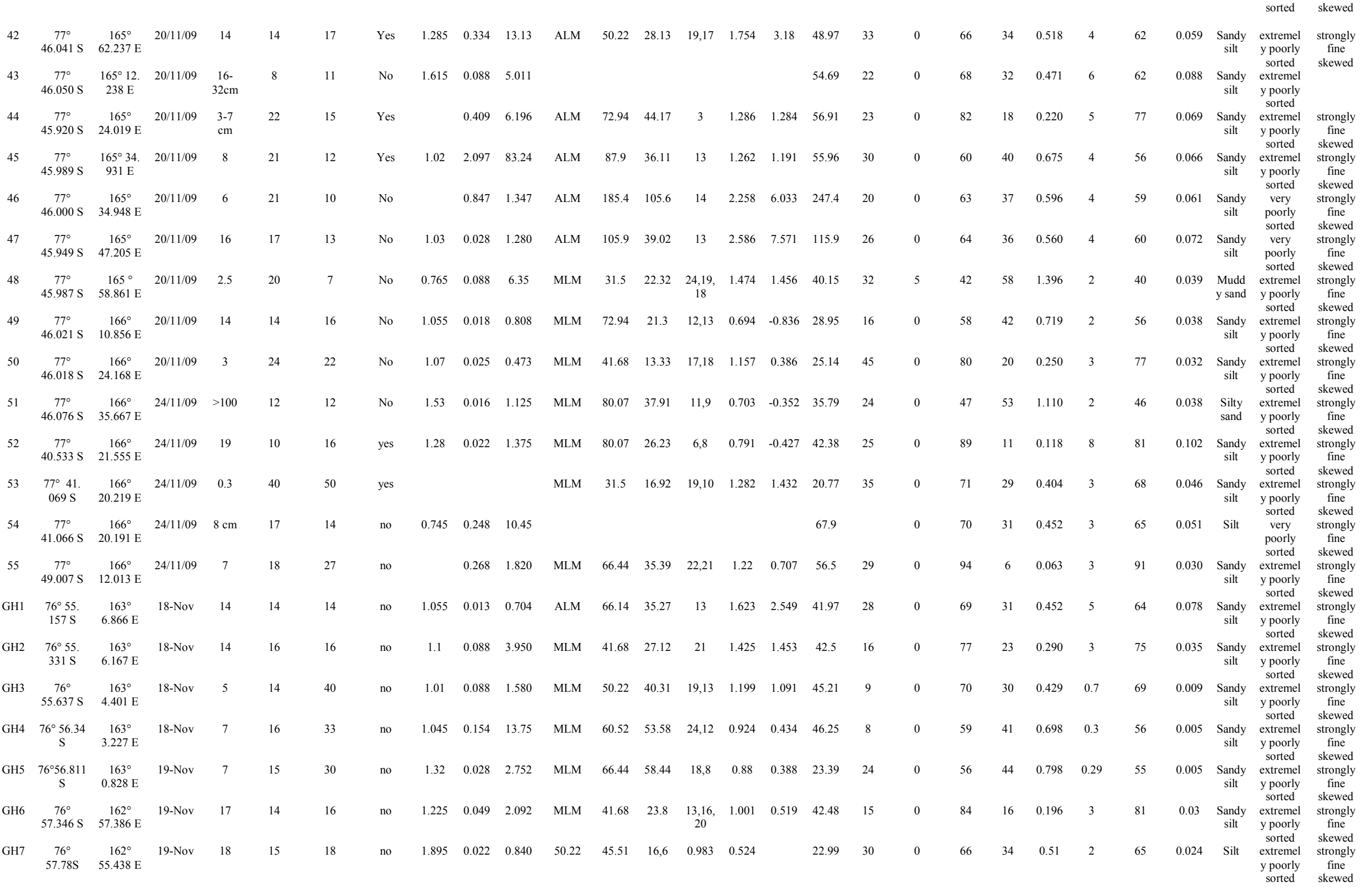




\begin{tabular}{|c|c|c|c|c|c|c|c|c|c|c|c|c|c|c|c|c|c|c|c|c|c|c|c|c|c|c|c|c|}
\hline GH8 & $\begin{array}{c}76^{\circ} \\
58.357 \mathrm{~S}\end{array}$ & $\begin{array}{c}162^{\circ} \\
52.782 \mathrm{E}\end{array}$ & 19-Nov & 5 & 60 & 18 & no & 0.77 & 0.019 & 0.643 & MLM & 37.97 & 21.02 & 17,18 & 1.122 & 1.026 & 32.09 & 24 & 0 & 92 & 8 & 0.088 & 8 & 88 & 0.042 & $\begin{array}{l}\text { Sandy } \\
\text { silt }\end{array}$ & $\begin{array}{l}\text { extremel } \\
\text { y poorly } \\
\text { sorted }\end{array}$ & $\begin{array}{c}\text { strongly } \\
\text { fine } \\
\text { skewed }\end{array}$ \\
\hline GH9 & $\begin{array}{c}76^{\circ} \\
58.359 \mathrm{~S}\end{array}$ & $\begin{array}{c}162^{\circ} \\
52.803 \mathrm{E}\end{array}$ & 19-Nov & 7 & 34 & 15 & no & 1.095 & 0.006 & 0.134 & MLM & 41.68 & 30.59 & 11,14 & 1.035 & 0.507 & & & 0 & 80 & 20 & 0.244 & 3 & 77 & 0.040 & & & \\
\hline $\begin{array}{c}\mathrm{GH} 1 \\
0\end{array}$ & $\begin{array}{c}76^{\circ} \\
59.161 \mathrm{~S}\end{array}$ & $\begin{array}{l}162^{\circ} \\
47.93\end{array}$ & 19-Nov & 8 & 17 & 28 & no & 1.095 & 0.004 & 0.345 & MLM & 31.5 & 24.55 & 17 & 1.865 & 3.538 & 34.61 & 23 & 0 & 85 & 15 & 0.172 & 1 & 84 & 0.013 & $\begin{array}{l}\text { Sandy } \\
\text { silt }\end{array}$ & $\begin{array}{l}\text { extremel } \\
\text { y poorly } \\
\text { sorted }\end{array}$ & $\begin{array}{c}\text { strongly } \\
\text { fine } \\
\text { skewed }\end{array}$ \\
\hline
\end{tabular}




\section{Appendix 2: Beckman Coulter-Counter Multi-sizer 3 methodology}

\section{Beckman Coulter-Counter Multi-sizer 3}

The Multi-sizer 3 (MS3) works by detection of an electrical signal generated by changes in resistance caused by particles suspended in an electrolyte that are forced to flow through a small aperture tube. Table A1 illustrates the size range of particles that different aperture tubes are capable of measuring. The advantages of this method are:

- Size distribution and particle concentration are obtained simultaneously, in contrast to direct weighing and LLS because the number of particles as well as their size are determined in each sample. If grain density is assumed, then the mass of particulates in each sample can be calculated without having to be physically weighed.

- It gives very high resolution in silt and clay size ranges.

- It can measure samples with very low ( $\mathrm{ppb})$ concentrations of particulates.

Table A1: Aperture tube diameters for Coulter Counter analysis. Source: Beckman-Coulter Inc. operators manual (2005).

\begin{tabular}{cc}
\hline $\begin{array}{c}\text { Aperture tube } \\
(\mu \mathrm{m})\end{array}$ & $\begin{array}{c}\text { Analysis range } \\
(\mu \mathrm{m})\end{array}$ \\
\hline 400 & $8.0-240$ \\
280 & $5.6-168$ \\
100 & $0.2-60$ \\
50 & $1.0-30$ \\
30 & $0.6-18.0$ \\
20 & $0.4-12.0$ \\
\hline
\end{tabular}

Unlike ice cores from the polar plateau (e.g. North Greenland Ice core Drilling Project (NGRIP), Greenland and Dome C and Vostok, East Antarctica) where the particle size range is small (e.g. Delmonte, 2002), coastal ice cores contain a wider range of particles (e.g. 0.2 $150 \mu \mathrm{m}$; Dunbar et al., 2009). The standard MS3 method is therefore not readily applicable to the McMurdo Sound samples and an invitation to the Glaciology Laboratory, University of Milano-Biccoca, Italy was accepted to modify the technique and measure dust in ice samples where the particles fall outside of the standard aperture size range $(0.2 \mu \mathrm{m}-60 \mu \mathrm{m})$. However, because of the large size range and high concentrations of McMurdo Sound 
particles in samples it was difficult to obtain a representative aliquot for measurement. Therefore, filtering/weighing in conjunction with the Beckman Coulter LS13320 Particle Size Analyser, which has a dynamic range of $0.4-2000 \mu \mathrm{m}$, was considered more appropriate for these samples. Furthermore, directly measuring the weight of sediment from melted and filtered snow or ice sediment allows the sample to be reclaimed from the filter for subsequent petrographic studies.

\section{Methodology}

Sixteen surface snow samples were analysed in the Glaciology Laboratory at the University of Milano-Bicocca, Italy with the intention that the remainder would be analysed at the National Ice Core Facility, GNS Science. The traditional method for particle size and concentration in ice cores from the polar plateau is described in detail in Delmonte et al. (2002), however this method was modified to accommodate for the larger particle size distribution of coastal samples which cannot be measured using a single aperture tube. Aliquots of melted surface snow samples were diluted and made conductive by adding pre filtered $20 \mathrm{wt} \% \mathrm{NaCl}$ electrolyte solution giving a $1 \%$ concentration to the final solution. The samples were mechanically stirred before the analysis and continuously stirred during analysis in order to prevent particles settling. At least three consecutive counts were performed on each volume of $0.5 \mu \mathrm{l}$ using the $100 \mu \mathrm{m}$ and $280 \mu \mathrm{m}$ aperture tubes in order to capture the entire particle size distribution. The instrument was set for measurements of particles with diameters from $1.65 \mu \mathrm{m}$ to $60 \mu \mathrm{m}$ for the $100 \mu \mathrm{m}$ tube and $5.6 \mu \mathrm{m}$ to $200 \mu \mathrm{m}$ for the $280 \mu \mathrm{m}$ tube in 256 channels.

The particle size is expressed by the diameter of a sphere with an equivalent volume. The mass was calculated from the measured volume assuming a particle density of $2.5 \mathrm{~g} \mathrm{~cm}^{-3}$. The overall size distribution for each sample was calculated by averaging the values in the overlapping size bins of the 100 and $280 \mu \mathrm{m}$ tubes and taking the $<5.6 \mu \mathrm{m}$ spectrum from the $100 \mu \mathrm{m}$ tube and $>60 \mu \mathrm{m}$ spectrum from the $280 \mu \mathrm{m}$ tube. 
Appendix 3A: Grain size data for Marble Point-Cape Roberts dust samples.

\begin{tabular}{|c|c|c|c|c|c|}
\hline $\begin{array}{c}\text { Diameter } \\
\mu \mathrm{m}\end{array}$ & MPR13-5 & NPN11-5 & MPK10 & CREG8 & CREG6 \\
\hline 0.37512 & 0.06070215 & 0 & 0 & 0 & 0 \\
\hline 0.4118 & 0.1079725 & 0 & 0 & 0 & 0 \\
\hline 0.45206 & 0.159364 & 0 & 0 & 0 & 0 \\
\hline 0.49625 & 0.222389 & 0 & 0 & 0 & 0 \\
\hline 0.54477 & 0.2672885 & 0 & 0 & 0 & 0 \\
\hline 0.59803 & 0.298508 & 0 & 0 & 0 & 0 \\
\hline 0.65649 & 0.3211005 & 0 & 0 & 0 & 0 \\
\hline 0.72068 & 0.335416 & 0 & 0 & 0 & 0 \\
\hline 0.79113 & 0.334412 & 0 & 0 & 0 & 0 \\
\hline 0.86848 & 0.318961 & 0 & 0 & 0 & 0 \\
\hline 0.95338 & 0.2961065 & 0.003796224 & 0 & 0 & 0.001091068 \\
\hline 1.0466 & 0.2756995 & 0.024596333 & 0.002887996 & 0 & 0.008749016 \\
\hline 1.1489 & 0.2598145 & 0.0841328 & 0.016258673 & 0 & 0.035651847 \\
\hline 1.2612 & 0.2554235 & 0.188029333 & 0.049592797 & 0 & 0.08899105 \\
\hline 1.3845 & 0.2655275 & 0.324887 & 0.116420567 & 0.00882898 & 0.166902023 \\
\hline 1.5199 & 0.3059155 & 0.479991333 & 0.230405 & 0.0489099 & 0.266911517 \\
\hline 1.6685 & 0.378866 & 0.648156 & 0.399742333 & 0.136353 & 0.406857087 \\
\hline 1.8316 & 0.491232 & 0.824457 & 0.608779667 & 0.261818 & 0.603771577 \\
\hline 2.0107 & 0.6318305 & 0.99324 & 0.817653667 & 0.38876 & 0.840081983 \\
\hline 2.2072 & 0.796742 & 1.149783333 & 1.000583667 & 0.502921 & 1.0740705 \\
\hline 2.423 & 0.9762355 & 1.272173333 & 1.127963333 & 0.589534 & 1.2576925 \\
\hline 2.6599 & 1.154525 & 1.366356667 & 1.201663333 & 0.652137 & 1.368572667 \\
\hline 2.92 & 1.31704 & 1.425066667 & 1.219416667 & 0.687075 & 1.393753833 \\
\hline 3.2054 & 1.42975 & 1.458323333 & 1.202856667 & 0.702235 & 1.3560155 \\
\hline 3.5188 & 1.494205 & 1.473646667 & 1.16698 & 0.700537 & 1.289126667 \\
\hline 3.8628 & 1.49181 & 1.473933333 & 1.131983333 & 0.694838 & 1.236630667 \\
\hline 4.2405 & 1.451825 & 1.48389 & 1.11899 & 0.69996 & 1.238123833 \\
\hline 4.6551 & 1.381305 & 1.484033333 & 1.11496 & 0.707491 & 1.279011833 \\
\hline 5.1102 & 1.31497 & 1.507103333 & 1.134873333 & 0.725879 & 1.362421833 \\
\hline 5.6098 & 1.272175 & 1.509943333 & 1.137946667 & 0.727238 & 1.416881 \\
\hline 6.1582 & 1.248115 & 1.539393333 & 1.156023333 & 0.738903 & 1.462914833 \\
\hline 6.7603 & 1.25954 & 1.565446667 & 1.159786667 & 0.746399 & 1.463423 \\
\hline 7.4212 & 1.258505 & 1.634253333 & 1.1879 & 0.778078 & 1.481337167 \\
\hline 8.1467 & 1.291315 & 1.71993 & 1.21886 & 0.809387 & 1.509228 \\
\hline 8.9432 & 1.30767 & 1.822996667 & 1.262603333 & 0.837371 & 1.57047 \\
\hline 9.8175 & 1.366685 & 1.968773333 & 1.346293333 & 0.879084 & 1.696817667 \\
\hline 10.777 & 1.41634 & 2.13237 & 1.45537 & 0.937007 & 1.861328333 \\
\hline 11.831 & 1.47459 & 2.38085 & 1.643103333 & 1.06744 & 2.113066667 \\
\hline 12.988 & 1.56237 & 2.607563333 & 1.819626667 & 1.21725 & 2.329211667 \\
\hline 14.257 & 1.6631 & 2.816316667 & 1.979913333 & 1.37641 & 2.515375 \\
\hline 15.651 & 1.85361 & 2.87396 & 2.013223333 & 1.43701 & 2.560803333 \\
\hline 17.181 & 2.029085 & 2.809486667 & 1.924146667 & 1.38263 & 2.513875 \\
\hline 18.861 & 2.21218 & 2.691403333 & 1.772036667 & 1.2597 & 2.446746667 \\
\hline 20.705 & 2.290605 & 2.61935 & 1.640023333 & 1.15048 & 2.426896667 \\
\hline 22.729 & 2.290655 & 2.725586667 & 1.66186 & 1.17259 & 2.556573333 \\
\hline 24.951 & 2.24912 & 2.980923333 & 1.85511 & 1.33631 & 2.781818333 \\
\hline 27.391 & 2.23753 & 3.36193 & 2.234853333 & 1.64118 & 3.093131667 \\
\hline
\end{tabular}




\begin{tabular}{|c|c|c|c|c|c|}
\hline 30.068 & 2.33942 & 3.712866667 & 2.69748 & 1.98358 & 3.39156 \\
\hline 33.008 & 2.57436 & 3.955296667 & 3.127146667 & 2.2676 & 3.631138333 \\
\hline 36.235 & 2.922025 & 4.07281 & 3.42771 & 2.44929 & 3.80195 \\
\hline 39.778 & 3.280675 & 4.11061 & 3.562786667 & 2.55093 & 3.909583333 \\
\hline 43.667 & 3.55559 & 4.150896667 & 3.58176 & 2.65978 & 3.976703333 \\
\hline 47.936 & 3.641155 & 4.200023333 & 3.545303333 & 2.8326 & 3.984003333 \\
\hline 52.622 & 3.559355 & 4.202466667 & 3.51405 & 3.0801 & 3.87349 \\
\hline 57.767 & 3.34583 & 4.033296667 & 3.507523333 & 3.34564 & 3.477966667 \\
\hline 63.414 & 3.12069 & 3.566383333 & 3.506883333 & 3.51763 & 2.8265 \\
\hline 69.614 & 2.97587 & 2.58753 & 3.47096 & 3.50096 & 2.129707 \\
\hline 76.42 & 2.950035 & 1.40288 & 3.356993333 & 3.27001 & 1.586245917 \\
\hline 83.891 & 3.051325 & 0.476501333 & 3.165623333 & 2.90783 & 1.167183 \\
\hline 92.092 & 3.17585 & 0.0932379 & 2.927106667 & 2.57222 & 0.787025167 \\
\hline 101.1 & 3.18931 & 0.008836267 & 2.686056667 & 2.39619 & 0.575305333 \\
\hline 110.98 & 2.938185 & 0 & 2.472873333 & 2.45393 & 0.739438867 \\
\hline 121.83 & 2.353015 & 0 & 2.267796667 & 2.72638 & 1.221631427 \\
\hline 133.74 & 1.487035 & 0 & 2.01831 & 3.12613 & 1.64172325 \\
\hline 146.81 & 0.6663555 & 0 & 1.719971 & 3.51426 & 1.71565 \\
\hline 161.17 & 0.1730158 & 0 & 1.3411234 & 3.74353 & 1.374085 \\
\hline 176.92 & 0.022034645 & 0 & 0.945990203 & 3.71944 & 0.780426667 \\
\hline 194.22 & 0.00073091 & 0 & 0.584372667 & 3.40444 & 0.281336667 \\
\hline 213.21 & 0 & 0 & 0.304037467 & 2.84715 & 0.049769 \\
\hline 234.05 & 0 & 0 & 0.113951972 & 2.17109 & 0.003193967 \\
\hline 256.94 & 0 & 0 & 0.0216232 & 1.53276 & 0 \\
\hline 282.06 & 0 & 0 & 0.001665763 & 1.06621 & 0 \\
\hline 309.63 & 0 & 0 & 0 & 0.804574 & 0 \\
\hline 339.9 & 0 & 0 & 0 & 0.697525 & 0 \\
\hline 373.13 & 0 & 0 & 0 & 0.657331 & 0 \\
\hline 409.61 & 0 & 0 & 0 & 0.582785 & 0 \\
\hline 449.66 & 0 & 0 & 0 & 0.395172 & 0 \\
\hline 493.62 & 0 & 0 & 0 & 0.178824 & 0 \\
\hline 541.88 & 0 & 0 & 0 & 0.0383484 & 0 \\
\hline 594.85 & 0 & 0 & 0 & 0.00336616 & 0 \\
\hline 653.01 & 0 & 0 & 0 & 0 & 0 \\
\hline 716.85 & 0 & 0 & 0 & 0 & 0 \\
\hline 786.93 & 0 & 0 & 0 & 0 & 0 \\
\hline 863.87 & 0 & 0 & 0 & 0 & 0 \\
\hline 948.32 & 0 & 0 & 0 & 0 & 0 \\
\hline 1041 & 0 & 0 & 0 & 0 & 0 \\
\hline 1142.8 & 0 & 0 & 0 & 0 & 0 \\
\hline 1254.5 & 0 & 0 & 0 & 0 & 0 \\
\hline 1377.2 & 0 & 0 & 0 & 0 & 0 \\
\hline 1511.8 & 0 & 0 & 0 & 0 & 0 \\
\hline 1659.6 & 0 & 0 & 0 & 0 & 0 \\
\hline 1821.9 & 0 & 0 & 0 & 0 & 0 \\
\hline 2000 & 0 & 0 & 0 & 0 & 0 \\
\hline
\end{tabular}


Appendix 3B: Grain size data for Granite Harbour dust samples.

\begin{tabular}{|c|c|c|c|c|c|c|c|c|c|c|}
\hline $\begin{array}{c}\text { Diameter } \\
\mu \mathrm{m}\end{array}$ & GH_1 & GH_2 & GH_3 & GH_4 & GH_5 & GH_6 & GH_7 & GH_8 & GH_9 & GH_10 \\
\hline 0.4 & 0 & 0 & 0 & 0 & 0 & 0 & 0 & 0 & 0.0047285 & 0 \\
\hline 0.4 & 0 & 0 & 0 & 0 & 0 & 0 & 0 & 0 & 0.00834605 & 0 \\
\hline 0.5 & 0 & 0 & 0 & 0 & 0 & 0 & 0 & 0 & 0.012280467 & 0 \\
\hline 0.5 & 0 & 0 & 0 & 0 & 0 & 0 & 0 & 0 & 0.016621083 & 0 \\
\hline 0.5 & 0 & 0 & 0 & 0 & 0 & 0 & 0 & 0 & 0.019010667 & 0 \\
\hline 0.6 & 0 & 0 & 0 & 0 & 0 & 0 & 0 & 0 & 0.019744333 & 0 \\
\hline 0.7 & 0 & 0 & 0 & 0 & 0 & 0 & 0 & 0 & 0.019570167 & 0 \\
\hline 0.7 & 0 & 0 & 0 & 0 & 0 & 0 & 0 & 0 & 0.018254667 & 0 \\
\hline 0.8 & 0 & 0 & 0 & 0 & 0 & 0 & 0 & 0 & 0.015137167 & 0 \\
\hline 0.9 & 0 & 0 & 0 & 0 & 0 & 0 & 0 & 0.002302845 & 0.010824987 & 0 \\
\hline 1.0 & 0 & 0 & 0 & 0 & 0.002802507 & 0.001780508 & 0.005147965 & 0.01533125 & 0.007711778 & 0 \\
\hline 1.0 & 0 & 0 & 0 & 0 & 0.017456573 & 0.011402405 & 0.03159125 & 0.05284115 & 0.012770612 & 0 \\
\hline 1.1 & 0.00765059 & 0 & 0 & 0 & 0.0569619 & 0.0380759 & 0.099485 & 0.1186955 & 0.040514909 & 0 \\
\hline 1.3 & 0.04720175 & 0 & 0 & 0 & 0.130152469 & 0.0851182 & 0.211604 & 0.206824 & 0.11307016 & 0.0030771 \\
\hline 1.4 & 0.147211 & 0.00800709 & 0.002482215 & 0.002721399 & 0.230375313 & 0.145142 & 0.3501875 & 0.3083775 & 0.238575983 & 0.0236839 \\
\hline 1.5 & 0.310661 & 0.04205595 & 0.01545795 & 0.01525612 & 0.3529693 & 0.214087 & 0.5070755 & 0.42505 & 0.415838167 & 0.0915773 \\
\hline 1.7 & 0.5031905 & 0.1091885 & 0.04813145 & 0.043307888 & 0.485412225 & 0.2874255 & 0.6737445 & 0.54819 & 0.623933167 & 0.204563 \\
\hline 1.8 & 0.7074075 & 0.20514 & 0.10265015 & 0.088543638 & 0.627808425 & 0.3670195 & 0.8492775 & 0.677759 & 0.85899 & 0.347208 \\
\hline 2.0 & 0.9026525 & 0.3023045 & 0.1654465 & 0.139695675 & 0.765476 & 0.444046 & 1.0162515 & 0.8002695 & 1.0979305 & 0.48526 \\
\hline 2.2 & 1.084875 & 0.4029475 & 0.230607 & 0.19399225 & 0.895532 & 0.5180775 & 1.17099 & 0.9139865 & 1.3357165 & 0.624887 \\
\hline 2.4 & 1.23494 & 0.495508 & 0.289213 & 0.2432845 & 1.00131025 & 0.579632 & 1.29261 & 1.0061675 & 1.550843333 & 0.751812 \\
\hline 2.7 & 1.352235 & 0.5829435 & 0.3440135 & 0.289483125 & 1.0839515 & 0.6302455 & 1.38541 & 1.079685 & 1.734995 & 0.868667 \\
\hline 2.9 & 1.426505 & 0.661388 & 0.3923235 & 0.330432625 & 1.141215 & 0.6698925 & 1.447035 & 1.13342 & 1.875703333 & 0.969526 \\
\hline 3.2 & 1.46317 & 0.72485 & 0.431531 & 0.36326825 & 1.17499175 & 0.6971145 & 1.481745 & 1.16687 & 1.96446 & 1.04575 \\
\hline 3.5 & 1.48008 & 0.780025 & 0.463991 & 0.39085575 & 1.19718775 & 0.7192565 & 1.502555 & 1.1909 & 2.01553 & 1.11204 \\
\hline 3.9 & 1.48352 & 0.822912 & 0.487346 & 0.41020925 & 1.20426 & 0.7306765 & 1.505625 & 1.19966 & 2.024748333 & 1.16432 \\
\hline 4.2 & 1.502625 & 0.8774055 & 0.518233 & 0.43576875 & 1.2222115 & 0.7491375 & 1.521905 & 1.217725 & 2.027516667 & 1.23679 \\
\hline 4.7 & 1.51648 & 0.9265105 & 0.5463795 & 0.458186125 & 1.2331215 & 0.7620735 & 1.53026 & 1.227565 & 2.00989 & 1.30321 \\
\hline 5.1 & 1.549795 & 0.9889985 & 0.584575 & 0.48943225 & 1.264437 & 0.7888855 & 1.565085 & 1.256685 & 2.008731667 & 1.3912 \\
\hline 5.6 & 1.56309 & 1.03388 & 0.610504 & 0.51034275 & 1.28170825 & 0.8070125 & 1.58373 & 1.27353 & 1.999708333 & 1.45698 \\
\hline
\end{tabular}




\begin{tabular}{|c|c|c|c|c|c|c|c|c|c|c|}
\hline 6.2 & 1.59128 & 1.08458 & 0.6405725 & 0.535457625 & 1.31550675 & 0.836014 & 1.624025 & 1.30703 & 2.012766667 & 1.5358 \\
\hline 6.8 & 1.613155 & 1.129305 & 0.6672175 & 0.558168125 & 1.34723275 & 0.864597 & 1.66148 & 1.339725 & 2.034611667 & 1.60748 \\
\hline 7.4 & 1.66333 & 1.19425 & 0.7112585 & 0.596698125 & 1.409099 & 0.913598 & 1.736065 & 1.400305 & 2.07583 & 1.70682 \\
\hline 8.1 & 1.729165 & 1.2738 & 0.76863 & 0.647713 & 1.491447 & 0.977829 & 1.834255 & 1.480065 & 2.140995 & 1.82183 \\
\hline 8.9 & 1.805835 & 1.36395 & 0.8379925 & 0.710362375 & 1.5913975 & 1.055215 & 1.95179 & 1.575275 & 2.208085 & 1.94664 \\
\hline 9.8 & 1.910805 & 1.479105 & 0.930164 & 0.79447275 & 1.72577 & 1.157615 & 2.107065 & 1.701195 & 2.318068333 & 2.10076 \\
\hline 10.8 & 2.012625 & 1.59625 & 1.031345 & 0.88786425 & 1.8654575 & 1.265295 & 2.26313 & 1.827605 & 2.42566 & 2.25229 \\
\hline 11.8 & 2.16218 & 1.75218 & 1.167885 & 1.01376525 & 2.0563475 & 1.407005 & 2.476695 & 1.99506 & 2.581818333 & 2.45649 \\
\hline 14.3 & 2.409035 & 2.044485 & 1.441925 & 1.26872125 & 2.40894 & 1.666785 & 2.85259 & 2.28049 & 2.846368333 & 2.85306 \\
\hline 15.7 & 2.43827 & 2.13468 & 1.538785 & 1.36053125 & 2.5078975 & 1.739695 & 2.93361 & 2.342125 & 2.925451667 & 3.01378 \\
\hline 17.2 & 2.38308 & 2.17438 & 1.59649 & 1.417795 & 2.5391725 & 1.76226 & 2.91593 & 2.33345 & 2.950536667 & 3.16871 \\
\hline 18.9 & 2.26638 & 2.160525 & 1.61016 & 1.4371225 & 2.51365 & 1.73808 & 2.819865 & 2.26501 & 2.971251667 & 3.31542 \\
\hline 20.7 & 2.14423 & 2.12967 & 1.60845 & 1.4472825 & 2.4876025 & 1.70942 & 2.722235 & 2.192635 & 2.971111667 & 3.47879 \\
\hline 22.7 & 2.12417 & 2.16535 & 1.6633 & 1.5176675 & 2.5658825 & 1.76482 & 2.76096 & 2.226645 & 3.00732 & 3.68806 \\
\hline 25.0 & 2.21778 & 2.295135 & 1.80891 & 1.68234 & 2.7731975 & 1.94244 & 2.96923 & 2.40645 & 3.023961667 & 3.87756 \\
\hline 30.1 & 2.73157 & 2.946695 & 2.539845 & 2.45188625 & 3.5939275 & 2.78728 & 3.86115 & 3.275485 & 3.016808333 & 4.05618 \\
\hline 33.0 & 2.98731 & 3.363545 & 3.066285 & 2.99480375 & 4.024435 & 3.33975 & 4.281365 & 3.78553 & 3.000488333 & 3.95465 \\
\hline 36.2 & 3.14268 & 3.733095 & 3.605405 & 3.54902875 & 4.3213675 & 3.84695 & 4.486045 & 4.17038 & 3.022513333 & 3.74363 \\
\hline 39.8 & 3.17392 & 3.990995 & 4.07689 & 4.038365 & 4.4230325 & 4.224395 & 4.407385 & 4.335225 & 3.083831667 & 3.47711 \\
\hline 43.7 & 3.122515 & 4.132505 & 4.44784 & 4.435595 & 4.34362 & 4.453995 & 4.085735 & 4.273575 & 3.160458333 & 3.2181 \\
\hline 47.9 & 3.030495 & 4.16752 & 4.69667 & 4.7235725 & 4.12469 & 4.541755 & 3.60752 & 4.028025 & 3.18842 & 2.97959 \\
\hline 52.6 & 2.939675 & 4.144445 & 4.85126 & 4.9290325 & 3.8439475 & 4.53994 & 3.08972 & 3.699235 & 3.079876667 & 2.75853 \\
\hline 57.8 & 2.852925 & 4.08517 & 4.925345 & 5.05817375 & 3.547735 & 4.47906 & 2.60943 & 3.374905 & 2.641628333 & 2.51858 \\
\hline 63.4 & 2.74355 & 3.98585 & 4.920215 & 5.10250875 & 3.254285 & 4.36831 & 2.195525 & 3.11558 & 2.0605295 & 2.22411 \\
\hline 69.6 & 2.579105 & 3.82203 & 4.823415 & 5.03832625 & 2.9472475 & 4.197285 & 1.83435 & 2.9412 & 1.493414667 & 1.86916 \\
\hline 76.4 & 2.347085 & 3.55506 & 4.607725 & 4.83504875 & 2.585455 & 3.94255 & 1.488135 & 2.820555 & 1.1740174 & 1.50041 \\
\hline 83.9 & 2.09117 & 3.18495 & 4.275075 & 4.50290625 & 2.151225 & 3.615245 & 1.133435 & 2.69679 & 0.947096 & 1.20479 \\
\hline 92.1 & 1.887365 & 2.77263 & 3.87742 & 4.0965325 & 1.6614165 & 3.266495 & 0.774803 & 2.493205 & 0.759329567 & 1.05877 \\
\hline 101.1 & 1.801225 & 2.40115 & 3.488475 & 3.70534625 & 1.17834375 & 2.95504 & 0.4524925 & 2.148605 & 0.551571333 & 1.08246 \\
\hline
\end{tabular}




\begin{tabular}{|c|c|c|c|c|c|c|c|c|c|c|}
\hline 133.7 & 1.88137 & 1.90212 & 2.72542 & 2.9941875 & 0.434579873 & 2.25395 & 0.012318807 & 0.5132045 & 0.027097333 & 1.31528 \\
\hline 146.8 & 1.617915 & 1.70669 & 2.39023 & 2.670965 & 0.361905457 & 1.853425 & 0 & 0.16557495 & 0.002354817 & 1.03517 \\
\hline 161.2 & 1.1017795 & 1.338255 & 1.87524 & 2.1226025 & 0.2755925 & 1.27669 & 0 & 0.028094355 & 0 & 0.590033 \\
\hline 176.9 & 0.5384205 & 0.801046 & 1.212095 & 1.4470675 & 0.16262425 & 0.6658575 & 0 & 0.002008695 & 0 & 0.217179 \\
\hline 194.2 & 0.160868 & 0.3251035 & 0.5828085 & 0.809402375 & 0.06503625 & 0.23010055 & 0 & 0 & 0 & 0.0392075 \\
\hline 213.2 & 0.02461524 & 0.0648229 & 0.18150835 & 0.353656263 & 0.01279665 & 0.04165802 & 0 & 0 & 0 & 0.00262865 \\
\hline 234.1 & 0.001369405 & 0.00529888 & 0.029490785 & 0.103229696 & 0.001008483 & 0.00325728 & 0 & 0 & 0 & 0 \\
\hline 256.9 & 0 & 0 & 0.001910725 & 0.015606681 & 0 & 0 & 0 & 0 & 0 & 0 \\
\hline 282.1 & 0 & 0 & 0 & 0.00080117 & 0 & 0 & 0 & 0 & 0 & 0 \\
\hline 309.6 & 0 & 0 & 0 & 0 & 0 & 0 & 0 & 0 & 0 & 0 \\
\hline 339.9 & 0 & 0 & 0 & 0 & 0 & 0 & 0 & 0 & 0 & 0 \\
\hline 373.1 & 0 & 0 & 0 & 0 & 0 & 0 & 0 & 0 & 0 & 0 \\
\hline 409.6 & 0 & 0 & 0 & 0 & 0 & 0 & 0 & 0 & 0 & 0 \\
\hline 449.7 & 0 & 0 & 0 & 0 & 0 & 0 & 0 & 0 & 0 & 0 \\
\hline 493.6 & 0 & 0 & 0 & 0 & 0 & 0 & 0 & 0 & 0 & 0 \\
\hline 541.9 & 0 & 0 & 0 & 0 & 0 & 0 & 0 & 0 & 0 & 0 \\
\hline 594.9 & 0 & 0 & 0 & 0 & 0 & 0 & 0 & 0 & 0 & 0 \\
\hline 653.0 & 0 & 0 & 0 & 0 & 0 & 0 & 0 & 0 & 0 & 0 \\
\hline 716.8 & 0 & 0 & 0 & 0 & 0 & 0 & 0 & 0 & 0 & 0 \\
\hline 786.9 & 0 & 0 & 0 & 0 & 0 & 0 & 0 & 0 & 0 & 0 \\
\hline 863.9 & 0 & 0 & 0 & 0 & 0 & 0 & 0 & 0 & 0 & 0 \\
\hline 948.3 & 0 & 0 & 0 & 0 & 0 & 0 & 0 & 0 & 0 & 0 \\
\hline 1041.0 & 0 & 0 & 0 & 0 & 0 & 0 & 0 & 0 & 0 & 0 \\
\hline 1142.8 & 0 & 0 & 0 & 0 & 0 & 0 & 0 & 0 & 0 & 0 \\
\hline 1254.5 & 0 & 0 & 0 & 0 & 0 & 0 & 0 & 0 & 0 & 0 \\
\hline 1377.2 & 0 & 0 & 0 & 0 & 0 & 0 & 0 & 0 & 0 & 0 \\
\hline 1511.8 & 0 & 0 & 0 & 0 & 0 & 0 & 0 & 0 & 0 & 0 \\
\hline 1659.6 & 0 & 0 & 0 & 0 & 0 & 0 & 0 & 0 & 0 & 0 \\
\hline 1821.9 & 0 & 0 & 0 & 0 & 0 & 0 & 0 & 0 & 0 & 0 \\
\hline 2000.0 & 0 & 0 & 0 & 0 & 0 & 0 & 0 & 0 & 0 & 0 \\
\hline
\end{tabular}


Appendix 3C: Grain size data for Southern McMurdo Sound MIS dust samples.

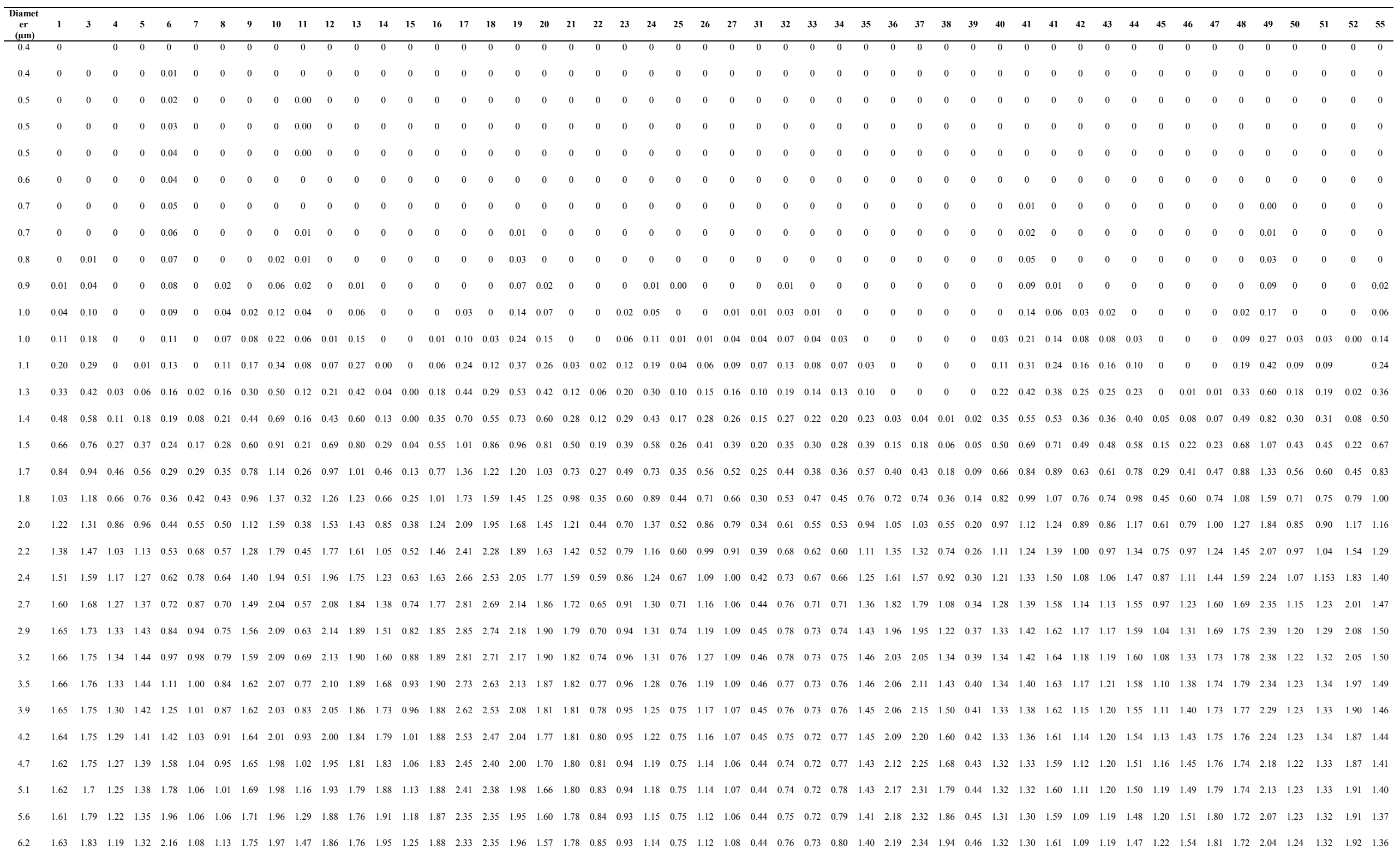




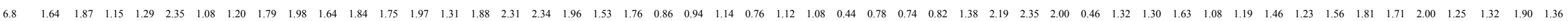

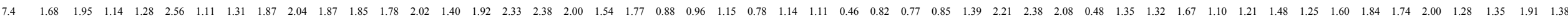

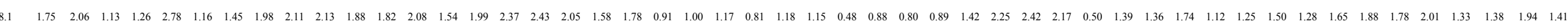

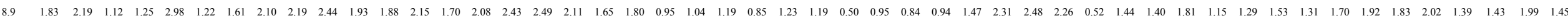

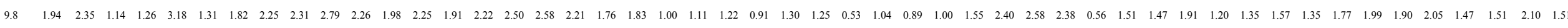

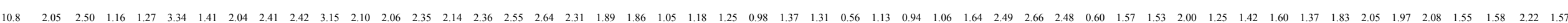

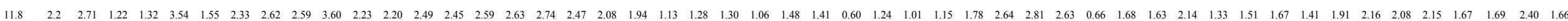

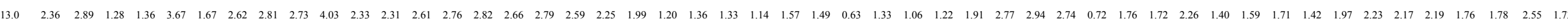

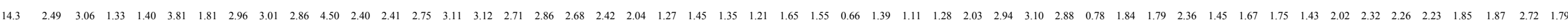

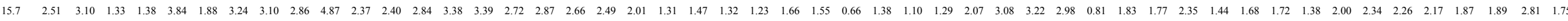

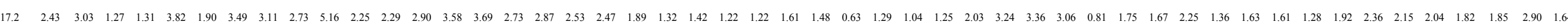

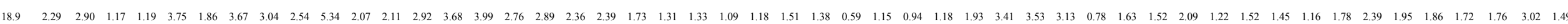

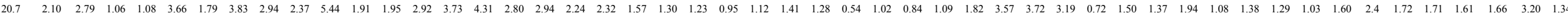

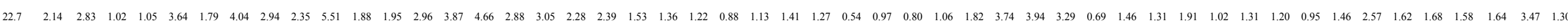

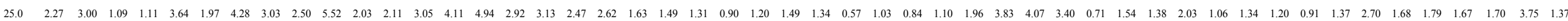

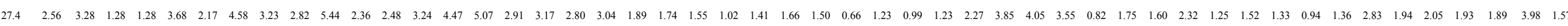

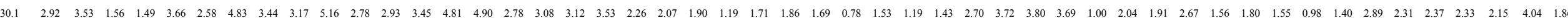

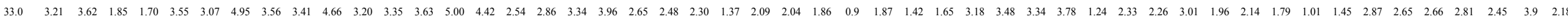

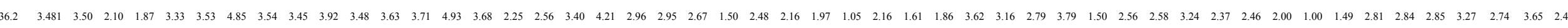

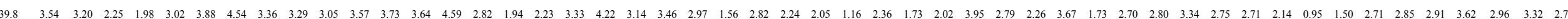

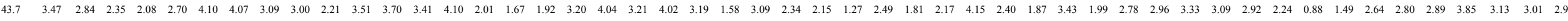

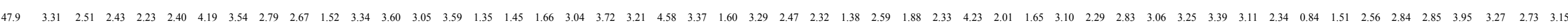

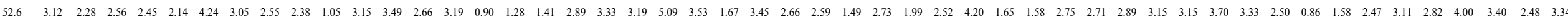

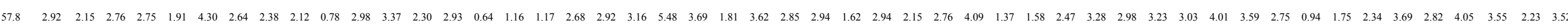

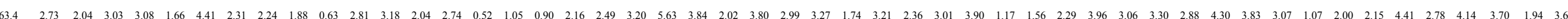

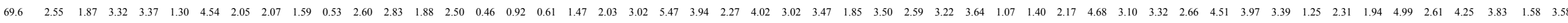

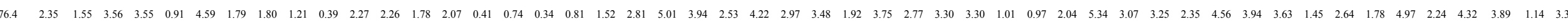

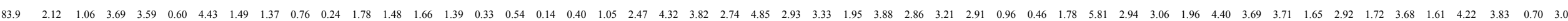

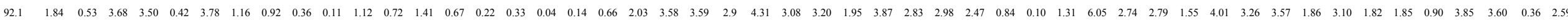

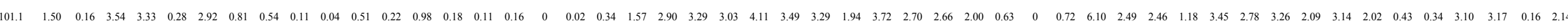

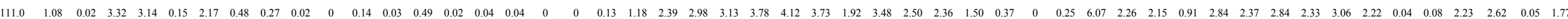

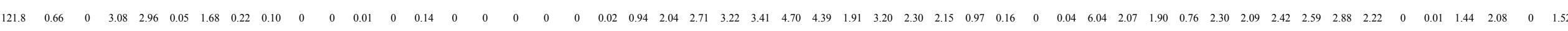

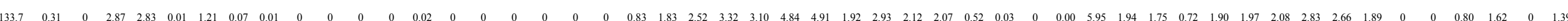




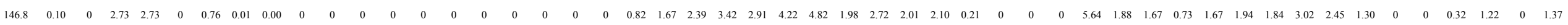

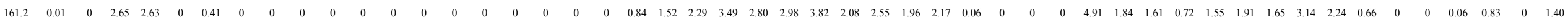

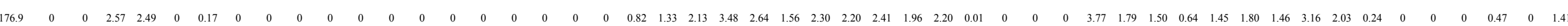

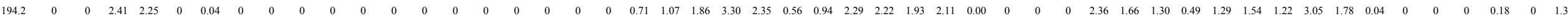

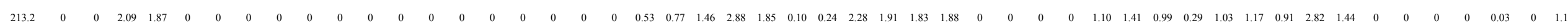

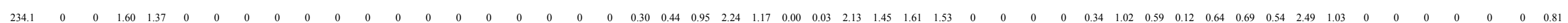

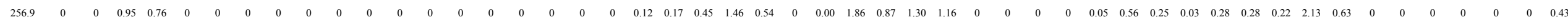

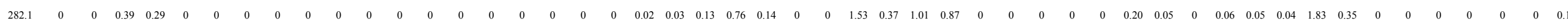

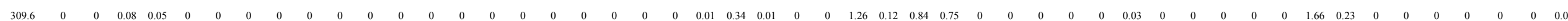

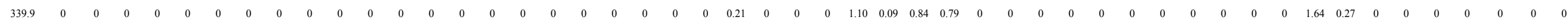

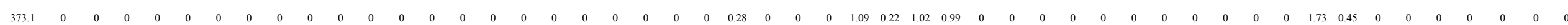

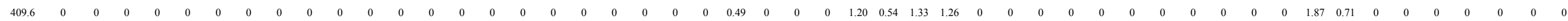

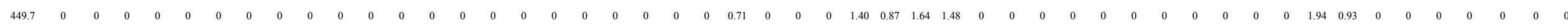

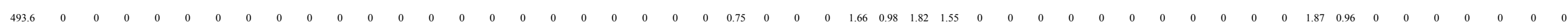

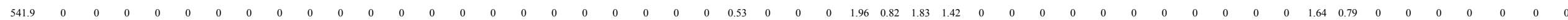

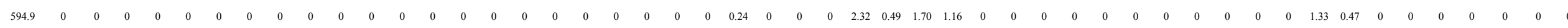

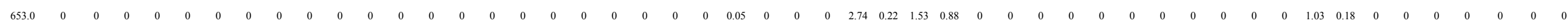

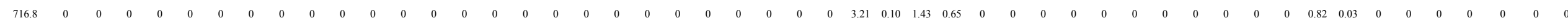

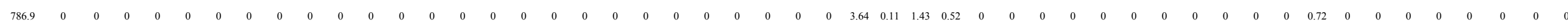

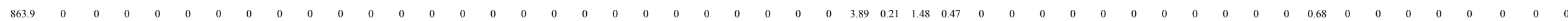

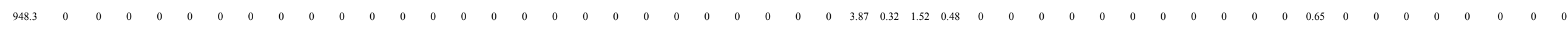

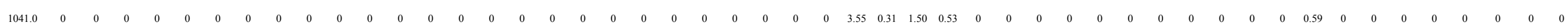

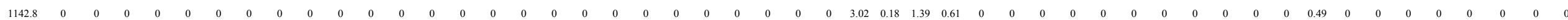

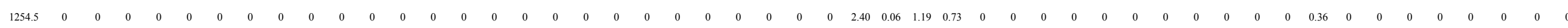

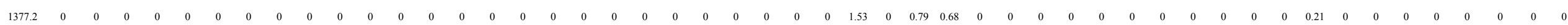

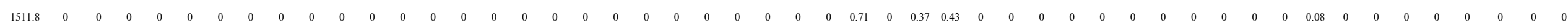

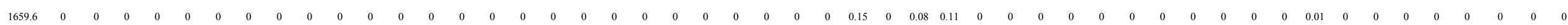

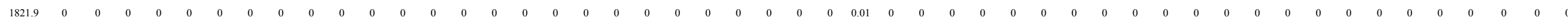

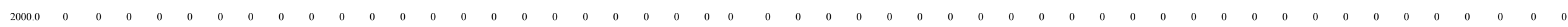


Appendix 3D: Grain size data from dust samples from southern McMurdo Sound Source: Atkins and Dunbar (2009).

\begin{tabular}{|c|c|c|c|c|}
\hline $\begin{array}{c}\text { Diameter } \\
\mu \mathrm{m}\end{array}$ & South_S23_32 & South_S9_07 & Central_C1_53 & North_N16_23 \\
\hline 0.4 & 0 & 0.000 & 0.000 & 0.000 \\
\hline 0.4 & 0 & 0.000 & 0.000 & 0.000 \\
\hline 0.5 & 0 & 0.000 & 0.000 & 0.000 \\
\hline 0.5 & 0 & 0.000 & 0.000 & 0.000 \\
\hline 0.5 & 0 & 0.000 & 0.000 & 0.000 \\
\hline 0.6 & 0 & 0.000 & 0.000 & 0.000 \\
\hline 0.7 & 0.00421186 & 0.003 & 0.000 & 0.001 \\
\hline 0.7 & 0.0228649 & 0.022 & 0.008 & 0.014 \\
\hline 0.8 & 0.0629379 & 0.073 & 0.046 & 0.074 \\
\hline 0.9 & 0.121231 & 0.158 & 0.140 & 0.202 \\
\hline 1.0 & 0.184594 & 0.260 & 0.288 & 0.384 \\
\hline 1.0 & 0.247468 & 0.362 & 0.451 & 0.565 \\
\hline 1.1 & 0.30418 & 0.448 & 0.593 & 0.720 \\
\hline 1.3 & 0.348122 & 0.510 & 0.687 & 0.826 \\
\hline 1.4 & 0.380796 & 0.551 & 0.740 & 0.889 \\
\hline 1.5 & 0.403312 & 0.577 & 0.769 & 0.927 \\
\hline 1.7 & 0.42327 & 0.600 & 0.804 & 0.967 \\
\hline 1.8 & 0.440148 & 0.619 & 0.834 & 0.999 \\
\hline 2.0 & 0.454 & 0.633 & 0.860 & 1.025 \\
\hline 2.2 & 0.460261 & 0.635 & 0.860 & 1.026 \\
\hline 2.4 & 0.460737 & 0.630 & 0.850 & 1.017 \\
\hline 2.7 & 0.4618 & 0.627 & 0.848 & 1.014 \\
\hline 2.9 & 0.4663 & 0.632 & 0.868 & 1.031 \\
\hline 3.2 & 0.479972 & 0.653 & 0.920 & 1.081 \\
\hline 3.5 & 0.494856 & 0.680 & 0.984 & 1.142 \\
\hline 3.9 & 0.515832 & 0.718 & 1.072 & 1.227 \\
\hline 4.2 & 0.532387 & 0.749 & 1.151 & 1.301 \\
\hline 4.7 & 0.552874 & 0.787 & 1.242 & 1.388 \\
\hline 5.1 & 0.566487 & 0.814 & 1.312 & 1.453 \\
\hline 5.6 & 0.579862 & 0.841 & 1.379 & 1.516 \\
\hline 6.2 & 0.584454 & 0.854 & 1.419 & 1.553 \\
\hline 6.8 & 0.584399 & 0.859 & 1.441 & 1.577 \\
\hline 7.4 & 0.5774 & 0.854 & 1.443 & 1.587 \\
\hline 8.1 & 0.562706 & 0.838 & 1.420 & 1.584 \\
\hline 8.9 & 0.543971 & 0.817 & 1.388 & 1.586 \\
\hline 9.8 & 0.518338 & 0.789 & 1.340 & 1.588 \\
\hline 10.8 & 0.495868 & 0.767 & 1.306 & 1.624 \\
\hline 11.8 & 0.473946 & 0.746 & 1.278 & 1.686 \\
\hline 13.0 & 0.459902 & 0.734 & 1.267 & 1.784 \\
\hline 14.3 & 0.45261 & 0.729 & 1.266 & 1.902 \\
\hline 15.7 & 0.456529 & 0.729 & 1.273 & 2.014 \\
\hline 17.2 & 0.475143 & 0.739 & 1.295 & 2.106 \\
\hline 18.9 & 0.504718 & 0.751 & 1.324 & 2.149 \\
\hline 20.7 & 0.541645 & 0.764 & 1.363 & 2.143 \\
\hline 22.7 & 0.572364 & 0.767 & 1.397 & 2.087 \\
\hline 25.0 & 0.596762 & 0.762 & 1.424 & 2.000 \\
\hline 27.4 & 0.620807 & 0.757 & 1.458 & 1.921 \\
\hline 30.1 & 0.6617 & 0.769 & 1.515 & 1.880 \\
\hline 33.0 & 0.739549 & 0.819 & 1.630 & 1.913 \\
\hline 36.2 & 0.862392 & 0.912 & 1.814 & 2.020 \\
\hline 39.8 & 1.04621 & 1.054 & 2.083 & 2.188 \\
\hline 43.7 & 1.28893 & 1.230 & 2.420 & 2.372 \\
\hline 47.9 & 1.60282 & 1.434 & 2.802 & 2.525 \\
\hline 52.6 & 1.98651 & 1.656 & 3.182 & 2.613 \\
\hline 57.8 & 2.43628 & 1.898 & 3.515 & 2.630 \\
\hline 63.4 & 2.94485 & 2.175 & 3.764 & 2.606 \\
\hline 69.6 & 3.4765 & 2.489 & 3.894 & 2.568 \\
\hline
\end{tabular}




\begin{tabular}{|c|c|c|c|c|}
\hline 76.4 & 3.9964 & 2.845 & 3.895 & 2.542 \\
\hline 83.9 & 4.44139 & 3.212 & 3.759 & 2.520 \\
\hline 92.1 & 4.75592 & 3.550 & 3.496 & 2.476 \\
\hline 101.1 & 4.90123 & 3.812 & 3.139 & 2.378 \\
\hline 111.0 & 4.86458 & 3.968 & 2.739 & 2.216 \\
\hline 121.8 & 4.67963 & 4.028 & 2.366 & 2.018 \\
\hline 133.7 & 4.40165 & 4.036 & 2.087 & 1.833 \\
\hline 146.8 & 4.09697 & 4.050 & 1.924 & 1.698 \\
\hline 161.2 & 3.81187 & 4.105 & 1.861 & 1.615 \\
\hline 176.9 & 3.5535 & 4.188 & 1.828 & 1.539 \\
\hline 194.2 & 3.29052 & 4.221 & 1.728 & 1.405 \\
\hline 213.2 & 2.9692 & 4.095 & 1.501 & 1.174 \\
\hline 234.1 & 2.55195 & 3.713 & 1.146 & 0.860 \\
\hline 256.9 & 2.05162 & 3.062 & 0.702 & 0.489 \\
\hline 282.1 & 1.54491 & 2.234 & 0.314 & 0.196 \\
\hline 309.6 & 1.14149 & 1.407 & 0.080 & 0.039 \\
\hline 339.9 & 0.914122 & 0.776 & 0.010 & 0.003 \\
\hline 373.1 & 0.874963 & 0.433 & 0.000 & 0.000 \\
\hline 409.6 & 0.984298 & 0.322 & 0.000 & 0.000 \\
\hline 449.7 & 1.15213 & 0.351 & 0.000 & 0.000 \\
\hline 493.6 & 1.24711 & 0.420 & 0.000 & 0.000 \\
\hline 541.9 & 1.16089 & 0.428 & 0.000 & 0.000 \\
\hline 594.9 & 0.879086 & 0.301 & 0.000 & 0.000 \\
\hline 653.0 & 0.487854 & 0.135 & 0.000 & 0.000 \\
\hline 716.8 & 0.17684 & 0.028 & 0.000 & 0.000 \\
\hline 786.9 & 0.0316358 & 0.002 & 0.000 & 0.000 \\
\hline 863.9 & 0.00212926 & 0.000 & 0.000 & 0.000 \\
\hline 948.3 & 0 & 0.000 & 0.000 & 0.000 \\
\hline 1041.0 & 0 & 0.000 & 0.000 & 0.000 \\
\hline 1142.8 & 0 & 0.000 & 0.000 & 0.000 \\
\hline 1254.5 & 0 & 0.000 & 0.000 & 0.000 \\
\hline 1377.2 & 0 & 0.000 & 0.000 & 0.000 \\
\hline 1511.8 & 0 & 0.000 & 0.000 & 0.000 \\
\hline 1659.6 & 0 & 0.000 & 0.000 & 0.000 \\
\hline 1821.9 & 0 & 0.000 & 0.000 & 0 \\
\hline 2000.0 & 0 & 0 & 0 & 0 \\
\hline
\end{tabular}


Appendix 3E: Grain size data for Windless Bight core 1.

\begin{tabular}{|c|c|c|c|c|c|c|c|c|c|c|c|c|c|c|c|c|c|c|c|c|c|c|c|c|c|c|}
\hline $\begin{array}{l}\text { Diamt } \\
(\mu \mathrm{m})\end{array}$ & WB1_1-2 & WB1_3 & WB1_4 & WB 1_5 & WB1_6 & $\begin{array}{c}\text { WB1 } 17 \\
{ }_{9-14}\end{array}$ & WB1_8 & WB1_12 & WB1_15-21 & WB1_22-24 & $\begin{array}{l}\text { WB1_25 } \\
31 \_33 \\
\end{array}$ & WB1_26 & WB1_27 & WB1_28 & $\begin{array}{c}\text { WB1_30 } \\
\text { 32 }\end{array}$ & $\begin{array}{c}\text { WB1_3 } \\
{ }_{3} 33^{-1}\end{array}$ & WB1_34 & $\begin{array}{c}\text { WB1_35 } \\
-38\end{array}$ & $\begin{array}{c}\text { WB1_29 } \\
-43\end{array}$ & WB1_40 & WB1_44 & WB1_45 & WB1_46 & WB1_47 & $\begin{array}{c}\text { WB1_4 } \\
8-53\end{array}$ & $\begin{array}{c}\text { WB1_54 } \\
-58\end{array}$ \\
\hline 0.37 & 0 & 0 & 0 & 0 & 0 & 0 & 0 & 0 & 0 & 0 & 0 & 0 & 0 & 0 & 0 & 0 & 0 & 0 & 0 & 0 & 0 & 0 & 0 & 0 & 0 & 0 \\
\hline 0.41 & 0 & 0 & 0 & 0 & 0 & 0 & 0 & 0 & 0 & 0 & 0 & 0 & 0 & 0 & 0 & 0 & 0 & 0 & 0 & 0 & 0 & 0 & 0 & 0 & 0 & 0 \\
\hline 0.45 & 0 & 0 & 0 & 0 & 0 & 0 & 0 & 0 & 0 & 0 & 0 & 0 & 0 & 0 & 0 & 0 & 0 & 0 & 0 & 0 & 0 & 0 & 0 & 0 & 0 & 0 \\
\hline 0.49 & 0 & 0 & 0 & 0 & 0 & 0 & 0 & 0 & 0 & 0 & 0 & 0 & 0 & 0 & 0 & 0 & 0 & 0 & 0 & 0 & 0 & 0 & 0 & 0 & 0 & 0 \\
\hline 0.54 & 0 & 0 & 0 & 0 & 0 & 0 & 0 & 0 & 0 & 0 & 0 & 0 & 0 & 0 & 0 & 0 & 0 & 0 & 0 & 0 & 0 & 0 & 0 & 0 & 0 & 0 \\
\hline 0.59 & 0 & 0 & 0 & 0 & 0 & 0 & 0 & 0 & 0 & 0 & 0 & 0 & 0 & 0 & 0 & 0 & 0 & 0 & 0 & 0 & 0 & 0 & 0 & 0 & 0 & 0 \\
\hline 0.65 & 0 & 0 & 0 & 0 & 0 & 0 & 0 & 0 & 0 & 0 & 0 & 0 & 0 & 0 & 0 & 0 & 0 & 0 & 0 & 0 & 0 & 0 & 0 & 0 & 0 & 0 \\
\hline 0.72 & 0 & 0 & 0 & 0 & 0 & 0 & 0 & 0 & 0 & 0 & 0 & 0 & 0 & 0 & 0 & 0 & 0 & 0 & 0 & 0 & 0 & 0 & 0 & 0 & 0 & 0 \\
\hline 0.79 & 0 & 0 & 0 & 0 & 0 & 0 & 0 & 0 & 0 & 0 & 0 & 0 & 0 & 0 & 0 & 0 & 0 & 0 & 0 & 0 & 0 & 0 & 0 & 0 & 0 & 0 \\
\hline 0.86 & 0 & 0 & 0 & 0 & 0 & 0 & 0 & 0 & 0 & 0 & 0 & 0 & 0 & 0 & 0 & 0 & 0 & 0 & 0 & 0 & 0 & 0 & 0 & 0 & 0 & 0 \\
\hline 0.95 & 0 & 0 & 0 & 0 & 0 & 0 & 0 & 0 & 0 & 0 & 0 & 0 & 0 & 0 & 0 & 0 & 0 & 0 & 0 & 0 & 0 & 0 & 0 & 0 & 0 & 0 \\
\hline 1.04 & 0 & 0 & 0 & 0 & 0 & 0 & 0 & 0 & 0 & 0 & 0 & 0 & 0 & 0 & 0.005 & 0 & 0 & 0 & 0 & 0 & 0 & 0 & 0 & 0 & 0 & 0 \\
\hline 1.14 & 0 & 0 & 0 & 0 & 0 & 0 & 0 & 0 & 0 & 0 & 0 & 0 & 0.003 & 0.015 & 0.046 & 0.016 & 0 & 0.001 & 0 & 0 & 0.003 & 0 & 0 & 0 & 0 & 0 \\
\hline 1.26 & 0 & 0 & 0 & 0.014 & 0 & 0 & 0 & 0 & 0 & 0 & 0 & 0 & 0.032 & 0.118 & 0.198 & 0.100 & 0.005 & 0.015 & 0.006 & 0 & 0.023 & 0 & 0 & 0 & 0 & 0 \\
\hline 1.38 & 0 & 0.010 & 0 & 0.085 & 0 & 0.005 & 0 & 0 & 0 & 0.007 & 0 & 0 & 0.136 & 0.453 & 0.480 & 0.313 & 0.032 & 0.082 & 0.040 & 0 & 0.081 & 0.007 & 0.007 & 0.007 & 0.011 & 0.003 \\
\hline 1.51 & 0.008 & 0.057 & 0.013 & 0.261 & 0 & 0.032 & 0 & 0 & 0.004 & 0.039 & 0.1818 & 0 & 0.336 & 1.053 & 0.853 & 0.655 & 0.103 & 0.228 & 0.134 & 0.001 & 0.187 & 0.040 & 0.040 & 0.0507 & 0.064 & 0.022 \\
\hline 1.66 & 0.045 & 0.155 & 0.092 & 0.539 & 0 & 0.089 & 0 & 0 & 0.035 & 0.107 & 0.440 & 0 & 0.596 & 1.807 & 1.232 & 1.050 & 0.217 & 0.434 & 0.281 & 0.021 & 0.318 & 0.114 & 0.115 & 0.164 & 0.179 & 0.062 \\
\hline 1.83 & 0.129 & 0.295 & 0.319 & 0.853 & 0.004 & 0.175 & 0 & 0 & 0.133 & 0.208 & 0.779 & 0.001 & 0.875 & 2.561 & 1.611 & 1.448 & 0.350 & 0.651 & 0.456 & 0.112 & 0.462 & 0.224 & 0.232 & 0.352 & 0.350 & 0.126 \\
\hline 2.01 & 0.244 & 0.439 & 0.679 & 1.158 & 0.084 & 0.265 & 0 & 0 & 0.298 & 0.317 & 1.1029 & 0.020 & 1.152 & 3.249 & 1.959 & 1.812 & 0.481 & 0.861 & 0.625 & 0.301 & 0.604 & 0.343 & 0.360 & 0.567 & 0.528 & 0.197 \\
\hline 2.20 & 0.364 & 0.579 & 1.0895 & 1.449 & 0.328 & 0.357 & 0 & 0.025 & 0.493 & 0.434 & 1.403 & 0.120 & 1.431 & 3.830 & 2.263 & 2.128 & 0.608 & 1.063 & 0.792 & 0.575 & 0.746 & 0.465 & 0.492 & 0.781 & 0.703 & 0.273 \\
\hline 2.4 & 0.470 & 0.701 & 1.443 & 1.705 & 0.616 & 0.442 & 0 & 0.380 & 0.658 & 0.549 & 1.652 & 0.374 & 1.686 & 4.248 & 2.489 & 2.359 & 0.719 & 1.239 & 0.942 & 0.855 & 0.875 & 0.574 & 0.612 & 0.972 & 0.855 & 0.344 \\
\hline 2.65 & 0.563 & 0.806 & 1.721 & 1.923 & 0.800 & 0.521 & 0 & 1.143 & 0.784 & 0.663 & 1.853 & 0.761 & 1.921 & 4.475 & 2.619 & 2.495 & 0.819 & 1.386 & 1.075 & 1.114 & 0.989 & 0.676 & 0.723 & 1.143 & 0.987 & 0.413 \\
\hline 2.92 & 0.637 & 0.890 & 1.909 & 2.088 & 0.831 & 0.589 & 0 & 1.390 & 0.863 & 0.772 & 1.996 & 1.188 & 2.122 & 4.482 & 2.650 & 2.519 & 0.897 & 1.490 & 1.183 & 1.309 & 1.081 & 0.762 & 0.824 & 1.286 & 1.090 & 0.476 \\
\hline 3.20 & 0.693 & 0.947 & 2.019 & 2.191 & 0.717 & 0.641 & 0 & 0.666 & 0.902 & 0.867 & 2.077 & 1.555 & 2.287 & 4.311 & 2.600 & 2.452 & 0.950 & 1.549 & 1.254 & 1.440 & 1.146 & 0.828 & 0.908 & 1.395 & 1.162 & 0.528 \\
\hline 3.51 & 0.736 & 0.985 & 2.093 & 2.260 & 0.549 & 0.682 & 0 & 0.062 & 0.923 & 0.953 & 2.121 & 1.825 & 2.443 & 4.046 & 2.512 & 2.337 & 0.983 & 1.580 & 1.308 & 1.528 & 1.200 & 0.878 & 0.982 & 1.477 & 1.210 & 0.574 \\
\hline 3.86 & 0.767 & 1.009 & 2.156 & 2.304 & 0.472 & 0.710 & 0 & 0 & 0.936 & 1.022 & 2.147 & 1.949 & 2.582 & 3.758 & 2.399 & 2.204 & 1.000 & 1.589 & 1.344 & 1.600 & 1.237 & 0.913 & 1.041 & 1.537 & 1.239 & 0.608 \\
\hline 4.24 & 0.809 & 1.045 & 2.270 & 2.371 & 0.576 & 0.745 & 0.002 & 0.017 & 0.973 & 1.098 & 2.207 & 1.947 & 2.754 & 3.506 & 2.305 & 2.095 & 1.022 & 1.607 & 1.394 & 1.686 & 1.284 & 0.959 & 1.118 & 1.615 & 1.285 & 0.648 \\
\hline 4.65 & 0.850 & 1.079 & 2.406 & 2.428 & 0.860 & 0.773 & 0.029 & 0.476 & 1.017 & 1.162 & 2.271 & 1.860 & 2.918 & 3.260 & 2.207 & 1.988 & 1.034 & 1.610 & 1.434 & 1.754 & 1.319 & 0.998 & 1.192 & 1.688 & 1.328 & 0.683 \\
\hline 5.11 & 0.902 & 1.123 & 2.567 & 2.500 & 1.184 & 0.809 & 0.158 & 2.090 & 1.080 & 1.233 & 2.361 & 1.771 & 3.116 & 3.039 & 2.138 & 1.909 & 1.049 & 1.624 & 1.485 & 1.809 & 1.368 & 1.047 & 1.288 & 1.776 & 1.387 & 0.728 \\
\hline 5.60 & 0.932 & 1.145 & 2.665 & 2.533 & 1.375 & 0.825 & 0.4213 & 3.042 & 1.112 & 1.282 & 2.410 & 1.750 & 3.282 & 2.798 & 2.059 & 1.814 & 1.038 & 1.609 & 1.512 & 1.791 & 1.398 & 1.073 & 1.362 & 1.826 & 1.418 & 0.758 \\
\hline 6.15 & 0.959 & 1.167 & 2.731 & 2.568 & 1.373 & 0.845 & 0.833 & 2.536 & 1.140 & 1.330 & 2.463 & 1.7899 & 3.463 & 2.587 & 2.003 & 1.746 & 1.035 & 1.601 & 1.545 & 1.764 & 1.438 & 1.102 & 1.444 & 1.871 & 1.451 & 0.794 \\
\hline
\end{tabular}




\begin{tabular}{|c|c|c|c|c|c|c|c|c|c|c|c|c|c|c|c|c|c|c|c|c|c|c|c|c|c|c|}
\hline 6.76 & 0.974 & 1.180 & 2.743 & 2.589 & 1.245 & 0.857 & 1.304 & 1.598 & 1.146 & 1.369 & 2.498 & 1.881 & 3.627 & 2.387 & 1.953 & 1.684 & 1.029 & 1.586 & 1.571 & 1.705 & 1.472 & 1.122 & 1.516 & 1.898 & 1.476 & 0.825 \\
\hline 7.42 & 1.005 & 1.214 & 2.771 & 2.634 & 1.133 & 0.885 & 1.824 & 0.509 & 1.180 & 1.420 & 2.566 & 1.899 & 3.810 & 2.238 & 1.944 & 1.672 & 1.051 & 1.599 & 1.620 & 1.686 & 1.527 & 1.165 & 1.616 & 1.949 & 1.526 & 0.875 \\
\hline 8.14 & 1.048 & 1.264 & 2.811 & 2.695 & 1.077 & 0.925 & 2.271 & 0.034 & 1.237 & 1.483 & 2.652 & 1.892 & 4.001 & 2.135 & 1.974 & 1.700 & 1.086 & 1.636 & 1.687 & 1.683 & 1.600 & 1.222 & 1.738 & 2.016 & 1.601 & 0.941 \\
\hline 8.94 & 1.102 & 1.325 & 2.855 & 2.759 & 1.116 & 0.975 & 2.570 & 0.011 & 1.323 & 1.553 & 2.739 & 1.832 & 4.168 & 2.073 & 2.034 & 1.766 & 1.129 & 1.695 & 1.769 & 1.725 & 1.688 & 1.293 & 1.873 & 2.092 & 1.696 & 1.023 \\
\hline 9.81 & 1.177 & 1.409 & 2.922 & 2.846 & 1.270 & 1.048 & 2.689 & 0.100 & 1.457 & 1.645 & 2.847 & 1.875 & 4.331 & 2.069 & 2.145 & 1.886 & 1.198 & 1.796 & 1.887 & 1.857 & 1.807 & 1.395 & 2.037 & 2.197 & 1.827 & 1.134 \\
\hline 10 & 1.257 & 1.496 & 2.963 & 2.911 & 1.464 & 1.129 & 2.619 & 0.382 & 1.615 & 1.737 & 2.930 & 2.001 & 4.421 & 2.072 & 2.254 & 2.021 & 1.287 & 1.909 & 2.011 & 2.069 & 1.929 & 1.508 & 2.197 & 2.296 & 1.965 & 1.257 \\
\hline 11 & 1.372 & 1.623 & 3.049 & 3.012 & 1.728 & 1.246 & 2.437 & 1.328 & 1.847 & 1.866 & 3.054 & 2.223 & 4.500 & 2.117 & 2.419 & 2.222 & 1.439 & 2.087 & 2.184 & 2.421 & 2.092 & 1.675 & 2.404 & 2.445 & 2.160 & 1.423 \\
\hline 12 & 1.475 & 1.742 & 3.082 & 3.071 & 2.099 & 1.362 & 2.126 & 2.788 & 2.074 & 1.985 & 3.130 & 2.491 & 4.468 & 2.122 & 2.554 & 2.400 & 1.572 & 2.264 & 2.344 & 2.712 & 2.239 & 1.841 & 2.589 & 2.572 & 2.342 & 1.589 \\
\hline 14 & 1.584 & 1.871 & 3.091 & 3.118 & 2.593 & 1.495 & 1.772 & 4.119 & 2.318 & 2.124 & 3.191 & 2.719 & 4.360 & 2.103 & 2.684 & 2.570 & 1.665 & 2.466 & 2.520 & 2.933 & 2.399 & 2.036 & 2.785 & 2.710 & 2.540 & 1.780 \\
\hline 15 & 1.639 & 1.937 & 2.979 & 3.062 & 3.004 & 1.588 & 1.416 & 4.858 & 2.470 & 2.221 & 3.143 & 2.989 & 4.090 & 2.004 & 2.711 & 2.622 & 1.628 & 2.593 & 2.633 & 2.938 & 2.497 & 2.190 & 2.897 & 2.776 & 2.659 & 1.934 \\
\hline 17 & 1.648 & 1.943 & 2.774 & 2.917 & 2.895 & 1.645 & 1.184 & 2.544 & 2.528 & 2.287 & 3.007 & 3.231 & 3.69 & 1.849 & 2.645 & 2.565 & 1.535 & 2.637 & 2.687 & 2.892 & 2.544 & 2.329 & 2.935 & 2.780 & 2.700 & 2.060 \\
\hline 18 & 1.609 & 1.896 & 2.542 & 2.707 & 2.287 & 1.656 & 1.158 & 0.243 & 2.491 & 2.301 & 2.816 & 3.629 & 3.215 & 1.685 & 2.513 & 2.442 & 1.493 & 2.595 & 2.665 & 2.969 & 2.530 & 2.452 & 2.891 & 2.720 & 2.652 & 2.140 \\
\hline 20 & 1.547 & 1.833 & 2.358 & 2.482 & 1.557 & 1.641 & 1.371 & 0.123 & 2.403 & 2.280 & 2.615 & 3.997 & 2.698 & 1.553 & 2.376 & 2.321 & 1.574 & 2.519 & 2.595 & 3.202 & 2.490 & 2.594 & 2.801 & 2.619 & 2.554 & 2.196 \\
\hline 22 & 1.531 & 1.833 & 2.345 & 2.340 & 1.293 & 1.662 & 1.847 & 1.778 & 2.381 & 2.278 & 2.493 & 4.284 & 2.230 & 1.522 & 2.346 & 2.350 & 1.807 & 2.522 & 2.567 & 3.623 & 2.500 & 2.818 & 2.749 & 2.558 & 2.504 & 2.287 \\
\hline 24 & 1.591 & 1.898 & 2.479 & 2.270 & 1.974 & 1.741 & 2.455 & 5.992 & 2.469 & 2.314 & 2.425 & 4.177 & 1.775 & 1.569 & 2.423 & 2.502 & 2.002 & 2.630 & 2.621 & 3.857 & 2.581 & 3.119 & 2.740 & 2.555 & 2.536 & 2.436 \\
\hline 27 & 1.792 & 2.050 & 2.748 & 2.280 & 3.513 & 1.927 & 3.056 & 10.36 & 2.736 & 2.441 & 2.414 & 3.467 & 1.351 & 1.683 & 2.617 & 2.784 & 1.970 & 2.879 & 2.824 & 3.746 & 2.780 & 3.525 & 2.808 & 2.662 & 2.714 & 2.699 \\
\hline 30 & 2.122 & 2.218 & 3.018 & 2.285 & 4.640 & 2.185 & 3.482 & 12.433 & 3.130 & 2.625 & 2.387 & 2.639 & 0.943 & 1.791 & 2.823 & 3.071 & 1.620 & 3.173 & 3.126 & 3.355 & 3.033 & 3.955 & 2.881 & 2.847 & 2.985 & 3.036 \\
\hline 33 & 2.564 & 2.353 & 3.198 & 2.240 & 4.382 & 2.489 & 3.748 & 10.495 & 3.580 & 2.850 & 2.312 & 2.013 & 0.592 & 1.854 & 2.969 & 3.266 & 1.131 & 3.435 & 3.469 & 2.967 & 3.292 & 4.368 & 2.916 & 3.084 & 3.291 & 3.415 \\
\hline 36 & 3.042 & 2.417 & 3.243 & 2.134 & 3.722 & 2.777 & 3.967 & 8.063 & 3.963 & 3.059 & 2.186 & 2.173 & 0.384 & 1.859 & 3.005 & 3.325 & 0.862 & 3.584 & 3.739 & 3.005 & 3.472 & 4.688 & 2.863 & 3.300 & 3.536 & 3.762 \\
\hline 39 & 3.462 & 2.419 & 3.174 & 2.010 & 3.938 & 3.008 & 4.257 & 10.448 & 4.170 & 3.212 & 2.037 & 3.074 & 0.326 & 1.817 & 2.945 & 3.280 & 0.976 & 3.617 & 3.853 & 3.603 & 3.526 & 4.876 & 2.722 & 3.431 & 3.642 & 4.016 \\
\hline 43 & 3.758 & 2.422 & 3.063 & 1.956 & 4.920 & 3.184 & 4.704 & 8.745 & 4.175 & 3.311 & 1.919 & 4.697 & 0.452 & 1.756 & 2.852 & 3.221 & 1.734 & 3.620 & 3.799 & 4.749 & 3.460 & 4.928 & 2.549 & 3.450 & 3.609 & 4.168 \\
\hline 47 & 3.882 & 2.477 & 2.951 & 2.025 & 5.373 & 3.320 & 5.225 & 1.205 & 4.003 & 3.363 & 1.851 & 6.434 & 0.756 & 1.677 & 2.768 & 3.210 & 3.294 & 3.66 & 3.618 & 6.110 & 3.299 & 4.834 & 2.395 & 3.353 & 3.467 & 4.216 \\
\hline 52 & 3.857 & 2.621 & 2.844 & 2.229 & 4.708 & 3.450 & 5.660 & 0 & 3.770 & 3.408 & 1.832 & 7.342 & 1.188 & 1.558 & 2.701 & 3.261 & 5.468 & 3.817 & 3.414 & 6.887 & 3.105 & 4.619 & 2.305 & 3.197 & 3.306 & 4.203 \\
\hline 57 & 3.748 & 2.835 & 2.688 & 2.500 & 4.263 & 3.584 & 5.810 & 0 & 3.603 & 3.461 & 1.824 & 5.989 & 1.565 & 1.373 & 2.597 & 3.294 & 7.584 & 4.044 & 3.267 & 5.659 & 2.923 & 4.298 & 2.284 & 3.042 & 3.210 & 4.152 \\
\hline 63 & 3.668 & 3.036 & 2.405 & 2.691 & 4.912 & 3.696 & 5.569 & 0 & 3.594 & 3.519 & 1.776 & 3.245 & 1.70 & 0.944 & 2.371 & 3.189 & 8.925 & 4.195 & 3.219 & 3.110 & 2.787 & 3.893 & 2.290 & 2.946 & 3.223 & 4.071 \\
\hline 69 & 3.750 & 3.121 & 1.679 & 2.637 & 5.478 & 3.736 & 5.017 & 0 & 3.778 & 3.554 & 1.655 & 0.794 & 1.326 & 0.459 & 1.683 & 2.366 & 9.059 & 4.080 & 3.247 & 0.769 & 2.725 & 3.437 & 2.269 & 2.932 & 3.346 & 3.950 \\
\hline 76 & 4.077 & 2.992 & 0.823 & 2.218 & 4.380 & 3.630 & 4.382 & 0 & 4.066 & 3.523 & 1.467 & 0.086 & 0.685 & 0.103 & 0.836 & 1.223 & 7.931 & 3.513 & 3.240 & 0.084 & 2.733 & 2.964 & 2.171 & 2.935 & 3.497 & 3.761 \\
\hline 83 & 4.659 & 2.651 & 0.187 & 1.400 & 1.702 & 3.336 & 3.833 & 0 & 4.256 & 3.390 & 1.247 & 0 & 0.159 & 0.010 & 0.191 & 0.285 & 6.005 & 2.310 & 3.065 & 0 & 2.804 & 2.522 & 2.006 & 2.849 & 3.526 & 3.498 \\
\hline 92 & 5.353 & 2.270 & 0.018 & 0.592 & 0.233 & 2.872 & 3.422 & 0 & 4.104 & 3.157 & 1.088 & 0 & 0.016 & 0 & 0.019 & 0.029 & 3.972 & 1.042 & 2.611 & 0 & 2.883 & 2.134 & 1.873 & 2.527 & 3.283 & 3.204 \\
\hline 101 & 5.811 & 2.033 & 0 & 0.120 & 0.456 & 2.355 & 2.995 & 0 & 3.435 & 2.851 & 1.024 & 0 & 0 & 0 & 0 & 0 & 2.403 & 0.224 & 1.876 & 0 & 2.861 & 1.786 & 1.872 & 1.724 & 2.680 & 2.936 \\
\hline 110 & 5.666 & 2.150 & 0 & 0.009 & 2.390 & 2.020 & 2.339 & 0 & 2.177 & 2.526 & 1.013 & 0 & 0 & 0 & 0 & 0 & 1.451 & 0.020 & 1.012 & 0 & 2.622 & 1.439 & 2.094 & 0.810 & 1.710 & 2.759 \\
\hline 121 & 4.695 & 2.684 & 0 & 0 & 3.174 & 2.033 & 1.391 & 0 & 0.937 & 2.226 & 0.943 & 0 & 0 & 0 & 0 & 0 & 0.923 & 0 & 0.357 & 0 & 2.094 & 1.053 & 2.487 & 0.179 & 0.767 & 2.662 \\
\hline 133 & 2.913 & 3.513 & 0 & 0 & 1.204 & 2.509 & 0.532 & 0 & 0.194 & 1.945 & 0.745 & 0 & 0 & 0 & 0 & 0 & 0.544 & 0 & 0.062 & 0 & 1.275 & 0.607 & 2.858 & 0.016 & 0.183 & 2.548 \\
\hline
\end{tabular}




\begin{tabular}{|c|c|c|c|c|c|c|c|c|c|c|c|c|c|c|c|c|c|c|c|c|c|c|c|c|c|c|}
\hline 146 & 1.220 & 4.225 & 0 & 0 & 0.085 & 3.346 & 0.099 & 0 & 0.016 & 1.666 & 0.437 & 0 & 0 & 0 & 0 & 0 & 0.232 & 0 & 0.004 & 0 & 0.529 & 0.241 & 2.927 & 0 & 0.020 & 2.298 \\
\hline 161 & 0.2474 & 4.355 & 0 & 0 & 0 & 4.170 & 0.006 & 0 & 0 & 1.330 & 0.167 & 0 & 0 & 0 & 0 & 0 & 0.049 & 0 & 0 & 0 & 0.106 & 0.047 & 2.500 & 0 & 0 & 1.818 \\
\hline 176 & 0.020 & 3.652 & 0 & 0 & 0 & 4.446 & 0 & 0 & 0 & 0.929 & 0.031 & 0 & 0 & 0 & 0 & 0 & 0.003 & 0 & 0 & 0 & 0.008 & 0.003 & 1.579 & 0 & 0 & 1.103 \\
\hline 194 & 0 & 2.460 & 0 & 0 & 0 & 3.851 & 0 & 0 & 0 & 0.500 & 0.002 & 0 & 0 & 0 & 0 & 0 & 0 & 0 & 0 & 0 & 0 & 0 & 0.668 & 0 & 0 & 0.458 \\
\hline 213 & 0 & 1.328 & 0 & 0 & 0 & 2.435 & 0 & 0 & 0 & 0.180 & 0 & 0 & 0 & 0 & 0 & 0 & 0 & 0 & 0 & 0 & 0 & 0 & 0.136 & 0 & 0 & 0.093 \\
\hline 234 & 0 & 0.582 & 0 & 0 & 0 & 1.024 & 0 & 0 & 0 & 0.032 & 0 & 0 & 0 & 0 & 0 & 0 & 0 & 0 & 0 & 0 & 0 & 0 & 0.011 & 0 & 0 & 0.007 \\
\hline 256 & 0 & 0.178 & 0 & 0 & 0 & 0.208 & 0 & 0 & 0 & 0.002 & 0 & 0 & 0 & 0 & 0 & 0 & 0 & 0 & 0 & 0 & 0 & 0 & 0 & 0 & 0 & 0 \\
\hline 282 & 0 & 0.028 & 0 & 0 & 0 & 0.017 & 0 & 0 & 0 & 0 & 0 & 0 & 0 & 0 & 0 & 0 & 0 & 0 & 0 & 0 & 0 & 0 & 0 & 0 & 0 & 0 \\
\hline 309 & 0 & 0.001 & 0 & 0 & 0 & 0 & 0 & 0 & 0 & 0 & 0 & 0 & 0 & 0 & 0 & 0 & 0 & 0 & 0 & 0 & 0 & 0 & 0 & 0 & 0 & 0 \\
\hline 339 & 0 & 0 & 0 & 0 & 0 & 0 & 0 & 0 & 0 & 0 & 0 & 0 & 0 & 0 & 0 & 0 & 0 & 0 & 0 & 0 & 0 & 0 & 0 & 0 & 0 & 0 \\
\hline 373 & 0 & 0 & 0 & 0 & 0 & 0 & 0 & 0 & 0 & 0 & 0 & 0 & 0 & 0 & 0 & 0 & 0 & 0 & 0 & 0 & 0 & 0 & 0 & 0 & 0 & 0 \\
\hline 409 & 0 & 0 & 0 & 0 & 0 & 0 & 0 & 0 & 0 & 0 & 0 & 0 & 0 & 0 & 0 & 0 & 0 & 0 & 0 & 0 & 0 & 0 & 0 & 0 & 0 & 0 \\
\hline 449 & 0 & 0 & 0 & 0 & 0 & 0 & 0 & 0 & 0 & 0 & 0 & 0 & 0 & 0 & 0 & 0 & 0 & 0 & 0 & 0 & 0 & 0 & 0 & 0 & 0 & 0 \\
\hline 493 & 0 & 0 & 0 & 0 & 0 & 0 & 0 & 0 & 0 & 0 & 0 & 0 & 0 & 0 & 0 & 0 & 0 & 0 & 0 & 0 & 0 & 0 & 0 & 0 & 0 & 0 \\
\hline 541 & 0 & 0 & 0 & 0 & 0 & 0 & 0 & 0 & 0 & 0 & 0 & 0 & 0 & 0 & 0 & 0 & 0 & 0 & 0 & 0 & 0 & 0 & 0 & 0 & 0 & 0 \\
\hline 594 & 0 & 0 & 0 & 0 & 0 & 0 & 0 & 0 & 0 & 0 & 0 & 0 & 0 & 0 & 0 & 0 & 0 & 0 & 0 & 0 & 0 & 0 & 0 & 0 & 0 & 0 \\
\hline 653 & 0 & 0 & 0 & 0 & 0 & 0 & 0 & 0 & 0 & 0 & 0 & 0 & 0 & 0 & 0 & 0 & 0 & 0 & 0 & 0 & 0 & 0 & 0 & 0 & 0 & 0 \\
\hline 716 & 0 & 0 & 0 & 0 & 0 & 0 & 0 & 0 & 0 & 0 & 0 & 0 & 0 & 0 & 0 & 0 & 0 & 0 & 0 & 0 & 0 & 0 & 0 & 0 & 0 & 0 \\
\hline 786 & 0 & 0 & 0 & 0 & 0 & 0 & 0 & 0 & 0 & 0 & 0 & 0 & 0 & 0 & 0 & 0 & 0 & 0 & 0 & 0 & 0 & 0 & 0 & 0 & 0 & 0 \\
\hline 863 & 0 & 0 & 0 & 0 & 0 & 0 & 0 & 0 & 0 & 0 & 0 & 0 & 0 & 0 & 0 & 0 & 0 & 0 & 0 & 0 & 0 & 0 & 0 & 0 & 0 & 0 \\
\hline 948 & 0 & 0 & 0 & 0 & 0 & 0 & 0 & 0 & 0 & 0 & 0 & 0 & 0 & 0 & 0 & 0 & 0 & 0 & 0 & 0 & 0 & 0 & 0 & 0 & 0 & 0 \\
\hline 1041 & 0 & 0 & 0 & 0 & 0 & 0 & 0 & 0 & 0 & 0 & 0 & 0 & 0 & 0 & 0 & 0 & 0 & 0 & 0 & 0 & 0 & 0 & 0 & 0 & 0 & 0 \\
\hline 1142 & 0 & 0 & 0 & 0 & 0 & 0 & 0 & 0 & 0 & 0 & 0 & 0 & 0 & 0 & 0 & 0 & 0 & 0 & 0 & 0 & 0 & 0 & 0 & 0 & 0 & 0 \\
\hline 1254 & 0 & 0 & 0 & 0 & 0 & 0 & 0 & 0 & 0 & 0 & 0 & 0 & 0 & 0 & 0 & 0 & 0 & 0 & 0 & 0 & 0 & 0 & 0 & 0 & 0 & 0 \\
\hline 1377 & 0 & 0 & 0 & 0 & 0 & 0 & 0 & 0 & 0 & 0 & 0 & 0 & 0 & 0 & 0 & 0 & 0 & 0 & 0 & 0 & 0 & 0 & 0 & 0 & 0 & 0 \\
\hline 1511 & 0 & 0 & 0 & 0 & 0 & 0 & 0 & 0 & 0 & 0 & 0 & 0 & 0 & 0 & 0 & 0 & 0 & 0 & 0 & 0 & 0 & 0 & 0 & 0 & 0 & 0 \\
\hline 1659 & 0 & 0 & 0 & 0 & 0 & 0 & 0 & 0 & 0 & 0 & 0 & 0 & 0 & 0 & 0 & 0 & 0 & 0 & 0 & 0 & 0 & 0 & 0 & 0 & 0 & 0 \\
\hline 1821 & 0 & 0 & 0 & 0 & 0 & 0 & 0 & 0 & 0 & 0 & 0 & 0 & 0 & 0 & 0 & 0 & 0 & 0 & 0 & 0 & 0 & 0 & 0 & 0 & 0 & 0 \\
\hline 2000 & 0 & 0 & 0 & 0 & 0 & 0 & 0 & 0 & 0 & 0 & 0 & 0 & 0 & 0 & 0 & 0 & 0 & 0 & 0 & 0 & 0 & 0 & 0 & 0 & 0 & 0 \\
\hline
\end{tabular}


Appendix 3F: Grain size data for Windless Bight core 3.

\begin{tabular}{|c|c|c|c|c|c|c|c|c|c|c|c|c|}
\hline $\begin{array}{c}\text { Diameter } \\
\mu \mathrm{m}\end{array}$ & WB3_001 & WB3_007 & WB3_013 & WB3_020 & WB3_021 & WB3_027 & WB3_029 & WB3_030 & WB3_033 & WB3_039 & WB3_046 & WB3_051 \\
\hline 0.375198 & 0 & 0 & 0.00423828 & 0.016307 & 0.0881826 & 0.0282872 & 0 & 0.0129001 & 0.0217507 & 0 & 0 & 0.0559309 \\
\hline 0.411878 & 0 & 0 & 0.00887354 & 0.0283269 & 0.155672 & 0.0500998 & 0 & 0.0229124 & 0.0389898 & 0 & 0 & 0.0992226 \\
\hline 0.452145 & 0 & 0 & 0.0173708 & 0.0420321 & 0.220206 & 0.074453 & 0 & 0.0340263 & 0.0570009 & 0 & 0 & 0.148075 \\
\hline 0.496347 & 0 & 0 & 0.0286612 & 0.0618667 & 0.294754 & 0.108488 & 0 & 0.0493319 & 0.0813677 & 0 & 0 & 0.216046 \\
\hline 0.544872 & 0 & 0 & 0.043352 & 0.0825825 & 0.33711 & 0.140686 & 0 & 0.0633155 & 0.103234 & 0 & 0 & 0.278276 \\
\hline 0.59814 & 0 & 0 & 0.0617337 & 0.104733 & 0.352849 & 0.172478 & 0 & 0.0767912 & 0.124888 & 0 & 0 & 0.335515 \\
\hline 0.656615 & 0 & 0 & 0.0836302 & 0.130484 & 0.348934 & 0.205222 & 0 & 0.0903236 & 0.1475 & 0 & 0 & 0.389003 \\
\hline 0.720807 & 0 & 0 & 0.108622 & 0.161711 & 0.331843 & 0.240632 & 0 & 0.104653 & 0.172881 & 0 & 0 & 0.440136 \\
\hline 0.791275 & 0 & 0 & 0.136165 & 0.199991 & 0.30251 & 0.277445 & 0 & 0.118951 & 0.201046 & 0 & 0 & 0.483179 \\
\hline 0.868632 & 0 & 0 & 0.165824 & 0.245572 & 0.26655 & 0.314763 & 0 & 0.132861 & 0.233273 & 0 & 0 & 0.510448 \\
\hline 0.953552 & 0 & 0 & 0.197794 & 0.300078 & 0.239748 & 0.352771 & 0 & 0.146466 & 0.272548 & 0 & 0 & 0.519303 \\
\hline 1.04677 & 0 & 0 & 0.233235 & 0.365112 & 0.235812 & 0.391649 & 0 & 0.159792 & 0.320522 & 0 & 0.00106552 & 0.507694 \\
\hline 1.14911 & 0.00201208 & 0 & 0.274019 & 0.442057 & 0.26729 & 0.431972 & 0 & 0.173037 & 0.378984 & 0 & 0.0162656 & 0.478209 \\
\hline 1.26145 & 0.0268682 & 0 & 0.322245 & 0.530495 & 0.336397 & 0.472692 & 0.00207315 & 0.185604 & 0.445839 & 0.0147756 & 0.0951822 & 0.43023 \\
\hline 1.38477 & 0.149312 & 0 & 0.379863 & 0.627029 & 0.445382 & 0.513726 & 0.0322428 & 0.197471 & 0.521739 & 0.0961844 & 0.285916 & 0.36817 \\
\hline 1.52015 & 0.424038 & 0 & 0.447544 & 0.729233 & 0.590483 & 0.555229 & 0.19044 & 0.208529 & 0.605426 & 0.319107 & 0.590265 & 0.301927 \\
\hline 1.66876 & 0.846508 & 0 & 0.523727 & 0.834178 & 0.766899 & 0.59858 & 0.566774 & 0.219268 & 0.696493 & 0.694515 & 0.957004 & 0.245236 \\
\hline 1.8319 & 1.31708 & 0 & 0.603796 & 0.941798 & 0.961428 & 0.644313 & 1.13088 & 0.229613 & 0.790885 & 1.1385 & 1.34572 & 0.211276 \\
\hline 2.011 & 1.76585 & 0 & 0.680135 & 1.0477 & 1.15228 & 0.693018 & 1.71524 & 0.239704 & 0.883636 & 1.55347 & 1.71011 & 0.204611 \\
\hline 2.2076 & 2.1104 & 0 & 0.743497 & 1.14821 & 1.31874 & 0.745472 & 2.20124 & 0.249728 & 0.972708 & 1.87098 & 1.99803 & 0.231835 \\
\hline 2.42342 & 2.29969 & 0 & 0.785456 & 1.23916 & 1.44363 & 0.803112 & 2.50659 & 0.260099 & 1.05634 & 2.05162 & 2.17623 & 0.297434 \\
\hline 2.66033 & 2.31685 & 0 & 0.803211 & 1.32106 & 1.52451 & 0.867487 & 2.59392 & 0.271334 & 1.13723 & 2.07948 & 2.22736 & 0.411616 \\
\hline 2.92042 & 2.16081 & 0.00149202 & 0.802538 & 1.39854 & 1.56091 & 0.93931 & 2.46284 & 0.283807 & 1.212 & 1.9554 & 2.15119 & 0.573066 \\
\hline 3.20592 & 1.85822 & 0.0177553 & 0.799233 & 1.4735 & 1.55827 & 1.01884 & 2.15781 & 0.298057 & 1.2814 & 1.70719 & 1.97692 & 0.769268 \\
\hline 3.51934 & 1.48518 & 0.0942233 & 0.815879 & 1.54916 & 1.52601 & 1.10606 & 1.76416 & 0.314417 & 1.3459 & 1.39946 & 1.75468 & 0.97427 \\
\hline 3.8634 & 1.16068 & 0.234056 & 0.873468 & 1.623 & 1.48267 & 1.20167 & 1.41217 & 0.33356 & 1.41156 & 1.12772 & 1.55315 & 1.16165 \\
\hline 4.2411 & 0.992794 & 0.405421 & 0.983727 & 1.70441 & 1.45822 & 1.30566 & 1.21143 & 0.355934 & 1.48659 & 0.968498 & 1.42932 & 1.32361 \\
\hline 4.65572 & 1.03377 & 0.53949 & 1.14133 & 1.79827 & 1.46968 & 1.41721 & 1.22419 & 0.382137 & 1.56789 & 0.962569 & 1.4187 & 1.45169 \\
\hline 5.11087 & 1.28269 & 0.677472 & 1.32406 & 1.9118 & 1.52533 & 1.53431 & 1.45477 & 0.412638 & 1.65204 & 1.11075 & 1.52557 & 1.54763 \\
\hline 5.61052 & 1.66411 & 0.854727 & 1.5004 & 2.03958 & 1.60675 & 1.65604 & 1.84311 & 0.447932 & 1.7236 & 1.37331 & 1.71708 & 1.60112 \\
\hline 6.15902 & 2.02543 & 1.06876 & 1.64338 & 2.16929 & 1.7007 & 1.78249 & 2.26947 & 0.488545 & 1.78604 & 1.68027 & 1.93919 & 1.62193 \\
\hline 6.76114 & 2.22446 & 1.30168 & 1.74759 & 2.30033 & 1.79933 & 1.91379 & 2.57121 & 0.534973 & 1.84057 & 1.92528 & 2.11239 & 1.64095 \\
\hline 7.42212 & 2.23709 & 1.43174 & 1.82964 & 2.43151 & 1.90572 & 2.04807 & 2.67524 & 0.588315 & 1.89114 & 2.04924 & 2.19464 & 1.6931 \\
\hline 8.14773 & 2.16717 & 1.50431 & 1.92702 & 2.58146 & 2.02503 & 2.181 & 2.59452 & 0.649857 & 1.93417 & 2.04183 & 2.18836 & 1.80947 \\
\hline 8.94427 & 2.14851 & 1.48858 & 2.0859 & 2.72713 & 2.11941 & 2.30865 & 2.41418 & 0.721881 & 1.93738 & 1.95674 & 2.14707 & 1.94563 \\
\hline 9.81869 & 2.25214 & 1.53465 & 2.33615 & 2.85869 & 2.18543 & 2.42854 & 2.30562 & 0.80684 & 1.93276 & 1.9351 & 2.17731 & 2.08457 \\
\hline 10.7786 & 2.44928 & 1.6116 & 2.68151 & 2.95066 & 2.2171 & 2.54025 & 2.34672 & 0.907904 & 1.94827 & 2.0563 & 2.32863 & 2.20301 \\
\hline 11.8323 & 2.62039 & 1.74636 & 3.04093 & 3.01437 & 2.28581 & 2.63807 & 2.63412 & 1.02872 & 2.07219 & 2.40989 & 2.63101 & 2.38154 \\
\hline 12.9891 & 2.66116 & 1.95151 & 3.19732 & 3.14653 & 2.50312 & 2.7147 & 3.1294 & 1.17476 & 2.37565 & 2.94236 & 3.00834 & 2.76392 \\
\hline 14.2589 & 2.58106 & 2.2183 & 3.01373 & 3.33514 & 2.86155 & 2.76663 & 3.66409 & 1.35339 & 2.79336 & 3.44153 & 3.27777 & 3.35358 \\
\hline 15.6529 & 2.50467 & 2.70113 & 2.74319 & 3.59837 & 3.33349 & 2.80633 & 4.05826 & 1.57339 & 3.24045 & 3.68597 & 3.31838 & 4.10654 \\
\hline 17.1832 & 2.58954 & 3.29089 & 2.8695 & 3.76423 & 3.66886 & 2.8631 & 4.07419 & 1.84495 & 3.47562 & 3.49244 & 3.08209 & 4.65978 \\
\hline 18.863 & 2.9044 & 4.07375 & 3.52988 & 3.70207 & 3.72857 & 2.96328 & 3.71335 & 2.17812 & 3.45899 & 3.0163 & 2.76832 & 4.74396 \\
\hline 20.7071 & 3.40146 & 4.70462 & 4.09042 & 3.52628 & 3.64249 & 3.11681 & 3.29512 & 2.58169 & 3.42682 & 2.70788 & 2.69171 & 4.50258 \\
\hline 22.7315 & 3.91395 & 5.06583 & 3.93054 & 3.34907 & 3.5985 & 3.30024 & 3.05915 & 3.05751 & 3.59855 & 2.83779 & 3.02297 & 4.20239 \\
\hline 24.9538 & 4.24171 & 4.96623 & 3.75843 & 3.50087 & 3.95939 & 3.46234 & 3.35371 & 3.59639 & 4.3192 & 3.75957 & 3.93663 & 4.37696 \\
\hline 27.3934 & 4.33109 & 4.51575 & 4.42545 & 4.02824 & 4.74843 & 3.55881 & 4.09348 & 4.17403 & 5.50695 & 5.29457 & 5.20524 & 5.11232 \\
\hline 30.0714 & 4.27986 & 4.17995 & 5.26813 & 4.79998 & 5.78784 & 3.57411 & 5.05161 & 4.75129 & 6.85439 & 7.00837 & 6.39563 & 6.24512 \\
\hline
\end{tabular}




\begin{tabular}{|c|c|c|c|c|c|c|c|c|c|c|c|c|}
\hline 33.0113 & 4.26057 & 4.21247 & 5.29323 & 5.51938 & 6.65802 & 3.55433 & 5.7618 & 5.28122 & 7.78249 & 8.09266 & 6.9389 & 7.29937 \\
\hline 36.2385 & 4.40729 & 4.97578 & 5.52993 & 5.58534 & 6.72143 & 3.57608 & 5.66848 & 5.71509 & 7.66035 & 7.92207 & 6.46243 & 7.42641 \\
\hline 39.7813 & 4.71965 & 6.25378 & 6.40555 & 4.24094 & 5.07795 & 3.69593 & 4.16901 & 6.00919 & 5.64239 & 5.75245 & 4.52376 & 5.62415 \\
\hline 43.6704 & 5.04475 & 7.60953 & 5.51387 & 2.1928 & 2.61563 & 3.92186 & 2.08871 & 6.13153 & 2.84276 & 2.8442 & 2.17693 & 2.88212 \\
\hline 47.9397 & 4.15149 & 8.3049 & 3.18555 & 0.523377 & 0.621553 & 4.1748 & 0.487969 & 6.06499 & 0.665317 & 0.6564 & 0.493537 & 0.682976 \\
\hline 52.6264 & 2.34111 & 7.77533 & 2.68398 & 0.0559853 & 0.0655907 & 4.29026 & 0.0506928 & 5.81515 & 0.0689353 & 0.066479 & 0.0490253 & 0.0719355 \\
\hline 57.7713 & 0.582813 & 5.44106 & 2.07617 & 0 & 0 & 4.10029 & 0 & 5.41191 & 0 & 0 & 0 & 0 \\
\hline 63.4192 & 0.0620939 & 2.60253 & 0.299362 & 0 & 0 & 2.97741 & 0 & 4.89772 & 0 & 0 & 0 & 0 \\
\hline 69.6192 & 0 & 0.586654 & 0 & 0 & 0 & 1.5006 & 0 & 4.32122 & 0 & 0 & 0 & 0 \\
\hline 76.4253 & 0 & 0.0576777 & 0 & 0 & 0 & 0.344904 & 0 & 3.71569 & 0 & 0 & 0 & 0 \\
\hline 83.8969 & 0 & 0 & 0 & 0 & 0 & 0.0346528 & 0 & 3.07547 & 0 & 0 & 0 & 0 \\
\hline 92.0988 & 0 & 0 & 0 & 0 & 0 & 0 & 0 & 2.39759 & 0 & 0 & 0 & 0 \\
\hline 101.103 & 0 & 0 & 0 & 0 & 0 & 0 & 0 & 1.68559 & 0 & 0 & 0 & 0 \\
\hline 110.987 & 0 & 0 & 0 & 0 & 0 & 0 & 0 & 0.940841 & 0 & 0 & 0 & 0 \\
\hline 121.837 & 0 & 0 & 0 & 0 & 0 & 0 & 0 & 0.370332 & 0 & 0 & 0 & 0 \\
\hline 133.748 & 0 & 0 & 0 & 0 & 0 & 0 & 0 & 0.0727115 & 0 & 0 & 0 & 0 \\
\hline 146.824 & 0 & 0 & 0 & 0 & 0 & 0 & 0 & 0.00598825 & 0 & 0 & 0 & 0 \\
\hline 161.177 & 0 & 0 & 0 & 0 & 0 & 0 & 0 & 0 & 0 & 0 & 0 & 0 \\
\hline 176.935 & 0 & 0 & 0 & 0 & 0 & 0 & 0 & 0 & 0 & 0 & 0 & 0 \\
\hline 194.232 & 0 & 0 & 0 & 0 & 0 & 0 & 0 & 0 & 0 & 0 & 0 & 0 \\
\hline 213.221 & 0 & 0 & 0 & 0 & 0 & 0 & 0 & 0 & 0 & 0 & 0 & 0 \\
\hline 234.066 & 0 & 0 & 0 & 0 & 0 & 0 & 0 & 0 & 0 & 0 & 0 & 0 \\
\hline 256.948 & 0 & 0 & 0 & 0 & 0 & 0 & 0 & 0 & 0 & 0 & 0 & 0 \\
\hline 282.068 & 0 & 0 & 0 & 0 & 0 & 0 & 0 & 0 & 0 & 0 & 0 & 0 \\
\hline 309.644 & 0 & 0 & 0 & 0 & 0 & 0 & 0 & 0 & 0 & 0 & 0 & 0 \\
\hline 339.916 & 0 & 0 & 0 & 0 & 0 & 0 & 0 & 0 & 0 & 0 & 0 & 0 \\
\hline 373.147 & 0 & 0 & 0 & 0 & 0 & 0 & 0 & 0 & 0 & 0 & 0 & 0 \\
\hline 409.626 & 0 & 0 & 0 & 0 & 0 & 0 & 0 & 0 & 0 & 0 & 0 & 0 \\
\hline 449.672 & 0 & 0 & 0 & 0 & 0 & 0 & 0 & 0 & 0 & 0 & 0 & 0 \\
\hline 493.633 & 0 & 0 & 0 & 0 & 0 & 0 & 0 & 0 & 0 & 0 & 0 & 0 \\
\hline 541.892 & 0 & 0 & 0 & 0 & 0 & 0 & 0 & 0 & 0 & 0 & 0 & 0 \\
\hline 594.869 & 0 & 0 & 0 & 0 & 0 & 0 & 0 & 0 & 0 & 0 & 0 & 0 \\
\hline 653.025 & 0 & 0 & 0 & 0 & 0 & 0 & 0 & 0 & 0 & 0 & 0 & 0 \\
\hline 716.866 & 0 & 0 & 0 & 0 & 0 & 0 & 0 & 0 & 0 & 0 & 0 & 0 \\
\hline 786.949 & 0 & 0 & 0 & 0 & 0 & 0 & 0 & 0 & 0 & 0 & 0 & 0 \\
\hline 863.883 & 0 & 0 & 0 & 0 & 0 & 0 & 0 & 0 & 0 & 0 & 0 & 0 \\
\hline 948.338 & 0 & 0 & 0 & 0 & 0 & 0 & 0 & 0 & 0 & 0 & 0 & 0 \\
\hline 1041.05 & 0 & 0 & 0 & 0 & 0 & 0 & 0 & 0 & 0 & 0 & 0 & 0 \\
\hline 1142.83 & 0 & 0 & 0 & 0 & 0 & 0 & 0 & 0 & 0 & 0 & 0 & 0 \\
\hline 1254.55 & 0 & 0 & 0 & 0 & 0 & 0 & 0 & 0 & 0 & 0 & 0 & 0 \\
\hline 1377.2 & 0 & 0 & 0 & 0 & 0 & 0 & 0 & 0 & 0 & 0 & 0 & 0 \\
\hline 1511.84 & 0 & 0 & 0 & 0 & 0 & 0 & 0 & 0 & 0 & 0 & 0 & 0 \\
\hline 1659.64 & 0 & 0 & 0 & 0 & 0 & 0 & 0 & 0 & 0 & 0 & 0 & 0 \\
\hline 1821.89 & 0 & 0 & 0 & 0 & 0 & 0 & 0 & 0 & 0 & 0 & 0 & 0 \\
\hline 2000 & 0 & 0 & 0 & 0 & 0 & 0 & 0 & 0 & 0 & 0 & 0 & 0 \\
\hline
\end{tabular}


Appendix 3G: Grain size data for Windless Bight core 5.

\begin{tabular}{|c|c|c|c|c|c|c|c|c|c|c|}
\hline $\begin{array}{c}\text { Diameter } \\
\mu \mathrm{m}\end{array}$ & WB5_14-15 & WB5_27-28 & WB5_3-4 & WB5_10-11 & WB5_21-24 & WB5_30-31 & WB5_32 & WB5_33-34 & WB5_36-37 & WB5_48-50 \\
\hline 0.37512 & 0 & 0 & 0 & 0 & 0 & 0 & 0 & 0 & 0 & 0 \\
\hline 0.4118 & 0 & 0 & 0 & 0 & 0 & 0 & 0 & 0 & 0 & 0 \\
\hline 0.45206 & 0 & 0 & 0 & 0 & 0 & 0 & 0 & 0 & 0 & 0 \\
\hline 0.49625 & 0 & 0 & 0 & 0 & 0 & 0 & 0 & 0 & 0 & 0 \\
\hline 0.54477 & 0 & 0 & 0 & 0 & 0 & 0 & 0 & 0 & 0 & 0 \\
\hline 0.59803 & 0 & 0 & 0 & 0 & 0 & 0 & 0 & 0 & 0 & 0 \\
\hline 0.65649 & 0 & 0 & 0 & 0 & 0 & 0 & 0 & 0 & 0 & 0 \\
\hline 0.72068 & 0 & 0 & 0 & 0 & 0 & 0 & 0 & 0 & 0 & 0 \\
\hline 0.79113 & 0 & 0 & 0 & 0 & 0 & 0 & 0 & 0 & 0 & 0 \\
\hline 0.86848 & 0 & 0 & 0 & 0 & 0 & 0 & 0 & 0 & 0 & 0 \\
\hline 0.95338 & 0 & 0 & 0 & 0 & 0 & 0 & 0 & 0 & 0 & 0 \\
\hline 1.0466 & 0 & 0 & 0 & 0 & 0 & 0 & 0 & 0 & 0 & 0 \\
\hline 1.1489 & 0 & 0 & 0 & 0 & 0 & 0 & 0 & 0 & 0 & 0 \\
\hline 1.2612 & 0 & 0 & 0 & 0 & 0 & 0 & 0 & 0.00430447 & 0.00020282 & 0 \\
\hline 1.3845 & 0 & 0 & 0 & 0.0036938 & 0.00242647 & 0.0010002 & 0.00797784 & 0.0286845 & 0.00513163 & 0 \\
\hline 1.5199 & 0 & 0.00665773 & 0 & 0.0251999 & 0.017593 & 0.0137803 & 0.0451083 & 0.0975732 & 0.0373392 & 0.00530207 \\
\hline 1.6685 & 0 & 0.0377997 & 0 & 0.0849111 & 0.0622828 & 0.0731882 & 0.126922 & 0.207005 & 0.132982 & 0.0325539 \\
\hline 1.8316 & 0 & 0.111685 & 0 & 0.186162 & 0.142336 & 0.198693 & 0.252469 & 0.338236 & 0.297973 & 0.104481 \\
\hline 2.0107 & 0 & 0.219861 & 0.00919468 & 0.304162 & 0.23992 & 0.367494 & 0.388085 & 0.466774 & 0.491666 & 0.213399 \\
\hline 2.2072 & 0 & 0.340076 & 0.0508136 & 0.422073 & 0.342494 & 0.539052 & 0.528695 & 0.594892 & 0.679612 & 0.337617 \\
\hline 2.423 & 0 & 0.450521 & 0.141062 & 0.524836 & 0.438357 & 0.695154 & 0.656558 & 0.712536 & 0.838003 & 0.449444 \\
\hline 2.6599 & 0 & 0.551526 & 0.281288 & 0.616464 & 0.531895 & 0.841672 & 0.775856 & 0.818773 & 0.974563 & 0.553193 \\
\hline 2.92 & 0 & 0.634497 & 0.430292 & 0.691284 & 0.61798 & 0.971095 & 0.880795 & 0.907121 & 1.08109 & 0.644477 \\
\hline 3.2054 & 0 & 0.699195 & 0.570443 & 0.747723 & 0.692407 & 1.08113 & 0.965131 & 0.968914 & 1.15951 & 0.722539 \\
\hline 3.5188 & 0 & 0.7473 & 0.682206 & 0.788413 & 0.755214 & 1.17426 & 1.03576 & 1.01623 & 1.21409 & 0.792015 \\
\hline 3.8628 & 0 & 0.777584 & 0.774916 & 0.814785 & 0.802413 & 1.24727 & 1.08836 & 1.04796 & 1.25142 & 0.845663 \\
\hline 4.2405 & 0 & 0.814966 & 0.869355 & 0.852677 & 0.855266 & 1.33481 & 1.15543 & 1.0889 & 1.31085 & 0.913865 \\
\hline 4.6551 & 0 & 0.84507 & 0.948601 & 0.888939 & 0.900312 & 1.4165 & 1.21588 & 1.12006 & 1.37292 & 0.97891 \\
\hline 5.1102 & 1.47784 & 0.88827 & 1.01593 & 0.939146 & 0.953261 & 1.51464 & 1.29424 & 1.15856 & 1.45826 & 1.06602 \\
\hline 5.6098 & 3.27424 & 0.904679 & 1.0255 & 0.968322 & 0.9845 & 1.57941 & 1.34791 & 1.17624 & 1.51053 & 1.12939 \\
\hline 6.1582 & 1.56714 & 0.92507 & 1.01699 & 0.999796 & 1.01522 & 1.64185 & 1.40752 & 1.19727 & 1.56459 & 1.19765 \\
\hline 6.7603 & 0 & 0.934669 & 0.984993 & 1.02329 & 1.0356 & 1.68525 & 1.45745 & 1.21272 & 1.60257 & 1.25365 \\
\hline 7.4212 & 0 & 0.977941 & 0.985598 & 1.07174 & 1.07311 & 1.75292 & 1.53523 & 1.24596 & 1.67509 & 1.34096 \\
\hline 8.1467 & 0 & 1.04453 & 1.0079 & 1.13908 & 1.1245 & 1.83915 & 1.63556 & 1.29328 & 1.76984 & 1.45363 \\
\hline 8.9432 & 0 & 1.13688 & 1.05934 & 1.2218 & 1.1875 & 1.93933 & 1.75454 & 1.35074 & 1.88027 & 1.58897 \\
\hline 9.8175 & 0 & 1.26993 & 1.16045 & 1.33178 & 1.27449 & 2.07115 & 1.91003 & 1.43521 & 2.02419 & 1.76251 \\
\hline 10.777 & 0 & 1.42543 & 1.30265 & 1.44874 & 1.36681 & 2.20161 & 2.07139 & 1.52554 & 2.17331 & 1.94437 \\
\hline 11.831 & 0 & 1.65643 & 1.54546 & 1.61166 & 1.50129 & 2.38464 & 2.28907 & 1.65693 & 2.39076 & 2.18831 \\
\hline 12.988 & 1.80882 & 1.89434 & 1.81067 & 1.76469 & 1.63381 & 2.54988 & 2.49648 & 1.77811 & 2.59416 & 2.4238 \\
\hline 14.257 & 4.46166 & 2.16532 & 2.10169 & 1.92503 & 1.78808 & 2.73022 & 2.72979 & 1.90971 & 2.8051 & 2.68175 \\
\hline 15.651 & 6.38383 & 2.36734 & 2.2829 & 2.01472 & 1.89644 & 2.82855 & 2.89596 & 1.98689 & 2.90797 & 2.85597 \\
\hline 17.181 & 5.56961 & 2.52254 & 2.35838 & 2.04265 & 1.96371 & 2.84481 & 3.0032 & 2.02106 & 2.9159 & 2.94875 \\
\hline 18.861 & 2.15898 & 2.6377 & 2.36378 & 2.01643 & 1.98136 & 2.77215 & 3.03262 & 2.01026 & 2.85094 & 2.95098 \\
\hline 20.705 & 0 & 2.76523 & 2.38859 & 1.97771 & 1.97894 & 2.65897 & 3.02018 & 1.98185 & 2.77697 & 2.92093 \\
\hline 22.729 & 0 & 2.98878 & 2.56723 & 2.0129 & 2.02808 & 2.62728 & 3.05519 & 2.00729 & 2.80388 & 2.98214 \\
\hline 24.951 & 4.12738 & 3.29471 & 2.89541 & 2.13733 & 2.14323 & 2.72639 & 3.16153 & 2.10511 & 2.92399 & 3.18286 \\
\hline 27.391 & 7.50235 & 3.70601 & 3.37228 & 2.39726 & 2.36424 & 3.02423 & 3.39148 & 2.33723 & 3.15779 & 3.5888 \\
\hline 30.068 & 7.32704 & 4.13355 & 3.85545 & 2.73837 & 2.63383 & 3.44412 & 3.66583 & 2.67493 & 3.40809 & 4.10994 \\
\hline
\end{tabular}




\begin{tabular}{|c|c|c|c|c|c|c|c|c|c|c|}
\hline 33.008 & 6.17764 & 4.54938 & 4.24863 & 3.1252 & 2.91166 & 3.88784 & 3.91683 & 3.11117 & 3.62985 & 4.64809 \\
\hline 36.235 & 5.9043 & 4.91648 & 4.50073 & 3.50118 & 3.14405 & 4.20196 & 4.03883 & 3.59469 & 3.79016 & 5.04779 \\
\hline 39.778 & 6.70764 & 5.21846 & 4.63416 & 3.82808 & 3.31253 & 4.28573 & 3.9766 & 4.06941 & 3.89817 & 5.20526 \\
\hline 43.667 & 8.70182 & 5.45661 & 4.74607 & 4.11486 & 3.45735 & 4.14209 & 3.75738 & 4.5055 & 4.00928 & 5.10522 \\
\hline 47.936 & 7.12393 & 5.57179 & 4.90147 & 4.35693 & 3.61199 & 3.84191 & 3.44448 & 4.85236 & 4.1297 & 4.7785 \\
\hline 52.622 & 5.07964 & 5.50581 & 5.13179 & 4.56879 & 3.81205 & 3.52021 & 3.15 & 5.09508 & 4.24753 & 4.3239 \\
\hline 57.767 & 4.41647 & 5.18205 & 5.38081 & 4.73192 & 4.03392 & 3.26962 & 2.95497 & 5.19815 & 4.28348 & 3.8273 \\
\hline 63.414 & 0 & 4.57403 & 5.53784 & 4.82203 & 4.19527 & 3.12657 & 2.89261 & 5.13384 & 4.12049 & 3.35242 \\
\hline 69.614 & 10.2297 & 3.73526 & 5.46075 & 4.81009 & 4.18753 & 3.04849 & 2.94301 & 4.87957 & 3.66652 & 2.93037 \\
\hline 76.42 & 0 & 2.8218 & 5.03061 & 4.67672 & 3.91909 & 2.91397 & 3.01202 & 4.42737 & 2.9034 & 2.54079 \\
\hline 83.891 & 0 & 2.03219 & 4.2294 & 4.42346 & 3.41728 & 2.61156 & 2.96132 & 3.80415 & 1.93276 & 2.14576 \\
\hline 92.092 & 0 & 1.47491 & 2.76167 & 4.027 & 2.87324 & 2.08215 & 2.67183 & 3.05359 & 0.970184 & 1.70874 \\
\hline 101.1 & 0 & 1.14636 & 1.27245 & 3.46388 & 2.50187 & 1.37459 & 2.09976 & 2.24769 & 0.320533 & 1.21727 \\
\hline 110.98 & 0 & 0.925656 & 0.277221 & 2.71611 & 2.45859 & 0.67548 & 1.26405 & 1.463 & 0.0532023 & 0.682493 \\
\hline 121.83 & 0 & 0.633135 & 0.0263083 & 1.81741 & 2.7026 & 0.211299 & 0.522802 & 0.754366 & 0.0031966 & 0.265427 \\
\hline 133.74 & 0 & 0.303884 & 0 & 0.923457 & 3.01632 & 0.0331768 & 0.105779 & 0.27303 & 0 & 0.0514662 \\
\hline 146.81 & 0 & 0.0692575 & 0 & 0.305452 & 3.06417 & 0.00169246 & 0.00892868 & 0.0502414 & 0 & 0.0040715 \\
\hline 161.17 & 0 & 0.00645314 & 0 & 0.0506351 & 2.59996 & 0 & 0 & 0.0037594 & 0 & 0 \\
\hline 176.92 & 0 & 0 & 0 & 0.00288182 & 1.6259 & 0 & 0 & 0 & 0 & 0 \\
\hline 194.22 & 0 & 0 & 0 & 0 & 0.677341 & 0 & 0 & 0 & 0 & 0 \\
\hline 213.21 & 0 & 0 & 0 & 0 & 0.136961 & 0 & 0 & 0 & 0 & 0 \\
\hline 234.05 & 0 & 0 & 0 & 0 & 0.0113428 & 0 & 0 & 0 & 0 & 0 \\
\hline 256.94 & 0 & 0 & 0 & 0 & 0 & 0 & 0 & 0 & 0 & 0 \\
\hline 282.06 & 0 & 0 & 0 & 0 & 0 & 0 & 0 & 0 & 0 & 0 \\
\hline 309.63 & 0 & 0 & 0 & 0 & 0 & 0 & 0 & 0 & 0 & 0 \\
\hline 339.9 & 0 & 0 & 0 & 0 & 0 & 0 & 0 & 0 & 0 & 0 \\
\hline 373.13 & 0 & 0 & 0 & 0 & 0 & 0 & 0 & 0 & 0 & 0 \\
\hline 409.61 & 0 & 0 & 0 & 0 & 0 & 0 & 0 & 0 & 0 & 0 \\
\hline 449.66 & 0 & 0 & 0 & 0 & 0 & 0 & 0 & 0 & 0 & 0 \\
\hline 493.62 & 0 & 0 & 0 & 0 & 0 & 0 & 0 & 0 & 0 & 0 \\
\hline 541.88 & 0 & 0 & 0 & 0 & 0 & 0 & 0 & 0 & 0 & 0 \\
\hline 594.85 & 0 & 0 & 0 & 0 & 0 & 0 & 0 & 0 & 0 & 0 \\
\hline 653.01 & 0 & 0 & 0 & 0 & 0 & 0 & 0 & 0 & 0 & 0 \\
\hline 716.85 & 0 & 0 & 0 & 0 & 0 & 0 & 0 & 0 & 0 & 0 \\
\hline 786.93 & 0 & 0 & 0 & 0 & 0 & 0 & 0 & 0 & 0 & 0 \\
\hline 863.87 & 0 & 0 & 0 & 0 & 0 & 0 & 0 & 0 & 0 & 0 \\
\hline 948.32 & 0 & 0 & 0 & 0 & 0 & 0 & 0 & 0 & 0 & 0 \\
\hline 1041 & 0 & 0 & 0 & 0 & 0 & 0 & 0 & 0 & 0 & 0 \\
\hline 1142.8 & 0 & 0 & 0 & 0 & 0 & 0 & 0 & 0 & 0 & 0 \\
\hline 1254.5 & 0 & 0 & 0 & 0 & 0 & 0 & 0 & 0 & 0 & 0 \\
\hline 1377.2 & 0 & 0 & 0 & 0 & 0 & 0 & 0 & 0 & 0 & 0 \\
\hline 1511.8 & 0 & 0 & 0 & 0 & 0 & 0 & 0 & 0 & 0 & 0 \\
\hline 1659.6 & 0 & 0 & 0 & 0 & 0 & 0 & 0 & 0 & 0 & 0 \\
\hline 1821.9 & 0 & 0 & 0 & 0 & 0 & 0 & 0 & 0 & 0 & 0 \\
\hline 2000 & 0 & 0 & 0 & 0 & 0 & 0 & 0 & 0 & 0 & 0 \\
\hline
\end{tabular}


Appendix 4a: Down core profile of stable isotopes, dust mass and grain size statistics for WB1.

\begin{tabular}{|c|c|c|c|c|c|c|c|c|c|c|c|c|c|c|c|c|c|c|c|c|c|c|c|c|c|c|c|c|c|}
\hline & & & & & $\begin{array}{l}\text { Dust } \\
\text { mass }\end{array}$ & & $\begin{array}{c}\text { Stable } \\
\text { isotopes }\end{array}$ & & & & Grain size statistics & & & & & & & & & & & & & & & & & & \\
\hline Sample & $\begin{array}{c}\text { Age } \\
\text { model }\end{array}$ & $\begin{array}{c}\text { Snow } \\
(\mathrm{g})\end{array}$ & $\begin{array}{l}\text { Area } \\
\left(\mathrm{cm}^{2}\right)\end{array}$ & $\begin{array}{c}\text { Snow } \\
\text { density } \\
\left(\mathrm{g} \mathrm{cm}^{3}\right)^{2}\end{array}$ & $\begin{array}{c}\text { Dust } \\
\text { weight } \\
\text { (mg) }\end{array}$ & $\begin{array}{c}\text { Dust conc. } \\
\left(\mathrm{g} \mathrm{m}^{2}\right)\end{array}$ & $\begin{array}{l}\delta^{818} \mathbf{O} \\
(\% 0)\end{array}$ & $\begin{array}{l}\delta \text { D } \\
(\%)\end{array}$ & $\begin{array}{c}\begin{array}{c}\text { Stdev } \\
\text { dD }\end{array} \\
\text { 作 }\end{array}$ & $\underset{\text { excess }}{d}$ & Sample & $\begin{array}{c}\text { Grain } \\
\text { size } \\
\text { Module }\end{array}$ & $\begin{array}{c}\text { Mode } \\
(\mu \mathrm{m})\end{array}$ & $\underset{(\mu \mathrm{Median})}{\text { Met }}$ & obs & $\begin{array}{l}\text { Skew- } \\
\text { ness (all } \\
\text { skewed } \\
\text { to the } \\
\text { right) }\end{array}$ & Kurtosis & $\begin{array}{l}\text { Stdev } \\
(\mu \mathrm{m})\end{array}$ & $\begin{array}{l}\text { Stdev } \\
\text { (phi) }\end{array}$ & $\%<10 \mu \mathrm{m}$ & $\begin{array}{c}\text { dust } \\
\text { conc. } \\
<10 \\
<\mathbf{1 0}(\mathbf{g} \\
\left.\mathbf{m}^{-2}\right)\end{array}$ & $\underset{\text { gravel }}{\%}$ & $\%$ mud & $\begin{array}{l}\% \\
\text { sand }\end{array}$ & $\begin{array}{l}\text { sand: } \\
\text { mud }\end{array}$ & $\begin{array}{c}\% \\
\text { clay) }\end{array}$ & $\%$ silt & clay: silt & sorting \\
\hline 1 & 2008 & 0 & 22.765 & 0 & 0.1 & 0.04392 & -33.83 & -267 & 0.5 & 2.74 & WB1_1-2 & MLM & 105.9 & 52.25 & 4 & 0.412 & -0.937 & 179.7 & 2.47633 & 14.4241 & 0.00633 & 0 & 57.9159 & 42.0840 & 0.72664 & 0.18401 & 57.7319 & 0.00318 & $\begin{array}{c}\text { very } \\
\text { poorly } \\
\text { portd }\end{array}$ \\
\hline 2 & & 37.097 & 22.765 & 0.19288 & 0.6 & 0.26356 & -35.11 & -278 & 1.3 & 2.48 & WB1_1-2 & MLM & 105.9 & 52.25 & 4 & 0.412 & -0.937 & 179.7 & 2.47633 & 14.4241 & 0.03801 & 0 & 57.9159 & 42.0840 & 0.72664 & 0.18401 & 57.7319 & 0.00318 & $\begin{array}{c}\text { sorted } \\
\text { very } \\
\text { poorly }\end{array}$ \\
\hline 3 & & 57.3382 & 22.765 & 0.29813 & 10.3 & 4.52448 & -29.61 & -233 & 0.1 & 3.88 & WB1_3 & MLM & 168.9 & 46.53 & & 0.911 & -0.276 & 63.5 & 3.97709 & 18.8357 & 0.85222 & 0 & 58.7300 & 41.2699 & 0.70270 & 0.52008 & 58.2099 & 0.00893 & $\begin{array}{l}\text { very } \\
\text { poorly } \\
\text { sorted }\end{array}$ \\
\hline 4 & & 72.3281 & 22.765 & 0.37607 & 2 & 0.87854 & -26.13 & -206 & 0.2 & 2.74 & WB1_4 & MLM & 37.97 & 145.54 & 15 & 1.094 & 0.255 & 19.74 & 5.66273 & 40.2863 & 0.35393 & 0 & 94.8852 & 5.11478 & 0.05390 & 0.42668 & 94.4585 & 0.00451 & $\begin{array}{l}\text { sortremely } \\
\text { poorly } \\
\text { sorted }\end{array}$ \\
\hline 5 & 2007 & 57.4969 & 22.765 & 0.29895 & 9.4 & 4.12914 & -31.59 & -253 & 0.7 & -1.08 & WB1_5 & MLM & 66.44 & 16.92 & & 1.512 & 2.063 & 30.68 & 5.02655 & 42.7681 & 1.76595 & 0 & 90.3277 & 9.67227 & 0.10707 & 1.75820 & 88.5695 & 0.01985 & $\begin{array}{l}\text { extremely } \\
\text { poorly } \\
\text { sorted }\end{array}$ \\
\hline 6 & & 62.4694 & 22.765 & 0.32481 & 2 & 0.87854 & -34.47 & -273 & 0.6 & 2.76 & & & - & & & & & & & & & 0 & 75.9808 & 24.0191 & 0.31612 & 0.00498 & 75.9758 & 0 & \\
\hline 7 & & 37.82 & 22.765 & 0.19664 & 0.6 & 0.26356 & -34.5 & -269 & 0.4 & 6.7 & $\mathrm{WB1}_{-} 7,9,10,11,13,14$ & MLM & 185.4 & 56.69 & 4 & 0.879 & -0.331 & 65.58 & 3.93060 & 13.2075 & 0.03481 & 0 & 54.3069 & 45.6930 & 0.84138 & 0.30422 & 54.0027 & 0.00563 & $\begin{array}{c}\text { very } \\
\text { poorly }\end{array}$ \\
\hline 8 & & 47.82 & 22.765 & 0.24864 & 0.7 & 0.30748 & -35.5 & -280 & 0.1 & 3.2 & WB1_8 & MLM & 60.52 & 44.57 & 0 & 0.034 & -0.264 & 31.67 & 4.98073 & 12.1064 & 0.03722 & 0 & 70.4097 & 29.5901 & 0.42025 & 0 & 70.4097 & 0 & $\begin{array}{l}\text { Sorted } \\
\text { very } \\
\text { poorly } \\
\text { sorted }\end{array}$ \\
\hline 9 & & 62.6973 & 22.765 & 0.32599 & 0.2 & 0.08785 & -38.18 & -305 & 0.3 & 0.44 & $\mathrm{WB1}_{-} 7,9,10,11,13,14$ & MLM & 185.4 & 56.69 & 4 & 0.879 & -0.331 & 65.58 & 3.93060 & 13.2075 & 0.01160 & 0 & 54.3069 & 45.6930 & 0.84138 & 0.30422 & 54.0027 & 0.00563 & $\begin{array}{l}\text { very } \\
\text { poorly } \\
\text { sorted }\end{array}$ \\
\hline 10 & & 67.8489 & 22.765 & 0.35278 & 0 & 0 & -36.56 & -289 & 1 & 2.78 & $\mathrm{WB1}_{-} 7,9,10,11,13,14$ & MLM & 185.4 & 56.69 & 4 & 0.879 & -0.331 & 65.58 & 3.93060 & 13.2075 & 0 & 0 & 54.3069 & 45.6930 & 0.84138 & 0.30422 & 54.0027 & 0.00563 & $\begin{array}{l}\text { very } \\
\text { poorly } \\
\text { sorted }\end{array}$ \\
\hline 11 & & 52.789 & 22.765 & 0.27447 & 0.1 & 0.04392 & -35.09 & -273 & 0.9 & 6.92 & $\mathrm{WBI}_{-} 7,9,10,11,13,14$ & MLM & 185.4 & 56.69 & 4 & 0.879 & -0.331 & 65.58 & 3.93060 & 13.2075 & 0.00580 & 0 & 54.3069 & 45.6930 & 0.84138 & 0.30422 & 54.0027 & 0.00563 & $\begin{array}{l}\text { very } \\
\text { poorly } \\
\text { sorted }\end{array}$ \\
\hline 12 & & 58.2453 & 22.765 & 0.30284 & 3.6 & 1.58137 & -33.66 & -261 & 0.3 & 7.58 & $\mathrm{WB1}_{-} 7,9,10,11,13,14$ & MLM & 185.4 & 56.69 & 4 & 0.879 & -0.331 & 65.58 & 3.93060 & 13.2075 & 0.20886 & 0 & 54.3069 & 45.6930 & 0.84138 & 0.30422 & 54.0027 & 0.00563 & $\begin{array}{l}\text { very } \\
\text { poorly } \\
\text { sorted }\end{array}$ \\
\hline 13 & & 62.2733 & 22.765 & 0.32379 & 0.2 & 0.08785 & -32.47 & -251 & 0.8 & 8.26 & $\mathrm{WB1}_{-} 7,9,10,11,13,14$ & MLM & 185.4 & 56.69 & 4 & 0.879 & -0.331 & 65.58 & 3.93060 & 13.2075 & 0.01160 & 0 & 54.3069 & 45.6930 & 0.84138 & 0.30422 & 54.0027 & 0.00563 & $\begin{array}{l}\text { very } \\
\text { poorly } \\
\text { sorted }\end{array}$ \\
\hline 14 & 2006 & 52.7238 & 22.765 & 0.27413 & 10 & 4.39270 & -32.44 & -252 & 1 & 7.32 & $\mathrm{WB1}_{-} 7,9,10,11,13,14$ & MLM & 185.4 & 56.69 & 4 & 0.879 & -0.331 & 65.58 & 3.93060 & 13.2075 & 0.58016 & 0 & 54.3069 & 45.6930 & 0.84138 & 0.30422 & 54.0027 & 0.00563 & $\begin{array}{l}\text { solcu } \\
\text { pery } \\
\text { poorly } \\
\text { sorted }\end{array}$ \\
\hline 15 & & 72.5451 & 22.765 & 0.37720 & 0 & 0 & -33.48 & -260 & 0.7 & 7.14 & WB1_15-21 & MLM & 87.9 & 36.46 & 12 & 0.695 & -0.494 & 32.78 & 4.93104 & 17.7022 & 0 & 0 & 54.3069 & 45.6930 & 0.84138 & 0.30422 & 54.0027 & 0.00563 & $\begin{array}{l}\text { extremely } \\
\text { poorly } \\
\text { sorted }\end{array}$ \\
\hline 16 & & 67.4598 & 22.765 & 0.35075 & 0.3 & 0.13178 & -34.32 & -268 & 0.3 & 6.36 & WB1_15-21 & MLM & 87.9 & 36.46 & 12 & 0.695 & -0.494 & 32.78 & 4.93104 & 17.7022 & 0.02332 & 0 & 73.4371 & 26.5628 & 0.36170 & 0.17390 & 73.2632 & 0.00237 & $\begin{array}{l}\text { extremely } \\
\text { poorly } \\
\text { sorted }\end{array}$ \\
\hline 17 & & 67.5441 & 22.765 & 0.35119 & 0.3 & 0.13178 & -34.01 & -264 & 1 & 7.18 & WB1_15-21 & MLM & 87.9 & 36.46 & 12 & 0.695 & -0.494 & 32.78 & 4.93104 & 17.7022 & 0.02332 & 0 & 73.4371 & 26.5628 & 0.36175 & 0.17390 & 73.2632 & 0.00237 & $\begin{array}{l}\text { extremely } \\
\text { poorly } \\
\text { sorted }\end{array}$ \\
\hline 18 & & 62.5245 & 22.765 & 0.32509 & 0.1 & 0.04392 & -33.43 & -261 & 0 & 6.04 & WB1_15-21 & MLM & 87.9 & 36.46 & 12 & 0.695 & -0.494 & 32.78 & 4.93104 & 17.7022 & 0.00777 & 0 & 73.4371 & 26.5628 & 0.36170 & 0.17390 & 73.2632 & 0.00237 & $\begin{array}{l}\text { extremely } \\
\text { poorly } \\
\text { sorted }\end{array}$ \\
\hline 19 & 2005 & 57.6678 & 22.765 & 0.29984 & 0 & 0 & -33.52 & -264 & 1.3 & 3.26 & WB1_15-21 & MLM & 87.9 & 36.46 & 12 & 0.695 & -0.494 & 32.78 & 4.93104 & 17.7022 & 0 & 0 & 73.4371 & 26.5628 & 0.36170 & 0.17390 & 73.2632 & 0.00237 & $\begin{array}{l}\text { extremely } \\
\text { poorly } \\
\text { sorted }\end{array}$ \\
\hline 20 & & 62.2328 & 22.765 & 0.32358 & 0.2 & 0.08785 & -35.47 & -282 & 0.2 & 1.76 & WB1_15-21 & MLM & 87.9 & 36.46 & 12 & 0.695 & -0.494 & 32.78 & 4.93104 & 17.7022 & 0.01555 & 0 & 73.4371 & 26.5628 & 0.36170 & 0.17390 & 73.2632 & 0.00237 & $\begin{array}{l}\text { extremely } \\
\text { poorly } \\
\text { sorted }\end{array}$ \\
\hline 21 & & 92.1759 & 22.765 & 0.47927 & 0.4 & 0.17570 & -36.69 & -290 & 0.9 & 3.22 & WB1_15-21 & MLM & 87.9 & 36.46 & 12 & 0.695 & -0.494 & 32.78 & 4.93104 & 17.7022 & 0.03110 & 0 & 73.4371 & 26.5628 & 0.36170 & 0.17390 & 73.2632 & 0.00237 & $\begin{array}{l}\text { extremely } \\
\text { poorly } \\
\text { sorted }\end{array}$ \\
\hline 22 & & 57.3825 & 22.765 & 0.29836 & 9.8 & 4.30485 & -35.7 & -282 & 1 & 3.4 & WB1_22-24 & MLM & 72.94 & 37.58 & 3 & 1.194 & 0.995 & 45.09 & 4.47104 & 19.5239 & 0.84047 & 0 & 68.6575 & 31.3424 & 0.45650 & 0.36417 & 68.2934 & 0.00533 & $\begin{array}{c}\text { extremely } \\
\text { poorly }\end{array}$ \\
\hline
\end{tabular}


$\begin{array}{lllllllll}52.0033 & 22.765 & 0.27039 & 0.2 & 0.08785 & -34.14 & -269 & 0.6 & 3.22\end{array}$

$\begin{array}{lllllllll}52.6934 & 22.765 & 0.46293 & 0.6 & 0.26356 & -32.8 & -260 & 0.1 & 2.4\end{array}$

$\begin{array}{llllllllll}2004 & 57.0234 & 15.7 & 0.29649 & 13.4 & 8.53503 & -30.52 & -248 & 0 & -4.04\end{array}$ $\begin{array}{llllllll}22.765 & 0 & 1.7 & 0.74676 & -31.25 & -252 & 0.1 & -2.4\end{array}$

$\begin{array}{lllllllll}47.6 & 22.765 & 0.24749 & 4.4 & 1.93279 & -31.8 & -254 & 0.7 & -0.1\end{array}$

$\begin{array}{lllllllll}82.2387 & 22.765 & 0.42760 & 1 & 0.43927 & -31.48 & -255 & 0.4 & -3.96\end{array}$ $\begin{array}{lllllllll}82.534 & 22.765 & 0.42913 & - & 0 & -31.36 & -253 & 0.4 & -2.72\end{array}$

$\begin{array}{lllllllll}85 & 22.765 & 0.44196 & 2.1 & 0.92246 & -30.9 & -248 & 1.3 & -1.7\end{array}$

$\begin{array}{lllllllll}77.5396 & 22.765 & 0.40316 & 0 & 0 & -30.6 & -245 & 1.4 & -0.9\end{array}$

$\begin{array}{llllllllll}2003 & 71.8187 & 22.765 & 0.37342 & 2.3 & 1.01032 & -30.96 & -248 & 0.5 & -1.02\end{array}$

$\begin{array}{lllllllll}73.27 & 22.765 & 0.38096 & 0 & 0 & -31.69 & -255 & 0.1 & -2.28\end{array}$

$\begin{array}{lllllllll}72.1054 & 22.765 & 0.37491 & 1.1 & 0.48319 & -32.27 & -260 & 0.1 & -2.54\end{array}$

$\begin{array}{lllllllll}67.6179 & 22.765 & 0.35158 & 0.4 & 0.175708324 & -32.33 & -262 & 1 & -3.86\end{array}$

$\begin{array}{lllllllll}67.623 & 22.765 & 0.35160 & 0 & 0 & -31.14 & -250 & 0.4 & -1.38\end{array}$

$\begin{array}{lllllllll}82.3627 & 22.765 & 0.42824 & 0.4 & 0.17570 & -30.17 & -244 & 0.4 & -2.84\end{array}$

$\begin{array}{llllllllll}2002 & 82.6447 & 22.765 & 0.42971 & 0.4 & 0.17570 & -30.27 & -244 & 1.1 & -1.84\end{array}$

$\begin{array}{lllllllll}82.3838 & 22.765 & 0.42835 & 0.6 & 0.26356 & -31.22 & -251 & 0.6 & -1.74\end{array}$

$\begin{array}{lllllllll}52.5589 & 22.765 & 0.27328 & 0.3 & 0.13178 & -32.03 & -258 & 0.1 & -2.16\end{array}$

$\begin{array}{llllllllll}51.8985 & 22.765 & 0.26984 & 0.2 & 0.08785 & -33.01 & -265 & 0.3 & -1.32\end{array}$

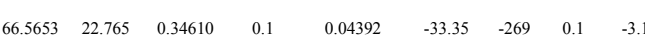

$\begin{array}{lllllllll}72.3287 & 22.765 & 0.37607 & 2.2 & 0.96639 & -33.18 & -266 & 1.4 & -1.26\end{array}$

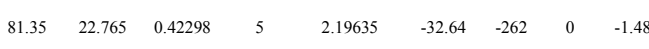

$\begin{array}{llllllllll}2001 & 77.38 & 22.765 & 0.40233 & 3.4 & 1.493520756 & -32.95 & -264 & 0.7 & -0.8\end{array}$

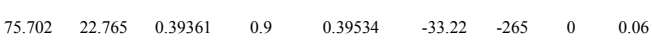

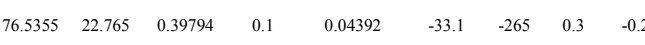

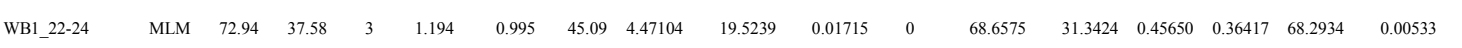

WB1_22-24

WB1 25

WB1_26

WB1 27

WB1_28

WB1_30,32

WB1_31-33

WB1_30,32

WB1_31-33

WB1_34

WB1_35-38

WB1_35-38

WB1_35-38

WB1_35-38

WB1_39,41-43

WB1_40

WB1_39,41-4

WB1_39,41-43

WB1_44

WB1_45

WB1_46

WB1_47 $\begin{array}{llllllllllllllll}72.94 & 37.58 & 3 & 1.194 & 0.995 & 45.09 & 4.47104 & 19.5239 & 0.05145 & 0 & 68.6575 & 31.3424 & 0.45650 & 0.36417 & 68.2934 & 0.005332529\end{array}$ $\begin{array}{llllllllllllllll}\text { MLM } & 14.91 & 14.28 & 2.204 & 4.109 & 30.57 & 5.03173 & 40.8237 & 3.48432 & 0 & 88.3998 & 11.6001 & 0.13122 & 1.44890 & 86.9509 & 0.01666\end{array}$

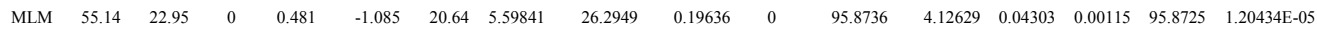
$\begin{array}{llllllllllllllll}\text { MLM } & 14.94 & 12.45 & 2.219 & 4.965 & 16.44 & 5.92664 & 43.5588 & 0.84190 & 0 & 96.3484 & 3.65155 & 0.03789 & 1.55961 & 94.7888 & 0.01645\end{array}$ $\begin{array}{lllllllllllllllll}\text { MLM } & 3.059 & 5.959 & 18 & 1.77 & 2.419 & 15.92 & 5.97301 & 64.5075 & 0.28336 & 0 & 98.4820 & 1.51793 & 0.01541 & 6.00990 & 92.4721 & 0.06499\end{array}$

$\begin{array}{lllllllllllllllll}\text { MLM } & 37.97 & 13.31 & 10 & 1.119 & 0.3 & 20.18 & 5.63093 & 44.68663335 & 0.41222 & 0 & 94.8983 & 5.10169 & 0.05375 & 4.42829 & 90.4700 & 0.04894\end{array}$ $\begin{array}{lllllllllllllllll}\text { MLM } & 37.97 & 16.01 & 10 & 0.919 & -0.23 & 21.63 & 5.53082 & 40.1612 & 0 & 0 & 92.9062 & 7.09374 & 0.07635 & 3.58578 & 89.3204 & 0.04014\end{array}$ $\begin{array}{lllllllllllllllll}\text { MLM } & 37.97 & 13.31 & 10 & 1.119 & 0.3 & 20.18 & 5.63093 & 44.6866 & 0.45147 & 0 & 94.8983 & 5.10169 & 0.05375 & 4.42829 & 90.4700 & 0.04894\end{array}$ $\begin{array}{lllllllllllllllll}\text { MLM } & 37.97 & 16.01 & 10 & 0.919 & -0.23 & 21.63 & 5.53082 & 40.1612 & 0 & 0 & 92.9062 & 7.09374 & 0.07635 & 3.58578 & 89.3204 & 0.04014\end{array}$ $\begin{array}{lllllllllllllllll}\text { MLM } & 72.94 & 56.91 & 0 & 0.179 & -0.769 & 33.71 & 4.89067 & 17.8460 & 0.08623 & 0 & 58.4958 & 41.5041 & 0.70952 & 0.70881 & 57.7870 & 0.01226\end{array}$ $\begin{array}{lllllllllllllllll}\text { MLM } & 66.44 & 24.83 & 8 & 0.713 & -0.57 & 25.75 & 5.27928 & 28.5444 & 0.05015 & 0 & 84.6120 & 15.3879 & 0.18186 & 1.41376 & 83.1983 & 0.01699\end{array}$ $\begin{array}{lllllllllllllllll}\text { MLM } & 59.94 & -7.25 & 16 & 1.247 & -0.371 & 25.75 & 5.27928 & 39.2428 & 0 & 0 & 84.6120 & 15.3879 & 0.18186 & 1.41376 & 83.1983 & 0.01699\end{array}$ $\begin{array}{lllllllllllllllll}\text { MLM } & 53.44 & -39.33 & 24 & 1.781 & -0.172 & 25.75 & 5.27928 & 49.9413 & 0.08775 & 0 & 84.6120 & 15.3879 & 0.18186 & 1.41376 & 83.1983 & 0.01699\end{array}$ $\begin{array}{lllllllllllllllll}\text { MLM } & 46.94 & -71.41 & 32 & 2.315 & 0.027 & 25.75 & 5.27928 & 60.6397 & 0.10654 & 0 & 84.6120 & 15.3879 & 0.18186 & 1.41376 & 83.1983 & 0.01699\end{array}$ $\begin{array}{lllllllllllllllll}\text { MLM } & 41.68 & 27.22 & 4 & 0.927 & 0.021 & 29.61 & 5.07777 & 25.3552 & 0.06682 & 0 & 81.3013 & 18.6985 & 0.22999 & 0.91905 & 80.3823 & 0.01143\end{array}$ $\begin{array}{lllllllllllllllll}\text { MLM } & 55.13 & 23.67 & 0 & 0.453 & -1.07 & 19.9 & 5.65108 & 24.4699 & 0.03224 & 0 & 90.3757 & 9.62421 & 0.10649 & 0.02283 & 90.3529 & 0\end{array}$ $\begin{array}{lllllllllllllllll}\text { MLM } & 41.68 & 27.22 & 4 & 0.927 & 0.021 & 29.61 & 5.07777 & 25.3552 & 0.02227 & 0 & 81.3013 & 18.6985 & 0.22999 & 0.91905 & 80.3823 & 0.01143\end{array}$

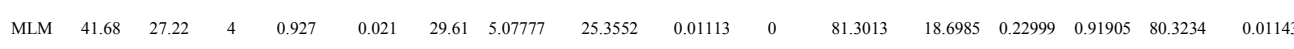
$\begin{array}{lllllllllllllllll}\text { MLM } & 41.68 & 29.63 & 7 & 1.079 & 0.335 & 36.56 & 4.77359 & 23.8670 & 0.23065 & 0 & 76.5659 & 23.4340 & 0.30606 & 1.07722 & 75.4887 & 0.01427\end{array}$

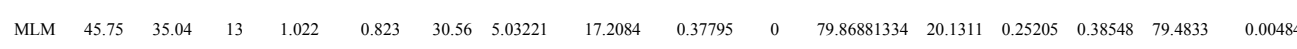

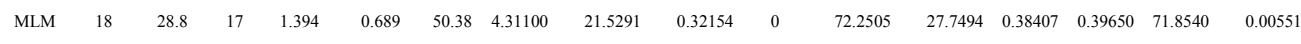
$\begin{array}{lllllllllllllllll}\text { MLM } & 45.75 & 23.08 & 7 & 1.012 & 0.124 & 28.91 & 5.11228 & 28.6728 & 0.11335 & 0 & 83.0781 & 16.9218 & 0.20368 & 0.57580 & 82.5023 & 0.00697\end{array}$ $\begin{array}{lllllll}0 & 77.7605 & 22.2394 & 0.28599 & 0.60649 & 77.1541 & 0.0078\end{array}$ 


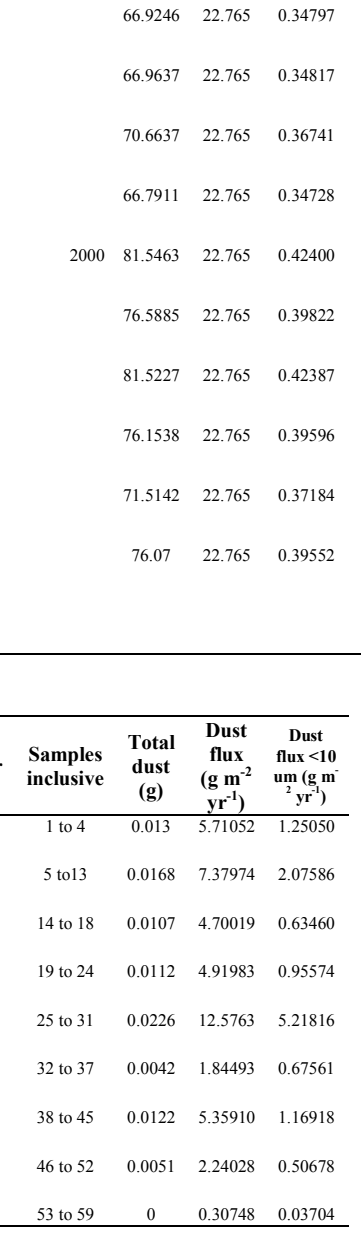

\begin{tabular}{|c|c|c|c|c|c|c|c|c|c|c|c|c|c|c|c|c|c|c|}
\hline WB1_49-53 & MLM & 41.68 & 29.37 & 11 & 6.887 & -0.15 & 32.05 & 4.96353 & 23.3801 & 0.01027 & 0 & 77.7605 & 22.2394 & 0.28599 & 0.60649 & 77.1541 & 0.00786 & $\begin{array}{c}\text { extremely } \\
\text { poorly }\end{array}$ \\
\hline WB1_49-53 & MLM & 41.68 & 29.37 & 11 & 6.887 & -0.15 & 32.05 & 4.96353 & 23.3801 & 0.03081 & 0 & 77.7605 & 22.2394 & 0.28599 & 0.60649 & 77.1541 & 0.00786 & $\begin{array}{l}\text { soltrencly } \\
\text { extreorly } \\
\text { poorly } \\
\text { sorted }\end{array}$ \\
\hline WB1_49-53 & MLM & 41.68 & 29.37 & 11 & 6.887 & -0.15 & 32.05 & 4.96353 & 23.3801 & 0.01027 & 0 & 77.7605 & 22.2394 & 0.28599 & 0.60649 & 77.1541 & 0.00786 & $\begin{array}{c}\text { extremely } \\
\text { poorly }\end{array}$ \\
\hline WB1_49-53 & MLM & 41.68 & 29.37 & 11 & 6.887 & -0.15 & 32.05 & 4.96353 & 23.3801 & 0.02054 & 0 & 77.7605 & 22.2394 & 0.28599 & 0.60649 & 77.1541 & 0.00786 & $\begin{array}{l}\text { sorted } \\
\text { extremely } \\
\text { poorly }\end{array}$ \\
\hline WB1_49-53 & MLM & 41.68 & 29.37 & 11 & 6.887 & -0.15 & 32.05 & 4.96353 & 23.3801 & 0 & 0 & 77.7605 & 22.2394 & 0.28599 & 0.60649 & 77.4105 & 0.00786 & $\begin{array}{l}\text { sorted } \\
\text { extremely } \\
\text { poorly }\end{array}$ \\
\hline WB1_54-58 & MLM & 50.22 & 45.63 & 7 & 1.051 & 0.535 & 45.09 & 4.47104 & 12.0479 & 0.00529 & 0 & 64.8278 & 35.1721 & 0.54254 & 0.21553 & 64.6123 & 0.00333 & $\begin{array}{l}\text { soltrencly } \\
\text { extreorly } \\
\text { poorly } \\
\text { sorted }\end{array}$ \\
\hline WB1_54-58 & MLM & 50.22 & 45.63 & 7 & 1.051 & 0.535 & 45.09 & 4.47104 & 12.0479 & 0.01587 & 0 & 64.8278 & 35.1721 & 0.54254 & 0.21553 & 64.6123 & 0.00333 & $\begin{array}{l}\text { extremely } \\
\text { poorly } \\
\text { sorted }\end{array}$ \\
\hline WB1_54-58 & MLM & 50.22 & 45.63 & 7 & 1.051 & 0.535 & 45.09 & 4.47104 & 12.0479 & 0.01058 & 0 & 64.8278 & 35.1721 & 0.54254 & 0.21553 & 64.6123 & 0.00333 & $\begin{array}{l}\text { extremely } \\
\text { poorly } \\
\text { sorted }\end{array}$ \\
\hline WB1_54-58 & MLM & 50.22 & 45.63 & 7 & 1.051 & 0.535 & 45.09 & 4.47104 & 12.0479 & 0.01587 & 0 & 64.8278 & 35.1721 & 0.54254 & 0.21553 & 64.6123 & 0.00333 & $\begin{array}{c}\text { extremely } \\
\text { poorly } \\
\text { sorted }\end{array}$ \\
\hline WB1_44-58 & MLM & 50.22 & 45.63 & 7 & 1.051 & 0.535 & 45.09 & 4.47104 & 12.0479 & & 0 & 64.8278 & 35.1721 & 0.54254 & 0.21553 & 64.612 & 0.00333 & $\begin{array}{l}\text { extremely } \\
\text { poorly } \\
\text { sorted }\end{array}$ \\
\hline
\end{tabular}

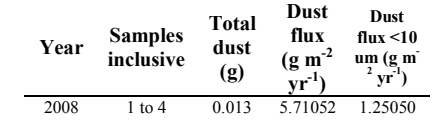

$$
\begin{aligned}
& \begin{array}{lllll}
2007 & 5 \text { to13 } & 0.0168 & 7.37974 & 2.07586
\end{array} \\
& \begin{array}{llllll}
2006 & 14 \text { to } 18 & 0.0107 & 4.70019 & 0.63460
\end{array} \\
& \begin{array}{llllll}
2005 & 19 \text { to } 24 & 0.0112 & 4.91983 & 0.95574
\end{array} \\
& \begin{array}{llllll}
2004 & 25 \text { to } 31 & 0.0226 & 12.5763 & 5.21816
\end{array} \\
& \begin{array}{llllll}
2003 & 32 \text { to } 37 & 0.0042 & 1.84493 & 0.67561
\end{array} \\
& \begin{array}{lllll}
2001 & 46 \text { to } 52 & 0.0051 & 2.24028 & 0.50678
\end{array} \\
& \begin{array}{lllll}
2000 & 53 \text { to } 59 & 0 & 0.30748 & 0.03704 \\
\hline
\end{array}
\end{aligned}
$$


Appendix 4b: Down core profile of stable isotopes, dust mass and grain size statistics for WB3.

\begin{tabular}{|c|c|c|c|c|c|c|c|c|c|c|c|c|c|c|c|c|c|c|c|c|c|c|c|c|c|c|c|c|}
\hline & & & & $\begin{array}{l}\text { Dust } \\
\text { conc. }\end{array}$ & $\begin{array}{c}\text { Stable } \\
\text { isotopes }\end{array}$ & & & & $\begin{array}{c}\text { Grain } \\
\text { size } \\
\text { statistics }\end{array}$ & & & & & & & & & & & & & & & & & & & \\
\hline Sample & $\begin{array}{c}\text { Age } \\
\text { model }\end{array}$ & $\begin{array}{c}\text { Snow } \\
\text { (g) }\end{array}$ & $\begin{array}{l}\text { Area } \\
\left(\mathbf{c m}^{2}\right)\end{array}$ & $\begin{array}{l}\text { Dust } \\
\text { conc. } \\
\left(\mathrm{g} \mathrm{m}^{-2}\right)\end{array}$ & $\begin{array}{l}\delta^{18} \mathbf{O} \\
(\%)\end{array}$ & $\begin{array}{l}\delta \text { D } \\
(\%)\end{array}$ & $\begin{array}{l}\text { Stdev } \\
\text { dD }\end{array}$ & $\begin{array}{c}\text { d } \\
\text { excess }\end{array}$ & Sample & $\begin{array}{c}\text { Grain } \\
\text { size } \\
\text { Module }\end{array}$ & $\begin{array}{c}\text { Mode } \\
(\mu \mathrm{m})\end{array}$ & $\underset{(\mu \mathrm{m})}{\text { Median }}$ & Obs & $\begin{array}{c}\text { Skew- } \\
\text { ness }\end{array}$ & $\begin{array}{c}\text { Skew- } \\
\text { ness } \\
\text { direction }\end{array}$ & $\begin{array}{c}\text { Kurt- } \\
\text { osis }\end{array}$ & $\begin{array}{l}\text { Stdev } \\
(\mu \mathrm{m})\end{array}$ & $\begin{array}{l}\text { Stdev } \\
\text { (phi) }\end{array}$ & $\begin{array}{l}\% \\
<10 \\
\mu \mathrm{m}\end{array}$ & $\begin{array}{c}\text { dust conc. } \\
<10 \text { um. } \\
\left(\mathrm{g} \mathrm{m}^{-2}\right)\end{array}$ & $\begin{array}{c}\% \\
\text { gravel }\end{array}$ & $\begin{array}{c}\% \\
\text { mud }\end{array}$ & $\begin{array}{c}\% \\
\text { sand }\end{array}$ & $\begin{array}{c}\text { sand: } \\
\text { mud }\end{array}$ & $\begin{array}{c}\% \\
\text { clay }\end{array}$ & $\%$ silt & $\begin{array}{c}\text { clay: } \\
\text { silt }\end{array}$ & sorting \\
\hline 1 & 2008 & 16.6 & 9.6162 & 0.1039 & -35.16 & -277 & 1.4 & 3.58 & & & & & & & & & & & & & & 99 & 0 & 0 & 2 & 97 & 0 & \\
\hline 2 & & & 9.6162 & 0 & -34.26 & -267 & 0.5 & 6.68 & & & & & & & & & & & & & & & & & & & & \\
\hline 3 & & 17.7 & 9.6162 & 0 & -30.95 & -240 & 0.3 & 7.1 & & & & & & & & & & & & & & & & & & & & \\
\hline 4 & & & 9.6162 & 0.1039 & -30.99 & -237 & 0.2 & 10.42 & & & & & & & & & & & & & & & & & & & & \\
\hline 5 & & & 9.6162 & 0 & -27.57 & -211 & 0.8 & 9.56 & & & & & & & & & 118.6 & 3.0758 & & & & & & & & & & $\begin{array}{l}\text { very } \\
\text { poorly } \\
\text { sorted }\end{array}$ \\
\hline 6 & & 19.2 & 9.6162 & 0.1039 & -24.92 & -192 & 0.6 & 7.36 & & & & & & & & & & & & & & & & & & & & \\
\hline 7 & 2007 & 18.6 & 9.6162 & 0.1039 & -24.98 & -193 & 0.3 & 6.14 & WB3_7 & MLM & 50.23 & 31.48 & 1 & 0.177 & $\mathrm{R}$ & -1.04 & 17.32 & 5.8514 & 11.154 & 0.0115 & 0 & 96 & 3 & 0 & 0 & 99 & 0 & $\begin{array}{c}\text { extremely } \\
\text { poorly } \\
\text { sorted }\end{array}$ \\
\hline 8 & & & 9.6162 & 0 & -28.24 & -224 & 0.3 & 1.22 & & & & & & & & & & & & & & & & & & & & \\
\hline 9 & & & 9.6162 & 0 & -32.29 & -257 & 0.3 & 0.82 & & & & & & & & & 200.6 & 2.3176 & & & & & & & & & & $\begin{array}{c}\text { very } \\
\text { poorly } \\
\text { sorted }\end{array}$ \\
\hline 10 & & & 9.6162 & 0 & -31.2 & -244 & 0.4 & 5.5 & & & & & & & & & & & & & & & & & & & & \\
\hline 11 & & & 9.6162 & 0 & -30.75 & -241 & 0.2 & 4.8 & & & & & & & & & & & & & & & & & & & & \\
\hline 12 & & 14.5 & 9.6162 & 0.1039 & -30.59 & -239 & 0.7 & 5.72 & & & & & & & & & & & & & & & & & & & & \\
\hline 13 & & 17.9 & 9.6162 & 0.20792 & -30.25 & -235 & 0.1 & 6.9 & & & & & & & & & & & & & & 99 & 0.3 & 0 & 3 & 96 & 0 & \\
\hline 14 & 2006 & & 9.6162 & 0 & -30.97 & -239 & 0.5 & 7.96 & & & & & & & & & & & & & & & & & & & & \\
\hline 15 & & 17.2 & 9.6162 & 0 & -32.12 & -249 & 1 & 7.26 & & & & & & & & & & & & & & & & & & & & \\
\hline 16 & & 14.4 & 9.6162 & 0 & -32.84 & -255 & 0.5 & 6.82 & & & & & & & & & & & & & & & & & & & & \\
\hline 17 & & & 9.6162 & 0 & -31.69 & -246 & 0.2 & 7.52 & & & & & & & & & & & & & & & & & & & & \\
\hline 18 & & & 9.6162 & 0 & -29.9 & -231 & 0.9 & 7.7 & & & & & & & & & & & & & & & & & & & & \\
\hline 19 & 2005 & 15.2 & 9.6162 & 0 & -33.02 & -245 & 0.5 & & & & & & & & & & & & & & & & & & & & & \\
\hline 20 & & 20.3 & 9.6162 & 0.1039 & -32.66 & -255 & 0.2 & 5.78 & WB3_20 & MLM & 37.97 & 16.11 & 3 & 0.554 & $\mathrm{R}$ & -0.84 & 12.63 & 6.3070 & 39.166 & 0.0407 & 0 & 100 & 0 & 0 & 5 & 94 & 0 & $\begin{array}{c}\text { extremely } \\
\text { poorly } \\
\text { sorted }\end{array}$ \\
\hline 21 & & 19.9 & 9.6162 & 0.3119 & -32.84 & -256 & 0.2 & 6.42 & WB3_21 & MLM & 37.97 & 10.06 & 4 & 0.313 & $\mathrm{R}$ & -1.09 & 13.09 & 6.2553 & 35.904 & 0.1120 & 0 & 99 & 0 & 0 & 6 & 93 & 0 & $\begin{array}{l}\text { extremely } \\
\text { poorly } \\
\text { sorted }\end{array}$ \\
\hline 22 & & & 9.6162 & 0 & -31.23 & -243 & 0.2 & 6.34 & & & & & & & & & & & & & & & & & & & & \\
\hline 23 & & & 9.6162 & 0 & -28.5 & -221 & 0.4 & 7 & & & & & & & & & & & & & & & & & & & & \\
\hline
\end{tabular}




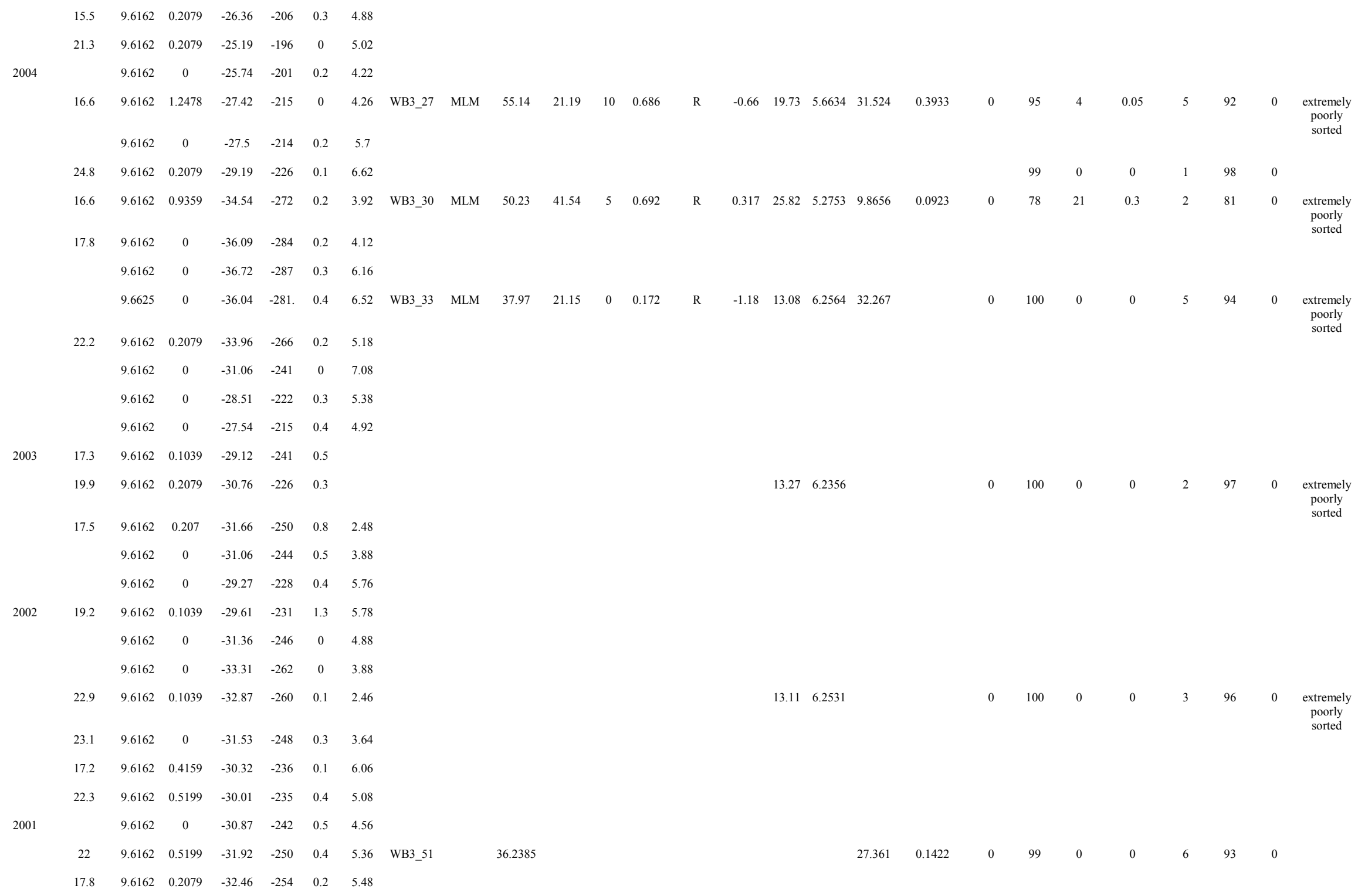

$13.27 \quad 6.2356$

$13.11 \quad 6.2531$ 


$\begin{array}{lccccccc}53 & & 9.6162 & 0 & -32.4 & -253 & 0.5 & 6.1 \\ 54 & 20.5 & 9.6162 & 0.2079 & -31.91 & -249 & 0.9 & 5.68 \\ 55 & & 9.6162 & 0 & -31.02 & -241 & 0.4 & 6.46 \\ 56 & & 9.6162 & 0 & -30.48 & -237 & 1 & 5.94\end{array}$

\begin{tabular}{cccc}
\hline Year & $\begin{array}{c}\text { Samples } \\
\text { inclusive }\end{array}$ & $\begin{array}{c}\text { Total } \\
\text { dust (g) }\end{array}$ & $\begin{array}{c}\text { Dust } \\
\text { flux } \\
\left(\mathbf{m}^{-2}\right. \\
\left.\mathbf{y r}^{-1}\right)\end{array}$ \\
\hline 2008 & 1 to 6 & 0 & 0.3119 \\
2007 & 7 to 13 & 0 & 0.4159 \\
2006 & 14 to 18 & 0 & 0 \\
2005 & 19 to 25 & 0 & 0.8319 \\
2004 & 26 to 37 & 0.0025 & 2.5997 \\
2003 & 38 to 42 & 0.0005 & 0.5199 \\
2002 & 43 to 49 & 0.0011 & 1.1438 \\
2001 & 50 to 56 & 0 & 0.9359 \\
\hline
\end{tabular}


Appendix 4c: Down core profile of stable isotopes, dust mass and grain size statistics for WB5.

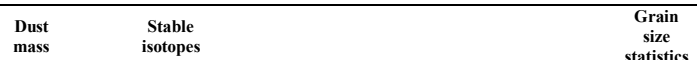

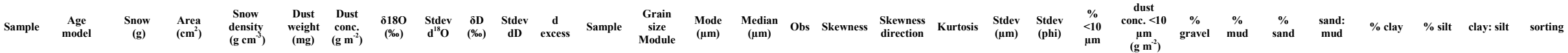

2008

$\begin{array}{llllllllll}41.744 & 22.765 & 0.2170 & 0 & 0 & -30.67 & 0.07 & -244 & 0.4 & 0.46\end{array}$

$\begin{array}{llllllllll}38.082 & 22.765 & 0.1980 & 0 & 0 & -31.58 & 0.08 & -252 & 0.2 & -0.06\end{array}$

$\begin{array}{lllllllllllllllllllllllllllllll}541.77 & 22.765 & 2.8169 & 0 & 0 & -33.74 & 0.08 & -270 & 0.4 & -0.58 & \text { WB5_3.4 } & \text { MLM } & 66.44 & 39.3 & 0 & 0.417 & \text { R } & -0.756 & 27.04 & 5.2087 & 21.466 & 0 & 0 & 75.403 & 24.596 & 0.3261 & 0 & 75.403 & 0\end{array}$

$32.042 \quad 22.765 \quad 0.1606$

$\begin{array}{lllllllllll}42.031 & 22.765 & 0.2185 & 0 & 0 & -35.33 & 0.07 & -285 & 0 & -3.06\end{array}$

$\begin{array}{lllllllllll}43.116 & 22.765 & 0.2241 & 0 & 0 & -35.22 & 0.07 & -282 & 0.2 & -0.44\end{array}$

$\begin{array}{lllllll}0 & 0 & -35.44 & 0.09 & -283 & 0.3 & 0.42\end{array}$

$\begin{array}{lllllll}0 & 0 & -34.73 & 0.01 & -278 & 0.1 & -0.36\end{array}$

$\begin{array}{lllllllllll}48.07 & 22.765 & 0.2499 & 0 & 0 & -28.46 & 0.08 & -226 & 0.1 & 0.88\end{array}$

$\begin{array}{llllllllllllllllllllllllllllllll}52.98 & 22.765 & 0.2754 & 0.3 & 0.0131 & -29.87 & 0.06 & -236 & 0.4 & 2.66 & \text { WB5 } 10- & \text { MLM } & 66.44 & 43.48 & 9 & 0.62 & \text { R } & -0.404 & 35.01 & 4.8360 & 6.3192 & 0 & 0 & 67.960 & 32.039 & 0.4714 & 0.3001 & 67.660 & 0.0044 & \text { extremely } \\ \text { poorly }\end{array}$

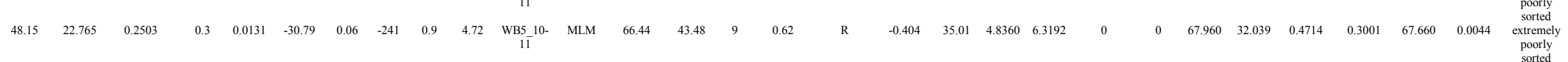

$\begin{array}{llllllllll}47.724 & 22.765 & 0.2481 & 0 & 0 & -32.15 & 0.05 & -252 & 0 & 4.9\end{array}$

$\begin{array}{lllllllllll}47.174 & 22.765 & 0.2452 & 0 & 0 & -32.1 & 0.03 & -253 & 0.2 & 3.5\end{array}$

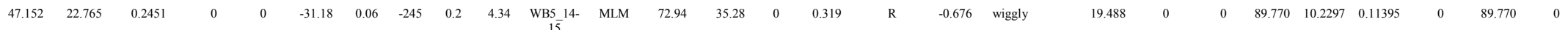

$\begin{array}{lllllllllllllllllllllllllllllll}2007 & 52.367 & 22.765 & 0.2722 & 0 & 0 & -22.46 & 0.05 & -175 & 0.2 & 4.38 & \text { WBS } 14 . & \text { MLM } & 72.94 & 35.28 & 0 & 0.319 & \text { R } & -0.676 & \text { wiggly } & 19.48 & 0 & 0 & 89.770 & 10.229 & 0.1139 & 0 & 89.770 & 0\end{array}$

$\begin{array}{llllllllll}32.561 & 22.765 & 0.1693 & 0 & 0 & -23.63 & 0.04 & -185 & 0.2 & 3.34\end{array}$

$\begin{array}{llllllllll}72.232 & 22.765 & 0.3755 & 0 & 0 & -27.01 & 0.08 & -216 & 0.2 & -0.62\end{array}$

$\begin{array}{lllllllllll}52.407 & 22.765 & 0.2724 & 0 & 0 & -29.75 & 0.02 & -240 & 0.4 & -2.1\end{array}$

$\begin{array}{llllllllll}51.457 & 22.765 & 0.2675 & 0 & 0 & -30.83 & 0.03 & -248 & 0.1 & -1.66\end{array}$

$\begin{array}{llllllllll}57.404 & 22.765 & 0.2984 & 0 & 0 & -30.9 & 0.07 & -247 & 0.1 & -0.5\end{array}$

$\begin{array}{llllllllllllllllllllllllllllll}52.782 & 22.765 & 0.2744 & 0.2 & 0.0087 & -29.89 & 0.08 & -240 & 0.3 & -1.28 & \text { WB_21- } & \text { MLM } & 60.44 & 46.51 & 0 & 0.993 & \text { R } & 0.207 & 49.11 & 4.3478 & 14.319 & 0.0012 & 0 & 62.612 & 37.387 & 0.5971 & 0.2247 & 62.387 & 0.0036 & 0\end{array}$

$\begin{array}{llllllllllllllllllllllllllllllll}2006 & 42.095 & 22.765 & 0.2188 & 0 & 0 & -29.71 & 0.04 & -239 & 0.5 & -1.62 & \text { WB } 21- & \text { MLM } & 60.44 & 46.51 & 0 & 0.993 & \text { R } & 0.207 & 49.11 & 4.3478 & 14.319 & 0 & 0 & 62.612 & 37.387 & 0.5971 & 0.2247 & 62.387 & 0.0036 & 0\end{array}$ $\begin{array}{llllllllllllllllllllllllllllll}42.089 & 22.765 & 0.218844664 & 0 & 0 & -29.89 & 0.07 & -241 & 0.2 & -1.98 & \text { WB_21- } & \text { MLM } & 60.44 & 46.51 & 0 & 0.993 & \text { R } & 0.207 & 49.11 & 4.3478 & 14.319 & 0 & 0 & 62.612 & 37.387 & 0.5971 & 0.2247 & 62.387 & 0.0036 & 0\end{array}$ 


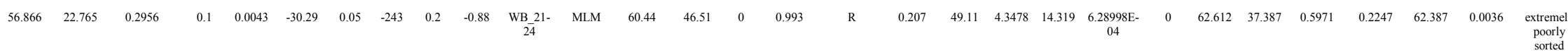
$\begin{array}{llllllllll}57.835 & 22.765 & 0.3007 & 0 & 0 & -30.41 & 0.08 & -242 & 0.1 & 0.68\end{array}$ 
$\begin{array}{llllllllll}68.908 & 22.765 & 0.3582 & 0 & 0 & -33.81 & 0.01 & -268 & 0.1 & 1.58\end{array}$

$\begin{array}{llllllllll}62.401 & 22.765 & 0.3262 & 0 & 0 & -32.44 & 0.08 & -256 & 0.1 & 2.72\end{array}$

$\begin{array}{lllllllllll}57.832 & 22.765 & 0.3007 & 0 & 0 & -31.02 & 0.16 & -244 & 0.3 & 3.36\end{array}$

$\begin{array}{lllllllllll}2004 & 47.898 & 22.765 & 0.2490 & 0 & 0 & -30.04 & 0.1 & -238 & 0.4 & 1.62\end{array}$

$\begin{array}{llllllllll}58.577 & 22.765 & 0.3045 & 0 & 0 & -30.71 & 0.03 & -243 & 0.1 & 2.18\end{array}$

$\begin{array}{lllll}-31.34 & 0.09 & -249 & 0.2 & 1.22\end{array}$

$\begin{array}{lllll}-31.72 & 0.07 & -252 & 0.4 & 1.16\end{array}$

$\begin{array}{lllll}-31.38 & 0.11 & -252 & 0.3 & -0.96\end{array}$

$\begin{array}{lllll}-29.64 & 0.06 & -239 & 0.5 & -1.88\end{array}$

$\begin{array}{llllll}-29.75 & 0.04 & -239 & 0.3 & -1.3\end{array}$

$\begin{array}{lllll}-30.61 & 0.04 & -246 & 0.1 & -1.12\end{array}$

$\begin{array}{rrrrr}-31.69 & 0.06 & -256 & 0.1 & -3.28\end{array}$

$\begin{array}{lllll}-32.97 & 0.09 & -266 & 0.3 & -2.54\end{array}$

$\begin{array}{lllll}-33.95 & 0.09 & -274 & 0 & -3.2\end{array}$

$\begin{array}{lllll}-34.92 & 0.01 & -282 & 0.1 & -3.44\end{array}$

$\begin{array}{lllll}-35.69 & 0.02 & -289 & 0.4 & -3.78\end{array}$

$\begin{array}{lllll}-36.15 & 0.04 & -293 & 0.2 & -4.1\end{array}$

$\begin{array}{llllll}-36.33 & 0.03 & -291 & 1 & -0.96\end{array}$

$\begin{array}{lllll}-36.3 & 0.12 & -294 & 0.7 & -4.3\end{array}$

$\begin{array}{lllll}-35.33 & 0.1 & -285 & 0.2 & -3.16\end{array}$

$\begin{array}{lllll}-34.7 & 0.1 & -279 & 0.2 & -1.9\end{array}$

$\begin{array}{llllll}-33.48 & 0.17 & -269 & 0.2 & -1.66\end{array}$

$\begin{array}{lllll}-31.96 & 0.05 & -257 & 0.2 & -1.62\end{array}$

$\begin{array}{llllll}-30.26 & 0.17 & -243 & 1.4 & -1.52\end{array}$

$\begin{array}{llllll}-28.67 & 0.05 & -230 & 0.2 & -1.44\end{array}$

$\begin{array}{llllll}-26.93 & 0.11 & -217 & 0.2 & -2.26\end{array}$

$\begin{array}{lllll}-25.96 & 0.09 & -207 & 0.3 & 0.08\end{array}$

$\begin{array}{llllll}-24.62 & 0.12 & -198 & 0.1 & -1.04\end{array}$

$\begin{array}{lllll}-24.33 & 0.04 & -195 & 0.2 & -0.86\end{array}$

$\begin{array}{lllll}-24.19 & 0.02 & -193 & 0.1 & -0.18\end{array}$ 


\begin{tabular}{|c|c|c|c|c|c|}
\hline 2003 & -24.12 & 0.06 & -194 & 0.1 & -1.74 \\
\hline & -24.52 & 0.13 & -199 & 0.3 & -2.84 \\
\hline & -25.25 & 0.06 & -205 & 0.2 & -3.1 \\
\hline & -26.88 & 0.04 & -217 & 0.2 & -2.76 \\
\hline & -28.18 & 0.07 & -227 & 0.2 & -2.06 \\
\hline & $-29,55$ & 0.08 & -237 & 0.3 & -1.5 \\
\hline & -30.24 & 0.1 & -246 & 0.4 & -4.58 \\
\hline & -30.37 & 0.08 & -251 & 1.1 & -8.04 \\
\hline & -30.8 & 0.07 & -253 & 0.2 & -7.1 \\
\hline & -30.77 & 0.03 & -252 & 0.1 & -6.54 \\
\hline & -30.73 & 0.02 & -251 & 0.3 & -5.86 \\
\hline & -30.45 & 0.1 & -248 & 0.1 & -4.9 \\
\hline & -30 & 0.1 & -242 & 0.2 & -2.8 \\
\hline & -29.41 & 0.02 & -237 & 0.1 & -1.92 \\
\hline 2002 & -28.86 & 0.09 & -232 & 0.6 & -1.82 \\
\hline & -28.89 & 0.08 & -232 & 0.2 & -1.08 \\
\hline & -29.46 & 0.02 & -237 & 0.1 & -1.32 \\
\hline & -30.3 & 0.04 & -244 & 0.4 & -2.5 \\
\hline & -31.11 & 0.1 & -253 & 0.1 & -4.32 \\
\hline & -31.86 & 0.07 & -260 & 0.1 & -5.22 \\
\hline & -32.47 & 0.08 & -265 & 0.2 & -5.34 \\
\hline & -32.98 & 0.15 & -266 & 0.3 & -2.66 \\
\hline & -32.76 & 0.06 & -264 & 0.3 & -2.42 \\
\hline & -31.98 & 0.05 & -258 & 0.1 & -2.86 \\
\hline & -31.17 & 0.07 & -251 & 0.1 & -2.14 \\
\hline & -30.12 & 0.06 & -242 & 0.1 & -1.54 \\
\hline & -29.61 & 0.12 & -236 & 0.2 & -0.02 \\
\hline 2001 & -27.34 & 0.13 & -220 & 1 & -1.38 \\
\hline & -27.56 & 0.11 & -222 & 0.6 & -2.12 \\
\hline & -27.81 & 0.05 & -224 & 0.3 & -1.52 \\
\hline & -27.87 & 0.07 & -226 & 0.2 & -3.24 \\
\hline & -21.78 & 0.02 & -174 & 0.3 & -0.56 \\
\hline
\end{tabular}




\begin{tabular}{|c|c|c|c|c|}
\hline \multirow[b]{2}{*}{ Year } & \multirow[b]{2}{*}{$\begin{array}{l}\text { Samples } \\
\text { inclusive }\end{array}$} & \multicolumn{3}{|c|}{ Dust } \\
\hline & & $\begin{array}{c}\text { Total } \\
\text { dust (g) }\end{array}$ & $\begin{array}{l}\text { flux } \\
\text { (g m } \\
2 \mathrm{yr}^{-} \\
\text {i }\end{array}$ & 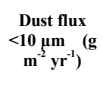 \\
\hline 2008 & $1-15$ & 0 & 10 & 0 \\
\hline 2007 & $16-22$ & 0 & 0.0263 & 0.0016 \\
\hline 2006 & $23-38$ & 0 & 0 & 0 \\
\hline 2005 & $39-56$ & 0 & 0.0131 & 0.0018 \\
\hline 2004 & $57-84$ & 0.0018 & 0.0790 & 0.0122 \\
\hline 2003 & $85-98$ & 0 & 0.0043 & 0.0010 \\
\hline 2002 & $99-111$ & 0 & 0.0087 & 0.0021 \\
\hline 2001 & $112-115$ & 0 & 0 & 0 \\
\hline
\end{tabular}


Appendix 5: Iron concentrations in Antarctic surface snow and sea ice. Data from surface snow (atmospheric Fe flux), sea ice, aerosols, global dust distribution models and estimates using Al concentrations.

\begin{tabular}{|c|c|c|c|c|c|c|c|}
\hline & Location & $\begin{array}{c}\text { Total } \\
\text { Dissolved } \\
\text { Fe }\left(\mathrm{pg} \mathrm{g}^{-1}\right)\end{array}$ & $\begin{array}{l}\text { Atmos. Fe } \\
\text { Flux } \\
\left(\mathrm{mg} \mathrm{m}^{-2} \mathrm{yr}^{-1}\right)\end{array}$ & $\begin{array}{c}\text { Atmos. Fe } \\
\text { Flux } \\
\left(\mu \mathrm{mol} \mathrm{m}^{-2} \mathrm{yr}^{-1}\right)\end{array}$ & $\begin{array}{c}\text { Dissolved } \\
\text { Fe } \\
\left(\mathrm{nmol} \mathrm{L}^{-1}\right)\end{array}$ & $\begin{array}{c}\text { Fe } \\
\text { solubility } \\
(\%)\end{array}$ & Reference \\
\hline \multirow[t]{6}{*}{$\begin{array}{c}\text { Snow } \\
\text { sampling }\end{array}$} & $\begin{array}{c}\text { Ross Sea } \\
\text { (Nov-Dec } \\
1994)\end{array}$ & $749-982$ & $0.07-0.01$ & & & $9-89$ & $\begin{array}{l}\text { Edwards } \\
\text { and } \\
\text { Sedwick } \\
(2001)\end{array}$ \\
\hline & $\begin{array}{c}\text { Dumnont } \\
\text { d'Durville } \\
\text { Sea (Aug } \\
1995 \text { ) }\end{array}$ & $42-85$ & $0.02-0.03$ & 0.30 & & $9-89$ & $\begin{array}{l}\text { Edwards } \\
\text { and } \\
\text { Sedwick } \\
(2001)\end{array}$ \\
\hline & $\begin{array}{c}\text { Prydz Bay } \\
\text { (Sept } \\
\text { 1994) }\end{array}$ & $376-505$ & $0.06-0.11$ & 0.11 & & $9-89$ & $\begin{array}{l}\text { Edwards } \\
\text { and } \\
\text { Sedwick } \\
(2001)\end{array}$ \\
\hline & $\begin{array}{l}\text { Princess } \\
\text { Elizabeth } \\
\text { Land } \\
\text { (Nov-Dec } \\
\text { 1994) }\end{array}$ & $503-1158$ & $0.03-0.08$ & & & $9-89$ & $\begin{array}{l}\text { Edwards } \\
\text { and } \\
\text { Sedwick } \\
(2001)\end{array}$ \\
\hline & $\begin{array}{l}\text { Weddell } \\
\text { Sea } \\
\text { Nov2004- } \\
\text { Jan } 2005\end{array}$ & & & & $1.9 \mathrm{nM}$ & & $\begin{array}{c}\text { Lannuzel } \\
\text { et al. } \\
(2008)\end{array}$ \\
\hline & $\begin{array}{c}\text { Wilkes } \\
\text { Land }\end{array}$ & & & & $1.2-31.7 \mathrm{nM}$ & & $\begin{array}{l}\text { Lannuzel } \\
\text { et al. } \\
(2007)\end{array}$ \\
\hline Pack ice & $\begin{array}{c}\text { East } \\
\text { Antarctica, } \\
\text { Weddell } \\
\text { Sea, } \\
\text { Bellingsha } \\
\text { usen Sea }\end{array}$ & & & & $1.1-36.8$ & & $\begin{array}{l}\text { Lannuzel } \\
\text { et al. } \\
(2010)\end{array}$ \\
\hline $\begin{array}{l}\text { Land fast } \\
\text { ice }\end{array}$ & $\begin{array}{c}\text { East } \\
\text { Antarctica }\end{array}$ & & & & $0.7-4.3$ & & $\begin{array}{l}\text { Van der } \\
\text { Merwe et } \\
\text { al. (2009) }\end{array}$ \\
\hline & $\begin{array}{c}\text { Ross Sea, } \\
\text { Terror } \\
\text { Nova Bay }\end{array}$ & & & & $\begin{array}{c}1.1-6.0 \text { or } \\
2.12-5.78 \\
\mu \mathrm{mol} \mathrm{Fe} \mathrm{m}{ }^{-3}\end{array}$ & & $\begin{array}{l}\text { Grotti et al. } \\
(2005)\end{array}$ \\
\hline
\end{tabular}




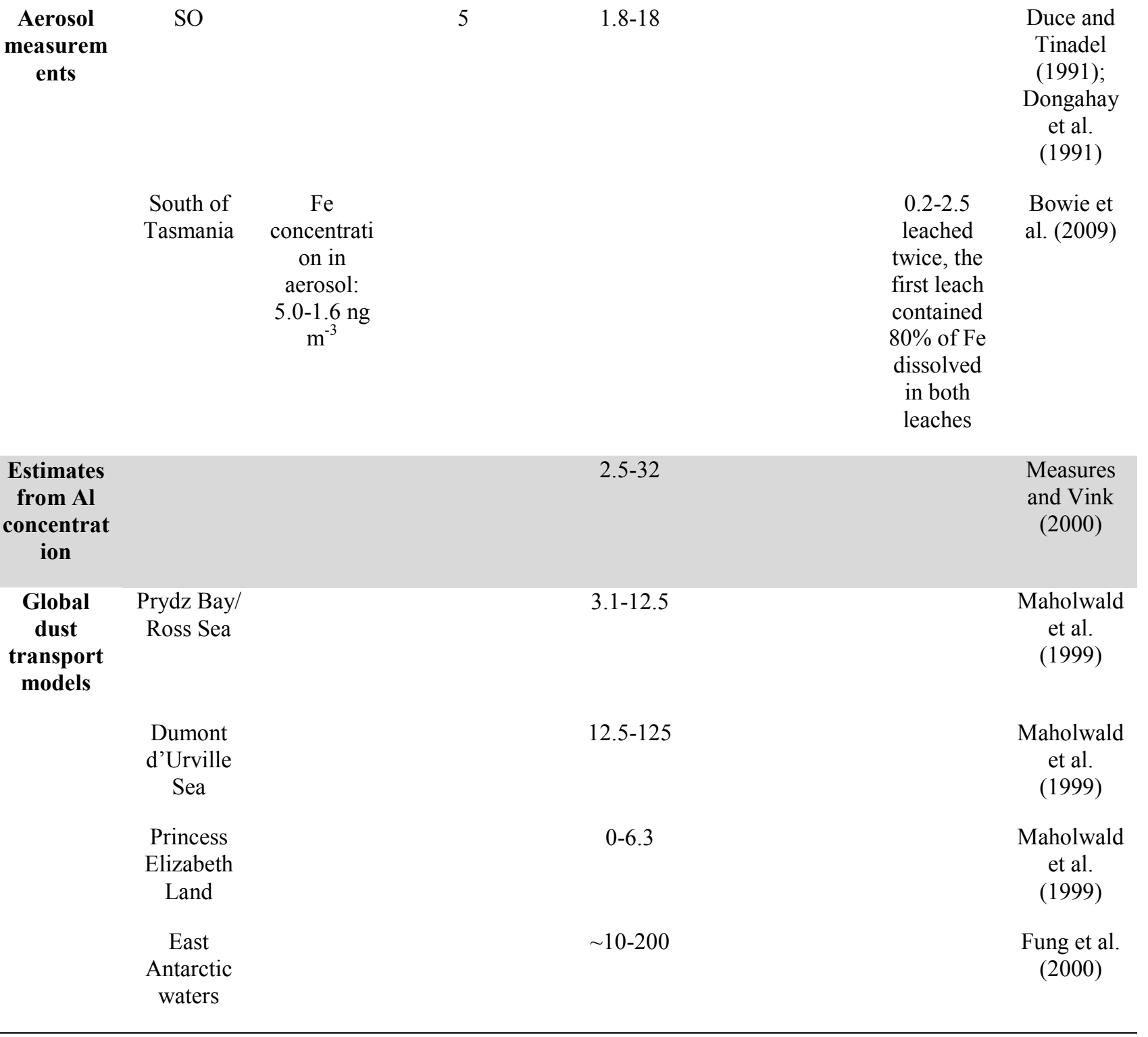


Appendix 6: Other Fe sources to the Southern Ocean.

\begin{tabular}{|c|c|c|c|c|}
\hline Fe Source & $\begin{array}{c}\text { Dissolved Fe } \\
\left(\mu \mathrm{mol} \mathrm{m} \mathrm{m}^{-2} \mathrm{yr}^{-1}\right)\end{array}$ & 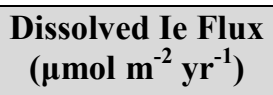 & 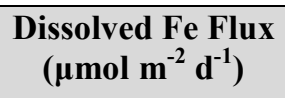 & Reference \\
\hline $\begin{array}{l}\text { Extra-terrestrial Fe } \\
\text { (assumed } 100 \% \\
\text { soluble) }\end{array}$ & & 0.3 & 0.0008 & Johnson, 2001 \\
\hline Upwelling & $8-16$ & 43.2 & 0.12 & $\begin{array}{l}\text { Watson et al., 2000; } \\
\text { Lannuzel et al., } 2008\end{array}$ \\
\hline $\begin{array}{l}\text { Aerosol Fe (assumed } \\
4.3 \% \text { Fe content; } 5 \% \\
\text { soluble) }\end{array}$ & & 0.3 & 0.0008 & Lannuzel et al., 2008 \\
\hline Vertical diffusion & & 3.6 & 0.01 & Lannuzel et al., 2008 \\
\hline $\begin{array}{l}\text { Total Fe input to } \\
\text { Antarctic Ocean }\end{array}$ & & 100.8 & 0.28 & Lannuzel., 2007 \\
\hline
\end{tabular}


Appendix 7: Soluble and particulate iron concentrations in McMurdo Sound sand and dust.

\begin{tabular}{|c|c|c|c|c|c|c|c|c|c|c|c|c|c|c|c|c|c|c|c|}
\hline Leach & Sample & Latitude & $\begin{array}{l}\text { Longti- } \\
\text { tude }\end{array}$ & $\begin{array}{l}\text { Area of } \\
\text { snow } \\
\left(\mathrm{cm}^{2}\right)\end{array}$ & $\begin{array}{c}\text { Mass of } \\
\text { dust } \\
(\mathrm{mg})\end{array}$ & $\begin{array}{c}\text { Dust } \\
\text { flux }(\mathrm{g} \\
\left.\mathrm{m}^{-2} \mathrm{yr}^{-1}\right)\end{array}$ & $\begin{array}{l}\text { Mass of } \\
\text { dust } \\
<10 \mu \mathrm{m} \\
(\mathrm{mg})\end{array}$ & $\begin{array}{c}\text { Dust } \\
\text { flux } \\
<10 \mu \mathrm{m} \\
\left(\mathrm{g} \mathrm{m}^{-2}\right. \\
\left.\mathrm{yr}^{-1}\right) \\
\end{array}$ & $\begin{array}{l}\text { MQ } \\
\text { leach } \\
(\mathrm{g})\end{array}$ & $\begin{array}{c}\mathrm{Fe} \\
\text { leached } \\
(\mathrm{ppb})\end{array}$ & $\begin{array}{c}\mathrm{Fe} \\
\text { leached } \\
(\mu \mathrm{mol} \\
\left.\mathrm{L}^{-1}\right)\end{array}$ & $\begin{array}{c}\text { Parti- } \\
\text { culate } \\
\mathrm{Fe}(\mu \mathrm{g} \\
\left.\mathrm{g}^{-1}\right)\end{array}$ & $\begin{array}{l}\text { Total Fe } \\
\text { (wt \%) }\end{array}$ & $\begin{array}{c}\text { Sol Fe } \\
\%\end{array}$ & $\begin{array}{l}\text { Cumu- } \\
\text { lative } \\
\text { sol \% }\end{array}$ & $\begin{array}{l}\text { Fe Flux } \\
\left(\mathrm{mg} \mathrm{m}^{-2}\right. \\
\left.\mathrm{yr}^{-1}\right)\end{array}$ & $\begin{array}{c}\text { Fe Flux } \\
<10 \mu \mathrm{m} \\
\left(\mathrm{mg} \mathrm{m}^{-2}\right. \\
\left.\mathrm{yr}^{-1}\right)\end{array}$ & $\begin{array}{c}\text { Fe Flux } \\
(\mu \mathrm{mol} \\
\left.\mathrm{m}^{-2} \mathrm{yr}^{-1}\right)\end{array}$ & $\begin{array}{c}\text { Fe Flux } \\
(\mu \text { mol } \\
\mathrm{m}^{-2} \text { day } \\
1)\end{array}$ \\
\hline $\begin{array}{c}\text { Snow } \\
\text { melt }\end{array}$ & $\begin{array}{l}\text { WB1 } \\
2004 \\
\text { dust }\end{array}$ & $\begin{array}{c}78^{\circ} \\
03.273 \\
\mathrm{~S}\end{array}$ & $\begin{array}{c}166^{\circ} \\
57.604 \\
E\end{array}$ & 22.77 & 64.33 & 28.26 & 1.53 & 0.67 & 66.87 & 385.64 & 0.45 & 47751 & 4.78 & 0.84 & 0.84 & 189.82 & 6.61 & $\begin{array}{c}\text { Coarse } \\
3389.59\end{array}$ & 9.29 \\
\hline leach 2 & $\mathrm{a}$ & & & & & & & & 40.02 & 174.81 & 0.12 & 69740 & 6.97 & 6.54 & 7.38 & & & $\begin{array}{c}\text { Fine } \\
117.99\end{array}$ & 0.32 \\
\hline leach 3 & $\mathrm{~b}$ & & & & & & & & 40.03 & 99.38 & 0.07 & 69740 & 6.97 & 3.72 & 11.10 & & & & \\
\hline leach 4 & $\mathrm{c}$ & & & & & & & & 40.02 & 79.21 & 0.06 & 69740 & 6.97 & 2.96 & 14.07 & & & & \\
\hline $\begin{array}{c}\text { Snow } \\
\text { melt }\end{array}$ & MIS44 & $\begin{array}{c}77^{\circ} \\
45.920 \\
\mathrm{~S}\end{array}$ & $\begin{array}{c}165^{\circ} \\
24.019 \\
\mathrm{E}\end{array}$ & 33.17 & 97.90 & 29.52 & 8.03 & 2.42 & 180.62 & 521.49 & 1.68 & 61788 & 6.18 & 1.56 & 1.56 & 75.19 & 5.29 & $\begin{array}{c}\text { Coarse } \\
1342.73\end{array}$ & 3.68 \\
\hline leach 2 & $\mathrm{a}$ & & & & & & & & 40.05 & 180.25 & 0.13 & 53012 & 5.30 & 1.70 & 3.25 & & & $\begin{array}{c}\text { Fine } \\
94.49\end{array}$ & 0.26 \\
\hline leach 3 & $\mathrm{~b}$ & & & & & & & & 40.08 & 56.45 & 0.04 & 53012 & 5.30 & 0.53 & 3.78 & & & & \\
\hline leach 4 & $\mathrm{c}$ & & & & & & & & 40.01 & 36.01 & 0.03 & 53012 & 5.30 & 0.34 & 4.12 & & & & \\
\hline $\begin{array}{c}\text { Snow } \\
\text { melt }\end{array}$ & $\begin{array}{l}\text { MPR13 } \\
-5\end{array}$ & $\begin{array}{c}77^{\circ} \\
35.440 \\
\mathrm{~S}\end{array}$ & $\begin{array}{c}164^{\circ} \\
50.180 \\
\mathrm{E}\end{array}$ & 33.17 & 5.37 & 1.62 & 1.03 & 0.31 & 301.47 & 98.55 & 0.32 & 35964 & 3.60 & 15.39 & 15.39 & 10.54 & 1.91 & $\begin{array}{l}\text { Coarse } \\
188.17\end{array}$ & 0.52 \\
\hline leach 2 & $\mathrm{a}$ & & & & & & & & 40.13 & 14.16 & 0.01 & 33885 & 3.39 & 1.62 & 17.02 & & & $\begin{array}{c}\text { Fine } \\
34.12\end{array}$ & 0.09 \\
\hline leach 3 & b & & & & & & & & 40.01 & 4.83 & 0.00 & 33885 & 3.39 & 0.55 & 17.57 & & & & \\
\hline leach 4 & $\mathrm{c}$ & & & & & & & & 39.97 & 4.71 & 0.00 & 33885 & 3.39 & 0.54 & 18.11 & & & & \\
\hline $\begin{array}{l}\text { Snow } \\
\text { melt }\end{array}$ & $\begin{array}{l}\text { MPN11 } \\
-5\end{array}$ & $\begin{array}{c}77^{\circ} \\
24.520 \\
S\end{array}$ & $\begin{array}{c}164^{\circ} \\
24.810 \\
\mathrm{E}\end{array}$ & 33.17 & 24.77 & 7.47 & 14.87 & 4.48 & 269.37 & 39.39 & 0.19 & 4577 & 0.46 & 9.36 & 9.36 & 6.81 & 2.29 & $\begin{array}{l}\text { Coarse } \\
121.63\end{array}$ & 0.33 \\
\hline leach 2 & $\mathrm{a}$ & & & & & & & & 40.01 & 36.08 & 0.03 & 2567 & 0.26 & 3.78 & 13.14 & & & $\begin{array}{l}\text { Fine } \\
40.94\end{array}$ & 0.11 \\
\hline leach 3 & $\mathrm{~b}$ & & & & & & & & 40.03 & 48.03 & 0.03 & 2567 & 0.26 & 5.04 & 18.18 & & & & \\
\hline leach 4 & $\mathrm{c}$ & & & & & & & & 40.13 & 16.60 & 0.01 & 2567 & 0.26 & 1.75 & 19.93 & & & & \\
\hline
\end{tabular}




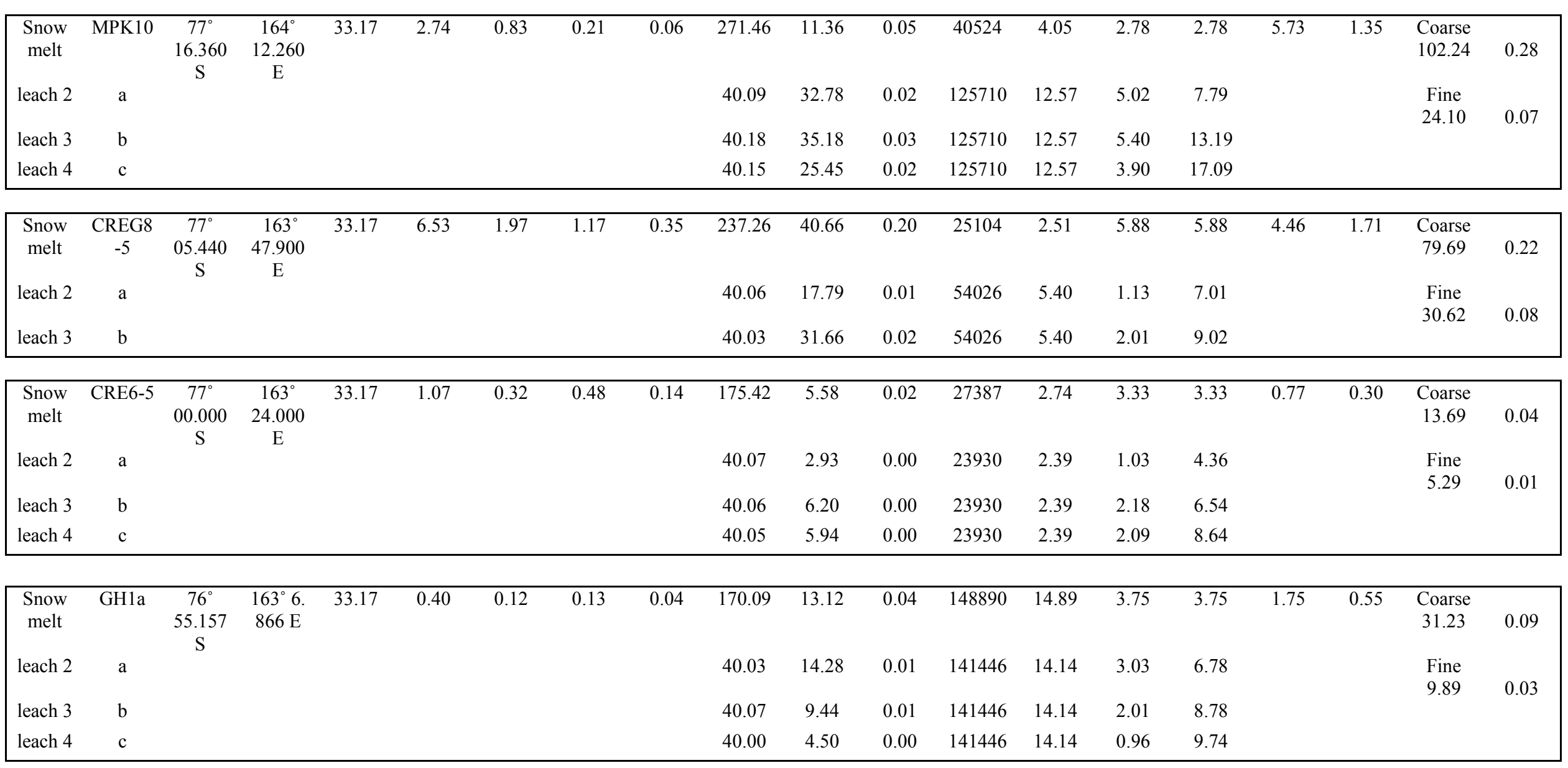




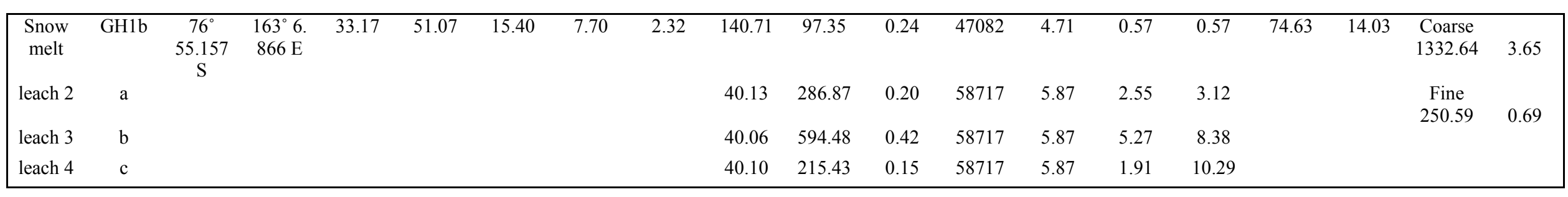


Appendix 8: Dust, chlorophyll- $a$ and methylsuphonate concentration data for the Ross Sea.

\begin{tabular}{|c|c|c|c|c|}
\hline Date & Age Model & $\begin{array}{c}\text { MS } \\
\left(\mathbf{u g} \mathbf{L}^{-1}\right)\end{array}$ & $\begin{array}{c}\text { Dust accumulation } \\
\text { rate } \\
\left(\mathrm{g} \mathrm{m}^{-2}\right) \\
\end{array}$ & $\begin{array}{l}\text { Chlorophyll- } a \\
\quad\left(\mathrm{mg} \mathrm{m}^{-3}\right)\end{array}$ \\
\hline Nov-97 & 1997.833333 & & & 681 \\
\hline Dec-97 & 1997.916667 & & & 8946 \\
\hline Jan-98 & 1998 & & & 8157 \\
\hline Feb-98 & 1998.083333 & & & 15688 \\
\hline Mar-98 & 1998.166667 & & & 434 \\
\hline Apr-98 & 1998.25 & & & 0 \\
\hline May-98 & 1998.333333 & & & 0 \\
\hline Jun-98 & 1998.416667 & & & 0 \\
\hline Jul-98 & 1998.5 & & & 0 \\
\hline Aug-98 & 1998.583333 & & & 0 \\
\hline Sep-98 & 1998.666667 & & & 0 \\
\hline Oct-98 & 1998.75 & & & 0 \\
\hline Nov-98 & 1998.833333 & & & 4720 \\
\hline Dec-98 & 1998.916667 & & & 19132 \\
\hline Jan-99 & 1999 & & & 14873 \\
\hline Feb-99 & 1999.083333 & & & 12953 \\
\hline Mar-99 & 1999.166667 & & & 323 \\
\hline Apr-99 & 1999.25 & & & 0 \\
\hline May-99 & 1999.333333 & & & 0 \\
\hline Jun-99 & 1999.416667 & & & 0 \\
\hline Jul-99 & 1999.5 & & & 0 \\
\hline Aug-99 & 1999.583333 & & & 0 \\
\hline Sep-99 & 1999.666667 & & & 0 \\
\hline Oct-99 & 1999.75 & & & 0 \\
\hline Nov-99 & 1999.833333 & & & 1798 \\
\hline Dec-99 & 1999.916667 & & & 20312 \\
\hline Jan-00 & 2000 & 8.24 & & 14894 \\
\hline Feb-00 & 2000.083333 & 3.88 & & 16856 \\
\hline Mar-00 & 2000.166667 & 26.99 & & 204 \\
\hline Apr-00 & 2000.25 & 28.38 & 0.1207 & 0 \\
\hline May-00 & 2000.333333 & 8.49 & 0.1024 & 0 \\
\hline Jun-00 & 2000.416667 & 3.39 & 0.0915 & 0 \\
\hline Jul-00 & 2000.5 & 0 & 0.1098 & 0 \\
\hline Aug-00 & 2000.583333 & 0 & 0.1281 & 0 \\
\hline Sep-00 & 2000.666667 & 0 & 0.0732 & 0 \\
\hline Oct-00 & 2000.75 & 0 & 0 & 0 \\
\hline Nov-00 & 2000.833333 & 1.49 & 0 & 440 \\
\hline Dec-00 & 2000.916667 & 6.24 & 0 & 2530 \\
\hline Jan-01 & 2001 & 7.61 & 0 & 6633 \\
\hline Feb-01 & 2001.083333 & 18.50 & 0.051 & 13451 \\
\hline Mar-01 & 2001.166667 & 24.45 & 0.0802 & 303 \\
\hline Apr-01 & 2001.25 & 5.28 & 0.0546 & 0 \\
\hline May-01 & 2001.333333 & 1.66 & 0.0736 & 0 \\
\hline Jun-01 & 2001.416667 & 0 & 0.1248 & 0 \\
\hline Jul-01 & 2001.5 & 0 & 0.0875 & 0 \\
\hline Aug-01 & 2001.583333 & 0 & 0.0439 & 0 \\
\hline Sep-01 & 2001.666667 & 0 & 0.0439 & 0 \\
\hline Oct-01 & 2001.75 & 0 & 0.1225 & 0 \\
\hline Nov-01 & 2001.833333 & 0 & 0.3043 & 3539 \\
\hline
\end{tabular}




\begin{tabular}{|c|c|c|c|c|}
\hline Dec-01 & 2001.916667 & 0 & 0.7614 & 22111 \\
\hline Jan-02 & 2002 & 6.90 & 1.4935 & 16133 \\
\hline Feb-02 & 2002.083333 & 5.47 & 1.962 & 15191 \\
\hline Mar-02 & 2002.166667 & 8.35 & 1.7863 & 2691 \\
\hline Apr-02 & 2002.25 & 9.79 & 0.9663 & 0 \\
\hline May-02 & 2002.333333 & 2.45 & 0.3514 & 0 \\
\hline Jun-02 & 2002.416667 & 0 & 0.0585 & 0 \\
\hline Jul-02 & 2002.5 & 0 & 0.0878 & 0 \\
\hline Aug-02 & 2002.583333 & 0 & 0.1171 & 0 \\
\hline Sep-02 & 2002.666667 & 4.63 & 0.1757 & 0 \\
\hline Oct-02 & 2002.75 & 4.22 & 0.2635 & 1 \\
\hline Nov-02 & 2002.833333 & 0 & 0.2049 & 86 \\
\hline Dec-02 & 2002.916667 & 3.01 & 0.1757 & 1185 \\
\hline Jan-03 & 2003 & 7.77 & 0.1757 & 1946 \\
\hline Feb-03 & 2003.083333 & 16.8 & 0.0878 & \\
\hline Mar-03 & 2003.166667 & 0 & 0 & 0 \\
\hline Apr-03 & 2003.25 & 0 & 0.0880 & 0 \\
\hline May-03 & 2003.333333 & 0 & 0.1763 & 0 \\
\hline Jun-03 & 2003.416667 & 0 & 0.3297 & 0 \\
\hline Jul-03 & 2003.5 & 0 & 0.4831 & 0 \\
\hline Aug-03 & 2003.583333 & 3.40 & 0.2406 & 0 \\
\hline Sep-03 & 2003.666667 & 1.95 & 0.0040 & 0 \\
\hline Oct-03 & 2003.75 & 1.48 & 0.5081 & 0 \\
\hline Nov-03 & 2003.833333 & 4.37 & 1.0083 & 1 \\
\hline Dec-03 & 2003.916667 & 6.95 & 0.5041 & 9791 \\
\hline Jan-04 & 2004 & 9.25 & & 6981 \\
\hline Feb-04 & 2004.083333 & 19.49 & 0.4630 & 14120 \\
\hline Mar-04 & 2004.166667 & 15.41 & 0.9187 & 0 \\
\hline Apr-04 & 2004.25 & 0.02 & 0.4584 & 0 \\
\hline May-04 & 2004.333333 & 3.08 & 0 & 0 \\
\hline Jun-04 & 2004.416667 & 9.83 & 0.2200 & 0 \\
\hline Jul-04 & 2004.5 & 8.72 & 0.4392 & 0 \\
\hline Aug-04 & 2004.583333 & 5.25 & 1.2171 & 0 \\
\hline Sep-04 & 2004.666667 & 6.44 & 1.8973 & 0 \\
\hline Oct-04 & 2004.75 & 7.64 & 1.4541 & 0 \\
\hline Nov-04 & 2004.833333 & 34.41 & 1.0109 & 7493 \\
\hline Dec-04 & 2004.916667 & 86.07 & 2.9878 & 16041 \\
\hline Jan-05 & 2005 & 137.73 & 8.5350 & 8537 \\
\hline Feb-05 & 2005.083333 & 93.64 & 3.7148 & 4736 \\
\hline Mar-05 & 2005.166667 & 9.35 & 0.2344 & 578 \\
\hline Apr-05 & 2005.25 & 0 & 0.1318 & 0 \\
\hline May-05 & 2005.333333 & 0 & 1.4944 & 0 \\
\hline Jun-05 & 2005.416667 & 0 & 3.9554 & 0 \\
\hline Jul-05 & 2005.5 & 0 & 2.2388 & 0 \\
\hline Aug-05 & 2005.583333 & 0.27 & 0.1683 & 0 \\
\hline Sep-05 & 2005.666667 & 2.01 & 0.1170 & 0 \\
\hline Oct-05 & 2005.75 & 0.76 & 0.0657 & 0 \\
\hline Nov-05 & 2005.833333 & 7.36 & 0.0145 & 6799 \\
\hline Dec-05 & 2005.916667 & 17.47 & 0.0183 & 16990 \\
\hline Jan-06 & 2006 & 17.25 & 0.0439 & 11420 \\
\hline Feb-06 & 2006.083333 & 24.89 & 0.0805 & 13529 \\
\hline Mar-06 & 2006.166667 & 4.54 & 0.1171 & 3415 \\
\hline Apr-06 & 2006.25 & 3.11 & 0.1317 & 0 \\
\hline
\end{tabular}




\begin{tabular}{|c|c|c|c|c|}
\hline May-06 & 2006.333333 & 3.20 & 0.1317 & 0 \\
\hline Jun-06 & 2006.416667 & 0 & 0.1207 & 0 \\
\hline Jul-06 & 2006.5 & 0.96 & 0.0658 & 0 \\
\hline Aug-06 & 2006.583333 & 1.65 & 0.0109 & 0 \\
\hline Sep-06 & 2006.666667 & 2.97 & 1.4642 & 0 \\
\hline Oct-06 & 2006.75 & 3.21 & 3.2945 & 0 \\
\hline Nov-06 & 2006.833333 & 5.816 & 3.6752 & 10785 \\
\hline Dec-06 & 2006.916667 & 59.18 & 1.8815 & 18372 \\
\hline Jan-07 & 2007 & 39.74 & & 14181 \\
\hline Feb-07 & 2007.083333 & 8.48 & 1.2193 & 8205 \\
\hline Mar-07 & 2007.166667 & 0.76 & 0.7893 & 2973 \\
\hline Apr-07 & 2007.25 & 0 & 0.0319 & 0 \\
\hline May-07 & 2007.333333 & 0 & 0.0026 & 0 \\
\hline Jun-07 & 2007.416667 & 0 & 0.0692 & 0 \\
\hline Jul-07 & 2007.5 & 0 & 0.2076 & 0 \\
\hline Aug-07 & 2007.583333 & 2.32 & 0.2941 & 0 \\
\hline Sep-07 & 2007.666667 & 6.60 & 0.3008 & 0 \\
\hline Oct-07 & 2007.75 & 3.78 & 0.7667 & 0 \\
\hline Nov-07 & 2007.833333 & & 2.7500 & 949 \\
\hline Dec-07 & 2007.916667 & & 3.1358 & 10197 \\
\hline Jan-08 & 2008 & & 0.8785 & 3 \\
\hline Feb-08 & 2008.083333 & & 2.0938 & 0 \\
\hline Mar-08 & 2008.166667 & & 3.3091 & 0 \\
\hline Apr-08 & 2008.25 & & 4.5244 & 0 \\
\hline May-08 & 2008.333333 & & 3.1041 & 0 \\
\hline Jun-08 & 2008.416667 & & 1.6838 & 0 \\
\hline Jul-08 & 2008.5 & & 0.2635 & 0 \\
\hline Aug-08 & 2008.583333 & & 0.1903 & 0 \\
\hline Sep-08 & 2008.666667 & & 0.1171 & 0 \\
\hline Oct-08 & 2008.75 & & 0.0439 & 0 \\
\hline Nov-08 & 2008.833333 & & & 2638 \\
\hline Dec-08 & 2008.916667 & & & 9252 \\
\hline Jan-09 & 2009 & & & 8535 \\
\hline Feb-09 & 2009.083333 & & & 9077 \\
\hline Mar-09 & 2009.166667 & & & 2691 \\
\hline Apr-09 & 2009.25 & & & 0 \\
\hline May-09 & 2009.333333 & & & 0 \\
\hline Jun-09 & 2009.416667 & & & 0 \\
\hline Jul-09 & 2009.5 & & & 0 \\
\hline Aug-09 & 2009.583333 & & & 0 \\
\hline Sep-09 & 2009.666667 & & & 0 \\
\hline Oct-09 & 2009.75 & & & 0 \\
\hline Nov-09 & 2009.833333 & & & 717 \\
\hline Dec-09 & 2009.916667 & & & 22725 \\
\hline Jan-10 & 2010 & & & 19965 \\
\hline
\end{tabular}

